\title{
Functional analysis of the Arabidopsis thaliana glutaredoxin ROXY9
}

\author{
Dissertation \\ for the award of the degree \\ "Doctor rerum naturalium" \\ of the Georg-August-Universität Göttingen \\ within the doctoral program Microbiology and Biochemistry \\ of the Georg-August University School of Science (GAUSS)
}

\author{
submitted by \\ Katrin Treffon
}

from Radolfzell (Bodensee)

Göttingen 2019 



\section{Thesis Committee}

Prof. Dr. Christiane Gatz, Department for Plant Molecular Biology and Physiology, Albrecht-von-Haller-Institut, Georg-August-Universität Göttingen

Prof. Dr. Ivo Feußner, Department for Plant Biochemistry, Albrecht-vonHaller-Institut, Georg-August-Universität Göttingen

Dr. Marcel Wiermer, Department for Molecular Biology of Plant-Microbe Interactions, Albrecht-von-Haller-Institut, Georg-August-Unviersität Göttingen

\section{Members of the Examination Board}

Referee

Prof. Dr. Christiane Gatz, Department for Plant Molecular Biology and Physiology, Albrecht-von-Haller-Institut, Georg-August-Universität Göttingen

$2^{\text {nd }}$ Referee

Prof. Dr. Ivo Feußner, Department for Plant Biochemistry, Albrecht-vonHaller-Institut, Georg-August-Universität Göttingen

\section{Further members of the Examination Board}

Prof. Dr. Andrea Polle, Department for Forest Botany and Tree Physiology, Büsgen-Institut, Georg-August-Universität Göttingen

Prof. Dr. Volker Lipka, Department for Plant Cell Biology, Albrecht-vonHaller-Institut, Georg-August-Universität Göttingen

PD Dr. Thomas Teichmann, Department for Plant Cell Biology, Albrecht-vonHaller-Institut, Georg-August-Universität Göttingen

Date of oral examination: 25.03.2019 


\section{Declaration}

I hereby declare that I prepared the dissertation entitled "Functional analysis of the Arabidopsis thaliana glutaredoxin ROXY9" independently and without any unauthorized help. I confirm that I did not apply for a Ph. D. or Dr. rer. nat. at any other University. Neither the entire dissertation nor parts of this dissertation have been presented to another examination board.

Katrin Treffon

Göttingen, 08.02.2019 


\section{Summary}

Glutaredoxins are nearly ubiquitious small enzymes which protect the thiol groups of cellular proteins from oxidative stress, contribute to iron-sulfur cluster biogenesis or regulate protein activities via redox modification. Three different classes of glutatreoxins are distinguished by their active site motif: CPYC-type glutaredoxins catalyze the reduction and oxidation of thiol groups and mediate defense against oxidative stress; CGFS-type glutaredoxins are weak catalysts but associate with iron-sulfur clusters, possibly transferring them to target proteins. Whereas CPYC- and CGFS-type glutaredoxins are found in all types of organisms, CC-type glutaredoxins, which are called ROXYs in Arabidopsis thaliana, are restricted to land plants. In contrast to their relatively well characterized relatives, CC-type glutaredoxins are biochemically poorly understood. Information about these glutaredoxins is only available from in vivo studies. Mutant analysis and studies with plants ectopically expressing ROXYs have shown that they modulate TGACG binding (TGA) factor activity. As an example, the CC-type glutaredoxin ROXY9 was shown to repress TGA1mediated hyponastic growth when overexpressed. Since TGA1 was shown to be redox-regulated in plants treated with the defense hormone salicylic acid, it was speculated that ROXY9 represses TGA1 via catalytic activity. Therefore, this study was set up to analyze whether recombinant ROXY9 exhibits oxidoreductase and/or iron-sulfur cluster binding activity. Up to now, difficulties in the purification of CC-type glutaredoxins has been the bottleneck for their biochemical characterization. Similarly, ROXY9 could not be purified in a soluble, non-aggregated state when using Escherichia coli as an expression host. Strikingly, expression in insect cells resulted in large amounts of monomeric and soluble ROXY9 fused to a strep-MBP-tag. However, the protein turned out to oxidize quickly under aerobic conditions. Reduction of the oxidized protein by glutathione might be inefficient, which could explain the inactivity of ROXY9 towards the typical glutaredoxin substrates bis(2hydroxyethyl)disulphide (HEDS), insulin, and the redox-sensitive green fluorescent protein (roGFP). Still, a weak reductase activity of ROXY9 towards glutathionylated glyceraldehyde 3-phosphate dehydrogenase (GAPDH) was observed, suggesting that ROXY9 in principle can reduce target proteins. In contrast to its weak reductase activity, ROXY9 turned out to glutathionylate roGFP efficiently with the help of glutathione disulfide, suggesting that 
ROXY9 might repress TGA1 in vivo via glutathionylation. However, because all these observations might have been influenced by the oxidation of the protein, the catalytic activity of ROXY9 has to be reproduced with fully reduced protein. Aside from catalysis, ROXY9 appeared to bind an iron-sulfur cluster under anaerobic conditions. This raises the alternative hypothesis that it could repress TGA1 via recruitment of an iron-sulfur cluster-dependent repression complex. In vivo analysis of the repression capacity of overexpressed mutated ROXY9 versions suggested that ROXY9 requires the second cysteine and the tyrosine of its extended active site motif CCLCY for its activity. Future experiments under anaerobic conditions will have to clarify whether ROXY9 requires these amino acids for catalysis or for iron-sulfur cluster binding. In an initial experiment to address the repression mechanism of TGA1 by ROXY9 in vivo, a TGA1 version with mutated cysteine residues was constructed and expressed in the tga 1 tga 4 mutant. This mutant protein is resistant to oxidation and was as active in vivo as wildtype TGA1 regarding hyponastic growth and flowering. However, the experimental setup might not allow the detection of a weak contribution of the TGA1 redox state to hyponastic growth and flowering; thus, this experiment does currently not allow to conclude whether TGA1 is redox-controlled. To test this hypothesis in the future, ROXY9 will have to be overexpressed in tga1 tga4 mutants complemented with wildtype TGA1 and the TGA1 cysteine mutant to find out, whether ROXY9 can still repress the TGA1 cysteine mutant. 


\section{Contents}

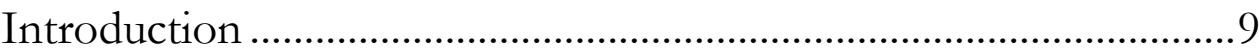

Redox signaling via thiol groups ....................................................10

Thioredoxins and glutaredoxins.....................................................13

TGA transcription factors ...............................................................38

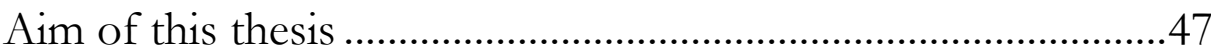

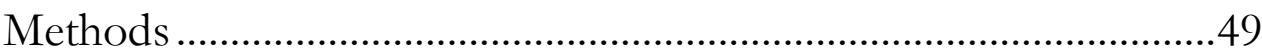

Work with organisms........................................................................

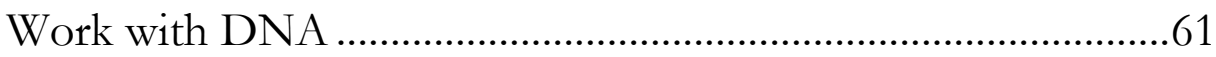

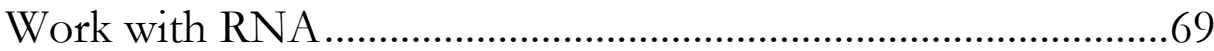

Work with proteins - basic methods.................................................73

Production and analysis of recombinant proteins .........................83

Characterization of glutaredoxins ................................................. 101

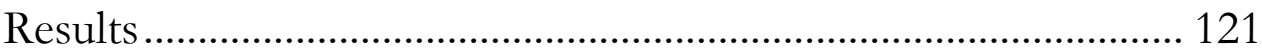

Amino acids required for ROXY9 in vivo activity ..................... 122

In vitro characterization of ROXY9............................................... 131

Towards a mechanism of regulation of TGA1 by ROXY9 ... 164

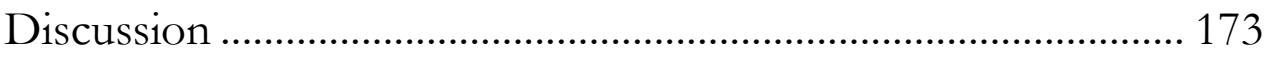

The CC-type glutaredoxin ROXY9 as an iron-sulfur cluster binding oxidase ......................................................................... 174

Potential regulatory mechanisms of TGA1 by ROXY9 ......... 187 


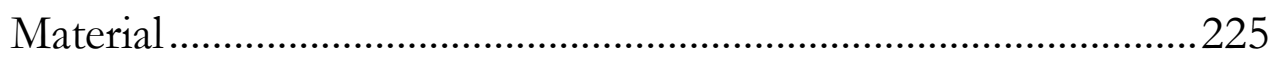

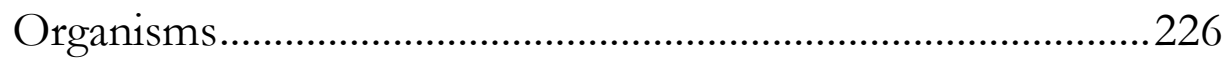

Oligo nucleotides ..........................................................................228

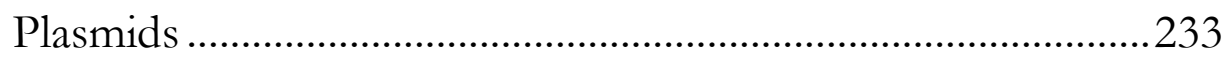

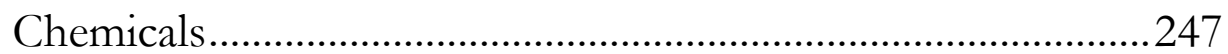

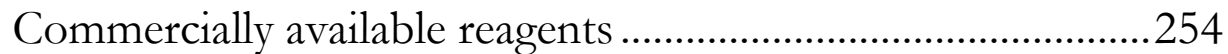

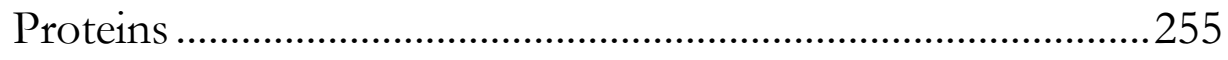

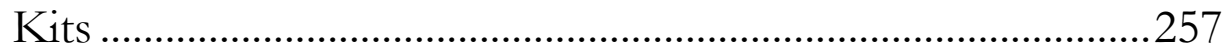

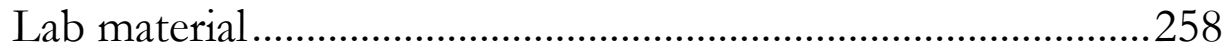

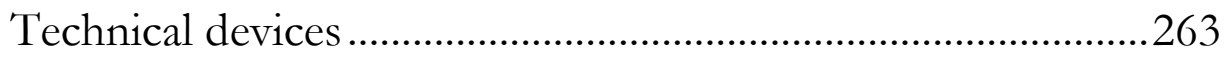

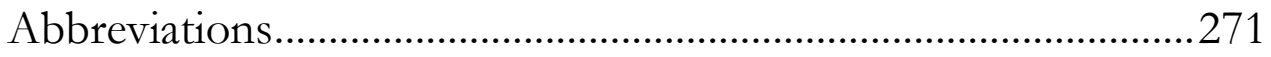

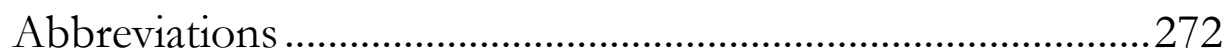

Units and natural constants ......................................................276

Amino acids and bases ...............................................................277

References and Acknowledgements ..........................................279

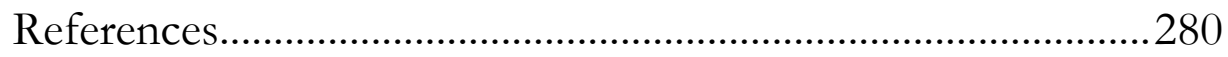

Acknowledgments ...........................................................................304 
Introduction 


\section{Redox signaling via thiol groups}

With the evolution of photosynthesis and oxygen-based cellular respiration, organisms became exposed to the byproducts of these two processes, namely reactive oxygen and reactive nitrogen species (ROS and RNS). On the one hand, both, ROS and RNS can damage biological macromolecules by oxidation, such as proteins, lipids and DNA and thereby interfere with normal metabolism. On the other hand, controlled redox modification of proteins allows changes in their activity, which serves as a means of signal transduction in many fundamental processes, such as development or stress responses [113].

During redox signaling, protein cysteine residues are of particular importance: Cysteines exhibit a thiol group which can undergo a variety of redox modifications (Figure I1). These modifications range from inter- or intramolecular disulfide bridges to nitrosylation and the oxidation to sulfenic acid. In addition, cysteine residues can react with the ubiquitous antioxidant glutathione ( $\gamma$-L-glutamyl-L-cysteinyl-glycine, GSH) (Figure I2A) leading to the formation of a glutathione-mixed disulfide (Figure I1). Moreover, sulfenic acid groups can be converted into sulfinic acid groups. While all aforementioned redox modifications are usually reversible, sulfinic acid groups can be converted irreversibly into sulfonic acid groups by further oxidation (Figure I1) $[5,7,8,11,12]$. Importantly, not every cysteine residue allows these modifications. Whether and which modifications occur at a specific cysteine residue is currently not fully understood. The ability of the thiol group to deprotonate contributes to its reactivity. The $\mathrm{pK}_{\mathrm{a}}$ value of free cysteine is ca. 8.3; thus the equilibrium of the thiol form and the thiolate form of free cysteine is shifted to the protonated state at cellular $\mathrm{pH}$ [1]. The extent of deprotonation of a cysteine in a protein depends on the steric and electrostatic environment of this specific cysteine residue. In addition, enzymes specific for certain cysteine residues can catalyze redox modifications $[1,5,6,11,14-16]$.

Several redox modifications have been implied in signaling. Prominent examples are the transcription factors OxyR in Escherichia coli (E. coli) [17-23], Yap1 in yeast [24-26], and NF- $\varkappa \mathrm{B}$ in mammals [27,28]. In plants, the DNA binding of TCP transcription factors and certain homeodomain transcription 


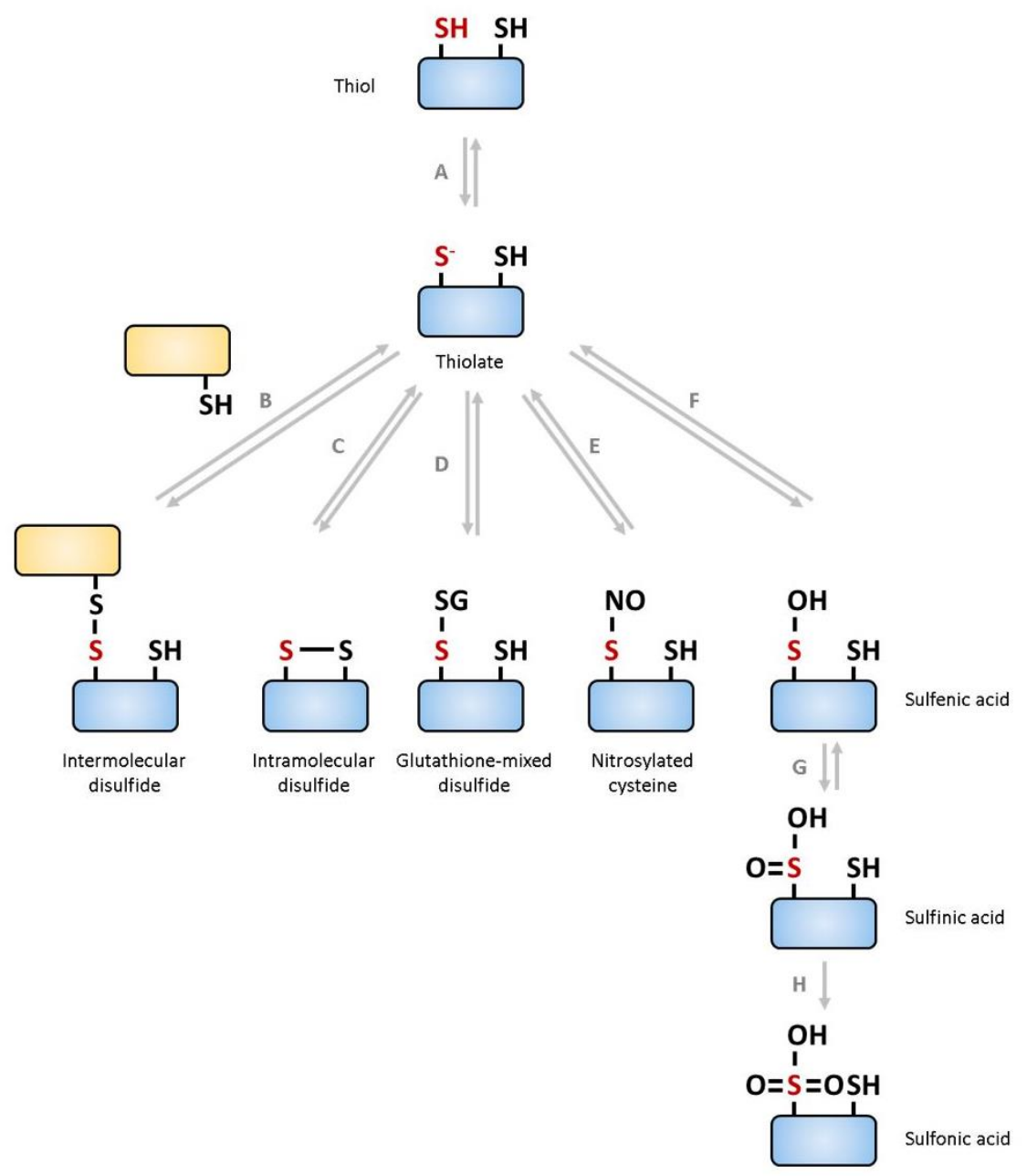

Figure I1. Redox modifications on protein cysteine residues. The different modifications which can occur on protein cysteine residues are shown as structural formulas; the names of the different modifications are shown below the schemes. Double arrows indicate reversible reactions, single arrows indicate irreversible reactions. The thiol group is at equilibrium with ist thiolate, the more reactive species (A). It can react with another thiol group from a different protein $(\mathbf{B})$ or another thiol from the same protein $(\mathbf{C})$. Reaction with glutathione $(\mathbf{D})$ or nitrosylating species $(\mathbf{E})$ is possible, as well. In addition, different states of reversible or irreversible oxidation can occur $(\mathbf{F}, \mathbf{G}$, and $\mathbf{H})$. The figure was taken from Zaffagnini et al. 2012 [7] and modified. 
factors depends on redox sensitive cysteine residues [29,30] and the transcriptional co- activator NPR1 has been proposed to be redox-regulated by disulfide bridge formation and nitrosylation [31-34]. In addition, metabolic processes such as glycolysis, gluconeogenesis, the Calvin-Benson and the tricarboxylic acid cycle, as well as mitochondrial respiration and photosynthetic electron transport are redox-regulated [35-43]. The dehydrogenase activity of plant glyceraldehyde-3-phosphate dehydrogenase (GAPDH) was shown to be inactivated by glutathionylation and nitrosylation [37,44-46]; still, these redox modifications are thought to initiate moonlighting activities of this enzyme $[37,46-48]$.

Certain redox modifications on cysteine residues, such as glutathionylation, can occur spontaneously $[2,5,7,15]$. However, a controlled way of redox signaling also requires the action of specific enzymes $[5,6,8,11,15,49]$. Two important types of enzymes involved in redox signaling are thioredoxins and glutaredoxins. Originally, both types of enzymes were described to transfer electrons to ribonucleotide reductase and to reduce different disulfide containing molecules [50-61]. Later, both, thioredoxins and glutaredoxins, were found to regulate the activity of target proteins specifically to transmit signals. For instance, the plant homedomain transcription factors Hahb-10 and HAHR1 and the transcriptional co-activator NPR1 can be reduced by thioredoxins [30,33], while plastidial GAPDH is reduced by thioredoxins and glutaredoxins $[47,48,62,63]$. 


\section{Thioredoxins and glutaredoxins}

Thioredoxins and glutaredoxins are widely distributed members of the thioredoxin superfamily, a group of proteins sharing the so-called thioredoxin fold (Figure I2B and $\mathrm{C}$ ). The thioredoxin fold consists of four $\beta$-strands assembled into a mixed $\beta$-sheet. This $\beta$-sheet is sandwiched by three $\alpha$-helices. The beginning of helix $\alpha_{1}$ and the loop between helix $\alpha_{1}$ and strand $\beta_{1}$ comprise the active site, a four amino-acid motif. This basic structure of the thioredoxin fold can be extended by additional $\alpha$-helices and $\beta$-strands $[1,2,7,9,64-68]$.

The active site motif of thioredoxins and glutaredoxins contains cysteine residues important for enzyme activity. In both, thioredoxins and glutaredoxins, a conserved cysteine residue is present at the $\mathrm{N}$-terminal end of the active site motif. Many reasons for its reactivity have been considered, such as stabilization of the thiolate by the dipole of helix $\alpha_{1}$ [69-72]. In case of human Grx1 and pig liver thioltransferase, the positively charged environment of the N-terminal active site cysteine residue was found to stabilize its thiolate form [73,74]. This effect was recently confirmed for other glutaredoxins by Begas et al. (2017) and attributed to a specific positively charged amino acid (frequently a lysine) preceeding the active site $\left(\mathrm{K}^{28}\right.$ in poplar GRXC1, Figure I2C, [75]).

Despite having the same fold and a similar active site, thioredoxins and glutaredoxins differ in their redox potential and thus, the electron donors they use. While thioredoxins usually exhibit a redox potential of around $-300 \mathrm{mV}$, glutaredoxins frequently show redox potentials ranging from -170 to $-270 \mathrm{mV}$. [1,76-78]. Thus, thioredoxins are directly reduced by reduced nicotineamide adenine dinucleotide phosphate (NADPH, redox potential ca. $-315 \mathrm{mV}$ ), whereas glutaredoxins use the weaker reductant glutathione. The oxidized form of glutathione is called glutathione disulfide (GSSG, Figure I2A). The redox potential of GSH and GSSG was measured to be ca. $-240 \mathrm{mV}$ at $\mathrm{pH} 7.0$ $[1,66,76]$. 


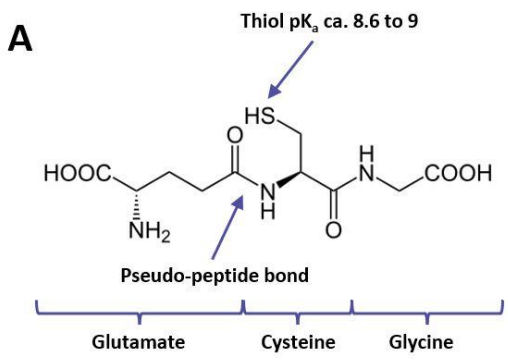

GSH

B

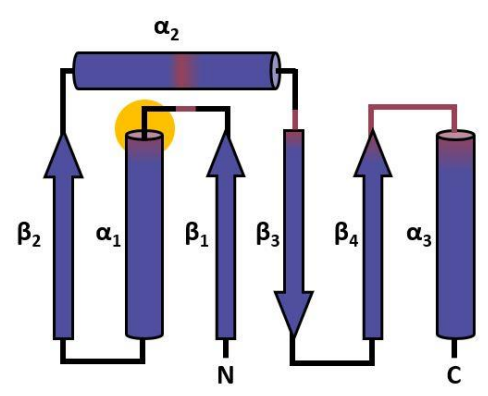<smiles>N[C@@H](CCC(=O)NC(CSSCC(NC(=O)CC[C@H](N)C(=O)O)C(=O)NCC(=O)O)C(=O)NCC(=O)O)C(=O)O</smiles>

GSSG

C

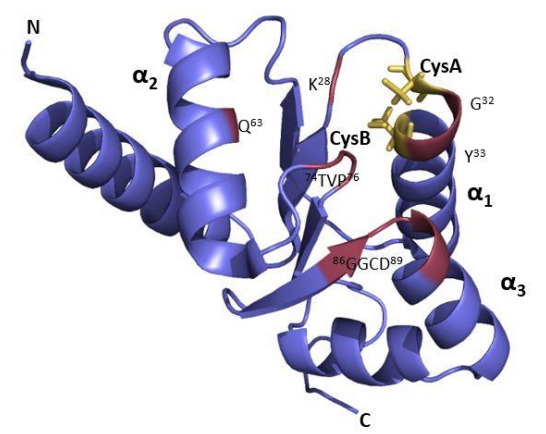

Figure I2. Glutathione and the thioredoxin fold. (A) Structural formula of glutathione (GSH) and glutathione disulfide (GSSG). In case of GSH, the $\mathrm{pK}_{\mathrm{a}}$ value of the cysteine thiol is indicated and the pseudo-peptide bond between the $\gamma$-carboxyl group of glutamate and the $\alpha$-amino group of cysteine is highlighted [1]. Images were taken from www.wikipedia.org. (B) Schematic representation of the core thioredoxin fold found in bacteria. Please note, that the number of $\alpha$-helices is higher in plant glutaredoxins. The location of the active site is marked by a yellow dot. Sites important for glutathione binding are highlighted in red $[1,79,80]$. (C) The solution structure solved by nuclear magnetic resonance (NMR) spectroscopy of the poplar CPYC-type glutaredoxin GRXC1 (CGYC acitve site) was determined by Feng et al. (2006) (PDB accession: 1Z7P [81]). The PDB data file was used to prepare the picture of the ribbon structure in PyMOL. The first and the last active site cysteine (CysA and CysB, respectively) are shown as yellow stick representations. A red coloring marks the sites of residues important for glutathione binding $[1,79]$. The figure was in parts taken from Lillig et al. (2008) and Gutsche et al. (2015) and modified [79,80]. 


\section{Thioredoxins}

In thioredoxins, the consensus sequence of the active site motif is CGPC [66]. Using both active site cysteine residues, they reduce protein disulfide bonds [50] and possibly glutathionylated proteins [82]. They can also reduce nitrosylated thiol groups $[33,34,83]$. Disulfide bridge reduction follows the socalled dithiol mechanism or disulfide exchange reaction: It is initiated by the $\mathrm{N}$ terminal active site cysteine residue attacking the disulfide bridge of the target protein(s). The target disulfide bridge is resolved, and a new disulfide bridge formed between the $\mathrm{N}$-terminal active site cysteine residue of the thioredoxin and one of the target cysteines. This intermolecular disulfide is then resolved by the $\mathrm{C}$-terminal active site cysteine of the thioredoxin, releasing a reduced target protein and leading to a disulfide bridge between the two active site cysteines of the thioredoxin [69]. This intramolecular disulfide is reduced by thioredoxin reductase (TR), transferring electrons from NADPH to the thioredoxin active site cysteines [51,54]. Still, some plastidial thioredoxins have been shown to receive electrons from Ferredoxin thioredoxin reductase (FTR) $[78,84,85]$.

\section{Glutaredoxins}

\section{Glutathione binding and reaction mechanisms of glutaredoxins}

In contrast to thioredoxins, glutaredoxins bind glutathione using it as an electron donor $[74,86]$. They harbor at least one cysteine at the $\mathrm{N}$-terminal end of the active site; sometimes, another cysteine is present at the $\mathrm{C}$-terminal end of the active site [1]. Glutaredoxins reduce disulfide bridges as well as glutathione-mixed disulfides [52,61]. In addition, they are able to glutathionylate protein cysteinyl residues $[87,88]$. Some glutaredoxins were even shown to associate with iron-sulfur clusters [89,90]. Importantly, not every glutaredoxin shows all these activities; still, they depend on glutathionebinding to fulfill their specific functions [1,2,7,9]. Starting in 1991 [86], a multitude of structural studies on glutaredoxins from different organisms 
Thioredoxins and Glutaredoxins

A

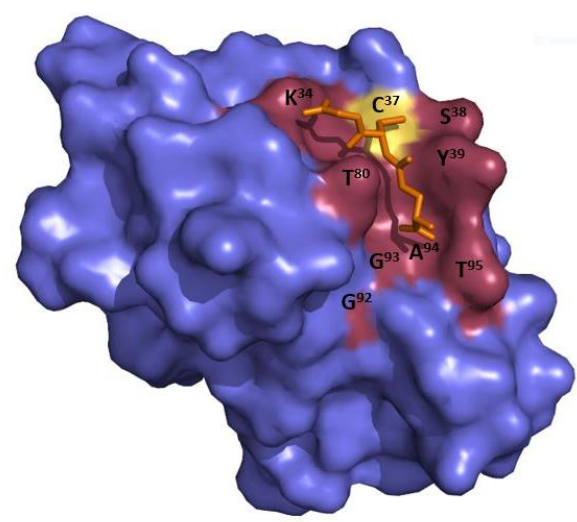

B

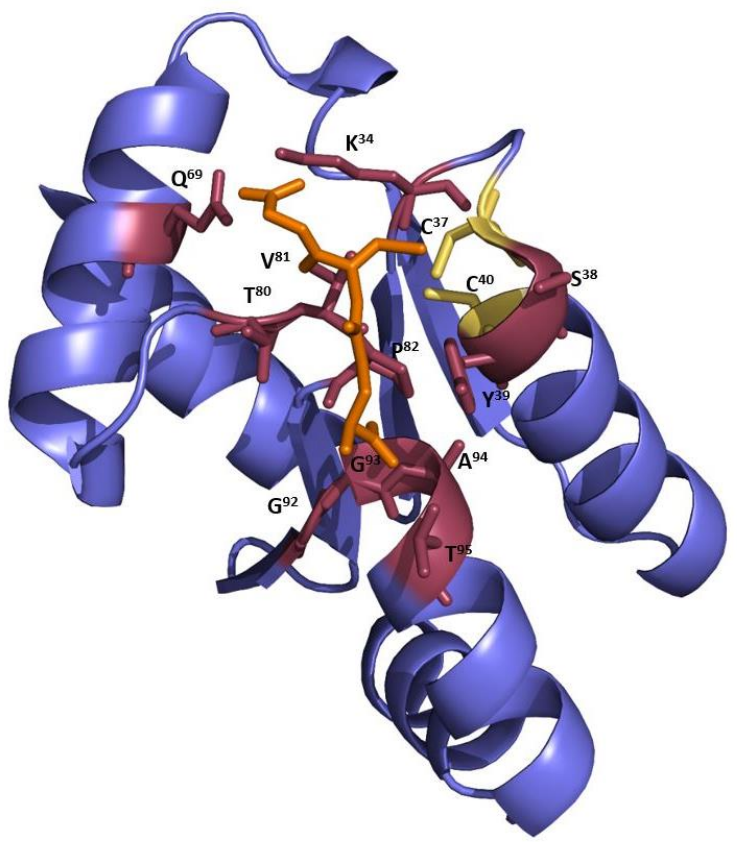

Figure I3, part I. Glutathione binding by the CPYC-type glutaredoxin Grx2 from human. The crystal structure of human Grx2 bound non-covalently to glutathione was solved by Johansson et al. (2006) to a resolution of $2.05 \AA$ (PDB accession: 2FLS). The structure pictures were produced from the PDB file using PyMOL. (A) Surface representation visualizing the glutathione binding groove, (B) Secondary and tertiary structure with selected residues depicted as sticks to show the glutathione binding mode. The active site cysteines are shown in yellow, glutathione binding motifs and residues are coloured in red. Glutathione is shown as stick model in orange. 
C

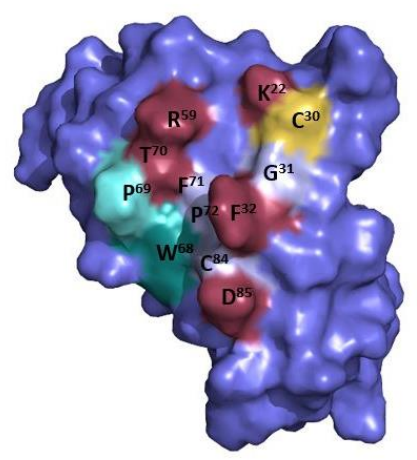

D

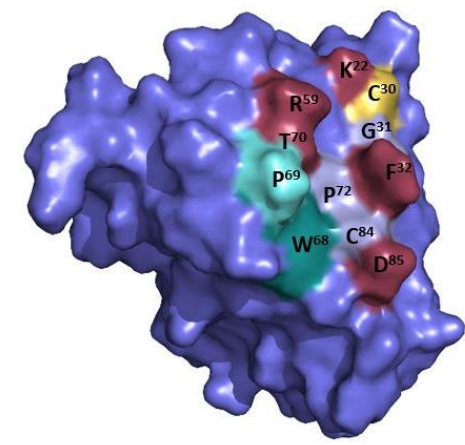

E

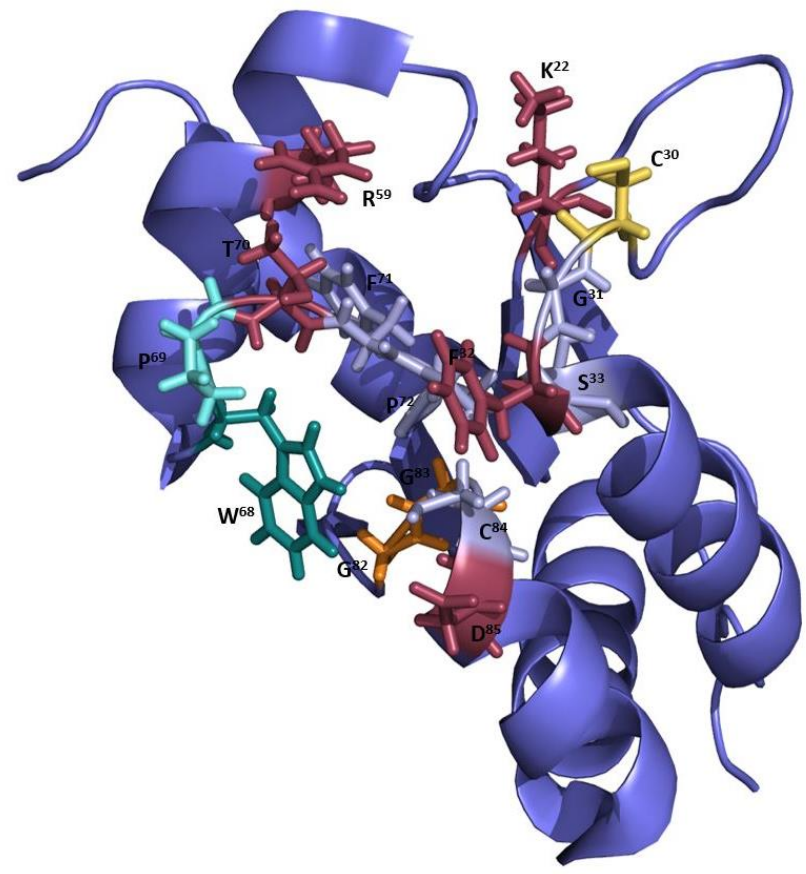

Figure I3, part II. Glutathione binding residues of the CGFS-type glutaredoxin Grx4 from $\boldsymbol{E}$. coli. The NMR structure of E. coli Grx4 was solved by Fladvad et al. (2005) [91]. The structure pictures were produced from the PDB file (accession: 1YKA) using PyMOL. (C and D) Surface representation visualizing the glutathione binding groove from two different angles; (D) shows the glutaredoxin from approximately the same view as human Grx 2 in Figure I3A and B. (E) Secondary and tertiary structure with selected residues depicted as sticks to show the putative glutathione binding mode. The active site cysteines are shown in yellow. Please note that the glutathione binding mode presented here derives from alignments and steric analysis. Potential glutathione binding residues inferred from comparisons with E. coli Grx3 are coloured in red. $\mathrm{W}^{68}$ and $\mathrm{P}^{69}$ (dark and light cyan) of the WP motif were speculated to determine the binding of glutathione in place of the buried GG motif. All other residues belonging to glutathione binding motifs are shown in light blue. $\mathrm{Lys}^{22}, \mathrm{Arg}^{59}, \mathrm{Phe}^{71}, \mathrm{Cys}^{84}$ and Asp ${ }^{85}$ were found to bind glutathione in holo Grx4 (Figure I6C) [92]. 
F

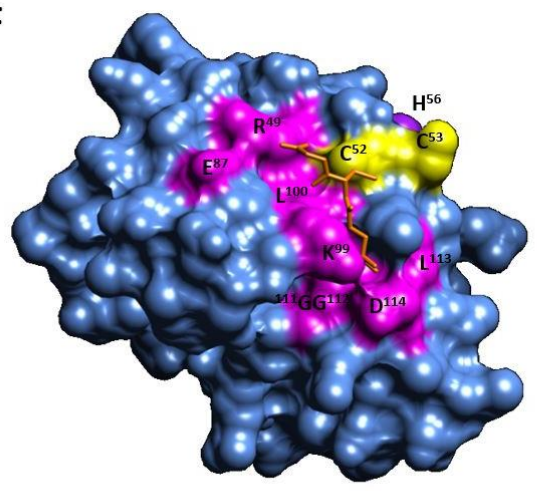

G

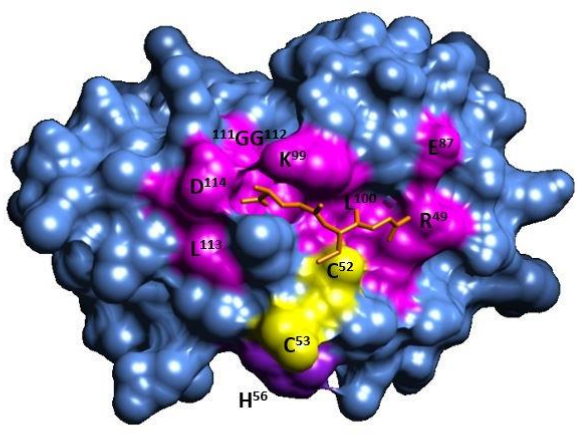

H

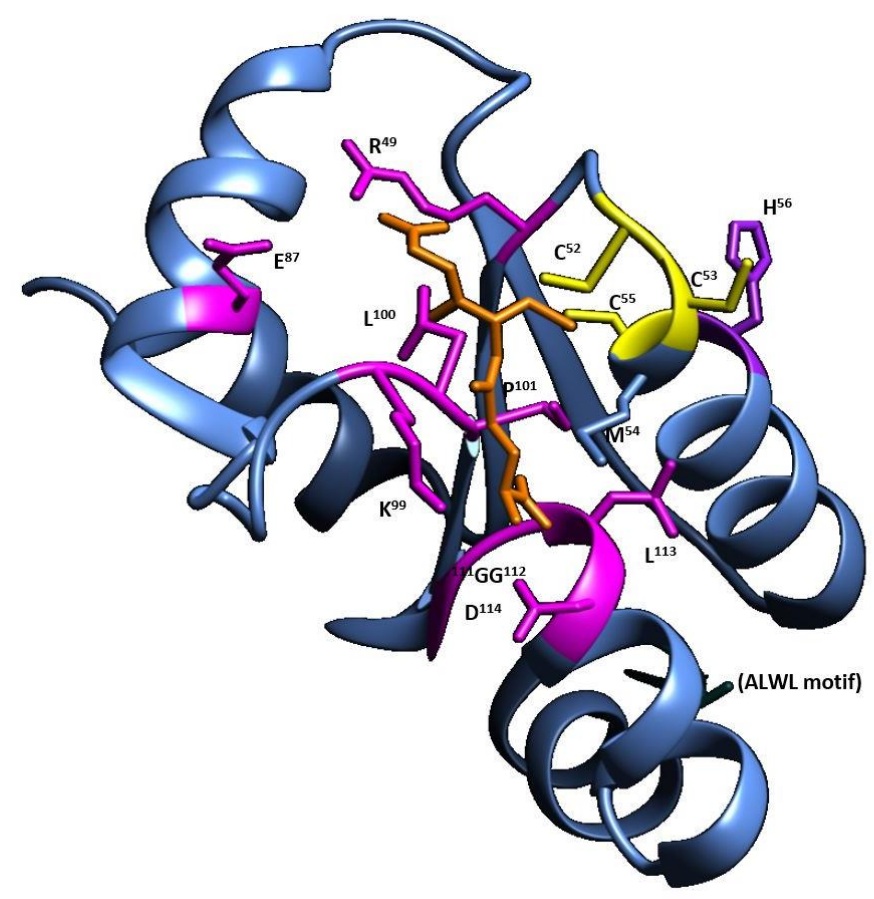

Figure I3, part III. Glutathione binding by the CC-type glutaredoxin ROXY19 from Arabidopsis thaliana. The structure was modeled using Chimera by Gutsche et al. (2015) [80]. ( $\mathbf{F}$ and $\mathbf{G})$ Surface representation visualizing the glutathione binding groove from two different angles; (F) shows the glutaredoxin from approximately the same view as human Grx2 in Figure I3A and B. (H) Secondary and tertiary structure with selected residues depicted as sticks to show the putative glutathione binding mode. The active site cysteines are shown in yellow, glutathione binding motifs and residues are coloured in magenta. The conserved histidine residue following the active site motif is shown in purple. Glutathione is depicted as a stick model in orange. Residues $\mathrm{K}^{99}$ and $\mathrm{D}^{114}$ seem to block the glutathione binding groove in contrast to the CPYC-type glutaredoxin Grx2 from human (Figure I3A and B). 
revealed that glutathione associates with a groove on the protein surface (Figure I2B and C and I3A, C and D) [79-81,93-100]. Together, these studies show that the glutathione-binding groove is lined by several polar residues. The glycine of glutathione is located at the end of the groove pointing upwards in the structural representations in Figures I3A and B. This part of the groove is formed by amino acids surrounding the active site motif in the primary structure. The glycine moiety is often bound by hydrogen bonds and salt bridges from polar and/or positively charged amino acids. In contrast, the glutamyl moiety binds in the region of the so-called GG motif and its following amino acids located at the opposite end of the groove. The region of the GG motif contains neutral and/or polar or charged amino acids. The center of the groove harbors a highly conserved TV/FP motif. The backbone of the protein in the region of the TV/FP motif and its surrounding residues interacts with the backbone of glutathione. They also associate with the glutamate and/or the cysteine of glutathione. The cysteine moiety of glutathione is located close to the active site of the glutaredoxin, next to the TV/FP motif $[9,80,93]$. In some glutaredoxin structures, the cysteine of glutathione is covalently bound to an invariant cysteine of the active site (Figure SIC) [94,96]. Only few structures show glutathione bound non-covalently to the groove of the glutaredoxin (Figure I3A) [Johansson et al., 2006; published in PDB] [9].

The glutathione binding mode described above represents a prototype model. Glutathione is a rather flexible molecule, and some glutaredoxins might use a slightly altered way to bind glutathione (Figure I3C to H) [9]. For instance, E. coli Grx4 and related glutaredoxins were speculated to employ a conserved WP motif shortly before the TFP motif for glutathione binding, since the GG motif is buried beneath the tryptophane and thus, not accessible for an interaction with glutathione (Figure I3C to E, [75,91]). Likewise, a structural model of the glutaredoxin ROXY19 suggests that glutathione binding in the same mode as shown in Figure I3A and B would cause steric conflicts (Figure I3F to H, [80]).

The covalently bound glutathione molecule represents an intermediate during the catalysis of disulfide reduction. To reduce protein disulfide bridges or glutathione-mixed disulfides, glutaredoxins either employ the monothiol or the dithiol mechanism. Dithiol and monothiol mechanism are distinguished by the number of cysteines used for catalysis (Figure I4A and B) [1,9,74,79,93,94,101103]. The reaction sequence of both mechanisms starts with the oxidative half reaction, during which the substrate becomes reduced and the glutaredoxin oxidized. Afterwards, the glutaredoxin is recycled during the reductive half reaction [9]. 
The monothiol mechanism (Figure I4A) relies only on the $\mathrm{N}$-terminal active site cysteine, which attacks the glutathione-mixed disulfide or the protein disulfide (Figure I4A and $\mathrm{B}$, step $A$ ). When a glutathione-mixed disulfide is used as a substrate, the glutaredoxin $\mathrm{N}$-terminal active site cysteine takes over the glutathione moiety from the substrate in a disulfide exchange reaction. As a result, the N-terminal active site cysteine residue of the glutaredoxin becomes covalently linked to the cysteine of glutathione (Figure SIC) and a free thiol on the target protein is formed (Figure I4A, step $A$ ). In case a protein disulfide bridge serves as a substrate, the glutaredoxin first resolves the disulfide by forming an intermolecular disulfide bridge with one of the target cysteines. The intermolecular disulfide bridge is then reduced by a molecule of glutathione. This leads to a glutathionylated glutaredoxin and thiol groups on the target protein (Figure I4B, step B). Independent of the initial substrate, the glutathionylated glutaredoxin is regenerated by another molecule of glutathione: Its thiol group attacks the disulfide bond between the first glutathione molecule and the cysteine of the glutaredoxin. This results in formation of GSSG and a reduced glutaredoxin (Figure SIA, Figure I4A, steps $B$ and $C$, Figure I4B, steps $C$ and D). GSSG is converted into two molecules of GSH by glutathione reductase using the reducing power of NADPH.

The dithiol mechanism of glutaredoxins (Figure I4A and B) involves additional cysteine residues, either located at the C-terminal end of the active site motif, or sometimes outside the active site. Disulfide reduction via the dithiol mechanism shares the first step with the monothiol mechansim (Figure I4A and $\mathrm{B}$, step A). However, the release of the reduced target and the regeneration of the glutaredoxin proceed differently and depend on the type of disulfide substrate used. As described above, during reduction of a glutathione-mixed disulfide (Figure I4A, step $A$ ) or a protein disulfide by the glutaredoxin with the help of glutathione (Figure I4B, step B), the glutaredoxin becomes covalently bound to glutathione. In the dithiol mechanism, this glutathione-mixed disulfide bond on the glutaredoxin is either resolved by the second active site cysteine or an external cysteine residue of the glutaredoxin. This gives rise to an intramolecular disulfide bridge within the glutaredoxin (Figure SIB, Figure I4A, step $D$ ). In most cases, such an intramolecular disulfide bridge is reduced by two molecules of glutathione, the first one binding to the $\mathrm{N}$-terminal active site cysteine because of a disulfide exchange (Figure I4A, step E). The second glutathione molecule reacts with the newly formed glutathione-mixed disulfide according to the monothiol mechanism (Figure I4A, steps $B$ and $C$ ). Alternatively, the intramolecular disulfide bonds resulting from the dithiol mechanism are reduced by ferredoxin thioredoxin reductase in the chloroplast 
or thioredoxin reductase (Figure I4A step F). In case a protein disulfide bridge is reduced by the glutaredoxin, the glutaredoxin can immediately reduce the disulfide bridge by a disulfide exchange reaction using the N-terminal active site cysteine and an additional cysteine. Glutathione is not used as an electron donor during this step (Figure I4B, step E). The resulting intramolecular disulfide bridge of the glutaredoxin is subsequently reduced by two molecules of glutathione (Figure I4B, steps $G, C$, and D) or by TR or FTR (Figure I4B, step H) $[1,9,74,78,79,93,94,101-106]$.

Whether the dithiol or the monothiol mechanism or both are used for catalysis, is specific for a given glutaredoxin. Recently, Ukuwela et al. (2018) demonstrated that E. coli Grx1 and human Grx1 are able to catalyze disulfide reduction employing the monothiol and the dithiol mechanism in parallel [102]. In contrast, the plant glutaredoxin AtGRXC5 was shown to operate only via the monothiol mechanism though it contains a second active site cysteine as well as external cysteines [94]. The role of the second active site or external cysteine residues represent still confusing aspects of glutaredoxin catalysis. In some cases, the second active site cysteine lowers the deglutathionylation activity [77,94], whereas in other glutaredoxins, it enhances catalysis [107]. Various potential explanations, such as influencing the reactivity of the $\mathrm{N}$ terminal active site cysteine residue, protecting it under oxidative conditions or allowing a distracting reaction via the dithiol mechanism have been discussed. $[9,77,94]$. The cysteine resolving model represents a logical explanation for the function of this second active site cysteine residue (or addional external cysteines) in agreement with the evolution of glutaredoxin catalysis [9]. However, further studies are necessary to substantiate this hypothesis. As for the second active site cysteine, the functions of external cysteines are not fully understood. External cysteine residues can fulfill functions other than catalysis. With respect to GRXC1 and GRXC2 from poplar, an external cysteine enables dimerization of two glutaredoxin subunits via an intermolecular disulfide bridge [77,98]. Couturier et al. (2011) showed that the external cysteine of AtGRXC5 can be glutathionylated [94]. Thus, external cysteines were suggested to regulate some glutaredoxins or to play a role in signaling $[77,98]$.

The example of the additional cysteine residues within glutaredoxins indicates that the details of glutaredoxin catalysis are currently not well understood. Interestingly, there are different classes of glutaredoxins with differential catalytic activity. Whereas glutaredoxins with an active site of the consensus CPYC are usually catalytically active, glutaredoxins with a CGFS active site motif are almost inactive. In an attempt to explain these differences, the role 
A

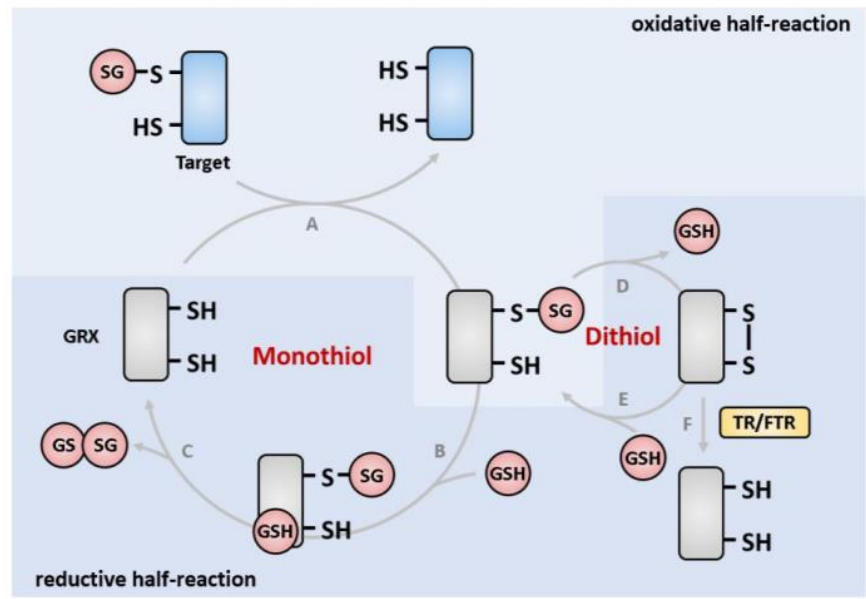

B

reductive half-reaction

oxidative half-reaction

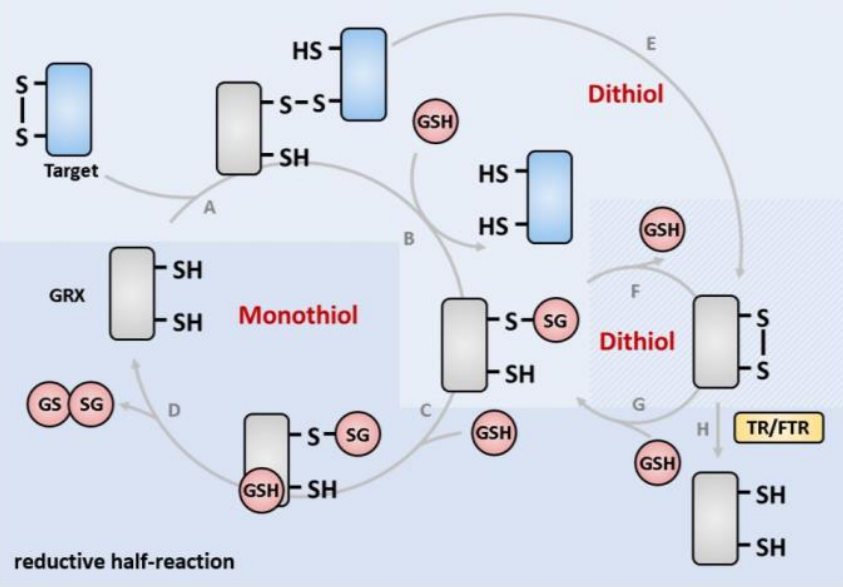

Figure I4, part I. Reaction mechanisms used by glutaredoxins. Glutaredoxins deglutathionylate other proteins via the monothiol or the dithiol mechanism (A). During deglutathionylation via the monothiol mechansim, a reduced glutaredoxin takes over the glutathione moiety (-SG) from the glutathionylated target protein (step $A$ ) and then deglutathionylates itself with the help of another glutathione molecule $(\mathrm{GSH})$, releasing glutathione disulfide (GSSG, steps $B$ and $C$ ). When using the dithiol mechanism for degluathionylation, the glutaredoxin is deglutathionylated forming an intramolecular disulfide $($ step $D)$, which is subsequently resolved by two molecules of glutathione (steps $E, B$, and $C$ ) or thioredoxins (TR) or ferredoxin thioredoxin reductase (FTR, step F). (B) shows the reduction of disulfide bridges in target proteins via the mono- or dithiol mechanism. During the dithiol mechanism, an intramolecular disulfide is formed within the glutaredoxin in the course of a disulfide exchange (steps $A$ and $E$ ), whereas in the monothiol mechanism, the glutaredoxin undergoes glutathionylation and deglutathionylation only (steps $A$ to $D$ ). However, after step $B$ of the monothiol mechanism, the glutaredoxin can also enter the dithiol mechanism (steps $F, G$ and $H$ ). During the dithiol mechanism, the disulfide bridge is either resolved by TR or FTR (step $H$ ) or via two molecules of GSH, releasing GSSG (steps $G, C$ and $D$ ). When viewed in terms of the reaction sequence steps $A, B, F, H$ or $A, B, F, G, C, D$, the reaction product of step $F$ belongs to the reductive half-reaction. When viewed from the reaction sequence beginning with steps $A$ and $E$, it belongs to the oxidative half reaction. The schemes were taken from Rouhier et al. (2008) [1], Deponte (2013) [9], and Ukuwela et al. (2018) [102] and modified. 
C

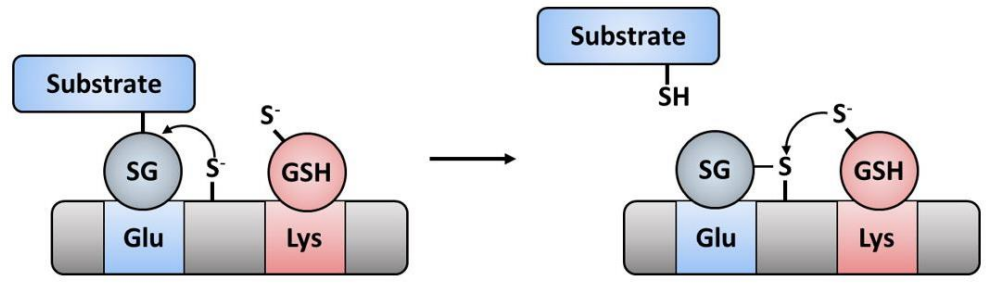

D

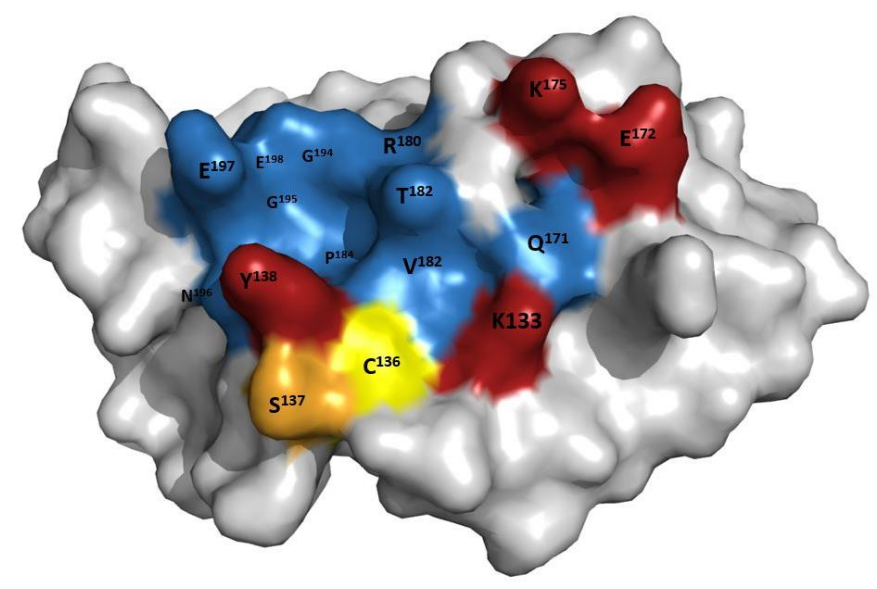

Figure I4, part II. The glutathione scaffold and acitvation site of glutaredoxins. The glutathione binding groove is divided in a scaffold (blue) and an activation site (red). In (C), the basic functions of the activation and the scaffold site of glutaredoxins during catalysis are illustrated. The glutaredoxin is shown in grey. The scaffold site containing a glutamate residue ( $\mathrm{E}^{197}$ in SoGrx6) binds the glutathione moiety of the substrate (blue), which is attacked by the thiolate of the active site cysteine (-S) ( $\mathrm{C}^{136}$ in SoGrx6). After release of the deglutathionylated substrate, the thiolate from the glutathione molecule bound by the activation site (containing a conserved lysine, $\mathrm{K}^{133}$ in $S_{0}$ Grx6, shown in red) reacts with the disulphide between the active site cysteine and glutathione from the substrate. For simplicity, the figure does not show the actual transition states occuring during catalysis. Therefore, it should be noted that glutaredoxin catalysis probably occurs via an $\mathrm{S}_{\mathrm{N}} 2$ reaction [9]. For details, the reader is referred to reference [75]. (D) shows the glutathione binding groove in the crystal structure of Saccharomyces cerevisiae Grx6. Residues belonging to the potential glutathione scaffold site associating with the glutathionylated substrate are highlighted in blue. The putative glutathione activation site is depicted in red. The N-terminal acitve site cysteine is shown in yellow and active site serine residues in orange. The structure was solved by Luo et al. (2010) [108] to a resolution of $1.5 \AA$. The representation was prepared according to the findings by Begas et al. (2017) in PyMOL using the PDB file (accession: $3 \mathrm{~L} 4 \mathrm{~N}$ ). $\mathrm{K}^{133}, \mathrm{Y}^{138}$, and $\mathrm{E}^{197}$ are homologous to the residues in SoGrx7 characterized by Begas et al. (2017) [75]. 
of two distinct residues of the glutathione binding groove during catalysis was discovered $[9,75,109]$. By analysing the reaction kinetics of mutant Saccharomyces cerevisiae (S. cerevisiae) Grx7 in comparison to the wildtype enzyme, Begas et al (2017) could show that the conserved lysine preceding the active site binds the recycling glutathione and activates the cysteine of glutathione, as well as the $\mathrm{N}$ terminal active site cysteine residue of the glutaredoxin. In contrast, a glutamate in the region of the GG motif was found to support the binding of the glutathionylated substrate. Homologous residues in a Plasmodium falciparum (P. falciparum) glutaredoxin (PfGrx) were found to play the same role [75]. This suggested that the glutathione binding groove revealed by structural studies might rather consist of two glutathione binding sites: a scaffold site associating with the glutamyl moiety of the glutathione moiety of the glutathionylated substrate and an activation site binding the recycling glutathione molecule via the glycine and cysteine part of glutathione. In addition, the activation site might deprotonate the thiol groups of the enzyme and glutathione (Figure I4C and D). Already a few years before, Li et al. (2010) noticed that replacement of the aspartate homologous to the scaffold glutamate by a serine in yeast Grx1 (Figure SIA and B) results in a lower $\mathrm{K}_{\mathrm{m}}$ for glutathione but higher turnover number and a higher specific activity [110]. Aside from the conserved lysine and the glutamate residue, initial data indicated that the tyrosine found in the active site of active glutaredoxins (CPYC active site) also belongs to the activation site, but might fulfill other functions, as well [75]. Interestingly, this residue adopts different rotamers in the structures of glutaredoxins with different oxidation states (Figures I3B and SIA to C). Because the structures represent intermediates during catalysis, it was already speculated that this residue might change its conformation during catalysis, thereby contributing to the displacement of GSSG from the active site [91,110,111]. However, Begas et al. (2017) did not discover a change in the concentrations of glutathionylated and non-glutathionylated enzyme during catalysis, when the active site tyrosine was mutated. Based on further sequence comparisons, Begas et al. (2017) assigned additional amino acids to the activation and the scaffold region of the glutathione binding groove. In summary, the glutathione activation site could be located at the end of the groove harboring the active site cysteine and the conserved lysine residue. The other part of the groove composed of the GG and the TV/FP motif could form the scaffold site (Figure I4D) [75]. 
Aside from disulfide reduction, some glutaredoxins were found to bind ironsulfur clusters [81,95,112-117]. These cofactors are composed of iron and sulfide ions, forming either a rhombic $[2 \mathrm{Fe}-2 \mathrm{~S}]$ or a cubic $[4 \mathrm{Fe}-4 \mathrm{~S}]$ cluster (Figure I5B and C). [3Fe-4S] clusters exhibit either a linear or a cuboidal structure and can even include other metal ions than those of iron (Figure I5D, $\mathrm{E}$, and F). Iron-sulfur clusters are frequently bound to the cysteines of proteins. Apart from the basic forms of iron-sulfur clusters shown in Figure I5, ironsulfur clusters are part of complex cofactor assemblies, such as siroheme cofactors or the FeMo cofactor and the $[8 \mathrm{Fe}-7 \mathrm{~S}]$ cluster found in nitrogenase (Figure I5G, H, and I) [118-120]. Single iron ions ligated by cysteine residues are referred to as mononuclear iron sulfur clusters (Figure I5A) [121]. The biogenesis of iron sulfur clusters is accomplished by a set of enzymes. First, iron ions and sulfide ions - the latter derived from free cysteine and provided by cysteine desulfurases - are assembled on a scaffold protein. Finally, a carrier protein takes over the assembled cluster and donates it to the apo-form of the target protein. Within their target proteins, iron sulfur clusters serve for electron transport or for catalysis [118].

Initially, all glutaredoxins were found to coordinate [2Fe-2S] clusters. However, in 2013, Zhang et al. proposed that glutaredoxins previously thought to bind solely $[2 \mathrm{Fe}-2 \mathrm{~S}]$ clusters might also coordinate $[3 \mathrm{Fe}-4 \mathrm{~S}]^{+}$and mixtures of $[2 \mathrm{Fe}-$ $2 \mathrm{~S}]$ and $[3 \mathrm{Fe}-4 \mathrm{~S}]^{+}$clusters [90]. In case of the well-studied [2Fe-2S] glutaredoxin complexes, the cluster bridges two glutaredoxin subunits and is ligated by the N-terminal active site cysteine residues and the thiol group of two glutathione molecules, one bound to each subunit (Figure I6A to C) [81,92,94,95,97,113115,122-125]. As for the catalytic activity, differences in the structure of ironsulfur cluster-bound glutaredoxins depend on the active site motif: In glutaredoxins with a CPYC active site motif, these subunits are - with respect to the central $\beta$-sheet - either assembled in a perpendicular (Figure I6A and B) $[94,113,124]$, whereas they are arranged in a co-planar way for glutaredoxins with a CGFS active site (Figure I6C and D) [92,95,124,125]. In some cases, two of these dimers might further assemble into tetramers. This was, among others, described for human Grx5 (Figure I6D); however, later, contradicting results were published $[122,124,125]$. An exception from this concept represents the zebrafish glutaredoxin Grx2. Albeit Grx2 closely resembles other glutaredoxins in its structure, it coordinates a [2Fe-2S] cluster as a monomer via cysteine residues outside the active site [126]. 
Thioredoxins and glutaredoxins

A<smiles>[B][PH]([Sb])([SbH])[SbH2]</smiles>

B<smiles>[R5][P+]1([SeH])S[Pb]([B])([SeH])S1</smiles>

C

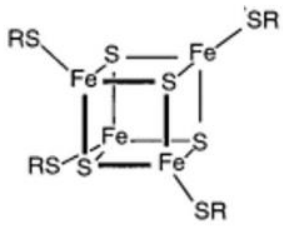

D

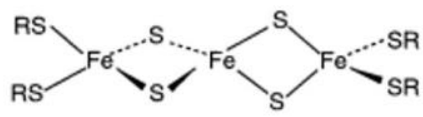

E

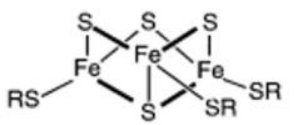

G

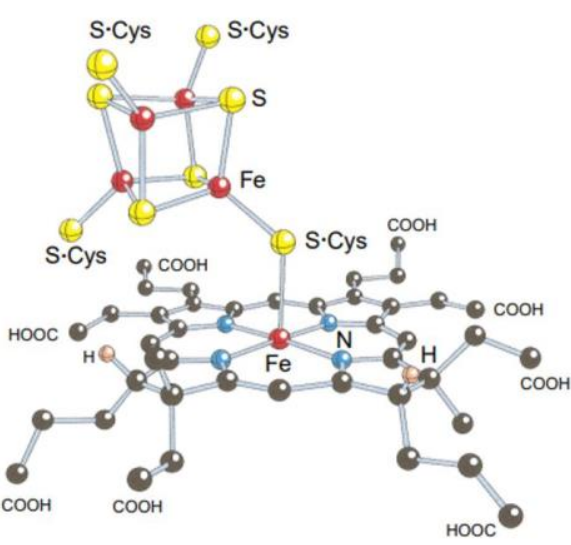

$\mathbf{F}$

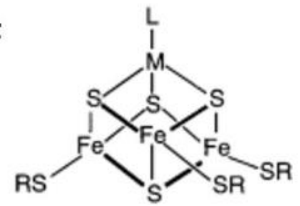

H
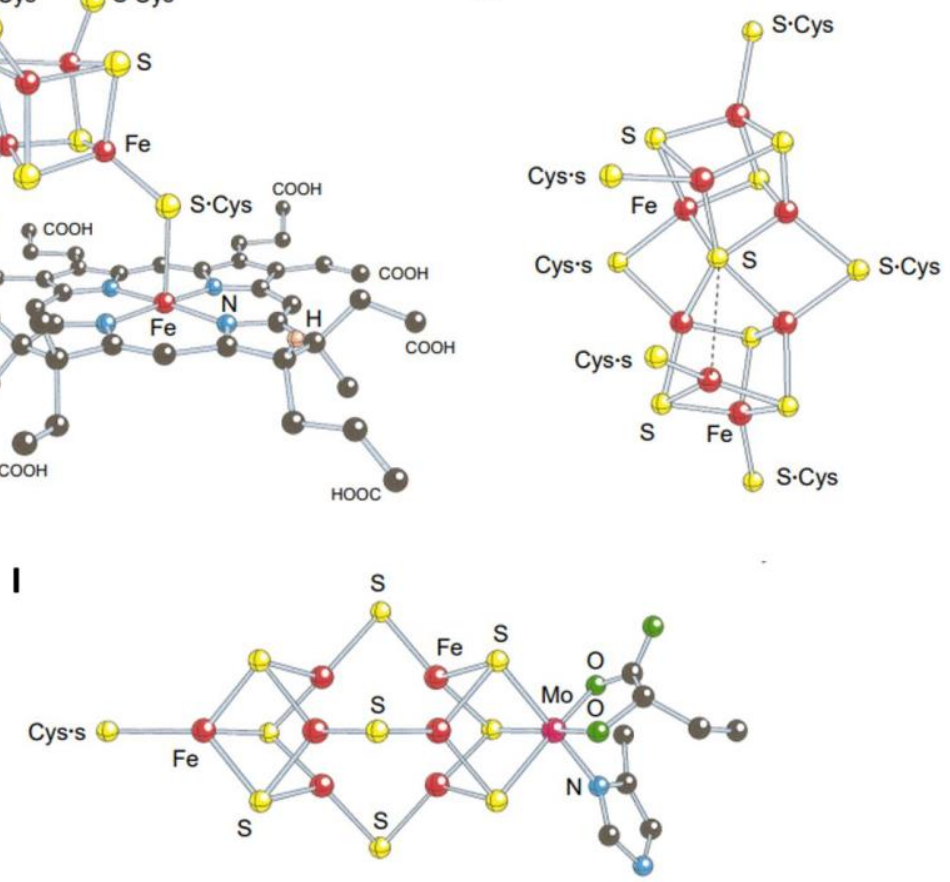

Figure I5. Different types of iron sulfur clusters. The figure shows different types of ironsulfur clusters and their coordination. (A) Mononuclear clusters, (B) $[2 \mathrm{Fe}-2 \mathrm{~S}]$ clusters, $(\mathbf{C})[4 \mathrm{Fe}-$ 4S] clusters, and (D) linear [3Fe-4S] clusters need four ligands, which are frequently cysteines (RS). Cuboidal [3Fe-4S] clusters (E) are complexed by three ligands, but can incorporate other metal ions $(M)$ bound by a fourth ligand $(L)$, as shown in $(\mathbf{F})$. Iron sulfur clusters can form complex cofactor structures: (G) Siroheme cluster composed of a [4Fe-4S] cluster bridged to a heme found e.g. in E. coli sulfite reductase; [8Fe-7S] P cluster $(\mathbf{H})$ and FeMo cofactor with a part of the coordinating homocitrate (I) found in nitrogenase. The iron-coordinating cysteine residues are indicated by Cys-S. The carboxyl groups of heme are indicated by $\mathrm{COOH}$. Colour code: red - iron ions $(\mathrm{Fe})$, yellow - sulphide ions $(\mathrm{S})$, black - carbon, blue - nitrogen $(\mathrm{N})$, pink - hydrogen $(\mathrm{H})$, purple - molybdenum $(\mathrm{Mo})$, green - oxygen $(\mathrm{O})$. The figure was taken from Beinert et al. (1997) [119] and modified. 
Interestingly, there are only minor changes in the conformation of the glutaredoxin and the mode of glutathione binding upon iron-sulfur cluster incorporation [124]. While the overall thioredoxin fold of glutaredoxins remains after incorporation of an iron-sulfur cluster, E. coli Grx4 and human Grx5 adopt a more "extended" conformation (Figure 6C and D) [92,125]. Similarly, certain loops and the $\mathrm{N}$-terminal part of helix $\alpha_{4}$ of AtGRXC5 are bent backward in comparison to the apo-form and, thus, point away from the subunit interface upon cluster binding (Figure 6A and B) [94]. As for the conformation of the glutaredoxin, the way of glutathione binding upon assembly into the cluster-bridged oligomers only changes weakly. Which changes in the glutathione binding mode occur depends again on the active site of the glutaredoxin. These differences were worked out by Couturier et al. (2015) using Arabidopsis thaliana (A. thaliana) GRXC5 and E. coli Grx4 as examples: In glutaredoxins with a CPYC active site, such as $A$. thaliana GRXC5, the side chains of the active site residues change their orientations (Figure I6E) [124]. The last active site residue associates with the conserved lysine residue before the active site, thereby stabilizing the loop containing the active site for cluster binding [94,124]. Active site residues in addition to the N-terminal cysteine were found to stabilize the position of glutathione [81,94,124] (Figure I6A, B and E). In addition, cross-contacts between glutathione of one glutaredoxin subunit and the active site residues of the other subunit were described $[81,94,124]$ (Figures I3 and I6). In contrast, in glutaredoxins with a CGFS-active site, the conformation of some active site residues does not change strongly upon cluster binding (Figure I6F). Still, the last active site serine interacts with the conserved lysine residue before the active site, as observed for CPYC glutaredoxins [124]. In addition, loop regions including the loop containing the $\mathrm{N}$-terminal active site cysteine change their conformation upon cluster binding in E. coli Grx4 (Figures I3E and I6C and F) [92]. Moreover, several contacts between the two glutaredoxin subunits independent of glutathione are established (Figure I6C and F) [92,124,125].

Consequently, the active site composition and additional residues outside the active site determine the ability to bind iron-sulfur clusters. To date, the precise requirements allowing iron-sulfur cluster binding are still not fully understood $[94,97,112,113,124]$. The function of cluster binding might vary among glutaredoxins, ranging from putative sensory and regulatory functions to the assembly and the transfer or the storage of these cofactors [81,94,97,113,114,116,117,124,127-131]. 
A

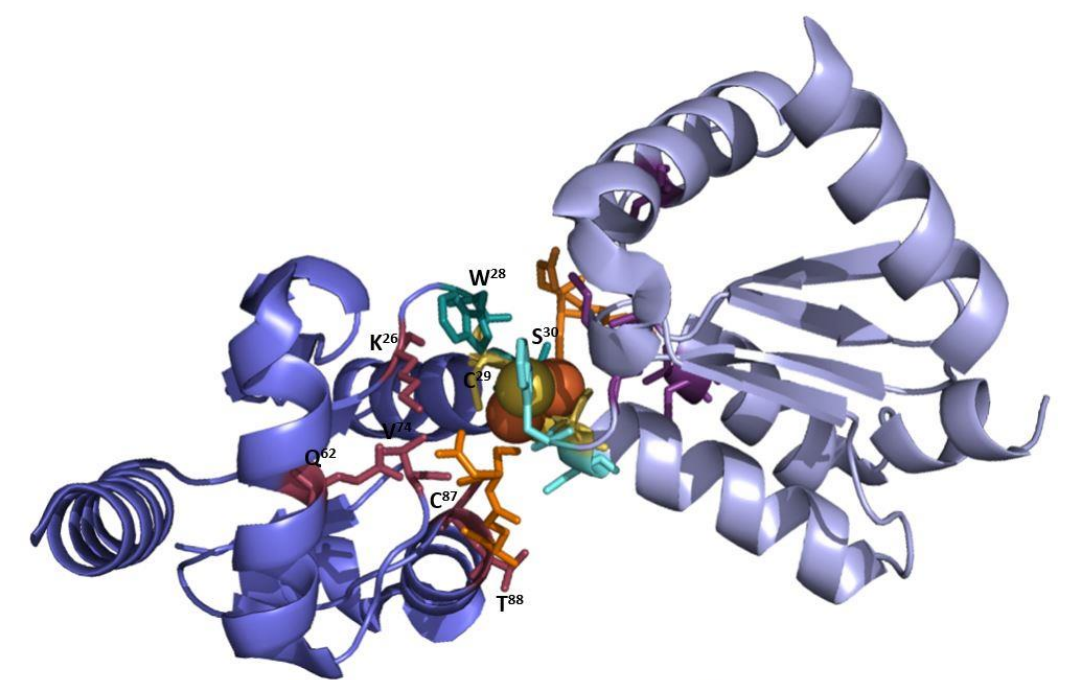

B

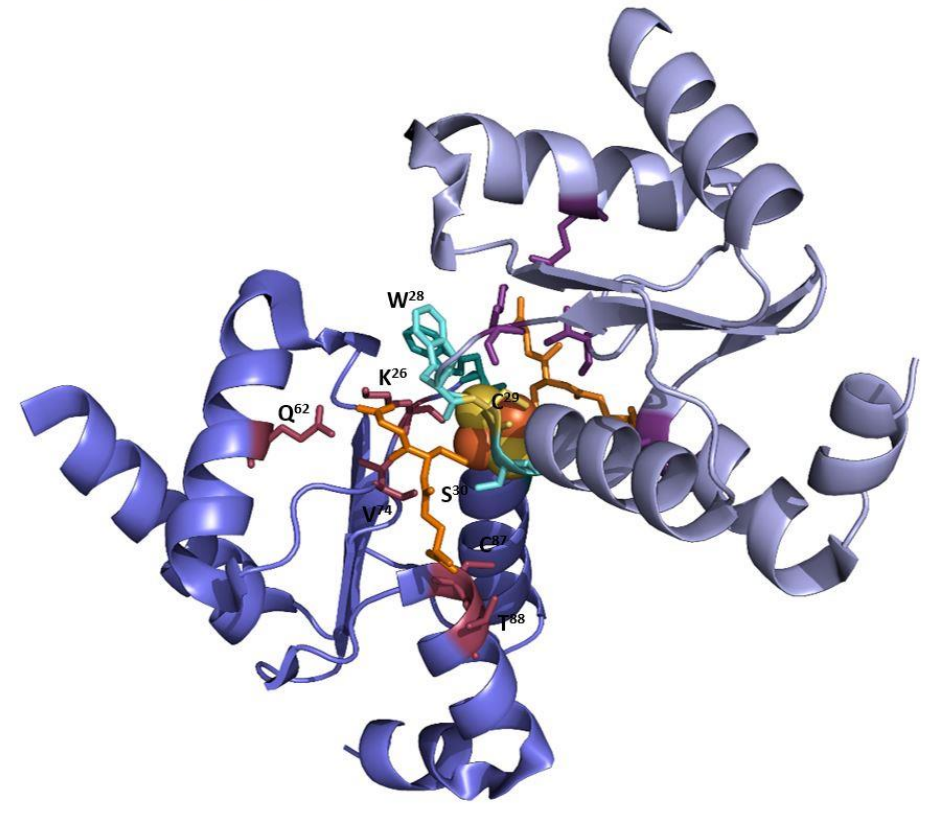

Figure I6, part I. Iron sulfur cluster binding by CPYC glutaredoxins. The crystal structure of holo GRXC5 from $A$. thaliana, a CPYC-type glutaredoxin, was solved by Couturier et al. (2011) [94] to a resolution of $2.4 \AA$. The structure pictures were produced from the PDB file (accession: 3RHC) using PyMOL according to the findings by Couturier et al. (2011) [94] and Couturier et al. (2015) [124]. The two glutaredoxin subunits of the dimer are shown in blue and grey, respectively. The first active site cysteines of both subunits are depicted in yellow and residues important for glutathione binding are shown in red and purple, respectively. Residues forming contacts to the glutathione molecule of the other subunit are coloured in light and dark cyan, respectively. The glutathione molecule bound to each subunit is presented as a stick model in orange. The [2Fe-2S] cluster is shown as spheres (yellow: sulfur, orange: iron). (A) and (B) show the same structure from different angles. 
C

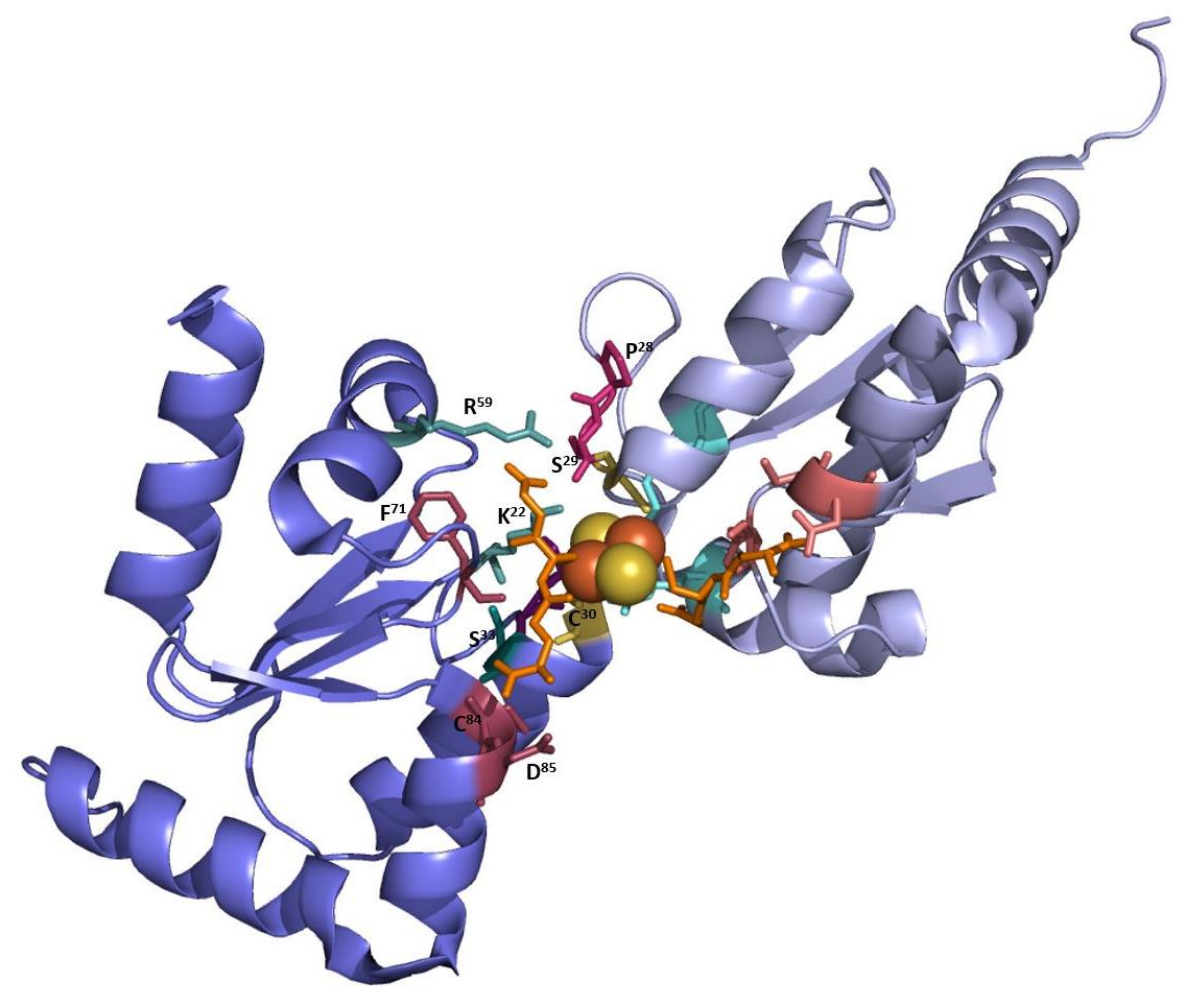

Figure I6, part II. Iron sulfur cluster binding by CGFS glutaredoxins. The crystal structure of holo Grx4 from E. coli, a CGFS glutaredoxin, was solved by Iwema et al. (2009) [92] to a resolution of $1.9 \AA$. The structure picture was produced from the PDB file (accession: 2WCI) according to the results of Iwema et al. (2009) [92] using PyMOL. The two glutaredoxin subunits of the dimer are shown in blue and grey, respectively. The first active site cysteines of both subunits are depicted in yellow and residues important for glutathione binding are shown in light red and dark red, respectively. Residues establishing contacts to the other subunit are shown in light and dark cyan, respectively. Residues binding to both, glutathione and the other subunit are depicted in purple and pink, respectively. The glutathione molecule bound to each subunit is presented as a stick model in orange. The [2Fe-2S] cluster is shown as spheres (yellow: sulfur, orange: iron). 


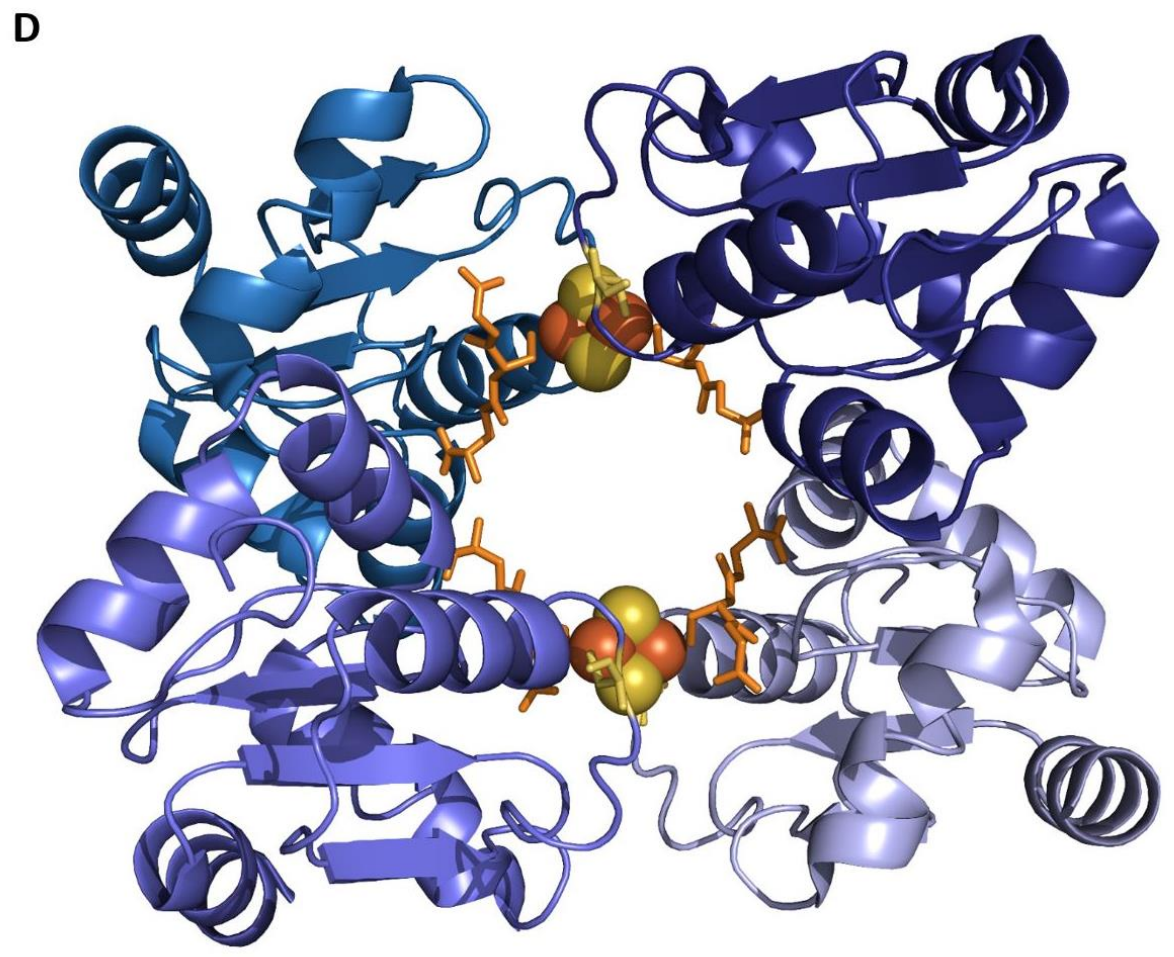

Figure I6, part III. Iron sulfur cluster binding by CGFS glutaredoxins. The crystal structure of human holo Grx5, a CGFS glutaredoxin, was solved by Johansson et al. (2011) [125] to a resolution of $2.4 \AA$. The structure picture was produced from the PDB file (accession: 2WUL) using PyMOL. The four glutaredoxin subunits of the dimer are shown in different shades of blue and grey, respectively. The first active site cysteine of each subunit is depicted in yellow. The glutathione molecule bound to each subunit is presented as a stick model in orange. The two [2Fe-2S] cluster are shown as spheres (yellow: sulfur, orange: iron). For reasons of simplicity, neither glutathione binding residues nor intersubunit contacts were highlighted. 
E

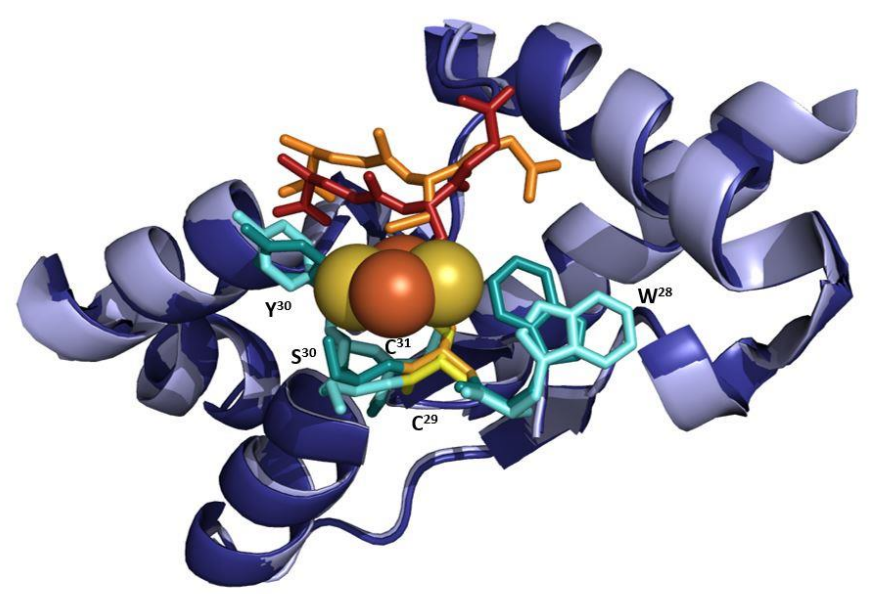

$\mathbf{F}$

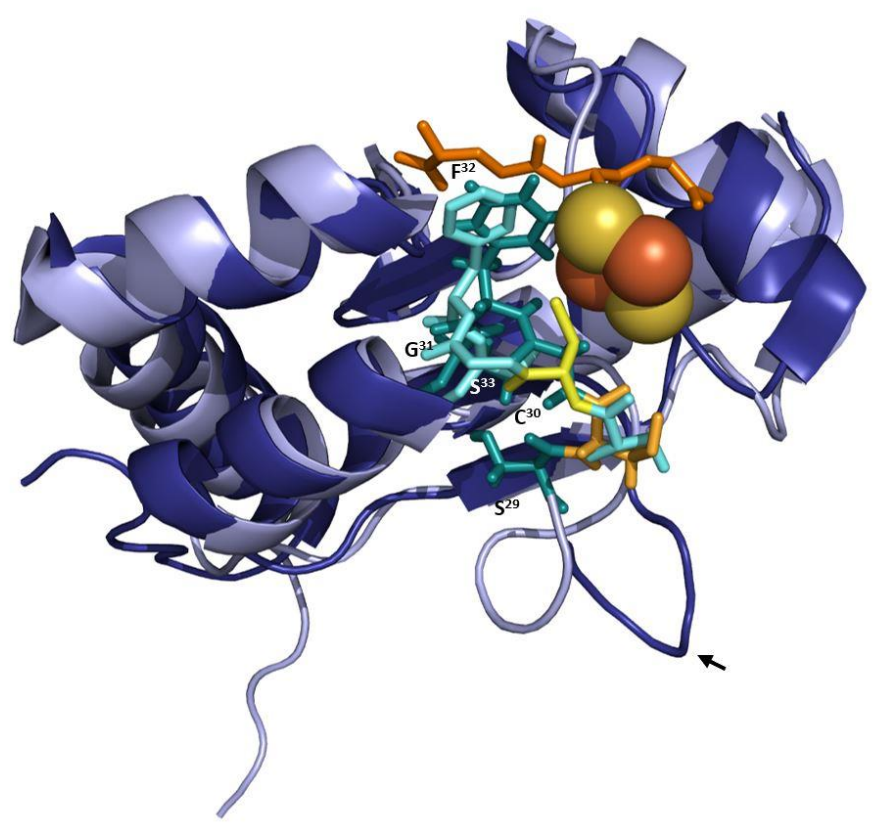

Figure I6, part IV. Rearrangements in the active site of CPYC and CGFS-type glutaredoxins upon iron-sulfur cluster binding. (E) Overlay of the crystal structures of AtGRXC5 apo-protein bound to glutathione and one subunit of the AtGRXC5 holo-protein; AtGRXC5 is a CPYC-glutaredoxin. (F) Overlay of the crystal structures of EcGrx4 apo-protein and one subunit of the EcGrx4 holo-protein; EcGrx4 belongs to the CGFS-type glutaredoxins. The iron and sulfur ions of the iron-sulfur cluster are shown as orange and yellow spheres, respectively. The apo-protein is shown in dark blue, the holo-protein in light blue. Amino acids of the active site region are highlighted in light cyan for the holo-protein and in dark cyan for the apo-protein and represented as stick models. Glutathione is shown as an orange (belonging to holo-protein) or red (belonging to apo-protein) stick model. The $\mathrm{N}$-terminal active site cysteine of the holo-protein is shown in yellow, whereas the one of the apo-protein is depicted in light orange. The loop containing the active site cysteine is indicated by an arrow in $(\mathbf{F})$. The figures were created from the PDB files (accessions: 3RHB, 3RHC, 1YKA, 2WCI) in PyMOL and are based on the articles by Couturier et al. (2015) [124], Couturier et al. (2011) [94], and Iwema et al. (2009) [92]. 


\section{Different classes of glutaredoxins in plants and their functions}

Compared to other organisms, plants harbor an extraordinarily large number of glutaredoxins [132-134]. Thus, while E. coli, S. cerevisiae and humans contain only about a handful of glutaredoxin genes, 31 glutaredoxin genes have been identified in $A$. thaliana. Similar numbers have been described for poplar (36) and rice (27) [134]. Phylogenetic analysis of the numerous plant glutaredoxins revealed three major groups. As mentioned before, each group is characterized by a conserved active site motif. According to the predominant amino acids in this motif, the three major glutaredoxin classes are termed "CPYC-“, "CGFS" and "CC-type glutaredoxins". While CPYC- and CGFS-type glutaredoxins are found in most prokaryotes and eukaryotes, CC-type glutaredoxins only evolved in land plants [132-135]. Within land plants, the number of CPYCand CGFS-type glutaredoxins remained relatively constant from mosses to angiosperms and does not differ strongly from the number of glutaredoxins in other organisms. However, the class of CC-type glutaredoxins underwent multiple events of gene duplication during the evolution of higher land plants. Thus, the large number of glutaredoxins in plants results from the increase in the number of the CC-type glutaredoxins [134-136].

\section{CPYC-type glutaredoxins}

Plant CPYC glutaredoxins (Figures I2C, I3A and B, I6A and B, and SI1C) exhibit active site motifs of the consensus sequence $\mathrm{C}(\mathrm{P} / \mathrm{G} / \mathrm{S})(\mathrm{Y} / \mathrm{F})(\mathrm{C} / \mathrm{S})$ [135] and efficiently catalyze the reduction of protein disulfides and glutathione-mixed disulfides [77,88,94,96,137]. A few studies indicate, that CPYC glutaredoxins are also able to transfer glutathione to the cysteines of target proteins $[87,88]$ and can catalyze the oxidation of redox-sensitiv GFP (roGFP) [97].

Higher plants contain four cytosolic CPYC glutaredoxins: GRXC1, GRXC2, GRXC3 and GRXC4, and at least one chloroplastic CPYC glutaredoxin named GRXS12. In Brassicacea, an additional CPYC glutaredoxin, GRXC5, is located in the chloroplast $[77,94,96,113,137]$. GRXC1 and GRXC2 differ in their properties slightly from GRXC3 and GRXC4, forming two subclasses of CPYC glutaredoxins. While GRXC1 and GRXC2 might dimerize via a disulfide bridge, the other glutaredoxins do not. This might result in different reaction mechanisms [77]. GRXC1 and GRXC2 have been studied extensively at the biochemical level and by mutant analysis. They are indispensable for plant 
viability $[77,81,112,113]$. They contribute strongly to the cellular glutaredoxin activity and might be involved in the protection of the cell from oxidative damage [112]. Besides, GRX370 was found to modulate the activity of the kinase BAK1 via glutathionylation, implicating a function of this glutaredoxin in brassinosteroid signaling [88]. Interestingly, GRXC1 was shown to dimerize via an iron-sulfur cluster coordinated by the $\mathrm{N}$-terminal active site cysteine residues and the cysteine moiety of glutathione [112,113]. Later, the same mode of iron-sulfur cluster coordination was also described for chloroplastic GRXC5 [94]. Because GRXC1 is catalytically active only in its apoform and because GRXC5 cannot transfer clusters to other proteins, the binding to the ironsulfur cluster observed for both proteins was proposed to play a regulatory role: During oxidative stress, the cluster might be destroyed, releasing catalytically active glutaredoxins, which help to protect the cell from oxidative damage $[94,112,138]$.

\section{CGFS-type glutaredoxins}

In contrast to CPYC-type glutaredoxins, the active site of CGFS glutaredoxins (Figures I3C, D, and E, I6C and D) is perfectly conserved [135]. Interestlingly, all these glutaredoxins exhibit only weak activity in the classical biochemical assays to assess the catalytic activity of glutaredoxins: They are less efficient in the reduction of the artificial compound bis(2-hydroxyethyl)disulfide (HEDS) and the reduction of dehydroascorbate (DHA) than CPYC glutaredoxins. They show an equally low activity towards protein substrates such as glutathionylated GAPDH, insulin or roGFP $[75,97,116,117]$. Moreover, for GRXS15 from $A$. thaliana, a weak activity regarding roGFP oxidation was observed $[75,97]$. Thus, catalysis of redox reactions appears not to be their main function in the cell. A possible role of these glutaredoxins emerged in 2002. In this year, RodríguezManzaneque et al. showed that the mitochondrial glutaredoxin Grx5 in $S$. cerevisiae contributes to the assembly of iron-sulfur clusters [89]. Subsequently, the CGFS-type glutaredoxins from plants, namely GRXS14, GRX15, GRXS16, and GRXS17, were found to complement the $S$. cerevisiae grx5 mutant at least partially. This suggested a contribution of plant CGFS-type glutaredoxins to iron-sulfur cluster assembly. All of them were shown to bind [2Fe-2S] clusters as dimers with the help of glutathione [94,95,97,114,116,117,123,129,139], but - as mentionend before - the possibility to associate with $[3 \mathrm{Fe}-4 \mathrm{~S}]^{+}$clusters should not be excluded [90]. 
In plants, the cytoplasm, plastids and mitochondria harbor their own ironsulfur cluster assembly machinery which are partly interconnected [118]. Similarly, plant CGFS-type glutaredoxin localize specifically to the three compartments. GRXS15 represents the only mitochondrial glutaredoxin in plants and is therefore essential. It possibly contributes to the maturation of mitochondrial iron-sulfur cluster proteins [97]. The plastidial glutaredoxins GRXS14 and GRXS16 are non-essential; they mediate chlorophyll maintenance in chloroplasts $[131,140]$. At least GRXS16 can take over ironsulfur clusters from another assembly protein [129]. The cytosolic CGFS glutaredoxin GRXS17 is a diverging representative of the CGFS-type glutaredoxins: It contains three glutaredoxin domains and dimerizes via $[2 \mathrm{Fe}-$ 2S] clusters bound to each of the glutaredoxin modules, presumably using glutathione for coordination. Though the second glutaredoxin domain was shown to complement the yeast gr $x 5$ mutant, the influence of GRXS17 on cytosolic iron-sulfur proteins in planta is at best weak [123,128]. In contrast, GRXS17 was linked with plant development and stress responses $[123,128,141-144]$. The protein is localized in the cytosol and in the nucleus, despite its molecular weight above the exclusion limit of the nuclear pore complex and the lack of an import signal. As it interacts with the transcription factor $\mathrm{NF}-\mathrm{YC} 11 / \mathrm{NC} 2 \alpha$, it was speculated to regulate gene expression via redox modulation of NF-YC11/NC2 $\alpha$ in response to environmental stimuli [123].

\section{CC-type glutaredoxins}

In contrast to the well-studied CPYC- and CGFS-type glutaredoxins, the CCtype glutaredoxins (Figures I3 F, G and H) are poorly understood. A. thaliana has 21 CC-type glutaredoxins, also named ROXYs [135,145]. Their active site motif is characterized by a conserved cysteine directly following the $\mathrm{N}$-terminal active site cysteine (CC motif; consensus: CC(M/C)(C/S/G), Figure SI2) $[135,136]$. In 2005 , the first CC-type glutaredoxin, ROXY1, was described as a regulator of flower development [146]. Later, it was shown that it interacts physically with the TGA transcription factor PERIANTHIA (PAN) in the nucleus to restrict the number of petals in the flower of $A$. thaliana [145]. About the same time, ROXY19, together with the TGA transcription factors TGA2, 5 and 6 , was proposed to mediate the crosstalk between the signaling pathways of the plant hormones salicylic acid (SA) and jasmonic acid (JA)/ethylene (ET) [147]. Since then, many studies described CC-type glutaredoxins as regulators of various developmental processes and stress responses in different plant species [146-167]. A subset of these studies also linked their function with 
TGA transcription factors [145-148,151,152,155-157,159,160,162-164,168172]. Though the interaction specificity of CC-type glutaredoxins with TGA transcription factors is broad [145,147,168,170] [M. Willmer, unpublished], there are specific pairs of the two types of proteins regarding the regulation of certain processes [145-148,151,152,155-157,159,162-164,168-171]. For some parts of the plant, such as flowers, specificity might rely on tissue- and/or organ-specific expression patterns which overlap for certain TGA factors and certain CC-type glutaredoxins [145,162,164,169,170]. However, the mechanisms conferring specificity to those pairs of ROXYs and TGA transcription factors expressed in parallel in leaves are unknown [152]. TGA transcription factors seem to be repressed by CC-type glutaredoxins in their capacity to regulate transcription [145,147,151,160,162,164,169,173]. Interestingly, CC-type glutaredoxins require their active site for regulation of TGA factors. ROXY19 has a CCMC active site. Using ROXY19overexpressing plants, it could be shown that only the first active site cysteine residue might be necessary for its function, but the highly conserved second cysteine of the CC motif and the last active site cysteine might be dispensable [152,174]. ROXY1 has the same active site as ROXY19. However, contradicting results regarding the requirement of its cysteine residues have been published: While Xing et al. (2005) showed, that ROXY1 requires the first cysteine residue for its activity, Ziemann (2010) noticed that an SSMS mutant of ROXY1 is active. However, these discrepancies could be attributed to different experimental conditions, such as the different expression constructs used in these studies: Whereas Xing et al. (2005) employed the CaMV35S promoter, Ziemann (2010) used the ROXY1 promoter which more likely reflects the natural expression intensities of ROXY1 [146,175]. Since some glutaredoxins contribute to the regulation of transcription factors and since TGA transcription factors contain conserved cysteine residues (Figure I8 and SI3), CC-type glutaredoxins were speculated repeatedly to redox-modulate TGA transcription factor, thereby controlling their activity [145149,151,153,155,160,163,164,166,168-173]. However, biochemical studies characterizing the catalytic abilities of CC-type glutaredoxins are scarce. The biochemical characterization of these glutaredoxins has been hampered by the inability to purify them after expression in E. coli. Only low amounts of poplar GrxS7.2 deleted for its hydrophobic C-terminus harboring a motif mediating transcriptional repression could be obtained. Interestingly, the purified protein showed a brownish colour, indicative of iron-sulfur cluster binding. Because of the difficulties to express and purify CC-type glutaredoxins for biochemical analyses, Couturier et al., (2010) constructed chimeric proteins to mimick CC- 
type glutaredoxins. For this, they exchanged the active site of CPYC glutaredoxins with the active site of a CC-type glutaredoxin. Though these chimeric proteins were catalytically only weakly active, they were able to associate with an [2Fe-2S] cluster, forming a dimer. Because other glutaredoxins bind iron-sulfur clusters frequently with the help of glutathione, it was speculated that CC-type glutaredoxins might also require glutathione for cluster incorporation [176]. The glutathione binding site is only partially conserved in CC-type glutaredoxins, as indicated by structural modelling approaches (Figure I3 and SI2) $[80,158,176]$. The glutathione scaffold site appears to be blocked in the structural model of ROXY19 (Figure I3F to H). In addition, the activation site contains a negatively charged glutamate (residue 87) where CPYC- and CGFS-type glutaredoxins harbor a positively charged residue (Figure I3A to E) [80]. However, ROXY19 was shown to bind glutathione with a dissociation constant comparable to a CPYC glutaredoxin and both, ROXY19 and ROXY1, require an intact GG motif for their activity, suggesting that glutathione binding is possible [158,168,174].

Aside from the lack of evidence for a catalytic activity in vitro, in vivo data argues against a redox modulation in the case of ROXY19. The single cysteine found in the ROXY19-interacting TGA5 (Figure I8 and SI3) could be mutated into a serine without noticeable effect on the activity of the transcription factor [152]. Instead, a hydrophobic ALWL motif at the very C-terminal end of ROXY19 and many other CC-type glutaredoxins (Figure SI2) was shown to mediate repression $[145,168,170]$. When present, this motif enables the interaction with the transcriptional co-repressor TOPLESS (TPL). TPL recruits chromatin modifiers leading to gene silencing [177]. Thus, the recruitment of corepressors might provide a better explanation for a repressive mechanism. Instead of acting as a redox modulator, CC-type glutaredoxins might represent targets of redox control [152]. In support of this idea, the interaction of the Marchantia polymorpha TGA transcription factor MpTGA with the CC-type glutaredoxins MpROXY1 and MpROXY2 appears to be rather stable, as it could be visualized in EMSA experiments. Interestingly, the interaction depends on a reduced ROXY protein and a reduced TGA factor [160].

Still, the ALWL motif is not conserved in all CC-type glutaredoxins found in A. thaliana. ROXY6, 7, 8, 9 and 20 lack this motif, and are not able to repress TGA2 and PAN. Interestingly, ROXY 9 and ROXY8 were shown to repress the activity of TGA1 and TGA4 [145,155-157,168,170]. In this context, Willmer (2014) could show that several CC-type glutaredoxins from $A$. thaliana are able to interact with so-called TIFY proteins [178]. TIFY-proteins are 
characterized by a protein-interaction domain containing a conserved motif of the amino acids TIFY. The transcriptional repressors from jasmonate signaling, the JAZ proteins, are part of the TIFY family $[179,180]$. Several JAZ proteins could interact with CC-type glutaredoxins [178]. JAZ proteins were shown to interact directly or indirectly via NINJA with the corepressor TPL $[179,181]$. This raised the hypothesis of whether CC-type glutaredoxins could recruit TPL via the JAZ proteins to the TGA transcription factors, thereby mediating the repression [178]. 


\section{TGA transcription factors}

\section{The TGA transcription factor family in Arabidopsis thaliana}

TGA transcription factors constitute a group of plant-specific basic leucine zipper (bZIP) transcription factors. In bZIP transcription factors, an $\alpha$-helical domain containing basic amino acids mediates binding to the DNA, whereas the leucine zipper allows dimerization of these transcription factors (Figure I7A). The leucine zipper consists of an $\alpha$-helix in which every seventh amino acid is a leucine. Usually, such a heptad is repeated about nine times. The leucine heptad repeats result in a hydrophobic side of the helix. Contacts between these hydrophobic regions of two monomers leads to dimerization of these transcription factors. Homodimerization, but sometimes also heterodimer formation is possible. Since bZIP transcription factors act as dimers, they usually recognize specifically palindromic DNA sequences [182]. TGA transcription factors, for instance, associate with the palindromic sequence TGACGTCA. The first five basepairs of this sequence are sufficient for binding [183-185]. The bZIP domain in TGA transcription factors is rather short since they harbor only three of the leucine heptad repeats (Figure I7B and SI3). In addition, TGA factors contain an $\mathrm{N}$-terminal extension $(\mathrm{N})$ of variable length. The $\mathrm{N}$-terminal domain of TGA transcription factors mediates at least partially transcriptional regulation [186-189]. C-terminally of the bZIP domain are two glutamine-rich regions (Q1 and Q2) [182,184,190]. Glutaminerich regions usually mediate transcriptional activation [191]. Regarding TGA factors, the precise function of these domains is not yet understood. However, the regions C-terminally of the bZIP domain might contribute to the interaction with transcriptional regulators [170,189,190,192-194].

In $A$. thaliana, there are ten TGA transcription factors (Figure $\mathrm{I} 7 \mathrm{C}$ ). Based on their sequence homology, they are divided into five different clades. Clade I consists of TGA1 and TGA4, clade II contains TGA2, TGA5 and TGA6. TGA3 and TGA7 form clade III. Clade IV is composed of TGA9 and TGA10, while clade V has only one member called PAN ("TGA8") [190]. Each clade 
A

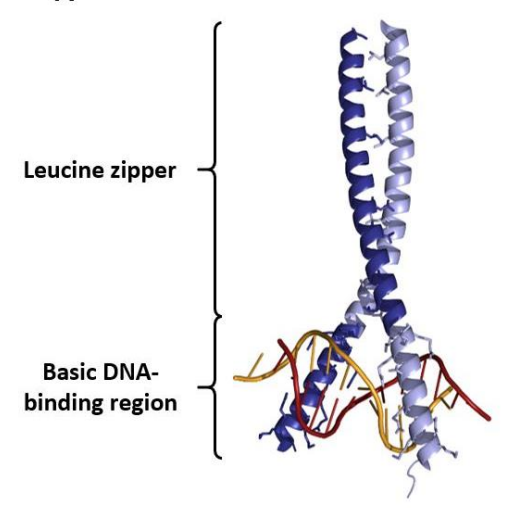

C

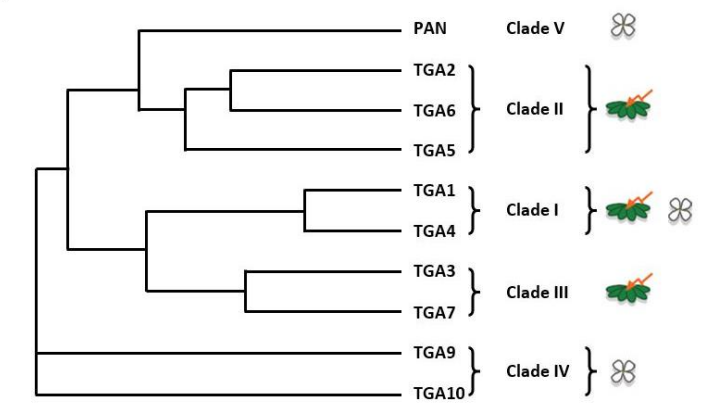

B

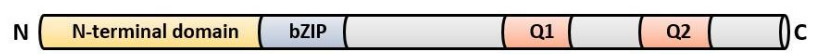

Figure I7. TGA transcription factors are bZIP transcription factors. (A) Structure of a basic leucine zipper (bZIP) transcription factor exemplified by the crystal structure of yeast Pap1 shown as a dimer (blue) bound to DNA (orange and red). The leucine zipper and the DNA binding region of each monomer are shown in different shades of blue. The leucine residues within the leucine zipper domain are pictured as sticks whereas the sticks in the basic DNA binding region represent lysines and arginines. The structure was solved by Fujii et al. (2000) [195] to a resolution of $2 \AA$, and the image created from the PDB file (accession: 1GD2) in PyMOL. (B) Overview of the domain structure of TGA transcription factors. Q1 and Q2 represent the two glutamine-rich regions. (C) Unrooted cladogramm of the TGA transcription factors from $A$. thaliana. The icons behind the names of the TGA factors represent the function of the corresponding class: rosette leaves with lightning - environmental stress response, white flower - flowering. The figure was taken from Gutsche et al. (2015) [80] and modified.

has a different function within the plant. Clade II TGA factors were extensively studied with respect to their function in the plant defense response "systemic acquired resistance". To regulate the defense program against biotrophic pathogens, clade II TGA factors interact with the SA-binding transcriptional regulators NPR1, NPR3 and NPR4 [192,193,196-201]. NPR1 and TGA2 were found to bind constitutively to the promoter of the defense gene PR-1. However, under non-inducing conditions, TGA2 is bound in high-order complexes to the DNA. These complexes are formed via its $\mathrm{N}$-terminal domain and do not interact with NPR1, thereby preventing PR-1 transcription 
[189,202,203]. In parallel, clade II TGA factors interact with the corepressors of defense gene expression, NPR3 and NPR4 [201]. Upon infection by a biotrophic pathogen, salicylic acid is produced by the plant [204] which binds to NPR1, NPR3 and NPR4 [199,200]. On the one hand, SA-binding inhibits the corepressors NPR3 and NPR4 [201]. On the other hand, SA-binding by NPR1 enables the protein to interact with TGA2 to repress the TGA2 Nterminus via its BTB/POZ domain, thereby resolving the high-order TGA2 complexes. An enhanceosome consisting probably of a TGA2 and an NPR1 dimer is formed on the DNA. This enhanceosome activates PR-1 transcription via a C-terminal transactivation domain in NPR1 [189,200,203]. Interestingly, this domain contains two oxidized cysteine residues [203].

The response towards biotrophic pathogens has to be balanced with the defense against necrotrophic pathogens which is controlled by the plant hormones jasmonic acid (JA) and ethylene (ET) [205]. Clade II TGA factors not only activate SA-dependent genes, but also JA/ET-dependent genes. This activation can be repressed by overexpressing the CC-type glutaredoxin ROXY19. Further experiments showed a protein-protein interaction between ROXY19 and clade II TGA factors, as well as activation of ROXY19 transcription by SA. Thus, it was concluded that ROXY19 might mediate the repression of clade II TGA factors during JA/ET signaling. However, loss-offunction evidence for this assumption is still missing [147,168]. Aside from defense responses against pathogens, TGA2, 5 and 6 also mediate the detoxification of xenobiotics. This response is controlled by interaction with a coactivator protein called SCL14 [206]. In addition, ectopically expressed ROXY19 acts again as an inhibitor of clade II TGA factors in the induction of the detoxification program [152].

The clade IV and V TGA factors - TGA9, TGA10, and PAN - have been associated with flower development. PAN restricts the number of petals in the flower of $A$. thaliana to four [207]. Since mutation of ROXY1 leads to less than four petals and since ROXY1 interacts with PAN, it might repress PAN during petal development. Interestingly, a cysteine in the C-terminus of PAN is required for PAN function and its $\mathrm{N}$-terminus contains several cysteines sensitive to oxidative modifications. Thus, it was speculated about a potential redox control of PAN by ROXY1. However, as outlined before, functional analysis of ROXY1 active site mutants provided conflicting results regarding this assumption [145,146,172,175]. TGA9 and 10 control anther development and are therefore indespensable for male fertility. Interestingly, they are not only regulated by ROXY1, but also by ROXY2 [169]. Recently, TGA9 and 
TGA10 were linked with defense responses against pathogens; however, coregulators have not yet been identified [208].

Knowledge about clade III TGA factors is relatively scarce. TGA3 interacts strongly with NPR1 and participates with clade II TGA factors in pathogen defense [193,209]. Its role in immunity has repeatedly been observed [210-214]. In addition, it plays a role in heavy metal tolerance [215,216]. TGA3 interacts with some CC-type glutaredoxins [145,170]; [M. Willmer, unpublished]. Moreover, it is constitutively bound to the ROXY19 promoter and, in addition to clade II transcription factors, contributes to the activation of ROXY19 transcription after SA treatment [151]. Like TGA3, TGA7 interacts with NPR1 and CC-type glutaredoxins [145,217,218] [M. Willmer, unpublished]. The interaction with NPR1 enhances the DNA-binding activity of TGA7 in vitro, The DNA fragments used for this study included elements of the PR-1 promoter. However, a function of TGA7 in defense gene expression remains to be demonstrated [218].

For clade I TGA transcription factors, first, a function in basal resistance was observed. Basal resistance includes the sensing of and initial defense responses to a pathogen in order to prevent its spread. Examples for such initial defense responses are cell wall reinforcement and the production of ROS and toxic proteins $[219,220]$. Both, TGA1 and TGA4, control this process redundantly [221]. The tga 1 tga4 double mutant is defective in callose deposition and the production of PR genes, though these processes could not be linked to transcriptional events [222]. TGA1 and TGA4 regulate only a small number of genes involved in defense and operate mainly independently of NPR1 [223]. Still, the interaction between clade I TGA factors and NPR1 was proposed to depend on the redox state of the TGA factors [224]. TGA1 contains four conserved cysteine residues located in or around the glutatmine-rich regions (C172, C260, C266 and C287, Figure I8 and SI3). Després et al. showed in 2003 that the pool of TGA1 and TGA4 in A. thaliana cells is partly reduced and 
Introduction

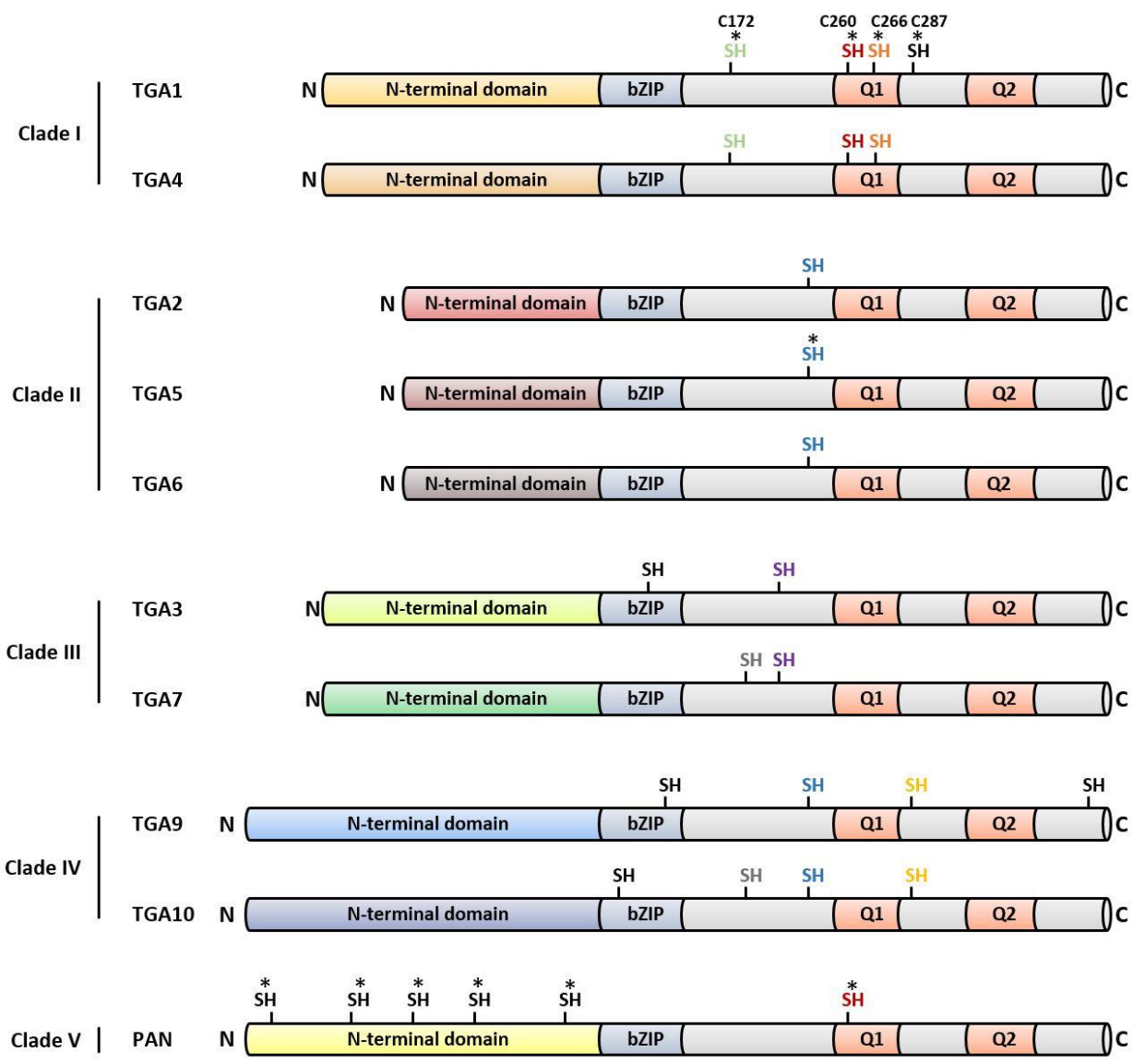

Figure I8. Conserved cysteine residues in TGA transcription factors from $\boldsymbol{A}$. thaliana. All cysteine residues found in the 10 TGA factors from $A$. thaliana are indicated by thiol groups (-SH) along the domain structure of the proteins. Cysteine residues conserved among different TGA factors are indicated by the same colours. Residues for which a redox modification or a function in regulating the activity of the TGA factors has been speculated or investigated, are marked by asterisks. The schemes of the TGA factors were grouped according to their clades and numbering. A detailed alignment highlighting all cysteine residues in the'TGA transcription factors is shown in Figure SI3. For TGA1, the positions of the cysteine residues are indicated above the thiol groups. The scheme was taken from Gatz (2012) [190] and modified. 
partly oxidized. Upon treatment with salicylic acid, the pool shifts completely to the reduced state. Further experiments involving in vitro translated TGA1 treated with the reducing agent $\beta$-mercaptoethanol or left in its oxidized state suggested the formation of a disulfide bridge between C260 and C266. This disulfide bridge might be present under non-inducing conditions, preventing the interaction with NPR1. According to the model, reduction of the disulfide bridge leads to the interaction with NPR1 and subsequent enhancement of the TGA1 DNA-binding activity [224]. However, because the overlap between SAinduced genes regulated by TGA1 and NPR1 is rather small, this redox regulation was speculated to affect the transcription of only a small subset of genes [223]. A few years later, Lindermayr et al. (2010) examined the reactivity of the TGA1 cysteine residues towards nitrosoglutathione (GSNO). GSNO occurs naturally in cells, as it arises from the reaction of glutathione with NO. It can act as both, a nitrosyl donor and as a glutathionyl donor [225,226]. In contrast to the study by Després et al., recombinant TGA1 was found to contain a disulfide bridge between cysteines 172 and 287 after purification and when treated with hydrogen peroxide in vitro. In both cases, this disulfide bridge was broken by reaction with GSNO. While C172 can be glutathionylated, C260 and C266 can be glutathionylated and nitrosylated. C287 was only modified when high concentrations of GSNO were used. The reaction with GSNO enhanced the DNA binding of TGA1 even in the absence of NPR1 [32]. However, phenotypic evidence for a redox-based regulation of TGA1 and/or TGA4 in innate immunity is still lacking [32]. Despite this and the discrepancies regarding the location of the disulfide bridge in TGA1 between the mentioned studies, Després et al. (2003) and Lindermayr et al. (2010) show that the redox state of the cysteine residues in TGA1 changes in vivo, as well as in vitro.

\section{Connections between clade I TGA transcription factors and CC-type glutaredoxins}

Beside their function in response to biotic stress [157,159,221], TGA1 and TGA4 also regulate hyponastic growth $[155,156]$, a response towards low light conditions, flooding or heat. Normally, the rosette leaves of $A$. thaliana are spread flat on the soil. Since in this state, $A$. thaliana is sensitive to the aforementioned environmental stresses, it raises its leaves in order to reach 
regions of stronger light or to allow aeration of the rosette leaves [227]. The increase in the petiole angle during hyponastic growth is caused by an asymmetric cell growth: Since cells at the abaxial side expand more strongly than at the adaxial side of the petiole, the petiole bends upwards [228].

So far, the control of hyponastic growth by TGA1 and TGA4 has not yet been correlated with co-activators regulating clade I TGA factors directly, but recent work established a connection to the CC-type glutaredoxin ROXY9 $[155,156]$. ROXY9 interacts directly with TGA1 and TGA4 [156,157]. As mentioned before, it belongs to the CC-type glutaredoxins lacking the ALWL motif $[145,168,170]$. ROXY9 can repress low light-induced hyponastic growth. So far, its function in hyponastic growth has only been confirmed by analyzing overexpression lines of this glutaredoxin. A roxy9 knock-out mutant does not differ from the wildtype regarding hyponastic growth $[155,156]$. However, this effect might be caused by the existence of redundant proteins. ROXY9 is most closely related to ROXY8, 7 and 6, all of them lacking the ALWL motif. In ROXY20, the ALWL motif is replaced by a non-functional ALWA sequence $[145,168,170]$. In addition, ROXY8 exerts the same repressive effect on hyponasty when overexpressed. Thus, some or all these CC-type glutaredoxins might be redundant with ROXY9. This assumption is further supported by the observation, that all these ROXYs as well as ROXY20 undergo changes in expression similar to that of ROXY9 during hyponastic growth $[155,156]$ : Under control light conditions of 100 to $120 \mu \mathrm{mol} \mathrm{s} \mathrm{m}^{-2}$, ROXY9 transcript levels are low. They decrease even further when $A$. thaliana encouters low light conditions. Interestingly, upon backshift of low light-treated plants to control light conditions, ROXY9 transcription is strongly induced. This induction is mediated by TGA1 and TGA4.

In support of the connection between clade I TGA factors and ROXY9, an overlap between the genes repressed by ROXY9 and activated by TGA1 and TGA4 during hyponastic growth has been observed. These genes included auxin-dependent genes and genes for cell growth. Altogether, these results suggested, that TGA1 and TGA4 activate hyponastic growth during low light by inducing cell growth at the abaxial side of the petiole. When normal light conditions return, TGA1 and TGA4 induce strongly ROXY9 transcription, thereby producing their own repressor in a negative feedback loop. Repression of TGA1 and TGA4-regulated genes by ROXY9 causes the leaves to resume their usual horizontal position $[155,156]$.

To repress hyponastic growth, ROXY9 requires its active center composed of the amino acid sequence CCLC. The CC motif, but not the C-terminal active 
site cysteine, are indispensable for repression by ROXY9. The C-terminal end of the protein comprising the amino acids SILY does not influence its repressive mechanism [155]. Since ROXY9 cannot interact with TPL [168,177] and since TGA1 might be redox-regulated [32,224], the assumption that ROXY9 might change the redox state of the TGA1 cysteines was investigated. However, complementation analysis of the tga 1 tga 4 mutant suggested that the two central cysteines in TGA1 (C260 and C266) were not necessary for controlling hyponastic growth [155].

Motivated by the finding of the interaction of clade I TGA factors and ROXY9, the involvement of TGA1 and TGA4 in innate immunity was revisited regarding a functional correlation to ROXY9 [159]: TGA1 and TGA4 have been shown to control the expression of defense genes early after infection or induction by SA, but not at later timepoints [159,223]. Interestingly, a similar observation was also made for plants overexpressing ROXY9, further supporting the functional relationship between both proteins [159].

In addition to their function in biotic and abiotic stresses, TGA1 and TGA4 are involved in plant development: Flowering is delayed in the tga 1 tga4 double mutant and its leaves are curled and arranged in a windmill-like fashion [223]. Both phenotypes also occur in ROXY9 overexpression lines. Although the delay in flowering of the tga 1 tga 4 mutant could not be observed by Muthreich (2014), a cross between the tga 1 tga4 mutant and ROXY9 overexpression plants showed that the delay in flowering seen for ROXY9 overexpression depends on clade I TGA factors [157].

Analysis of hyponastic growth and flowering provides direct links between ROXY9 and clade I TGA factors. However, an additional function of clade I transcription factors in nitrate-dependent root growth is reminiscent of functions reported for CC-type glutaredoxins [154,166,229,230]:

Transcription of TGA1 and TGA4 is induced in roots by nitrate [229]. Both transcription factors regulate the expression of almost all nitrate-responsive genes. The nitrate uptake systems NRT2.1 and NRT2.2, however, are only weakly induced by TGA1 and TGA4. Consistent with this, nitrate uptake is not affected in the tga 1 tga 4 double mutant. Instead, clade I TGA factors activate primary and lateral root growth in response to nitrate [229]. In addition, they control root hair development [230]. Interestingly, ROXY9 was recently identified as a mobile protein involved in controlling the root growth on soil with unevenly distributed nitrogen sources: Roots growing in soil containing too low amounts of nitrogen produce a peptide hormone traveling to the shoot. 
This peptide hormone induces the transcription of ROXY9 and its paralogue ROXY6 in the leaves. Both, ROXY9 and ROXY6, are transported via the phloem to the roots in those regions of the soil rich in nitrogen. This serves to activate NRT2.1 [154]. This activation of NRT2.1 is, however, in contrast to the repressive effect of ROXY9 on clade I TGA factors observed for other functions of the two proteins [155-157,159]. Aside from ROXY9, the CC-type glutaredoxins ROXY11, 12, 13 and 15 have been associated with root growth in response to nitrate [166,231]. These four CC-type glutaredoxins contain an ALWL motif $[145,168,170]$ and are encoded in a cluster on chromosome 4 $[136,166,231]$. Their transcription is induced by nitrate [166]. In soil rich in nitrate, they enhance lateral root growth but limit primary root growth, in order to increase the surface needed for the uptake of nitrate [166,231]. Interestingly, ROXY11, 12, 13 and 15 were shown to be transcriptionally regulated under basal conditions by clade I TGA factors and ROXY9. While the four cluster ROXYs are repressed by TGA1 and TGA4, their transcription is activated by ROXY9 [155,156]. 


\section{Aim of this thesis}

The aim of this study was to find out whether the peculiar CCLCY active site of the CC-type glutaredoxin ROXY9 allows the catalysis of redox reactions or enables the binding of an iron-sulfur cluster for the formation of iron-sulfur cluster-dependent protein complexes. For this, the following experiments were set up:

- The function of overexpressed mutant variants of ROXY9 carrying a CCLCA or CPYC active site should be examined in planta.

- Recombinant ROXY9 should be produced to analyze its catalytic activity in standard glutaredoxin activity assays and its ability to bind iron-sulfur clusters by in vitro reconstitution experiments.

- The ROXY9 interaction partner TGA1 should be mutated in its four cysteine residues and the functionality of such a protein in vivo should be analyzed. 
Methods 


\section{Work with organisms}

\section{Cultivation of Escherichia coli}

E. coli was grown on Luria-Bertani (LB) plates or in LB or dYT broth. If applicable, the medium was supplied with the required antibiotic. For liquid cultures, flasks or test tubes were used. The cultures were incubated at $37^{\circ} \mathrm{C}$, shaking at $220 \mathrm{rpm}$. Plates were incubated over night $(\mathrm{ON})$ at $37^{\circ} \mathrm{C}$ and stored at $4^{\circ} \mathrm{C}$.

Cryo-cultures of E. coli were prepared by either mixing $100 \mu \mathrm{DMSO}$ with $900 \mu \mathrm{l}$ of an overnight culture, or by mixing an aliquot of an overnight culture with glycerol to a final concentration of $15 \%$ glycerol. Alternatively, cells were scraped off from a plate and transferred to $15 \%$ glycerol in LB. Both, DMSO and glycerol stocks were thoroughly mixed, frozen in $\mathrm{N}_{2}(\mathrm{l})$ and stored at $-70^{\circ} \mathrm{C}$.

\section{LB medium}

\begin{tabular}{lc} 
Component & Concentration \\
\hline Tryptone/peptone from casein & $1 \%(\mathrm{w} / \mathrm{v})$ \\
Yeast extract & $0.5 \%(\mathrm{w} / \mathrm{v})$ \\
$\mathrm{NaCl}$ & $1 \%(\mathrm{w} / \mathrm{v})$ \\
\hline
\end{tabular}

For plates, $1.2 \%(\mathrm{w} / \mathrm{v})$ agar was added.

The medium was autoclaved.

\section{dYT medium}

\begin{tabular}{lc} 
Component & Concentration \\
\hline Tryptone/peptone from caseine & $1.6 \%(\mathrm{w} / \mathrm{v})$ \\
Yeast extract & $1 \%(\mathrm{w} / \mathrm{v})$ \\
$\mathrm{NaCl}$ & $0.5 \%(\mathrm{w} / \mathrm{v})$ \\
\hline
\end{tabular}

The $\mathrm{pH}$ was adjusted to 7.0 to 7.2 using $1 \mathrm{M} \mathrm{NaOH}$.

The medium was autoclaved. 
Antibiotic concentrations used to select for $E$. coli transformants

\begin{tabular}{llcc}
\hline Antibiotic & Solvent & $\begin{array}{c}\text { Stock } \\
\text { concentration }\end{array}$ & $\begin{array}{c}\text { Selective } \\
\text { concentration }\end{array}$ \\
\hline Ampicillin & $\mathrm{dH}_{2} \mathrm{O}$ & $100 \mathrm{mg} / \mathrm{ml}$ & 100 to $200 \mu \mathrm{g} / \mathrm{ml}$ \\
Spectinomycin & $\mathrm{dH}_{2} \mathrm{O}$ & $50 \mathrm{mg} / \mathrm{ml}$ & $100 \mu \mathrm{g} / \mathrm{ml}$ \\
Gentamicin & $\mathrm{dH}_{2} \mathrm{O}$ & $25 \mathrm{mg} / \mathrm{ml}$ & $25 \mu \mathrm{g} / \mathrm{ml}$ \\
\hline
\end{tabular}

\section{Preparation of chemically competent E. coli cells}

To prepare chemically competent E. coli cells, a modified version of the protocol by Inoue et al. (1990) [232] was applied.

First, a single colony was inoculated in $5 \mathrm{ml} \mathrm{SOC}+$ and incubated over day at $37^{\circ} \mathrm{C} .2 \mathrm{ml}$ of this preculture were used to inoculate $300 \mathrm{ml} \mathrm{SOC}+$ medium. The cells were grown at $18^{\circ} \mathrm{C}$ over night until an optical densitiy at $600 \mathrm{~nm}$ $\left(\mathrm{OD}_{600 \mathrm{~nm}}\right)$ of 0.5 was reached. The culture flask was placed in an ice-waterbath for 10 min prior to distributing the culture to six sterile $50 \mathrm{ml}$ falcon tubes. The cells were harvested by centrifugation at $4000 \mathrm{rpm}$ at $4^{\circ} \mathrm{C}$ for $10 \mathrm{~min}$. The supernatant was poured off and $15 \mathrm{ml}$ ice-cold Inoue transformation buffer were added to each tube. The cells were resuspended gently in the buffer. The suspensions from three tubes each were pooled, resulting in two tubes with $45 \mathrm{ml}$ suspension. The cells were spun down at $4000 \mathrm{rpm}$ for $10 \mathrm{~min}$ at $4^{\circ} \mathrm{C}$. The supernatant was decanted, and the remaining buffer was removed by placing the tubes top-down on paper towels. Each pellet was carefully resuspended in $15 \mathrm{ml}$ ice-cold Inoue transformation buffer. $1050 \mu \mathrm{l}$ DMSO were added and mixed with the suspension by swirling. The suspension was stored on ice for $10 \mathrm{~min} .200 \mu \mathrm{l}$ aliquots of the suspensions were distributed to pre-cooled sterile reaction tubes. The aliquots were immediately frozen in liquid nitrogen and stored at $-70^{\circ} \mathrm{C}$ until transformation. 


\section{SOC medium}

\begin{tabular}{lc} 
Component & Concentration \\
\hline Trypton/Peptone from caseine & $2 \%(\mathrm{w} / \mathrm{v})$ \\
Yeast extract & $0.5 \%(\mathrm{w} / \mathrm{v})$ \\
$\mathrm{NaCl}$ & $10 \mathrm{mM}$ \\
$\mathrm{KCl}$ & $2.5 \mathrm{mM}$ \\
\hline
\end{tabular}

The solution was filled up with $\mathrm{dH}_{2} \mathrm{O}$ to the final volume and autoclaved for 10 min at $121^{\circ} \mathrm{C}$. After cooling down, SOC medium was completed to obtain $\mathrm{SOC}+$. For this, $\mathrm{MgCl}_{2}$ and $\mathrm{MgSO}_{4}$ were added to a final concentration of $10 \mathrm{mM}$ each. Additionally, the medium was supplied with glucose to a final concentration of $20 \mathrm{mM}$. To avoid large changes in the volume, 1000x stock solutions of $\mathrm{MgCl}_{2}, \mathrm{MgSO}_{4}$, and glucose were used.

\section{Transformation buffer}

\begin{tabular}{lc} 
Component & Concentration \\
\hline PIPES pH 6.7 & $10 \mathrm{mM}$ \\
$\mathrm{KCl}$ & $250 \mathrm{mM}$ \\
$\mathrm{CaCl}_{2} \times 2 \mathrm{H}_{2} \mathrm{O}$ & $15 \mathrm{mM}$ \\
$\mathrm{MnCl}_{2} \times 4 \mathrm{H}_{2} \mathrm{O}$ & $55 \mathrm{mM}$ \\
\hline
\end{tabular}

$\mathrm{CaCl}_{2}, \mathrm{KCl}$ and PIPES were dissolved in $\mathrm{H}_{2} \mathrm{O}$ to $96 \%$ of the final volume. The $\mathrm{pH}$ was adjusted with $1 \mathrm{M} \mathrm{KOH}$. Afterwards, $\mathrm{MnCl}_{2}$ was added from a $2 \mathrm{M}$ stock solution and the solution filled up to the final volume. The buffer was filtered through a wetted bottle top filter $(0.45 \mu \mathrm{m}$ pore size $)$ into sterile bottles.

\section{Preparation of fresh chemically competent E. coli cells using $\mathrm{CaCl}_{2}$}

To prepare fresh competent cells, the desired E. coli strain was inoculated in LB media and incubated over night at $37^{\circ} \mathrm{C}$, shaking at $220 \mathrm{rpm}$. The next day, the $\mathrm{OD}_{600 \mathrm{~nm}}$ was measured and $10 \mathrm{ml} \mathrm{LB}$ inoculated to an $\mathrm{OD}_{600 \mathrm{~nm}}$ of 0.1 . This main culture was incubated at $37^{\circ} \mathrm{C}$ and $220 \mathrm{rpm}$ until it reached an $\mathrm{OD}_{600 \mathrm{~nm}}$ of 0.3 . The cells were harvested by centrifugation at $4000 \mathrm{rpm}$ at $4^{\circ} \mathrm{C}$ for $5 \mathrm{~min}$ in sterile tubes. The media was discarded, and the pellet resuspended in $10 \mathrm{ml}$ 
sterile $50 \mathrm{mM} \mathrm{CaCl}_{2}$. The cells were incubated on ice for ca. $30 \mathrm{~min}$ and then spun down again (4000 rpm, $4^{\circ} \mathrm{C}, 5 \mathrm{~min}$ ). The supernatant was removed, and the cells resuspended in ca. $1 \mathrm{ml} 50 \mathrm{mM} \mathrm{CaCl}$. $100 \mu \mathrm{l}$ aliquots of this suspension were used for transformation following the usual protocol.

\section{Reactivation of chemically competent E. coli using $\mathrm{CaCl}_{2}$}

When chemically competent BL21 cells started to lose their competence, the cells were made competent again by incubating them in $\mathrm{CaCl}_{2}$. The cell aliquot was first thawed on ice. Once thawed, it was spun down briefly, the supernatant was removed, and the cell pellet was resuspended in $1 \mathrm{ml}$ sterile $50 \mathrm{mM} \mathrm{CaCl}_{2}$ solution. The cells were incubated for ca. 30 to $60 \mathrm{~min}$ on ice. After brief centrifugation, the supernatant was removed. The cells were resuspended in

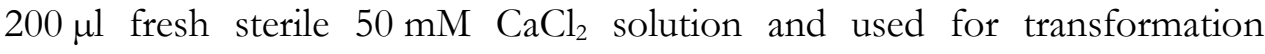
according to the standard protocol.

\section{Transformation of chemically competent E. coli cells}

Aliquots of chemically competent E. coli cells were thawed on ice. For transformation, an aliquot was mixed with a small amount of the desired plasmid, ligation mix or ligation-indepenendet cloning (LIC) mix. As a negative control, another aliquot of the competent E. coli cells was left untreated. Next, the samples were incubated on ice for ca. $30 \mathrm{~min}$. Then, the cells were heatshocked at $42^{\circ} \mathrm{C}$ for ca. $1.5 \mathrm{~min}$ and cooled down on ice for ca. $5 \mathrm{~min} .800 \mu \mathrm{l}$ $\mathrm{LB}$ or $\mathrm{dY}$ T medium were added and the cells incubated for at least $45 \mathrm{~min}$ at $37^{\circ} \mathrm{C}$, shaking. Afterwards, the cells were spun down briefly and the supernatant was decanted. The remaining drop of supernatant was used to resuspend the cell pellet. The suspension was plated on LB plates supplied with the required antibiotic. The plates were incubated overnight at $37^{\circ} \mathrm{C}$. 


\section{Cultivation of Agrobacterium tumefaciens}

Agrobacterium tumefaciens (A. tumefaciens) was cultivated in YEB medium or streaked out on YEB plates. Broth culures were incubated at $29^{\circ} \mathrm{C}$ for one or two days, shaking them at $220 \mathrm{rpm}$. Plates were incubated at $29^{\circ} \mathrm{C}$ for one or two days. If necessary, the YEB medium was supplemented with antibiotics.

\section{YEB medium}

\begin{tabular}{lc} 
Component & Concentration \\
\hline Meat extract & $1 \%(\mathrm{w} / \mathrm{v})$ \\
Yeast extract & $0.2 \%(\mathrm{w} / \mathrm{v})$ \\
Tryptone/Peptone from caseine & $0.5 \%(\mathrm{w} / \mathrm{v})$ \\
Sucrose & $0.5 \%(\mathrm{w} / \mathrm{v})$ \\
\hline
\end{tabular}

The $\mathrm{pH}$ was adjusted to 7.0 using $1 \mathrm{M} \mathrm{NaOH}$. For plates, $1.2 \%(\mathrm{w} / \mathrm{v})$ agar was added.

The medium was autoclaved. After autoclaving, sterile $\mathrm{MgSO}_{4}$ was added to a final concentration of $2 \mathrm{mM}$.

\section{Antibiotic concentrations used to select for A.tumefaciens transformants}

\begin{tabular}{lccc}
\hline Antibiotic & Solvent & $\begin{array}{c}\text { Stock } \\
\text { concentration }\end{array}$ & $\begin{array}{c}\text { Selective } \\
\text { concentration }\end{array}$ \\
\hline Rifampicin & $\begin{array}{c}\mathrm{dH}_{2} \mathrm{O} \\
\text { acidified } \\
\text { with } \mathrm{HCl}\end{array}$ & $10 \mathrm{mg} / \mathrm{ml}$ & $50 \mu \mathrm{g} / \mathrm{ml}$ \\
Spectinomycin & $\mathrm{dH}_{2} \mathrm{O}$ & $50 \mathrm{mg} / \mathrm{ml}$ & $100 \mu \mathrm{g} / \mathrm{ml}$ \\
Gentamicin & $\mathrm{dH}_{2} \mathrm{O}$ & $25 \mathrm{mg} / \mathrm{ml}$ & $25 \mu \mathrm{g} / \mathrm{ml}$ \\
\hline
\end{tabular}

\section{Preparation of electro-competent $A$. tumefaciens cells}

To prepare electrocompetent $A$. tumefaciens GV3101 cells, the strain was grown in $400 \mathrm{ml} \mathrm{YEB}+$ in a 21 flask for two days at $29^{\circ} \mathrm{C}$, shaking at $220 \mathrm{rpm}$. The culture was distributed to $50 \mathrm{ml}$ falcon tubes. The tubes were centrifuged at 
$4000 \mathrm{rpm}$ to harvest the cells. The supernatant was discarded, and each pellet was washed three times with $\mathrm{dH}_{2} \mathrm{O}$ and finally with $15 \%$ glycerol. After the last wash step, the cell pellet was resuspended in 1 to $3 \mathrm{ml} 15 \%$ glycerol. The cell suspensions were pooled. Finally, $300 \mu \mathrm{l}$ aliquots were distributed to reaction tubes and frozen in liquid nitrogen. The cell aliquots were stored at $70^{\circ} \mathrm{C}$ until transformation.

\section{Transformation of $A$. tumefaciens}

Aliquots of electro-competent $A$. tumefaciens cells were thawed on ice and mixed with 100 ng plasmid DNA. To include a negative control, one cell aliquot underwent the transformation procedure without addition of DNA. The cells were incubated on ice for at least $30 \mathrm{~min}$. Subsequently, the cells were transferred to sterilized cuvettes and subjected to an electric pulse $(2.5 \mathrm{kV}$, $200 \Omega, 25 \mu \mathrm{F})$. Imediately after the electroshock, the cells were mixed with $1 \mathrm{ml}$ YEB medium and transferred back to the reaction tubes. The samples were incubated for $2 \mathrm{~h}$ at $29^{\circ} \mathrm{C}$ on a shaker. Finally, the cells were centrifuged. After decanting the supernatant, the cells were resuspended in the remaining drop of liquid and plated on selective plates. The plates were incubated for two days at $29^{\circ} \mathrm{C}$.

\section{Cultivation of Arabidopsis thaliana}

A. thaliana seeds were surface-sterilized in a chlorine gas atmosphere in a dessicator. For this, $50 \mathrm{ml}$ of a $12 \%$ sodium hypochlorite solution were mixed with $2.5 \mathrm{ml} 32 \% \mathrm{HCl}$ in a jar placed in the dessicator together with reaction tubes containing the seeds for sterilization. For storage of surface-sterilized seeds, the chlorine gas was allowed to evaporate from the tubes for approximately 30 to $60 \mathrm{~min}$.

A. thaliana was grown on soil (Fruhstorpfer Topferde Typ T Struktur 1 fein) in round pots (diameter: $6 \mathrm{~cm}$ ) or square pots $(9 \mathrm{~cm} \times 9 \mathrm{~cm})$. For this, seeds were surface-sterilized for at least $2 \mathrm{~h}$ using chlorine gas, placed on the soil surface 
and moistened with tab water. Subsequently, the sown seeds were stratified in the cold room over night or up to a week. After stratification, the pots were transferred to climate chambers or Percival incubators and cultivated under the indicated fluence rate, day length, temperature and humidity.

To grow $A$. thaliana on Murashige-Skoog (MS) plates, seeds were sterilized for at least $5 \mathrm{~h}$ using chlorine gas. The clorine gas was allowed to evaporate under a clean bench. The seeds were placed on the plate surface using a sterile tooth pick. Alternatively, the sterizlized seeds were resuspended in $0.1 \%(\mathrm{w} / \mathrm{v})$ plant agar in $\mathrm{dH}_{2} \mathrm{O}$ and transferred to the plate with the help of a $200 \mu \mathrm{l}$ pipette using autoclaved cut tips.

\section{Transformation of $A$. thatiana and generation of stably transformed plants}

\section{Transformation}

A. thaliana was transformed using the flower dip method [233]. Per T-DNA to be transformed, approximatley ten square pots with nine $A$. thaliana plants each were grown under long day conditions $(16 \mathrm{~h}$ light with $60 \%$ humidity at $22^{\circ} \mathrm{C} / 8 \mathrm{~h}$ dark with $60 \%$ humidiy at $18^{\circ} \mathrm{C}$, fluence rate: 100 to $120 \mu \mathrm{mol} \cdot \mathrm{m}^{-2} \cdot \mathrm{s}^{-1}$ ) until flowering. Alternatively, approximately four seeds were sown on round pots. In this case, approximately 15 pots were needed for the transformation of a single T-DNA. Optionally, once the plants started to flower, the first flowers were cut off to allow the formation of more floral stems.

When the $A$. thaliana plants were flowering, cultures of $A$. tumefaciens GV3101 transformed with the desired T-DNA vector were prepared. First, a preculture was prepared. For this, 10 to $20 \mathrm{ml}$ YEB medium with antibiotics in $100 \mathrm{ml}$ flasks were inoculated from plates and grown at $29^{\circ} \mathrm{C}$, shaking them at $220 \mathrm{rpm}$ for one to two days. 8 to $10 \mathrm{ml}$ of these pre-cultures were used to inoculate 300 to $400 \mathrm{ml}$ main cultures in 2 1-flasks. After one or two days at $29^{\circ} \mathrm{C}$ and $220 \mathrm{rpm}$, the cells were harvested by centrifugation at $5000 \mathrm{rpm}$ for $10 \mathrm{~min}$ at room temperature (RT), using a Sorvall RC6+ centrifuge (Rotor SLA-3000). The supernatant was discarded, and the cell pellet resuspended in ca. $40 \mathrm{ml}$ sucrose solution. Next, the $\mathrm{OD}_{600 \mathrm{~nm}}$ of these cell suspensions was determined, 
and $500 \mathrm{ml}$ cell suspensions of an $\mathrm{OD}_{600 \mathrm{~nm}}=0.8$ were prepared in beakers. The flowers of the $A$. thaliana plants were dipped into the cell suspension for approximately 10 to $20 \mathrm{sec}$. Finally, the dipped plants were covered with a plastic hood and incubated overnight under long day conditions. Afterwards, the hoods were removed, and the plants cultivated until seed formation was finished.

\section{T1 genereation}

The seeds of the dipped plants were collected and an appropriate number of them was sown on soil. The plants (T1 generation) were grown for approximately one week under long day conditions in a climate chamber. During the second week, the plants were sprayed two to three times with Basta solution to select for transformants. Transformants were then transferred to individual pots and cultivated further in a long day climate chamber. Optionally, before or during the onset of flowering, leaf material for a pre-characterization of the plants by Western Blot or qPCR was taken. With this precharacterization, the expression of the transgene was checked. As soon as they started to form floral stems, the plants were covered with ARACONs (Betatech) to allow self-fertilization only. The seeds of the individual plants were harvested.

\section{T2 generation}

Seeds of T1 plants ( $\mathrm{T} 2$ generation) expressing the transgene were used to test whether the mother plant had obtained only a single T-DNA or more T-DNAs during transformation. For this, seeds of the T1 generation were subjected to selection via the Basta-active substance phosphinotricine (PTT). About 100 plants were sown on $2 \mathrm{MS}$ plates containing PTT. The plates were incubated in a $14 \mathrm{~h}$ light/10 h dark regime in a climate chamber without control of air humidity. The fluence rate was $120 \mu \mathrm{mol} \cdot \mathrm{m}^{-2} \cdot \mathrm{s}^{-1}$. As a negative control, a PTTsensitive plant was included. A PT'T-resistant plant was used as a positive control. After 10 to 14 days, the percentage of PTT surviving T2 plants and the rate of PTT-sensitive T2 plants was determined by counting. Roughly $75 \%$ of surviving T2 plants indicated a single T-DNA insertion, whereas roughly $93 \%$ of survivors suggested the insertion of two T-DNAs. 
Optionally, if not done in the T1 genration, a mixture of T2 plants was used for a pre-characterization regarding expression of the transgene. For this, approximately 30 seeds of a single T1 plant were sown on a single pot on soil and harvested after 3 weeks for a pre-characterization by qRT-PCR or Western Blot to detect expression of the transgene. Those plants expressing the transgene were selected for addressing the question of the number of T-DNAs present in the mother plant, following the procedure described above.

All plants showing expression of the transgene from a single T-DNA were selected for propagation. For this, 20 to 40 seeds were sown on individual pots and the plants grown under long day conditions. Once floral stems were visible, the plants were covered with ARACONs for self-fertilization. Finally, the ripe seeds were harvested.

\section{T3 generation}

The T2 generation contains homozygous plants. To identify homozygous plants, the seeds of the T2 generation (T3 generation) were harvested. Approximately 100 seeds per plant were sown on 2MS plates for PTT selection as described for the T2 generation. In case of a T2 plant homozygous for the transgene, all decendants of this plant should be resistant to PT'T. A T2 plant heterozygous for the transgene should only produce $75 \%$ resistant decendants. Loss of the transgene in a T2 plant would result only in plants sensitive to PTT. Seeds from homozygous transgenic plants were sown on soil for futher propagation and/or directly used for experiments. Seeds from plants in which the transgene was lost during segregation after self-fertilization were kept as a control for experiments.

\section{Sucrose solution}

\begin{tabular}{lc} 
Component & Concentration \\
\hline Sucrose & $5 \%(\mathrm{w} / \mathrm{v})$ \\
Silwet L77 & $0.02 \%(\mathrm{v} / \mathrm{v})$ \\
\hline
\end{tabular}

Sucrose solution was always prepared freshly. 


\section{Basta solution}

\begin{tabular}{lc} 
Component & Concentration \\
\hline Silwet L77 & $0.02 \%(\mathrm{v} / \mathrm{v})$ \\
Basta & $0.2 \%(\mathrm{v} / \mathrm{v})$ \\
\hline
\end{tabular}

Basta solution was pepared using tab water and stored at $4^{\circ} \mathrm{C}$.

2MS plates

\begin{tabular}{lc} 
Component & Concentration \\
\hline MS & $0.44 \%(w / v)$ \\
Sucrose & $2 \%(w / v)$ \\
\hline
\end{tabular}

The $\mathrm{pH}$ of the solution was adjusted to $5.7 \mathrm{using} \mathrm{KOH}$. Then, $0.68 \%(\mathrm{w} / \mathrm{v})$ plant agar were added and the mixture autoclaved. After autoclaving, the solution was allowed to cool down and PTT was added to a final

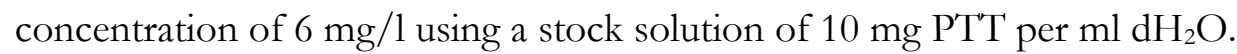

\section{Hyponastic growth experiment}

Hyponastic growth experiments were carried out as described previously $[155,156]$. Briefly, plants were grown on individual pots at $12 \mathrm{~h}$ light $/ 12 \mathrm{~h}$ dark cycle at a fluence rate of 100 to $120 \mu \mathrm{mol} \cdot \mathrm{m}^{-2} \cdot \mathrm{s}^{-1}$ and $60 \%$ humidity during both, day and night. After approximately 28 days, the plants were subjected to low light (LL) treatment to induce hyponasty. For this, the plants were splitted into three groups with ca. 10 plants per genotype: One group served as a control and was therefore incubated under control light (CL) conditions (fluence rate: $\left.100-120 \mu \mathrm{mol} \cdot \mathrm{m}^{-2} \cdot \mathrm{s}^{-1}\right)$ throughout the entire experiment. The second group was used to analyze the onset of hyponasty; these plants were placed in low light (fluence rate: 10 to $20 \mu \mathrm{mol} \cdot \mathrm{m}^{-2} \cdot \mathrm{s}^{-1}$ ) 1.5 hours after beginning of the photoperiod. They were kept under these conditions until 7.5 hours after onset of the photoperiod on the next day had passed. Using the third group, the reversal of the hyponastic response was assessed. For this, the third group of plants was first treated with low light, starting 1.5 hours after beginning of the photoperiod until the onset of night. Shortly after the onset of the next photoperiod, the plants were placed again in control light conditions for $7.5 \mathrm{~h}$. 
Finally, the plants were photographed in the side view using a Canon PowerShot G11 camera and the angle of the petiole of the seventh or eighth leaf measured using the angle tool in Image 1.x [234].

\section{Analysis of the leaf morphology}

To analyze the overall leaf morphology and the petiole length, individual plants were grown in a climate chamber with a $12 \mathrm{~h}$ day and a $12 \mathrm{~h}$ night rhythm at $22^{\circ} \mathrm{C}$ and $60 \%$ relative humidity. The fluence rate was $100-120 \mu \mathrm{mol} \cdot \mathrm{m}^{-2} \cdot \mathrm{s}^{-1}$. After 28 days, leaf 7 and leaf 8 were detached at the petiole base and photographed in the top view using a Canon PowerShot G11 camera. Using these pictures, the length of the petiole and the leaf blade of either leaf 7 or leaf 8 were measured using the line tool in image J 1.x [234]. The total leaf length was obtained by adding the petiole length to the length of the leaf blade. The ratio of petiole length to total leaf length was computed and plotted in a bar chart. For this experiment, ca. 9 replicates for each plant line were examined. A replicate corresponds to one plant from which either leaf 7 or leaf 8 was used for the measurement.

\section{Flowering experiment}

To analyze the duration until flowering, ca. 6 plants were cultivated on individual pots under long day conditions in a climate chamber $(16 \mathrm{~h}$ day at $22^{\circ} \mathrm{C}, 8 \mathrm{~h}$ night at $18^{\circ} \mathrm{C}$, relative humididy at day and night: $60 \%$, fluence rate: $\left.100-120 \mu \mathrm{mol} \cdot \mathrm{m}^{-2} \cdot \mathrm{s}^{-1}\right)$. As soon as a floral stem of about $1 \mathrm{~cm}$ in length could be observed, the number of days from the start of incubation in the climate chamber to this day was determined. In addition, the leaves of the plant were counted to find out whether the plant is generally delayed in development or shows a delay particularly in flowering. 


\section{Work with DNA}

\section{Polymerase chain reaction (PCR)}

To prepare a PCR, the following components were mixed in a PCR tube:

\begin{tabular}{|c|c|c|}
\hline Component & Volume & Final concentration \\
\hline Primer fwd $(10 \mu \mathrm{M})$ & $2.5 \mu \mathrm{l}$ & $0.5 \mu \mathrm{M}$ \\
\hline Primer rev $(10 \mu \mathrm{M})$ & $2.5 \mu \mathrm{l}$ & $0.5 \mu \mathrm{M}$ \\
\hline dNTPs $(10 \mathrm{mM})$ & $1 \mu \mathrm{l}$ & $0.2 \mathrm{mM}$ \\
\hline $\begin{array}{l}\text { 5x Phusion HF Buffer containing } \\
7.5 \mathrm{mM} \mathrm{MgCl}_{2}\end{array}$ & $10 \mu \mathrm{l}$ & $1 \mathrm{x}$ \\
\hline Template DNA & \multicolumn{2}{|c|}{ Depending on template } \\
\hline $\begin{array}{l}\text { Phusion High-Fidelity DNA } \\
\text { Polymerase }(2 \mathrm{U} / \mu \mathrm{l})\end{array}$ & $0.5 \mu \mathrm{l}$ & $0.02 \mathrm{U} / \mu \mathrm{l}$ \\
\hline Total volume & Ad $50 \mu \mathrm{ldH}$ & \\
\hline
\end{tabular}

The standard PCR programm used was:

\begin{tabular}{lccc}
\hline Step & $\begin{array}{c}\text { Temperature } \\
{\left[{ }^{\circ} \mathrm{C}\right]}\end{array}$ & Duration [min] & $\begin{array}{c}\text { Number of } \\
\text { cycles }\end{array}$ \\
\hline Initial denaturation & 98 & 1 & 1 \\
Denaturation & 98 & 0.25 & 35 \\
Annealing & $\begin{array}{c}\text { Depending on } \\
\text { Tongation }\end{array}$ & 0.5 & 35 \\
Tin of primers & & \\
Final Elongation & 72 & $0.5 / \mathrm{kb}$ & 35 \\
Hold & 72 & 10 & 1 \\
\hline
\end{tabular}

The annealing temperature $\left(T_{A}\right)$ depended on the melting temperatures of the primers used $\left(\mathrm{T}_{\mathrm{M}}\right)$ for the PCR and was ideally calculated according to the formula:

$$
T_{A}=\frac{T_{M}(\text { Primer } f w d)+T_{M}(\text { Primer rev })}{2}
$$


A no template-control was employed to ensure that all components used were free of contaminating DNA. For this, the volume of the template DNA was replaced by an equal volume of $\mathrm{dH}_{2} \mathrm{O}$.

\section{Agarose gel electrophoresis}

Agarose gels were prepared using 1xTAE buffer. The agarose was dissolved in 1xTAE buffer by heating in a microwave oven oven for ca. 5 min at maximum power. Once prepared, agarose solutions in $1 \mathrm{xTAE}$ were stored at 60 to $65^{\circ} \mathrm{C}$ in an incubator. To prepare the gel, the agarose solution was poured into horizontal gel chambers supplied with a comb. After the gel had solidified, it was placed in a running chamber filled with 1xTAE. The comb was removed, and the samples were loaded into the wells. As a size standard, Gene Ruler DNA Ladder Mix $0.5 \mu \mathrm{g} / \mu \mathrm{l}$ (Thermo Scientific) was used. The DNA was separated in an electric field applying a constant voltage of 120 to $130 \mathrm{~V}$. Afterwards, the gel was stained in an ethidium bromide bath $(0.1 \%(\mathrm{w} / \mathrm{v})$ ethidium bromide in $\mathrm{dH}_{2} \mathrm{O}$ ) and destained in a water bath. Finally, the bands were visualized under ultraviolet (UV) light $(260 \mathrm{~nm}$ for documentation and $366 \mathrm{~nm}$ for gel extractions) and a picture taken using an Intas gel documentation system.

\section{1xTAE buffer}

\begin{tabular}{lc} 
Component & Concentration \\
\hline TRIS $\mathrm{pH} 8.4$ to 8.5 & $0.8 \mathrm{M}$ \\
Acetic acid & $2.28 \%(\mathrm{v} / \mathrm{v})$ \\
$\mathrm{Na}_{2}$-EDTA & $20 \mathrm{mM}$ \\
\hline
\end{tabular}

The $\mathrm{pH}$ was adjusted using $\mathrm{HCl}$. 


\section{Purification of DNA from reaction mixtures or from agarose gels}

The DNA of a PCR or a restriction digest was purified from the reaction mix or from an agarose gel using the kit „NucleoSpin Gel and PCR clean-up“ (Macherey \& Nagel), according to the instructions described in the manual. The DNA was eluted in an appropriate volume of $\mathrm{dH}_{2} \mathrm{O}$.

\section{Determination of nucleic acid concentrations}

The concentration of RNA or DNA was determined photometrically using NanoDrop 2000 Spectrophotometer (Peqlab). Purity was checked considering the absorption ratios at $260 \mathrm{~nm}$ and $280 \mathrm{~nm}$ or at $260 \mathrm{~nm}$ and $230 \mathrm{~nm}$. Alternatively, DNA concentrations could be determined by separating a known volume of the DNA fragment in an agarose gel and estimating the amount of DNA in the respective band by comparing its intensity to the band intensities of the size standard (Gene Ruler DNA Ladder Mix $0.5 \mu \mathrm{g} / \mu \mathrm{l}$ (Thermo Scientific)).

\section{Cloning using restriction enzymes and ligation}

\section{Restriction digests}

Restriction digests were performed using enzymes purchased from Thermo Scientific, New England Biolabs, MBI Fermentas, Boehringer Mannheim $\mathrm{GmbH}$, GIBCO BR, or Promega. The reaction composition and the reaction conditions were chosen according to the manufacturer's instructions. To estimate the amount of enzyme necessary for the digest of a DNA, the following formula was used:

$$
\text { Units }(\text { Enzyme })=2 \times \frac{\% \text { Max }(\text { Enzyme }) \times S(\text { Plasmid }) \times m(\text { Plasmid }) \times l(\lambda)}{t(\text { Digest }) \times \% B u f(\text { Enzyme }) \times S(\lambda) \times m(\lambda) \times l(\text { Plasmid })}
$$


$\% \operatorname{Max}($ Enzyme $)$ - maximal activity of the enzyme [\%]; \%Max (Enzyme $)=100 \%$

$\%$ Buf(Enzyme) - activity of the enzyme in the buffer used [\%]

$\mathrm{S}$ (Plasmid), $\mathrm{S}(\lambda)$ - number of restriction sites of the enzyme in the plasmid or in the $\lambda$-DNA, respectively

$\mathrm{m}$ (Plasmid), $\mathrm{m}(\lambda)$ - amount of plasmid used or amount of $\lambda$-DNA $[\mu \mathrm{g}] ; \mathrm{m}(\lambda)=1 \mu \mathrm{g}$

1 (Plasmid), $1(\lambda)$ - length of plasmid or $\lambda$-DNA [bp]; $1(\lambda)=48500 \mathrm{bp}$

$\mathrm{t}($ Digest $)$ - duration of the digest $[\mathrm{h}]$

\section{Ligation}

Ligations were performed using T4 DNA Ligase $5 \mathrm{Weiss} \mathrm{U} / \mu \mathrm{l}$ and 10x T4 DNA Ligase Buffer provided by Thermo Scientific. Usually, the amount of vector DNA used for a ligation depended on the size of the fragment: per 1000 bp length, $10 \mathrm{ng}$ of DNA were used. For the insert, a threefold molar excess of insert DNA over vector DNA was used. The reaction mixture was incubated for at least $20 \mathrm{~min}$ at room temperature and half of the prepared volume used for the transformation of E. coli. Multiple fragment ligations were performed in the same way, using all DNA fragments considered as inserts in a threefold molar excess over the single backbone fragment (vector). After selection of succsessfully transformed E. coli clones, positive clones were identified by test restriction digests.

\section{Cloning using the Gateway® system}

The Gateway ${ }^{\circledR}$ cloning system was obtained from LifeTechnologies. Cloning was performed as recommended in the manual. In short, entry clones were created in BP reactions. For this, an insert containing at $\mathrm{B}$ sites was prepared by PCR. 100 to $250 \mathrm{ng}$ of the insert and of the desired donor plasmid were prepared in a total volume of $8 \mu \mathrm{l}$ and added to $2 \mu \mathrm{l}$ BP enzyme mixture, vortexed, and incubated for $1 \mathrm{~h}$ at RT. Then, a part or the entire reaction mixture was used for an E. coli transformation. Positive clones were identified by Mini Plasmid Preparation and subsequent test restriction digests of the isolated plasmids. To transfer the insert from an entry vector to a destination vector, 100 to $250 \mathrm{ng}$ of each, the destination vector and the entry vector, were 
combined with 1 to $2 \mu \mathrm{l}$ of LR clonase mix in a total volume of 5 to $10 \mu \mathrm{l}$, vortexed and incubated for $1 \mathrm{~h}$ at RT. Finally, the reaction mixture was transformed in E. coli, as for a BP reaction. Again, positive clones were identified using restriction digest of isolated plasmids.

\section{Ligation-independent cloning (LIC)}

The ligation-independent cloning (LIC) was developed by QB3 MacroLab at the University of California, Berkeley. This cloning strategy uses the 3'-exonuclease activity of T4 polymerase to degrade one strand at the end of a linear DNA fragment, thereby creating 5'-overhangs. The overhangs of the vector and the insert are complementary. When annealed, the overlap is long enough to allow ligation of the nicks in E. coli, therefore circumventing a ligation step.

For ligation-independent cloning, the LIC vector was linearized using an appropriate restriction site. The vector was purified from an agarose gel to ensure that only fully linearized plasmid was used for the following steps. PCR was used to generate the LIC insert, fusing the gene of interest to LIC sites. Afterwards, 5'-overhangs were created in LIC reactions with the help of T4 polymerase for both, the LIC vector and LIC insert. A LIC reaction contained:

\begin{tabular}{|c|c|c|}
\hline Component & Volume & Final concentration \\
\hline PCR product or vector $\mathrm{DNA}$ & $\begin{array}{l}50 \text { to } 150 \text { ng } \\
\text { in } 10 \mu \mathrm{ld} \mathrm{dH}_{2} \mathrm{O}\end{array}$ & 25 to $75 \mathrm{ng}$ \\
\hline $\mathrm{dNTP}(25 \mathrm{mM})^{*}$ & $2 \mu \mathrm{l}$ & $2.5 \mathrm{mM}$ \\
\hline $\begin{array}{l}5 \mathrm{x} \text { Buffer for } \mathrm{T} 4 \text { DNA } \\
\text { Polymerase }\end{array}$ & $4 \mu \mathrm{l}$ & $1 \mathrm{x}$ \\
\hline T4 DNA Polymerase $(5 \mathrm{u} / \mu \mathrm{l})$ & $0.4 \mu \mathrm{l}$ & $0.1 \mathrm{u} / \mu \mathrm{l}$ \\
\hline DTT $(100 \mathrm{mM})$ & $1 \mu \mathrm{l}$ & $5 \mathrm{mM}$ \\
\hline Total volume & $20 \mu \mathrm{l}$ & \\
\hline
\end{tabular}


The LIC reaction was prepared in a PCR tube and incubated at $22^{\circ} \mathrm{C}$ for $30 \mathrm{~min}$ in a thermal cycler. The reaction was heat-inactivated by incubating at $75^{\circ} \mathrm{C}$ for $20 \mathrm{~min}$.

Next, $2 \mu \mathrm{l}$ of LICed vector and $2 \mu \mathrm{l}$ LICed PCR product were mixed with $6 \mu \mathrm{l}$ $\mathrm{dH}_{2} \mathrm{O}$ and allowed to anneal for $10 \mathrm{~min}$ at RT. After incubation, this mixture was transformed in E. coli. Plasmids were isolated from selected E. coli clones and checked in restriction digests.

\section{Mini plasmid preparation from E. coli}

For extraction of small amounts of plasmid DNA, an E. coli clone transformed with the desired plasmid was inoculated in LB or dYT media mixed with the correct antibiotic in sterile test tubes or flasks and incubated over night at $37^{\circ} \mathrm{C}$ at $220 \mathrm{rpm}$. The plasmid DNA was isolated from the cells using the „NucleoSpin Plasmid“ kit (Macherey \& Nagel), following the manual instructions. The plasmid DNA bound to the column was eluted with $\mathrm{dH}_{2} \mathrm{O}$.

\section{Midi plasmid preparation from E. coli}

For preparation of large amounts of plasmid DNA, an E. coli clone containing the plasmid of interest was inoculated in ca. $4 \mathrm{ml}$ dYT media containing the appropriate antibiotic in a sterile test tube and cultivated over day at $37^{\circ} \mathrm{C}$, shaking at $220 \mathrm{rpm}$. Ca. $300 \mu \mathrm{l}$ of this pre-culture were used to inoculate $300 \mathrm{ml}$ dYT supplied with the required antibiotic in a 21 flask. This main culture was incubated over night at $220 \mathrm{rpm}$ at $37^{\circ} \mathrm{C}$. The next day, the E. coli cells were harvested by centrifugation at $6000 \mathrm{rpm}$ for $15 \mathrm{~min}$ at RT (SLA-3000 rotor, Sorvall RC6 + centrifuge). The supernatant was discarded. Plasmid DNA was extracted using the kit „NucleoBond XtraMidi“ (Macherey \& Nagel), following the instructions of the manufacturer. 


\section{Plasmid isolation from $A$. tumefaciens}

Plasmids were isolated from $A$.tumefaciens with the help of the NucleoBond Plasmid kit (Macherey \& Nagel). The protocol recommended by the manufacturer was modified as follows:

Cultures were prepared by inoculating a single $A$. tumefaciens clone harboring the desired plasmid in ca. $10 \mathrm{ml}$ YEB supplied with the appropriate antibiotics. The cultures were incubated for 1 or 2 days at $29^{\circ} \mathrm{C}$ at $220 \mathrm{rpm}$. 6 to $10 \mathrm{ml}$ of the cultures were harvested for plasmid isolation by centrifugation $(20 \mathrm{~min}$, $4000 \mathrm{rpm}$, RT or $4^{\circ} \mathrm{C}$; Rotina 380R centrifuge equipped with 1798 4000rpm 4 x $300 \mathrm{~g}$ rotor). The supernatant was removed, and the pellet resuspended in $300 \mu \mathrm{l}$ buffer A1 containing RNase A. Additional $10 \mu \mathrm{l}$ of a $10 \mu \mathrm{g} / \mu \mathrm{l}$ RNase A stock were added to the sample. Then, $300 \mu \mathrm{l}$ buffer A2 were added and the sample mixed by inversion. Subsequently, the sample was incubated for $5 \mathrm{~min}$ at room temperature. Lysis was stopped by adding $350 \mu$ l neutralization buffer (A3). The sample was centrifuged for $20 \mathrm{~min}$ at $13000 \mathrm{rpm}$ at RT. After centrifugation, the procedure corresponded exactly to the protocol described in the manual until elution. The bound plasmid DNA was eluted in 18 to $20 \mu \mathrm{l}$ $\mathrm{dH}_{2} \mathrm{O}$.

\section{Extraction of total DNA from $A$. thaliana}

Total DNA was extracted from $A$. thaliana using the DNeasy Plant Mini Kit (Qiagen) following the instrucitons of the manufacturer. As starting material, approximately $500 \mu \mathrm{g}$ of rosette leaf powder were used and resuspended in $500 \mu \mathrm{l}$ buffer AP1. $100 \mu \mathrm{l}$ of this mixture were diluted with $300 \mu \mathrm{l} \mathrm{AP1}$. This dilution was supplied with $4 \mu \mathrm{l}$ RNase A. After that, the extraction procedure followed the instructions in the manual. 
Work with DNA

\section{DNA sequencing}

Purified DNA such as plasmids or PCR products were sent to Microsynth (SeqLab) in Göttingen or to Eurofins GATC (Konstanz) for sequencing according to the chain termination method by Sanger et al. (1977) [235] using specific primers. The DNA template and the primer were mixed according to the instructions of the sequencing company. The sequences retrieved were analyzed using the program Geneious Pro 5.3.6. (Biomatters Ltd; [236]). 


\section{Work with RNA}

\section{RNA extraction from $A$. thaliana}

For RNA extraction, leaf material was frozen in $\mathrm{N}_{2}(\mathrm{l})$ and grinded in a mortar to obtain a fine powder. Approximately 200 to $500 \mu \mathrm{g}$ of leaf powder were transferred to $2 \mathrm{ml}$ reaction tubes precooled in liquid nitrogen. The tubes were frozen in $\mathrm{N}_{2}(\mathrm{l})$ and stored at $-70^{\circ} \mathrm{C}$ until RNA isolation.

To extract the RNA, the phenol-chloroform method was applied. First, the still-frozen leaf powder was mixed with $1.4 \mathrm{ml}$ Trizol buffer and vortexed for at least 5 to $10 \mathrm{~min}$. Then, $300 \mu \mathrm{l}$ chloroform were added and again vortexed for 5 to $10 \mathrm{~min}$. The samples were centrifuged at $13000 \mathrm{rpm}$ for 30 to $60 \mathrm{~min}$ in a table top centrifuge at $4^{\circ} \mathrm{C}$. After centrifugation, 920 to $950 \mu \mathrm{l}$ supernatant were mixed $400 \mu \mathrm{l}$ HSP precipitation buffer and $400 \mu$ isopropanol in fresh $2 \mathrm{ml}$ reaction tubes. The tubes were inverted several times, incubated for $5 \mathrm{~min}$ at room temperature and centrifuged again for 30 to $60 \mathrm{~min}$ at $13000 \mathrm{rpm}$ at $4^{\circ} \mathrm{C}$ to precipitate the RNA. After centrifugation, the supernatant was decanted. $500 \mu \mathrm{l} 70 \%$ ethanol were added to the pellet and the samples inverted. Then, the samples were spun for 2 to $3 \mathrm{~min}$ (no longer than $5 \mathrm{~min}$ ) at $13000 \mathrm{rpm}$ at $4^{\circ} \mathrm{C}$. Afterwards, the ethanol was removed as fast as possible using a pipette. Another $500 \mu \mathrm{l} 70 \%$ ethanol were added to the pellet and both, ethanol and pellet, transferred to a fresh $1.5 \mathrm{ml}$ reaction tube. The centrifugation step for 2 to 3 min was repeated and the supernatant taken off using a pipette. The samples were spun for another minute to collect and remove the remaining supernatant. The whitish pellets were dried for ca. $30 \mathrm{~min}$ at room temperature under the hood until they became transparent. Finally, the pellets were dissolved in 30 to $100 \mu$ autoclaved $\mathrm{dH}_{2} \mathrm{O}$ by incubating for $10 \mathrm{~min}$ at $65^{\circ} \mathrm{C}$, vortexing in between. The dissolved RNA was stored at $-20^{\circ} \mathrm{C}$. 
Work with RNA

\section{HSP buffer}

\begin{tabular}{lc} 
Component & Concentration \\
\hline $\mathrm{NaCl}$ & $1.2 \mathrm{M}$ \\
$\mathrm{Na}_{3}$-citrate $\times 2 \mathrm{H}_{2} \mathrm{O}$ & $0.8 \mathrm{M}$ \\
\hline
\end{tabular}

\section{Trizol buffer}

\begin{tabular}{lc} 
Component & Concentration \\
\hline Phenol containing 0.1 M citrate buffer & $38 \%(\mathrm{v} / \mathrm{v})$ \\
Guanidinium thiocyanate & $0.8 \mathrm{M}$ \\
Ammoniumthiocyanate & $0.4 \mathrm{M}$ \\
$3 \mathrm{M} \mathrm{Na}-$ Acetat pH 5.2 & $3.3 \%(\mathrm{v} / \mathrm{v})$ \\
$100 \%$ Glycerol & $5 \%(\mathrm{v} / \mathrm{v})$ \\
\hline
\end{tabular}

\section{cDNA synthesis}

The concentration of the RNA was determined using a NanoDrop 2000 spectrophotometer. To prepare the cDNA, $1 \mu \mathrm{g}$ of RNA in a volume of $8 \mu \mathrm{l}$ $\mathrm{dH}_{2} \mathrm{O}$ was prepared in PCR stripes. An additional control was prepared

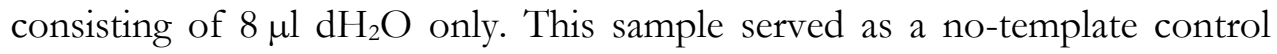
during quantitative Realtime PCR later.

First, the remaining DNA was digested using DNase I. For this, the following master mix was prepared and $2 \mu \mathrm{l}$ added to each sample.

\begin{tabular}{lc}
\hline Component & Volume for 1 reaction \\
\hline $10 \mathrm{x}$ Reaction buffer with $\mathrm{MgCl}_{2}$ for & $1 \mu \mathrm{l}$ \\
DNase I & \\
DNase I, RNase-free, $1 \mathrm{U} / \mu \mathrm{l}$ & $1 \mu \mathrm{l}$ \\
\hline
\end{tabular}

The stripes were incubated in a PCR cycler for $30 \mathrm{~min}$ at $37^{\circ} \mathrm{C}$. 
Then, $1 \mu \mathrm{l} 25 \mathrm{mM}$ EDTA (1:2 dilution of $50 \mathrm{mM}$ EDTA solution provided together with DNase I) were added and the samples incubated at $65^{\circ} \mathrm{C}$ for 10 min to stop the DNase I digest.

Secondly, cDNA synthesis was primed using an oligo dT-primer. For this, the following master mix was prepared and $1.2 \mu$ added to each sample:

\begin{tabular}{lc}
\hline Component & Volume for 1 reaction \\
\hline Oligo dT-primer $(100 \mu \mathrm{M})$ & $0.2 \mu \mathrm{l}$ \\
$\mathrm{dH}_{2} \mathrm{O}$ & $1 \mu \mathrm{l}$ \\
\hline
\end{tabular}

The samples were heated at $70^{\circ} \mathrm{C}$ for $10 \mathrm{~min}$.

Finally, a third master mix containing the remaining components necessary for cDNA synthesis was prepared and $7.8 \mu \mathrm{l}$ added to each sample. The master mix contained:

\begin{tabular}{lc}
\hline Component & Volume for 1 reaction \\
\hline $\mathrm{dH}_{2} \mathrm{O}$ & $1.5 \mu \mathrm{l}$ \\
$5 \mathrm{x}$ Reaction Buffer for RT & $4 \mu \mathrm{l}$ \\
dNTPs $(10 \mathrm{mM})$ & $2 \mu \mathrm{l}$ \\
Revert Aid $\mathrm{H}$ Minus Reverse & $0.3 \mu \mathrm{l}$ \\
Transcriptase $200 \mathrm{U} / \mu \mathrm{l}$ & \\
\hline
\end{tabular}

cDNA elongation took place at $42^{\circ} \mathrm{C}$ for $70 \mathrm{~min}$ and the reaction was heatinactivated by incubating at $70^{\circ} \mathrm{C}$ for $10 \mathrm{~min}$. The cDNA was stored at $-20^{\circ} \mathrm{C}$.

\section{Quantitative realtime PCR}

For quantitative realtime PCR (qRT-PCR), the cDNA was diluted 10-fold in $\mathrm{dH}_{2} \mathrm{O}$ and $1 \mu \mathrm{l}$ of this dilution used as a template during the PCR. In addition, a primer mix was prepared containing both, forward and reverse primer, at a concentration of $4 \mu \mathrm{M}$. Using this primer mix and the remaining components, 
a master mix was prepared. $49 \mu \mathrm{l}$ of the master mix were added to $1 \mu \mathrm{l}$ of the template cDNA. All in all, a single qRT-PCR reaction contained:

\begin{tabular}{lcc}
\hline Component & Volume & Final concentration \\
\hline $\mathrm{dH}_{2} \mathrm{O}$ & $17.2 \mu \mathrm{l}$ & \\
10xNH4 Reaction Buffer (No & $2.5 \mu \mathrm{l}$ & $1 \mathrm{x}$ \\
$\mathrm{MgCl} 2)$ & $1 \mu \mathrm{l}$ & $2 \mathrm{mM}$ \\
$50 \mathrm{mM} \mathrm{MgCl}{ }_{2}$ Stock Solution & & \\
(provided together with & & \\
BIOTAQ polymerase) & $0.25 \mu \mathrm{l}$ & $100 \mu \mathrm{M}$ \\
dNTPs $(10 \mathrm{mM})$ & $2.5 \mu \mathrm{l}$ & $0.4 \mu \mathrm{M}$ \\
Primer mix $(4 \mu \mathrm{M}]$ & $0.25 \mu \mathrm{l}$ & $1: 100000$ \\
SybrGreen $(1: 1000)$ & $0.25 \mu \mathrm{l}$ & $10 \mathrm{nM}$ \\
Fluorscein $(1 \mu \mathrm{M})$ & $0.05 \mu \mathrm{l}$ & $0.25 \mathrm{u}$ \\
BIOTAQ DNA Polymerase & & \\
5 u/ $\mu$ l & $1 \mu \mathrm{l}$ & \\
cDNA $(1: 10$ dilution) & & \\
\hline
\end{tabular}

The reactions were incubated in a thermal cycler according to the following protocol:

\begin{tabular}{lccc}
\hline Step & $\begin{array}{c}\text { Temperature } \\
{\left[{ }^{\circ} \mathrm{C}\right]}\end{array}$ & Duration [min] & $\begin{array}{c}\text { Number of } \\
\text { cycles }\end{array}$ \\
\hline Initial denaturation & 95 & 1.5 & 1 \\
Denaturation & 95 & 0.33 & 39 \\
Annealing & 55 & 0.33 & 39 \\
Elongation & 72 & 0.66 & 39 \\
Final Elongation & 72 & 4 & 1 \\
\hline
\end{tabular}




\section{Work with proteins - basic methods}

\section{Preparation of protein extracts from $A$. thaliana in urea buffer}

Protein extracts for sodium dodecylsulfate polyacrylamide gel electrophoresis (SDS-PAGE) were prepared by freezing leaf material in liquid nitrogen and grinding it in a tissue lyser (Retsch ${ }^{\circledR}$ MM301) for 1 min at $20 \mathrm{~Hz}$ using acidwashed stainless-steel beads (diameter: $5 \mathrm{~mm}$ ). Afterwards, the bead was removed and approximately $100 \mathrm{mg}$ powder were mixed with $200 \mu \mathrm{l}$ urea buffer and heated for $10 \mathrm{~min}$ at $65^{\circ} \mathrm{C}$. The samples were centrifuged for $15 \mathrm{~min}$ at $17000 \mathrm{~g}$ in a table top centrifuge at RT. The supernatant corresponding to the protein extract was transferred to a new tube and stored at $-20^{\circ} \mathrm{C}$. Protein concentration in the extracts was determined in a Pierce 660 assay using the ionic detergent compatability reagent (IDCR). For SDS-PAGE, the samples prepared in urea buffer were boiled at $65^{\circ} \mathrm{C}$ for $10 \mathrm{~min}$ and loaded on the gel.

\section{Urea buffer}

\begin{tabular}{lc} 
Component & Concentration \\
\hline Urea & $4 \mathrm{M}$ \\
Glycerol & $16.7 \%(\mathrm{v} / \mathrm{v})$ \\
SDS & $5 \%(\mathrm{w} / \mathrm{v})$ \\
Bromophenolblue & $0.06 \mathrm{~g} / \mathrm{l}$ \\
\hline
\end{tabular}




\section{TCA precipitation of proteins}

An equal volume of ice-cold $20 \%(\mathrm{w} / \mathrm{v})$ TCA was added to the protein sample. The sample vortexed and then incubated at $-20^{\circ} \mathrm{C}$ for $10 \mathrm{~min}$, followed by centrifugation at $17000 \mathrm{~g}$ at $4^{\circ} \mathrm{C}$ for $10 \mathrm{~min}$. The supernatant was removed and the pellet washed twice with ice-cold $100 \%$ acetone. For this, $1 \mathrm{ml}$ acetone was added to the pellet, the sample was inverted and then centrifuged at $17000 \mathrm{~g}$ at $4^{\circ} \mathrm{C}$ for $5 \mathrm{~min}$. The supernatant was decanted. In the second washing step, the sample was spun again briefly to collect any residual acetone at the bottom of the tube. This residual volume was removed using a pipette tip. The pellet was dried briefly on ice.

\section{Determination of protein concentration according to Bradford}

To measure protein amounts in the range of 1 to $6 \mu \mathrm{g}$, the Bradford method [237] was applied. Quantification was done with the help of a BSA standard curve. For the standard curve, bovine serum albumin (BSA) dilutions of 0, 0.1, $0.2,0.3,0.45$, and $0.6 \mu \mathrm{g} / \mu \mathrm{l}$ in $\mathrm{dH}_{2} \mathrm{O}$ were prepared and $10 \mu \mathrm{l}$ added to the wells of a microtiter plate. Since the protein samples to be measured were dissolved in a buffer but BSA in $\mathrm{dH}_{2} \mathrm{O}$, the respective amount of buffer was added to all

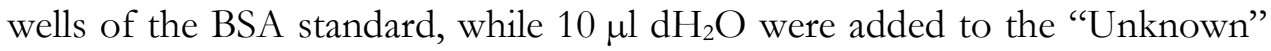
samples. Finally, all samples were mixed with $200 \mu$ l Roti ${ }^{\circledR}$-Quant Bradford solution diluted 1:5 in $\mathrm{dH}_{2} \mathrm{O}$, and incubated at $\mathrm{RT}$ for 5 min. Then, the absorption at $595 \mathrm{~nm}$ was measured in a plate reader (Synergy HT). The standard curve was calculated by plotting the values obtained for $A_{595 n m}$ against the mass of BSA in the sample. Linear regression was performed, and the equation retrieved was used to calculate the protein amount in the test samples. 


\section{Determination of protein concentration using Pierce 660 reagent}

To quantify protein amounts in the range of 1 to $9 \mu \mathrm{g}$, Pierce ${ }^{\circledR} 660$ reagent was used. In case the buffer of the protein samples contained detergent, IDCR was dissolved in the Pierce ${ }^{\circledR} 660$ solution according to the manufacturer's instructions. A BSA standard consisting of 1, 3, 6, and $9 \mu \mathrm{g}$ samples and a blank control consisting of $\mathrm{dH}_{2} \mathrm{O}$ only were prepared. For this, $10 \mu \mathrm{l}$ of $0,0.1,0.3$, 0.6 , and $0.9 \mu \mathrm{g} / \mu \mathrm{l} \mathrm{BSA}$ solution in $\mathrm{dH}_{2} \mathrm{O}$ were added to the wells of a microtiter

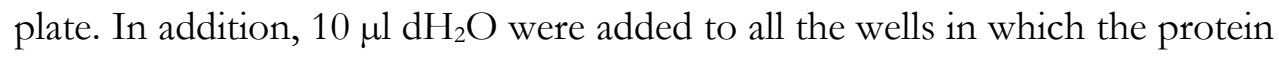
sample to be measured should be prepared. Then, a known volume of the protein samples of interest was mixed with $\mathrm{dH}_{2} \mathrm{O}$ aliquots. To the blank and the BSA standard samples, the same volume of buffer was added. Finally, $150 \mu$ Pierce ${ }^{\circledR} 660$ (optionally supplied with IDCR) were distributed to the wells. The plate was incubated for $5 \mathrm{~min}$ at RT. Then, $\mathrm{A}_{660 \mathrm{~nm}}$ was measured in a plate reader (Synergy HT). A standard curve was obtained by plotting the absorbtion intensity against the BSA amount and by performing a linear regression. This yielded a formula used to calculate the protein amount in the test samples.

\section{Denaturing discontinouus polyacrylamide gel electrophoresis (reducing and non-reducing)}

Discontinouus polyacrylamide gels were casted in home-made gel chambers $(7 \mathrm{~cm} \times 18.4 \mathrm{~cm})$ or in chambers of the Mini-PROTEAN® Tetra Cell System ( 0.75 or $1.5 \mathrm{~mm}$ thickness). First, the gel solutions were prepared omitting only ammonium peroxodisulfate (APS), tetramethylethylenediamine (TEMED) and acrylamide. These three components were added immediately before pouring the gels. First, the resolving gel solution was completed and poured into the assembled gel chamber. To obtain an even surface, the resolving gel was covered with $100 \%$ isopropanol. After the resolving gel had solidified, isopropanol was washed off with $\mathrm{dH}_{2} \mathrm{O}$ and the stacking gel solution was poured on top of the resolving gel. A comb was pressed between the glass plates to create the wells. After solidifying, the gels were used immediately for electrophoresis or wrapped in wet tissues and foil to store them in the fridge. 
Work with proteins - basic methods

Gel solutions for denaturing polyacrylamide gels

Resolving gels $(6 \%, 8 \%, 12 \%, 15 \%)$

\begin{tabular}{lcccc}
\hline Percentage of acrylamide & $8 \%$ & $10 \%$ & $12 \%$ & $15 \%$ \\
\hline Component & 4.6 & 4.0 & 3.3 & 2.3 \\
\hline $\mathrm{dH}_{2} \mathrm{O}$ & 2.7 & 3.3 & 4.0 & 5.0 \\
$30 \%$ Acrylamide solution & 2.5 & 2.5 & 2.5 & 2.5 \\
$(37.5: 1)$ & 0.05 & 0.05 & 0.05 & 0.05 \\
$1.5 \mathrm{M}$ TRIS pH 8.8 & 0.1 & 0.1 & 0.1 & 0.1 \\
$20 \%(w / v)$ SDS & 0.006 & 0.004 & 0.004 & 0.004 \\
$10 \%(w / v)$ APS & \multicolumn{5}{c}{$10 \mathrm{ml}$} \\
TEMED & \multicolumn{5}{c}{} \\
\hline Total volume &
\end{tabular}

Stacking gel (5\%)

\begin{tabular}{lc}
\hline Percentage of acrylamide & $5 \%$ \\
\hline Component & Volume [ml] \\
\hline $\mathrm{dH}_{2} \mathrm{O}$ & 6.8 \\
$30 \%$ Acrylamide solution & 1.7 \\
$1.5 \mathrm{M}$ TRIS pH 6.8 & 1.25 \\
$20 \%(\mathrm{w} / \mathrm{v}) \mathrm{SDS}$ & 0.05 \\
$10 \%(\mathrm{w} / \mathrm{v})$ APS & 0.1 \\
TEMED & 0.01 \\
\hline Total volume & 10 \\
\hline
\end{tabular}

As an alternative to running self-made gels, Mini-PROTEAN® Precast TGX Gels 4-20\% (BioRad) were used.

For electrophoresis, the gel was assembled in the running chamber filled with 1x SDS running buffer. The comb was removed. To prepare the samples for SDS-PAGE, the protein was mixed with $2 x$ RPB. Samples for reducing PAGE were mixed with reducing $2 \mathrm{x}$ RPB. In case a non-reducing PAGE was performed, the samples were mixed with non-reducing $2 \mathrm{x}$ RPB lacking dithiothreitol (DTT). The samples were boiled for 5 to $20 \mathrm{~min}$ at $95^{\circ} \mathrm{C}$, spun down briefly and loaded into the wells of the gel. As a size standard, Page Ruler ${ }^{\text {TM }}$ Prestained Protein Ladder (Thermo Scientific) was applied to the gel. The gels were run at 80 to $160 \mathrm{~V}$. 
Reducing and non-reducing 2x RPB

\begin{tabular}{lc} 
Component & Concentration \\
\hline TRIS pH 6.8 & $100 \mathrm{mM}$ \\
SDS & $4 \%(\mathrm{w} / \mathrm{v})$ \\
Glycerol & $20 \%(\mathrm{w} / \mathrm{v})$ \\
Bromophenol blue & A spatula \\
\hline
\end{tabular}

To prepare reducing RPB, $200 \mathrm{mM}$ DTT were included in the mixture. For non-reducing RPB, the DTT was omitted. Reducing and non-reducing RPB was stored at $-20^{\circ} \mathrm{C}$.

\section{0x SDS running buffer}

\begin{tabular}{lc} 
Component & Concentration \\
\hline TRIS & $250 \mathrm{mM}$ \\
Glycine & $1.9 \mathrm{M}$ \\
SDS & $1 \%(\mathrm{w} / \mathrm{v})$ \\
\hline
\end{tabular}

\section{Native PAGE}

For native PAGE, Mini-PROTEAN® Precast TGX Gels 4-20\% (BioRad) were assembled in running chambers containing $1 \mathrm{x}$ Native running buffer. The running chamber was placed on ice to prevent heating of the gel during the run. Samples were mixed with native loading buffer and immediately loaded on the gel without boiling them. NativeMark ${ }^{\mathrm{TM}}$ Unstained Protein Standard (Invitrogen) was used as a size standard. The gel was run strictly at $80 \mathrm{~V}$.

\section{5x Native loading dye}

\begin{tabular}{lc} 
Component & Concentration \\
\hline TRIS-HCl pH 6.8 & $187.5 \mathrm{mM}$ \\
Glycerol & $7.5 \%(\mathrm{v} / \mathrm{v})$ \\
\hline
\end{tabular}

The solution was prepared using a stock solution of TRIS-HCl buffer $\mathrm{pH}$ 6.8. The final mixture was supplied with bromophenol blue until the solution turned light blue. 


\section{0x Native running buffer}

\begin{tabular}{lc} 
Component & Concentration \\
\hline TRIS-base & $250 \mathrm{mM}$ \\
Glycine & $1.92 \mathrm{M}$ \\
\hline
\end{tabular}

The buffer was diluted 10x prior to use.

\section{Coomassie staining of polyacrylamide gels}

To stain native or denaturing SDS gels with Coomassie, the gel was incubated in different solutions on a shaker at room temperature. The gel was exposed to Coomassie fixing solution for at least $10 \mathrm{~min}$. Then, the gel was incubated in Coomassie staining solution until it became dark blue. The staining solution was exchanged for Coomassie destaining solution, in which the gel was incubated until the bands were clearly visible. Optionally, gels were destained with Coomassie fixation solution. Gels were digitalized using an Epson Perfection V700 Photoscanner with the SilverFast-SE v6.6.1r2b software (LaserSoft Imaging).

\section{Coomassie fixing solution}

\begin{tabular}{lc} 
Component & Concentration \\
\hline Acetic acid & $10 \%(\mathrm{v} / \mathrm{v})$ \\
Isopropanol & $20 \%(\mathrm{v} / \mathrm{v})$ \\
\hline
\end{tabular}

\section{Coomassie staining solution}

\begin{tabular}{lc} 
Component & Concentration \\
\hline Acetic acid & $10 \%(\mathrm{v} / \mathrm{v})$ \\
Coomassie Brilliant Blue G250 & $\begin{array}{c}\text { Added until solution } \\
\text { was dark blue }\end{array}$ \\
\hline
\end{tabular}




\section{Coomassie destaining solution}

\begin{tabular}{lc} 
Component & Concentration \\
\hline Acetic acid & $10 \%(\mathrm{v} / \mathrm{v})$ \\
\hline
\end{tabular}

\section{Colloidal Coomassie staining of polyacrylamide gels}

Colloidal Coomassie staining was used in the course of sample preparation for mass spectrometry and performed using a protocol obtained from Dr. Oliver Valerius and Dr. Kerstin Schmitt (Service Unit LCMS Protein Analytics, Georg August University Göttingen). The protocol represented a modified version of the protocol described by Kang et al. (2002) [238].

After the run, the SDS gel was incubated for ca. $1 \mathrm{~h}$ in Colloidal Coomassie fixing solution. This incubation step, as well as all following steps, were done at room temperature on a shaker. Afterwards, the gel was washed $2 \mathrm{x}$ for ca. 10 min with $\mathrm{dH}_{2} \mathrm{O}$. Then, the gel was stained for some time (not over night) in Colloidal Coomassie staining solution. When the bands of interest were visible, the gel was washed $3 \mathrm{x}$ for ca. 10 min with $\mathrm{dH}_{2} \mathrm{O}$. The gel was scanned using an Epson Perfection V700 Photoscanner via the SilverFast-SE v6.6.1 r2b software (LaserSoft Imaging).

\section{Colloidal Coomassie fixing solution}

\begin{tabular}{lc} 
Component & Concentration \\
\hline Ethanol $(\mathrm{EtOH})$ & $40 \%(\mathrm{v} / \mathrm{v})$ \\
Acetic acid & $10 \%(\mathrm{v} / \mathrm{v})$ \\
\hline
\end{tabular}




\section{Colloidal Coomassie staining solution}

\begin{tabular}{lc} 
Component & Concentration \\
\hline Aluminium sulfate hexadeca hydrate & $5 \%(\mathrm{w} / \mathrm{v})$ \\
Methanol & $10 \%(\mathrm{v} / \mathrm{v})$ \\
Coomassie Brilliant Blue G250 & $0.1 \%(\mathrm{w} / \mathrm{v})$ \\
O-Phosphoric acid & $2 \%(\mathrm{v} / \mathrm{v})$ \\
\hline
\end{tabular}

The order of preparation is desicive for the sensitivity of the solution: First, aluminium sulfate was dissolved in water, then methanol was added while stirring. After mixing, Coomassie was added. When Coomassie appeared to be dissolved, phosphoric acid was mixed into the solution creating the Coomassie colloidal particels. Eventually, the solution was filled up to the final volume using $\mathrm{dH}_{2} \mathrm{O}$.

\section{Western Blot}

Western Blots were used to specifically detect a protein via an antibody after SDS-PAGE and subsequent transfer to a polyvinyldiflouride (PVDF) membrane. First, the protein samples were separated by SDS-PAGE. 6 pieces of Blotting paper and a PVDF membrane, all cut to the size of the resolving gel, were prepared. Three layers of Blotting paper were soaked with transfer buffer and placed on the cathode of the blotting chamber. The membrane was activated in methanol, then incubated in transfer buffer and placed on top of the Blotting paper layers. Now, the SDS gel was stacked on top of the membrane. Finally, three more layers of Blotting paper were wetted in transfer buffer and placed on top of the SDS gel. Bubbles were removed by sliding a roller over the stack. The blotting chamber was closed, placing the anode on top of the stack. A constant amperage was applied. The blotting time depended on the amperage used, the size of the gel and the protein to be detected. 


\begin{tabular}{lcc}
\hline Protein & \multicolumn{2}{c}{ Blotting conditions } \\
\cline { 2 - 3 } & $\begin{array}{c}\text { Biorad MiniProtean } \\
\text { Gels } 0.75 \text { or } 1.5 \mathrm{~mm} \\
\text { thickness }\end{array}$ & Self-made gels \\
\hline 3xHA-ROXY9 & - & $125 \mathrm{~mA}, 60 \mathrm{~min}$ \\
GFP-TGA1 & $135 \mathrm{~mA}, 45 \mathrm{~min}$ & - \\
Strep-MBP-ROXY9 & $135 \mathrm{~mA}, 45 \mathrm{~min}$ & - \\
Strep-MBP & $135 \mathrm{~mA}, 45 \mathrm{~min}$ & - \\
His-MBP-JAZ9 & $135 \mathrm{~mA}, 45 \mathrm{~min}$ & - \\
\hline
\end{tabular}

After disassembly of the blot, the membrane was optionally rinsed with methanol. Then, the membrane was incubated with different solutions on a shaker. First, it was blocked with blocking solution for at least $1 \mathrm{~h}$ at room temperature or over night in the cold room. The blocking solution was decanted, and the membrane incubated with the primary antibody for $2 \mathrm{~h}$ at RT or over night in the cold room. Next, the membrane was washed three times with $1 \mathrm{x}$ TBS-T for $10 \mathrm{~min}$ at RT and incubated with a horseradish peroxidase (HRP)-coupled secondary antibody for $2 \mathrm{~h}$ at RT. The membrane was washed five times for $5 \mathrm{~min}$ at RT with $1 \mathrm{x}$ TBS. Finally, HRP activity was detected using either Luminata ${ }^{\text {TM }}$ Forte Western HRP Substrate (Millipore) or SuperSignal ${ }^{\mathrm{TM}}$ West Femto Maximum Sensitivity Substrate (Thermo Scientific) and a luminescence imager (Intas ChemoStar Imager, ChemoStarProfessional software).

\begin{tabular}{|c|c|c|c|}
\hline $\operatorname{tag}$ & Blocking solution & Primary antibody & Secondary antibody \\
\hline HA & $\begin{array}{l}5 \%(\mathrm{w} / \mathrm{v}) \text { skimmed } \\
\text { milk powder in } 1 \mathrm{x} \\
\text { TBS or } 1 \mathrm{xTBS}-\mathrm{T}\end{array}$ & $\begin{array}{l}\alpha-\mathrm{HA}(1: 2000 \text { to } \\
4000 \text { in } 5 \%(\mathrm{w} / \mathrm{v}) \\
\text { skimmed milk } \\
\text { powder in } 1 \mathrm{xTBS}-\mathrm{T})\end{array}$ & $\begin{array}{l}\alpha-\text { rabbit }(1: 8000 \text { in } \\
5 \%(w / v) \text { skimmed } \\
\text { milk powder in } \\
1 \times \text { TBS-T })\end{array}$ \\
\hline Strep & $\begin{array}{l}30 \mu \mathrm{g} / \mathrm{ml} \text { BSA in } \\
1 \mathrm{xPBS} \text { supplied with } \\
0.5 \% \text { (v/v) Tween- } \\
20\end{array}$ & $\begin{array}{l}\alpha \text {-strep }(1: 10000 \text { in } \\
2 \mu \mathrm{g} / \mathrm{ml} \text { BSA in } \\
1 \mathrm{xPBS}-\mathrm{T})\end{array}$ & $\begin{array}{l}\alpha \text {-mouse }(1: 8000 \text { in } \\
2 \mu \mathrm{g} / \mathrm{ml} \text { BSA in } \\
1 \mathrm{xPBS}-\mathrm{T})\end{array}$ \\
\hline GFP & $\begin{array}{l}5 \%(\mathrm{w} / \mathrm{v}) \text { skimmed } \\
\text { milk powder in } \\
1 \times \text { TBS or } 1 \mathrm{xTBS}-\mathrm{T}\end{array}$ & $\begin{array}{l}\alpha-G F P(1: 1000 \text { in } \\
5 \%(w / v) \text { skimmed } \\
\text { milk powder in } \\
1 x \text { TBS-T })\end{array}$ & $\begin{array}{l}\alpha \text {-mouse }(1: 8000 \text { in } \\
5 \%(w / v) \text { skimmed } \\
\text { milk powder in } \\
1 \times \text { TBS-T })\end{array}$ \\
\hline His & $\begin{array}{l}5 \%(\mathrm{w} / \mathrm{v}) \text { skimmed } \\
\text { milk powder in } \\
1 \times \text { TBS or } 1 \mathrm{xTBS}-\mathrm{T}\end{array}$ & $\begin{array}{l}\alpha \text {-His }(1: 500 \text { in } \\
5 \%(\mathrm{w} / \mathrm{v}) \text { skimmed } \\
\text { milk powder in } \\
1 \times \text { TBS-T) }\end{array}$ & $\begin{array}{l}\alpha \text {-mouse }(1: 8000 \text { in } \\
5 \%(\mathrm{w} / \mathrm{v}) \text { skimmed } \\
\text { milk powder in } \\
1 \mathrm{xTBS}-\mathrm{T})\end{array}$ \\
\hline
\end{tabular}




\section{Transfer buffer}

\begin{tabular}{lc} 
Component & Concentration \\
\hline TRIS & $48 \mathrm{mM}$ \\
Glycin & $39 \mathrm{mM}$ \\
Methanol & $20 \%(\mathrm{v} / \mathrm{v})$ \\
SDS & $0.04 \%(\mathrm{w} / \mathrm{v})$ \\
\hline
\end{tabular}

\section{0x TBS, 1x TBS and 1x TBS-T}

\begin{tabular}{lc} 
Component & Concentration \\
\hline TRIS-base & $200 \mathrm{mM}$ \\
$\mathrm{NaCl}$ & $1.37 \mathrm{M}$ \\
\hline
\end{tabular}

To prepare $1 \mathrm{x}$ TBS, 10x TBS was diluted $1: 10$ in $\mathrm{dH}_{2} \mathrm{O}$. To prepare $1 \mathrm{x}$ PBST, 10x PBS was diluted $1: 10$ in $\mathrm{dH}_{2} \mathrm{O}$ and the solution mixed with $0.1 \%(\mathrm{v} / \mathrm{v})$ Tween-20.

\section{0x PBS, 1x PBS and 1x PBS-T}

\begin{tabular}{lc} 
Component & Concentration \\
\hline $\mathrm{KH}_{2} \mathrm{PO}_{4} \mathrm{pH} 7.4$ & $40 \mathrm{mM}$ \\
$\mathrm{Na}_{2} \mathrm{HPO}_{4} \times 2 \mathrm{H}_{2} \mathrm{O}$ & $160 \mathrm{mM}$ \\
$\mathrm{NaCl}$ & $1.150 \mathrm{M}$ \\
\hline
\end{tabular}

The $\mathrm{pH}$ of $10 \mathrm{x}$ PBS was adjusted to 7.4 using $\mathrm{KOH}$ and $\mathrm{H}_{3} \mathrm{PO}_{4}$ before filling up to the final volume. To prepare 1x PBS, 10x PBS was diluted 1:10 in $\mathrm{dH}_{2} \mathrm{O}$ and the $\mathrm{pH}$ readjusted to 7.4. To prepare $1 \mathrm{x}$ PBS-T, 10x PBS was diluted $1: 10$ in $\mathrm{dH}_{2} \mathrm{O}$, the $\mathrm{pH}$ readjusted to 7.4 and the solution mixed with $0.1 \%(\mathrm{v} / \mathrm{v})$ Tween-20. 


\section{Production and analysis of recombinant proteins}

\section{Expression of recombinant protein in E. coli}

To express recombinant proteins in E. coli BL21(DE3), the bacterium was transformed with an expression plasmid harboring the gene of interest under control of the $\mathrm{T} 7$ promoter and terminator. One or more clones were used to inocculate a flask containing an adequate volume of LB medium (10 to $20 \mathrm{ml}$ per main culture) supplemented with the correct antibiotic. This culture was grown over night at $37^{\circ} \mathrm{C}$, shaking at $220 \mathrm{rpm}$. The next day, the optical desity at $600 \mathrm{~nm}$ was measured in a 1:10 dilution using a Libra S11 spectrophotometer. Then, a main culture was prepared. For this, $400 \mathrm{ml} \mathrm{LB}$ medium filled in 21 flasks without baffles were mixed with the selective antibiotic and inocculated to an $\mathrm{OD}_{600 \mathrm{~nm}}$ of 0.1 using the pre-culture. The main culture was incubated at $37^{\circ} \mathrm{C}$ at $220 \mathrm{rpm}$ until it reached the $\mathrm{OD}_{600 \mathrm{~nm}}$ of induction. This density varied for each protein. A culture aliquot corresponding to an $\mathrm{OD}_{600 \mathrm{~nm}}$ of 2 was taken as a pre-induction sample for SDS-PAGE. The sample was stored on ice until processing it further. Expression of the main culture was induced by addition of isopropyl- $\beta$-D-thiogalactopyranoside (IPTG). The culture was incubated at those growth conditions allowing the expression of the recombinant protein. The expression conditions, the IPTG concentration, and the growth conditions after induction varied depending on the recombinant protein. The table below summarizes the expression conditions for each protein:

\begin{tabular}{lccc}
\hline Protein & OD $_{600 \mathrm{~nm}}$ (Induction) & $\mathrm{c}(\mathrm{IPTG})$ & Incubation \\
& {$[$ A.U.] } & {$[\mathrm{mM}]$} & \\
\hline Strep-SUMO-ROXY9 & $\geq 0.5$ & 0.75 & $30^{\circ} \mathrm{C}, 3 \mathrm{~h}$, \\
& $\geq 0.5$ & & $220 \mathrm{rpm}$ \\
Strep-SUMO-Grx1 & & 1 & $27^{\circ} \mathrm{C}, \mathrm{ON}$, \\
& & & $220 \mathrm{rpm}$ \\
\hline
\end{tabular}




\begin{tabular}{lccc}
\hline Protein & $\begin{array}{c}\text { OD }_{600 \mathrm{~nm}} \text { (Induction) } \\
\text { [A.U.] }\end{array}$ & $\begin{array}{c}\mathrm{c}(\mathrm{IPTG}) \\
{[\mathrm{mM}]}\end{array}$ & $\begin{array}{c}\text { Incubation } \\
\text { Strep-MBP-GRXC2 }\end{array}$ \\
& $\geq 0.5$ & 0.5 & $20^{\circ} \mathrm{C}, \mathrm{ON}$, \\
GFP-TGA1 & $\geq 0.5$ & 1 & $220 \mathrm{rpm}$ \\
& $\geq 1$ & 0.5 & $26^{\circ} \mathrm{C}, \mathrm{ON}$, \\
His-MBP-JAZ9 & & & $20^{\circ} \mathrm{C}, 2 \mathrm{~d}$, \\
& $\geq 0.7$ & 0.5 & $220 \mathrm{rpm}$ \\
Strep-MBP & & & $37^{\circ} \mathrm{C}, 2.5 \mathrm{~h}$, \\
& & & $220 \mathrm{rpm}$ \\
\hline
\end{tabular}

After expression, the optical density of the cultures was determined and a sample corresponding to an $\mathrm{OD}_{600 \mathrm{~nm}}$ of 2 was taken as a post-induction sample for SDS-PAGE. The sample was kept on ice until later processing. The remaining cells from the expression culture were harvested by centrifugation at $5000 \mathrm{rpm}$ at $4^{\circ} \mathrm{C}$ to $\mathrm{RT}$ for 15 to $20 \mathrm{~min}$ (Sorvall RC6+ centrifuge, rotor SLA3000). The supernatant was discarded, and the pellet resuspended in 10 to $20 \mathrm{ml}$ of harvesting buffer. The buffer depended on the type of the recombinant protein and is listed in the table below. The cell suspension was transferred to a $50 \mathrm{ml}$ falcon tube, which was centrifuged at $4000 \mathrm{rpm}$ for at least $20 \mathrm{~min}$. The supernatant was removed as completely as possible and the pellet frozen at $-20^{\circ} \mathrm{C}$.

Finally, the pre- and post-induction samples were spun down, and the supernatants were removed. The pellets were resuspended in $100 \mu \mathrm{l} 2 \mathrm{x}$ RPB. $20 \mu \mathrm{l}$ of these samples were loaded on an SDS gel to check expression of the recombinant protein.

\begin{tabular}{lll}
\hline Protein & Harvesting buffer & Buffer recipe \\
\hline $\begin{array}{l}\text { Strep-SUMO- } \\
\text { ROXY9 }\end{array}$ & $50 \mathrm{mM}$ Na phosphate buffer $\mathrm{pH} 8.0$ & Page 88 \\
$\begin{array}{l}\text { Strep-SUMO- } \\
\text { Grx1 }\end{array}$ & 1x Grx1 buffer & Pages 89, 90 \\
$\begin{array}{l}\text { Strep-MBP- } \\
\text { GRXC2 }\end{array}$ & 1x Grx1 buffer & Pages 89, 90 \\
GFP-TGA1 & 50 mM Na phosphate buffer pH 8.0 or 1x & Pages 88 to \\
& Grx1 buffer w/o DTT & 90 \\
His-MBP- & 1x MBP lysis buffer with 50 mM NaCl & Page 95 \\
JAZ9 & & \\
Strep-MBP & 1x MBP lysis buffer & Page 93 \\
\hline
\end{tabular}




\section{Expression of tobacco etch virus protease in E. coli using autoinduction media}

Tobacco etch virus (TEV) protease was expressed from the plasmid pMA/CLTEV-A in BL21(DE2) growing the cells in autoinduction media. This method was described by Sturdier et al. (2014) [239]. For expression of TEV protease, a simpler protocol published by the Lindstrom group of the imperial college (London) on www.openwetware.org was used. This expression method does not rely on IPTG for induction of the T7 polymerase gene placed under control of the lac promoter, but on the change in the use of carbon sources by E. coli. For this, the cells are grown in medium containing a mixture of glucose, glycerol and lactose. The cells first grow by consuming their preferred carbon source glucose, which represses the lac promoter. Once glucose becomes limiting, E. coli switches to the use of lactose for growth, which induces transcription from the lac promoter and, thereby, expression of the recombinant protein.

To express the TEV protease via this method, several of the transformed BL21 clones were inoculated in a small volume of autoinduction media and then distributed to 21 flasks without baffels which contained $400 \mathrm{ml}$ autoinduction media supplied with the correct antibiotic. These cultures were incubated for $6 \mathrm{~h}$ at $37^{\circ} \mathrm{C}$, shaking them at $220 \mathrm{rpm}$. Then, the cultures were transferred to $29^{\circ} \mathrm{C}$ and $220 \mathrm{rpm}$ and expression was continued over night.

The cells were harvested by centrifugation at $4^{\circ} \mathrm{C}$ and $5000 \mathrm{rpm}$ for $20 \mathrm{~min}$ in an SLA-3000 rotor (Sorvall RC6 + centrifuge). The pellets were resuspended in 1x Strep buffer and transferred to $50 \mathrm{ml}$ falcon tubes. The cells were pelleted again by centrifugation at $4000 \mathrm{rpm}$ at $4^{\circ} \mathrm{C}$ for $40 \mathrm{~min}$. The supernatant was decanted, and the pellets frozen in liquid nitrogen to store them at $-70^{\circ} \mathrm{C}$.

\section{Stock solutions of carbon sources}

\begin{tabular}{lc} 
Component & Concentration \\
\hline Glycerol & $60 \%(\mathrm{v} / \mathrm{v})$ \\
Glucose & $10 \%(\mathrm{w} / \mathrm{v})$ \\
Lactose & $8 \%(\mathrm{w} / \mathrm{v})$ \\
\hline
\end{tabular}

The solutions were prepared in $\mathrm{dH}_{2} \mathrm{O}$ and sterilized by filtration through a $0.2 \mu \mathrm{m}$ filter. 


\section{Autoinduction media}

\begin{tabular}{lc} 
Component & Concentration \\
\hline $\mathrm{Na}_{2} \mathrm{HPO}_{4}$ (water-free) & $0.6 \%(\mathrm{w} / \mathrm{v})$ \\
$\mathrm{KH}_{2} \mathrm{PO}_{4}$ (water-free) & $0.3 \%(\mathrm{w} / \mathrm{v})$ \\
Tryptone/peptone from caseine & $2 \%(\mathrm{w} / \mathrm{v})$ \\
Yeast extract & $0.5 \%(\mathrm{w} / \mathrm{v})$ \\
$\mathrm{NaCl}$ & $0.5 \%(\mathrm{w} / \mathrm{v})$ \\
Glycerol & $0.6 \%(\mathrm{v} / \mathrm{v})$ \\
Glucose & $0.05 \%(\mathrm{w} / \mathrm{v})$ \\
Lactose & $0.2 \%(\mathrm{w} / \mathrm{v})$ \\
\hline
\end{tabular}

The autoinduction media was prepared by dissolving $\mathrm{Na}_{2} \mathrm{HPO}_{4}, \mathrm{KH}_{2} \mathrm{PO}_{4}$, tryptone/peptone, yeast extract and $\mathrm{NaCl}$ in $\mathrm{dH}_{2} \mathrm{O}$ and adjusting the $\mathrm{pH}$ to 7.2 using $\mathrm{NaOH}$. The solution was filled up to $96 \%$ of the final volume and autoclaved. Before use, the medium was completed by adding the carbon sources using the stock solutions listed above.

\section{1x Strep buffer}

\begin{tabular}{lc} 
Component & Concentration \\
\hline TRIS pH 8.0 & $10 \mathrm{mM}$ \\
$\mathrm{NaCl}$ & $150 \mathrm{mM}$ \\
$\mathrm{Na}_{2}$-EDTA & $0.5 \mathrm{mM}$ \\
\hline
\end{tabular}

The buffer was prepared as a 10x stock solution, the $\mathrm{pH}$ was titrated to 8.0 at RT using $\mathrm{HCl}$. The stock solution was diluted adequately before use.

\section{Expression of recombinant protein in insect cells}

Strep-MBP-ROXY9 was produced via the bacculovirus system in Trichoplusia ni (T. ni) by Isaac Fianu from the Department of Molecular Biology (Prof. Dr. Patrick Cramer) at the MPI of Biohphysical Chemistry (Göttingen). The expression vector encoding strep-MBP-ROXY9 (438C_strep-ROXY9) was ligated into a bacmid with the help of DH10 Multibac cells. The isolated bacmid was used to transfect High Five insect cells. Expression cultures of $600 \mathrm{ml}$ were grown and harvested in this department, as well. The cells harvested after 
expression were frozen in liquid nitrogen, stored at $-70^{\circ} \mathrm{C}$, and transported on dry ice.

\section{Purification of strep-SUMO-ROXY9 from BL21(DE3)- star using gravity flow columns}

Strep-SUMO-ROXY9 was purified from a cell pellet resulting from a $400 \mathrm{ml}$ expression culture. The pellet was resuspended in $20 \mathrm{ml} \mathrm{Na}$ phosphate buffer $\mathrm{pH}$ 8.0. To this suspension, DTT and lysozyme were added to a final concentration of $1 \mathrm{mM}$ and $25 \mu \mathrm{g} / \mathrm{ml}$, respectively. The sample was incubated for ca. $1 \mathrm{~h}$ at $15 \mathrm{rpm}$ end-over-end in the cold room or for up to $2 \mathrm{~h}$ on ice without agitation. Next, the cells were lysed by sonication (Sonopuls sonifier, MS73 sonotrode: $15 \mathrm{~min}, 1 \mathrm{sec}$ pulse on, $2 \mathrm{sec}$ pulse off, $50 \%$ amplitude), under aerobic conditions or in a nitrogen atmosphere created in an atmosbag glove bag (Sigma-Aldrich) by evaporating liquid nitrogen. The lysate was centrifuged at $20000 \mathrm{rpm}$ in a SS-34 rotor (Sorvall RC6+ centrifuge) at RT to $4^{\circ} \mathrm{C}$ for $45 \mathrm{~min}$. The supernatant was kept as the crude extract for purification. $20 \mu \mathrm{l}$ of the crude extract were taken as a sample for SDS-PAGE and mixed with $20 \mu$ l reducing RPB.

To purify the recombinant protein from the crude extract, $750 \mu \mathrm{l} 50 \%$ StrepTactin slurry were added to a gravity flow column and equilibrated by washing the beads twice with $9 \mathrm{ml} \mathrm{Na}$ phosphate buffer $\mathrm{pH} 8.0+1 \mathrm{mM}$ DT'T. The crude extract was loaded and the flowthrough collected. A $20 \mu \mathrm{l}$ sample of the flowthrough was mixed with an equal volume of non-reducing RPB for SDS-PAGE. The column was washed four times with $2.5 \mathrm{ml} \mathrm{Na}$ phosphate buffer $\mathrm{pH} 8.0+1 \mathrm{mM}$ DTT. Again, a $20 \mu \mathrm{l}$ sample of the last wash step was taken for SDS-PAGE and mixed with non-reducing RPB. Finally, the protein was eluted in four steps, using $375 \mu \mathrm{l} \mathrm{Na}$ phosphate buffer $\mathrm{pH} 8.0+1 \mathrm{mM}$ DTT $+2.5 \mathrm{mM}$ D-desthiobiotin. $10 \mu \mathrm{l}$ of each fraction were taken as a sample for SDS-PAGE and supplied with an equal volume of reducing RPB.

The SDS-PAGE samples were stored at $-20^{\circ} \mathrm{C}$. The protein was stored at $4{ }^{\circ} \mathrm{C}$ or at $-20^{\circ} \mathrm{C}$. 
$50 \mathrm{mM}$ Na phosphate buffer $\mathrm{pH} 8.0$

\begin{tabular}{lc} 
Component & Concentration \\
\hline $\mathrm{Na}_{2} \mathrm{HPO}_{4} / \mathrm{NaH}_{2} \mathrm{PO}_{4} \mathrm{pH} 8.0$ & $50 \mathrm{mM}$ \\
$\mathrm{NaCl}$ & $200 \mathrm{mM}$ \\
\hline
\end{tabular}

This buffer was prepared by mixing $0.5 \mathrm{M} \mathrm{Na}_{2} \mathrm{HPO}_{4}$ and $0.5 \mathrm{M} \mathrm{NaH}_{2} \mathrm{PO}_{4}$ in the ratio $95: 5$ to achieve $\mathrm{pH} 8.0$ at a final concentration of $50 \mathrm{mM}$. After $\mathrm{NaCl}$ was added, the $\mathrm{pH}$ of the buffer was checked and readjusted using $\mathrm{NaOH}$ or $\mathrm{H}_{3} \mathrm{PO}_{4}$, if necessary.

\section{Purification of strep-SUMO-Grx1 from BL21(DE3)-star using gravity flow columns}

Strep-SUMO-Grx1 was purified from three to four E. coli pellets (BL21 or BL21-star), each resulting from a $400 \mathrm{ml}$ expression culture. Each pellet was resuspended in $15 \mathrm{ml}$ Grx1 lysis buffer; alternatively, two pellets were pooled in $15 \mathrm{ml}$ of this buffer. DTT and lysozyme were added to a final concentration of $1 \mathrm{mM}$ and 40 to $80 \mu \mathrm{g} / \mathrm{ml}$, respectively. Following incubation for $1 \mathrm{~h}$ at $15 \mathrm{rpm}$ in the cold room, the DT'T concentration was increased to $5-10 \mathrm{mM}$ in total. Then, the cell suspensions were lysed on ice using a Sonopuls sonifier equipped with an MS73 sonotrode (15 min, 1 sec pulse on, 2 sec pulse off, $50 \%$ amplitude). Afterwards, the cell suspension was transferred to a centrifugation tube and spun at $20000 \mathrm{rpm}$ for $45 \mathrm{~min}$ at $20^{\circ} \mathrm{C}$ (Sorvall RC6+ centrifuge, rotor SS-34). Finally, the supernatants were pooled in a fresh tube to obtain the crude extract for purification. A sample of the crude extract was taken for SDS-PAGE, mixed with $2 x$ reducing RPB and $20 \mu$ of this sample were loaded on SDS gels.

Strep-SUMO-Grx1 was purified via StrepTactin beads. The amount of the column material and the buffer volumes used for the purification depended on the number of pellets lysed. Thus, the amounts and volumes are summarized in the table below. To prepare the column, $50 \%$ StrepTactin slurry were loaded in a gravity flow column and the liquid was allowed to drain off. The Strep'Tactin beads were equilibrated by applying Grx1 wash buffer twice. Next, the crude extract was loaded. The flowthrough was collected in a fresh tube. 
An aliquot of the flowthrough was mixed with $2 \mathrm{x}$ RPB and $20 \mu$ of this mixture were analyzed by SDS-PAGE. The column was washed four times with Grx1 wash buffer. A $20 \mu \mathrm{l}$ sample of the last wash step was taken, supplied with $2 \mathrm{x}$ RPB and checked by SDS-PAGE. Ultimately, strep-SUMO-Grx1 was eluted in three steps using Grx1 elution buffer. A $10 \mu \mathrm{l}$ sample of each elution fraction was mixed with an equal volume of $2 \mathrm{x}$ reducing $\mathrm{RPB}$ and analyzed by SDSPAGE.

The amounts of StrepTactin slurry and the buffer volumes were:

\begin{tabular}{lcc} 
Number of pellets & 3 & 4 \\
\hline Number of gavity flow columns & 1 & 2 \\
V(50\% strepTactin slurry) per column & $750 \mu \mathrm{l}$ & $1 \mathrm{ml}$ \\
V(Wash buffer) for equilibration per column & $9 \mathrm{ml}$ & $12 \mathrm{ml}$ \\
V(Wash buffer) for washing per column & $2 \mathrm{ml}$ & $2.5 \mathrm{ml}$ \\
V(Elution buffer) for elution per column & $375 \mu l$ & $500 \mu l$ \\
\hline
\end{tabular}

Elution fractions, as well as the samples for SDS-PAGE were stored at $-20^{\circ} \mathrm{C}$.

\section{1x Grx1 lysis buffer}

\begin{tabular}{lc} 
Component & Concentration \\
\hline TRIS-HCl pH 7.5 & $50 \mathrm{mM}$ \\
$\mathrm{Na}_{2}$-EDTA & $1 \mathrm{mM}$ \\
\hline
\end{tabular}

This buffer was prepared as a $10 \mathrm{x}$ stock solution, adjusting the $\mathrm{pH}$ at $\mathrm{RT}$ to 7.5 using $\mathrm{HCl}$. The stock solution was autoclaved. To obtain $1 \mathrm{x}$ buffer, the $10 \mathrm{x}$ stock was diluted adequately and the $\mathrm{pH}$ readjusted to 7.5 using $\mathrm{NaOH}$ or HCl. 1x Grx1 lysis buffer was then used to prepare all other Grx1 purification buffers.

\section{1x Grx1 wash buffer}

\begin{tabular}{lc} 
Component & Concentration \\
\hline TRIS-HCl pH 7.5 & $50 \mathrm{mM}$ \\
$\mathrm{Na}_{2}$-EDTA & $1 \mathrm{mM}$ \\
DTT & $10 \mathrm{mM}$ \\
\hline
\end{tabular}

To prepare this buffer, $1 \mathrm{mM}$ DTT was added to 1x Grx1 lysis buffer. 


\section{1x Grx1 elution buffer}

\begin{tabular}{lc} 
Component & Concentration \\
\hline TRIS-HCl pH 7.5 & $\sim 50 \mathrm{mM}$ \\
Na2-EDTA & $\sim 1 \mathrm{mM}$ \\
Glycerol & $10 \%(\mathrm{w} / \mathrm{v})$ \\
DTT & $5-10 \mathrm{mM}$ \\
D-Desthiobiotin & $2.5 \mathrm{mM}$ \\
\hline
\end{tabular}

To prepare this buffer, $10 \mathrm{~g} \mathrm{100 \%} \mathrm{glycerol} \mathrm{were} \mathrm{filled} \mathrm{up} \mathrm{to} 100 \mathrm{ml}$ using $1 \mathrm{x}$ Grx1 lysis buffer and supplied freshly with DT'T and D-Desthiobiotin.

\section{Test purification of strep-MBP-ROXY9 from insect cells using amylose resin}

For the small-scale purification of strep-MBP-ROXY9 from insect cells, the cells from $25 \mathrm{ml}$ culture were concentrated in $1 \mathrm{ml}$ and stored at $-70{ }^{\circ} \mathrm{C}$. Prior to purification, a $1 \mathrm{ml}$ aliquot was thawed on ice or at RT and mixed with $0.2 \mathrm{~g}$ acid-washed glass beads with a diameter of 400 to $650 \mu \mathrm{m} .100 \mu \mathrm{l}$ x MBP lysis buffer supplied with $1 \mathrm{mM}$ DTT (page 93) an 1x Protease Inhibitor Cocktail were added. Then, the cells were lysed in a bead beater, shaking them three times for $30 \mathrm{sec}$ at $30 \mathrm{~Hz}$. In between, the cells were placed on ice. Afterwards, the lysate was spun for $15 \mathrm{~min}$ at $17000 \mathrm{~g}$ at $4^{\circ} \mathrm{C}$. The supernatant (crude extract) was used for the purification via amylose-coated beads. A $10 \mu \mathrm{l}$ sample of the crude extract was taken for SDS-PAGE and mixed with reducing RPB.

For the purification, amylose resin in $20 \% \mathrm{EtOH}$ (Biolabs) was used. The beads were centrifuged at RT at $12000 \mathrm{~g}$ throughout the entire procedure. $300 \mu \mathrm{l}$ resin were transferred into a $1.5 \mathrm{ml}$ reaction tube and centrifuged for $30 \mathrm{sec}$. The ethanol was removed. For equilibration, the beads were resuspended in $300 \mu \mathrm{l} 1 \mathrm{x}$ MBP lysis buffer wit $1 \mathrm{mM}$ DTT and 1x Protease Inhibitor Cocktail, centrifuged again for $10 \mathrm{sec}$. After removal of the supernatant, $600 \mu \mathrm{l}$ fresh lysis buffer were added, the beads resuspended and spun down at for $10 \mathrm{sec}$. The supernatant was removed and the washing with another fresh $600 \mu \mathrm{l}$ aliquot of the buffer repeated. In this last step, the beads 
were sedimented by centrifugation for $30 \mathrm{sec}$ and the supernatant was removed.

The equilibrated beads were mixed with the insect cell crude extract and incubated overnight in the cold room attached to a rotor, turning them at $10 \mathrm{rpm}$ end-over-end. The next day, the sample was centrifuged for $1 \mathrm{~min}$ and the supernatant corresponding to the flowthrough transferred to a new reaction tube. A $10 \mu$ sample of the flowthrough was taken for SDS-PAGE and mixed with reducing $\mathrm{RPB}$. The beads were washed twice with $1 \mathrm{ml} 1 \mathrm{x}$ MBP lysis buffer with $1 \mathrm{mM}$ DTT and 1x Protease Inhibitor Cocktail, centrifuging them for $10 \mathrm{sec}$ and discarding the supernatant. In the third washing step, the beads were pelleted for $1 \mathrm{~min}$ and the supernatant was collected in a fresh tube. A $20 \mu$ sample of this wash step was taken for SDS-PAGE, mixing it with reducing RPB. The protein bound to the amylose beads was eluted in three steps. For each elution step, the beads were resuspended in $300 \mu \mathrm{l} 1 \mathrm{x}$ MBP elution buffer containing $1 \mathrm{mM}$ DTT (page 93) and 1x Protease Inhibitor, incubated for $5 \mathrm{~min}$ on ice and finally centrifuged for $1 \mathrm{~min}$. The supernatant was transferred to a new reaction tube. Finally, a $20 \mu \mathrm{l}$ sample from each elution step was taken for SDS-PAGE analysis and mixed with reducing RPB.

\section{Purification of strep-MBP from BL21 using an ÄKTA purifier}

Strep-MBP was purified from E. coli BL21 cell pellets resulting from four $400 \mathrm{ml}$ expression cultures. Each pellet was thawed and resuspended in $10 \mathrm{ml}$ 1xMBP lysis buffer with $50 \mathrm{mM} \mathrm{NaCl}$ (page 95). Two suspensions were pooled and mixed with $2-3$ spatulas lysozyme. Lysozyme-mediated degradation of the bacterial cell wall took place in the cold room for $15 \mathrm{~min}$, rotating the samples at $15 \mathrm{rpm}$ end-over-end. Each suspension was lysed by sonication on ice for $15 \mathrm{~min}$ at $50 \%$ amplitude using 1 sec-pulses followed by 2 sec-breaks. Afterwards, the suspensions were centrifuged at $20000 \mathrm{rpm}$ at $4^{\circ} \mathrm{C}$ for $45 \mathrm{~min}$ (Sorvall RC6+, SS-34). The supernatants were pooled and filtered through a $0.45 \mu \mathrm{m}$ sterile filter. A $20 \mu \mathrm{l}$ sample of the resulting crude extract was taken for SDS-PAGE and mixed with an equal volume of reducing RPB. The rest of 
the crude extract was used for purification. For purification, two $5 \mathrm{ml}$ MBPTrap columns were connected to an ÄKTA prime plus device and equilibrated with 1 xMBP lysis buffer with $50 \mathrm{mM} \mathrm{NaCl}$. The crude extract was loaded with the help of a $50 \mathrm{ml}$ superloop at a flow rate of $1 \mathrm{ml} / \mathrm{min}$, collecting $10 \mathrm{ml}$ fractions. The column was washed with 1x MBP lysis buffer with $50 \mathrm{mM}$ $\mathrm{NaCl}$ at a flow rate of $1 \mathrm{ml} / \mathrm{min}$, collecting again $10 \mathrm{ml}$ fractions, until the $\mathrm{A}_{280 \mathrm{~nm}}$ reached the baseline level. For elution, $100 \%$ of 1x MBP elution buffer containing $50 \mathrm{mM} \mathrm{NaCl}$ (page 95) were passed at a flow rate of $1 \mathrm{ml} / \mathrm{min}$ over the column, fractionating the eluate in $2 \mathrm{ml}$ steps. $20 \mu \mathrm{l}$ of each flowthrough and wash fraction and $30 \mu \mathrm{l}$ of each elution fraction were mixed with an equal volume of non-reducing RPB and separated by SDS-PAGE.

\section{ÄKTA-based purification of strep-MBP-ROXY9 (insect cells)}

High Five insect cells expressing strep-MBP-ROXY9 from a $600 \mathrm{ml}$ culture were pelleted and resuspended in 30 to $50 \mathrm{ml}$ Buffer $\mathrm{L}$ at the department for Molecular Biology (Prof. Dr. Patrick Cramer, MPI for Biophysical Chemistry, Göttingen). Prior to lysis, the cells were briefly thawed by placing the tubes in a beaker containing water. Sonication was performed on ice employing a Sonopuls sonifier with an M73 sonotrode at an amplitude of $30 \%$ and alternating a $0.4 \mathrm{sec}$-pulse and a $0.6 \mathrm{sec}$-break over $5 \mathrm{~min}$. The resulting cell lysate was transferred to a centrifugation tube and centrifuged at $20000 \mathrm{rpm}$ for 45 min at RT to $4^{\circ} \mathrm{C}$ (Sorvall centrifuge RC6+, rotor SS-34). The supernatant was filtered through a $0.45 \mu \mathrm{m}$ sterile filter. A small aliquot was mixed with $2 \mathrm{x}$ RPB. $20 \mu$ of this mixture were subjected to SDS-PAGE.

To purifiy strep-MBP-ROXY9, an ÄKTA prime plus purifier (GE Healthcare) placed at 10 to $20^{\circ} \mathrm{C}$ was connected to two $5 \mathrm{ml}$ MBPTrap columns (GE Healthcare). The columns were equilibrated with 1x MBP lysis buffer. Then, the filtered crude extract was loaded at a flow rate of $1 \mathrm{ml} / \mathrm{min}$ using a $50 \mathrm{ml}$ superloop (GE Healthcare). The flowthrough was collected in $5 \mathrm{ml}$ fractions. After loading, the column was washed until the absorption at $280 \mathrm{~nm}$ returned to the baseline absorption of the $1 \mathrm{x}$ MBP lysis buffer. The flow rate during 
washing was $1 \mathrm{ml} / \mathrm{min}$ and the fraction size was $5 \mathrm{ml}$. Bound protein was eluted using $1 \mathrm{x}$ MBP elution buffer at a flow rate of $1 \mathrm{ml} / \mathrm{min}$, fractionating $2 \mathrm{ml}$. Protein-containing fractions were stored at $4^{\circ} \mathrm{C}$ in a fridge. To analyze the success of protein purification, $10 \mu \mathrm{l}$ of each fraction of interest were mixed with $2 x$ RPB and separated by SDS-PAGE.

\section{1x Buffer $L$}

\begin{tabular}{lc} 
Component & Concentration \\
\hline HEPES pH 7.5 & $20 \mathrm{mM}$ \\
$\mathrm{NaCl}$ & $300 \mathrm{mM}$ \\
$\mathrm{Na} 2$-EDTA & $1 \mathrm{mM}$ \\
Imidazol & $30 \mathrm{mM}$ \\
DT'T & $1 \mathrm{mM}$ \\
Protease Inhibitor Cocktail & $1 \mathrm{x}$ \\
\hline
\end{tabular}

\section{1x MBP lysis buffer}

\begin{tabular}{lc} 
Component & Concentration \\
\hline HEPES pH 7.5 & $20 \mathrm{mM}$ \\
$\mathrm{NaCl}$ & $300 \mathrm{mM}$ \\
$\mathrm{Na}_{2}$-EDTA & $2 \mathrm{mM}$ \\
Glycerol & $5 \%(\mathrm{w} / \mathrm{v})$ \\
DTT & $1 \mathrm{mM}$ \\
\hline
\end{tabular}

A 5x stock solution of this buffer was prepared omitting DTT and glycerol. The $\mathrm{pH}$ was adjusted at $\mathrm{RT}$ using $\mathrm{NaOH}$ and the stock solution was autoclaved. Prior to purification, the $5 \mathrm{x}$ buffer was diluted adequately mixing it with glycerol and DTT to the final concentrations. The $\mathrm{pH}$ of the final $1 \mathrm{x}$ buffer was not readjusted. For ÄKTA-based purification, the buffer was filtered through a Nylon membrane (pore size $0.2 \mu \mathrm{M}$ ) and degassed. 


\section{1x MBP elution buffer}

\begin{tabular}{lc} 
Component & Concentration \\
\hline HEPES pH 7.5 & $20 \mathrm{mM}$ \\
$\mathrm{NaCl}$ & $300 \mathrm{mM}$ \\
$\mathrm{Na}$-EDTA & $2 \mathrm{mM}$ \\
Glycerol & $5 \%(\mathrm{w} / \mathrm{v})$ \\
D-Maltose & $100 \mathrm{mM}$ \\
DTT & $1 \mathrm{mM}$ \\
\hline
\end{tabular}

To prepare this buffer, $5 \mathrm{x}$ MBP lysis buffer was diluted adequately, dissolving maltose, glycerol and DTT. The $\mathrm{pH}$ of the elution buffer was not readjusted. As for the 1x MBP lysis buffer, the buffer was filtered through a Nylon membrane (pore size $0.2 \mu \mathrm{M}$ ) and degassed.

\section{strep-MBP-GRXC2 from E. coli using an ÄKTA purifier}

Strep-MBP-GRXC2 was purified from four E. coli BL21-star pellets obtained by expression of $400 \mathrm{ml}$ cultures. The pellets were thawed by placing the tubes briefly in a beaker containing water. Two pellets were pooled by resuspending them in a total volume of $20 \mathrm{ml} 1 \mathrm{x}$ MBP lysis buffer containing $50 \mathrm{mM} \mathrm{NaCl}$. With the tip of a spatula, lysozyme was added to the cell suspension and the mixture incubated for ca. $30 \mathrm{~min}$ in the cold room, rotating the samples at $15 \mathrm{rpm}$. The cells were lysed by sonication on ice (Sonifier Sonopuls, sonotrode MS73; Programm: $15 \mathrm{~min}, 1 \mathrm{sec}$ pulse on, $2 \mathrm{sec}$ pulse off, $50 \%$ amplitude). The lysate was centrifuged at 10 to $4^{\circ} \mathrm{C}$ for $45 \mathrm{~min}$ at $20000 \mathrm{rpm}$ in a SS-34 rotor (Sorvall RC6+ centrifuge). After centrifugation, the supernatants were pooled and filtered through a $0.45 \mu \mathrm{m}$ sterile filter prior to purification via an ÄKTA prime plus purifier equipped with two $5 \mathrm{ml}$ MBPTrap columns. The columns were equilibrated with 1x MBP lysis buffer containing $50 \mathrm{mM} \mathrm{NaCl}$. After taking a small sample for SDS-PAGE, the crude extract was loaded using a $50 \mathrm{ml}$ superloop at a flow rate of $1 \mathrm{ml} / \mathrm{min}$, collecting $5 \mathrm{ml}$ fractions. The column was washed with $1 \mathrm{x}$ MBP lysis buffer containing $50 \mathrm{mM} \mathrm{NaCl}$ until the baseline absorption at $280 \mathrm{~nm}$ of the buffer was reached. Elution took place at $1 \mathrm{ml} / \mathrm{min}$, fractionating $2 \mathrm{ml}$, and using $1 \mathrm{x}$ MBP elution buffer containing $50 \mathrm{mM} \mathrm{NaCl}$. To analyze the purification, $20 \mu \mathrm{l}$ of each fraction of interest were mixed with 2x RPB and loaded on an SDS gel. 
1x MBP lysis buffer containing $50 \mathrm{mM} \mathrm{NaCl}$

\begin{tabular}{lc} 
Component & Concentration \\
\hline HEPES pH 7.5 & $20 \mathrm{mM}$ \\
$\mathrm{NaCl}$ & $50 \mathrm{mM}$ \\
$\mathrm{Na}_{2}$-EDTA & $2 \mathrm{mM}$ \\
Glycerol & $5 \%(\mathrm{w} / \mathrm{v})$ \\
DTT & $1-2 \mathrm{mM}$ \\
\hline
\end{tabular}

A $5 \mathrm{x}$ stock solution and $1 \mathrm{x}$ dilutions of this stock were prepared as described for the MBP lysis buffer used for purification of strep-MBP-ROXY9 (page 93).

\section{1x MBP elution buffer containing $50 \mathrm{mM} \mathrm{NaCl}$}

\begin{tabular}{lc} 
Component & Concentration \\
\hline HEPES pH 7.5 & $20 \mathrm{mM}$ \\
$\mathrm{NaCl}$ & $50 \mathrm{mM}$ \\
$\mathrm{Na}_{2}$-EDTA & $2 \mathrm{mM}$ \\
Glycerol & $5 \%(\mathrm{w} / \mathrm{v})$ \\
D-Maltose & $100 \mathrm{mM}$ \\
DTT & $1-2 \mathrm{mM}$ \\
\hline
\end{tabular}

This buffer was prepared as described for the MBP lysis buffer used for purification of strep-MBP-ROXY9 (page 93).

\section{Purification of TEV protease from E. coli cells using an ÄKTA purification system}

His-tagged TEV protease was purified from E. coli pellets derived from a $400 \mathrm{ml}$ expression culture. The pellets were thawed in water. Each pellet was resuspended in $20 \mathrm{ml} \mathrm{1x}$ TEV wash buffer. With the tip of a spatula, lyszyme was added and the cell suspensions incubated for approximately $30 \mathrm{~min}$ in the cold room at $15 \mathrm{rpm}$. Then, each pellet was lysed by sonication on ice for 15 min using a Sonopuls sonifier and an MS73 sonotrode. Over these $15 \mathrm{~min}$, the suspension was pulsed with $50 \%$ amplitude for $1 \mathrm{sec}$, followed by a break 
of $2 \mathrm{sec}$. The cell lysate was centrifuged for $45 \mathrm{~min}$ at $4^{\circ} \mathrm{C}$ in an SS-34 rotor (Sorval RC6+ centrifuge) at $20000 \mathrm{rpm}$. The supernatants were pooled and filtrered through an $0.45 \mu \mathrm{m}$ sterile filter.

Purification was performed using one $5 \mathrm{ml}$ HisTrap column per pellet. The column(s) were connected to an ÄKTA prime plus device (GE healthcare). The crude extract was loaded using a Superloop at a flow rate of $1 \mathrm{ml} / \mathrm{ml}$ collecting $5 \mathrm{ml}$ fractions. Then, the column was washed using $1 \mathrm{x}$ TEV wash buffer. Once the baseline of $A_{280 \mathrm{~nm}}$ was reached, a gradient ranging from 0 to $100 \%$ 1xTEV elution buffer over $25 \mathrm{ml}$ per pellet was applied. Finally, TEV protease was eluted using $100 \% 1 \mathrm{x}$ TEV elution bufffer. During elution, $2.5 \mathrm{ml}$ fractions per pellet were collected. Those fractions containing TEV protease were pooled and concentrated. To remove the imidazol, the protein was dialysed in the cold room for $3 \mathrm{~h}$ against a 1000-fold volume of $1 \mathrm{x}$ TEV wash buffer using a MEMBRA-CEL dialysis tubing ( $3 \mathrm{kDa}$ Molecular weight cut-off (MWCO)). After that, the buffer was exchanged, and dialysis continued over night against the ca. 1000-fold volume of fresh 1x TEV wash buffer. Alternatively, TEV protease was dialysed four times (three times for $45 \mathrm{~min}$ and once over night) against the 700 -fold buffer volume.

\section{1x TEV wash buffer}

\begin{tabular}{lc} 
Component & Concentration \\
\hline TRIS pH 8.0 & $50 \mathrm{mM}$ \\
$\mathrm{NaCl}$ & $150 \mathrm{mM}$ \\
Glycerol & $5 \%(\mathrm{w} / \mathrm{v})$ \\
\hline
\end{tabular}

The $\mathrm{pH}$ of the buffer was adjusted at $\mathrm{RT}$ using $\mathrm{HCl}$.

\section{1x TEV elution buffer}

\begin{tabular}{lc} 
Component & Concentration \\
\hline TRIS pH 8.0 & $50 \mathrm{mM}$ \\
$\mathrm{NaCl}$ & $150 \mathrm{mM}$ \\
Glycerol & $5 \%(\mathrm{w} / \mathrm{v})$ \\
Imidazol & $250 \mathrm{mM}$ \\
\hline
\end{tabular}

The $\mathrm{pH}$ of the buffer was adjusted at RT with $\mathrm{HCl}$. 


\section{TEV digest of strep-MBP-ROXY9}

To cleave off the tag from strep-MBP-ROXY9, His-TEV, freshly purified from E. coli, was used. Strep-MBP-ROXY9 was present in 1x MBP elution buffer (page 93), whereas TEV protease was present in TEV wash buffer (page 96). The protein concentrations were determined according to Bradford. $4.8 \mathrm{mg}$ strep-MBP-ROXY9 were mixed with $3.48 \mathrm{mg}$ His-TEV and filled up to $15 \mathrm{ml}$ with TEV wash buffer. A sample of $20 \mu \mathrm{l}$ was removed from the mixture and supplied with $20 \mu \mathrm{l}$ reducing RPB for analysis by SDS-PAGE. To initiate the cleavage reaction, $30 \mu \mathrm{l} 1 \mathrm{M}$ DTT were added to the remaining digest. Then, the digest was incubated over night at $4^{\circ} \mathrm{C}$. Afterwards, the digest was centrifuged at $4000 \mathrm{rpm}$ at $4^{\circ} \mathrm{C}$ for ca. 30 min to remove precipitated protein. A $20 \mu \mathrm{l}$ sample of the supernatant was taken for SDS-PAGE and mixed with reducing RPB. Subsequently, uncut protein, TEV protease and the strep-MBP tag were tried to be removed by several purification steps: First, a $5 \mathrm{ml}$ MBPTrap and a $5 \mathrm{ml}$ HisTrap column were connected to an ÄKTA purifier and equilibrated with TEV wash buffer. The digest was passed over the column using TEV wash buffer at a flow rate of $1 \mathrm{ml} / \mathrm{min}$, collecting $2 \mathrm{ml}$ fractions. All protein-containing fractions were pooled and a $30 \mu \mathrm{l}$ sample taken for SDSPAGE. To the remaining mixture, DTT was added in a final concentration of $1 \mathrm{mM}$. The digest was further purified via StrepTactin resin placed in a gravity flow column. For this, $2 \mathrm{ml}$ of the $50 \%$ StrepTactin slurry were added to a $12 \mathrm{ml}$-gravity flow column and equilibrated by filling up the column twice with TEV wash buffer. The pooled fractions were passed over the resin and the flowthrough was collected. Again, $30 \mu \mathrm{l}$ were removed as a sample for SDSPAGE and mixed with an equal volume of reducing RPB. Finally, the flowthrough was concentrated about 20 -fold at $4^{\circ} \mathrm{C}$ using a VivaSpin concentrator with an MWCO of $3 \mathrm{kDa}$. After dialysis against the desired buffer, this preparation was used for experiments. Of the samples taken during the preparation of ROXY9, $40 \mu \mathrm{l}$ were separated by $15 \%$ SDS-PAGE. 


\section{Dialysis}

Dialysis was either performed using ZelluTrans dialysis tubing (MWCO: 12 $14 \mathrm{kDa}$, Roth) or MEMBRA-CEL (Serva) dialysis tubing (MWCO: $3 \mathrm{kDa}$ ). The tubings were prepared for use according to the manufacturer's instructions: While ZelluTrans tubings were soaked in and rinsed with $\mathrm{dH}_{2} \mathrm{O}$, MEMBRACEL tubings were boiled for 2 to 3 times in $\mathrm{dH}_{2} \mathrm{O}$ for at least $20 \mathrm{~min}$. On account of the rather small enzyme volumes used for dialysis, dialysis was usually performed in reaction tubes, the lid of which was scooped out with a hot needle. The protein was added to these tubes, fixing a single layer of dialysis tubing under the hole in the lid. The tubes were placed upside-down in dialysis buffer. Dialysis took place in the cold room, stirring the buffer constantly. Buffer composition, buffer volume and duration of dialysis and dialysis tubing depended on the type of experiment. At the end of dialysis, 50 to $100 \mathrm{ml}$ of dialysis buffer were kept as a buffer control for experiments and protein concentration measurements.

\begin{tabular}{|c|c|c|c|c|}
\hline & Tubing & Buffer & Volume & Duration/Steps \\
\hline \multirow{4}{*}{$\begin{array}{l}\text { HEDS, } \\
\text { Insulin, and } \\
\text { GAPDH } \\
\text { assay }\end{array}$} & MembraCel & $\begin{array}{c}\mathrm{Na} / \mathrm{K} \text { phosphate } \\
\text { pH } 7.5\end{array}$ & $3333 x$ & $1 \mathrm{~h}+\mathrm{ON}$ \\
\hline & MembraCel & $\begin{array}{c}\mathrm{Na} / \mathrm{K} \text { phosphate } \\
\text { pH } 7.5\end{array}$ & $2500 x$ & $3 \mathrm{x} 1 \mathrm{~h}+1 \mathrm{x} O \mathrm{ON}$ \\
\hline & Zellutrans & $\begin{array}{c}\mathrm{Na} / \mathrm{K} \text { phosphate } \\
\mathrm{pH} 7.5+\mathrm{GSH}\end{array}$ & $1500 x$ & $\begin{array}{c}2 \mathrm{x} 2 \mathrm{~h}+1 \mathrm{x} 1 \mathrm{~h}, \\
1 \mathrm{x} \text { ON }\end{array}$ \\
\hline & MembraCel & $\begin{array}{c}\mathrm{Na} / \mathrm{K} \text { phosphate } \\
\mathrm{pH} 7.5+\mathrm{GSH}\end{array}$ & $\begin{array}{c}2333 x, \\
\text { then } \\
3333 x\end{array}$ & $\begin{array}{c}1 \mathrm{x} 4 \mathrm{~h}+\text { for } \\
2 \mathrm{~d}\end{array}$ \\
\hline $\begin{array}{l}\text { Ellman's } \\
\text { assay }\end{array}$ & Zellutrans & $\mathrm{Na} / \mathrm{K}$ phosphate & $1000 \mathrm{x}$ & $\begin{array}{c}2 \mathrm{x} 2 \mathrm{~h}+1 \mathrm{x} 1 \mathrm{~h}, \\
1 \mathrm{x} \text { ON }\end{array}$ \\
\hline
\end{tabular}

\section{$5 \mathrm{xNa} / \mathrm{K}$ phosphate buffer}

This buffer was prepared as described by Starke et al. (2003) [87]; however, $0.5 \mathrm{M}$ stock solutions of $\mathrm{Na}_{2} \mathrm{HPO}_{4}$ and $\mathrm{KH}_{2} \mathrm{PO}_{4}$ were used. A given volume of $0.5 \mathrm{M} \mathrm{Na}_{2} \mathrm{HPO}_{4}$ was mixed with $\mathrm{KH}_{2} \mathrm{PO}_{4}$ until a $\mathrm{pH}$ of 7.5 was reached. Before use, this $5 \mathrm{x}$ stock solution was diluted adequately. 


\section{Analytical gel filtration of strep-MBP-ROXY9}

Analytical gel filtration was performed in cooperation with the department for Molecular Enzymology (Prof. Dr. Kai Tittmann, University Göttingen). For this, a Superdex ${ }^{\text {TM }} 200$ 10/300 GL column connected to an ÄKTApure device equipped with a Fraction Collector F9-R was equilibrated with 1x MBP lysis buffer containing $1 \mathrm{mM} \mathrm{DTT}$ (page 93). Strep-MBP-ROXY9 was concentrated to approximately $7 \mu \mathrm{g} / \mu \mathrm{l}$. After centrifugation at $17000 \mathrm{~g}$ and $4^{\circ} \mathrm{C}$ to remove precipitated protein, ca. $150 \mu \mathrm{l}$ were injected into a $100 \mu \mathrm{l}$ loop and loaded on the column. For separation, the column was washed with 1x MBP lysis buffer at a flow rate of $0.5 \mathrm{ml} / \mathrm{min}$, fractionating $0.5 \mathrm{ml}$. Additionally, a gel filtration standard (BioRad \#1511901) was passed over the column in three replicates under the same conditions used for strep-MBP-ROXY9. The elution volume of all peaks observed during the separation of strep-MBP-ROXY9 and the gel filtration standard was determined using the software Unicorn 7.1 (GE Healthcare). For the size standard, the mean of the elution volume of each standard molecule was calculated. Then, the partition coefficient $\left(\mathrm{K}_{\mathrm{av}}\right)$ for each peak was determined using the following formula:

$$
K_{a v}=\frac{V_{e}-V_{0}}{V_{c}-V_{0}}
$$

$\mathrm{V}_{\mathrm{e}}$ corresponds to the volume after which the protein elutes. $\mathrm{V}_{0}$ is the void volume, corresponding to those proteins which are too big for separation and, thus, elute first from the column. $\mathrm{V}_{\mathrm{c}}$ can be approximated by the total volume of the column (24 ml for Superdex ${ }^{\mathrm{TM}} 200$ 10/300 GL).

The $\mathrm{K}_{\mathrm{av}}$ values for the size standard were plotted against $\log$ (Molecular weigth $(\mathrm{MW})$ ). Using the $\mathrm{K}_{\mathrm{av}}$ value for strep-MBP-ROXY9, its $\log (\mathrm{MW})$ was calculated and converted into the value of its molecular weight. 
Production and analysis of recombinant proteins

\section{Concentrating proteins}

Proteins were concentrated using concentrator tubes with a polyether sulfone membrane and an adequate MWCO (obtained from Corning or GE Healthcare). The concentrators loaded with the protein were spun at $4^{\circ} \mathrm{C}$ and $4000 \mathrm{rpm}$ in a Rotina $380 \mathrm{R}$ centrifuge, in which a $17984000 \mathrm{rpm} 4 \mathrm{x} 300 \mathrm{~g}$ rotor was fastened.

The following table lists the concentrators used for certain proteins:

\begin{tabular}{lc}
\hline Protein & Concentrator \\
\hline Strep-MBP-ROXY9 & Spin-X® UF 6; \\
& 30 or $50 \mathrm{kDa}$ MWCO \\
ROXY9 after TEV cleavage & (Corning) \\
& Vivaspin 20; \\
& $3 \mathrm{kDa}$ MWCO \\
His-TEV & (GE Healthcare) \\
& Vivaspin 20; \\
& $3 \mathrm{kDa}$ MWCO \\
\hline
\end{tabular}




\section{Characterization of glutaredoxins}

The catalytic activity of glutaredoxins was measured towards the substrates HEDS, insulin, and glutathionylated GAPDH (GAPDH-SG). For all those experiments, the glutaredoxins were dialyzed against $1 \mathrm{xNa} / \mathrm{K}$ phosphate buffer (page 98). Buffer without $1 \mathrm{mM}$ glutathione served to remove DTT from the purification process. Buffer supplemented with $1 \mathrm{mM}$ glutathione served to remove DTT while keeping the proteins reduced through the action of glutathione. The proteins were dialyzed over night, using ZelluTrans (Roth) or MEMBRA-CEL (Serva) tubings. The buffer was at least exchanged once. Buffer volumes were used such that the total purification buffer volume was diluted at least by a factor of 7777000 during the entire dialysis process. When removing only DTT, the glutaredoxin activity experiments were conducted within the next two days after dialysis. When exchanging DTT for GSH, the proteins were stored for a longer time. The protein concentration for the assays was determined according to Bradford. A $100 \mathrm{mM}$ stock of L-glutathione was always prepared freshly before the experiments.

\section{Bis(2-hydroxyethyl)disulfide (HEDS) assay}

During the HEDS assay, the artificial compound HEDS is reduced by the glutaredoxin, leading to the formation of GSSG. Coupling the reduction of HEDS to the reduction of GSSG by GSSG reductase allows photometric measurement of the reaction progress, since GSSG reductase requires electrons from NADPH for its catalysis. In contrast to NADPH, oxidized nicotinamide adenine dinucleotide phosphate $\left(\mathrm{NADP}^{+}\right)$does not absorb at $340 \mathrm{~nm}$. Thus, a decline in absorption at this wavelength indicates proceeding reduction of HEDS. However, the first steps of HEDS reduction by glutaredoxins are not fully clarified. HEDS might first react with GSH to GSSEtOH. Subsequent deglutathionylation of GSSEtOH by the glutaredoxin leads to formation of 
GSSG. In parallel, glutaredoxins might reduce HEDS directly and are recycled by glutathione, producing GSSG [56,75,101,107,240,241].

The protocol of the HEDS assay was adapted from Gallogly et al. (2008) [242] and modified. A single reaction of the HEDS assay contained:

\begin{tabular}{lcc} 
Component & Volume & Concentration \\
\hline Na/K phosphate buffer $(5 \mathrm{x})$ & $40 \mu \mathrm{l}$ & $1 \mathrm{x}$ \\
HEDS $(8.75 \mathrm{M})$ & $0.02 \mu \mathrm{l}$ & $0.875 \mathrm{mM}$ \\
NADPH $(20 \mathrm{mM})$ & $2 \mu \mathrm{l}$ & $0.2 \mathrm{mM}$ \\
GSSG reductase $(1.5 \mathrm{mg} / \mathrm{ml})$ & $0.25 \mu \mathrm{l}$ & $2 \mu \mathrm{g} / \mathrm{ml}$ \\
GSH $(100 \mathrm{mM})$ & $2 \mu \mathrm{l}$ & $1 \mathrm{mM}$ \\
Glutaredoxin $(0.1-20 \mu \mathrm{M}) /$ Dialysis & $10 \mu \mathrm{l}$ & $0.005-2 \mu \mathrm{M}$ \\
buffer & & \\
\hline & $\mathrm{Ad} 200 \mu \mathrm{l}$ & \\
& $\mathrm{dH}_{2} \mathrm{O}$ \\
\hline
\end{tabular}

$10 \mu$ lof the enyzme or the dialysis buffer in case of the no-enzyme control were pipetted into the wells of a microtiter plate. Then, a master mix containing all other components of the reaction was prepared and $190 \mu \mathrm{l}$ of it quickly distributed to each well. The absorption at $340 \mathrm{~nm}$ was measured imeadiately every $20 \mathrm{sec}$ over $20 \mathrm{~min}$.

\section{Insulin assay}

In the insulin assay, the reduction of insulin by glutaredoxins is measured. Insulin consists of two chains, A and B, which are linked by disulfide bridges. Glutaredoxins can reduce these disulfide bridges, thereby resolving the linkage between chain A and chain B. Chain B, however, is insoluble, when not bound to chain $\mathrm{A}$ and precipitates. Thus, ongoing insulin reduction can be monitored by measuring the turbidity of the solution at $650 \mathrm{~nm}[78,243]$.

The insulin assay was performed as described in Zaffagnini et al. (2008) [78]. A single reaction of the insulin assay contained: 


\begin{tabular}{lcc} 
Component & Volume/Amount & Concentration \\
\hline K phosphate buffer pH $7.0(0.5 \mathrm{M})$ & $40 \mu \mathrm{l}$ & $0.1 \mathrm{M}$ \\
$\mathrm{Na}_{2}$-EDTA pH $8.0(0.5 \mathrm{M})$ & $0.8 \mu \mathrm{l}$ & $2 \mathrm{mM}$ \\
Insulin & $0.015 \mathrm{mg}$ & $0.075 \mu \mathrm{g} / \mu \mathrm{l}$ \\
$\mathrm{GSH}(100 \mathrm{mM})$ & $2 \mu \mathrm{l}$ & $1 \mathrm{mM}$ \\
$\begin{array}{l}\text { Glutaredoxin }(0.1-20 \mu \mathrm{M}) / \text { Dialysis } \\
\text { buffer }\end{array}$ & $10 \mu \mathrm{l}$ & $0.005-2 \mu \mathrm{M}$ \\
\hline \multicolumn{3}{l}{$\mathrm{Ad} 200 \mu \mathrm{l} \mathrm{\textrm {dH } _ { 2 } \mathrm { O }}$} \\
\hline
\end{tabular}

First, a master mix containing $\mathrm{K}$ phosphate buffer, EDTA and $\mathrm{dH}_{2} \mathrm{O}$ was prepared. The required amount of insulin was weighed and dissolved in this mix, rotating it at $15 \mathrm{rpm}$ at room temperature. In the meantime, $10 \mu \mathrm{l}$ of the enzyme or dialysis buffer for the "No enzyme" control were pipetted into the wells of a microtiter plate. When dissolved, $188 \mu \mathrm{l}$ of the master mix were added to the wells. Finally, $2 \mu \mathrm{l} 100 \mathrm{mM}$ glutathione were added and the reactions mixed. The microtiter plate was incubated for $10 \mathrm{~h}$ at room temperature in a plate reader, shaking the plate before measuring $\mathrm{A}_{650 \mathrm{~nm}}$ every $10 \mathrm{~min}$.

\subsection{K phosphate buffer $\mathrm{pH} 7.0$}

To prepare this buffer, $0.5 \mathrm{M} \mathrm{K}_{2} \mathrm{HPO}_{4}$ were mixed with $0.5 \mathrm{M} \mathrm{KH}_{2} \mathrm{PO}_{4}$ until a $\mathrm{pH}$ of 7.0 at $\mathrm{RT}$ was reached.

\section{GAPDH assay}

The GAPDH assay is based on the inactivation of GAPDH by glutathionylation and its reactivation by glutathione. GAPDH catalyzes the conversion of glyceraldehyde-3- phosphate (GAP) to 1,3-bisphosphoglycerate (BPGA), thereby consuming inorganic phosphate and generating NADH. The reaction is reversible. The GAPDH assay makes use of the reverse reaction: First, GAPDH forms a covalent intermediate with BPGA via a catalytic cysteine residue. Then, electrons are transferred from NADH to this intermediate yielding the final reaction product GAP and releasing oxidized nicotinamide adenine dinucleotide $\left(\mathrm{NAD}^{+}\right)$. The catalytic cysteine residue of 
GAPDH has been shown to be glutathionylated, leading to the inactivation of the enzyme $[37,78]$.

The GAPDH assay was adapted from Zaffagnini et al. (2008) [78].

For this experiment, several solutions were prepared freshly:

- $250 \mu \mathrm{M}$ rabbit muscle GAPDH in Na phosphate buffer $\mathrm{pH} 8.0$ (page 88)

- $100 \mathrm{mM}$ GSH in $\mathrm{dH}_{2} \mathrm{O}$

- $150 \mathrm{mM}$ 3-phosphoglycerate in $\mathrm{dH}_{2} \mathrm{O}$ (protected from light)

- $100 \mathrm{mM}$ ATP in $\mathrm{dH}_{2} \mathrm{O}$

- $700 \mathrm{mM} \mathrm{H}_{2} \mathrm{O}_{2}$ in $\mathrm{dH}_{2} \mathrm{O}$ (protected from light)

First, glutathionylated GAPDH was prepared. For this, the following reaction mix was pipetted:

\begin{tabular}{lcc} 
Component & Volume/Amount & Concentration \\
\hline Na phosphate buffer $\mathrm{pH}=8.0$ & $4513 \mu \mathrm{l}$ & \\
$250 \mu \mathrm{M}$ GAPDH & $333 \mu \mathrm{l}$ & $15 \mu \mathrm{M}$ \\
$100 \mathrm{mM} \mathrm{GSH}$ & $150 \mu \mathrm{l}$ & $3 \mathrm{mM}$ \\
$700 \mathrm{mM} \mathrm{H}_{2} \mathrm{O}_{2}$ & $10 \mu \mathrm{l}$ & $1.4 \mathrm{mM}$ \\
\hline & $\sum 5 \mathrm{ml}$ \\
\hline
\end{tabular}

The reaction was incubated for at least $15 \mathrm{~min}$ at $\mathrm{RT}$, protecting it from light. In the mean time, two PD-10 desalting columns (GE Healthcare) were equilibrated by filling them up with $\mathrm{Na}$ phosphate buffer $\mathrm{pH} 8.0$ twice. Then, $2.5 \mathrm{ml}$ GAPDH mixture were loaded per column and the flowthrough discarded. GAPDH-SG was eluted with $3.5 \mathrm{ml} \mathrm{Na}$ phosphate buffer $\mathrm{pH} 8.0$ and both eluates were pooled. This step served to remove residual GSH and $\mathrm{H}_{2} \mathrm{O}_{2}$ from the reaction.

An additional control to address the maximal GAPDH activity was prepared by mixing $166.5 \mu \mathrm{l} 250 \mu \mathrm{M}$ GAPDH with $3.333 \mathrm{ml}$ Na phosphate buffer $\mathrm{pH}$ 8.0. This yielded a $12 \mu \mathrm{M}$ dilution of GAPDH which corresponds to the maximal possible GAPDH concentration that can be obtained after desalting.

In the second step of the experiment, the substrate of GAPDH during the first step of its reaction, BPGA, was prepared. For this, a mastermix consisting of 
the following components was prepared and incubated for at least $10 \mathrm{~min}$ at room temperature, protected from light:

\begin{tabular}{|c|c|c|}
\hline Component & $\begin{array}{l}\text { Volume/Amount } \\
\text { for a single reaction }\end{array}$ & Concentration \\
\hline $\mathrm{Na}$ phosphate buffer $\mathrm{pH}=8.0$ & $41.71 \mu \mathrm{l}$ & \\
\hline 150 mM 3-phosphoglycerate & $4.5 \mu \mathrm{l}$ & $13.5 \mathrm{mM}$ \\
\hline 100 mM ATP & $3 \mu \mathrm{l}$ & $6 \mathrm{mM}$ \\
\hline $1 \mathrm{M} \mathrm{MgCl}_{2}$ & $0.75 \mu \mathrm{l}$ & $15 \mathrm{mM}$ \\
\hline \multirow[t]{2}{*}{$\begin{array}{l}15 \mathrm{u} / \mu \mathrm{l} \text { 3-phosphoglyceric acid } \\
\text { phosphokinase }\end{array}$} & $0.04 \mu \mathrm{l}$ & $0.012 \mathrm{u} / \mu \mathrm{l}$ \\
\hline & $\sum 50 \mu \mathrm{l}$ & \\
\hline
\end{tabular}

$50 \mu \mathrm{l}$ aliquots of this master mix were distributed to the wells of a 96-well microtiter plate. They served to stop the deglutathionylation of GAPDH.

Then, the actual deglutathionylation reactions were prepared. The following samples were included into the assay:

\begin{tabular}{|c|c|c|c|c|}
\hline GAPDH & GAPDH-SG & $\begin{array}{c}\text { GAPDH-SG + } \\
\text { DTT }\end{array}$ & $\begin{array}{c}\text { GAPDH-SG + } \\
\text { GSH }\end{array}$ & $\begin{array}{c}\text { GAPDH-SG + } \\
\text { Glutaredoxin }\end{array}$ \\
\hline $\begin{array}{c}500 \mu \mathrm{l} \mathrm{GAPDH} \\
12 \mu \mathrm{M}\end{array}$ & $\begin{array}{c}500 \mu \mathrm{l} \\
\text { GAPDH-SG }\end{array}$ & $\begin{array}{c}500 \mu \mathrm{l} \\
\text { GAPDH-SG }\end{array}$ & $\begin{array}{c}500 \mu \mathrm{l} \\
\text { GAPDH-SG }\end{array}$ & $\begin{array}{c}500 \mu \mathrm{l} \\
\text { GAPDH-SG }\end{array}$ \\
\hline $\begin{array}{l}100 \mu \mathrm{l} \text { Dialysis } \\
\text { buffer }\end{array}$ & $\begin{array}{l}100 \mu \mathrm{l} \text { Dialysis } \\
\text { buffer }\end{array}$ & $\begin{array}{l}100 \mu \mathrm{l} \text { Dialysis } \\
\text { buffer }\end{array}$ & $\begin{array}{l}100 \mu \mathrm{l} \text { Dialysis } \\
\text { buffer }\end{array}$ & $\begin{array}{c}100 \mu \mathrm{l} \text { Enyzme } \\
(2-20 \mu \mathrm{M})\end{array}$ \\
\hline $25 \mu \mathrm{l} \mathrm{dH_{2 } \mathrm { O }}$ & $25 \mu \mathrm{ld} \mathrm{d}_{2} \mathrm{O}$ & $25 \mu \mathrm{l} 1 \mathrm{M}$ DTT & $\begin{array}{c}25 \mu \mathrm{l} 100 \mathrm{mM} \\
\mathrm{GSH}\end{array}$ & $\begin{array}{c}25 \mu \mathrm{l} 100 \mathrm{mM} \\
\mathrm{GSH}\end{array}$ \\
\hline
\end{tabular}

These reactions were incubated at RT and $50 \mu \mathrm{l}$ aliquots were removed and stopped by mixing them with $50 \mu \mathrm{l}$ BPGA mix in the microtiter plate at different time points. Once, all samples were taken and stopped, a third master mix was prepared by mixing the following components:

\begin{tabular}{lcc} 
Component & $\begin{array}{c}\text { Volume/Amount } \\
\text { for a single reaction }\end{array}$ & Concentration \\
\hline Na phosphate buffer $\mathrm{pH}=8.0$ & $48.2 \mu \mathrm{l}$ & \\
$0.5 \mathrm{M} \mathrm{Na}_{2}$-EDTA $\mathrm{pH}=8.0$ & $0.3 \mu \mathrm{l}$ & $3 \mathrm{mM}$ \\
$20 \mathrm{mM} \mathrm{NADH}$ & $1.5 \mu \mathrm{l}$ & $0.6 \mathrm{mM}$ \\
\hline & $\sum 50 \mu \mathrm{l}$ \\
\hline
\end{tabular}

This mastermix was also protected from light, until $50 \mu \mathrm{l}$ of it were distributed to each well fo the 96 -well plate containing a stopped sample of the reactions. 
Then, the absorption at $340 \mathrm{~nm}$ was measured every minute over $1 \mathrm{~h}$ to determine the activity of deglutathionylated GAPDH. For data evaluation, the absorption intensity was plotted over time and the slope of the linear range of the curve determined via linear regression in Excel. This yielded the velocity of GAPDH. The velocity of GAPDH was then plotted against the time points at which the aliquots for the final measurement were withdrawn. This yielded the glutaredoxin activity.

\section{RoGFP assay to determine deglutathionylation activity of glutaredoxins}

RoGFP is an oxidation-sensitive GFP variant. Reduced roGFP absorbs more strongly at ca. $480 \mathrm{~nm}$ whereas oxidized roGFP absorbs more strongly at $400 \mathrm{~nm}$. Independent of the wavelength used for excitation, roGFP emits fluorescence light at $510 \mathrm{~nm}$. The intensity of emission depends on the intensity of excitation. Thus, reduced roGFP will emit more strongly after excitation at $480 \mathrm{~nm}$ than after excitation at $400 \mathrm{~nm}$, whereas oxidized roGFP will emit less after excitation at $480 \mathrm{~nm}$ than after excitation at $400 \mathrm{~nm}$. Hence, the ratio of emission after excitation at the different wavelength reflects the oxidation state of roGFP [244].

RoGFP assays were carried out in coopreration with the department for Chemical Signalling (Prof. Dr. Andreas Meyer) at the Institut für Nutzpflanzenwissenschaft und Ressourcenschutz (INRES). Strep-MBPROXY9 was dialyzed against $\mathrm{K}$ phosphate buffer $\mathrm{pH} 8.0$ (dilution factor ca. 2700000) and send on ice to Lara Ostermann, who perfomed the experiment described below:

$100 \mathrm{mM}$ DTT and $10 \mathrm{mM}$ NADPH, as well as $10 \mathrm{U}$ glutathione reductase were prepared in $100 \mathrm{mM} \mathrm{K}$ phosphate buffer. In addition, a $25 \mathrm{mM} \mathrm{GSH}$ solution in potassium phosphate buffer was prepared. Importantly, the $\mathrm{pH}$ was readjusted to $\mathrm{pH}$ 8.0. $100 \mu \mathrm{M}$ stock solutions of the glutaredoxins to be tested and $10 \mu \mathrm{M}$ N-terminally His-tagged roGFP2 were prepared in the same buffer. The roGFP sample was splitted into two parts and one of them was treated

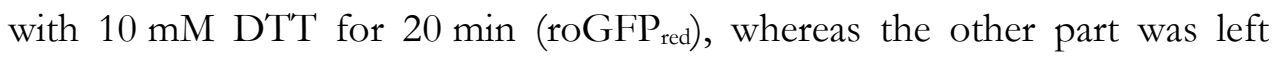


untreated (roGFP $\left.{ }_{\text {ox }}\right)$. The roGFP sample containing DTT was not desalted as it should stay reduced throughout the experiment. The glutaredoxins (GRXs) were used in the oxidized state in which they were purified.

Using these proteins, the reactions listed in the table below were prepared:

\begin{tabular}{|c|c|c|c|c|c|c|c|c|}
\hline Sample & Blank & NADPH & roGFP2 $2_{\text {red }}$ & roGFP2 $2_{\text {ox }}$ & \multicolumn{4}{|c|}{ GRX } \\
\hline Prepared in wells & & & & & & & & \\
\hline $\begin{array}{l}\mathrm{V}(100 \mathrm{mM} \mathrm{K} \\
\text { phosphate buffer } \mathrm{pH} \\
8.0)[\mu \mathrm{l}]\end{array}$ & 92 & 82 & 82 & 82 & 62 & 61 & 59 & 52 \\
\hline $\begin{array}{l}\text { V (10 mM NADPH }) \\
{[\mu \mathrm{l}]}\end{array}$ & - & 10 & - & - & 10 & 10 & 10 & 10 \\
\hline $\begin{array}{l}\mathrm{V}\left(10 \mu \mathrm{M} \text { roGFP2 } 2_{\mathrm{red}}\right) \\
{[\mu \mathrm{l}]}\end{array}$ & - & - & 10 & - & - & - & - & - \\
\hline $\begin{array}{l}\mathrm{V}\left(10 \mu \mathrm{M} \text { roGFP2 } 2_{\text {ox }}\right) \\
{[\mu l]}\end{array}$ & - & - & - & 10 & 10 & 10 & 10 & 10 \\
\hline $\mathrm{V}(10 \mathrm{U}$ GR $)[\mu \mathrm{l}]$ & - & - & - & 10 & 10 & 10 & 10 & 10 \\
\hline $\begin{array}{l}\mathrm{V}(100 \mu \mathrm{M} \mathrm{GRX})[\mu \mathrm{l}] \\
\text { Added via injector }\end{array}$ & - & - & - & - & - & 1 & 2 & 5 \\
\hline $\mathrm{V}(25 \mathrm{mM} \mathrm{GSH})[\mu \mathrm{l}]$ & - & - & - & - & 8 & 8 & 8 & 8 \\
\hline $\begin{array}{l}\mathrm{V}(100 \mathrm{mM} \mathrm{K} \\
\text { phosphate buffer } \mathrm{pH} \\
8.0)[\mu \mathrm{l}]\end{array}$ & 8 & 8 & 8 & 8 & - & - & - & - \\
\hline
\end{tabular}

With exception of the $8 \mu \mathrm{l}$ GSH or K phosphate buffer, the reactions were prepared in at least three replicates in a 96-well microtiter plate. The injection pumps of the plate reader were filled with $\mathrm{K}$ phosphate buffer or GSH solution. The measurement of the fluorescence emission at $510 \mathrm{~nm}$ after excitation at $400 \mathrm{~nm}$ and $482 \mathrm{~nm}$, respectively, was initiated and continued for $10 \mathrm{~min}$ to allow equilibration. Then, $8 \mu \mathrm{l}$ of GSH or buffer were injected to start the reactions. Fluorescence changes of the reactions were monitored over at least $60 \mathrm{~min}$. For data evaluation, the ratio of the emission after excitation at $400 \mathrm{~nm}$ and after excitation at $482 \mathrm{~nm}$ was computed and plotted against the time. 


\section{K phosphate buffer $\mathrm{pH} 8.0$}

\begin{tabular}{lc} 
Component & Volume \\
\hline $0.5 \mathrm{M} \mathrm{KH}_{2} \mathrm{PO}_{4}$ & $5 \%(\mathrm{v} / \mathrm{v})$ \\
$0.5 \mathrm{M} \mathrm{K}_{2} \mathrm{HPO}_{4}$ & $95 \%(\mathrm{v} / \mathrm{v})$ \\
\hline
\end{tabular}

The $\mathrm{pH}$ of the buffer was adjusted at RT with concentrated o-phosphoric acid or with $\mathrm{KOH}$.

\section{RoGFP assay to determine glutathionylation activity of glutaredoxins}

This assay was performed in analogy to the deglutathionylation assay with roGFP. The glutaredoxins were reduced prior to the experiment by treatment wit $10 \mathrm{mM}$ DTT for $45 \mathrm{~min}$, followed by desalting via ZebaSpin columns. Oxidized roGFP2 was added to the enyzeme reactions instead of reduced roGFP2. Moreover, instead of GSH, GSSG was injected to a final concentration of $5 \mathrm{mM}$.

\section{Analysis of glutathione and iron-sulfur cluster binding}

Thermophoresis and BioLayer Interferometry to determine the dissociation constant $\left(\mathrm{K}_{\mathrm{d}}\right)$ of strep-MBP-ROXY9 towards glutathione, were carried out by Dr. Sven-Andreas Freibert at the institute for Cytobiology and Cytopathology (Prof. Dr. Roland Lill, Institute for Cytobiology and Cytopathology, PhilippsUniversity Marburg). For this, DTT was removed via a ZebaSpin or a PD-10 column. 
Chemical reconstitution of a potential iron-sulfur cluster in strep-MBPROXY9 in the presence of glutathione and the subsequent determination of bound iron and sulfide ions via the ferene-based approach [245] and the methylene blue method [246] were performed by Dr. Freibert. Purified strepMBP-ROXY9 was dialyzed to remove EDTA and sent on wet ice to this institute.

\section{Differential labeling of reduced and oxidized cysteine residues in purified strep-MBP-ROXY9}

The experiment was carried out as described by Vollheyde (2014) [247]. In this experiment, the reduced thiol groups of strep-MBP-ROXY9 were labeled with iodoacetamide (IAM) while the modified thiol groups were labeled with an iodoacetyl Tandem Mass $\mathrm{Tag}^{\mathrm{TM}}$ (iodoTMT ${ }^{0}-126$, Thermo Scientific). To prevent loss of or changes in the cysteine modifications, the light was kept low during the experiment and incubation periods were carried out in the dark. Iodoacetamide, Tris(2-carboxyethyl)phosphine (TCEP), ascorbate and iodoTMT solutions were prepared freshly before starting the experiment.

First, about $90 \mu \mathrm{g}$ of purified protein were subjected to a TCA precipitation to remove all oxidizing or reducing agents from the buffer solution. The resulting pellet was resuspended in $500 \mu \mathrm{l}$ Urea buffer containing $100 \mathrm{mM}$ iodoacetamide. The mixture was incubated at $25^{\circ} \mathrm{C}$ for $15 \mathrm{~min}$, shaking it at $600 \mathrm{rpm}$, and precipitated again using TCA. This time, the pellet was dissolved in $300 \mu \mathrm{l}$ reduction buffer (either with $1 \mathrm{mM}$ ascorbate to prepare the labeling of nitrosylated cysteine residues only or with $20 \mathrm{mM}$ TCEP to allow labeling of all modified cysteine residues) and incubated at $30^{\circ} \mathrm{C}$ for $30 \mathrm{~min}$, shaking the sample at $600 \mathrm{rpm}$. After a third TCA precipitation, the pellet was resuspended in $20 \mu \mathrm{l}$ HENS buffer (Thermo Scientific). Then, $1 \mu$ liodoTMT solution was added and the samples incubated at $37^{\circ} \mathrm{C}$, shaking them for about $1 \mathrm{~h}$. 


\section{Urea buffer}

\begin{tabular}{lc} 
Component & Concentration \\
\hline TRIS-HCl pH 8.5 & $200 \mathrm{mM}$ \\
Urea & $6 \mathrm{M}$ \\
$\mathrm{Na}_{2}$-EDTA & $5 \mathrm{mM}$ \\
SDS & $0.005 \%(\mathrm{w} / \mathrm{v})$ \\
\hline
\end{tabular}

\section{Reduction buffer with TCEP}

\begin{tabular}{lc} 
Component & Concentration \\
\hline Urea buffer & $90 \%(\mathrm{v} / \mathrm{v})$ \\
$200 \mathrm{mM}$ TCEP & $10 \%(\mathrm{v} / \mathrm{v})$ \\
\hline
\end{tabular}

\section{Reduction buffer with ascorbate}

\begin{tabular}{lc} 
Component & Concentration \\
\hline Urea buffer & $90 \%(\mathrm{v} / \mathrm{v})$ \\
$20 \mathrm{mM} \mathrm{L}(+)$-ascorbic acid & $5 \%(\mathrm{v} / \mathrm{v})$ \\
$\mathrm{dH}_{2} \mathrm{O}$ & $5 \%(\mathrm{v} / \mathrm{v})$ \\
\hline
\end{tabular}

\section{Stock solutions to be prepared freshly}

\begin{tabular}{lc} 
Component & Concentration \\
\hline TCEP in $\mathrm{dH}_{2} \mathrm{O}$ & $200 \mathrm{mM}$ \\
$\mathrm{L}(+)$-ascorbic acid in $\mathrm{dH}_{2} \mathrm{O}$ & $20 \mathrm{mM}$ \\
Iodoacetamide in urea buffer & $100 \mathrm{mM}$ \\
iodoTMT ${ }^{0}-126$ in DMSO & $10 \mu \mathrm{g} / \mu \mathrm{l}$ \\
\hline
\end{tabular}

Treatment of strep-MBP-ROXY9 for Ellman's assay and redox titration

To prepare strep-MBP-ROXY9 in different oxidation states, protein purified in the presence of DTT was concentrated to $95 \mu \mathrm{M}$ and subjected to the following treatments: 
A sample of ca. $400 \mu \mathrm{l}$ strep-MBP-ROXY9 was dialyzed against $100 \mathrm{mM}$ HEPES pH 7.0 to remove DTT from the purification process. The dilution factor was ca. 1000000-fold. Dialysis took place in the cold room over night.

Oxidation by removal of DTT via a ZepaSpin column and oxidation with $\mathrm{H}_{2} \mathrm{O}_{2}$

$200 \mu \mathrm{l}$ strep-MBP-ROXY9 purified in the presence of DT'T were dialysed over night in the cold room against $\mathrm{K}$ phosphate buffer $\mathrm{pH} 8.0$ (as used for roGFP assays, page 108). The dilution factor during dialysis was ca. 4000000-fold. Then, the sample was splitted into two aliquots of $70 \mu$ l. One aliquot was treated for $20 \mathrm{~min}$ with $10 \mathrm{mM}$ DTT at RT, whereas the other aliquot was incubated with $10 \mathrm{mM} \mathrm{H}_{2} \mathrm{O}_{2}$ for the same time. Both samples were desalted via ZebaSpin columns equilibrated with Ellman's assay buffer (page 113).

\section{Determination of free thiol groups using 5,5-dithio-bis-2- nitrobenzoic acid (Ellman's assay [248])}

5,5-dithio-bis-2-nitrobenzoic acid (DTNB) was obtained from Thermo Scientific and the assay was conducted according to the manufacturer's instructions with some modifications. The final protocol was:

Strep-MBP-ROXY9 was subjected to the following treatments: Removal of DTT by dialysis or ZebaSpin column or oxidation with $\mathrm{H}_{2} \mathrm{O}_{2}$. The treatments are described in the section before. After the treatment, the protein concentration of all samples was determined according to Bradford and different dilutions of the protein within the detection range of the L-cysteine standard curve were made in $1 \mathrm{xNa} / \mathrm{K}$ phosphate buffer (page 98). The entire L-cysteine standard curve ranged from $0.025 \mathrm{mM}$ to $1.5 \mathrm{mM}$. For this standard, L-cysteine was dissolved freshly in DTNB assay buffer to a concentration of $1.5 \mathrm{mM}$. The standard samples were prepared by diluting the $1.5 \mathrm{mM} \mathrm{L}$ cysteine solution according to the following table: 


\begin{tabular}{cccc}
\hline Sample & $\begin{array}{c}\mathrm{V} \text { (Assay buffer) } \\
{[\mu \mathrm{l}]}\end{array}$ & $\begin{array}{c}\mathrm{V}(1.5 \mathrm{mM} \text { L-cysteine }) \\
{[\mu \mathrm{l}]}\end{array}$ & $\begin{array}{c}\mathrm{c} \text { (L-cysteine) } \\
{[\mathrm{mM}]}\end{array}$ \\
\hline $\mathrm{A}$ & - & $300(\mathrm{~A})$ & 1.5 \\
$\mathrm{~B}$ & 50 & $250(\mathrm{~A})$ & 1.25 \\
$\mathrm{C}$ & 100 & $200(\mathrm{~A})$ & 1 \\
$\mathrm{D}$ & 150 & $150(\mathrm{~A})$ & 0.75 \\
$\mathrm{E}$ & 200 & $100(\mathrm{~A})$ & 0.5 \\
F & 250 & $50(\mathrm{~A})$ & 0.25 \\
$\mathrm{G}$ & 150 & $150(\mathrm{~F})$ & 0.125 \\
$\mathrm{H}$ & 240 & $60(\mathrm{E})$ & 0.1 \\
$\mathrm{I}$ & 150 & $150(\mathrm{H})$ & 0.05 \\
J & 150 & $150(\mathrm{I})$ & 0.025 \\
K & 300 & - & 0 \\
\hline
\end{tabular}

Since L-cysteine is light-sensitive, all these samples were protected from light.

A $4 \mathrm{mg} / \mathrm{ml}$ DTNB stock solution was made freshly using assay buffer. A mastermix consisting of $2.5 \mu \mathrm{l}$ DTNB solution and $125 \mu \mathrm{l}$ DTNB assay buffer per sample was prepared. $125 \mu \mathrm{l}$ of this master mix were distributed to the wells of a microtiter plate. To the wells of the L-cysteine standard samples, $12.5 \mu \mathrm{l}$ $1 \mathrm{xNa} / \mathrm{K}$ phosphate buffer were added, whereas to the wells of the protein samples, $12.5 \mu \mathrm{l}$ DTNB assay buffer were added. Then, $12.5 \mu \mathrm{l}$ of the Lcysteine standard samples were distributed to their wells and $12.5 \mu \mathrm{l}$ of the different strep-MBP-ROXY9 dilutions. Each standard and each protein sample was prepared in triplicate. After the samples were mixed thoroughly, the plate was incubated for $15 \mathrm{~min}$ at room temperature. Eventually, the absorption at $420 \mathrm{~nm}$ was measured in a Synergy HT plate reader.

To determine the concentration of free thiols in the protein samples, a standard curve was obtained by plotting $A_{420 \mathrm{~nm}}$ against the concentration of free thiols of the standard samples (one free thiol per cysteine molecule) and by performing a linear regression. The resulting equation was used to calculate the concentration of free thiols in the protein samples. By dividing the concentration of free thiols by the protein concentration in each sample, the number of reduced cysteines in strep-MBP-ROXY9 was determined. 


\section{Ellman's assay buffer}

\begin{tabular}{lc} 
Component & Volume \\
\hline $50 \mathrm{mM} \mathrm{Na}$ phosphate buffer $\mathrm{pH} 8.0$ & $200 \mathrm{ml}$ \\
$0.5 \mathrm{M} \mathrm{Na}_{2}$-EDTA pH 8.0 & $400 \mu \mathrm{l}$ \\
\hline
\end{tabular}

\section{Redox titration using monobromobimane}

To determine the mid-point redox potential of strep-MBP-ROXY9, the method described by Couturier et al. (2013) [77] was employed. In this method, oxidized protein is incubated in the presence of different ambient redox potentials $\left(\mathrm{E}_{\mathrm{h}}\right)$ set by mixing DTT and dithiane (oxidized DTT) in defined ratios. The relation between the redox potential and the concentration of oxidized and reduced compounds is specified by the Nernst equation:

$$
E_{h}=E^{0}+\frac{R \times T}{n \times F} \times \ln \left(\frac{c(O x)}{c(\text { Red })}\right)
$$

$\mathrm{R}=8.314 \mathrm{~J} /(\operatorname{mol} \times K)$

$T=298 \mathrm{~K}\left(25^{\circ} \mathrm{C}\right)$;

$n=2$ (for DTT/dithiane; $n$ is the number of electrons exchanged between the oxidized and the reduced compound);

$F=96485.336 \mathrm{C} / \mathrm{mol}$

Thus, the entire measurement depends on the DT'T standard redox potential $\left(\mathrm{E}^{9}\right)$, which is $-0.33 \mathrm{~V}$ at $\mathrm{pH} 7.0$ at $25^{\circ} \mathrm{C}$ [77]. Therefore, the $\mathrm{pH}$ of the $100 \mathrm{mM}$ HEPES buffer used throughout the experiment was adjusted to 7.0 at $25^{\circ} \mathrm{C}$. The experiment was carried out as close to $25^{\circ} \mathrm{C}$, as possible.

The protein oxidation state adjusts over time to the ambient redox potential created by the different DT'T/dithiane concentrations. Finally, reduced cysteine residues are labeled with the fluorescing dye monobromobimane $(\mathrm{mBBr})$. Using the fluorescence intensity, the extent of reduction and oxidation of the protein in each sample is determined $[77,249]$. 
Oxidized strep-MBP-ROXY9 was prepared according to the protocol for removal of DTT by dialysis. After dialysis, the concentration of the sample was determined according to Bradford and dilutions of ca. $15 \mu \mathrm{M}$ in $100 \mathrm{mM}$ HEPES pH 7.0 were prepared.

To set the ambient redox potential, fresh stock solutions of DTT and dithiane were made by dissolving the compounds to a concentration of $4 \mathrm{mM}$ in $100 \mathrm{mM}$ HEPES pH 7.0. Using these stock solutions, different dilutions were pipetted to obtain the indicated ambient redox potential:

\begin{tabular}{cccc}
\hline $\mathrm{E}_{\mathrm{h}}[\mathrm{V}]$ & $\begin{array}{c}\mathrm{c}(\text { Dithiane }) / \\
\mathrm{c}(\mathrm{DT} T)\end{array}$ & $\begin{array}{c}\mathrm{V}(\text { Dithiane } \\
4 \mathrm{mM})\end{array}$ & $\mathrm{V}(\mathrm{DT} T \mathrm{mM})$ \\
\hline$-0,3$ & 10,3 & 3647 & 353 \\
$-0,29$ & 22,5 & 3829 & 171 \\
$-0,28$ & 49 & 3920 & 80 \\
$-0,27$ & 106,7 & 3962 & 38 \\
$-0,26$ & 232,5 & 3982 & 8 \\
$-0,25$ & 506,3 & 3992 & 4 \\
$-0,24$ & 1102,8 & 3996 & $100 \mu \mathrm{l}$ of sample "-0.26" \\
$-0,23$ & 2401,8 & 900 & $100 \mu \mathrm{l}$ of sample "-0.25" \\
$-0,22$ & 5231,1 & 900 & $100 \mu \mathrm{l}$ of sample "-0.24" \\
$-0,21$ & 11393,2 & 900 & $100 \mu \mathrm{l}$ of sample "-0.23" \\
$-0,2$ & 24814,1 & 900 & $100 \mu \mathrm{l}$ of sample "-0.22" \\
$-0,19$ & 54044,6 & 900 & $100 \mu \mathrm{l}$ of sample "-0.21" \\
$-0,18$ & 117708,1 & 900 & $100 \mu \mathrm{l}$ of sample "-0.20" \\
$-0,17$ & 256365,9 & 900 &
\end{tabular}

Finally, $100 \mu \mathrm{l}$ of the DTT/dithiane mixtures were added to $100 \mu \mathrm{l}$ aliquots of the $15 \mu \mathrm{M}$ protein dilution. The samples were incubated for approximately $2 \mathrm{~h}$ at ca. $25^{\circ} \mathrm{C}$. Then, $10 \mu \mathrm{l}$ of a $40 \mathrm{mM} \mathrm{mBBr}$ stock solution in $100 \% \mathrm{DMSO}$ were added. After the addition of the light-sensitive $\mathrm{mBBr}$, further processing took place in the dark. The samples were incubated with $\mathrm{mBBr}$ for another $20 \mathrm{~min}$ at $25^{\circ} \mathrm{C}$. The reaction was stopped by adding $210 \mu \mathrm{l} 20 \%$ ice-cold TCA. The protein was precipitated on ice for $30 \mathrm{~min}$, followed by a centrifugation at $17000 \mathrm{~g}$ at $4^{\circ} \mathrm{C}$ for $10 \mathrm{~min}$. The supernatant was removed with a pipette tip and $1 \mathrm{ml}$ of $100 \%$ ice-cold acetone was added to each pellet. To wash the pellet thoroughly, the tubes were inverted several times and then centrifuged for another $5 \mathrm{~min}$ at $4^{\circ} \mathrm{C}$ at $17000 \mathrm{~g}$. The supernatant was removed completely, and the pellets dried briefly at RT. Finally, the pellets were dissolved by adding $400 \mu 100 \mathrm{mM}$ TRIS pH 8.0 containing $1 \%(\mathrm{w} / \mathrm{v})$ SDS vortexing them for ca. $5 \mathrm{~min} .100 \mu \mathrm{l}$ of the solution were added to the wells of a 96-well plate. As a 
blank, $100 \mu$ l TRIS-SDS buffer were added to one well. Then, fluorescence was excited using a 360/40 filter in a Synergy HT plate reader and measured with a $528 / 40$ filter.

To analyze the data, the blank value was substracted from the sample values. Following the protocol described by Couturier et al. (2013) [77], the highest value measured was set to $100 \%$ reduced protein, whereas the lowest value measured was set to $0 \%$ reduced protein. All other values were assigned values for the percentage of reduced protein accordingly. The percentage of the reduced protein was plotted against the ambient redox potential and the inflection point of the resulting curve estimated to determine roughly the standard redox potential of the protein [77]. However, it should be noted that this way of data evaluation leads to errors when the protein used is not fully reduced or oxidized under the ambient redox potential chosen. Employing additional controls, namely fully reduced and fully oxidized protein, might be advisable. Still, since the experiment performed in this study was a first try to establish the method, it was performed exactly according to Couturier et al. (2013).

\section{Analysis of the cysteine redox state by mass spectrometry}

\section{Sample preparation}

Strep-SUMO-ROXY 9 was dialyzed against $1 \mathrm{xNa} / \mathrm{K}$ phosphate buffer $\mathrm{pH} 7.5$ (page 98), optionally containing the reducing reagents of interest. Of these preparations, a sample containing ca. $90 \mu \mathrm{g}$ of the protein was taken and immediately precipitated with TCA. Then, the reduced and oxidized cysteines of strep-MBP-ROXY9 were labeled with IAM or iodoTMT ${ }^{0}-126$, respectively. After incubation in HENS buffer (Thermo Scientific) containing the iodoTMT reagent, the samples were mixed with non-reducing $\mathrm{RPB}$ and stored at $-20^{\circ} \mathrm{C}$ until further processing.

The following table summarizes the preparation procedure for each sample analyzed in this work: 


\begin{tabular}{lccc}
\hline $\begin{array}{l}\text { Sample } \\
\text { ID }\end{array}$ & Sample name & $\begin{array}{c}\text { Buffer additive and } \\
\text { concentration }\end{array}$ & Dilution factor \\
\hline KT11 & $\begin{array}{c}\text { Strep-MBP-ROXY9 } \\
+ \text { DTT }\end{array}$ & $\begin{array}{c}\text { None } \\
\text { (used as purified) }\end{array}$ & $\begin{array}{c}\text { None } \\
\text { (used as purified) }\end{array}$ \\
& & & \\
KT07 & $\begin{array}{c}\text { Strep-MBP-ROXY9 } \\
\text { - DTT }\end{array}$ & none & $4.8 \times 10^{11}$ \\
& & & \\
& & & \\
KT09 & $\begin{array}{c}\text { Strep-MBP-ROXY9 } \\
+ \text { GSH }\end{array}$ & 1 mM GSH & \\
& & & \\
\hline
\end{tabular}

In addition to these samples, another sample was prepared to test how fast oxidized strep-MBP-ROXY9 can be reduced by GSH (KT17). For this, about $90 \mu \mathrm{g}$ strep-MBP-ROXY 9 were mixed with GSH to a final concentration of $1 \mathrm{mM}$, incubated for $10 \mathrm{~min}$ and instantly subjected to TCA precipitation. Reduced and oxidized cysteine residues were labeled with IAM and iodo $\mathrm{TMT}^{0}-126$, respectively.

Finally, all samples were loaded on $10 \%$ SDS gels (the samples were not boiled to protect the modifications) and separated at $80 \mathrm{~V}$ in fresh $1 \mathrm{x}$ SDS running buffer. Subsequently, the gels were stained with Colloidal Coomassie. After documentation using an Epson Perfection V700 Photo scanner, all bands of interest were cut out from the gel with a clean scalpel blade and transferred to fresh $2 \mathrm{ml}$ reaction tubes. The gel slices were destained with Colloidal Coomassie fixing solution, applying about $2 \mathrm{ml}$ of this solution to the gel slice and rotating it at $30 \mathrm{rpm}$ at room temperature for short time or overnight in the cold room. To destain the gel slices as completely as possible, destaining was performed over a duration of three days, exchanging the fixing solution every day. 


\section{Sample preparation for liquid chromatography-tandem mass spectrometry (LC-MS/MS)}

\section{In-gel trypsin digest}

The destained gel pieces were cut into $2 \mathrm{~mm}$ pieces using a clean scalpel. The gel pieces were transferred to a $2 \mathrm{ml}$ reaction tube, covered with acetonitrile and vortexed for $10 \mathrm{~min}$ at RT to dehydrate them. To rehydrate the gel pieces, acetonitrile was removed, and the gel pieces covered with $150 \mu 100 \mathrm{mM}$ $\mathrm{NH}_{4} \mathrm{HCO}_{3}$, shaking them again at $\mathrm{RT}$ for $10 \mathrm{~min}$. After removing $\mathrm{NH}_{4} \mathrm{HCO}_{3}$, $150 \mu \mathrm{l}$ acetonitrile were added, the gel pieces vortexed again for $10 \mathrm{~min}$ at RT, and the acetonitrile removed. This washing with $\mathrm{NH}_{4} \mathrm{HCO}_{3}$ and acetonitrile was repeated once. Finally, the gel pieces were dried in a speedvac at $50^{\circ} \mathrm{C}$ for ca. $10 \mathrm{~min}$. The gel pieces were covered with trypsin digestion buffer and incubated for $45 \mathrm{~min}$ on ice. After removing the trypsin digestion buffer, the gel pieces were covered with $25 \mathrm{mM} \mathrm{NH}_{4} \mathrm{HCO}_{3} \mathrm{pH} 8.0$ and incubated over night at $37^{\circ} \mathrm{C}$.

The sample was centrifuged at $13000 \mathrm{rpm}$ for $1 \mathrm{~min}$ at RT (table top centrifuge) and the supernatant transferred to a fresh LoBind Eppendorf tube ("collection tube"). To extract the acidic peptides, the gel pieces were overlain with $20 \mathrm{mM}$ $\mathrm{NH}_{4} \mathrm{HCO}_{3}$ and vortexed for $10 \mathrm{~min}$ at RT. The tube was spun at $13000 \mathrm{rpm}$ for $1 \mathrm{~min}$ at RT and the supernatant transferred to the collection tube. Next, basic peptides were extracted by repeating the following step three times: The gel pieces were covered with $50 \%$ acetonitrile $/ 5 \%$ formic acid and vortexted for $20 \mathrm{~min}$ at RT. Then, the gel pieces were spun down by centrifugation at $13000 \mathrm{rpm}$ for $1 \mathrm{~min}$ at RT and the supernatant transferred to the collection tube. Finally, the liquid in the collection tube was completely evaporated in a speedvac at $50^{\circ} \mathrm{C}$, leaving only the peptides in the pellet. This pellet could be stored $-20^{\circ} \mathrm{C}$.

\section{C18 stage tip purification}

Stage tips were prepared by placing two $0.5 \mathrm{~mm}$ plugs of C18 material in a $200 \mu \mathrm{l}$ tip. The stage tips were placed in an adaptor in a $2 \mathrm{ml}$ reaction tube without lid. To equilibrate the $\mathrm{C} 18$ material, $100 \mu \mathrm{l}$ methanol $/ 0.1 \%$ formic acid was loaded on the stage tip. The stage tip was spun down for $2 \mathrm{~min}$ at $17000 \mathrm{~g}$ at RT and the flow through discarded. Next, $100 \mu \mathrm{l} 70 \%$ acetonitrile/0.1\% 
formic acid were added to the C18 material and centrifugation repeated. The flowthrough was discarded. Finally, $100 \mu \mathrm{l} 0.1 \%$ formic acid were pipetted into the stage tip, centrifuged and the flow through discarded. This step was repeated once.

The pellet obtained after trypsin digestion was resuspended in an appropriate volume of fresh sample buffer by pipetting and by sonication in an ultrasonic bath for $3 \mathrm{~min}$ at maximum power. The samples were spun down briefly to sediment any residual non-dissolved pellet pieces and the supernatant was loaded on the equilibrated stage tip placed in a fresh tube, followed by incubation for 3 to $5 \mathrm{~min}$ at RT. The stage tip was centrifuged for $5 \mathrm{~min}$ at $4000 \mathrm{rpm}$ and the flowthrough reloaded, repeating incubation and centrifugation. The stage tip was washed twice by adding $100 \mu \mathrm{l} 0.1 \%$ formic acid, centrifuging for $2 \mathrm{~min}$ at $10000 \mathrm{rpm}$. Finally, the stage tip was transferred to a fresh LoBind Eppendorf tube and $60 \mu \mathrm{l} 70 \%$ acetonitrile/ $0.1 \%$ formic acid were pipetted onto the $\mathrm{C} 18$ material. The sample was centrifuged for $5 \mathrm{~min}$ at $4000 \mathrm{rpm}$ at RT. The eluate was dried in a speedvac at $50^{\circ} \mathrm{C}$ and the pellet was stored until LC-MS/MS analysis. Prior to LC-MS/MS analysis, the pellet was dissolved in $20 \mu \mathrm{l}$ fresh sample buffer by pipetting and sonification in an ultrasonic bath ( 3 min, maximum power). The sample was transferred into LCMS vials, making sure that there were no air bubbles or undissolved pellet pieces in the solution.

\section{Trypsin solution}

$25 \mu \mathrm{g}$ Trypsin were dissolved in $25 \mu \mathrm{l}$ resuspension buffer and mixed with $475 \mu \mathrm{l} 25 \mathrm{mM} \mathrm{NH}_{4} \mathrm{HCO}_{3} \mathrm{pH} 8.0$ (Trypsin solution). $750 \mu \mathrm{l} 25 \mathrm{mM} \mathrm{NH}_{4} \mathrm{HCO}_{3}$ $\mathrm{pH} 8.0$ were added before use to $500 \mu$ Trypsin solution.

\section{Sample buffer}

\begin{tabular}{lc} 
Component & Concentration \\
\hline $\mathrm{H}_{2} \mathrm{O}$ & $98 \%(\mathrm{v} / \mathrm{v})$ \\
Acetonitrile & $2 \%(\mathrm{v} / \mathrm{v})$ \\
Formic acid & $0.1 \%(\mathrm{v} / \mathrm{v})$ \\
\hline
\end{tabular}




\section{LC-MS/MS measurement and data analysis}

LC-MS/MS was performed by Dr. Kerstin Schmitt at the Service Unit LCMS Protein Analytics (Georg August University Göttingen) on a Q Exactive HF mass spectrometer using electron spray ionization. LC-MS/MS data was analyzed using the software Proteome Discoverer 2.2.0. (Thermo Scientific). To achieve a rough quantification, the number of different peptide spectrum matches (PSMs) observed for each modified peptide was divided by the number of (different) PSMs for a non-modified peptide resulting from the MBP tag of ROXY9. Two sets of normalized PSMs were produced, using two different non-modified MBP peptides. This data analysis allowed comparisons of the amount of the same peptide in different samples, but not between different peptides.

\section{Pulldown of recombinant GFP-TGA1 and His-MBP-JAZ9 using strep-MBP-ROXY9 from insect cells}

To analyze co-purification of GFP-TGA1 and His-MBP-JAZ9 with strepMBP-ROXY9 and strep-MBP as a control, recombinant proteins were used. Strep-MBP-ROXY9 was purified from insect cells whereas strep-MBP was purified from E. coli, as described in the sections before. Maltose was removed by dialyzing against $1 \mathrm{x}$ MBP lysis buffer with $50 \mathrm{mM} \mathrm{NaCl}$ (dilution factor ca. 5000000 , buffer recipe page 95). The protein concentrations were determined according to Bradford. GFP-TGA1 and His-MBP-JAZ9 were expressed in E. coli. A single $400 \mathrm{ml}$ pellet of E. coli cells expressing GFP-TGA1 and His-MBPJAZ9 was thawed and resuspended in $20 \mathrm{ml}$ 1x MBP lysis buffer containing $50 \mathrm{mM} \mathrm{NaCl}$. Two tablets EDTA-free protease inhibitor cocktail (Biomake) and a spatula of lysozyme were added. The samples were incubated for $20 \mathrm{~min}$ in the cold room, agitating them end-over-end at $15 \mathrm{rpm}$. Then, the cells were lysed by sonication (15 min, $1 \mathrm{sec}$ pulse on, $2 \mathrm{sec}$ pulse off, $50 \%$ amplitude). The samples were centrifuged at $4^{\circ} \mathrm{C}$ at $4000 \mathrm{rpm}$ for $30 \mathrm{~min}$ and the supernatants used for the pulldown experiment. 
To prepare a pulldown sample, $500 \mu \mathrm{l}$ GFP-TGA1 crude extract or His-MBPJAZ9 crude extract were mixed with a final concentration of $1 \mu \mathrm{M}$ strep-MBPROXY9 or strep-MBP and filled up to $1.480 \mu \mathrm{l}$ using 1x MBP lysis buffer with $50 \mathrm{mM} \mathrm{NaCl}$. $20 \mu \mathrm{l}$ TEV dialysis buffer (page 96) were added. The samples were incubated in the cold room, rotating them end-over-end at $15 \mathrm{rpm}$ over night.

The next day, $500 \mu \mathrm{l}$ aliquots of $50 \%$ StrepTactin slurry in $2 \mathrm{ml}$ reaction tubes were prepared. The beads were spun down at $17000 \mathrm{~g}$ at RT for $10 \mathrm{sec}$ and the supernatant was removed. Then, the beads were equilibrated by adding $1 \mathrm{ml} 1 \mathrm{x}$ MBP lysis buffer with $50 \mathrm{mM} \mathrm{NaCl}$, inverting, and centrifuging again at $17000 \mathrm{~g}$ at RT for $10 \mathrm{sec}$ and removing the supernatant. This step was repeated twice. Finally, each bead aliquot was resuspended in $250 \mu \mathrm{l} 1 \mathrm{x}$ MBP lysis buffer with $50 \mathrm{mM} \mathrm{NaCl}$ and all aliquots were pooled. $300 \mu \mathrm{l}$ of the equilibrated slurry were distributed to fresh $2 \mathrm{ml}$ tubes. After taking a $20 \mu \mathrm{l}$ sample ("Input") of the pulldown mixture and supplying it with non-reducing RPB, the beads were mixed with the residual pulldown mixture and incubated for ca. 30 min at RT, rotating them end-over-end at $15 \mathrm{rpm}$. After incubation, the mixture was transferred in $800 \mu \mathrm{l}$-steps on a BioRad Spin column and centrifuged at $1000 \mathrm{~g}$ at RT for $1 \mathrm{~min}$. The flowthrough was pooled in a tube and a $22 \mu \mathrm{l}$ sample taken for SDS-PAGE, mixing it with $22 \mu \mathrm{l}$ non-reducing RPB ("Unbound"). The beads were washed five times with $800 \mu \mathrm{l}$ 1x MBP lysis buffer with $50 \mathrm{mM}$ $\mathrm{NaCl}$, centrifuging at $1000 \mathrm{~g}$ for $1 \mathrm{~min}$ at RT. The flowthrough was discarded. After the last wash step, residual liquid in the spin column was removed by a blank spin for $1 \mathrm{~min}$ at $1000 \mathrm{~g}$ at RT. Finally, the bound protein was eluted by adding $400 \mu$ of a 1:1 mixture of non-reducing RPB and 1x MBP lysis buffer with $50 \mathrm{mM} \mathrm{NaCl}$. The samples were incubated at RT for $5 \mathrm{~min}$ and centrifuged at $1000 \mathrm{~g}$ for $1 \mathrm{~min}$ at RT. $20 \mu \mathrm{l}$ of the elution were separated together with $20 \mu \mathrm{l}$ of the input and $22 \mu \mathrm{l}$ of the unbound samples on precast SDS gels (4-20\% gradient) and blotted in a semi-dry Western blot on a PVDF membrane. For each pulldown, two gels were prepared, one to perform a Western blot with the anti-strep antibody, and the other one to perform a Western blot with the anti-His or anti-GFP antibody. 
Results 


\section{Amino acids required for ROXY9 in vivo activity}

\section{Construction and selection of stable ROXY9 overexpression plants}

ROXY9 (At2g47880, CEPD2) belongs to the glutaredoxins with a peculiar CC motif in their active site $[145,154]$. The ROXY9 active site is ${ }^{21} \mathrm{CCLC}^{24}$. The active site of the CC-type glutaredoxins is immediately followed by another, conserved residue: Either a histidine, or - as for ROXY9 - a tyrosine [133,145]. In a previous study, it was shown that ROXY9 can repress hyponastic growth when overexpressed in A. thaliana. Overexpression of mutated ROXY9 variants was used to investigate which of the three active site cysteines in ROXY9 are necessary for repression of hyponasty. To act as a repressor, ROXY9 requires the CC motif, but not the most C-terminal cysteine residue of the active site $[155,156]$.

In this work, the dissection of the active site in ROXY9 was continued by examining the function of the conserved tyrosine residue $\left(\mathrm{Tyr}^{25}\right)$ after the active site motif. To this end, a ROXY9 mutant construct was cloned in which the $\mathrm{Tyr}^{25}$ codon was exchanged for an alanine codon (ROXY9 CCLCA). In addition, it was tested whether the CC-type active site is indispensable for ROXY9 activity. Thus, the active site coding sequence of ROXY9 was exchanged for the coding sequence of an active site of a class I glutaredoxin (ROXY9 CPYC). Both mutant variants were placed under control of the CaMV35S promoter and the constructs were stably transformed in $A$. thaliana Col-0. As a control, a plant overexpressing wildtype ROXY9 from the same promoter was generated. For each transformation, several homozygous plants were isolated and analyzed by Western Blot for the expression of their transgene in petioles under different light regimes: Control light, low light treatment, and backshift to control light after low light treatment. As controls, petioles from Col-0, a former ROXY9 overexpression line (\#7-18, [155,156]) 
were included in the analysis. Col-0 $+H A-R O X Y 9 \# 17-4$ had lost the transgene during segregation and thus served as an internal control.

As can be seen in Figure R1, this internal control did not express HA-tagged ROXY9, as expected. The same observation was made for the wildtype. Col-0 + HA-ROXY9 \#7-18, in contrast, expressed the transgene. The newly generated line Col-0 + HA-ROXY9 \#5-18 expressed the transgene to similar levels, whereas Col-0 + HA-ROXY9 \#4-40 and \#7-25 showed an even higher expression. All other ROXY9 lines (\#2-22, \#17-3, \#29-1, \#30-7) displayed poor or no expression of HA-tagged ROXY9. In contrast to this, most of the ROXY9 CCLCA (lines \#1-3, \#9-10, and \#18-10) or ROXY9 CPYC (lines \#138, \#18-1, \#29-3, and \#31-4) overexpression lines showed an equal or a stronger expression than Col-0 + HA-ROXY9 \#7-18. Only lines Col-0 + HA-ROXY9 CCLCA \#29-2 and Col-0 + HA-ROXY9 CPYC \#19-10 had a weaker expression. Lines Col-0 + HA-ROXY9 CCLCA \#37-5, as well as Col-0 + HAROXY9 CPYC \#27-3 and \#38-5 could not be evaluated since Coomassie Brilliant Blue staining of the membrane indicated lower protein amounts in general. Regarding the different light regimes, the overall expression seemed not to depend on the light treatment; the variations observed might have been caused by uneven blotting (e.g. Figure R1, part II, central blot Col-O $\mathrm{HA}$ ROXY9 \#7-18).

Based on this result, the following plant lines were selected to analyze the activity of the different ROXY9 mutant variants in comparison to plants overexpressing wildtype ROXY9:

Col-0 + HA-ROXY9 \#2-22, \#4-40, \#5-18, and \#7-25

Col-0 + HA-ROXY9 CCLCA \#1-3, \#9-10, and \#18-10

Col-0 + HA-ROXY9 CPYC \#18-1, \#29-3, and \#31-4

As controls, the following plant lines were chosen:

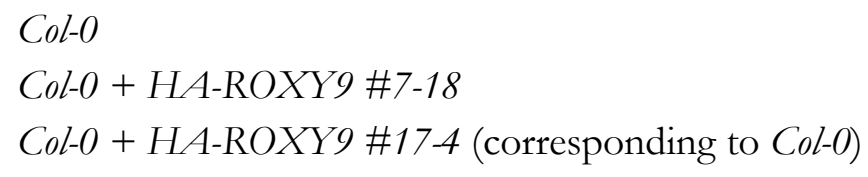



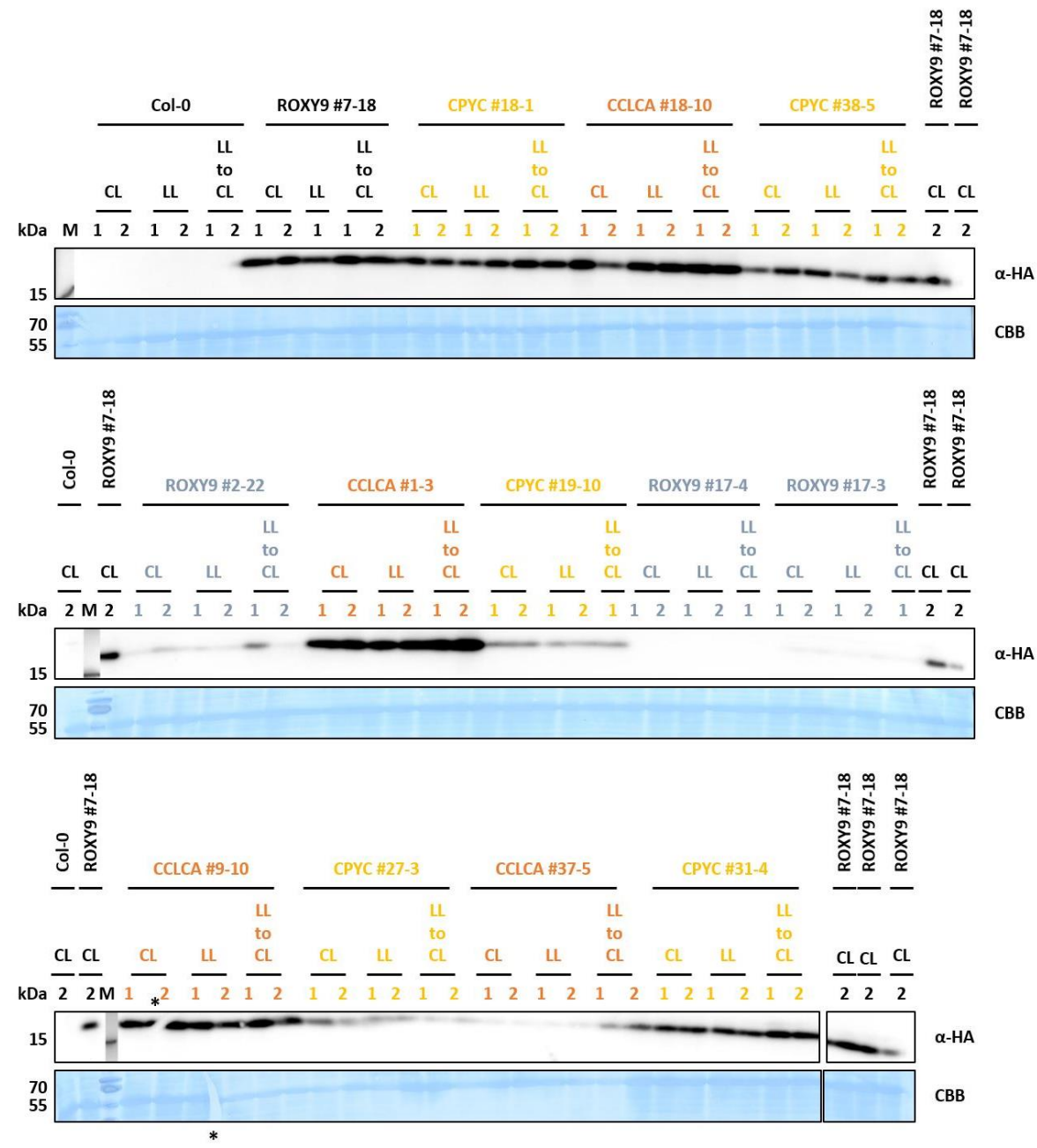

Figure R1, part I. Characterization of A. thaliana overexpressing HA-ROXY9 and mutant versions of $\boldsymbol{R O X Y 9}$ by Western Blot. Col-0 was transformed stably with pB2GW7HA-ROXY9, pB2GW7HA-ROXY9 CCLCA, or pB2GW7HA-ROXY9 CPYC. Homozygous plants were isolated and grown for 4 weeks in a $12 \mathrm{~h}$ light regime. Afterwards, the plants were subjected to different light treatments (control light (CL), low light (LL), low light followed by control light (LL to CL)) as described in Methods (page 59). Finally, the petioles of one or two plants per line were harvested to prepare urea extracts. $10 \mu \mathrm{g}$ of the protein extracts were separated by $15 \%$ SDS-PAGE and analyzed by Western Blot using an $\mathrm{HA}$ antibody. Afterwards, the membrane was stained with Coomassie Brilliant Blue (CBB) to control loading. ROXY9 - Col-0 + HA-ROXY9, CCLCA - Col-0 + HA-ROXY9 CCLCA, CPYC - Col-0 + HA-ROXY9 CPYC; 1 - plant 1, 2 - plant 2. The asterisk indicates a rupture in the gel. 


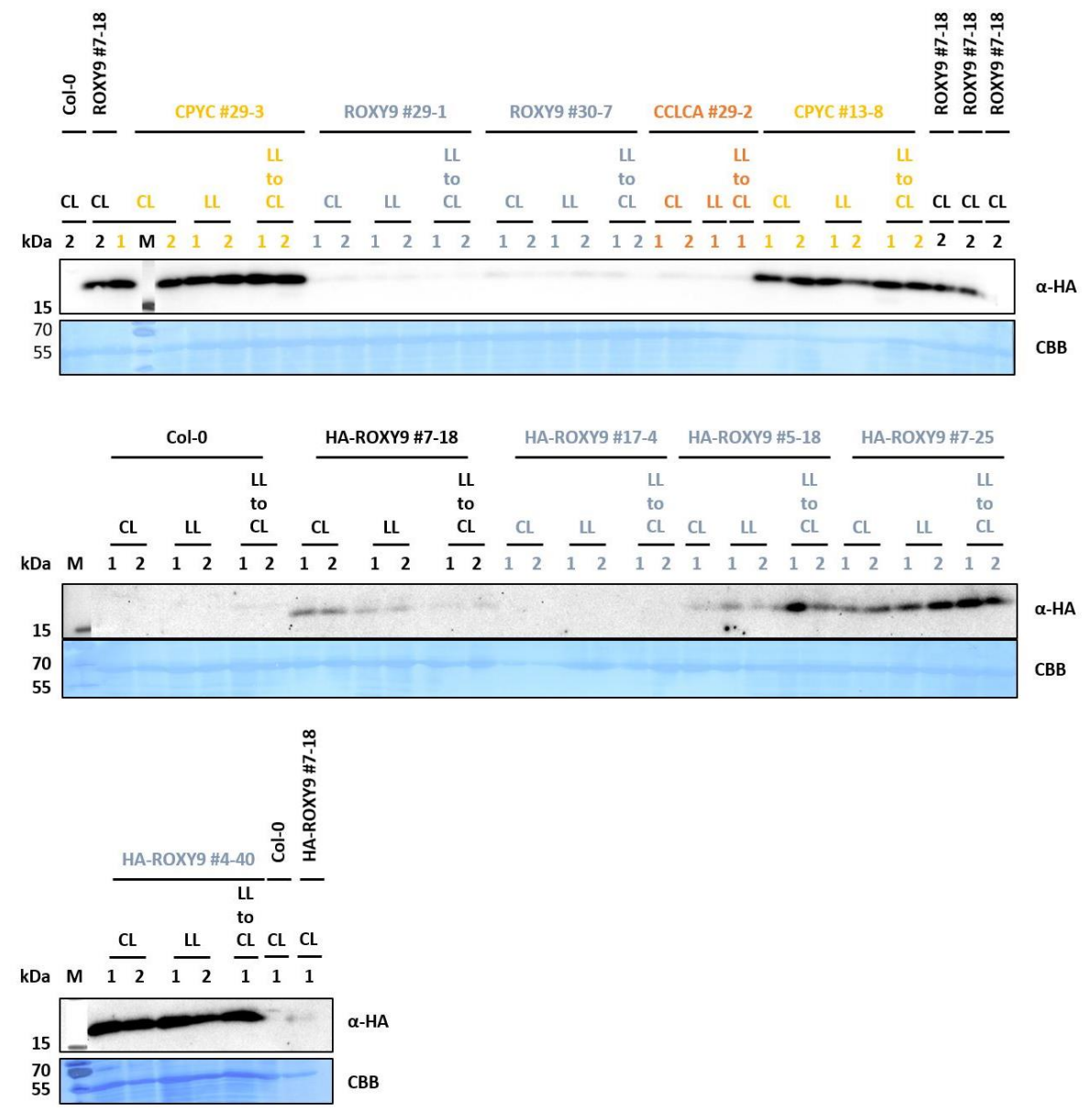

Figure R1, part II. Characterization of A. thaliana overexpressing HA-ROXY9 and mutant versions of $\boldsymbol{R O X Y \boldsymbol { Y }}$ by Western Blot. Col- 0 was transformed stably with pB2GW7HA-ROXY9, pB2GW7HA-ROXY9 CCLCA, or pB2GW7HA-ROXY9 CPYC. Homozygous plants were isolated and grown for 4 weeks in a $12 \mathrm{~h}$ light regime. Afterwards, the plants were subjected to different light treatments (control light (CL), low light (LL), low light followed by control light (LL to CL)) as described in Methods (page 59). Finally, the petioles of one or two plants per line were harvested to prepare urea extracts. $10 \mu \mathrm{g}$ of the protein extracts were separated by $15 \%$ SDS-PAGE and analyzed by Western Blot using an HA antibody. Afterwards, the membrane was stained with Coomassie Brilliant Blue (CBB) to control loading. ROXY9 - Col-0 + HA-ROXY9, CCLCA - Col-0 + HA-ROXY9 CCLCA, CPYC-Col-O + HA-ROXY9 CPYC; 1 - plant 1, 2 - plant 2. 


\section{The peculiar active site and $\mathrm{Tyr}^{25}$ of $\mathrm{ROXY} 9$ are indispensable to repress hyponasty}

To examine whether ROXY9 is active when its active site is mutated to CPYC or when $\mathrm{Tyr}^{25}$ is mutated into an alanine, three of the Arabidopsis lines overexpressing wildtype ROXY9, ROXY9 CCLCA or ROXY9 CPYC were examined for their ability to initiate and terminate low light-induced hyponastic growth. Col-0 + HA-ROXY9 \#17-4 (i.e. Col-0) served as a negative control, whereas Col-0 + HA-ROXY9 \#7-18 served as a positive control for the repressive effect of functional ROXY9 on hyponasty.

Figure R2 and Supplementary Figures SR1, part I to III summarize the results from three independent experiments:

The leaf angles of wildtype Arabidopsis ranged from 5 to $10^{\circ}$. The plants initiated hyponasty when placed in low light conditions leading to an increased leaf angle of 15 to $30^{\circ}$. With exception of one experiment (Figure R2C; Figure SR1, part III), they also terminated hyponasty when shifted to control light conditions after low light treatment. In contrast, Col-0 + HA-ROXY9 \#7-18 showed no or a reduced hyponastic growth, as reported earlier $[155,156]$. The newly generated plants overexpressing HA-ROXY9 showed a similar behaviour. In several cases, even negative angles could be observed.

Interestingly, both, Col-0 $+H A-R O X Y 9$ CCLCA and CPYC plant lines were able to perform hyponastic growth, reflecting the wildtype behaviour in two out of three experiments. In the experiment shown in Figure R2C (Figure SR1, part III), both plant lines lowered their leaves upon backshift to control light, while the wildtype did not.

Thus, ROXY9 seems to require its active site and $\mathrm{Tyr}^{25}$ for its repressive activity on hyponasty in vivo.

In consequence of the repressed circadian hyponastic growth, ROXY9 overexpressing plants exhibit a reduced petiole length [155]. To further support the results from the hyponastic growth experiments described before, the petiole length of the different overexpression lines in comparison to wildtype plants, Col-0 + HA-ROXY9 \#17-4 (i.e. Col-0) and Col-0 + HA-ROXY9 \#7-18 was studied. Since in this work, the leaves between the different plant lines varied in size, the petiole length relative to the entire leaf length was determined (Figure R3). The petioles of wildtype plants were about $45 \%$ of the entire leaf 

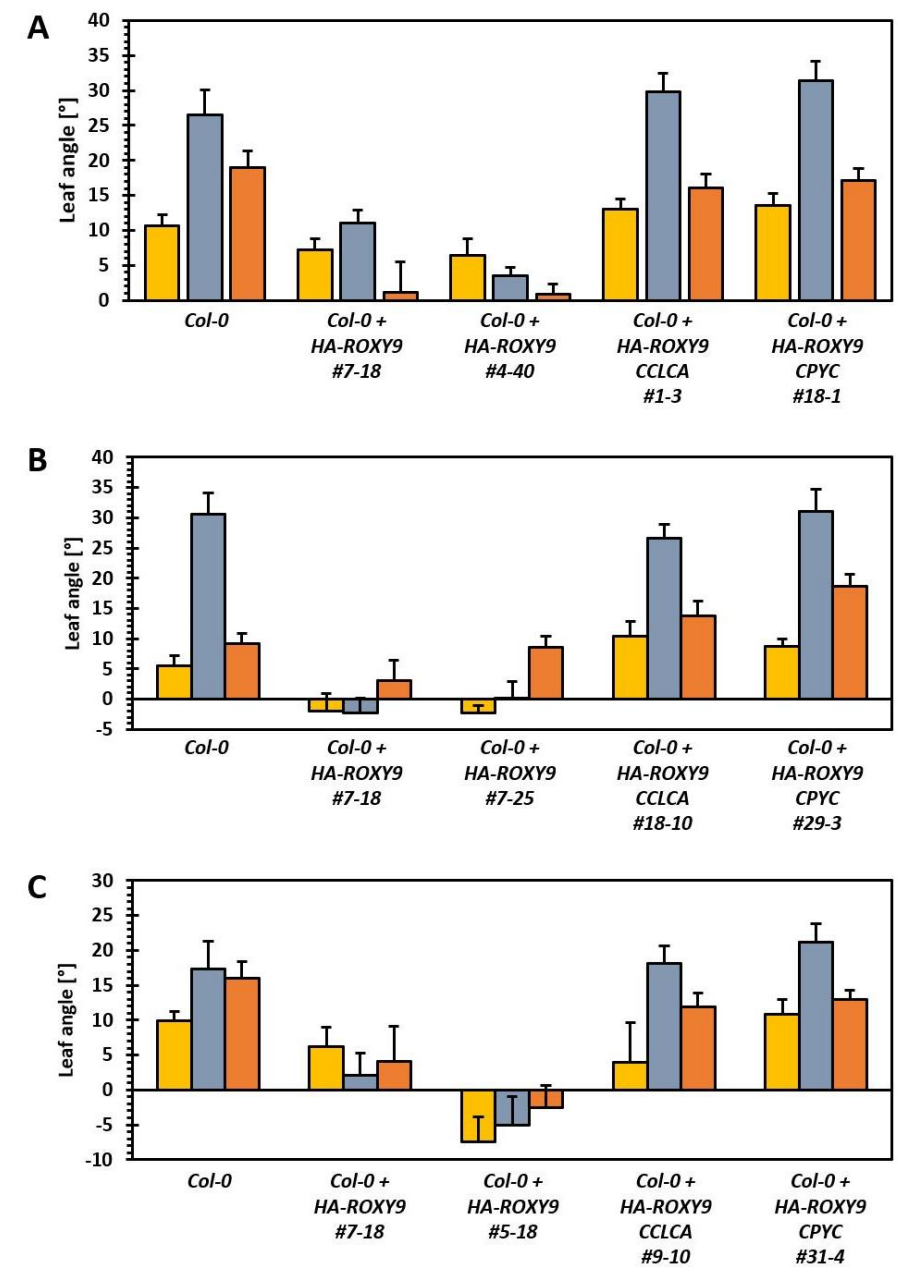

$\square \mathrm{CL}$

$\square$ LL

$\square \mathrm{LL}$ to $\mathrm{CL}$

Figure R2. Analysis of hyponastic growth of plants overexpressing $H A-R O X Y$, $H A$ ROXY9 CCLCA or HA-ROXY9 CPYC. Plants overexpressing HA-ROXY9, HA-ROXY9 CCLCA or HA-ROXY9 CPYC from the CaMV35S promoter were grown for 4 weeks in $12 \mathrm{~h}$ light $/ 12 \mathrm{~h}$ dark rhythm and then transferred to different light conditions as described in Methods (page 59). After the differential light treatments, pictures of the plants in the side view were taken with a Canon PowerShot G11 camera and angles of leaf 7 or 8 measured using Image J. Bars represent mean \pm standard error of the mean (SEM) from at least 4 replicates. $\mathbf{A}, \mathbf{B}$, and $\mathbf{C}$ show three independend experiments. $C L$ - control light, $L L-$ low light, $L L$ to $C L-$ shift from low light to control light; Col-0 corresponds to Col-0 + HA-ROXY9 \#17-4. 


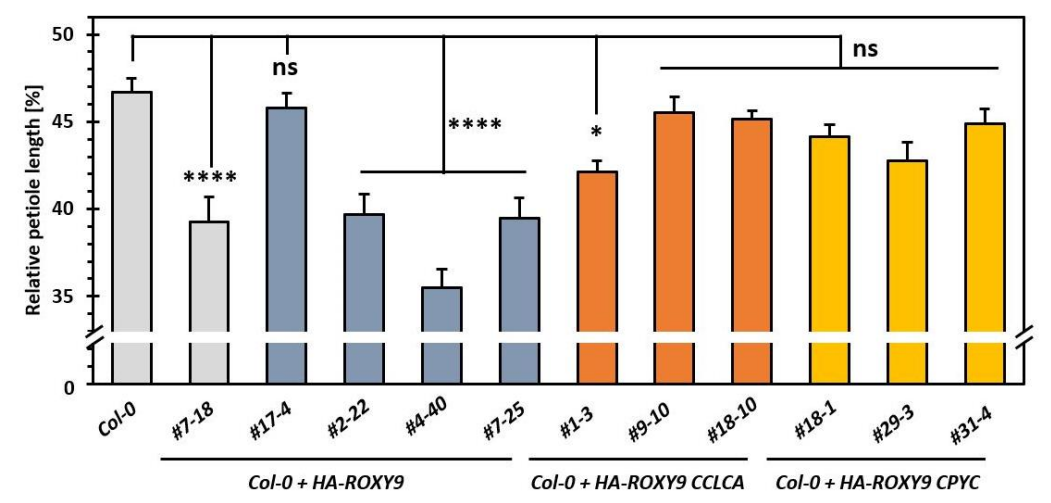

Figure R3. Determination of relative petiole length of plants overexpressing $\boldsymbol{H A}$ ROXY9, HA-ROXY9 CCLCA or HA-ROXY9 CPYC. Plants overexpressing HA-ROXY9, HA-ROXY9 CCLCA or HA-ROXY9 CPYC from the CaMV35S promoter were grown for 4 weeks in $12 \mathrm{~h}$ day. Either leaf 7 or 8 from at least 6 different plants was detached and photographed from the top with a Canon PowerShot G11 camera. The petiole length and the blade length were measured using Image $J$ and the petiole length relative to the entire leaf length computed. Bars represent mean \pm SEM. Statistical analysis (One-way-ANOVA and Tukey test) was performed using GraphPad Prism 8.0.1 assuming normally distributed data. Adjusted pvalues in comparison to Col- 0 are indicated above the bars: ns - non-significant, $\mathrm{p}>0.05, *^{*} \mathrm{p}$ $\leq 0.05, * *$ - p-value $\leq 0.01, * * *$ - p-value $\leq 0.001, * * * *$ - - -value $\leq 0.0001$.

length. A similar observation was made for Col-0 + HA-ROXY9 CPYC lines and two out of three Col-0 + HA-ROXY9 CCLCA lines. Col-0 + HA-ROXY9 CCLCA \#1-3 petioles were slightly, but significantly shorter. This pattern remained the same when analysing additional ROXY9 or ROXY9 mutant overexpression lines (Figure SR2). In addition to Col-0 + HA-ROXY9 CCLCA \#1-3, another plant line (\#29-2) exhibited a significantly reduced relative petiole length. Since expression of HA-ROXY9 CCLCA in this plant was comparatively low, whereas expression of HA-ROXY9 CCLCA in plant line \#1-3 was strong, the insertion site of the transgene in the genome could lead to this effect, since petiole growth is influenced by many factors [250-253]. Alternatively, ROXY9 CCLCA might be partially functional regarding certain processes or under certain conditions. 


\section{ROXY9 requires its peculiar active site and $\mathrm{Tyr}^{25}$ to delay flowering}

Aside from repressing hyponasty, overexpression of ROXY9 was found to delay flowering [157]. Thus, the number of days plants overexpressing $H A$ ROXY9, HA-ROXY9 CCLCA, or HA-ROXY9 CPYC needed to initiate a floral stem of ca. $1 \mathrm{~cm}$ length was determined. On this day, the number of leaves of each plant was counted to check whether the plant is generally retarded in development or whether it exhibits a specific delay in flowering. As controls, Col-0, Col-0 + HA-ROXY9 \#17-4 and Col-0 + HA-ROXY9 \#7-18 were used. The data is depicted in Figure R4 and Figures SR3.

Wildtype plants flowered after ca. 27 to 28 days, whereas plants overexpressing ROXY9 formed floral stems after ca. 35 days, a significant delay (Figure R4A). HA-ROXY9 CCLCA- or HA-ROXY9 CPYC-overexpressing plants flowered about the same time as wildtype plants. This suggests, that ROXY9 is inactive in delaying flowering when $\mathrm{Tyr}^{25}$ is mutated or when the active site is exchanged for CPYC.

While wildtype plants and HA-ROXY9 CCLCA or CPYC-overexpressing plants had at maximum 15 leaves on average at the onset of flowering, Col-0 + HA-ROXY9 \#7-18, \#2-22, \#4-40, \#5-18, and \#7-25 showed at least 16 leaves at this time point (Figure R4B). However, with exception of a single plant line (\#2-22), this difference was statistically not significant. Still, ROXY9 overexpressing plants formed additional, smaller leaves in between the $\geq 16$ leaves. This feature was also observed for two out of six plants of Col- $0+H A$ ROXY9 CCLCA \#1-3 though this plant exhibited 14.7 leaves at flowering. Despite the lack of a significant difference between the numbers of the counted leaves, the occurrence of these additional, smaller leaves might suggest that the delay in flowering is accompanied by an increase in the leaf number. This hints that ROXY9 delays flowering, but not development. Analysis of additional transgenic plant lines (Figure SR3) confirmed the observations and showed that the phenotype required a strong expression of the transgene in case of $H A$ ROXY9 transformants (Figures R1 and SR3): Whereas weakly expressing plants (\#17-3, \#29-1 and \#30-7) flowered like wild type plants, plants expressing the transgene strongly showed the retardation (e.g. \#2-22 and \#440). 
A

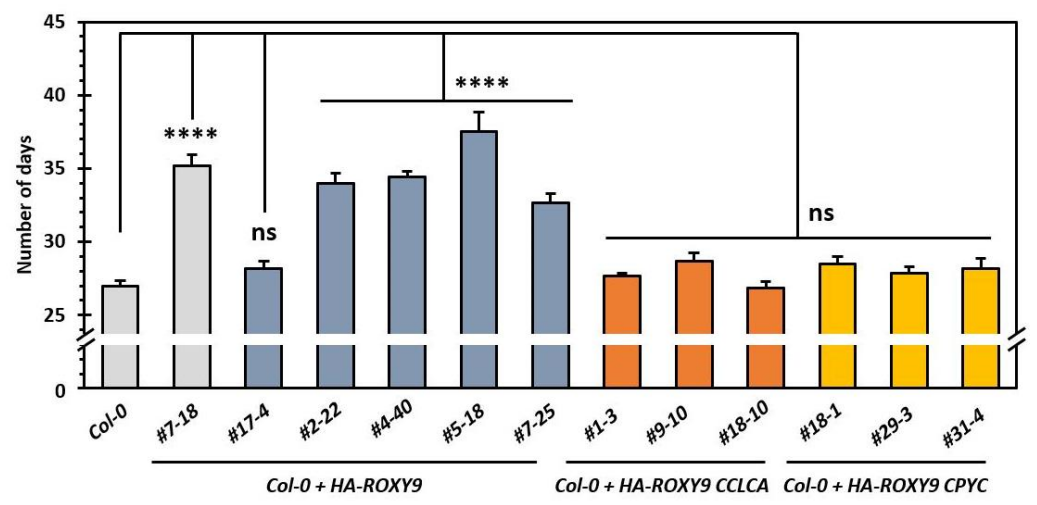

B

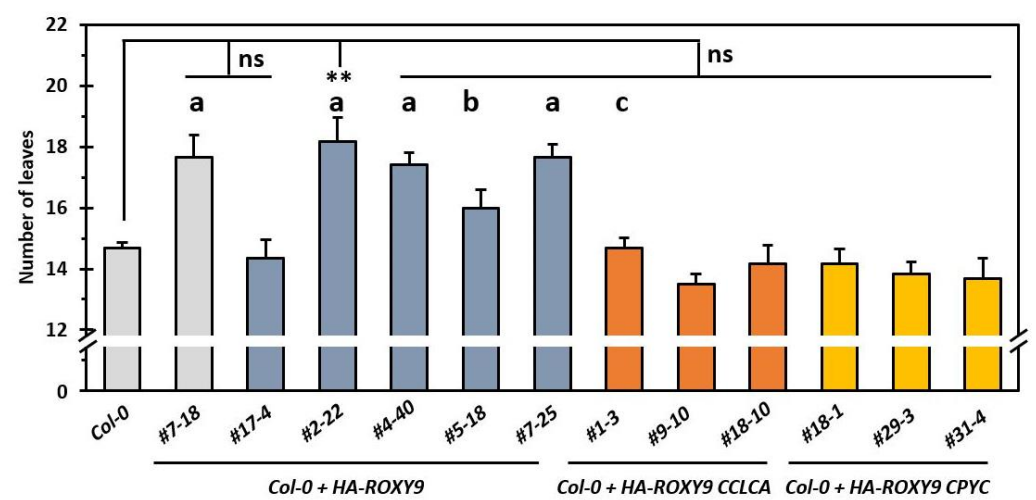

Figure R4. Flowering time point and leave number at flowering of plants overexpressing HA-ROXY9, HA-ROXY9 CCLCA or HA-ROXY9 CPYC. Plants overexpressing HA-ROXY9, HA-ROXY9 CCLCA or HA-ROXY9 CPYC from the CaMV35S promoter were grown under long day conditions. The number of days were counted until a $1 \mathrm{~cm}$ floral stem could be observed (A). At this time point, the number of leaves was determined $(\mathbf{B})$. Bars represent mean \pm SEM from at least 5 replicates. Statistical analysis (Oneway-ANOVA and Tukey test) was performed using GraphPad Prism 8.0.1 assuming normally distributed data. Adjusted p-values in comparison to Col-0 are indicated above the bars: ns non-significant, $\mathrm{p}>0.05, *$ - $\mathrm{p} \leq 0.05, * *$ - $\mathrm{p}$-value $\leq 0.01, * * *$ - $\mathrm{p}$-value $\leq 0.001, * * * *$ - $\mathrm{p}$-value $\leq$ 0.0001. $a$-smaller leaves in between counted leaves for all replicates, $b$-smaller leaves in between counted leaves for all except for 1 replicate, $c$-smaller leaves in between counted leaves for 2 replicates out of 6 


\section{In vitro characterization of ROXY9}

The composition of the active site in ROXY 9 and $\mathrm{Tyr}^{25}$ are important for the activity of the protein in vivo. Since CC-type glutaredoxins are poorly understood at the biochemical level [176], it cannot be deduced why these residues are indispensable for ROXY9 function. To approach the importance of these amino acids, a biochemical study of ROXY9 was initiated. For this, ROXY9 should be produced recombinantly and analyzed for typical glutaredoxin activities, such as reductase and oxidase activity, glutathione binding and the association with iron-sulfur clusters [1].

\section{Recombinant ROXY9 is aggregated when produced in E. coli, but can be purified from insect cells}

CC-type glutaredoxins are difficult to express and/or purify. The only biochemical study about CC-type glutaredoxins to date analyzed a chimeric protein composed of a CPYC glutaredoxin in which a CC-type active site was inserted and a CC-type glutaredoxin deleted for the ALWL motif at its Cterminus. Both proteins were produced in E. coli and purified via a His tag [176]. Having the expression difficulties in mind, ROXY9 was decided to be expressed as a SUMO fusion protein to support expression and solubility [254]. Thus, ROXY9 was cloned via the Gateway system in the expression vector pGP172-SUMO-GW, placing the gene in frame to an N-terminal strep-SUMO tag under control of the T7 promoter and terminator. The expression vector was transformed in E. coli BL21 or BL21-star, expressed at $30^{\circ} \mathrm{C}$ for 3 to $4 \mathrm{~h}$ and purified via its strep tag. As shown in Figure R5, the $27 \mathrm{kDa}$ fusion protein could be purified successfully. It migrated at $35 \mathrm{kDa}$ during SDS-PAGE, However, due to its low isoelectric point (pI) of 6.01 which is expected to accelerate the migration during SDS-PAGE, the reasons for this migration behaviour are unclear (Figure R5A). When separating strep-SUMO-ROXY9 by native PAGE (Figure R5B), two bands, one at $720 \mathrm{kDa}$ and one slightly below appeared. Moreover, a smear ranging from the band at $720 \mathrm{kDa}$ to the bottom 
A

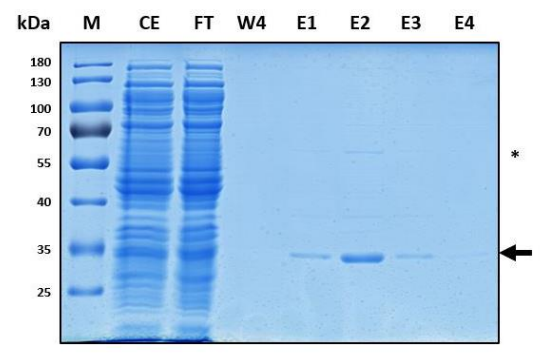

B

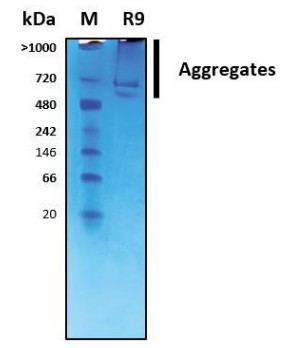

Figure R5. Purification of strep-SUMO-ROXY9 from $\boldsymbol{E}$. coli. Strep-SUMO-ROXY9 was expressed from pGP172-SUMO-ROXY9 in BL21-star. The protein was purified via a Strep'Tactin gravity flow column as described in Methods (page 87) and $10 \mu \mathrm{l}$ of CE, FT, and E1 to E4 and $20 \mu$ l of W4 were separated by $12 \%$ SDS-PAGE (A). The gel was stained with Coomassie Brilliant Blue. Prestained PageRuler was used as a size standard. The arrow points at the band of strep-SUMO-ROXY9. The asterisk marks a contamination from StrepTactinbased purification. To determine the oligomerization state of purified strep-SUMO-ROXY9, $50 \mu \mathrm{l}$ of fraction E2 were analyzed by native PAGE using a a 4 to $20 \%$ gradient gel (B). NativeMark Protein Standard Unstained (Fermentas) was used as a size standard. The gel underwent Coomassie staining. M-Marker, CE - crude extract, FT - flowthrough, W4 - wash 4, E1 to E4 - eluate 1 to 4, R9 - strep-SUMO-ROXY9.

of the well was observed. Altogether, this indicates that strep-SUMO-ROXY9 forms high-molecular weight aggregates.

Despite a multitude of attempts to optimize the purification of strep-SUMOROXY9 (such as different buffer conditions or renaturation, data not shown), it was impossible to obtain lower molecular weight species of strep-SUMOROXY9 from E. coli. Finally, the expression host was changed to insect cells (Trichoplusia ni). Thus, ROXY9 was cloned via the LIC method in the expression vector 438C_strep, fusing it N-terminally to a strep-MBP tag, and expressed in insect cells by Isaac Fianu (Department for Molecular Biology, Prof. Dr. Patrick Cramer, MPI for Biophysical Chemistry, Göttingen). The protein was purified via its MBP tag using an ÄKTA purifier. The chromatogram from the purification (Figure R6A) showed a broad absorption plateau for fractions 1 to 7, corresponding to the flowthrough (Figure R6B). After switching to $100 \%$ Buffer B for elution, a large peak with a maximum of 
A

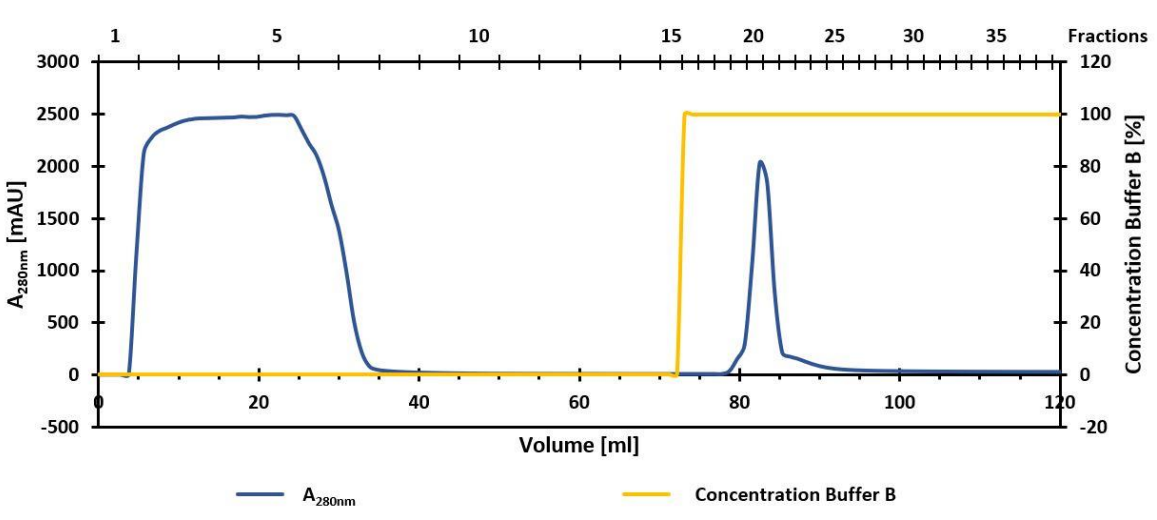

B
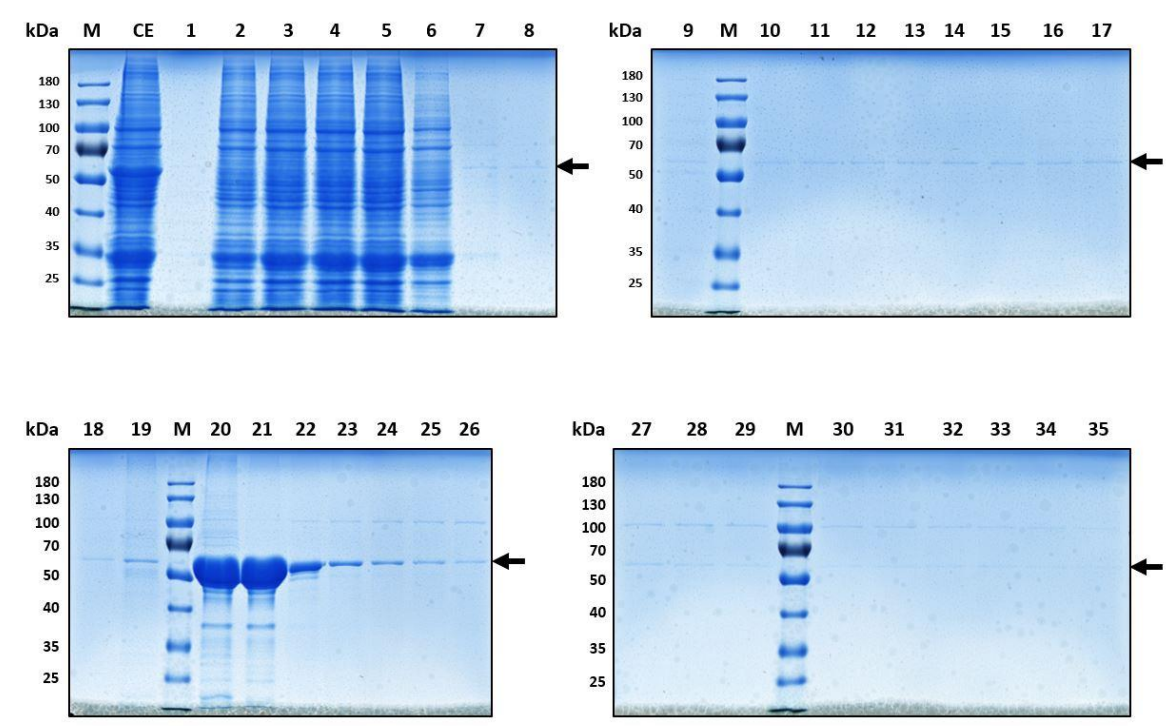

Figure R6. Purification of strep-MBP-ROXY9 from insect cells. Strep-MBP-ROXY9 was expressed in T. ni Hi5 cells and purified via an MBPTrap column using an ÄKTA system. (A) shows the chromatogram of the purification procedure. Buffer $\mathrm{B}$ corresponds to $1 \mathrm{x}$ MBP elution buffer. $10 \mu \mathrm{l}$ of each fraction were analyzed by $10 \%$ SDS-PAGE. The gels were stained with Coomassie Brilliant Blue (B). The arrow indicates the migration height of strep-MBPROXY9. Prestained PageRuler (Thermo Scientific) was used as a molecular weight standard. mAU - milli absorption units, $M$ - Marker, CE - crude extract, 1 to 35 - fraction number.

ca. $2000 \mathrm{mAU}$ in fractions 20 to 21 was observed (Figure R6A). As revealed by SDS-PAGE (Figure R6B), this peak corresponded to strep-MBP-ROXY9 (ca. $59 \mathrm{kDa}$ ). Concentration measurements according to Bradford indicated that ca. $20 \mathrm{mg}$ strep-MBP-ROXY 9 could be obtained from a $600 \mathrm{ml}$ insect cell culture with a concentration of ca. $5 \mathrm{mg} / \mathrm{ml}(80 \mu \mathrm{M})$ in the most highly concentrated 
fractions (data not shown). Importantly, when separating strep-MBP-ROXY9 purified from insect cells by native PAGE (Figure R7), a band at ca. $242 \mathrm{kDa}$ could be detected. Excluding anomalous migration in native PAGE, this band could correspond to a tetramer of strep-MBP-ROXY9. The distinct aggregates observed for strep-SUMO-ROXY9 purified from E. coli could not be detected anymore for strep-MBP- ROXY9. Only a faint smear could be observed above the band at $242 \mathrm{kDa}$.

To further confirm, that strep-MBP-ROXY9 is not present in aggregates, analytical gel filtration was performed in cooperation with the group of Prof. Dr. Kai Tittmann (Georg-August-University, Göttingen). The chromatogram of strep-MBP-ROXY9 (Figure R8A) showed two peaks, a smaller one with a maximum at $8.08 \mathrm{ml}$ and a larger one with a maximum at $14.71 \mathrm{ml}$. The first peak likely corresponds to aggregated protein and its maximum was thus used as the void volume for the calculation of the molecular weight. The second peak (fraction 31) corresponded to a species of strep-MBP-ROXY9 the size of which was within the range of the standard (Figure R8B). From this peak, a molecular weight of $61 \mathrm{kDa}$ could be calculated for strep-MBP-ROXY9, which is close to its theoretical molecular weigth of $59 \mathrm{kDa}$ (Figure $\mathrm{R} 8 \mathrm{C}$ and D). Consequently, analytical gel filtration indicated that strep-MBP-ROXY9 might form a monomer, which contrasts with the results from native PAGE (Figure R7). Despite the contrasting results from native PAGE and analytical gel filtration, it is evident that strep-MBP-ROXY9 purified from insect cells is largely soluble and non-aggregated.

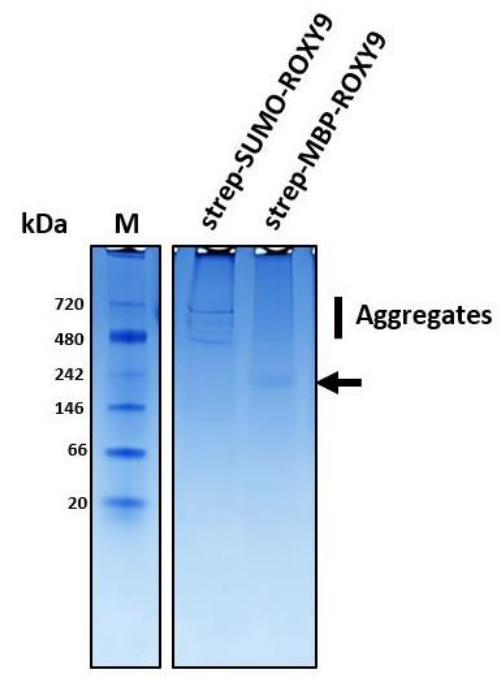

Figure R7. Analysis of the oligomerization state of strep-SUMO-ROXY9 and strep-MBP-ROXY9. StrepSUMO-ROXY9 was expressed in E. coli and purified via Strep'Tactin resin. Strep-MBP-ROXY9 was purified from insect cells via an amylose resin. $50 \mu \mathrm{l}$ of strep-SUMOROXY9 E2 and ca. $4.5 \mu \mathrm{g}$ of strep-MBP-ROXY9 were separated by native PAGE (4 - 20\% gradient). NativeMark Protein Standard Unstained (Fermentas) was loaded as a size standard. The gel was stained with Coomassie. The arrow point shows strep-MBP-ROXY9. $M$ - marker. 
A

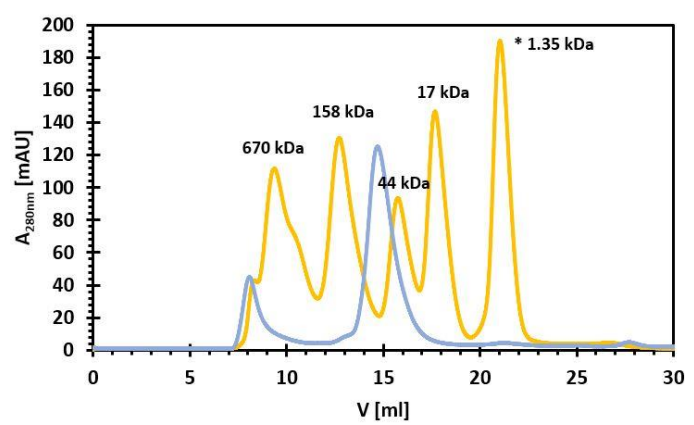

B

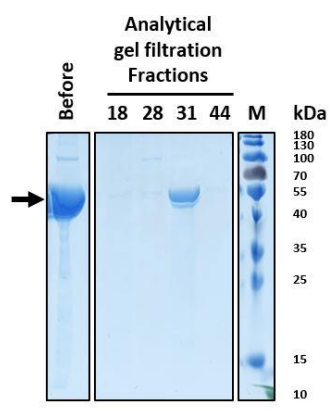

C

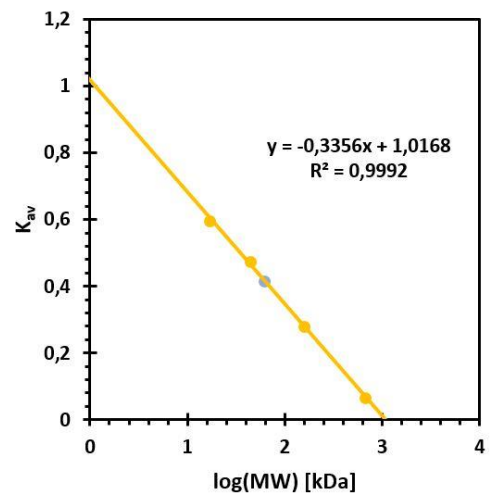

D

\begin{tabular}{lc}
\hline Calculations for strep-MBP-ROXY9 \\
\hline $\mathrm{V}(\mathrm{ml})$ & 14,71 \\
$\mathrm{~K}_{\mathrm{av}}$ & 0,42 \\
Calculated MW (KDa) & 61 \\
Theoretical MW (kDa) & 57 \\
\hline
\end{tabular}

Figure R8. Analytical gel filtration of strep-MBP-ROXY9. At least $700 \mu \mathrm{g}$ of strep-MBPROXY9 purified from insect cells were passed over a Superdex ${ }^{\text {TM }} 200$ 10/300 GL gel filtration column in 1x MBP lysis buffer with DTT. The column was calibrated by performing three runs of the BioRad gel filtration standard in the same buffer. (A) shows the overlay of the chromatograms for the single strep-MBP-ROXY9 run and one of the calibration runs. Black numbers above the peaks indicate the molecular weigths of the proteins in the standard. The asterisk marks the peak of vitamin $\mathrm{B}_{12}$ in the standard. Because this peak did not continue the linear correlation between $\mathrm{K}_{\mathrm{av}}$ and $\log (\mathrm{MW})$, it was not taken into account for linear regression. (C) shows the linear correlation between $\mathrm{K}_{\mathrm{av}}$ and $\log (\mathrm{MW})$. The equation obtained after linear regression and its $\mathrm{R}^{2}$ value are shown in the diagram. The data points of the standard are shown in yellow, the data point for strep-MBP-ROXY9 is shown in blue. Table (D) summarizes the calculation of the molecular weigth of strep-MBP-ROXY9 from the volume at its peak maximum. (B) shows a $12 \%$ SDS-PAGE gel on which a sample before gel filtration (Before) and the indicated gel filtration fractions were separated. The gel was stained with Coomassie. Prestained PageRuler was used as a size standard. The arrow points at strep-MBP-ROXY9. M - marker, $M W$ - molecular weigth. 


\section{Strep-MBP-ROXY9 purified from insect cells can interact with GFP-TGA1}

ROXY9 was shown previously to interact with the transcription factor TGA1 (At5g65210) in yeast-two-hybrid experiments and in pulldown experiments with proteins expressed in Arabidopsis leaf protoplasts [156,157]. Moreover, it interacts with the transcriptional repressor JAZ9 (At1g70700) in a yeast-twohybrid experiment [178]. This information was exploited to test whether purified strep-MBP-ROXY9 is expressed in a functional conformation. To this end, the purified protein should be tested for its interaction with recombinant TGA1 and JAZ9 via a pulldown experiment in vitro.

As a negative control for the pulldown experiment, strep-MBP should be used. Thus, strep-MBP was expressed from the plasmid pGP172-MBP in E. coli and purified via an amylose column using an ÄKTA purification system. The purification was checked by SDS-PAGE (Figure R9). The protein preparation was pure with exception of an equally strong band right below strep-MBP. The identiy of this protein is unknown. Still, the preparation was used for the pulldown experiment (Figure R10).
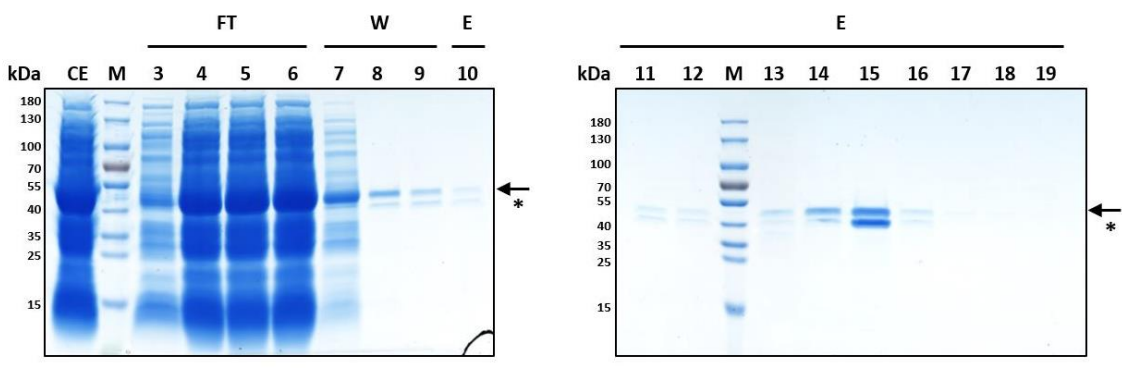

Figure R9. Purification of strep-MBP from $\boldsymbol{E}$. coli. Strep-MBP was expressed from the plasmid pGP172-MBP in E. coli and purified using an ÄKTA purification system supplied with an MBPTrap column. The success of the purification was analyzed by loading $20 \mu \mathrm{l}$ of CE, $20 \mu \mathrm{l}$ of fractions 3 to 9 , and $30 \mu \mathrm{l}$ of fractions 10 to 19 on a gradient SDS gel $(4-20 \%)$. Prestained PageRuler (Thermo Scientific) was used as a molecular weight standard. After the run, the gels were stained with Coomassie. The arrow marks the band most likely corresponding to strep-MBP while the asterisk marks a band belonging to an unknown protein which was co-purified. $M$-Marker, CE - crude extract, FT-flowthrough, $W$ - wash, E-elution, 1 to 19 - fraction number. 
A

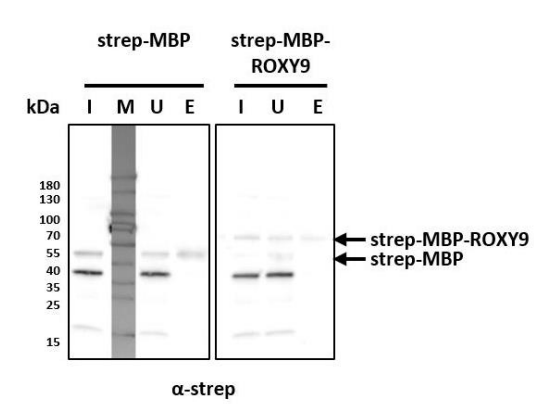

B

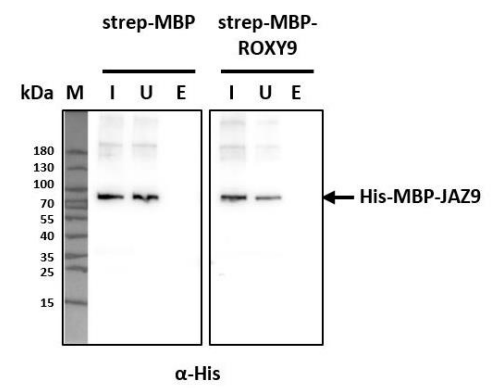

C

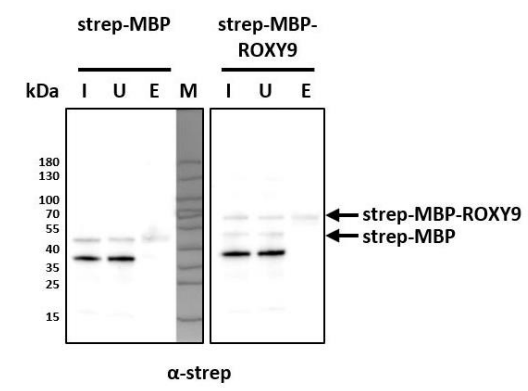

D

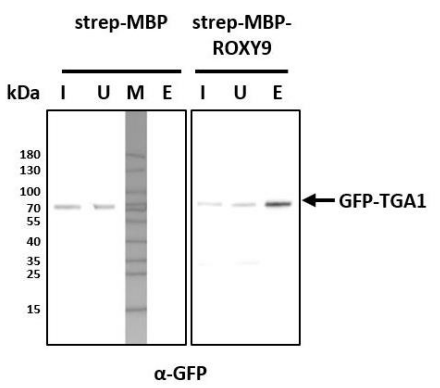

Figure R10. Co-purification of GFP-TGA1 and His-MBP-JAZ9 using strep-MBP or strep-MBP-ROXY9. $1 \mu \mathrm{M}$ purified strep-MBP and strep-MBP-ROXY9 was incubated with His-MBP-JAZ9 and GFP-TGA1 crude extracts prepared after expression of these proteins in E. coli. Strep-MBP and strep-MBP-ROXY9 were purified from the mixture using StrepTactin beads and co-purifucation of His-MBP-JAZ9 and GFP-TGA1 was analyzed. For this, $10 \mu$ of the Input (I) and Elution (E) fractions, and $11 \mu \mathrm{l}$ of the Unbound $(\mathrm{U})$ fraction were separated by non-reducing SDS-PAGE using precast gradient gels (4-20\%). Western Blots of the gels were made. His-MBP-JAZ9 and GFP-TGA1 were detected using a His antibody (B) and a GFP antibody (D), respectively. To control even amounts of strep-MBP and strep-MBPROXY9 in the different samples, Western Blots were detected with a strep antibody (A and C). $M-$ Marker.

For the pulldown, strep-MBP and strep-MBP-ROXY9 were coupled to StrepTactin sepharose and crude extracts from E. coli cells expressing either GFP-TGA1 or His-MBP-JAZ9 were passed over the columns. Input, Unbound and Elution fractions were analyzed by Western Blot. Strep-MBP and strep-MBP-ROXY9 were detected to ensure equal loading (Figure R10A and C). Similar amounts of both proteins were present in the different fractions, showing that they could be successfully purified. In contrast, His-MBP-JAZ9 could only be detected in the Input and in the Unbound fraction, but neither in the elution of the strep-MBP- or the strep-MBP-ROXY9-coupled beads 
(Figure R10B). Likewise, GFP-TGA1 was present in Input and Unbound samples of strep-MBP and strep-MBP-ROXY9-covered beads (Figure R10D). However, GFP-TGA1 could be co-purified with strep-MBP-ROXY9, but not with strep-MBP. This result suggests that strep-MBP-ROXY9 can interact with GFP-TGA1, but not with His-MBP-JAZ9. The lack of the co-purification of His-MBP-JAZ9 could have several reasons, ranging from compromised binding e.g. due to the large MBP tags fused to both proteins to a truly missing interaction. The interaction between ROXY9 and JAZ9 is not as well supported by experiments as the interaction between ROXY9 and TGA1. Since this well-known interaction could be reproduced, strep-MBP-ROXY9 was considered to be in a functional state when purified from insect cells and was thus used for further analyses.

\section{Strep-MBP-ROXY9 is partly oxidized after removal of DTT}

Strep-MBP-ROXY9 is a glutaredoxin containing five cysteines, three of which are in the active site. Since this type of protein depends on reduced cysteine residues for activity, strep-MBP-ROXY9 was purified in the presence of at least 1 mM DTT. However, DTT as a strong reductant interferes with assays to test glutaredoxin activity. Thus, DTT was removed by dialysis before the experiments to address the catalytic activity of strep-MBP-ROXY9. After removal of DT'T by dialysis, Ellman's reagent [248] was used to determine the number of reduced cysteines per molecule of strep-MBP-ROXY9 using an Lcysteine standard curve (Figure R11, sample A). A sample of $74 \mu \mathrm{M}$ strepMBP-ROXY9 contained $138 \mu \mathrm{M}$ free thiol groups, corresponding to approximately two out of five reduced cysteines per protein molecule. Thus, strep-MBP-ROXY9 was only partly reduced after purification and DTT removal. Preincubation of such a protein preparation with DTT for $20 \mathrm{~min}$ and removal of DT' immediately before the Ellman's assay also caused two reduced cysteines per strep-MBP-ROXY9 (Figure R11, sample B). However, treatment with $\mathrm{H}_{2} \mathrm{O}_{2}$ for $20 \mathrm{~min}$ and removing it by a desalting column increased the oxidation of strep-MBP-ROXY9 to one reduced cysteine per protein (Figure R11, sample C), suggesting that strep-MBP-ROXY9 after DT'T removal is not in its maximally oxidized state. 
A Different treatments of strep-MBP-ROXY9

\begin{tabular}{|c|c|c|c|}
\hline Sample & $\bar{A}$ & B & C \\
\hline Treatment & $\begin{array}{l}\text { Purification of protein in } \\
\text { prescence of } 1 \mathrm{mM} \text { DTT }\end{array}$ & $\begin{array}{l}\text { - Incubation for } 20 \mathrm{~min} \text { with } 10^{\circ} \\
\text { mM DTT }\end{array}$ & $\begin{array}{l}\text { Incubation for } 20 \text { min with } 10 \\
\mathrm{mM} \mathrm{H}_{2} \mathrm{O}_{2}\end{array}$ \\
\hline & - Removal of DTT via dialysis & $\begin{array}{l}\text { - Removal of DTT via ZebaSpin - } \\
\text { column }\end{array}$ & $\begin{array}{l}\text { Removal of } \mathrm{H}_{2} \mathrm{O}_{2} \text { via } \\
\text { ZebaSpin column }\end{array}$ \\
\hline
\end{tabular}

\section{B}

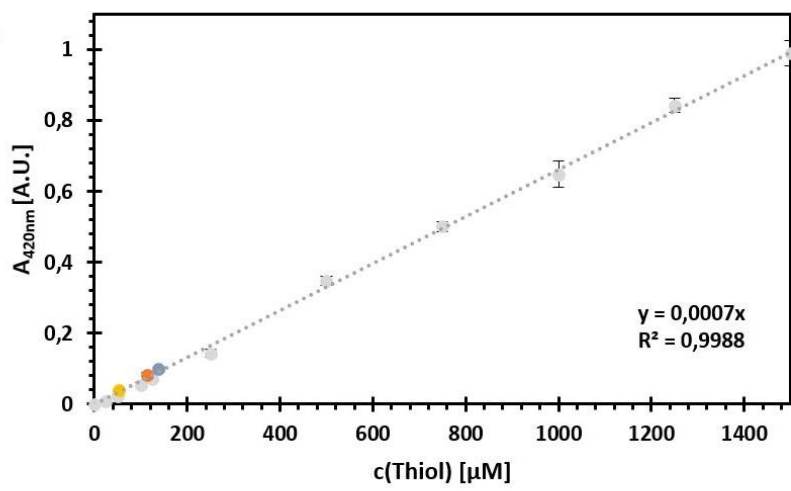

$\square$ L-Cysteine standard

$\square$ Sample A

$\square$ Sample B

$\square$ Sample C

C Calculations for differentially treated strep-MBP-ROXY9

\begin{tabular}{lrrr}
\hline Sample & A & \multicolumn{1}{c}{ B } \\
\hline$c($ ROXY9) $[\mu \mathrm{M}]$ & 74 & 67 & 70 \\
$\mathrm{~A}_{420 \mathrm{~nm}}$ (mean \pm SD) [A.U.] & $0,097 \pm 0,006$ & $0,080 \pm 0,009$ & $0,037 \pm 0,003$ \\
$c($ Thiol) $[\mu \mathrm{M}]$ & 139 & 114 & 53 \\
ratio $c($ Thiol)/c(ROXY9) & 1,9 & 1,7 & 0,8 \\
Number of reduced cysteines per & 2 & 2 & 1 \\
molecule of strep-MBP-ROXY9 & 3 & 3 & 4 \\
Number of oxidized cysteines per & 3 & \\
molecule of strep-MBP-ROXY9 & & & \\
\hline
\end{tabular}

Figure R11. Number of reduced cysteines in strep-MBP-ROXY9 under different oxidative and reductive conditions. Strep-MBP-ROXY9 was exposed to the three different treatments described in A. Afterwards, the number of reduced cysteines was determined using Ellman's reagent. A calibration curve with L-cysteine was prepared in triplicate (mean \pm standard deviation (SD) was plotted) (B) and used to calculate the number of reduced cysteines per protein $(\mathbf{C})$. The samples were measured in triplicate and the protein concentration was determined with Bradford solution. 


\section{Strep-MBP-ROXY9 does neither reduce HEDS, insulin nor glutathionylated GAPDH after removal of DT'T}

Despite strep-MBP-ROXY9 being partially oxidized, the protein was tested for its catalytic activity in vitro: The reduction capacity towards the artificial substrate HEDS and the deglutathionylation activity towards the protein substrate GAPDH, as well as the protein disulphide reduction activity towards insulin were assayed. As positive controls, the well characterized human glutaredoxin Grx1 [242,255-257] and the plant glutaredoxin GRXC2 from A. thaliana were chosen. The GRXC2 homolog in poplar was already characterized regarding its catalytic properties [77] and also for A. thaliana GRXC2, a catalytic activity was recently described [88].

Grx1 was expressed as a strep-SUMO fusion in E. coli and purified via its streptag (Figure R12). The $27 \mathrm{kDa}$ protein showed the same anomalous migration as already observed for strep-SUMO-ROXY9 during SDS-PAGE (Figure R5A). Since the purification necessitated the use of low-salt buffer, some contaminations with E. coli proteins remained in elution fractions 2 and 3 of strep-SUMO-Grx1. GRXC2 was fused to a strep-MBP-tag and expressed in E. coli, as well. The protein was purified via its MBP tag using an ÄKTA purifier (Figure R13). After switching to the elution buffer B, only two small peaks could be detected by measuring $\mathrm{A}_{280 \mathrm{~nm}}$ (Figure R13A). SDS-PAGE showed,

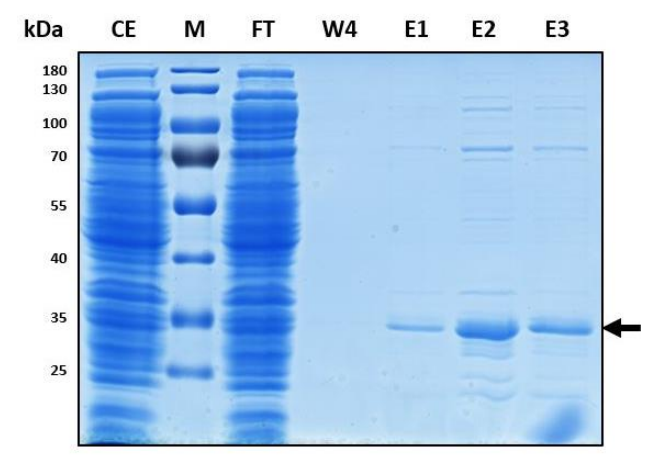

Figure R12. Purification of strepSUMO-Grx1 from E. coli. Strep-MBPGrx1 was expressed in E. coli BL21-star and purified using a StrepTactin gravitiy flow column. $12 \%$ SDS-PAGE and Coomassie staining visualized the course of the purification. $10 \mu \mathrm{l}$ of CE, FT, and E1 to E3, and $20 \mu$ l of W4 were loaded. Prestained PageRuler (Thermo Scientific) was employed as a size standard. The arrow shows the band of strep-SUMOGrx1. M-Marker, CE - crude extract, FT flowthrough, W4 - wash 4, E1 to E3 - eluate 1 to 3 
A

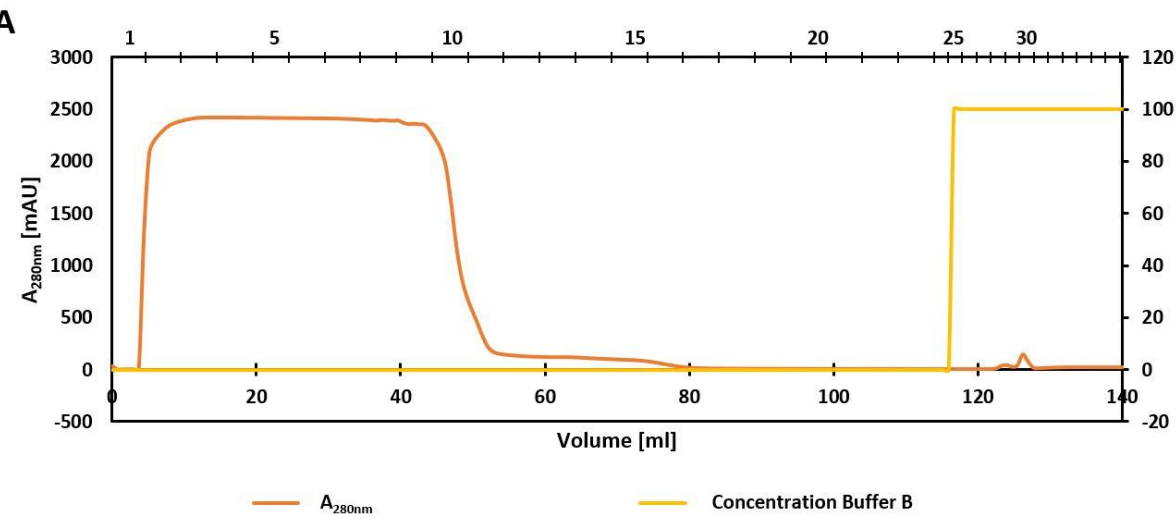

\section{B}
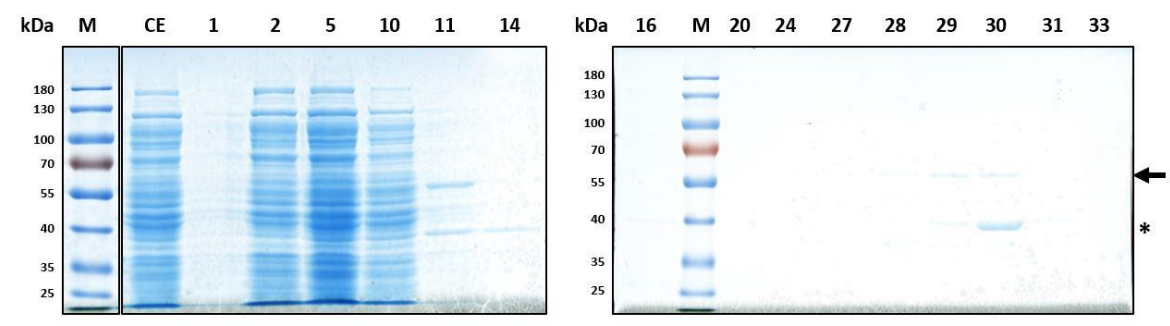

Figure R13. Purification of strep-MBP-GRXC2 from E. coli. Strep-MBP-GRXC2 was expressed in E. coli BL21-star and purified via its MBP tag using an ÄKTA purification system. Purification was monitiored by following $\mathrm{A}_{280 \mathrm{~nm}}(\mathbf{A})$. Buffer $\mathrm{B}$ represents 1x MBP elution buffer with $50 \mathrm{mM} \mathrm{NaCl} .20 \mu$ of each fraction were separated by $10 \%$ SDS-PAGE. The gels were stained with Coomassie (B). The arrow points at strep-MBP-GRXC2, whereas the asterisk marks the strep-MBP tag. Prestained PageRuler (Thermo Scientific) was used as a size standard. mAU - milli absorption units, $M$ - Marker, $C E$ - crude extract, 1 to 33 - fraction number.

that the first peak (fraction 28) corresponded to almost pure strep-MBPGRXC2 in very low amounts. The second peak belonged to a mixture of strepMBP-GRXC2 with strep-MBP tag (Figure R13B, fractions 29 and 30). For this reason, the concentration of strep-SUMO-Grx1 fraction E1 and strep-MBPGRXC2 Fraction 28 was determined using Bradford solution. When other elution fractions were used, the concentration was estimated by loading a known volume of the protein preparation on an SDS gel together with different, defined amounts of BSA. Both proteins were dialysed to remove DTT from the purification and tested along with strep-MBP-ROXY9 for catalytic activity. 
First, the deglutathionylation and/or disulphide reduction activity of all three proteins towards HEDS was addressed (Figure R14A and B). For both, strepSUMO-Grx1 and strep-MBP-GRXC2, 5, 25 and $50 \mathrm{nM}$ protein were tested. For strep-MBP-ROXY9, the ten-fold concentrations were used. As a negative control, a reaction without enzyme, but with glutathione was used. Reduction activity was visualized by coupling the glutaredoxin reaction to the NADPHconsuming reaction of GSSG reductase. As shown in Figure R14B, GSH alone led to a slow decline in the absorption at $A_{340 \mathrm{~nm}}$. Strep-SUMO-Grx1 and strepMBP-GRXC2 did not differ from the no-enzyme control when they were used at concentrations of $5 \mathrm{nM}$. At 25 and $50 \mathrm{nM}$, however, a deglutathionylation activity could be detected by the stronger decrease in $A_{340 \mathrm{~nm}}$. Strep-MBPROXY9 was not different from the no-enzyme control even though it was used in higher concentrations than the two control proteins, suggesting that it is unable to reduce HEDS.

Next, the capacity of all three proteins to reduce the intermolecular disulfides in insulin was addressed (Figure R14A, C and D). The concentrations of the proteins used were the same as for the HEDS assay. As a control served a sample containing GSH but lacking a glutaredoxin. Precipitation of the insoluble $B$ chain of bovine insulin was followed by the increase in $A_{650 \mathrm{~nm}}$. The negative control led to an increase in the absorption after $1.5 \mathrm{~h}$, indicating that GSH alone caused reduction of insulin to a small extent (Figure R14D). StrepSUMO-Grx1 and strep-MBP-GRXC2 were active at a concentration of 25 and $50 \mathrm{nM}$, but not or only weakly at a concentration of $5 \mathrm{nM}$ (Figure R14C and D). Similarly, strep-MBP-ROXY9 used at a concentration of $50 \mathrm{nM}$ did not reduce insulin to a higher extent than observed for the no-enzyme control. However, at 250 and $500 \mathrm{nM}$, strep-MBP-ROXY 9 even seemed to slow down the reduction rate observed for glutathione alone (Figure R14D).

Because we could only observe this so far unexplainable effect of strep-MBPROXY 9 towards insulin and no activity towards HEDS, we decided to use a protein substrate to test deglutathionylation. For this reason, strep-SUMOGrx1 and strep-MBP-ROXY9 were tested for their deglutathionylation capacity towards glutathionylated GAPDH. As outlined in Methods (page 103), this assay relies on the inactivation of GAPDH by glutathionylation of its catalytic cysteine residue (GAPDH-SG) and reactivation by deglutathionylation through glutaredoxins [37]. Deglutathionylation is indirectly determined via the activity of GAPDH, the reaction of which needs reduced nicotinamid adenine dinucleotide (NADH) [37,78]. Figure R14E displays the initial velocity of GAPDH and GAPDH-SG after different time points of incubation with or 
without glutaredoxin. As controls, non-glutathionylated GAPDH, glutathionylated GAPDH and GAPDH-SG treated with DTT were used. The deglutathionylation of GAPDH-SG caused by GSH alone was determined by omitting the enzyme in the reaction mix. Whereas non-glutathionylated GAPDH was active, GAPDH-SG was completely inactive in this assay. Reactivation of GAPDH-SG with an excess of DTT restored GAPDH activity, though not to the same levels as observed for GAPDH. This might be explained by partial reduction or by protein losses caused by the preparation procedure of GAPDH-SG, as the GAPDH activity measured for the nonglutathionylated control is caused by the maximum possible GAPDH concentration within the experiment. Glutathione treatment led to a much smaller enhancement of GAPDH activity over time, indicating that it restored GAPDH activity though not as efficiently as DTT. Strep-SUMO-Grx1 at a concentration of $40 \mathrm{nM}$ reactivated approximately twice as much GAPDH as glutathione. After removal of DTT by dialysis, strep-MBP-ROXY9, although used again at almost ten-fold higher concentrations than strep-SUMO-Grx1, exhibited a weak to no activity compared to glutathione.

Assuming that ROXY9 might only act on a subset of proteins, redox-sensitive GFP (roGFP) was selected as another target to test for deglutathionylation activity of strep-MBP-ROXY9. This experiment was performed by Lara Ostermann (INRES, Prof. Dr. Markus Schwarzländer, Bonn). During this experiment, roGFP is first oxidized (roGFP ${ }_{\text {ox }}$ ) and then incubated with GSH or GSH and glutaredoxin. The redox state of roGFP determines its fluorescence emission intensity at $510 \mathrm{~nm}$ after excitation at $400 \mathrm{~nm}$ and $482 \mathrm{~nm}$. Oxidation of the protein leads to an increase of its fluorescence emission after excitation at $400 \mathrm{~nm}$, whereas reduction causes an increase in the fluorescence emission after excitation at $482 \mathrm{~nm}$ [244]. Thus, redox reactions can be followed by monitoring the ratio of $F_{400 \mathrm{~nm}} / \mathrm{F}_{482 \mathrm{~nm}}$. Samples containing roGFP ${ }_{\text {ox }}$ showed a high fluorescence ratio, while samples containing roGFP red showed a low fluorescence ratio (Figure SR4). In this experiment, GRXC1 from Arabidopsis purified by the department for Chemical Signalling (INRES) in Bonn was used as a positive control. Strep-MBP-ROXY9 was dialysed against the reaction buffer, thereby removing DTT. This treatment leads to an oxidation of the protein (Figure R11, sample A). The control protein, GRXC1, was used in the oxidized state in which it was purified. 
A

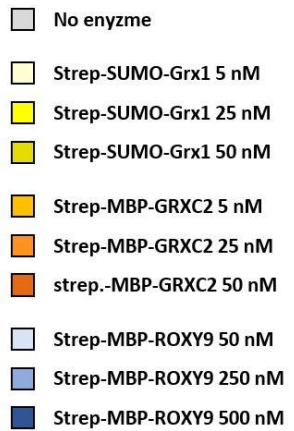

C

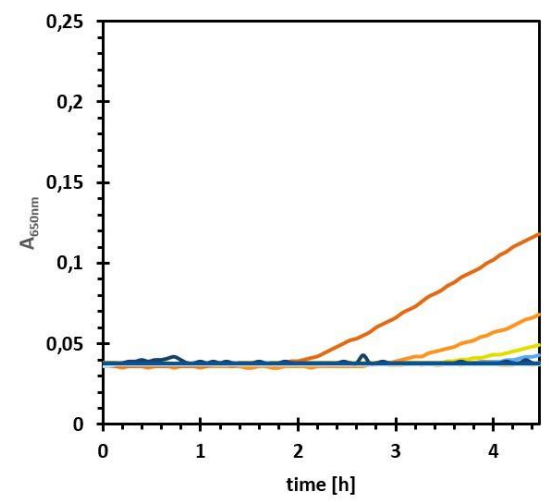

B

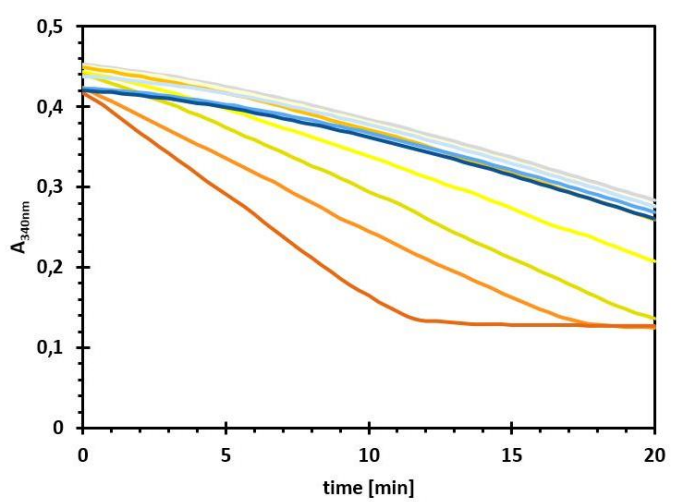

D

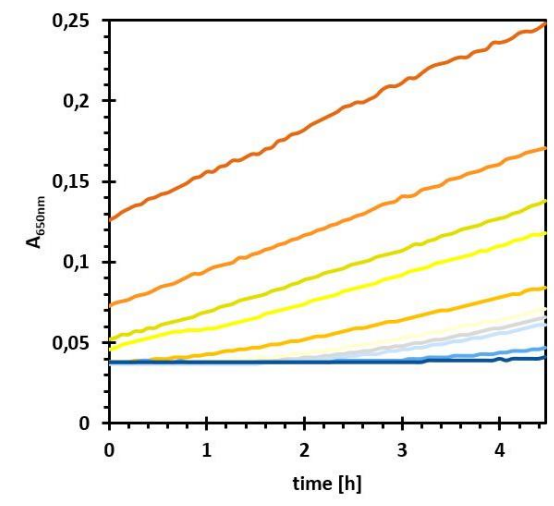

Figure R14, part I. In vitro reductase activity of strep-MBP-ROXY9 when DTT is removed by dialysis. Different concentrations of strep-SUMO-Grx1, strep-MBP-GRXC2 and strep-MBP-ROXY9 (A) were analyzed in the HEDS (B) and the insulin assay $(\mathbf{C}$ and $\mathbf{D})$ for reductase activity. The diagrams show the change in the absorption at $340 \mathrm{~nm}$ (HEDS) and $650 \mathrm{~nm}$ (insulin) over time. In case of the insulin assay, the measurement was continued after a break at $4.5 \mathrm{~h}(\mathbf{C})$ for another $4.5 \mathrm{~h}(\mathbf{D})$. Each reaction was prepared only once. 
E

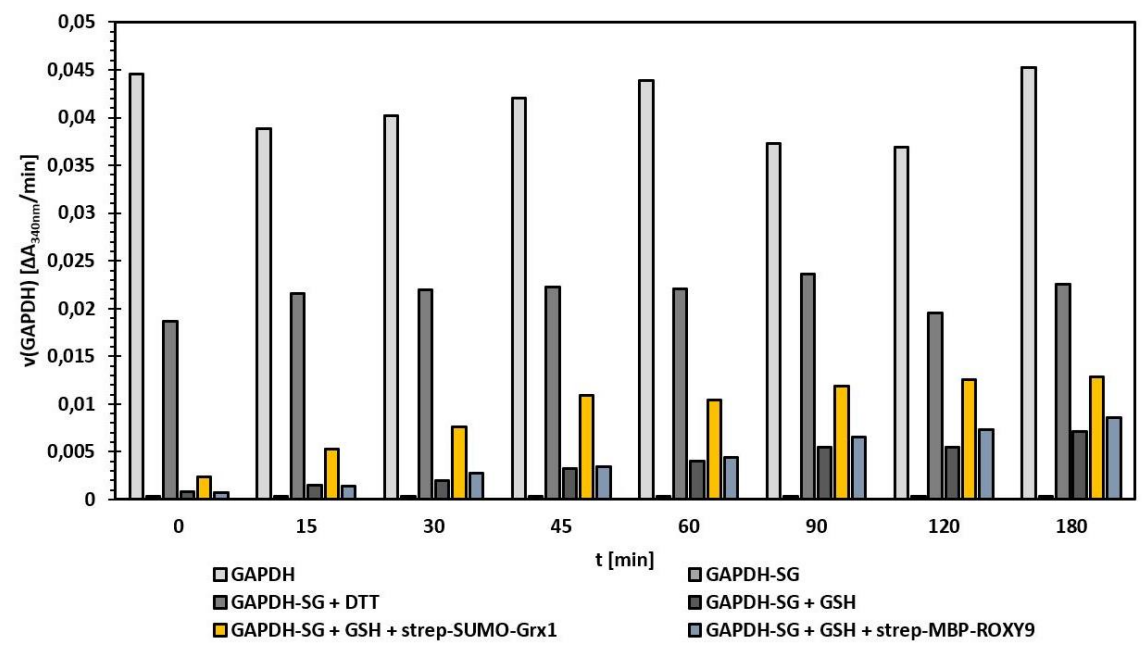

$\mathbf{F}$

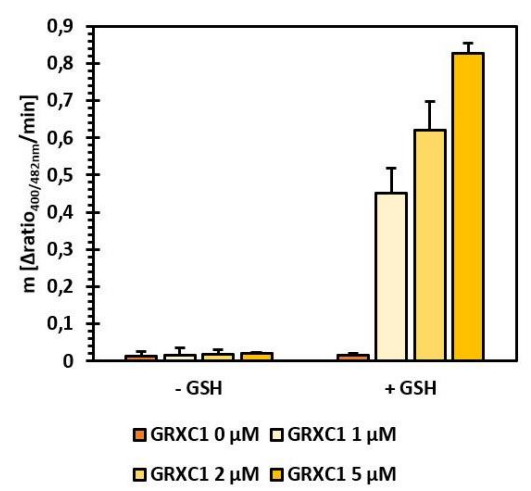

G

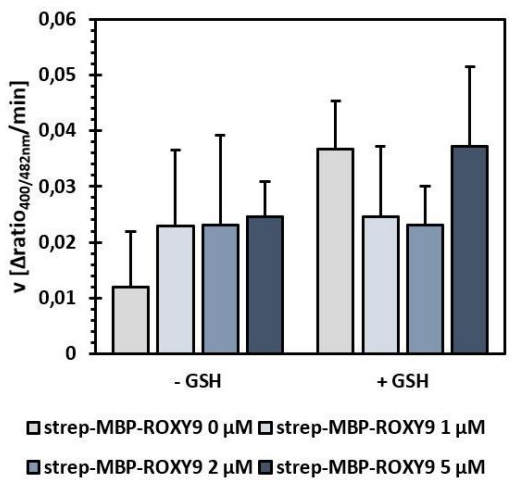

Figure R14, part II. In vitro reductase activity of strep-MBP-ROXY9 when DTT is removed by dialysis. (E) Strep-SUMO-Grx1 and strep-MBP-ROXY9 were compared in the GAPDH assay. For this, ca. $40 \mathrm{nM}$ of strep-SUMO-Grx1 and ca.300 nM strep-MBP-ROXY9 were added together with GSH to the reaction mixtures containing maximally $9.6 \mu \mathrm{M}$ glutathionylated GAPDH (GAPDH-SG). The GAPDH activity was assessed by withdrawing aliquots during the reaction and measuring the velocity of GAPDH $(\mathrm{v}(\mathrm{GAPDH}))$ in these samples. The initial velocity of GAPDH was plotted over the timepoints at which a sample was taken. Treatment of GAPDH-SG with DTT or GSH alone served as controls. Additionally, a sample of untreated GAPDH-SG and $9.6 \mu \mathrm{M}$ GAPDH were analyzed. All samples in the GAPDH assay were prepared only once. ( $\mathbf{F}$ and $\mathbf{G})$ strep-MBP-ROXY9 and Nterminally His-tagged GRXC1 were compared regarding the reduction of oxidized roGFP2. Strep-MBP-ROXY9 and GRXC1 were used in an oxidized state. The indicated amounts of GRXC1 (F) and strep-MBP-ROXY9 (G) were incubated with or without GSH and oxidized roGFP. The reactions were started after $10 \mathrm{~min}$ of preincubation. The initial velocity of these reactions measured in the linear reaction phase between minutes 13 and 15 was plotted in the bar charts. Values represent mean \pm SD. 
Figures R14F and G display the slope of the increasing reduction of roGFP for samples containing $0,1,2$, or $5 \mu \mathrm{M}$ of glutaredoxin, either with or without glutathione. The fluorescence changes over the entire experiments are shown in Figure SR4. GRXC1 (Figure R14F) led to a significant increase in the amount of reduced roGFP compared to the controls without glutathione and without enzyme, or with glutathione alone. However, the reduction of roGFP by GRXC1 depended on glutathione, as no activity was observed for samples containing enzyme, but lacking glutathione. In contrast, for strep-MBPROXY9, only the background activity caused by glutathione could be observed, even when protein was added to the reaction mixture (Figure R14G). Thus, while GRXC1 reduced roGFP ox, strep-MBP-ROXY9 did not.

\section{Strep-MBP-ROXY9 oxidizes quickly after removal of DTT}

The lack of a reductase activity for strep-MBP-ROXY9 could be caused by the partial oxidation of the protein. Because glutathione is a reductant and is present in the reaction mixtures of all glutaredoxin activity assays, strep-MBPROXY9 purified in the presence of DTT should be dialyzed against buffer containing glutathione in order to prevent oxidation of the protein. Because glutathione interferes with the Ellman's method, a first attempt to determine the oxidation state of strep-MBP-ROXY9 when dialyzed against glutathione by mass spectrometry was made. In addition, protein purified with DTT and protein from which DTT was removed by dialysis, as well as protein incubated for only 10 min with glutathione, was analyzed. At the end of the respective treatment, all samples were immediately precipitated with TCA and then labelled with IAM and iodoTMT: For this, first, all reduced cysteines were blocked with IAM. Subsequently, all oxidized cysteines were reduced with TCEP. Finally, all cysteine residues reduced by TCEP were labelled with iodoTMT. Thus, all originally reduced cysteine residues are marked by IAM whereas all originally oxidized cysteine residues are labelled with iodoTMT. For analysis of the MS data (Supplementary table SR1, part I to IV; Figures R15 and SR5), the number of independent identifications (PSMs) was normalized to the number of independent identifications of two different, unmodified peptides from the MBP tag. For normalization, only those peptides which were fully labelled and completely cleaved by trypsin were chosen, because they were 
present in all samples and corresponded to the most abundant peptides. This normalization allowed a comparison between different treatments for the same peptide. All other peptides, which were sometimes not found in all samples, were considered qualitatively.

Data analysis showed that protein containing DT' was the most strongly reduced preparation of all regarding the active site (Figure R15A to D and SR5A to D). Still, oxidized peptides were discovered in this sample, but their amount cannot be deduced. Removal of DT'T, as well as introducing GSH by dialysis while removing DTT resulted in an increase of strep-MBP-ROXY9 with one or none reduced cysteine residues within the active site compared to the DTT-treated sample (Figure R15C and D and SR5C and D). The sample treated for $10 \mathrm{~min}$ with GSH after removal of DTT showed constantly low amounts of the different peptides (Figure R15A to D and SR5A to D). Cys ${ }^{49}$ was partially oxidized and partially reduced. The extent of reduction or oxidation did not vary much between the different treatments (Figure R15E and $\mathrm{F}$ and SR5E and F). Similar observations were made for $\mathrm{Cys}^{61}$ with exception of the sample which was treated for 10 min with glutathione (Figure R15G and $\mathrm{H}$ and SR5G and H). As for the active site peptides, this $10 \mathrm{~min}$ glutathione treatment led to the lowest normalized PSMs for all oxidation states analyzed. However, the effect was less pronounced compared to the active site peptides. Because the initial PSM values are normalized to the amount of protein analyzed by MS, these low numbers of peptides cannot result from lower protein amounts in general. Additionally, the peptides could be detected in other samples excluding difficulties with separation and detection of these peptides during MS. Instead, the low normalized PSM values might result from incomplete labelling and/or incomplete cleavage. Several not fully labelled or cleaved peptides were detected in the four samples (Supplementary table SR1). Importantly, as not each of these peptides was detected throughout all samples, their number cannot be estimated. Thus, they represent an unknown variable in this analysis. Similarly, this analysis did not include a search for peptides linked by or containing disulphide bridges or cysteine residues oxidized to sulfonic acids. Whereas disulphide bridges can in principle be detected indirectly via reduction with TCEP and iodoTMT labelling, sulfonic acids cannot be reduced by TCEP and therefore escape labelling with iodoTMT [258]. However, enhanced sulfonic acid formation after $10 \mathrm{~min}$ treatment with the reducing agent glutathione seems unlikely.

Considering the whole dataset, the contribution of sulfonic acid formation to the number of oxidized peptides and of those modifications which cannot be 
A

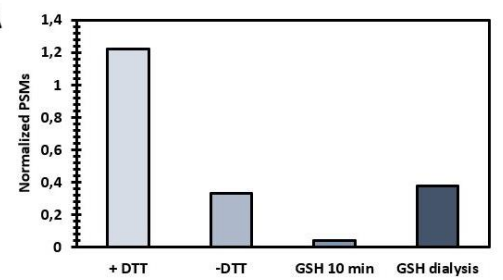

C

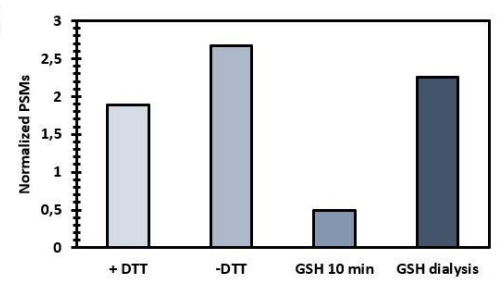

E

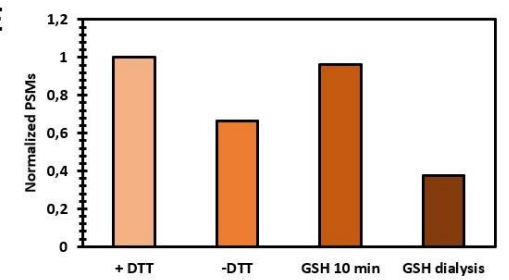

G

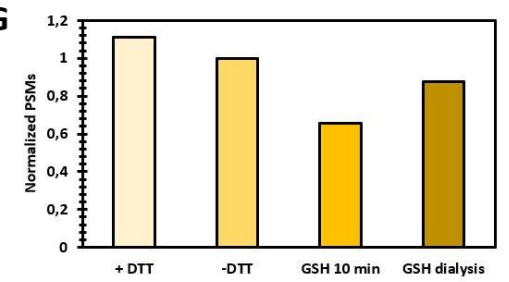

B

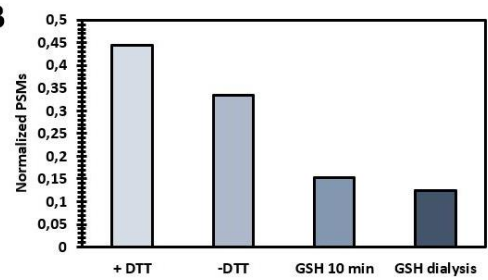

D
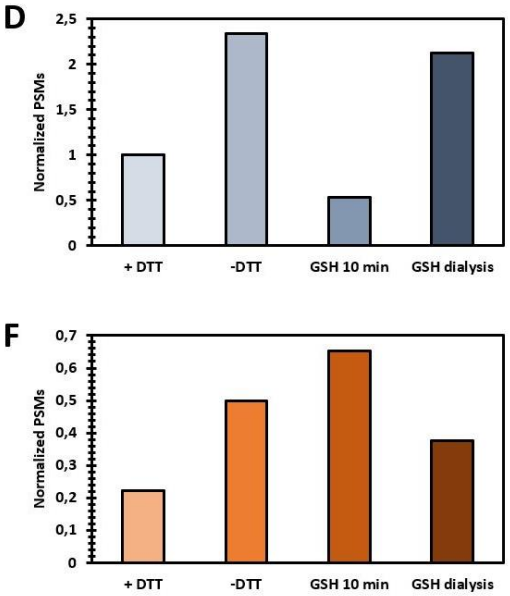

H

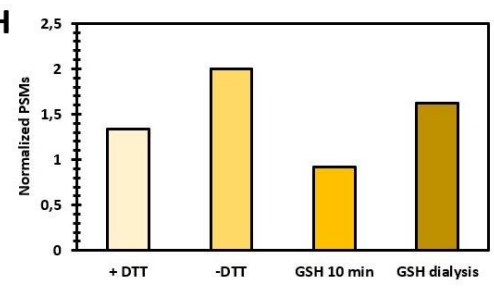

Figure R15. Analysis of the strep-MBP-ROXY9 redox state by LC-MS/MS. Strep-MBPROXY9 was purified from insect cells in the presence of DTT and subjected to different oxidizing and reducing treatments: (1) Dialysis to remove DTT; (2) Dialysis to remove DTT and then incubation for $10 \mathrm{~min}$ with GSH; (3) Dialysis against buffer containing GSH, to remove DTT while simultaneously applying GSH. All samples were labeled with IAM (reduced cysteines) and iodoTMT (oxidized cysteines), separated in a $10 \%$ non-reducing SDS-PAGE and analyzed by LC-MS/MS as described in Methods (pages 109-110 and 115-119). To allow quantification, the number of different PSMs for each peptide and modification was normalized to the number of PSMs of an unmodified peptide resulting from the MBP tag (LYPFTWDAVR [1267,64698 Da]). As this allows only comparison of the abundance of specific peptides between treatments, the values for the normalized PSMs were plotted accordingly: active site-containing peptides with none $(\mathbf{A})$, one $(\mathbf{B})$, two $(\mathbf{C})$ or three $(\mathbf{D})$ oxidized cysteines; peptides containing $\mathrm{Cys}^{49}$ in a reduced $(\mathbf{E})$ or oxidized $(\mathbf{F})$ state; peptides containing $\mathrm{Cys}^{61}$ in a reduced $(\mathbf{G})$ or oxidized state $(\mathbf{H})$. Please note that the diagrams show only the properly cleaved and fully labelled peptides of each sample. They are the most abundant peptides in each sample. All other peptides are listed in Supplementary table SR1. 
quantified is impossible to estimate. For these reasons, the entire the mass spectrometry analysis must be considered with caution. Despite these limitations, the data from the fully labelled and fully cleaved peptides suggested that strep-MBP-ROXY9 is only partially reduced, even in presence of DT'T, and oxidizes more strongly in the active site, when DTT is removed or exchanged for glutathione. However, the nature of these redox modifications and the precise proportion of oxidized protein in all samples except for that from which DTT was removed by dialysis (Figure R11, sample A) remain elusive.

Strep-MBP-ROXY9 can weakly deglutathionylate GAPDH-SG when dialyzed prior to the experiment against glutathione and when used in higher concentrations

Assuming that the increased oxidation observed for the active site in case of dialysis against GSH was caused by glutathionylation and disulphide bond formation which play a role during catalysis [74,101], strep-MBP-ROXY9 and the control proteins, strep-MBP-Grx1 and strep-MBP-GRXC2, were dialysed against glutathione before the reductase assays. To avoid an influence of the low glutathione amounts in the protein preparation after dialysis, all reactions without enzyme contained the same amount of dialysis buffer instead of glutaredoxin.

When analysing $1 \mu \mathrm{M}$ strep-MBP-ROXY 9 along with $20 \mathrm{nM}$ strep-SUMOGrx1 and $30 \mathrm{nM}$ strep-MBP-GRXC2 in the HEDS (Figure R16A and C) and in the insulin assay (Figure R16B and C), strep-SUMO-Grx1 and strep-MBPGRXC2 showed a reductase activity. This reductase activity, however, was not evident for strep-MBP-ROXY9. As before when dialysed to remove DTT, strep-MBP-ROXY9 showed apparently an inhibition of reduction in the insulin assay.

When the GAPDH assay was repeated under the new conditions (Figure R16D), again, strep-SUMO-Grx1 (70 nM) and strep-MBP-GRXC2 (100 nM) were able to reactivate glutathionylated GAPDH. Different amounts of strepMBP-ROXY9 ranging from ca. $3 \mu \mathrm{M}$ down to $300 \mathrm{nM}$ were analysed in 
A

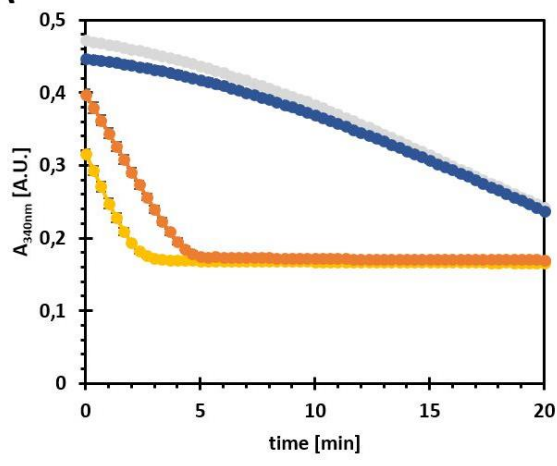

C $\square$ No enyzme
B

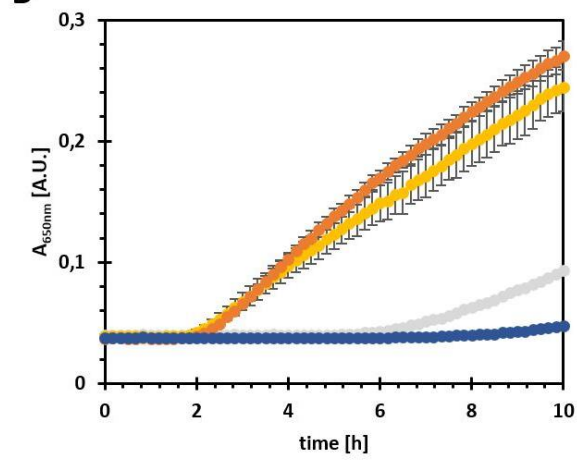

Strep-MBP-GRXC2 $30 \mathrm{nM}$

Strep-MBP-ROXY9 $1 \mu \mathrm{M}$

D

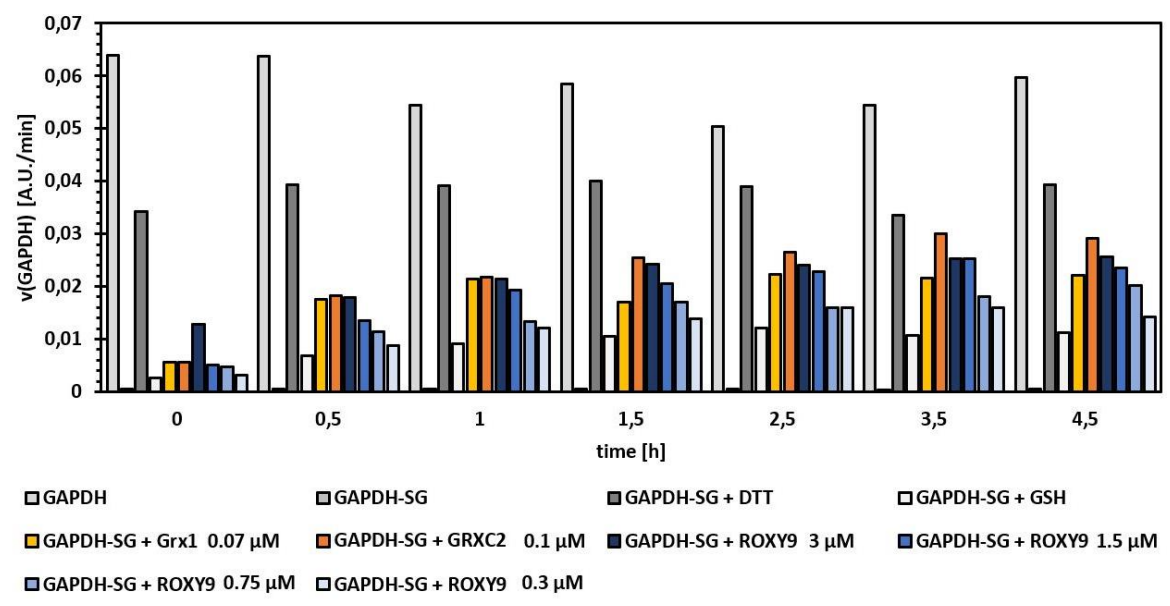

Figure R16. In vitro reductase activity of strep-MBP-ROXY9 when the protein is dialyzed against GSH. Strep-SUMO-Grx1, strep-MBP-GRXC2, and strep-MBP-ROXY9 were dialyzed against buffer containing GSH to remove DTT and concomittantly introducing GSH into the sample. Then, their activity was compared using the indicated protein concentrations $(\mathbf{C})$ in the HEDS $(\mathbf{A})$ and the insulin assay $(\mathbf{B})$. In $(\mathbf{A})$ and $(\mathbf{B})$, the absorption values represent the mean of three technical replicates $\pm \mathrm{SD}$, except for the no enzyme control for which only one reaction was prepared. The three proteins were also analyzed in the GAPDH assay (D). The concentrations used in the GAPDH assay are shown in the legend below the corresponding diagram. As described before, the reductase activty was measured by the decline in absorption at $340 \mathrm{~nm}$ for the HEDS assay and by the increase in absoprtion at $650 \mathrm{~nm}$ for the insulin assay. For the GAPDH assay, the initial velocity (v) of GAPDH in aliquots taken after the indicated timepoints was determined to assess the deglutathionylation activity. Note that dialysis buffer was added to those samples without enzyme to compensate for the glutathione amounts introduced by the dialysis buffer for all experiments shown above. All samples in the GAPDH assay were prepared only once. 
comparison. At $300 \mathrm{nM}$, the activity of ROXY9 was similar to the activity observed after removal of DT'T without introducing glutathione in the protein sample during dialysis (Figure R14E). Surprisingly, strep-MBP-ROXY9 was able to deglutathionylate GAPDH-SG at concentrations of $0.75,1.5$, and $3 \mu \mathrm{M}$. Only at 1.5 to $3 \mu \mathrm{M}$, strep-MBP-ROXY9 reached an activity comparable to the control proteins. Thus, strep-MBP-ROXY9 can deglutathionylate GAPDHSG albeit at a ca. 15 to 30-fold less efficiency in comparison to strep-SUMOGrx1 and strep-MBP-GRXC2. However, it is impossible to conclude whether this activity of strep-MBP-ROXY9 only results from the higher protein concentrations used in this experiment or whether it comes from the dialysis against glutathione or both, since the concentration of strep-MBP-ROXY9 was only titrated in this experiment (Figure R16D), but not after removal of DTT using dialysis buffer without glutathione (Figure R14E).

\section{The strep-MBP tag does not interfere with the catalytic activity of strep-MBP-ROXY9}

Aside from the redox state, the weak activity observed for strep-MBP-ROXY9 might result from inhibition of catalysis by the large strep-MBP tag. Because cloning of ROXY9 into 438C_strep introduced a TEV site between ROXY9 and the strep-MBP tag, TEV protease was expressed in E. coli and purified to homogeneity via its His tag (Figure SR6). The SDS gel in Figure R17 shows His-TEV and strep-MBP-ROXY9 alone, as well as the mixture of both proteins before overnight incubation at $4^{\circ} \mathrm{C}$. After incubation, a complete cleavage of the tag from ROXY9 was observed, as evidenced by the disappearing band of strep-MBP-ROXY9. Instead, a band of the strep-MBP tag at ca. $40 \mathrm{kDa}$ and ROXY9 at ca. $12 \mathrm{kDa}$ became visible. Despite several purification steps to remove the strep-MBP tag and His-TEV from the reaction mixture, only His-TEV could be removed successfully, whereas approximately half of the strep-MBP tag remained in the solution. Still, this protein mixture should suffice for activity measurements and was thus used in the HEDS, the insulin and in the GAPDH assay. The concentration of ROXY9 was estimated from an SDS gel by comparing the band intensities resulting from separation of a defined volume of the ROXY9 preparation to the band intensities of various defined amounts of BSA. 
Again, strep-SUMO-Grx1 and strep-MBP-GRXC2 were able to reduce HEDS and insulin, while strep-MBP-ROXY9 was not (Figure R18A, B, and C). As for strep-MBP-ROXY9, ROXY9 was not active in these assays, suggesting that the tag did not interfere with its catalytic activity. Interestingly, ROXY9 did not inhibit the reduction of insulin as did strep-MBP-ROXY9 (Figure R18B and C). When testing the glutaredoxins in the GAPDH assay, strep-SUMO-Grx1 and strep-MBP-GRXC2 deglutathionylated GAPDH-SG (Figure R18D). Strep-MBP-ROXY9 reactivated GAPDH-SG, although again with a weaker efficiency. After dialyzing both proteins against GSH, both, ROXY9 and strepMBP-ROXY9 exhibited a similar activity, showing that the tag does not inhibit ROXY9 deglutathionylation activity.

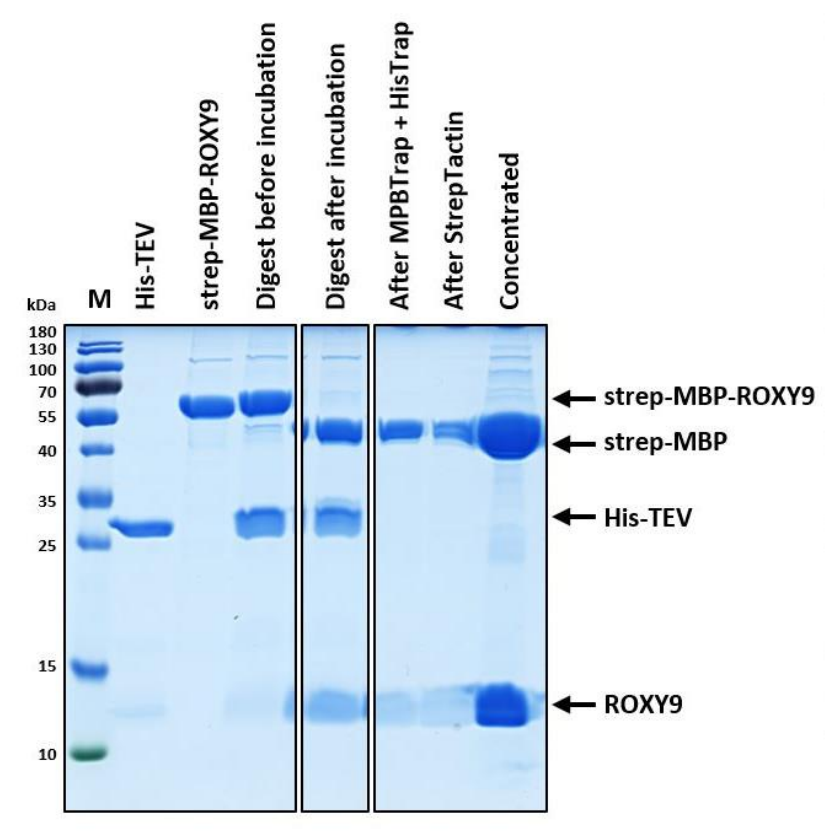

Figure R17. TEV digest of strep-MBP-ROXY9. Purified strep-MBP-ROXY9 was incubated with purified His-TEV protease over night at $4^{\circ} \mathrm{C}$. Subsequently, the strep-MBP tag, as well as uncleaved strep-MBP-ROXY9 and His-TEV were tried to be removed by several purification steps (MPBTrap + His'Trap, StrepTactin). The final mixture was concentrated. The proteins before mixing, the digestion mixture before and after incubation, and the purification steps were analyzed by running $4.4 \mu \mathrm{g}$ His-TEV, $6 \mu \mathrm{g}$ strep-MBP-ROXY9, and $20 \mu \mathrm{l}$ of the remaining fractions on a $15 \%$ SDS gel. The gel was stained with Coomassie. Prestained PageRuler (Thermo Scientific) was employed as a size standard. $M$ - Marker. 
A

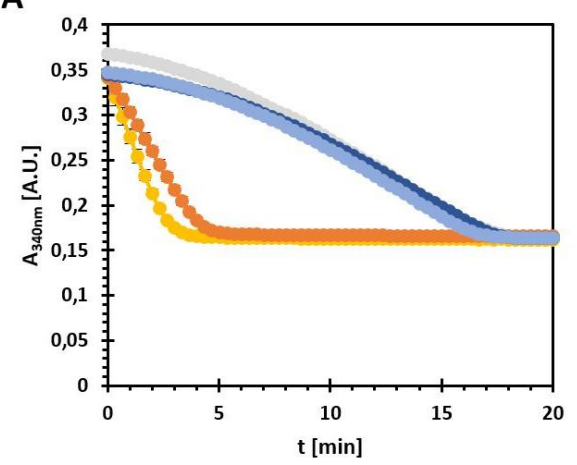

C

$\square$ No enyzme

$\square$ Strep-SUMO-Grx1 $20 \mathrm{nM}$

Strep-MBP-GRXC2 $30 \mathrm{nM}$
B

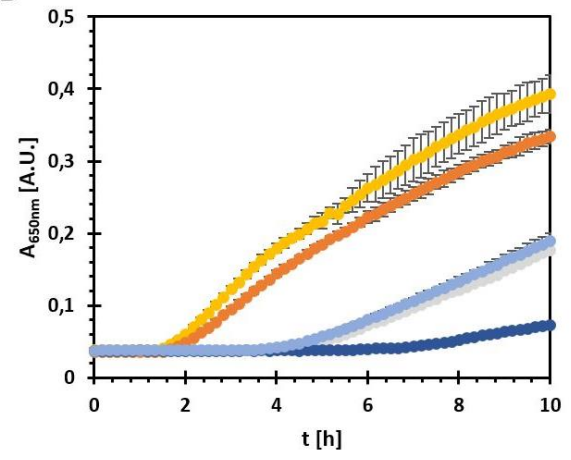

Strep-MBP-ROXY9 1 M

$\square$ ROXY9 $1 \mu \mathrm{M}$

D

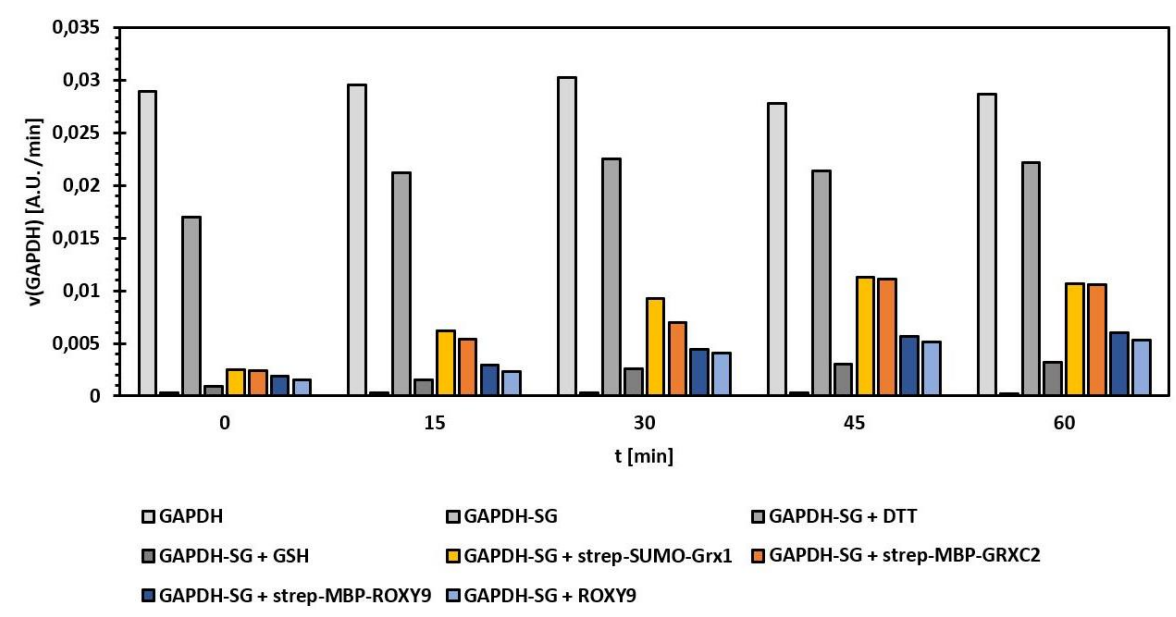

Figure R18. In vitro reductase activity of strep-MBP-ROXY9 and ROXY9. Strep-SUMOGrx1, strep-MBP-GRXC2, strep-MBP-ROXY9, and ROXY9 were dialyzed against buffer containing GSH, this way removing DTT, but including GSH. The reductase activity of all proteins was compared using the indicated protein concentrations $(\mathbf{C})$ in the HEDS (A) and the insulin assay (B). For the GAPDH assay (D), $70 \mathrm{nM}$ strep-SUMO-Grx1, $100 \mathrm{nM}$ strepMBP-GRXC2, and $400 \mathrm{nM}$ strep-MBP-ROXY9 or ROXY9 were used. For the HEDS assay, the mean absorption at $340 \mathrm{~nm} \pm \mathrm{SD}$ of technical triplicates is shown for strep-SUMO-Grx1, strep-MBP-GRXC2, and strep-MBP-ROXY9. For ROXY9, the mean of two technical replicates is plotted; for the no enzyme control, only a single reaction was prepared. For the insulin assay, the mean absorption at $650 \mathrm{~nm} \pm \mathrm{SD}$ from technical triplicates is depicted, except for the no enzyme control for which only one reaction was measured. In case of the GAPDH assay, the initial velocity of the GAPDH over $60 \mathrm{~min}$ of reactivation by deglutathionylation is plotted in a bar chart. In all experiments, dialysis buffer was added to those samples without enzyme to compensate for the glutathione amounts introduced by the dialysis buffer. All samples in the GAPDH assay were prepared only once. 


\section{Strep-MBP-ROXY9 and ROXY9 need glutathione to reactivate GAPDH-SG}

Strep-MBP-ROXY9 did only deglutathionylate GAPDH-SG but reduced neither HEDS nor roGFP ox. This might be explained by target specificity. Alternatively, ROXY9 might not be recycled by glutathione during catalysis. If this is the case, ROXY9 would only be active for one reaction cycle, and the amounts of GAPDH reduced by ROXY9 would correspond to the amounts of ROXY9 added to the reaction. To investigate whether ROXY9 is recycled by glutathione, strep-MBP-ROXY9 and ROXY9 were dialysed against glutathione and then subjected to the GAPDH assay. The activity of both proteins was addressed either with or without glutathione. To compensate for the glutathione present in the protein preparations, dialysis buffer was added in adequate amounts to all reactions without glutaredoxin.

As described before, a weak activity could be measured for both, strep-MBPROXY9 and ROXY9 (Figure R19A). Importantly, this activity depended on glutathione suggesting that ROXY9 deglutathionylates GAPDH-SG and is recycled by glutathione.

To further investigate the requirement of glutathione in the reaction mix, the redox potential of strep-MBP-ROXY9 against the DTT/dithiane redox system was determined with the help of monobromobimane (mBBr, Figure R19B). For this, strep-MBP-ROXY9 was dialyzed to remove DTT from the purification buffer. Unfortunately, the $\mathrm{E}_{\mathrm{h}}$ range chosen for this first experiment did not extend far enough into the low voltages. Thus, the saturation of the fluorescence observed in this region for other glutaredoxins [77] was not identified in this experiment. Considering that analysis of such a dataset would lead to an overestimation of the redox potential, the data was still evaluated.

The curve showed an inflection point at roughly $-0.27 \mathrm{~V}$. Thus, the redox potential of strep-MBP-ROXY9 was -0.27 V. As listed in Figure R19C, this value is in the range of the redox potentials measured for catalytically active CPYC glutaredoxins which are recycled by glutathione [77]. Thioredoxins which are not recycled by glutathione, exhibit lower redox potentials [76]. Taking the overestimation of the redox potential of strep-MBP-ROXY9 into account, its true redox potential might be closer to that of thioredoxins. However, since this type of analysis determines the average redox potential of all five cysteine residues in strep-MBP-ROXY9, it might be that the five cysteines in ROXY9 show different redox potentials. Consistent with this, MS 

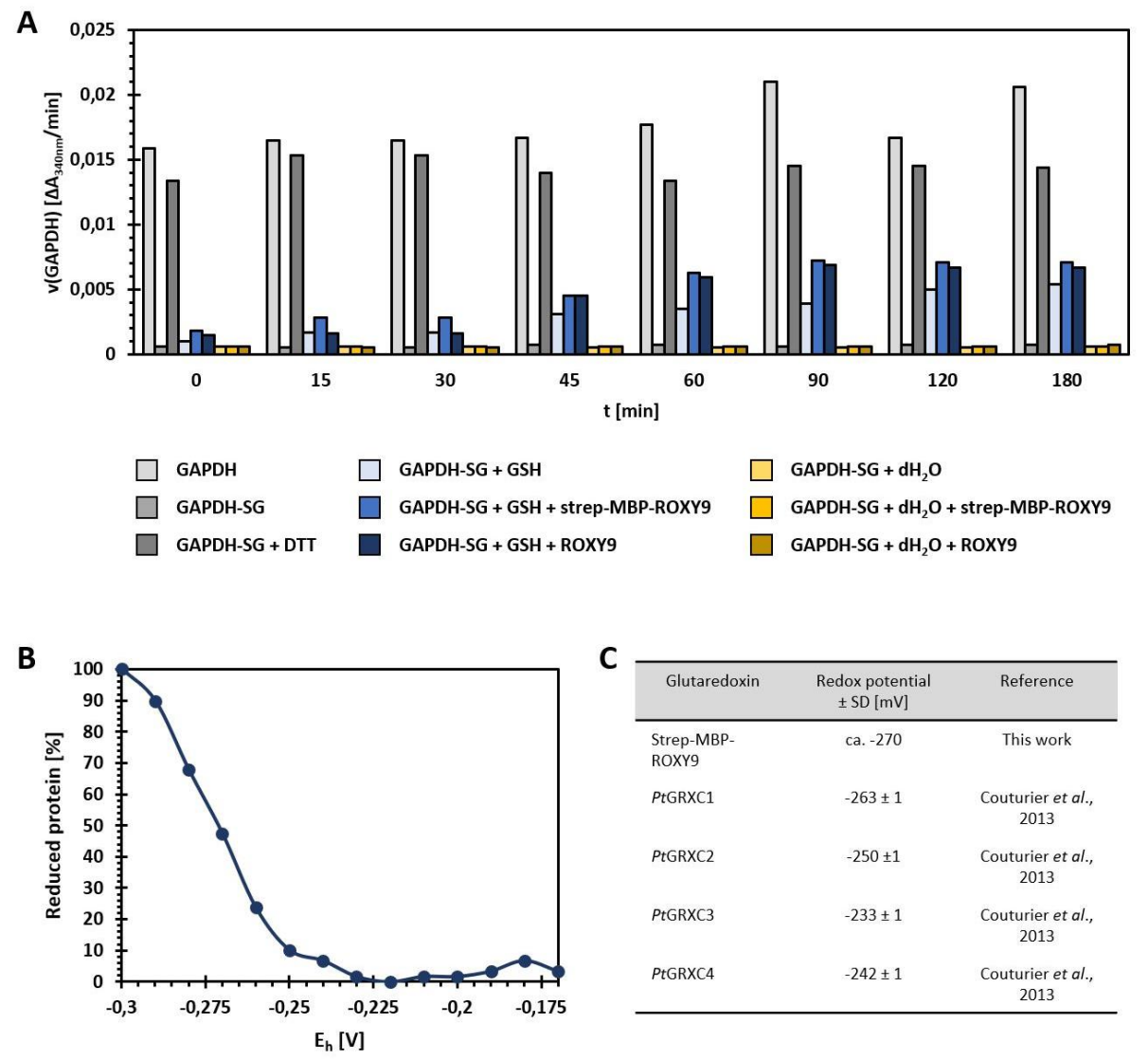

Figure R19. Strep-MBP-ROXY9 and ROXY9 require GSH to reduce GAPDH-SG. (A) Strep-MBP-ROXY9 and ROXY9 were dialyzed against buffer containing glutathione to remove DTT from the purification but to simultaneously include GSH to the protein sample. These protein preparations were tested for their activity in the GAPDH assay, either with or without glutathione as a cofactor. Approximately $400 \mathrm{nM}$ strep-MBP-ROXY9 or ROXY9 were used. Importantly, dialysis buffer was added to all samples without enzyme to compensate for the glutathione amounts introduced by this buffer. The initial GAPDH activity was plotted over the different time points, at which samples from the deglutathionylation reaction were taken. All samples in the GAPDH assay were prepared only once. (B) Strep-MBP-ROXY9 was purified in the presence of DTT. Prior to the redox titration experiment, DTT was removed by dialysis. Then, $7.5 \mu \mathrm{M}$ strep-MBP-ROXY9 were incubated in the presence of different ambient redox potentials $\left(\mathrm{E}_{\mathrm{h}}\right)$ set by mixing defined ratios of DTT and dithiane in $100 \mathrm{mM}$ HEPES pH 7.0. Finally, the percentage of reduced protein was determined using monobromobimane and plotted against the ambient redox potential to estimate the redox potential of strep-MBP-ROXY9. All values represent single measurements. The table in (C) summarizes the redox potentials of different glutaredoxins studied elsewhere and compares them to strep-MBP-ROXY9. 
data showed that even in the presence of $1 \mathrm{mM}$ DT'T, strep-MBP-ROXY9 was not fully reduced (Figure R15), suggesting that some residues might have rather low redox potentials. Consequently, other ROXY9 cysteine residues must have higher redox potentials and could thus be reduced by glutathione.

\section{Strep-MBP-ROXY9 oxidizes reduced roGFP}

Though strep-MBP-ROXY9 acted as a reductase towards glutathionylated GAPDH with glutathione as a reducing agent, its activity was much weaker than for control CPYC-type glutaredoxins. According to findings by Moseler et al. (2015) and Begas et al. (2017), the CGFS-type glutaredoxin GRXS15 does not catalyze reduction reactions but rather the reverse reaction, acting as an oxidase towards reduced roGFP. For this, it requires GSSG as a cosubstrate $[75,97,116,117,259,260]$. Similarly, the CPYC-type glutaredoxin GRXC1 acts as an oxidase for roGFP red in the presence of GSSG [75,97].

For this reason, the oxidase activity of strep-MBP-ROXY9 was tested in the roGFP assay using GRXC1 as a positive control. The assay was set up as described before for the reduction of oxidized roGFP, but reduced roGFP was replaced by oxidized roGFP and GSH was replaced by GSSG. Strep-MBPROXY9 was dialyzed against the assay buffer to remove DTT and received a DTT treatment right before the experiment. Thus, it contained two reduced cysteines per protein (Figure R11, sample B). GRXC1 was treated in the same way. Again, oxidized roGFP showed a weak fluorescence ratio, whereas reduced roGFP exhibited a high fluorescence ratio (Figure SR7). The slope of the oxidation of roGFP red caused by GSSG and/or the glutaredoxins is shown

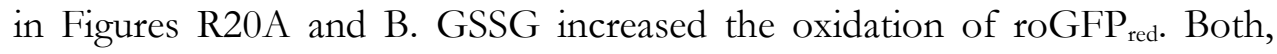
GRXC1 and strep-MBP-ROXY9 doubled the oxidation speed, but only in the presence of GSSG. Thus, strep-MBP-ROXY9 acted as an oxidase towards roGFP red using GSSG. Consequently, GSSG might transfer a glutathione to strep-MBP-ROXY9, which subsequently transfers this glutathione moiety on roGFP red. 
A

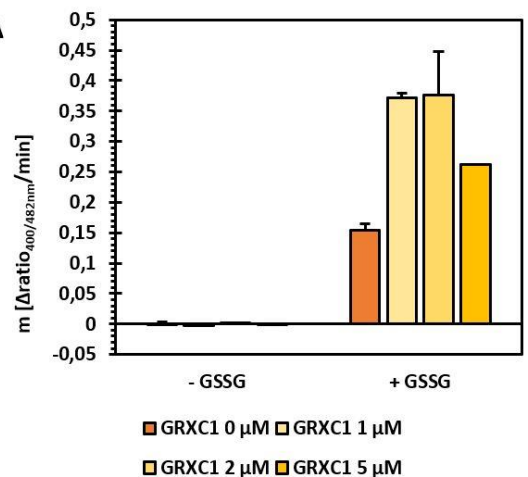

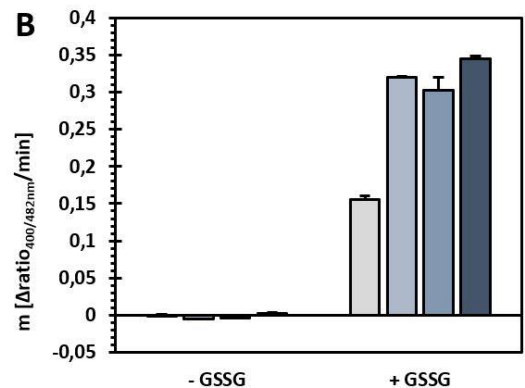

口 strep-MBP-ROXY9 $0 \mu \mathrm{M} \square$ strep-MBP-ROXY9 $1 \mu \mathrm{M}$

口strep-MBP-ROXY9 $2 \mu \mathrm{M} \square$ strep-MBP-ROXY9 $5 \mu \mathrm{M}$

Figure R20. Strep-MBP-ROXY9 oxidizes reduced roGFP2. Strep-MBP-ROXY9 (B) was dialyzed against buffer without DTT and tested for its ability to oxidize reduced roGFP2 in comparison with N-terminally His-tagged GRXC1. Both proteins received a DTT-treatment and were desalted immediately before the experiment via a column. (A). Different protein concentrations from 0 to $5 \mu \mathrm{M}$ were used. After $10 \mathrm{~min}$ of preincubation, GSSG was added to start the reactions, while buffer was added to the control samples without cofactor. The initial velocity was determined by measuring the change in the fluorescence ratio of roGFP2 between minutes 13 and 15 of the reaction and plotted in a bar chart. Values represent mean \pm SD. For $1 \mu \mathrm{M}$ GRXC1 - GSSG and $5 \mu \mathrm{M}$ GRXC1 + GSSG only two replicates were prepared. Thus, no SD could be calculated.

\section{Strep-MBP-ROXY9 binds glutathione probably via altered glutathione interaction sites}

A recent publication [75] suggested that the glutathione binding site of glutaredoxins consists of two functionally distinct regions: (1) an activation site stabilizing the thiolate of bound glutathione and of the $\mathrm{N}$-terminal cysteine of the active site and (2) a scaffold site interacting with the glutathione moiety from the substrate to be deglutathionylated. Differences in the amino acid composition of these two sites were proposed to account for the diverging activities observed for CPYC and CGFS glutaredoxins: Whereas in CPYC glutaredoxins both sites are functional, CGFS glutaredoxins have a nonfunctional activation site. Since strep-MBP-ROXY9 showed an activity pattern resembling that of CGFS-type glutaredoxins, its interaction with glutathione was examined. 
First, the dissociation constant of strep-MBP-ROXY9 and glutathione was quantified by Dr. Sven-Andreas Freibert (Prof. Dr. Roland Lill, PhillipsUniversität Marburg) using microscale thermophoresis (MST) and BioLayer Interferometry (Figure R21A and B). For this, DTT from the purification process of strep-MBP-ROXY9 was removed via a desalting column. Thus, the experiments were conducted with oxidized protein. Although both measurements represent initial experiments, they suggested that the $K_{d}$ of strep-MBP-ROXY9 for glutathione is around $146 \mu \mathrm{M}$ (Figure R21C). This value is ca. 10-fold higher than measured for the CPYC glutaredoxin strepMBP-GRXC2 $(12.6 \pm 3.6 \mu \mathrm{M})$ and about 30-fold higher than determined for the CC-type glutaredoxin strep-MBP-ROXY19 (4.6 $\pm 1 \mu \mathrm{M})$ [174]. Both values were obtained by MST. Wang et al. (2014) calculated the $\mathrm{K}_{\mathrm{d}}$ value for the plastidial CGFS glutaredoxin GRXS14 based on NMR data, yielding a value of ca. $5 \mathrm{mM}$ [95]. Thus, the $\mathrm{K}_{\mathrm{d}}$ value of strep-MBP-ROXY9 is in between that of a catalytically active CPYC glutaredoxin and a catalytically largely inactive CGFS glutaredoxin [88,174,259].

The higher dissociation constant of strep-MBP-ROXY9 compared to strepMBP-ROXY19 and strep-MBP-GRXC2 might be caused by the partial conservation of the glutathione binding site in CC-type glutaredoxins [80]. The analysis of the conservation of glutathione binding residues in CC-type glutaredoxins carried out by Gutsche et al. (2015) focussed on five sites in glutaredoxins required for glutathione binding [80]. At that time, it was still unknown that the glutathione binding site is divided into a glutathione activation site and a glutathione scaffold site (Figure I4C). However, Begas et al. (2017) did not include CC-type glutaredoxins when analysing the activation and scaffold site [75].

To examine the extent of conservation of the glutathione binding site ROXY9 identified by Gutsche et al. (2015) in the light of the study by Begas et al. (2017), the protein sequence of ROXY9 was aligned with all glutaredoxins used in this work and known to catalyse reductase reactions or for which the $K_{d}$ was determined. These glutaredoxins were Arabidopsis GrxC1, GrxC2 (GRX370), GRXS14, ROXY19, and human Grx1. In addition, S. cerevisiae Grx7 and Plasmodium falciparum (P. falciparum) Grx1 were included in the alignment, as these two glutaredoxins were dissected regarding their interaction with glutathione by Begas et al. (2017). Since they also analysed AtGRXS15, its sequence was added to the alignment [75]. Finally, S. cerevisiae Grx6 was chosen, since its crystal structure was solved [108] and compared to $S_{c}$ Grx7 by Begas et al. (2017) (Figure I4D) [75]. 


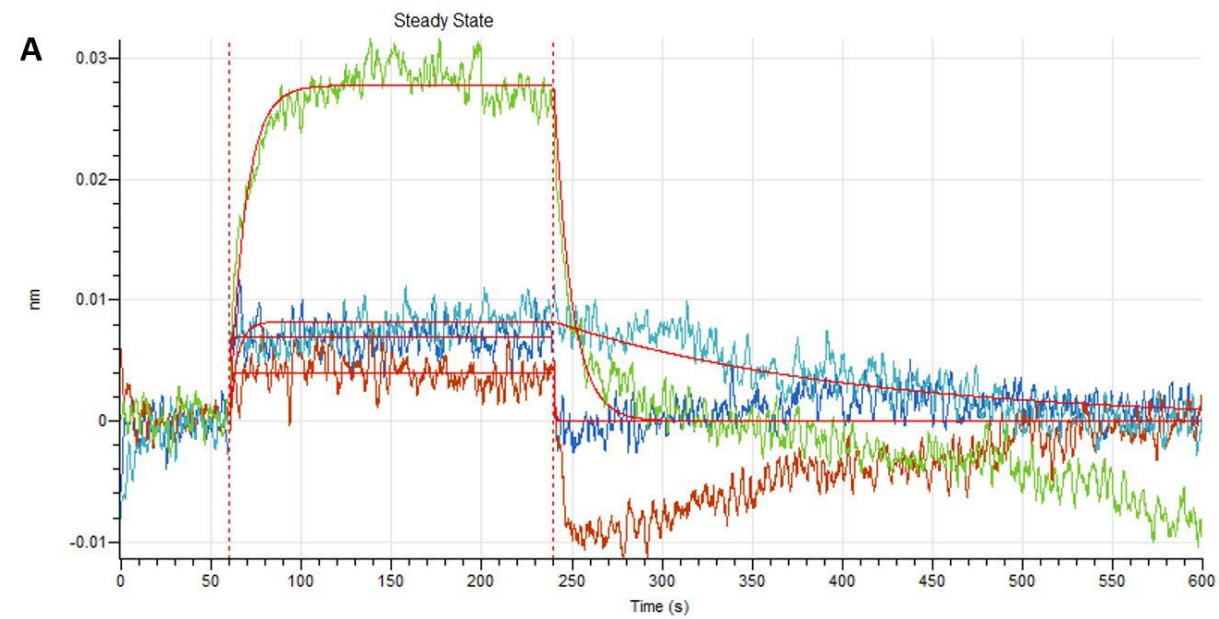

B

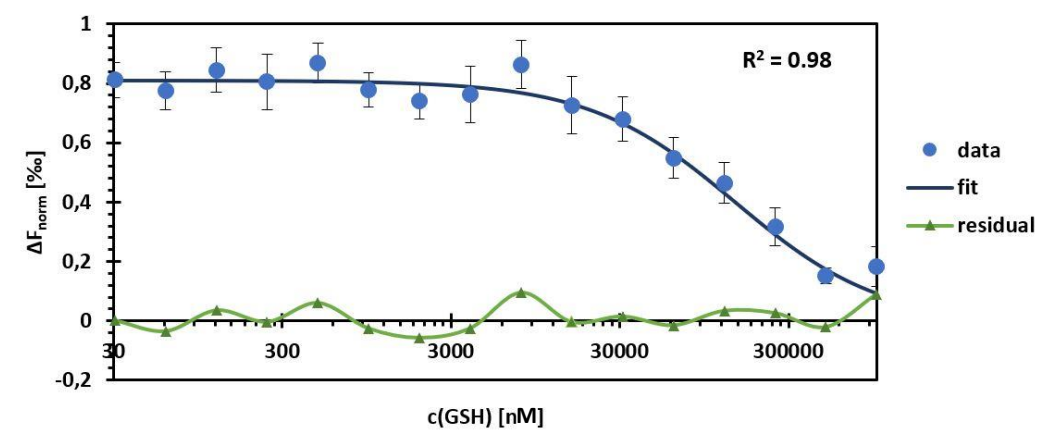

C

\begin{tabular}{lcccc}
\hline Protein (Expression host) & Glutaredoxin class & Method & $\mathrm{K}_{\mathrm{d}} \pm$ SD [uM] & Reference \\
\hline Strep-MBP-ROXY9 (insect cells) & CC-type & MST & $146.08 \pm 36.57$ & This work \\
& & BLI & $147.6 \pm 62.18$ & This work \\
Strep-MBP-ROXY19 (E. coli) & CC-type & MST & $4.6 \pm 1.0$ & Oberdiek (2018) \\
Strep-MBP-GRXC2 (E. coli) & CPYC-type & MST & $12.6 \pm 3.6$ & Oberdiek (2018) \\
GRXS14 & CGFS-type & NMR & 5000 & Wang et al. (2014) \\
\hline
\end{tabular}

Figure R21. strep-MBP-ROXY9 binds glutathione. Strep-MBP-ROXY9 purified in the presence of DTT was dialyzed to remove EDTA from the purification buffer and then purified via desalting columns to remove DTT. It was subsequently tested by BioLayer Interferometry (BLI, A) and microscale thermophoresis (MST, B) for its binding to glutathione. (A) shows the raw data from a single BioLayer Interferometry measurement. The dark red, green, light and dark blue curves correspond to the recorded interference shifts in $\mathrm{nm}$ for different GSH concentrations, wherease the light red lines show their fit. The diagram in (B) displays the mean of the normalized fluorescence of $200 \mathrm{nM}$ NHS-labeled ROXY9 \pm SD (dark blue points) of three technical replicates and the corresponding fit (light blue curve) including its residual plot (green). From both datasets, $\mathrm{K}_{\mathrm{d}}$ for strep-MBP-ROXY9 and GSH was computed and compared to published values for other glutaredoxins $(\mathbf{C})$. 
The alignment is shown in Figure R22. Only those residues for which a function in glutathione binding has been suggested based on experimental data $[75,79,80,92,94]$ are highlighted (Figures I3A and B, I6A, B, and C, SI1C). The activation site for binding of the recycling glutathione and the scaffold site for binding of the glutathionylated substrate are indicated in red and blue, respectively (for comparison see Figure I4C and D).

In comparison to CPYC- and CGFS-type glutaredoxins, a major difference of CC-type glutaredoxins found within the active site [80]. With respect to the active site, the tyrosine from the CPYC motif (A2) has been suggested as an activation site residue, though based on initial data. In CGFS-type glutaredoxins, this residue is replaced by a phenylalanine [75] and a leucine in ROXY9 or methionine in ROXY19, respectively [80]. Tyrosine harbors a hydroxyl group and can therefore form hydrogen bonds. Both, phenylalanine and tyrosine harbor a benzol ring allowing $\pi$-stacking interactions. In contrast, the side chains of leucine and methionine are stretched and can only form Vander-Waals interactions. Thus, the residue in CC-type glutaredoxins deviates regarding its properties compared to CPYC- and CGFS-type glutaredoxins. The lysine residue (A1) characterized with respect to its function in the activation site [75] is conserved in ROXY9 and ROXY19.

The major differences of ROXY9 and ROXY19 and glutaredoxins from the CPYC and CGFS class are restricted to the scaffold site, in particular the TVP (S3 to S5) and the GG motif (S6 and S7). In ROXY9, the TVP motif is replaced by ${ }^{64} \mathrm{AVP}^{66}$ and the GG motif by ${ }^{76} \mathrm{GS}^{77}$, whereas a KLP motif and the usual GG motif are found in ROXY19 [80]. The K of the KLP motif and the methionine found in the active site of ROXY19 appear to block the glutathione binding groove in the homology model of this protein (Figure I3F to $\mathrm{H}$ ) [80]; however, the smaller alanine found in ROXY9 at this site might narrow the groove less than the lysine in ROXY19. The negatively charged residue at the second or third position after the GG motif ( $\mathrm{S} 9$ or S10) was characterized regarding its function in the scaffold site [75]. Because at position S9, a negative residue is found in ROXY19 [80] and at positions S10 in ROXY9, this site seems to be conserved in both CC-type glutaredoxins. Residues S2 and S8 are difficult to evaluate with respect to conservation because they are variable even in CPYC-glutaredoxins.

Similarly, residues S1 and A3 cannot be evaluated regarding their conservation. The present alignment suggests that residue A3 is positively charged in CGFSand CC-type glutaredoxins but shows variable properties in CPYC-type 


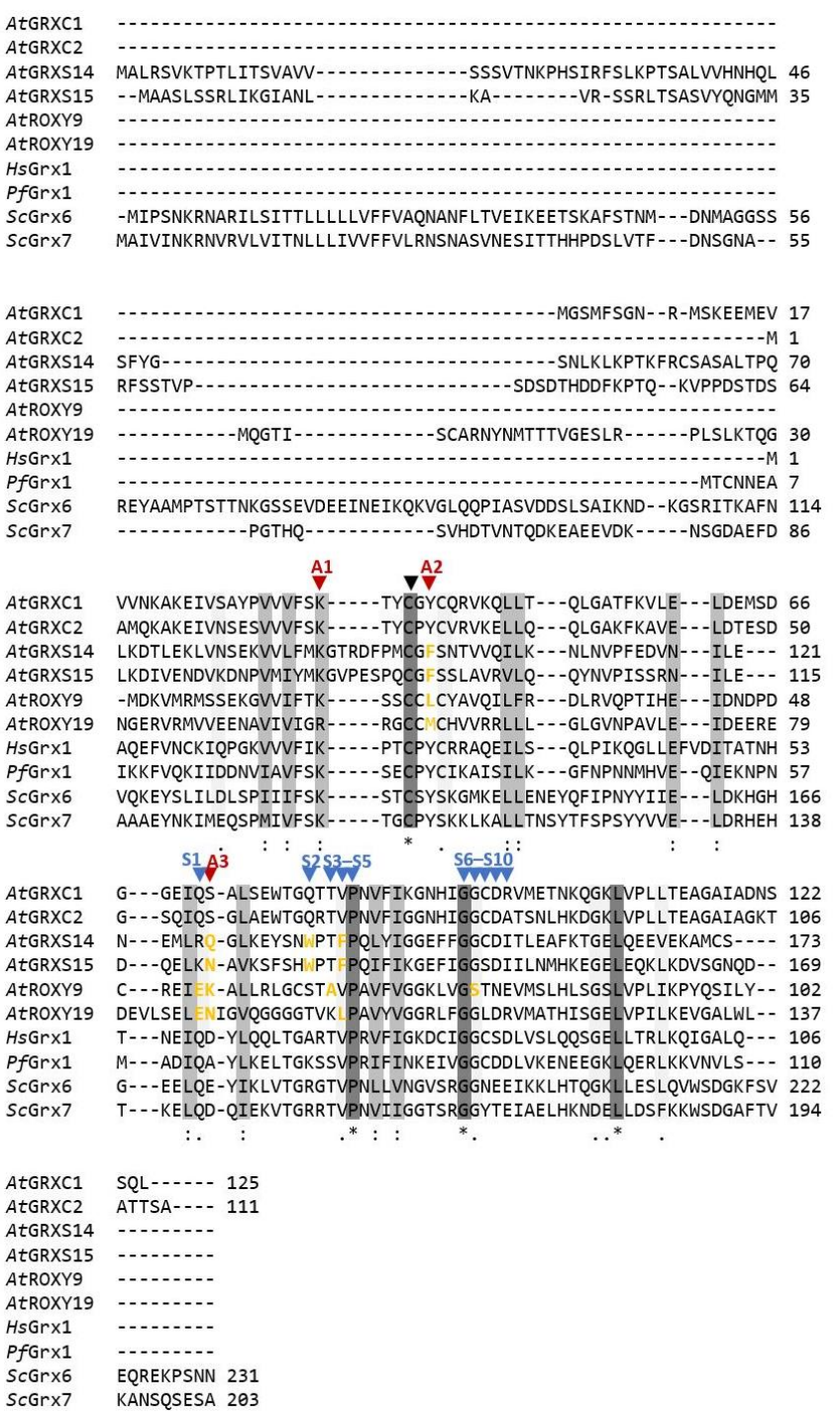

Figure R22. Potential glutathione-binding residues are substituted in ROXY9. The protein sequences of CC-type glutaredoxins ROXY9 and ROXY19, the CPYC-type glutaredoxins GRXC1, GRXC2, human Grx1 and the CGFS-type glutaredoxins GRXS14 and GRXS15, as well as Grx6 and Grx7 from baker's yeast and Grx1 from P. falciparum were aligned using CLUSTAL O (1.2.4) via the UniProt database (A). The degree of conservation of the different amino acids is highlighted by different gray values in the following order: dark grey $(*$, conserved) $>$ grey $(:)>$ light grey $()>$. white (not conserved). The glutathione-binding residues are marked by arrows: Red arrows point to those residues proposed to participate in the activation site (A1 to A3). Those residues belonging to the putative scaffold site are indicated by blue arrows (S1 to S10). A black arrow shows the active site cysteine. Residues which deviate from the residues observed for catalytically active glutaredoxins are shown in yellow. The figure was prepared according to Gutsche et al. (2015) [80] and Begas et al. (2015) [75]. 
glutaredoxins. Residue S1 is negatively charged in CC-type glutaredoxins and positively charged in all other glutaredoxins. However, the gaps found in front of this region might allow a different way of aligning these residues depending on the algorithms and/or the protein sequences used. Gutsche et al. (2015) and Begas et al. (2017) presented alignments deviating from the one shown here $[75,80]$. Thus, it is unclear whether S1 and A3 are conserved in CC-type glutaredoxins.

All in all, major differences in the glutathione binding site are restricted to the active site, which is part of the activation site, and the TVP and GG motif, forming in parts the scaffold site.

\section{Strep-MBP-ROXY9 could bind a [4Fe-4S] cluster}

Strep-MBP-ROXY9 was only weakly active as a reductase but catalyzed the oxidation of reduced roGFP efficiently. This observation is reminiscent of the CGFS-type glutaredoxin GRXS15. CGFS-type glutaredoxins are known to bind iron-sulfur clusters $[95,97,114,116,129,139]$. To test, whether strep-MBPROXY9 might bind an iron-sulfur cluster, as well, the protein was reconstituted non-enzymatically by Dr. Sven-Andreas Freibert and analyzed for iron-sulfur cluster binding. For this, strep-MBP-ROXY9 was dialyzed to remove EDTA from the purification buffer. During reconstitution, the protein was incubated in the presence of iron ions derived from ammonium iron (III) citrate, $\mathrm{FeCl}_{2}$, or $\mathrm{FeCl}_{3}$, lithium sulphide, glutathione and DTT. After incubation, the protein was purified from the reaction mix via a desalting column. Reconstitution took place under anaerobic conditions [261].

The reconstituted protein had a brownish colour and lost this colour quickly when exposed to air (S. Freibert, personal communication). Ultraviolet/Visible (UV/Vis)-spectral measurements (Figure R23A and B) showed a large peak at $280 \mathrm{~nm}$ caused by strep-MBP-ROXY9, which has an extinction coefficient of ca. $80000 \mathrm{M}^{-1} \mathrm{~cm}^{-1}$. Around 320, 420 and $590 \mathrm{~nm}$ shoulders indicative of ironsulfur cluster binding could be observed. The absorption at $420 \mathrm{~nm}$ was ca. 0.14 A.U. (Figure R23B) while it was 1.8 A.U. at $280 \mathrm{~nm}$ (Figure R23A), and therefore, 13-fold higher. Considering a single cluster bound per protein, a 
cluster with an extinction coefficient around $6000 \mathrm{M}^{-1} \mathrm{~cm}^{-1}$ at $420 \mathrm{~nm}$ would fit to this absorption ratio. Alternatively, an extinction coefficient of ca. $12000 \mathrm{M}^{-1} \mathrm{~cm}^{-1}$ would argue for one cluster bound by two proteins. These values are within the range observed usually for [4Fe-4S] clusters (3000 to $15000 \mathrm{M}^{-1} \mathrm{~cm}^{-1}$ [261]).

To determine the stoichiometry of the occupancy, the amount of iron ions and the number of sulphide ions bound per molecule of strep-MBP-ROXY9 was quantified using a feren-based method [245] or the methylene-blue method [246], respectively (Figure R23C). Approximately 5 iron ions and 3.4 sulphide ions could be determined per protein. Considering some non-specifically bound iron and slightly lower cysteine concentrations in the methylene blue standard, these data indicate that strep-MBP-ROXY9 might associate with a $[4 \mathrm{Fe}-4 \mathrm{~S}]$ cluster. However, the unclear oxidation state of the protein sample even in presence of reducing agents questions this calculation unless the number of oxidized proteins is much smaller than that of reduced proteins.
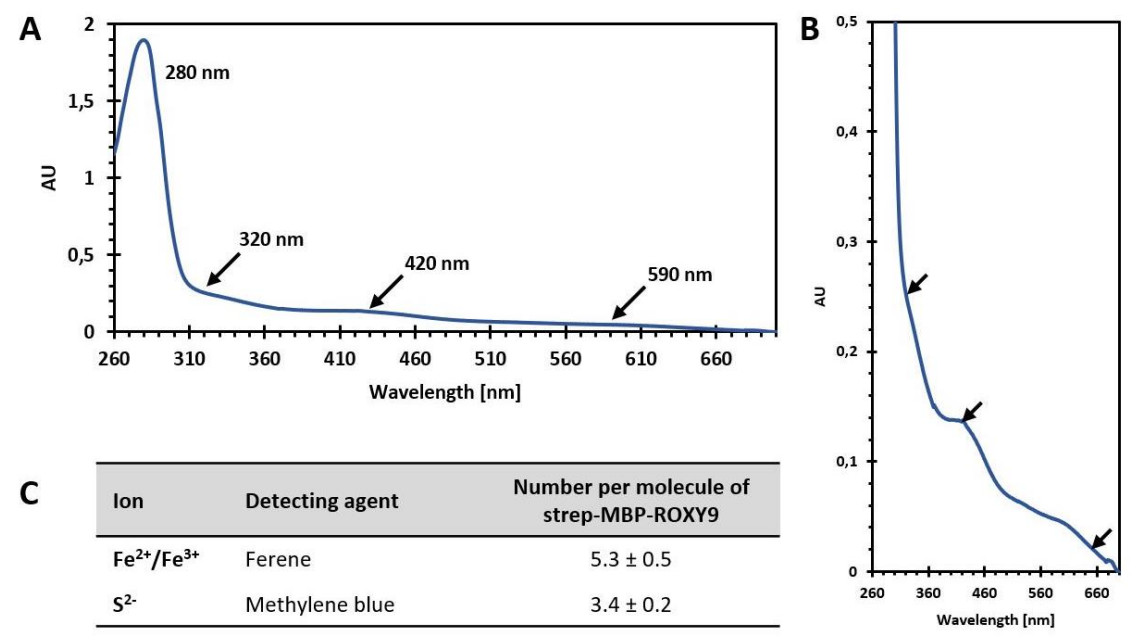

Figure R23. Strep-MBP-ROXY9 probably binds an iron-sulfur cluster after reconstitution. Strep-MBP-ROXY9 was dialyzed to remove EDTA from the purification buffer and then reconstituted non-enzymatically and anaerobically in the presence of glutathione. To test iron-sulfur cluster incorporation, a UV/Vis spectrum of the reconstituted protein was recorded (A). (B) shows an enlargement of this spectrum. Shoulders around $320 \mathrm{~nm}, 420 \mathrm{~nm}$, and $590 \mathrm{~nm}$ are indicated by arrows. In addition, the amount of iron ions and sulfide ions bound to reconstituted strep-MBP-ROXY9 was quantified (C) 


\section{Towards a mechanism of regulation of TGA1 by ROXY9}

\section{Complementation of the tga1 tga4 mutant with genomic TGA1 (TGA1g) constructs}

ROXY9 was shown to repress the activity of TGA1 regarding hyponastic growth $[155,156]$. A repressive function has also been published for several other CC-type glutaredoxins [145-147,151,162,164,168,169]. Redox modification of TGA transcription factors by CC-type glutaredoxins to change TGA factor activity has been frequently discussed [145149,151,153,155,163,164,166,168-171]. Especially, TGA1 has been reported as a redox-regulated transcription factor [32,224].

During in vitro characterization of strep-MBP-ROXY9, only a weak deglutathionylation activity of ROXY9 towards GAPDH-SG was observed but a stronger oxidase activity. To test whether ROXY9 can redox-modify TGA1 during hyponastic growth [155], the requirement of the cysteine residues of TGA1 for the regulation of hyponastic growth was addressed.

For this, complementation of the tgal tga4 mutant with three different constructs was tested. The complementation constructs are shown in Figure R24A. Since the cDNA sequence of TGA1 under control of the $35 \mathrm{~S}$ promoter does not complement $[32,157]$, the genomic coding sequence of TGA1 and approximately $2000 \mathrm{bp}$ upstream of the 5'-untranslated region (5'UTR) as well as ca. $300 \mathrm{bp}$ downstream of the TGA1 stop codon were cloned into the pB vector, as described previously [223,262]. In the course of cloning, a 1xHA tag was introduced right before the start codon of TGA1 leading to an $\mathrm{N}$ terminal tag. This construct represented the wildtype complementation construct [223,262]. To test the requirement of the cysteine residues of TGA1, a cysteine mutant complementation construct was generated. For this, all four cysteine codons in the genomic coding sequence of TGA1 were mutated. In analogy to Despres et al., 2003, the cysteines were mutated such that they 


\section{A}
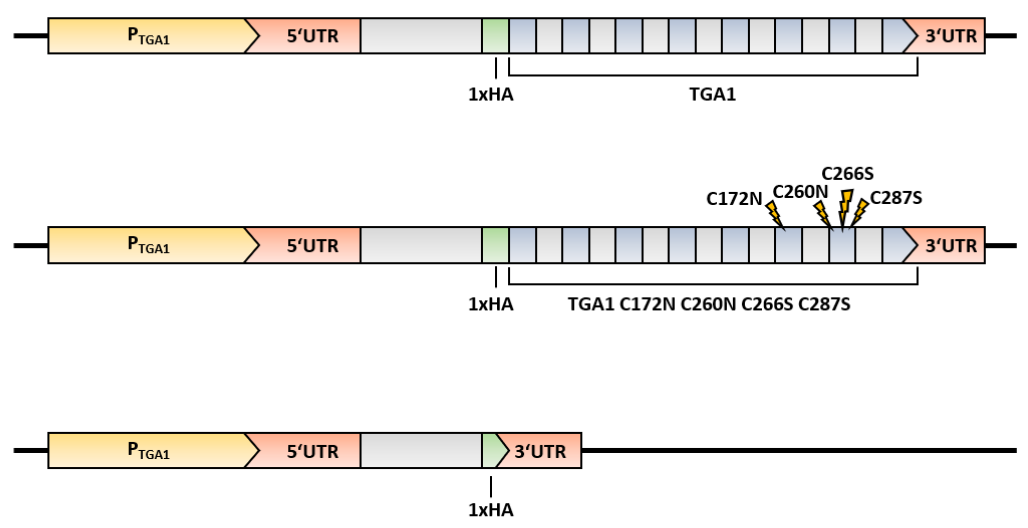

B
$\begin{array}{lccccccccr}163 & 172 & 178 & 252 & 260 & 266 & 275 & 282 & 287 & 296 \\ \text { I } & \text { I } & \text { I } & \text { I } & \text { I } & \text { I } & \text { I } & \text { I } & \text { I } & \text { । }\end{array}$
TGA1 ... WVEEQNRQICELRTVL ... TDQQLLDVCNLKQSCQQAEDALTQ ... HTLADCVAAG--QL-...---GEG ..
TGA2 ... WLEEKNKQMNELRSAL ... TERQLMGINNLQQTSQQAEDALSQ ... QSLADTLSSGTLGSSSS-.--GNVA ...
TGA3 ... WLEEQNRRVSEIRTAL ... TDQQLLEVRNLQQSSQQAEEALSQ ... QGLVESIAIQIKVV-.....--ESV ...
TGA4 ... WVEEQNRQICELRTVL ... TDQQLLDVCNLRQSCQQAEDALSQ ... HTLAESVAAG--KL-....--GEG ...
TGA5 ... WQEDKNRQMKELSSAI ... TEQQSLDINNLQQSSQQAEDALSQ ... QSLADTLSSGTLGSSSS---GNVA ...
TGA6 ... WLEEKNRQMNELRSAL ... TERQVMGINSLQQTSQQAEDALSQ ... QSLADTLSSGTLGSSSS-.--DNVA ...
TGA7 ... WLQEQSRRVSELRTAL .... TDQQILEVRNLQQSSQQAEDALSQ ... QSLAESIVIDA-VI--...-- EST ...
PAN ... WKEEHQRMINDLRSGV ... TDQQLIGICNLQQSSQQAEDALSQ ... QSLLETLSSASMGPNSS-.--ANVA ...
TGA9 ... WLEDDNRHMSEIRTGL ... TEQQLMGIYSLQHSSQQAEEALSQ ... QSLIDTLAAS-..........PVI ...
TGA10 ... WLEEQQRLLNELRVAT ... TEQQIVGICGLQQSTQEAEEALSQ ... QSLSDSIVSDSLPPASAPLPPHLS ...

Figure R24. Complementation of the tga1 tga4 mutant with genomic TGA1. (A) Complementation constructs used for stable transformation of the $A$. thaliana tga1 tga4 mutant. Introns are shown in grey; exons are depicted in blue. Mutations are shown as yellow lightnings. (B) Clustal O (1.2.4)-based alignment of the stretch in the TGA1 protein sequence containing the cysteine residues (yellow) with the corresponding sequences of TGA2 to TGA10. The alignment was done via UniProt. 
mimicked the reduced state of the protein but allowed integrity of the protein fold. Based on the amino acids found in other TGA factors from Arabidopsis at the sites of the cysteines in TGA1 (Figure R24B), the first two cysteine codons (C172N and $\mathrm{C} 260 \mathrm{~N})$ were converted into asparagine codons, whereas the second two cysteine codons were exchanged for serine codons (C266S and C287S) [224]. Again, this mutant construct was fused N-terminally to a single HA-tag. As a control construct, the single HA-tag was placed between the genomic TGA1 control regions. Unfortunately, later, a mutation of A192 in the 3'-untranslated region (3'UTR) in the control construct was found to be mutated to a guanine. The 3'UTR of the other two complementation constructs was intact (Figure SR8).

The three complementation constructs were subsequently transformed into the tga1 tga4 mutant via the flower dip method and homozygous transformants were isolated. Three lines for each construct were selected for complementation analysis. The selected lines were:

tga1 tga4 + pB1xHA-TGA1g \#5-6, \#15-3, \#21-9

tga1 tga4 + pB1xHA-TGA1g C172N C260N C266S C287S \#5-3, \#15-15, \#222

tga1 tga4 + pB1xHA \#10-3, \#11-2, \#21-9

As an internal control, line tga1 tga4 $+p B 1 \times H A-T G A 1 g$ \#22-7 was used. During segregation, it had lost its transgene and, thus, corresponded to a tga 1 tga 4 mutant. Further controls were Col-0 and the original tga 1 tga4 mutant.

\section{TGA1g complements the tga1 tga4 mutant when all four cysteines are mutated}

The above-mentioned complementation lines were tested sequentially in three independent hyponastic growth experiments (Figure R25 and SR9, part I to III). While wildtype plants initiated hyponastic growth under low light conditions, and lowered their leaves when shifted back to control light conditions, tga1 tga4 mutants were compromised in this response. Similar effects were observed for the control complementation lines tga 1 tga $4+$ 
A

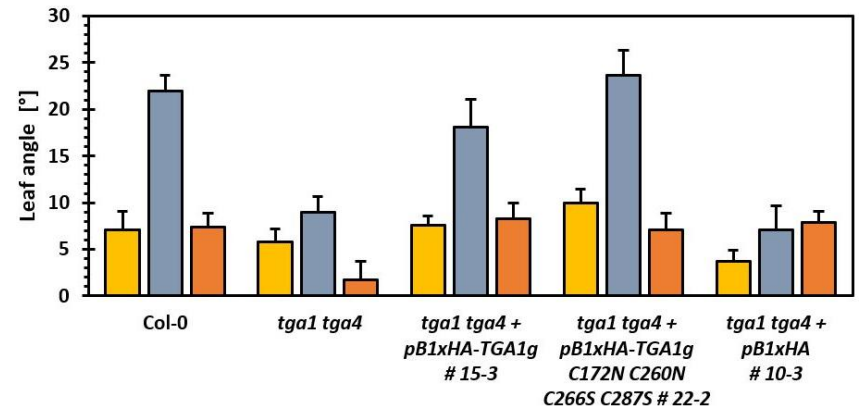

B

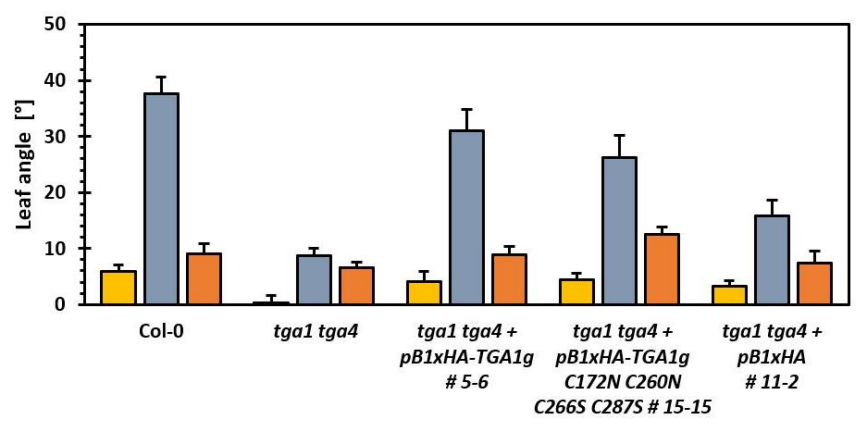

C

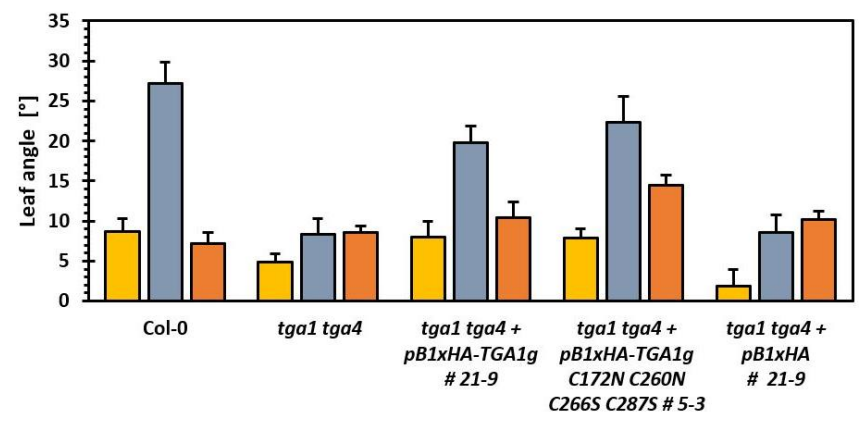

$\mathrm{cl}$

$\square$ แ

$\square \mathrm{Uto} \mathrm{CL}$

Figure R25. Analysis of hyponastic growth of the TGA1 complementation lines. Selected complementation lines of the tga 1 tga 4 mutant were grown together with the tga 1 tga4 mutant and Col-O wildtype plants for 4 weeks in $12 \mathrm{~h}$ day and then treated with CL, LL or first with LL followed by CL, as described in Methods (page 59). Pictures of the plants were taken with a Canon PowerShot G11 camera. Angles of leaf 7 or 8 were measured using ImageJ. Bars are mean \pm SEM from at least 6 replicates. (A), (B), and (C) represent three independet experiments. $C L$ - control light, $L L-$ low light, $L L$ to $C L-$ shift from low light to control light. tga1 tga4 corresponds to tga1 tga4 $+p B 1 \times H A-T G A 1 g$ \#22-7, which lost its transgene during segregation as a beterozygous plant. 
pB1xHA \#10-3, \#11-2, and \#21-9. In contrast, tga1 tga4 mutants complemented with $1 \times H A-T G A 1 g$ or $1 \times H A-T G A 1 g$ C172N C260N C266S $C 2875$ showed a hyponastic response similar to the wildtype. Complementation seemed sometimes partial (Figure R25B and C), which could be attributed to a reduced functionality of the resulting proteins or to differences in expression. In summary, it seemed that both, $1 \times H A-T G A 1 g$ and 1xHA-TGA1g C172N C260N C266S C287S-complemented the hyponastic growth deficit of the tga1 tga4 mutant, suggesting that the cysteine residues in TGA1 do not play a role during regulation of hyponasty.

To further substantiate these findings, the petiole length relative to the entire leaf length was determined. As shown in Figure R26 and SR10, Col-0 plants had petioles almost half as long as the entire leaf (ca. 49\%). The petioles of tga1 tga4 mutants and the internal control plant (tga1 tga4 + pB1xHA-TGA1g \#22-7) were only $40 \%$ as long as the whole leaf. This was also observed for the control complementation lines expressing only the $1 \mathrm{xHA}$ tag. When $1 \mathrm{xHA}-\mathrm{TGA} 1 \mathrm{~g}$ or

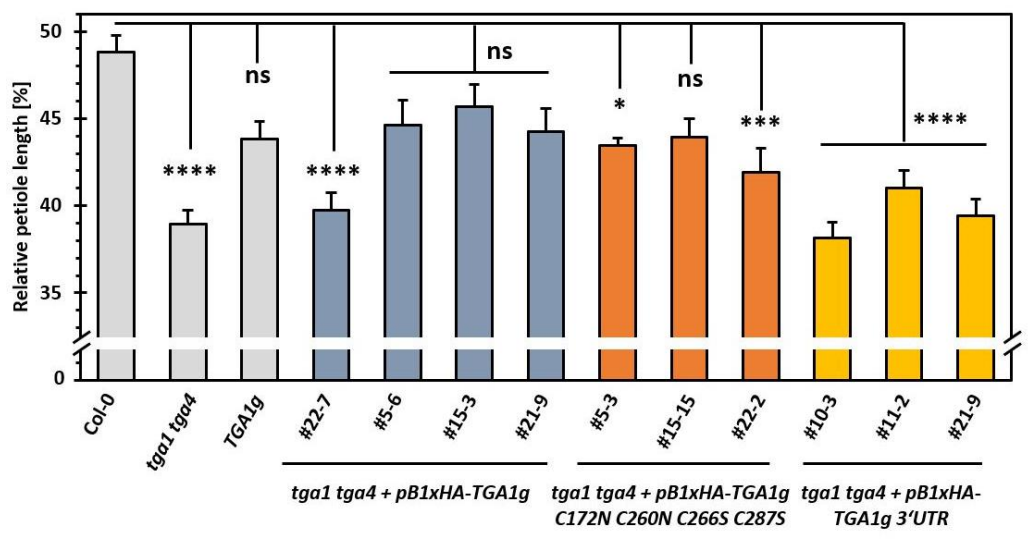

Figure R26. Measurement of the relative petiole length of the TGA1 complementation lines. Col-0, tga1 tga4, a complementation line (TGA1g) from the Department of Botany (Prof. Dr. Yuelin Zhang, UBC Vancouver [222,223,262]) and selected complementation lines constructed during this work were grown for 4 weeks in $12 \mathrm{~h}$ day. Either leaf 7 or 8 from at least 6 different plants was detached and photographed from the top with a Canon PowerShot G11 camera. The petiole length and the blade length were measured using Image $J$ and the petiole length relative to the entire leaf length computed. Bars represent mean \pm SEM. Statistical analysis (One-way-ANOVA and Tukey test) was performed using GraphPad Prism 8.0.1 assuming normally distributed data. Adjusted $\mathrm{p}$-values in comparison to Col-O are indicated above the bars: ns - non-significant, $\mathrm{p}>0.05,{ }^{*}-\mathrm{p} \leq 0.05$, ** - $\mathrm{p}$-value $\leq 0.01$, *** p-value $\leq 0.001, * * * *$ - -value $\leq 0.0001$. 
1xHA-TGA1g C172N C260N C266S C287S were expressed in tga1 tga4, the resulting relative petiole length was in between that of the tga 1 tga 4 mutant and that of Col-O. However, this appeared to be only significant for two out of three 1xHA-TGA1g C172N C260N C266S C287S complementation lines. Including additional plants into the analysis revealed that both, 1xHA-TGA1g and TGA1 cysteine mutant transformants, varied in their capacitiy to complement the reduced petiole length of the tga1 tgat mutant (Figure SR10). However, for the cysteine mutant complementation construct, more plants with a significantly reduced petiole length were observed than for the 1xHA-TGA1g complementation construct. Still, the differences in the petiole length between both types of complementation lines were not strong. Altogether, this might suggest only a partial complementation by both constructs. Thus, the complementation constructs might either not result in fully functional proteins or TGA1 might be redundant with TGA4 in controlling the petiole length. All in all, TGA1g seems to complement the phenotype independent of its cysteine residues at least partially.

In addition to controlling hyponasty and petiole length, the tga 1 tga 4 mutant was described to flower late [223]. Since this phenotype resembles the delay in flowering observed for ROXY9 overexpressing plants, it seems possible that ROXY9 represses TGA1 also during the induction of flowering [157]. Thus, the number of days, wildtype plants, tga 1 tgat mutants, and the internal control line TGA1g needed to start flowering was quantified along with those of the complementation lines generated for this study. Additionally, the number of leaves on the day of flowering was counted. The data is depicted in figures R27 and SR11, part I and II.

Col-0 flowered after ca. 35 days in this experiment and had ca. 19 leaves on the day of flowering. The tga 1 tga 4 mutant flowered three days later and had more leaves than the wildtype. As for the ROXY9 overexpressing plants, the formation of numerous smaller leaves among the counted leaves were observed. This indicates, that the late flowering phenotype of the tga1 tgat mutant is not a result of a generally delayed development. However, the internal control line (\#22-7) neither flowered significantly later than wildtype plants nor showed an increased leaf number. Similarly, only two out of three plants expressing the 1xHA tag showed a delay in flowering, and only one of them formed significantly more leaves. Consequently, flowering might be influenced by additional effects resulting e.g. from the construction process of these transgenic lines. Alternatively, the sample size used in this experiment might not have been high enough to clearly distinguish the small differences in the 
time point of flowering and leaf number between wildtype-like plants and tga1 tga4 mutant-like plants. This is supported by the observation of tendencies even in case of statistically insignificant differences, and the formation of additional, smaller leaves by tga 1 tga4 $+p B 1 x H A-T G A 1 g \# 22-7$, and those plants expressing 1xHA. Despite these discrepancies for some control plants, the TGA1g complementation construct seemed to successfully restore the initiation of flowering after ca. 35 days. Interestingly, this was only observed for two of the three cysteine mutant complementation lines tested. The other transformant flowered significantly earlier than the wildtype. However, the leaf number did not differ from wildtype leaves for TGA1g- and TGA1g C172N C260N C266S C2875-complementation lines. Analysis of additional plant lines confirmed these results (Figure SR11, part I and II).

Considering the influence of additional factors and the small sample size, it seems that the tga1 tgat mutant flowers later than Col-O but is not generally delayed in development. Moreover, the 1xHA-TGA1g seems to complement the phenotype of the tga 1 tga 4 mutant. Similarly, the TGA1g complementation construct carrying the cysteine mutations complements the phenotype, and sometimes even causes an earlier flowering. However, because neither the TGA1 protein levels nor the influence of the tag and the effect of the cysteine mutations on the stability of TGA1 were analyzed in planta, the cause of this observation cannot be evaluated.

All in all, the results from analyzing hyponastic growth contrast with a redox regulation of TGA1 regarding hyponasty [155] whereas for the flowering timepoint, a minor influence of the cysteines cannot be ruled out. 
A

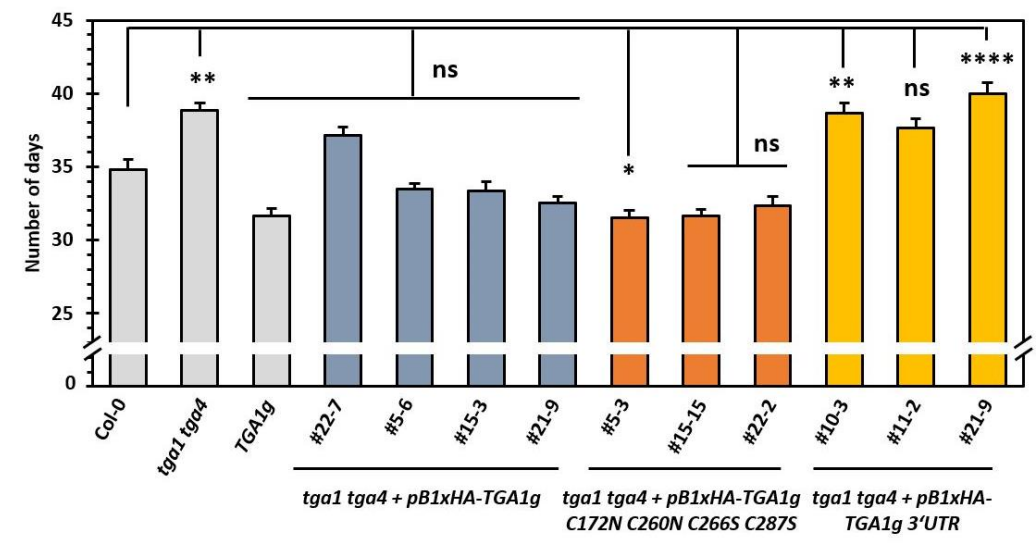

B

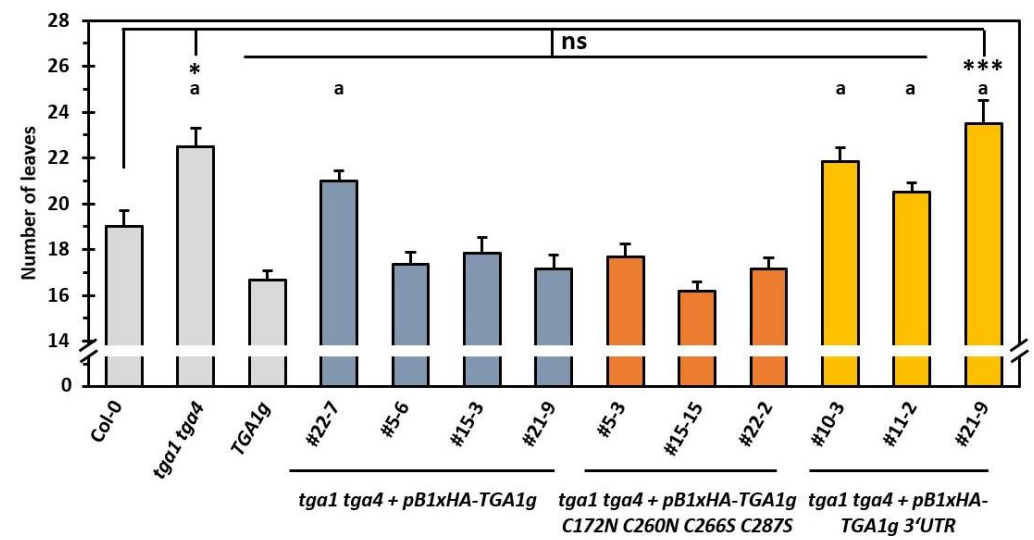

Figure R27. Flowering time point and leave number at flowering of TGA1g complementation lines. Col-0, tga1 tga4, TGA1g [222,223,262] and tga1 tga4 mutants complemented with the constructs shown in Figure 24A were grown in a long day climate chamber. The number of days was determined until a floral stem of $1 \mathrm{~cm}$ lenght was visible (A). At this time point, the number of leaves was counted, as well (B). Bars are mean \pm SEM from at least 5 replicates. Statistical analysis (One-way-ANOVA and Tukey test) was performed using GraphPad Prism 8.0.1 assuming normally distributed data. Adjusted p-values in comparison to Col- 0 are indicated above the bars: ns - non-significant, $\mathrm{p}>0.05$, * - $\mathrm{p} \leq 0.05$, ** - $\mathrm{p}$-value $\leq 0.01, * * *$ - $\mathrm{p}$-value $\leq 0.001, * * * *$ - $\mathrm{p}$-value $\leq 0.0001$. $a$ - smaller leaves in between counted leaves for all replicates. 


\section{Discussion}




\section{The CC-type glutaredoxin ROXY9 as an iron- sulfur cluster binding oxidase}

CC-type glutaredoxins are a land plant-specific group of glutaredoxins which have been associated with the regulation of different stress responses and developmental processes $[80,176]$. In these pathways, they mediate the repression of TGA transcription factors [80], but their precise function has remained enigmatic.

Because glutaredoxins are known to redox-modify proteins to regulate their activity, CC-type glutaredoxins have frequently been speculated to change the oxidation state of TGA transcription factors [145$149,151,153,155,160,163,164,166,168-173]$. Especially, the cellular pool of TGA1 was found to change its redox state after treatment with salicylic acid [224]. Thus, the regulation of TGA1 activity after hyponastic growth by ROXY9 was proposed to occur via redox regulation $[155,156]$. However, evidence for a catalytic activity of CC-type glutaredoxins is scarce $[174,176]$.

\section{Purified strep-MBP-ROXY9 is sensitive to oxidation}

This study was set up to characterize ROXY9 biochemically. This required the production of recombinant protein. First, a strep-SUMO-fusion of ROXY9 was expressed in E.coli. However, the protein turned out to be aggregated (Figure R5), and this condition could not be changed by optimizing the expression and purification procedure. This is similar to the published unsuccessfull attempts to express and purifiy CC-type glutaredoxins from E. coli $[174,176]$. Strikingly, switching the expression host for strep-MBP tagged ROXY9 from E.coli to insect cells resulted in large amounts of soluble, monomeric protein (Figure R6 to 8). Importantly, the well-known interaction between TGA1 and ROXY9 was reproduced in a pulldown experiment using strep-MBP-ROXY9 and recombinant GFP-TGA1 (Figure R10C and D) $[156,157]$. Because this pulldown experiment was not quantitative, it cannot be concluded whether the entire strep-MPB-ROXY9 preparation has led to the 
co-purification of GFP-TGA1. Thus, at least, a fraction of recombinant ROXY9 is functional.

Because glutaredoxins rely on their cysteine residues for catalysis and ironsulfur cluster binding [1,9], strep-MBP-ROXY9 was purified in the presence of the reducing agent DTT. Mass spectrometry revealed that even in the presence of DTT, strep-MBP-ROXY9 is partly oxidized, although the extent of this oxidation was not determined (Figure R15). Consequently, strep-MBP-ROXY9 was either present in an oxidized state in insect cells or oxidized after cell lysis during purification despite the presence of DTT. After removal of DTT by a desalting column or by dialysis, the thiol content of the protein preparation was oxidized to ca. $60 \%$ as suggested by performing Ellman's assay (Figure R11). Importantly, treatment with glutathione during dialysis did not lead to a reduction of the protein (Figure R15). Thus, glutathione in the dialysis buffer might not protect strep-MBP-ROXY9 entirely from oxidation, since glutathione is oxidized to GSSG in the absence of a recycling system [1,9]. Alternatively, it could modify strep-MBP-ROXY9 by glutathionylation $[1,9]$.

Rapid oxidation after purification was also observed for the CGFS-type glutaredoxin AtGRXS16, which oxidized during non-reducing gel electrophoresis and was also not reduced by glutathione [263]. The rapid oxidation of $A t G R X S 16$ possibly depends on its $\mathrm{N}$-terminal active site cysteine residue with a low $\mathrm{pK}_{\mathrm{a}}$ value [263]. Thus, it can be speculated that at least some cysteines of strep-MBP-ROXY9 have low $\mathrm{pK}_{\mathrm{a}}$ values, as well, making them reactive to oxidants. In the future, determination of the $\mathrm{pK}_{\mathrm{a}}$ values together with redox titration experiments and detailed quantitative mass spectrometric analyses of wild type strep-MBP-ROXY9 and strep-MBP-ROXY9 versions in which the cysteine residues are mutated might reveal the reason for the fast oxidation of strep-MBP-ROXY9. A physiological function of this sensitivity of strep-MBP-ROXY9 towards oxidation remains to be investigated. Prelimiary experiments with plants overexpressing HA-tagged ROXY9 showed that ROXY9 is present in a reduced state even when the plant is treated with hydrogen peroxide [N. Li, unpublished]. However, hydrogen peroxide is readily scavenged by antioxidant systems [264,265]. Therefore, further studies using high light $[264,265]$, menadione, paraquat or diamide to induce oxidative stress have to be conducted. 
Strep-MBP-ROXY9 was inactive in using glutathione to reduce different glutaredoxin substrates after removing DTT from the protein preparation by desalting columns or dialysis (Figure R14). Based on the above-mentioned results regarding the oxidation of strep-MBP-ROXY9, it could be speculated that glutathione from the assay mixtures is not able to reduce the CC-type glutaredoxin.

Introducing glutathione into the protein preparation while removing DT'T by dialysis and testing higher amounts of the CC-type glutaredoxin resulted in a weak reactivation of GAPDH, which is most likely caused by a ROXY9mediated deglutathionylation of GAPDH-SG (Figure R16D). However, it is unclear whether the antioxidant glutathione [1,9] preserved the catalytic activity of strep-MBP-ROXY9 or whether the higher protein concentrations used led to this effect. A weak reductase activity of strep-MBP-ROXY9 would be consistent with previous data showing that chimeric CPYC-type glutaredoxins carrying a CC-type active site slowly reduce glutaredoxin substrates [176]. However, in contrast to the chimeric glutaredoxins, strep-MBP-ROXY9 was not active towards the disulphide bridge-containing substrates HEDS and insulin [56,75,101,107,240,241,243,266] (Figure R16A to D), indicating that strep-MBP-ROXY9 might not reduce disulphide bridges. Recently, GAPDH was found to easily form disulphide bridges after glutathionylation of the active site [267]. If strep-MBP-ROXY9 only catalyzes deglutathionylation but not the reduction of other disulphides, only a part of the GAPDH pool serves as a substrate for strep-MBP-ROXY9. In addition, it might not use the artificial compound HEDS in its glutathionylated form as a substrate $[56,75,101,107,240,241,266]$. Thus, a selectivity of strep-MBP-ROXY9 for the substrate and/or the type of disulphide bond on the substrate could account for its weak activity in the GAPDH assay.

However, the sensitivity of strep-MBP-ROXY9 to oxidation most likely lowers the amounts of active protein in the preparation used. Hence, the oxidation state of strep-MBP-ROXY9 after purification with increased DTT concentrations, different reducing agents, or anaerobic conditions has to be tested. Once conditions under which strep-MBP-ROXY9 can be purified and analysed in a reduced state have been established, the catalytic activity of strepMBP-ROXY9 towards HEDS, insulin, GAPDH, and roGFP can be reassessed. This would allow a more precise analysis of the catalytic properties of ROXY9 and will support the evaluation of the observed catalytic activity towards glutathionylated GAPDH. 


\section{Strep-MBP-ROXY9 acts as an oxidase in vitro}

Aside from reductase reactions, glutaredoxins can catalyse the oxidation of certain proteins, such as roGFP, by glutathionylation $[1,9,75,87,88,263]$. During the oxidation of roGFP, the glutaredoxin first glutathionylates itself via GSSG (Figure D1, step $A$ ) and then transfers the glutathione moiety to roGFP (Figure D1, step $B$ and $C$ ). Glutathionylated roGFP forms a disulphide bridge, thereby releasing glutathione (Figure D1, step D) [268].

In contrast to the weak activity towards glutathionylated GAPDH, strep-MBPROXY9 oxidized reduced roGFP2 as strongly as the control glutaredoxin GRXC1 from the CPYC class (Figure R20). Strep-MBP-ROXY9 after removal of DT'T was used. Although strep-MBP-ROXY9 is partially oxidized under these conditions, the oxidation of roGFP $2_{\text {red }}$ by strep-MBP-ROXY9 depended on GSSG, suggesting that strep-MBP-ROXY9 was first glutathionylated by GSSG and then transferred the glutathione moiety to roGFP $2_{\text {red. }}$.

A glutathionylation activity has initially been described for the CPYC-type glutaredoxin Grx1 from humans [87]. On the one hand, this glutathionylation activity might play a role in the quenching of reactive oxygen species: Reaction with ROS leads to the formation of glutathionyl radicals, which might be glutathionylated by glutaredoxins, forming GSSG. On the other hand, glutaredoxins can glutathionylate other proteins, thereby regulating their activity [87]. In A. thaliana, GRXC2 was found by pulldown experiments and yeast-two-hybrid studies to interact with the receptor-like kinase BAK1. In vitro experiments showed that the glutaredoxin glutathionylates BAK1, which results in an inhibition of the BAK1 kinase activity [88]. However, in vivo evidence for the redox regulation of BAK1 is still missing. In contrast, the TGA transcription factor TGA1 has already been described as a redox-controlled protein in vivo and in vitro $[32,224]$. Because ROXY9 interacts with TGA1 in pulldown and yeast-two-hybrid experiments [155-157] and showed an oxidase activity towards roGFP2 $2_{\text {red, }}$ it is tempting to speculate that it might regulate TGA1 by glutathionylation. Thus, future experiments using TGA1 as a substrate could be conducted to test this hypothesis. TGA1 can be purified from E. coli or translated in vitro as described by Després et al. (2003) [224] and Lindermayr et al. (2010) [32]. Labelling of the oxidized and reduced residues via iodo'TMT and IAM could be used to detect changes in the redox state of TGA1 via Western Blots using iodoTMT and TGA1 antibodies. Alternatively, labelling of reduced cysteines via $\mathrm{mBBr}$ would allow to measure changes in the TGA1 redox state photometrically [249]. Finally, mass spectrometry could be 

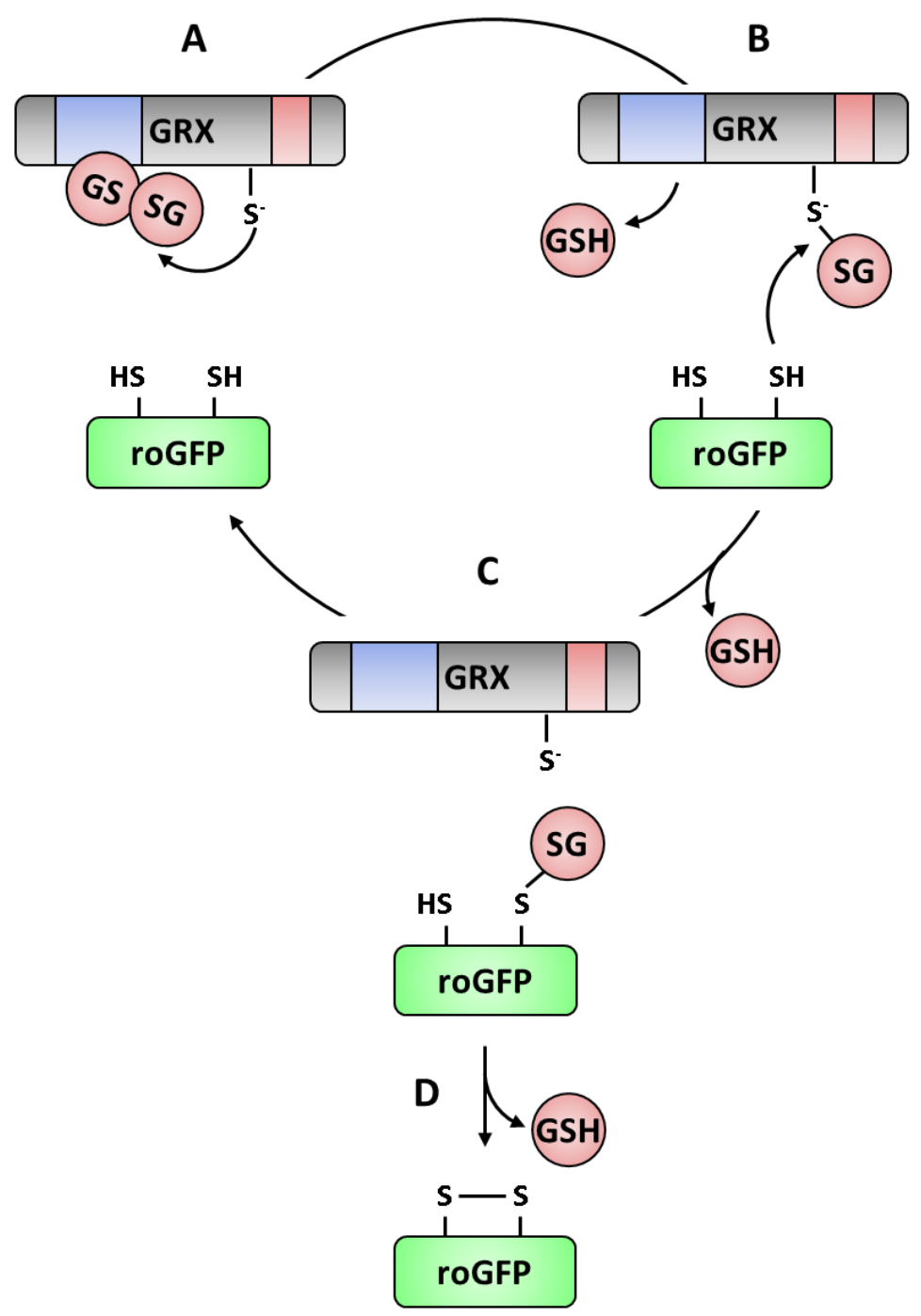

Figure D1. Oxidation of roGFP by glutaredoxins. In step $A$, the glutaredoxin (GRX, grey) binds GSSG via its scaffold site (blue) and then glutathionylates itself on its $\mathrm{N}$-terminal active site cysteine (step B). The weak activation site (red) in CGFS-type glutaredoxins prevents deglutathionylation of the glutaredoxin via another molecule of glutathione or additional cysteines from the glutaredoxin. RoGFP (green) than takes over the glutathione moiety (step C) which results in the formation of an intramolecular disulphide in the protein $($ step $D)$. The figure was prepared according to Meyer \& Dick (2010) [268], Begas et al. (2017) [75], and Zannini et al. (2019) [263]. 
used to identify the redox modifications occurring in TGA1 after incubation with strep-MBP-ROXY9 and GSSG.

The weak reductase/strong oxidase activity of ROXY9 might be determined by the glutathione binding site

As described above, strep-MBP-ROXY9 exhibited probably a weak reductase activity but a significant oxidase activity (Figures R16D and R20). It is interesting to note that such a differential activity has previously been described for the CGFS-type glutaredoxins GRXS15 and GRXS16 [75,97,116,117]. This differential activity was recently attributed to the composition of the glutathione binding site in GRXS15 and GRXS16:

The weak reductase activity of GRXS15 and GRXS16 might result from a weakened glutathione activation site (Figure I4C and D) [75,263]. This site binds and activates the recycling glutathione molecule during glutaredoxin catalysis [75]. Whereas both CGFS-type glutaredoxins might still be able to attack the substrate, forming a disulfide bridge via their $\mathrm{N}$-terminal acitve site cysteine to glutathione or the target itself, they inefficiently bind and activate the recycling glutathione molecule to resolve this linkage (Figure I4A and B). Thus, the slow recycling of the glutaredoxin becomes limiting regarding the overall reaction velocity $[75,263]$.

In CGFS-type glutaredoxins, binding and activation of glutathione is hampered by amino acid exchanges and an extended loop region before the active site motif $[75,263]$. Comparing the amino acid sequence of ROXY9 and other CCtype glutaredoxins to those of CPYC- and CGFS-type glutaredoxins (Figure R22; [80]) shows that the disturbing loop region is missing in ROXY9. Moreover, the conserved lysine residue of the activation site is present in ROXY9 [80]. However, in CPYC-type glutaredoxins, the tyrosine of the active site might contribute to the binding and activation of glutathione, as indicated by initial results [75]. This residue is replaced in both, CGFS-type glutaredoxins [75] and CC-type glutaredoxins [80]. Consequently, the leucine found at this site in ROXY9 might weaken the acitvation site in this protein, thereby lowering its reductase activity.

In contrast to the weak activation site, the scaffold site of CGFS-type gluaredoxins might still enable an interaction with GSSG and glutathionylation of the glutaredoxin $\mathrm{N}$-terminal active site cysteine residue (Figure D1, steps $A$ and $B$ ) [75]. Because the altered activation site prevents an efficient attack of 
recycling cysteine or a recycling glutathione molecule on the glutathione-mixed disulphide in the glutaredoxin active site, the protein remains glutathionylated [75,263]. This enables the transfer of the glutathione moiety to a target protein such as roGFP, resulting in its oxidation (Figure D1, step B and C) [75,263,269].

The scaffold site of glutaredoxins includes the TVP and the GG motif and a rather conserved glutamate residue after the GG motif (Figure I4D) [75]. The scaffold site glutamate appears to be conserved in ROXY9, but not the TVP and the GG motif (Figure R22) [80]. Thus, as for GRXS15 and GRXS16, the rather strong oxidase activity might result from the amino acid exchanges observed in the TVP and the GG motif in ROXY9. Because the oxidation of roGFP by ROXY9 is comparable to that of GRXC1 (Figure R20), but CGFStype glutaredoxins are weaker oxidases than GRXC1 [75,97,263], one could speculate that the differences in the ROXY9 glutathione binding site allow a more efficient association with GSSG and/or a less efficient recycling than the CGFS-glutaredoxin scaffold site. In addition to this, a reactive $\mathrm{N}$-terminal active site cysteine residue could be rapidly glutathionylated, as described for AtGRXS16 [263], and the weakened activation site might prevent that ROXY9 deglutathionylates itself [75,263].

Because the activities of ROXY9 described in this work resemble those of the CGFS-type glutaredoxins GRXS15 and GRXS16, it might be interesting to test mutant ROXY9 variants for their kinetic parameters regarding glutathione and the glutathionylated substrate during catalysis, as described by Begas et al. (2017) [75]. Such studies would address the influence of certain glutathione binding site residues on the efficiency of the association of ROXY9 with the substrate, as well as the binding and activation of the recycling glutathione [75]. Once designed and purified, the ROXY9 mutants could be tested for their activity in plate reader-based roGFP or GAPDH assays. However, because of the oxidation sensitivity of ROXY9, such experiments might require anaerobic conditions. By crystallization and NMR, the structure of the glutathione binding groove in ROXY9 could be examined. However, these structural studies will require optimization of the TEV cleavage of ROXY9 (Figure R17) regarding the purification of the cleaved protein. Both, mutant analysis and structural studies could reveal differences and similarities in the binding mode of glutathione between glutaredoxins from the CC class and the other classes and might therefore help to understand the deviations observed in the catalytic activity of strep-MBP-ROXY9 and the control glutaredoxins from the CPYC class. 


\section{Purified strep-MBP-ROXY9 might bind an iron-sulfur cluster}

Aside from catalytic activity, several glutaredoxins were found to incorporate $[2 \mathrm{Fe}-2 \mathrm{~S}]$ or $[3 \mathrm{Fe}-4 \mathrm{~S}]^{+}$clusters as dimers $[1,81,90,95,112-117,124]$. Similarly, a CC-type glutaredoxin from poplar associated with an iron-sulfur cluster. Using chimeric CPYC-glutaredoxins with a CC-type active site, Couturier et al. (2010) showed that CC-type glutaredoxins possibly bind [2Fe-2S] clusters [176]. In this study, strep-MBP-ROXY9 was reconstituted in the presence of DTT and glutathione under anaerobic conditions in vitro. As indicated by $\mathrm{UV} / \mathrm{Vis}$ spectroscopy and determination of the iron and sulfur amount bound per protein, strep-MBP-ROXY9 incorporated probably a [4Fe-4S] cluster per monomer (Figure R23). With exception of zebrafish Grx2, all glutaredoxins analyzed so far for cluster incorporation bind iron-sulfur clusters as dimers. Moreover, they use their active site and glutathione to coordinate these cofactors via the thiolate of cysteines $[1,81,90,95,112-117,124,126]$. Therefore, the active site represents a potential iron-sulfur cluster binding site in ROXY9. ROXY9 carries a CCLC active site extended by a tyrosine residue (CCLCY) (Figure SI2) [133,145]. In addition, it harbours two more cysteine residues, which are conserved in its most closely related orthologues, ROXY6, 7, and 8. As inferred from an alignment with ROXY19 and the ROXY19 homology model, one of them, $\mathrm{Cys}^{49}$, is probably located at the end of helix $\alpha_{2}$ placed rectangular to the helix containing parts of the active site $\left(\alpha_{1}\right)$ (Figures R22, SI2, and D2A). Thus, it might be situated close to the active site. Consequently, four cysteine residues in proximity to each other can be identified in ROXY9. Since both, [2Fe-2S] and [4Fe-4S] cluster require four ligands for coordination (Figure I5B and C) [118,119], they represent potential residues to test for cluster coordination, once cluster binding can be confirmed for ROXY9. Finally, since the cluster was reconstituted in the presence of glutathione, glutathione might represent an additional ligand, as observed for other glutaredoxins $[1,81,90,95,112-117,124,126]$. However, as can be seen in Figure D2A, the coordination of an iron-sulfur cluster by ROXY9 would require a partially altered orientation of the cysteine residues in the region of the active site motif and a different orientation of the cysteine of glutathione. Structural rearrangements within the active site and the glutathione binding mode upon cluster binding occur for both, CPYC- and CGFS-type glutaredoxins (Figure I6E and F) $[92,94,124]$ and are therefore not unlikely to occur in ROXY9.

Cluster association of strep-MBP-ROXY9 turned out to be unstable under aerobic conditions (S. Freibert, personal communication). Consequently, the cluster itself might be labile under these conditions [270], but the lability could 
Discussion

A

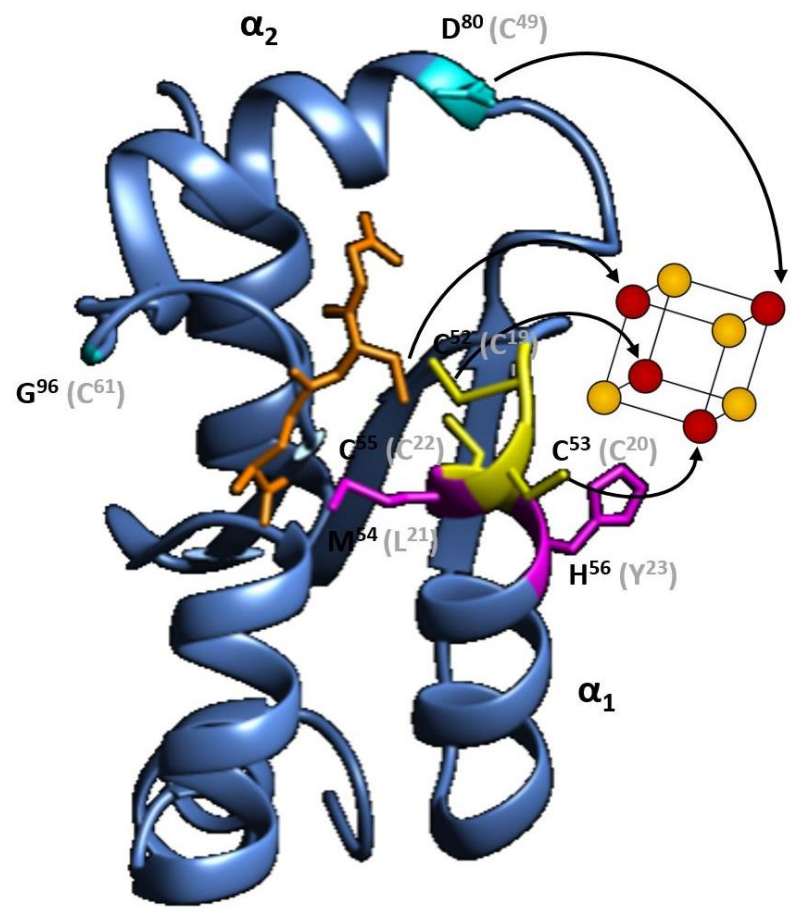

Figure D2, part I. Localization of the CCMCH motif in ROXY19 and residues homologous to the ROXY9 external cysteines and speculation about potential ironsulfur cluster binding modes for ROXY9. Due to the lack of a ROXY9 structural model, the figures were created from the ROXY19 homology model [80] using Chimera. Size and properties of most amino acids highlighted in the figure are similar in ROXY9 and ROXY19, allowing speculations about iron-sulfur cluster binding mode in ROXY9 based on the ROXY19 structure. (A) illustrates a potential coordination by the CC motif, Cys ${ }^{49}$, and the cysteine of glutathione. The amino acid one letter code and numbering are shown in black for ROXY19 and in grey for ROXY9. The active site cysteines of ROXY19 are shown in yellow, whereas the methionine and the histidine of the extended active site are shown in pink. Glutathione is depicted as a stick model in orange. The residues homologous to the external cysteines in ROXY9 are coloured in cyan. The precise alignment of strand $\beta_{1}$ (partly hidden by helix $\alpha_{1}$ ), helix $\alpha_{2}$ and the loop in between is impossible because the residues and the length of this region are not well conserved. In ROXY9, the loop might be shorter. 
B

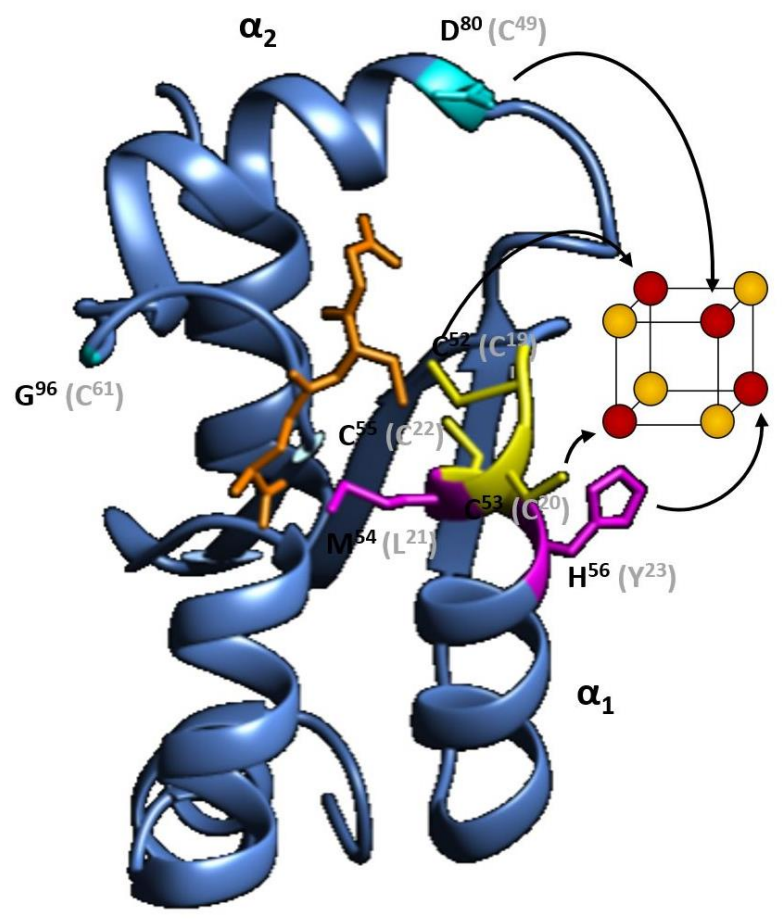

Figure D2, part II. Localization of the CCMCH motif in ROXY19 and residues homologous to the ROXY9 external cysteines and speculation about potential ironsulfur cluster binding modes for ROXY9. Due to the lack of a ROXY9 structural model, the figures were created from the ROXY19 homology model [80] using Chimera. Size and properties of most amino acids highlighted in the figure are similar in ROXY9 and ROXY19, allowing speculations about iron-sulfur cluster binding mode in ROXY9 based on the ROXY19 structure. (B) depicts a cluster binding mode via the CC motif, the tyrosine of the extended active site and $\mathrm{Cys}^{49}$. Colouring and labelling are the same es in Figure D2, part I. 
also be caused by the rapid oxidation of ROXY9 under aerobic conditions. The latter possibility questions the observed stoichiometry of the iron and sulphide ions bound to strep-MBP-ROXY9. However, because the DT'T concentrations used during reconstitution are higher than those used for the purification, the possibility exists that that strep-MBP-ROXY9 is fully reduced during the reconstitution. Thus, the oxidation state of strep-MBP-ROXY9 under reconstitution conditions has to be determined, before the experiment is reproduced. In case the binding of a [4Fe-4S] cluster by strep-MBP-ROXY9 can be confirmed, EPR and Mössbauer spectroscopy will have to be used to reasonably show the incorporation of an iron-sulfur cluster. Once, cluster incorporation has been demonstrated and the type of cluster has been determined, structural studies and mutant analysis could be used to find out how ROXY9 coordinates this cluster.

\section{Comparisons of ALWL- and non-ALWL CC-type glutaredoxins}

To clarify the function of CC-type glutaredoxins in planta, the requirement of the active site cysteine residues of ROXY9 and other CC-type glutaredoxins for their repressive activity has been studied before (Table D1) [146,152,155,174,175]. Lacking a corresponding knock-out mutant, ROXY9 and mutant constructs of ROXY9 were ectopically expressed in wildtype plants and their influence on hyponastic growth was examined. These experiments showed that ROXY9 requires its CC-motif for repression, but not the last active site cysteine residue. [155]. Similarly, in this study, two further ROXY9 mutants, ROXY9 CPYC and ROXY CCLCA were tested for their repressive activity regarding hyponastic growth and flowering.

Although the flowering and petiole length experiments might have suffered from a small sample size, the ROXY9 CPYC mutant protein appeared to be inactive in repressing both, hyponasty and flowering (Figure R1 to R4, SR1 to SR3). Since the CPYC motif lacks the second cysteine residue of the active site motif, the apparent inactivity of ROXY9 CPYC is consistent with previous data showing that this cysteine is essential for ROXY9 activity [155]. These findings contrast with the observations made for other CC-type glutaredoxins: Overexpression of the CC-type glutaredoxin ROXY19 and a CPYC mutant of ROXY19 in wild type plants showed that both proteins are active (Table D1) [152]. The same type of experiment revealed that the second cysteine of the CC motif in ROXY19 might be dispensable for its function [174]. When complementing the roxy1 mutant with ROXY1 and ROXY1 SSMS under the 
control of the ROXY1 promoter, Ziemann (2010) noticed that both proteins were functional (Table D1) [175]. Thus, whereas ROXY9 might require the CC motif for its activity, ROXY1 and ROXY19 do not. Aside from the active site requirements, ROXY1, ROXY19, and ROXY9 differ in their C-terminus. Whereas ROXY1 and ROXY19 carry an ALWL motif at this site, ROXY9 lacks this motif $[145,155,168,170]$. Interestingly, there are findings pointing to differences between the repressive mechanisms used by ALWL- and nonALWL CC-type glutaredoxins: Whereas ROXY19 interacts with TPL in transient protoplast assays via its ALWL motif and represses TGA2 during these assays, it is unable to repress TGA1-mediated hyponasty when overexpressed. ROXY9 shows exactly the opposite behaviour [155,168,177].

Thus, the difference between the requirement of the CC motif for repression might represent another difference between these two subgroups of CC-type glutaredoxins. Still, this questions the strict conservation of this motif within CC-type glutaredoxin $[133,134,136]$. One could speculate, that, whereas this cysteine is necessary for repression in non-ALWL CC-type glutaredoxins, it might fulfill an alternative function in ALWL-containing CC-type glutaredoxins. Thus, additional experiments involving more CC-type glutaredoxins from these two subgroups are necessary to uncover the role of the CC motif in both groups of CC-type glutaredoxins.

Aside from its CC motif, ROXY9 required the tyrosine extending the active site to repress both, flowering and hyponasty (Figure R1 to R4, SR1 toSR3). Measuring the shortening of petiole length caused by ROXY9 overexpression suggested that the CCLCA mutant protein might be partially functional regarding this phenotype (Figure SR2). Still, statistical effects resulting from the small sample size might have influenced the outcome of the petiole length and flowering experiments. Therefore, repetition of these experiments is necessary to confirm these results. Currently, the tyrosine residue appears to be important for repression, although perhaps not in general. This importance of the extended active site motif for ROXY9 contrasts with the findings for ROXY19: Mutation of the histidine extending the active site in ROXY19 does not disturb its repressive activity (Table D1) [174]. Interestingly, all ALWL-containing CCtype glutaredoxins in $A$. thaliana harbour a histidine after their active site motif, while a tyrosine is found in most non-ALWL glutaredoxins (Figure SI2) [133]. Thus, regarding ROXY9 and its most closely related proteins ROXY6, 7, and 8 [145], this tyrosine residue could constitute a further difference in the repressive mechanisms of ALWL- and non-ALWL CC-type glutaredoxins. 
Structural modelling of ROXY19 suggests that the side chain of the histidine residue of the extended active site is protruding from the protein surface [80]. The homologous residues from other glutaredoxins stick out from the surface, as well (Figures SI1A to C and D2) [94,110]. Thus, in ROXY9, the tyrosine of the CCLCY motif might also be situated on the surface of the protein. Regarding this surface localization, one could speculate that this residue might be modified or interact with other molecules. Interestingly, a tyrosine contributes to the binding of a mononuclear cluster in the iron-sulfur cluster assembly protein IscA $[118,271]$. Hence, this protruding tyrosine residue might represent an additional candidate for iron-sulfur cluster ligation in ROXY9 aside from the active site cysteines and the external $\mathrm{C}^{49}$.

All in all, the results presented here substantiate the view that the repressive mechansims of the non-ALWL CC-type glutaredoxin ROXY9 and the ALWL CC-type glutaredoxin ROXY19 differ [155,168,177]. However, this assumption is based on the overexpression of wildtype-like or mutant ROXY9 and ROXY19 in wildtype Arabidopsis plants [152,155,174]. Overexpression can lead to artificial effects. In contrast, the complementation of mutant plants of both CC-type glutaredoxins might provide more reliable results. Thus, in the future, knock-out plants for ROXY9 and ROXY19 and their redundant genes $[145,152,155,168]$ should be obtained and transformed with wildtype-like and mutant ROXY9 or ROXY19 variants.

\begin{tabular}{lccc}
\hline Glutaredoxin & ROXY1 & ROXY19 & ROXY9 \\
\hline Extended active site motif ${ }^{1}$ & CCMCH & CCMCH & CCLCY \\
ALWL motif & Yes & Yes & No \\
$1^{\text {st }} \mathrm{C}$ & $\mathrm{No}^{2}$ & Yes $^{4}$ & Yes $^{7}$ \\
$2^{\text {nd }} \mathrm{C}$ & $\mathrm{No}^{2}$ & $\mathrm{No}^{4}$ & $\mathrm{Yes}^{7}$ \\
$3^{\text {rd }} \mathrm{C}$ & $\mathrm{No}^{2,3}$ & $\mathrm{No}^{4}$ & $\mathrm{No}^{7}$ \\
$\mathrm{H}$ or Y & Not tested & $\mathrm{No}^{6}$ & Largely yes $^{8}$ \\
CPYC & Not tested & Active $^{5}$ & Inactive $^{8}$ \\
\hline
\end{tabular}

Table D1. Summary of the active site requirements of ALWL and non-ALWL CC-type glutaredoxins. The table shows the extended active site motif, the presence of an ALWL motif and the requirements of the $1^{\text {st }}, 2^{\text {nd }}$, and $3^{\text {rd }}$ active site cysteine, as well as the histidine or tyrosine of the extended active site. The outcome of mutating the active site to CPYC is shown, as well. 1 - Lemaire (2004) [133], 2 - Ziemann (2010) [175], 3 - Xing et al. (2005) [146], 4 - Oberdiek (2017) [174], 5 - Huang et al. (2016) [152], 6 - Oberdiek, unpublished, 7- Li (2017) [155], 8 - this work. 


\section{Potential regulatory mechanisms of TGA1 by ROXY9}

The TGA transcription factor TGA1 was found to change its redox state after treatment with salicylic acid in planta [224]. Since the tga1 tga4 mutant phenocopies ROXY9 overexpression plants with respect to hyponasty and because ROXY9 is a glutaredoxin, TGA1 was speculated to be redox-regulated by ROXY9 [155]. In an initial experiment to address this idea, an HA-tagged TGA1 version with mutations in all four cysteine residues mimicking the reduced state of the protein was expressed in tga 1 tga4 plants and compared to plant lines expressing either a tagged non-mutated TGA1 version or the tag only. As for the ROXY9 overexpression lines, the phenotype of the different lines with respect to hyponastic growth and flowering was examined (Figure R25 to 27, SR9 to 11$)$.

As discussed for the phenotypic analysis of ROXY9 overexpression plants, the measurement of the petiole length and flowering might have been influenced by a small sample size, and, therefore, requires repetition with a larger sample size (Figures R26, R27, SR10, and SR11). In addition, a partial complementation - resulting either from the HA tag fused to the expressed TGA1 protein or from a contribution of TGA4 [155-157,194,223,229,230,272] - must be taken into account regarding the measurement of the petiole length (Figures R26 and SR10). Still, the overall result suggested that the cysteine mutant of TGA1 mimicking the reduced state of the protein is at least as active as non-mutated TGA1 in planta regarding hyponasty and flowering. With respect to flowering (Figures R27 and SR11), the cysteine mutant of TGA1 appeared to complement the phenotype slightly stronger than the wild typelike TGA1 version. However, the earlier flowering might be caused by an increased stability of the protein. Therefore, this observation will have to be proven by analysing the abundance of the transgenic protein in the plant cells. Altogether, these findings are in accordance with the observation that a TGA1 version carrying mutations in only two of the four cysteine residues is active in planta [155]. 


\section{Redox-regulation of TGA1 by ROXY9}

At a first glance, the functionality of the TGA1 cysteine mutant might indicate that TGA1 is not redox-regulated during flowering and hyponasty. However, if ROXY-mediated repression of these processes via redox changes represents only an accessory mechanism, the redox state of the TGA1 cysteines might not contribute significantly to the overall termination process.

Regarding hyponastic growth, the experiments performed so far represent only end-point measurements; therefore, altered kinetics of the inititation and/or termination of hyponastic growth of the complementation line expressing the TGA1 cysteine mutant plants might have been missed. Experiments analysing the kinetics of initiation and termination of hyponastic growth [273] in the different complementation lines will help to clarify whether TGA1 indeed does not require its cysteines during the regulation of hyponastic growth or whether a potential fine-tuning of hyponastic growth via redox regulation of TGA1 occurs. Similar to the TGA1 cysteine mutant plant, roxy8 roxy9 and roxy6 roxy 7 roxy9 mutant plants have been analysed and were found to perform a wild typelike hyponasty [155] J. Budimir, unpublished]. Thus, these mutants should also be tested for the kinetics of hyponastic growth. Moreover, if these mutants show wildtype-like hyponastic growth kinetics, roxy6 roxy 7 roxy 8 roxy9 and even roxy6 roxy 7 roxy 8 roxy9 roxy 20 knockout plants could be included in the analysis $[145,155,168]$. This will clarify the extent of the contribution of ROXY9 and its relatives to the regulation of this process. In contrast to roxy 8 roxy9 and roxy6 roxy7 roxy9 mutants, ROXY9 overexpression plants show a clear repression of hyponastic growth. To further test, whether this repression is mediated by oxidation of TGA1, ROXY9 could be overexpressed in the different tga1 tga4 complementation lines to test whether the CC-type glutaredoxin still mediates repression when the cysteine residues in TGA1 are mutated. Finally, analysing the oxidation state of TGA1 in these plants via iodoTMT and IAM labelling and subsequent Western blotting will help to understand whether ROXY9 mediates redox-regulation of TGA1. Aside from examining hyponastic growth, the latter two experiments could be performed to test whether TGA1 is repressed by ROXY9 via redox regulation during flowering.

All in all, it is still possible to speculate about a redox regulation of TGA1 by ROXY9 during both, hyponastic growth and flowering. Because recombinant ROXY9 was found to oxidize roGFP efficiently and because TGA1 is active in its reduced state, it seems possible that ROXY9 might oxidize TGA1 to inhibit hyponastic growth and induction of flowering (Figure D3A). 
Repression of TGA1 by ROXY9 via complex formation involving co-repressors and an ironsulfur cluster

Although a minor or pathway-specific redox-regulation of TGA1 by ROXY9 might exist, the ability of the TGA1 cysteine mutant to complement the tga1 tga4 mutant raises the question of alternative mechanisms for ROXY9 to repress TGA1 regarding hyponasty and flowering. As discussed before, ROXY9 might incorporate an iron-sulfur cluster (Figure R23). Iron-sulfur cluster binding would open new possibilities for a repressive mechanism regarding TGA1, such as complex formation with TGA1 and perhaps additional proteins. Repression by ROXY19 is mediated by recruitment of the corepressor TPL [177]. ROXY9 does not bind to TPL directly [177], but JAZ proteins, which interact themselves or via the bridging protein NINJA with TPL [181], also associate with ROXY9 in yeast-two-hybrid experiments (Figure D3B) [178]. In this study, ROXY9 and JAZ9 did not interact in a pulldown experiment using recombinant proteins (Figure R10) but this interaction could have been disturbed by the large MBP tags fused to both proteins or could require specific conditions. However, treatment of plants with methyl jasmonate represses hyponastic growth [274]. Since JAZ proteins are degraded in the presence of jasmonic acid, these findings contradict with a role of JAZ proteins in repression of hyponasty [179]. Still, non-JAZ TIFY proteins, such as TIFY8, which also interact with NINJA and ROXY9 [178,181,275] might represent candidates for a potential repressive complex. Since ROXY9 and ROXY19 differ in the amino acids required for repression [152,155,168,174], potential repressive complexes formed by these two proteins might differ, as well. In conclusion, other repressive complexes with respect to hyponastic growth, with or without TPL, should be considered (Figure D3C). Because the data presented in this study suggests that ROXY9 is sensitive to oxidation and might associate with an iron-sulfur cluster, one could speculate that the regulation of such a complex might be mediated via redox sensitivity of ROXY9 and/or cluster incorporation [270]. Regarding iron-sulfur cluster binding, it is interesting to note, that sensing of oxidative stress by glutaredoxins via labile iron-sulfur clusters has been proposed before $[94,112,138]$. Thus, after excluding a redox-regulation of TGA1 by ROXY9, one could attempt the co-purification of complexes involving ROXY9 and TGA1 from plant cells to confirm whether TIFY proteins and TPL associate with these transcriptional regulators. In addition, simultaneous co-purification of enzymes mediating the assembly and transfer of iron-sulfur clusters to target proteins would represent a first indication, whether ROXY9 associates with an iron-sulfur cluster in planta [S. Freibert, personal communication]. 
A

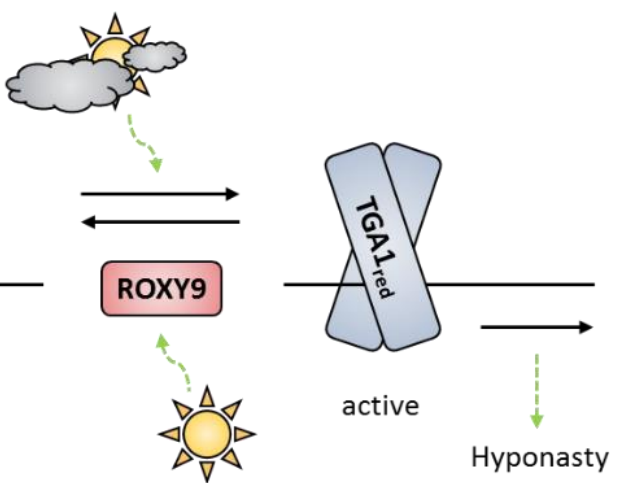

B
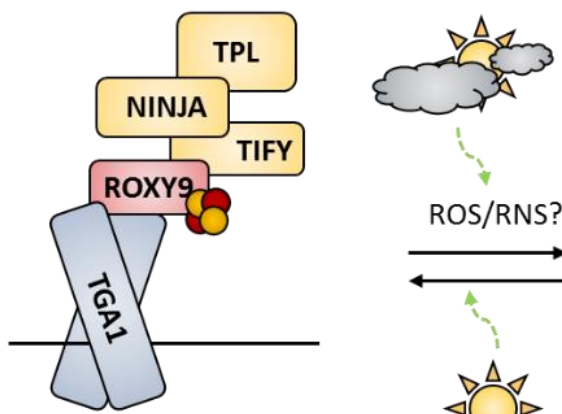

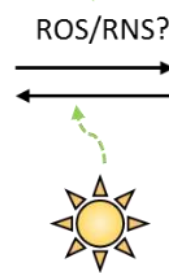

Light

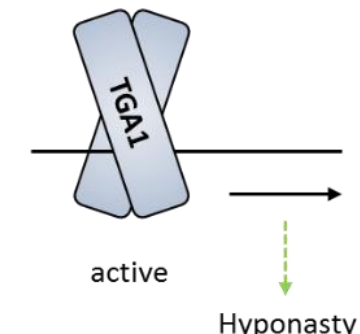

Hyponasty
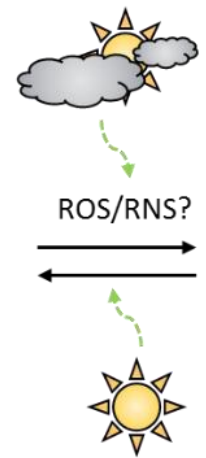

Light

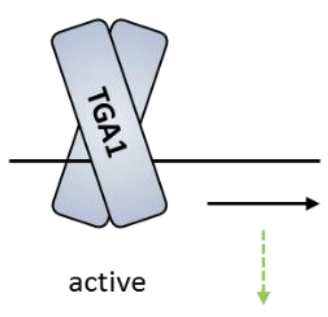

Hyponasty

Figure D3. Examples for potential regulatory mechanisms of ROXY9 during hyponastic growth. (A) TGA1 might become reduced under low light conditions, thereby activating hyponastic growth. Upon backshift to control light conditions, ROXY9 is expressed $[155,156]$ and might oxidize TGA1, thereby inactivating the protein. (B) TGA1 might be repressed by ROXY9 via iron-sulfur cluster binding which induces the association of ROXY9 with corepressors such as the TIFY protein-NINJA-TPL complex $[177,178,181]$. ROS/RNS might interfere with cluster binding by ROXY9 and therefore release the repression. (C) Instead of recruiting the TIFY protein-NINJA-TPL complex, ROXY9 could repress hyponasty by e.g. interacting with still unidentified corepressors. Derepression might occur as described in (B). Mechanisms similar to those shown in $(\mathbf{A}),(\mathbf{B})$, and $(\mathbf{C})$ could contribute to the regulation of flowering. However, the stimuli inducing these mechanisms are unknown. 
Supplementary figures 
Supplementary figures

A

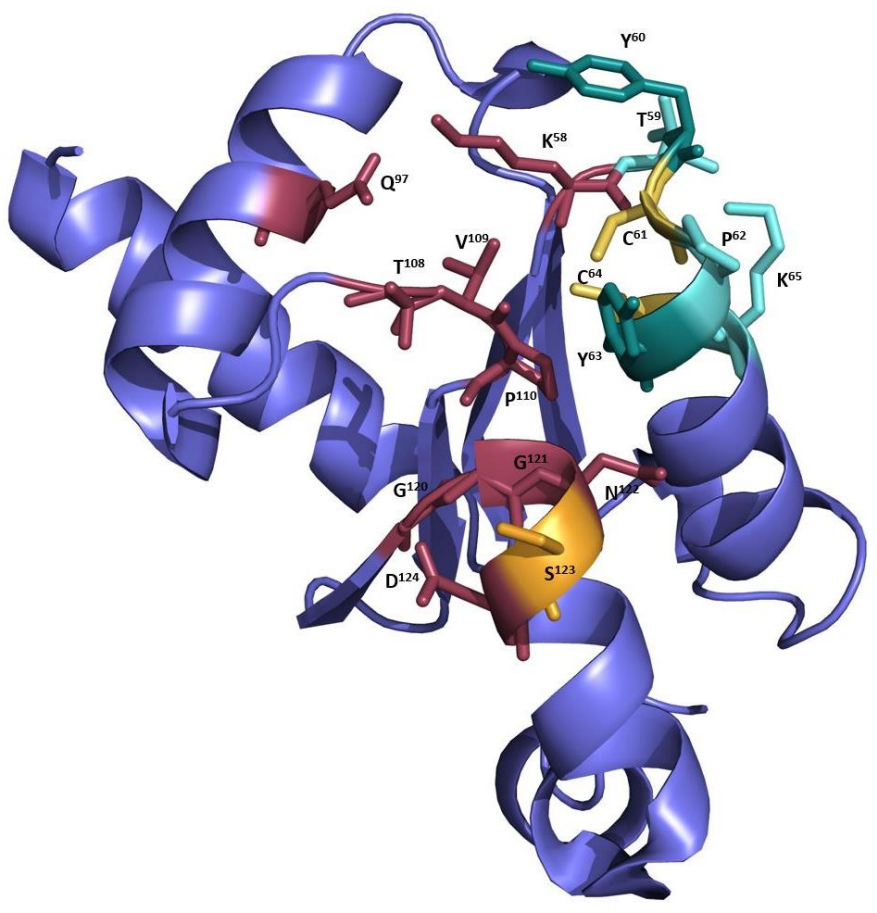

Figure SI1, part I. Different oxidation states of glutaredoxins occuring during catalysis. (A) The crystal structure of S. cerevisiae (Grx2) in its reduced form was solved by Li et al. (2010) [110] to a resolution of $1.5 \AA$. The structure pictures were produced from the PDB file (accession: 3CTG) using PyMOL. The cysteines of the CPYC active site are presented as thiols and shown in yellow; residues important for glutathione binding are shown in red. Residues changing their conformation upon oxidation are coloured in light or dark cyan, respectively. Residues speculated to contribute to catalysis are shown in dark cyan; residues demonstarted to determine the specific activity are shown in orange. 
B

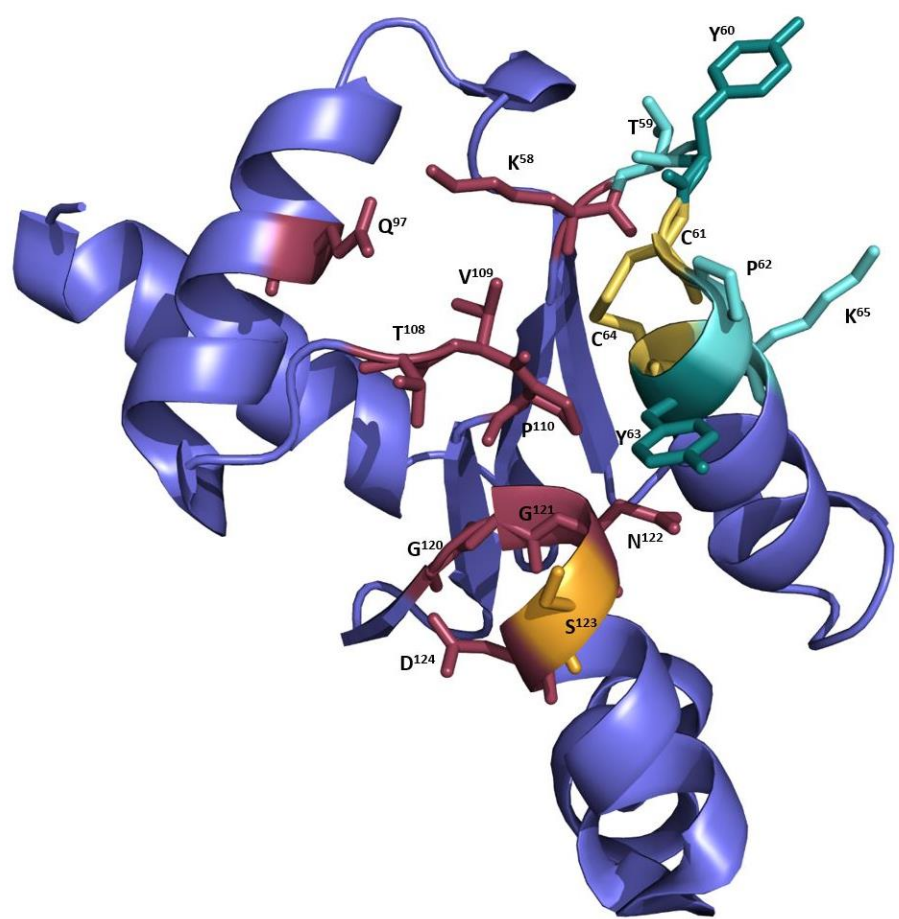

Figure SI1, part II. Different oxidation states of glutaredoxins occuring during catalysis. (B) The crystal structure of $S$. cerevisiae Grx2 in its oxidized form was solved by Li et al. (2010) [110] to a resolution of $2.1 \AA$. The structure pictures were produced from the PDB file (accession: 3CTF) using PyMOL. The active site cysteines of the CPYC glutaredoxin form an intramolecular disulfide and are shown in yellow; residues important for glutathione binding are shown in red. Residues changing their conformation upon oxidation are coloured in light or dark cyan, respectively. Residues speculated to contribute to catalysis are shown in dark cyan; residues demonstarted to determine the specific activity are shown in orange. 
Supplementary figures

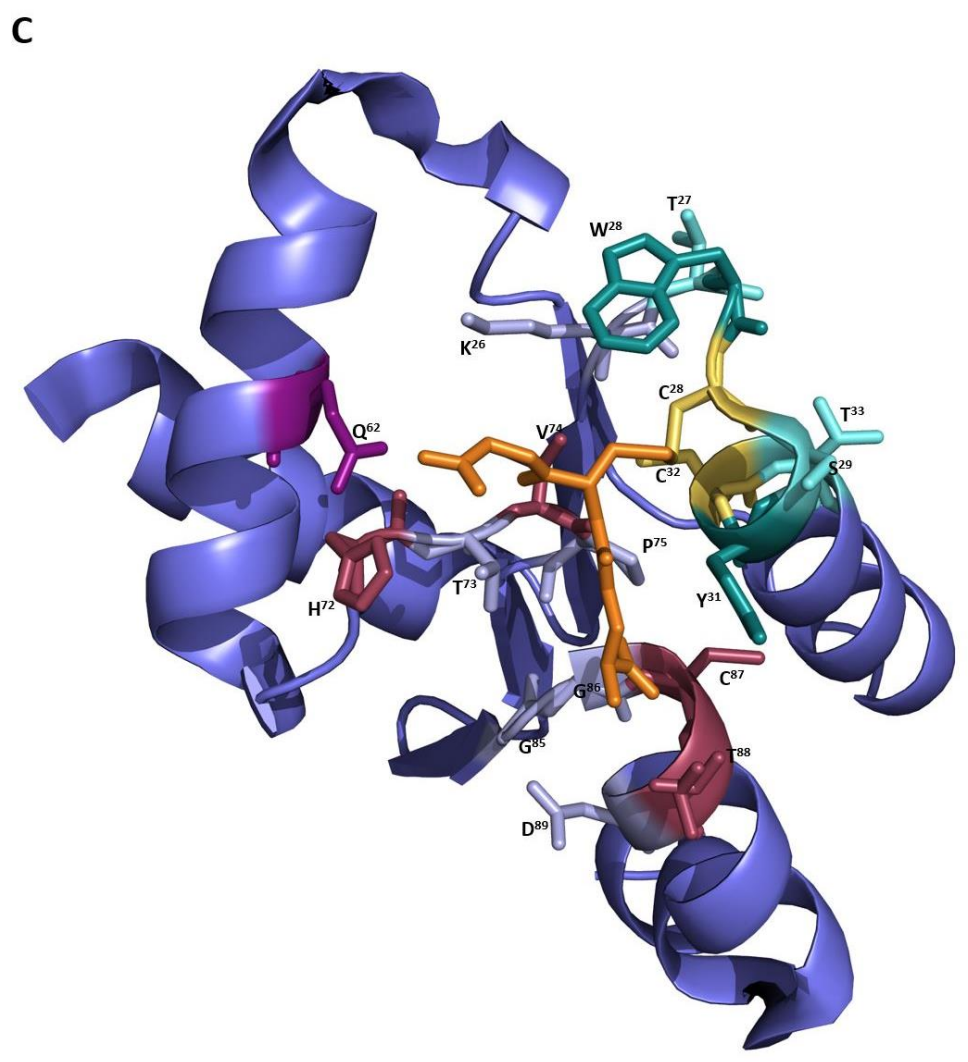

Figure SI1, part III. Different oxidation states of glutaredoxins occuring during catalysis. (C) The crystal structure of the apoform of $A$. thaliana GRXC5 covalently bound to glutathione via its first active site cysteine was solved by Couturier et al. (2011) [94] to a resolution of $1.2 \AA$. The structure pictures were produced from the PDB file (accession: 3RHB) using PyMOL. The active site cysteines of the CPYC glutaredoxin are shown in yellow; residues important for glutathione binding are shown in red. Residues found in other glutaredoxins to contribute to glutathione binding, but not mentioned in the publication about GRXC5, are shown in light blue. $\mathrm{Q}^{62}$ is shown in purple because it indirectly contributes to glutathione binding via TVP loop stabilization. Residues homologous to those changing their conformation in S. cerevisiae Grx2 upon oxidation are depicted in light and dark cyan. 

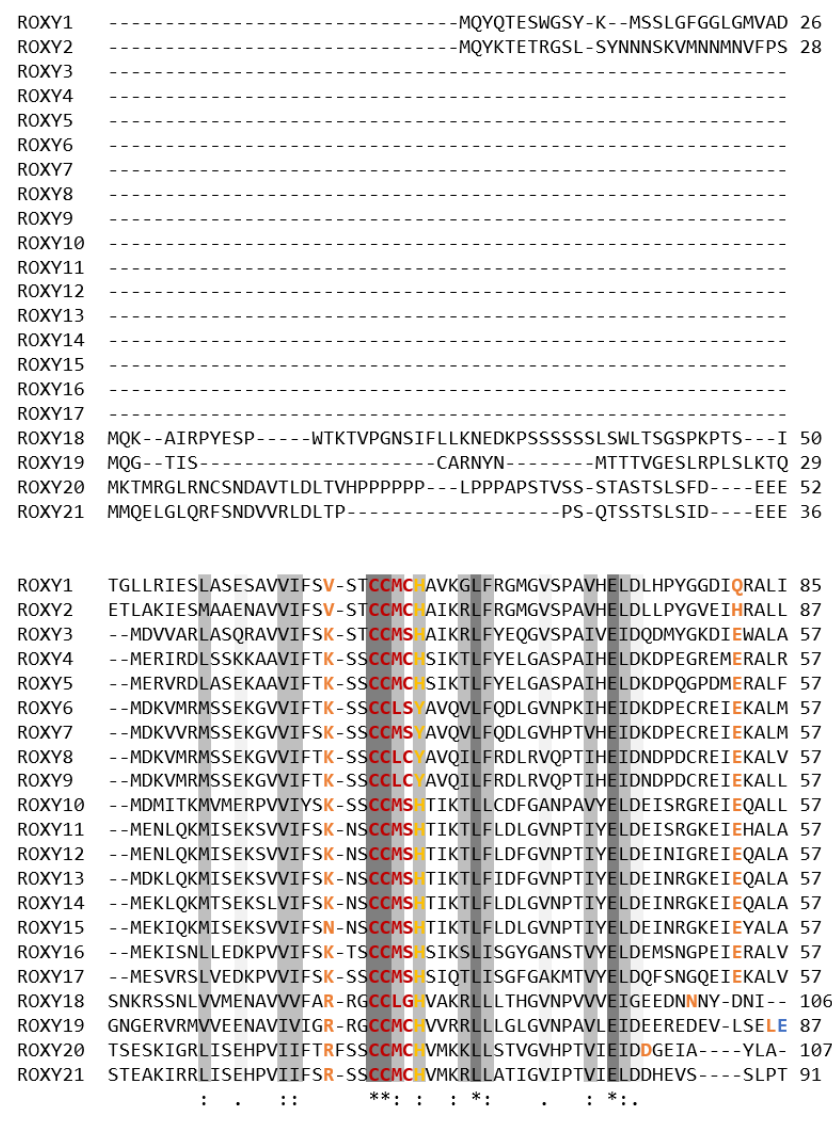

ROXY1 RLLGCS--GSSSPGSL VVFIGGKLV AMDRVMASHINGSEVPLLKDAGALWL 136

ROXY2 RLLGCSSGGATSPGAL VVFIGGKMV AMERVMASHINGSLVPLLKDAGALWL 140

ROXY3 RL-GCS-.-.--PTV AVFVGGKFV TANTVMTLHLNGSLKILLKEAGALWL 102

ROXY4 ALGSSN-...-.PAV AVFVGGRYI SAKDIISFHVDGSLKQMLKDAKAIWL 103

ROXY5 RVFGSN-...-PAV AVFVGGRYV SAKDVISFHVDGSLKQMLKASNAIWL 103

ROXY6 RL-GCS - ...-.-KPV AVFIGGKLV STNEVMSMHLSSSLVPLVKPYLC ... 99

ROCY7 RL-GCS--TPV AIFVGGKLI STNEVMSLHLSGSIVPLVKPFOANLC 102

ROCY7 RL-GCS-1.-TPV AIFVGGKLI STNEVMSLHLSGSLVPLVKPFQANLC 102

ROXY8 RL-GCA - -NAV AVFVSGKLV STNDVMSLHLSGSLVPLIKPYQSFHN 102

ROXY9 RL-GCS-..--TAV AVFVGGKLV STNEVMSLHLSGSLVPLIKPYQSILY 102

ROXY10 RL-GCS-

ROXY11 QL-GCS-...-.-PTV VVFIGGQLV GANQVMSLHLNRSLVPMLKRAGALWL 102

ROXY12 QL-GCS--.--PTV VVFIGGQLV GANQVMSLHLNRSLVPMLKRAGALWL 102

ROXY13 QL-GCS-..--.-PTV VVFIGGQLV GANQVMSLHLNRSLVPMLKRVGALWL 102

ROXY14 QL-GCS-....-PTV VVFIGGQLV GANQVMSLHLNRSLIPMLKRVGALWL 102

ROXY15 QL-GCS-...--.-PTV VVFIGGQLV GANQVMSLHLNRSLIPMLKRFGALWL 102

ROXY16 EL-GCK -.....-PTV AVFIGQELV GANQLMSLQVRNQLASLLRRAGAIWI 102

ROXY17 QM-GCK-...--PSV AVFIGQQFI GANQVMTLQVKNQLAAMLRRAGAIWV 102

ROXY18 - _....-VSDKEKL MMYIGGKLF GLENLMAAHINGDLVPTLRQAGALWL 150

ROXY19 - - NIGVQGGGGTVKL AVYVGGRLF GLDRVMATHISGELVPILKEVGALWL 137

ROXY20 -.......-VEAA VLFIGGTCV GFESLVALHLSGOLIPRLVEVGALWA 148

ROXY21 ALODEYSGGVSVVGPP AVFIGRECV GLESLVALHLSGOLPKLVQVGALUV 144

$*::: . *^{*} .:::::: .^{*}:$.

Figure SI2. Alignment of CC-type glutaredoxins from $\boldsymbol{A}$. thaliana. The sequences from all CC-type glutaredoxins in $A$. thaliana were obtained from and aligned with the Clustal $\mathrm{O}$ (1.2.4) algorithm on the UniProt database. The degree of conservation between the residues from weak to strong is indicated by colours and symbols as follows: light grey (.) > grey (:) > dark grey $(*)$. The active site motif is shown in red. The conserved residue following the active site is coloured in yellow. Glutathione binding residues and the GG motif are shown in orange, whereas the TVP motif is depicted in green. Functional ALWL motifs are shown in blue. Please note, that the homology towards residue 81 in ROXY1 is not clear for ROXY18 to 21. The homology proposed by Gutsche et al. (2015) [80] is indicated in orange; the homology for ROXY19 suggested by structural comparisons (Figure I3) are shown in blue. 
Supplementary figures

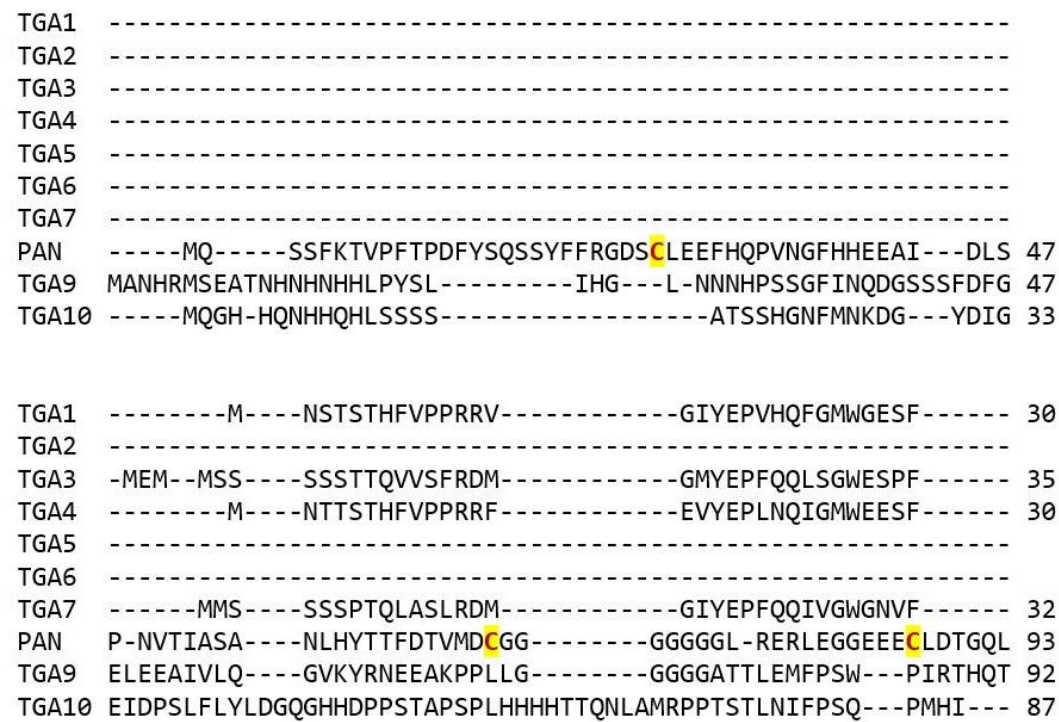

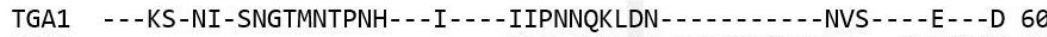

TGA2 -

TGA3 ---KS-DI-NNITSNQNNN---QSSSTTLEVDARPEA-----DDNNRVN- -YTS-- -V 75

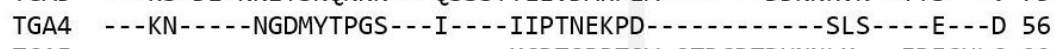

TGA5 -

TGA6 -

TGA7 -.-KS-DI-NDHSPNTA-...-.TSSIIQVDPRIDDH-...-NNNIKIN--YDS-.-S 69

PAN VYQKGTRLVGGGVGEVN-SSWCDSVSAMADNSQHTDT-STDIDTDDKTQLNGGHQGMLLA 151

TGA9 LPTES-SKSGGESSDSGSANFSGKAESQQPESPMSSKHHLMLQPHHNNMANSSSTSGLPS 151

TGA10 EPPPSSTH-.-.--NTDNTRLVPAAQPSGSTRPASDP-SMDL -TNHSQFHQ - - - - PPQ 133

TGA1 TSHGTA--GTPH--MFDQEASTSRHPDKIQRRLAQNREAARKSRLRKKAYVQQLETSRLK 116

TGA2 T-.....-AASDSSDRSKGKMDQKTLRRLAQNREAARKSRLRKKAYVQQLENSRLK 78

TGA3 YNNSLE--AEPS--SN-NDQDEDRINDKMKRRLAQNREAARKSRLRKKAHVQQLEESRLK 130

TGA4 TSHGTE--GTPH--KFDQEASTSRHPDKIQRRLAQNREAARKSRLRKKAYVQQLETSRLK 112

TGA5 I-...--GASDSSDRSKSKMDQKTLRRLAQNREAARKSRLRKKAYVQQLENSRLK 78

TGA6 A-1..-AASDSSDRSKDKLDQKTLRRLAQNREAARKSRLRKKAYVQQLENSRLK 78

TGA7 HNQIEA--EQPS--SNDNQDDDGRIHDKMKRRLAONREAARKSRLRKKAYVQQLEESRLK 125

PAN T-..--NCSD--QSNVKSSDQRTLRRLAQNREAARKSRLRKKAYVQQLENSRIR 198

TGA9 TSRTLA-PPKPSEDKRKATTSGKQLDAKTLRRLAQNREAARKSRLRKKAYVQQLESSRIK 210

TGA10 GSKSIKKEGNRKGLASSDHDIPKSSDPKTLRRLAQNREAARKSRLRKKAYVQQLESCRIK 193

bZIP

$* * * * * * * * * * * * * * * * * * *: * * * * * . *::$

TGA1 LIQLEQELDRARQQGFYVGNGIDTNSLG--.---FSETMNPGIAAFEMEYGHWVEEQNRQ 170

TGA2 LTQLEQELQRARQQGVFISG--TGDQA---H-----STGGNGALAFDAEHSRWLEEKNKQ 128

TGA3 LSQLEQELVRARQQGLCVRNSSDTSYLG--.-.-PAGNMNSGIAAFEMEYTHWLEEQNRR 184

TGA4 LIHLEQELDRARQQGFYVGNGVDTNALS--.-.-FSDNMSSGIVAFEMEYGHWVEEQNRQ 166

TGA5 LTQLEQELQRARQQGVFISS--SGDQA---H----STTAGDGAMAFDVEYRRWQEDKNRQ 128

TGA6 LTQLEQELQRARQQGVFISS--SGDQA---H----STGGNGALAFDAEHSRWLEEKNRQ 128

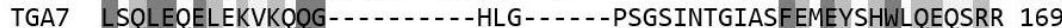

PAN LAQLEEELKRARQQGSLVERGVSADHT---H----LAAGNGVFSFELEYTRWKEEHQRM 250

TGA9 LSQLEQELQRARSQGLFMGGCGP-.-P-.-_-_-.-PNITSGAAIFDMEYGRWLEDDNRH 259

TGA10 LTQLEQEIQRARSQGVFFGGSLIGGDQQQGGLPIGPGNISSEAAVFDMEYARWLEEQQRL 253

* :**:*: : :.** ${ }^{*}: *^{*}::^{*}:: \ldots$ : 


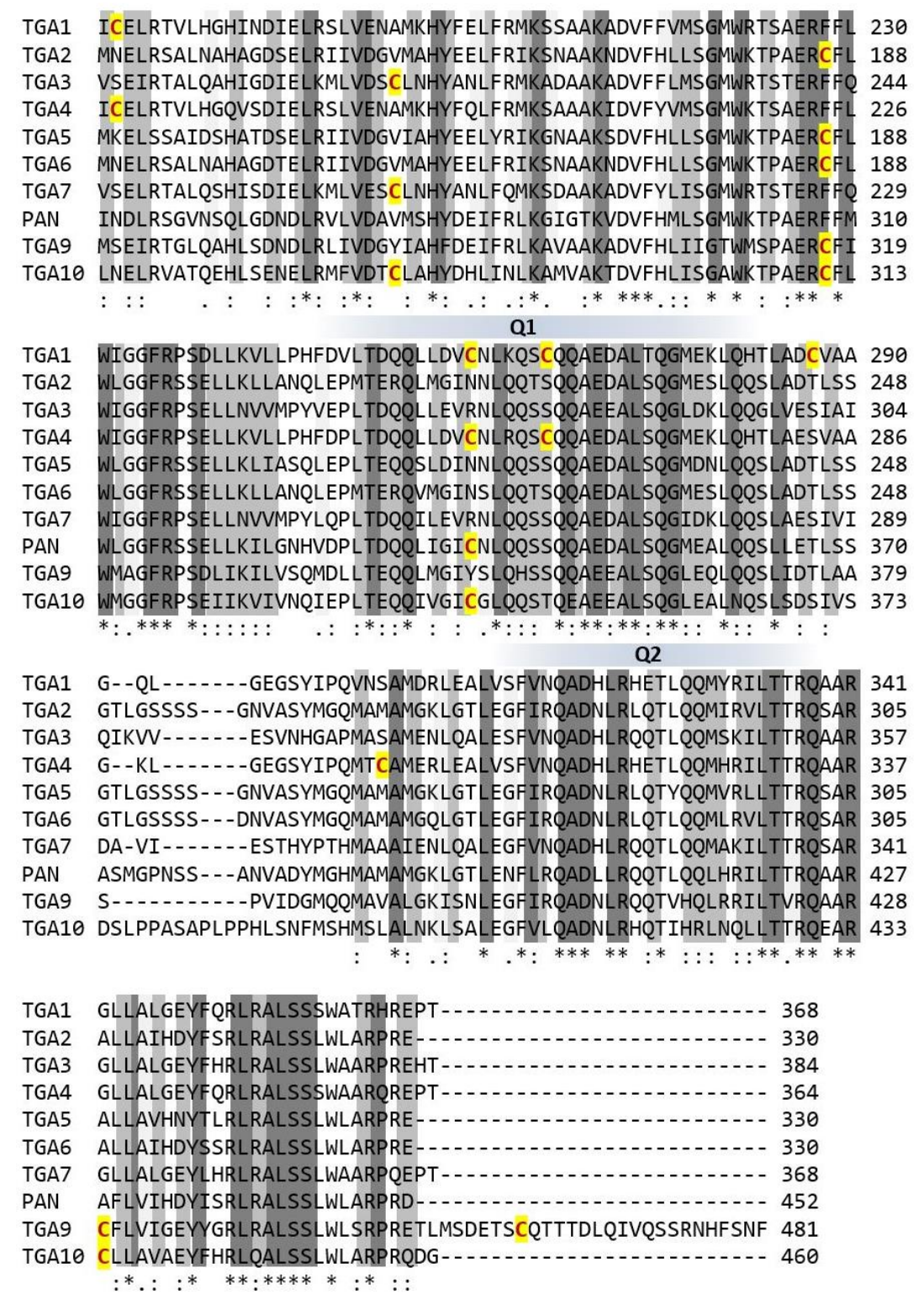

Figure SI3. Alignment of TGA transcription factors from $\boldsymbol{A}$. thaliana. The sequences from all TGA transcription factors in $A$. thaliana were obtained from and aligned with the Clustal O (1.2.4) algorithm on the UniProt database. The degree of conservation between the residues from weak to strong is indicated by colours and symbols as follows: light grey (.) > grey $(:)>$ dark grey $(*)$. The approximate position of the bZIP domain and the two glutaminerich regions (Q1 and Q2) are indicated by grey stripes above the alignment. Cysteine residues are shown in red and yellow. 
Supplementary figures
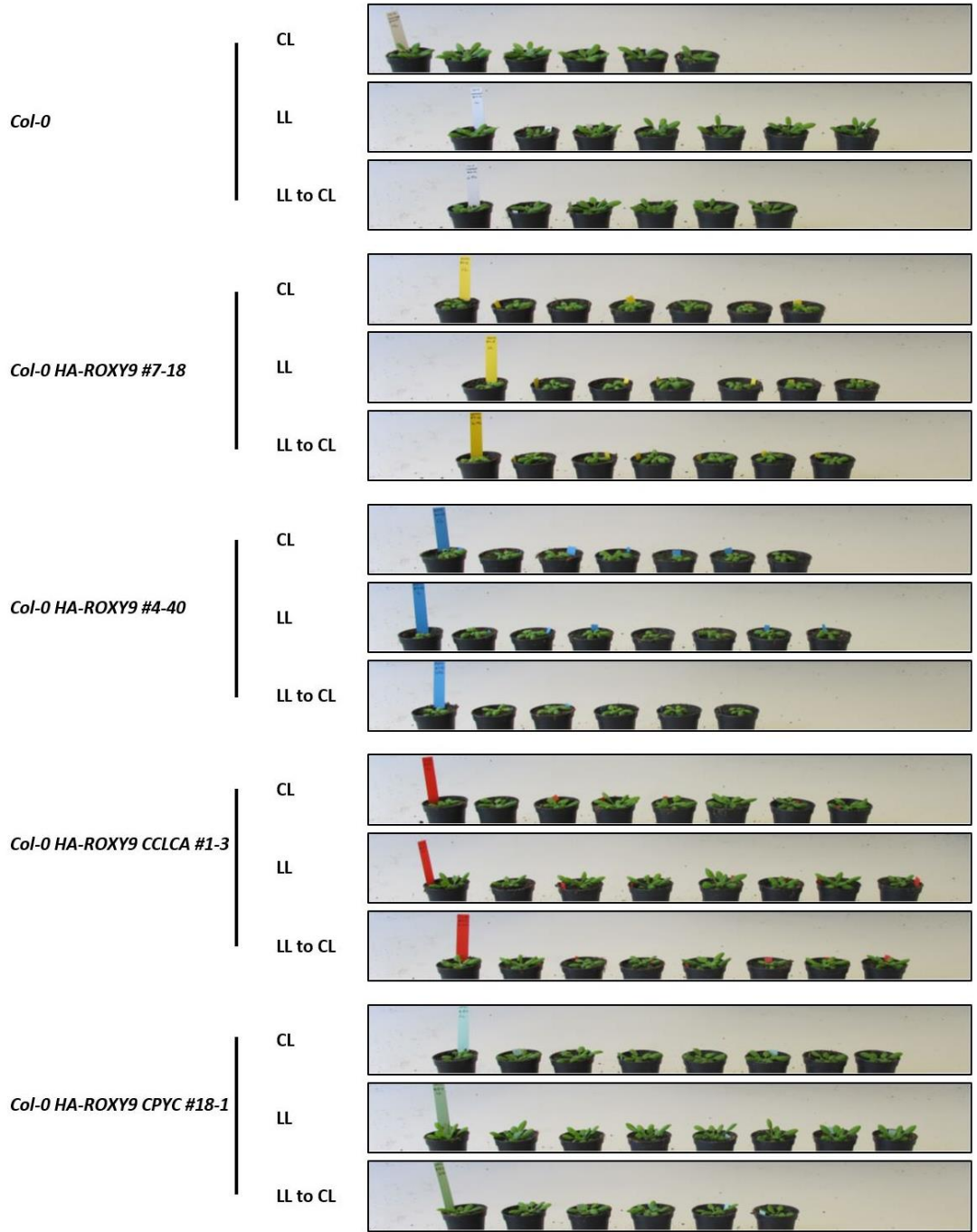

Figure SR1, part I. Analysis of hyponastic growth of plants overexpressing HA-ROXY9, HA-ROXY9 CCLCA or HA-ROXY9 CPYC. Plants overexpressing HA-ROXY9, HAROXY9 CCLCA or HA-ROXY9 CPYC from the CaMV35S promoter were grown for 4 weeks in $12 \mathrm{~h}$ light $/ 12 \mathrm{~h}$ dark rhythm and then transferred to different light conditions as described in Methods (page 59). Pictures of the plants were taken with a Canon PowerShot G11 camera. The images belong to the experiment shown in Figure R2A. CL - control light, $L L-$ low light, $L L$ to $C L$ - shift from low light to control light; Col-0 corresponds to Col-0 + HA-ROXY9 \#17-4. 

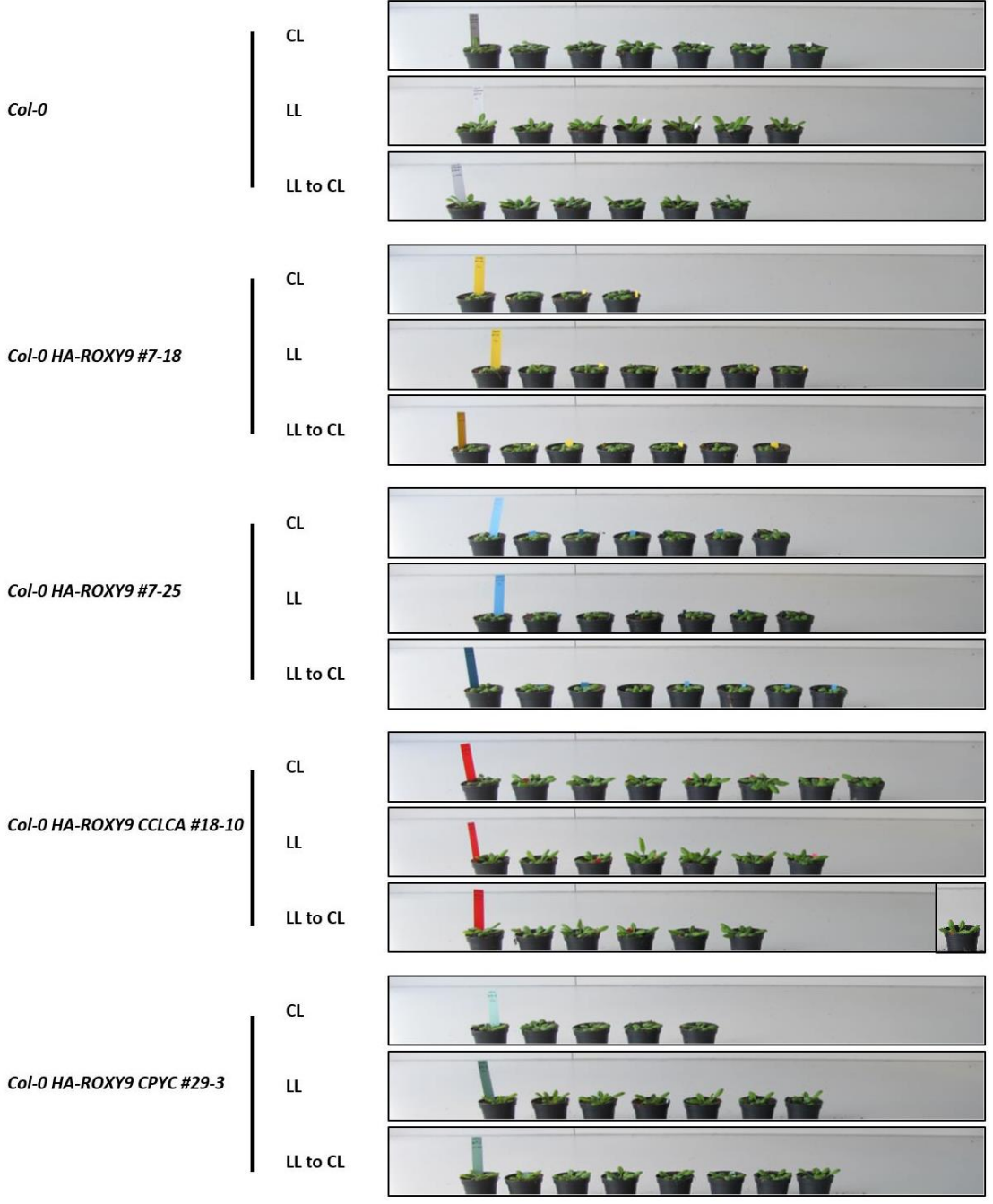

Figure SR1, part II. Analysis of hyponastic growth of plants overexpressing $\mathbf{H A}$ ROXY9, HA-ROXY9 CCLCA or HA-ROXY9 CPYC. Plants overexpressing HA-ROXY9, HA-ROXY9 CCLCA or HA-ROXY9 CPYC from the CaMV35S promoter were grown for 4 weeks in $12 \mathrm{~h}$ light $/ 12 \mathrm{~h}$ dark rhythm and then transferred to different light conditions as described in Methods (page 59). Pictures of the plants were taken with a Canon PowerShot G11 camera. The images belong to the experiment shown in Figure R2B. CL-control light, $L L-$ low light, $L L$ to $C L-$ shift from low light to control light; Col-0 corresponds to Col-0 + HA-ROXY9 \#17-4. 
Supplementary figures

\begin{tabular}{c|l} 
Col-o & $\mathrm{CL}$ \\
& $\mathrm{LL}$ \\
$\mathrm{LL}$ to CL
\end{tabular}

\begin{tabular}{l|l} 
COI-O HA-ROXY9 \#7-18 & $\mathrm{CL}$ \\
& $\mathrm{LL}$ \\
$\mathrm{LL}$ to CL
\end{tabular}

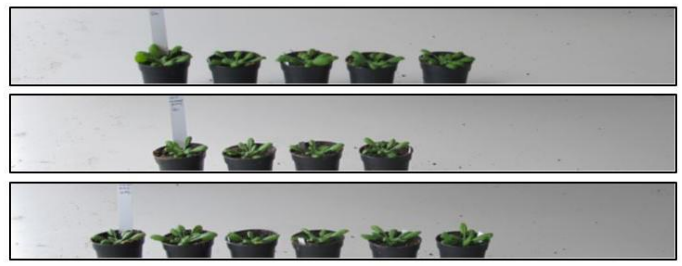

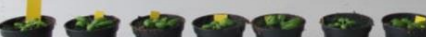
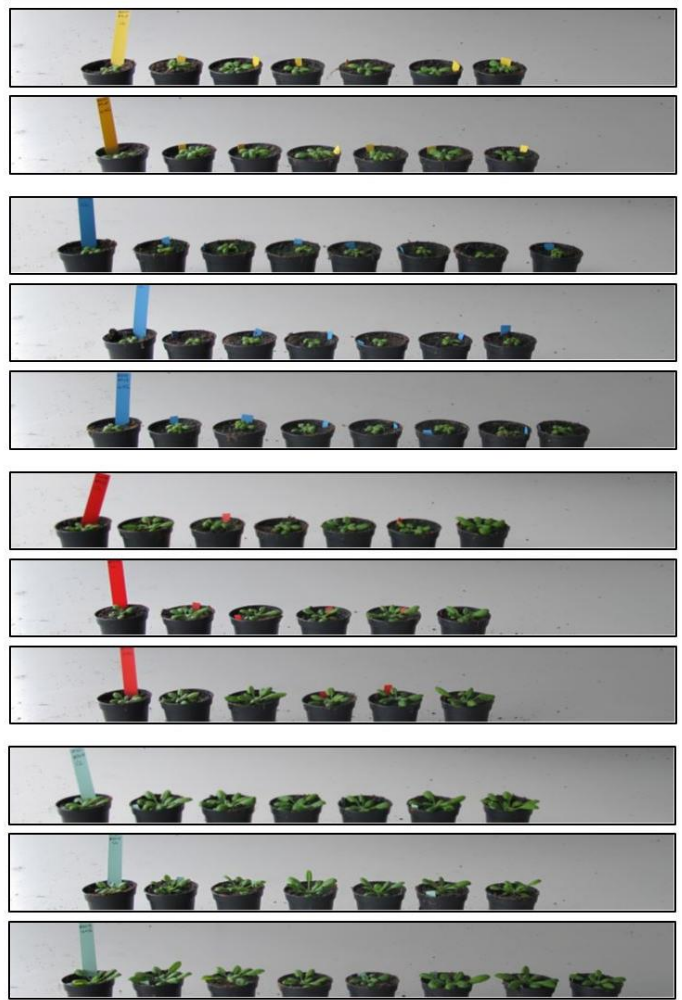

Figure SR1, part III. Analysis of hyponastic growth of plants overexpressing $\boldsymbol{H A}$ ROXY9, HA-ROXY9 CCLCA or HA-ROXY9 CPYC. Plants overexpressing HA-ROXY9, HA-ROXY9 CCLCA or HA-ROXY9 CPYC from the CaMV35S promoter were grown for 4 weeks in $12 \mathrm{~h}$ light $/ 12 \mathrm{~h}$ dark rhythm and then transferred to different light conditions as described in Methods (page 59). Pictures of the plants were taken with a Canon PowerShot G11 camera. The images belong to the experiment shown in Figure R2C. CL - control light, $L L-$ low light, LL to CL - shift from low light to control light; Col-0 corresponds to Col-O +HA-ROXY9 \#17-4. 


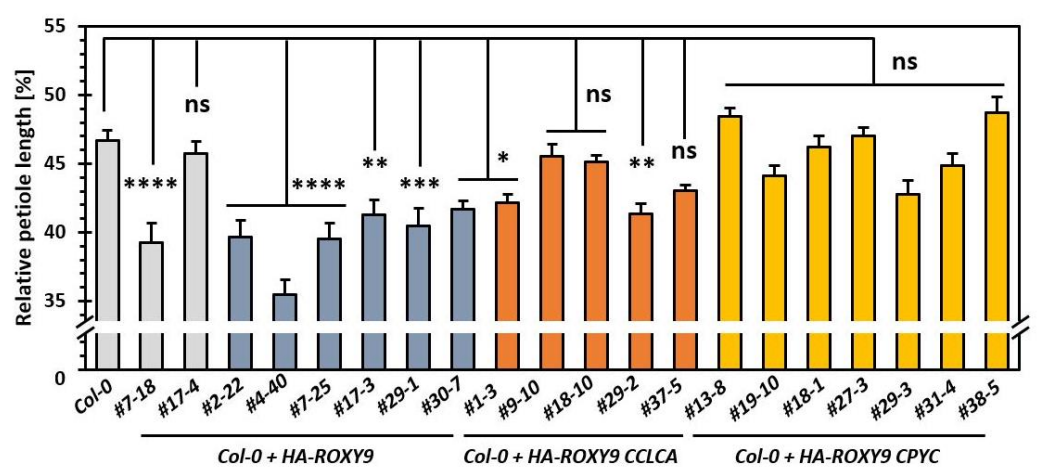

Figure SR2. Determination of relative petiole length of plants overexpressing $\boldsymbol{H A}$ ROXY9, HA-ROXY9 CCLCA or HA-ROXY9 CPYC. Plants overexpressing HA-ROXY9, HA-ROXY9 CCLCA or HA-ROXY9 CPYC from the CaMV35S promoter were grown for 4 weeks in $12 \mathrm{~h}$ day. Either leaf 7 or 8 from at least 6 different plants was detached and photographed from the top with a Canon PowerShot G11 camera. The petiole length and the blade length were measured using Image $J$ and the petiole length relative to the entire leaf length was computed. Bars represent mean \pm SEM. Statistical analysis (One-way-ANOVA and Tukey test) was performed using GraphPad Prism 8.0.1 assuming normally distributed data. Adjusted $\mathrm{p}$-values in comparison to Col-0 are indicated above the bars: ns - non-significant, $\mathrm{p}>0.05, *$ $\mathrm{p} \leq 0.05, * *$ - $\mathrm{p}$-value $\leq 0.01, * * *$ - $\mathrm{p}$-value $\leq 0.001,{ }^{*} * * *$ - $\mathrm{p}$-value $\leq 0.0001$. 
Supplementary figures

A

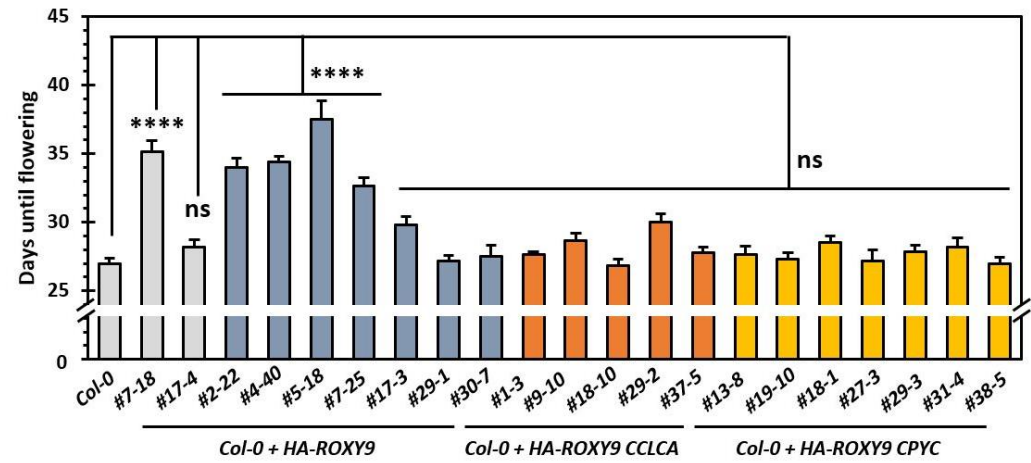

B

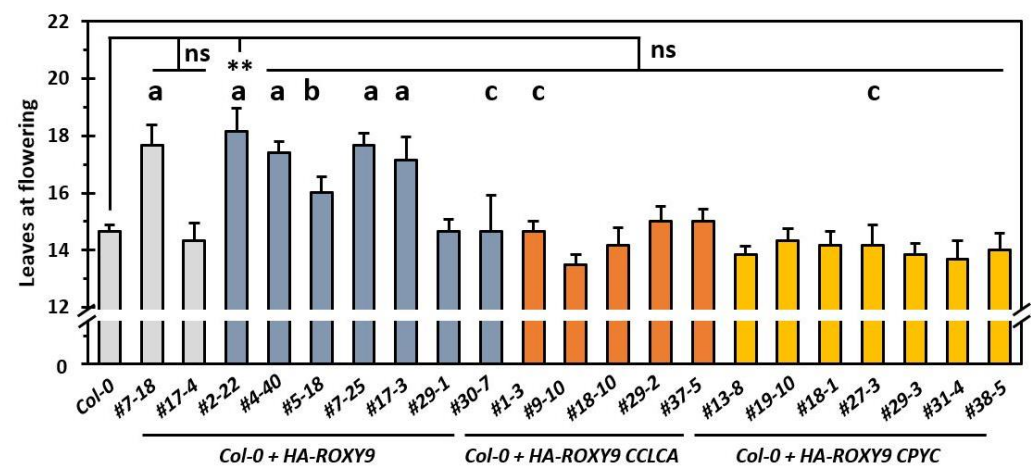

Figure SR3, part I. Flowering time point and leave number at flowering of plants overexpressing HA-ROXY9, HA-ROXY9 CCLCA or HA-ROXY9 CPYC. Plants overexpressing HA-ROXY9, HA-ROXY9 CCLCA or HA-ROXY9 CPYC from the CaMV35S promoter were grown under long day conditions. The number of days were counted until a $1 \mathrm{~cm}$ floral stem could be observed (A). At this time point, the number of leaves was determined (B). Bars represent mean \pm SEM from at least 5 replicates. $a$ - smaller leaves in between counted leaves for all replicates, $b$ - smaller leaves in between counted leaves for all except for 1 replicate, $c-$ smaller leaves in between counted leaves for 1 or 2 replicates out of 6 . 
Supplementary figures

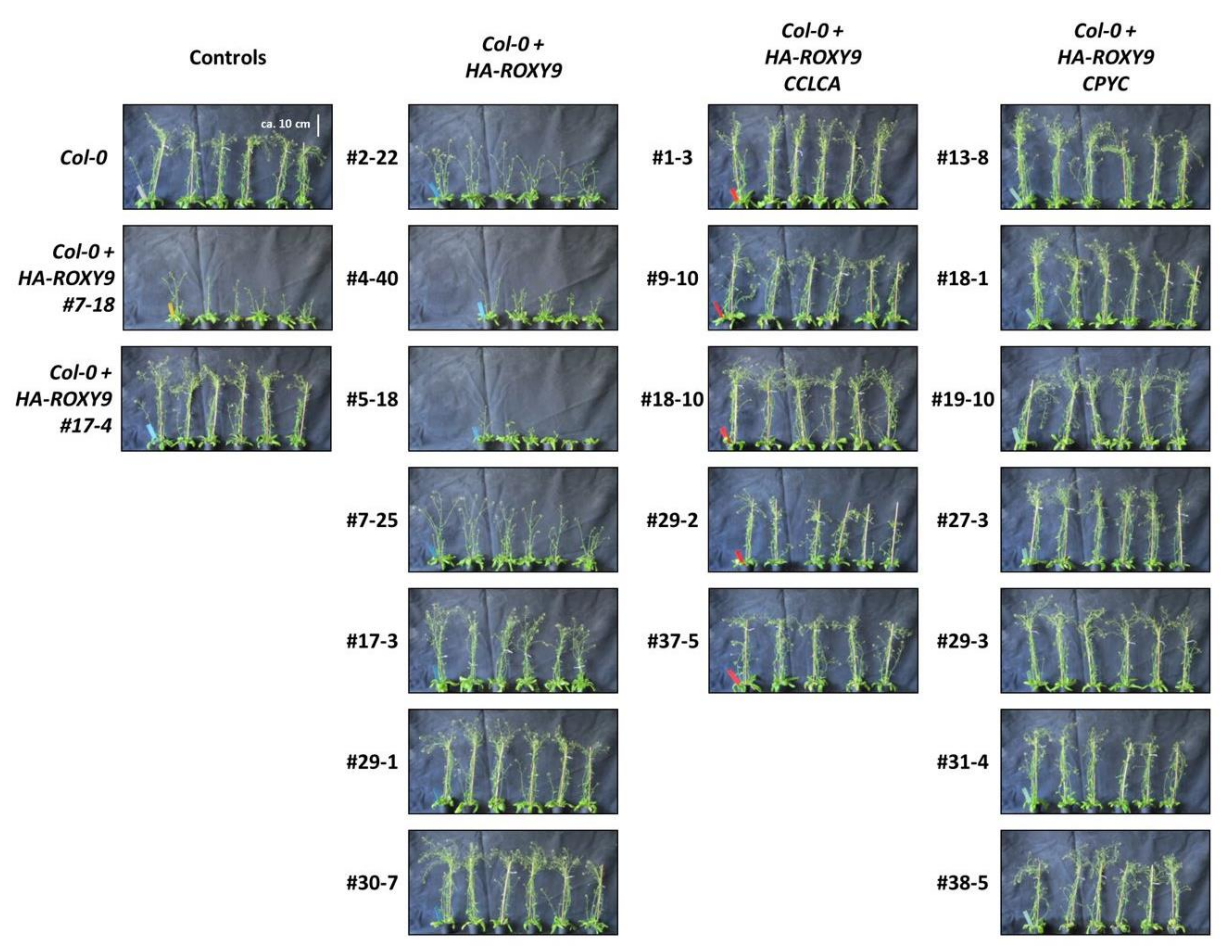

Figure SR3, part II. Comparison of floral stems of plants overexpressing HA-ROXY9, HA-ROXY9 CCLCA or HA-ROXY CPYC. Plants overexpressing HA-ROXY9, HAROXY9 CCLCA or HA-ROXY9 CPYC from the CaMV35S promoter were grown under long day conditions. When the last plant had flowered, the plants were photographed in the side view using a Canon PowerShot G11 camera. If necessary, large floral stems were tied to sticks. The scale bar corresponds to approximately $10 \mathrm{~cm}$. 
Supplementary figures

A

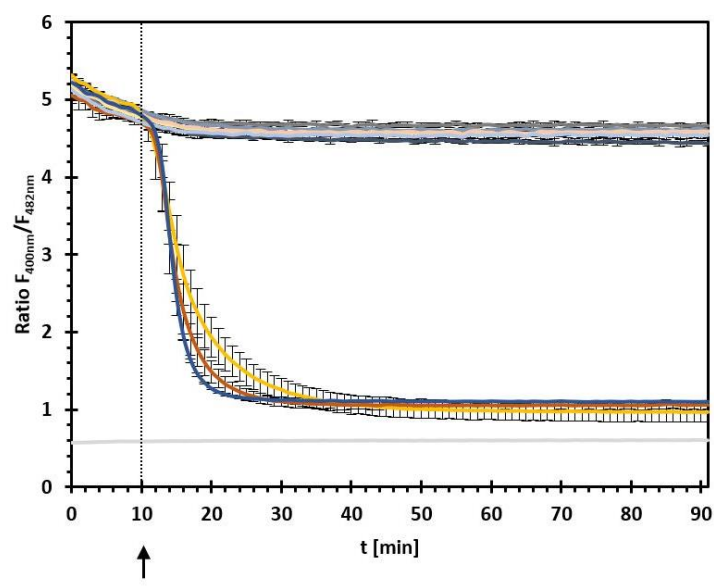

roGFP2 red

rOGFP2 ox

0 MM GRXC1 - GSH

0 - $\mu \mathrm{M}$ GRXC1 + GSH

$1 \mu \mathrm{M}$ GRXC1 - GSH

$1 \mu \mathrm{M}$ GRXC1 + GSH

$2 \mu \mathrm{M}$ GRXC1 - GSH

$2 \mu \mathrm{M} \mathrm{GRXC1}+\mathrm{GSH}$

$5 \mu \mathrm{M}$ GRXC1 - GSH

$-5 \mu \mathrm{M}$ GRXC1 + GSH

B

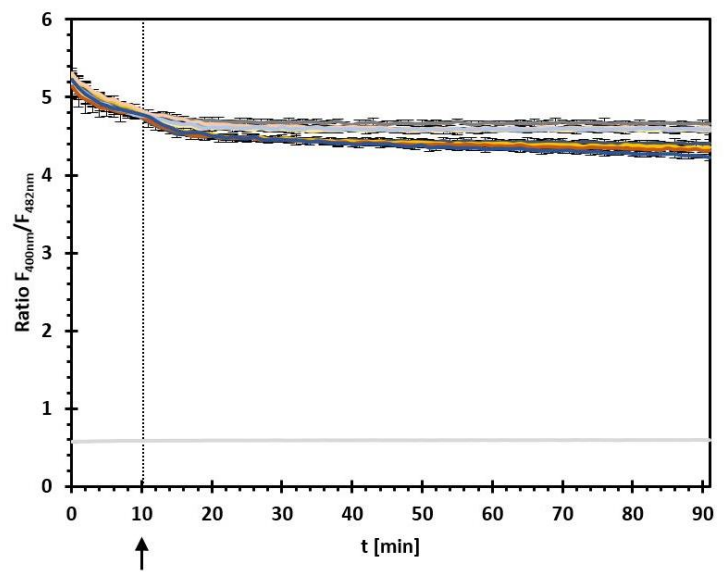

roGFP2 red

roGFP2 ox

$0 \mu \mathrm{M}$ ROXY9 - GSH

$0 \mu \mathrm{M}$ ROXY9 + GSH

$1 \mu \mathrm{M}$ ROXY9 - GSH

$1 \mu \mathrm{M}$ ROXY9 + GSH

$2 \mu \mathrm{M}$ ROXY9 - GSH

$-2 \mu \mathrm{M}$ ROXY9 + GSH

$5 \mu \mathrm{M}$ ROXY9 - GSH

$-5 \mu \mathrm{M}$ ROXY9 + GSH

Figure SR4. In vitro reductase activity of strep-MBP-ROXY9 towards roGFP2 when DTT is removed. Strep-MBP-ROXY9 was dialyzed against buffer without DTT. N-terminally His-tagged GRXC1 purified in an oxidized state was used as a control. Different amounts of enzyme were incubated with or without GSH and oxidized roGFP2. Oxidized roGFP2 and reduced roGFP2 alone served as controls. The reactions were equilibrated for $10 \mathrm{~min}$. After that, GSH or buffer were added to initate the reactions (arrow and dashed line). The change in the fluorescence ration was monitored over time. Values represent mean $\pm \mathrm{SD}$. 
A

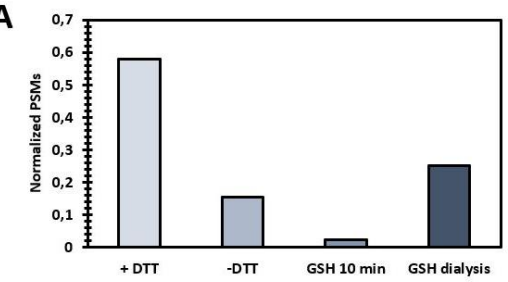

C

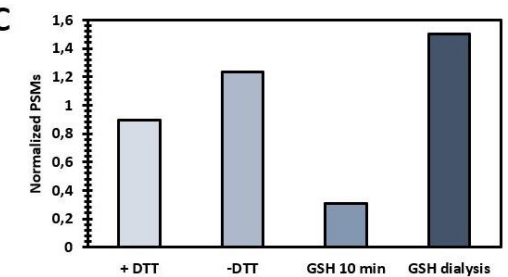

E

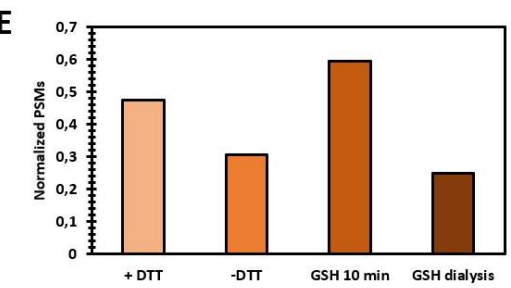

G

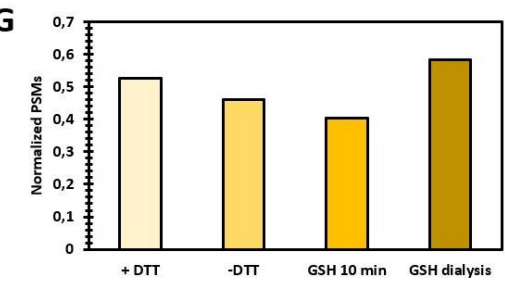

B

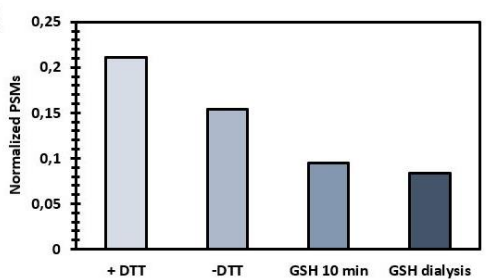

D

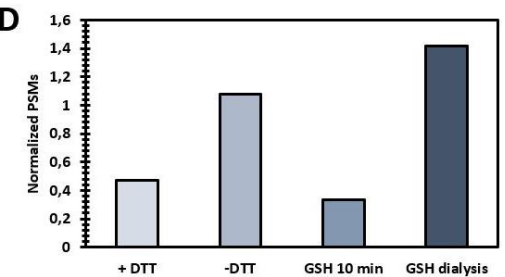

$F$

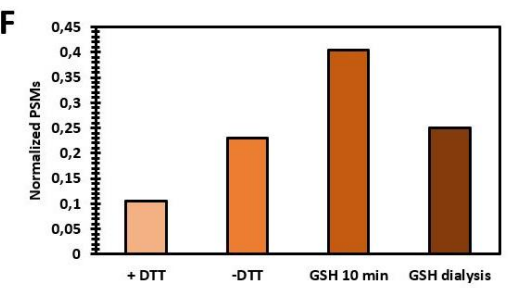

H

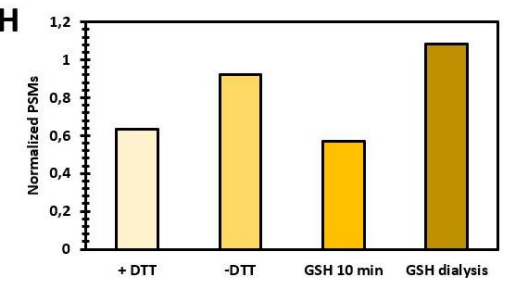

Figure SR5. Analysis of the strep-MBP-ROXY9 redox state by LC-MS/MS. Strep-MBPROXY9 was purified from insect cells in the presence of DTT and subjected to different oxidizing and reducing treatments: (1) Dialysis to remove DTT; (2) Dialysis to remove DTT and then incubated for 10 min with GSH; (3) Dialysis against buffer containing GSH, to remove DTT while simultaneously applying GSH. All samples were labeled with IAM (reduced cysteins) and iodoTMT (oxidized cysteines), separated by $10 \%$ non-reducing SDS-PAGE and analyzed by LC-MS/MS as described in Methods (pages 109-110 and 115-119). To allow quantification, the number of different PSMs for each peptide and modification was normalized to the number of PSMs of an unmodified peptide resulting from the MBP tag (VNYGVTVLPTFK [1337,74636 Da]). As this allows only comparison of the abundance of specific peptides between treatments, the values for the normalized PSMs were plotted accordingly: active site-containing peptides with three $(\mathbf{A})$, two $(\mathbf{B})$, one $(\mathbf{C})$ or none $(\mathbf{D})$ reduced cysteines; peptides containing $\mathrm{Cys}^{49}$ in a reduced $(\mathbf{E})$ or oxidized $(\mathbf{F})$ state; peptides containing $\mathrm{Cys}^{61}$ in a reduced $(\mathbf{G})$ or oxidized state $(\mathbf{H})$.). Please note that the diagrams show only the properly cleaved and fully labelled peptides of each sample. They are the most abundant peptides in each sample. All other peptides are listed in Supplementary table SR1 
Supplementary figures

A

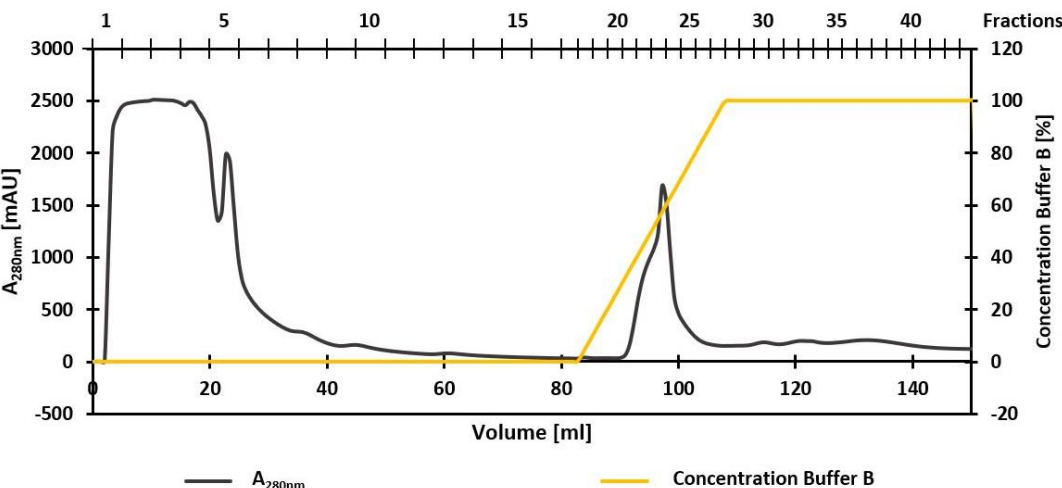

B

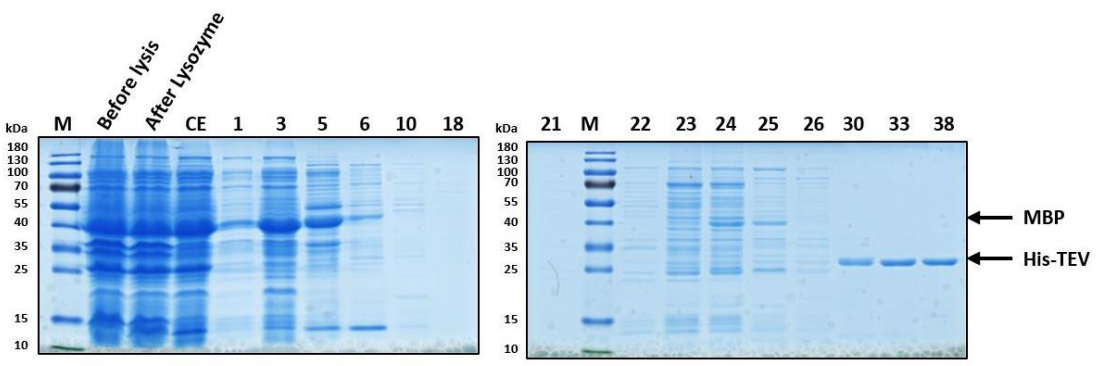

Figure SR6. Purification of His-TEV. His-TEV was expressed in E. coli BL21. Cells were lysed using lysozyme and sonication. The protein was purified via its His tag using an ÄKTA purification system. Purification was controlled by following the changes in $A_{280 \mathrm{~mm}}(\mathbf{A})$. Buffer B contains $250 \mathrm{mM}$ imidazole. $20 \mu \mathrm{l}$ of each fraction were separated by $12 \%$ SDS-PAGE (B). In additon, a cell suspension aliquot of $20 \mu \mathrm{l}$ prior to lysis and after lysozyme treatment were analyzed. Prestained PageRuler (Thermo Scientific) was used as a size standard. The gels were subsequently stained with Coomassie Brilliant Blue. $m A U$ - milli absorption units, $M-$ Marker, $C E$ - crude extract, 1 to 38 - fraction number. 
A

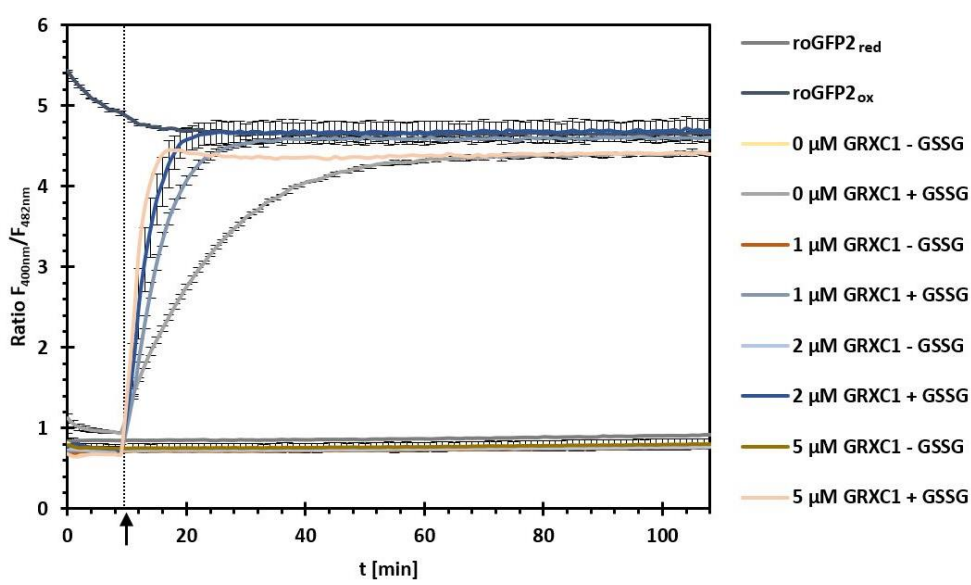

B
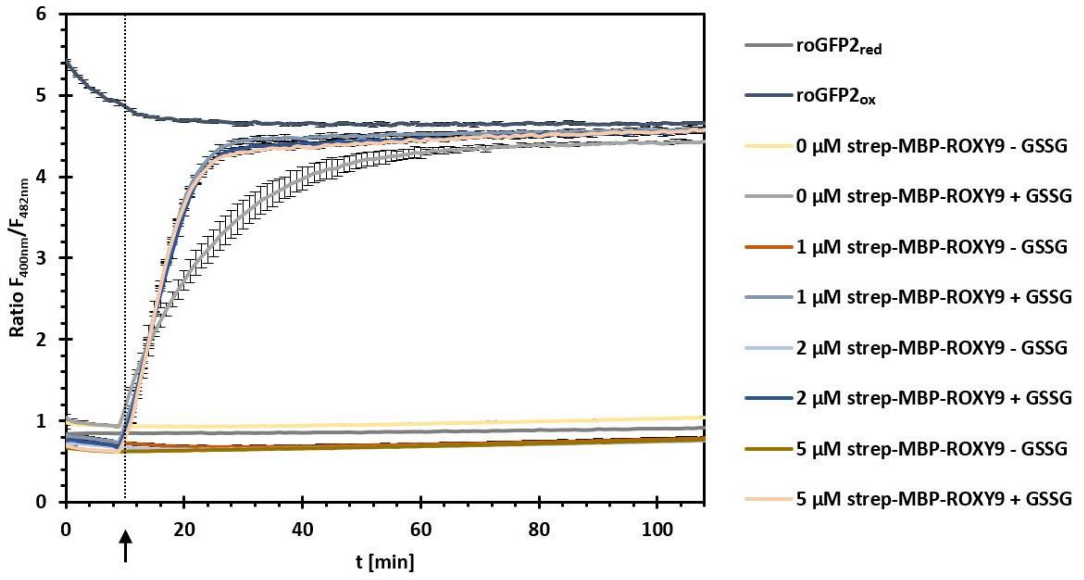

Figure SR7. In vitro oxidase activity of strep-MBP-ROXY9 towards roGFP2 when DTT is removed. Strep-MBP-ROXY9 was compared to N-terminally His-tagged GRXC1 regarding the oxidation of roGFP2. Both proteins received a DTT treatment prior to the experiment. After the treatment, DTT was removed via a desalting column. Different amounts of enzyme were incubated with or without GSSG and reduced roGFP2. Oxidized roGFP2 and reduced roGFP2 alone served as controls. The reactions were equilibrated for $10 \mathrm{~min}$. After that, GSSG or buffer were added to initate the reactions (arrow and dashed line). The change in the fluorescence ratio was monitored over time. Values represent mean $\pm \mathrm{SD}$. For roGFP $2_{\text {red, }}$, $1 \mu \mathrm{M}$ GRXC1 - GSSG, and $5 \mu \mathrm{M}$ GRXC1 + GSSG only two replicates were prepared. Thus, no SD could be calculated. 
Supplementary figures

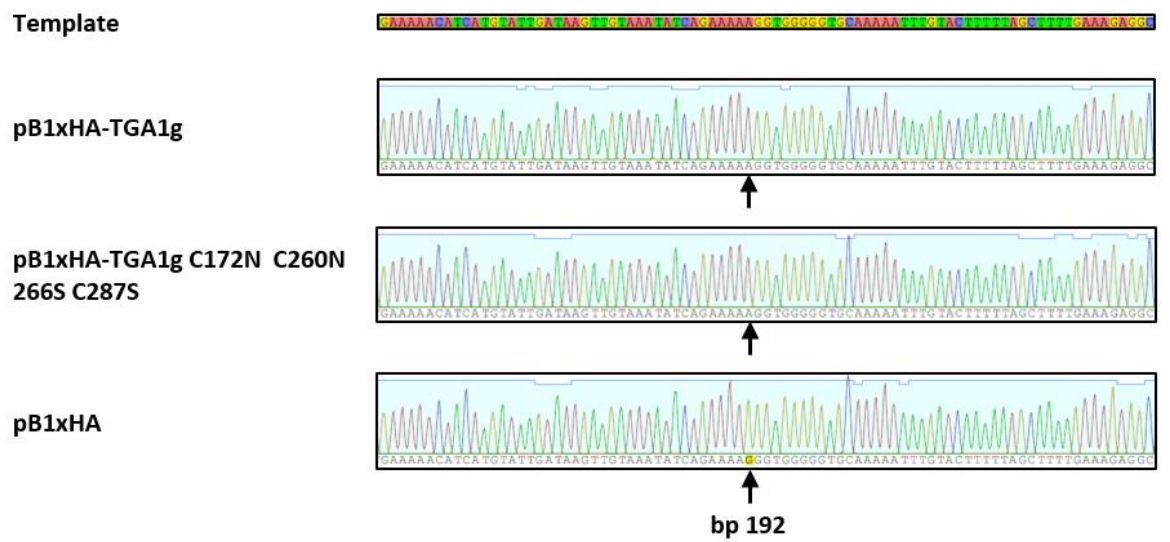

Figure SR8. Mutation occuring in the 3'UTR of the 1xHA complementation construct. The indicated plasmids were sequenced with primer rev23. Sequence analysis was done using Geneious. An excerpt of the alignments in the region of the 3'UTR is shown. The arrow points at the $192^{\text {nd }}$ bp after the stop codon of TGA1. 

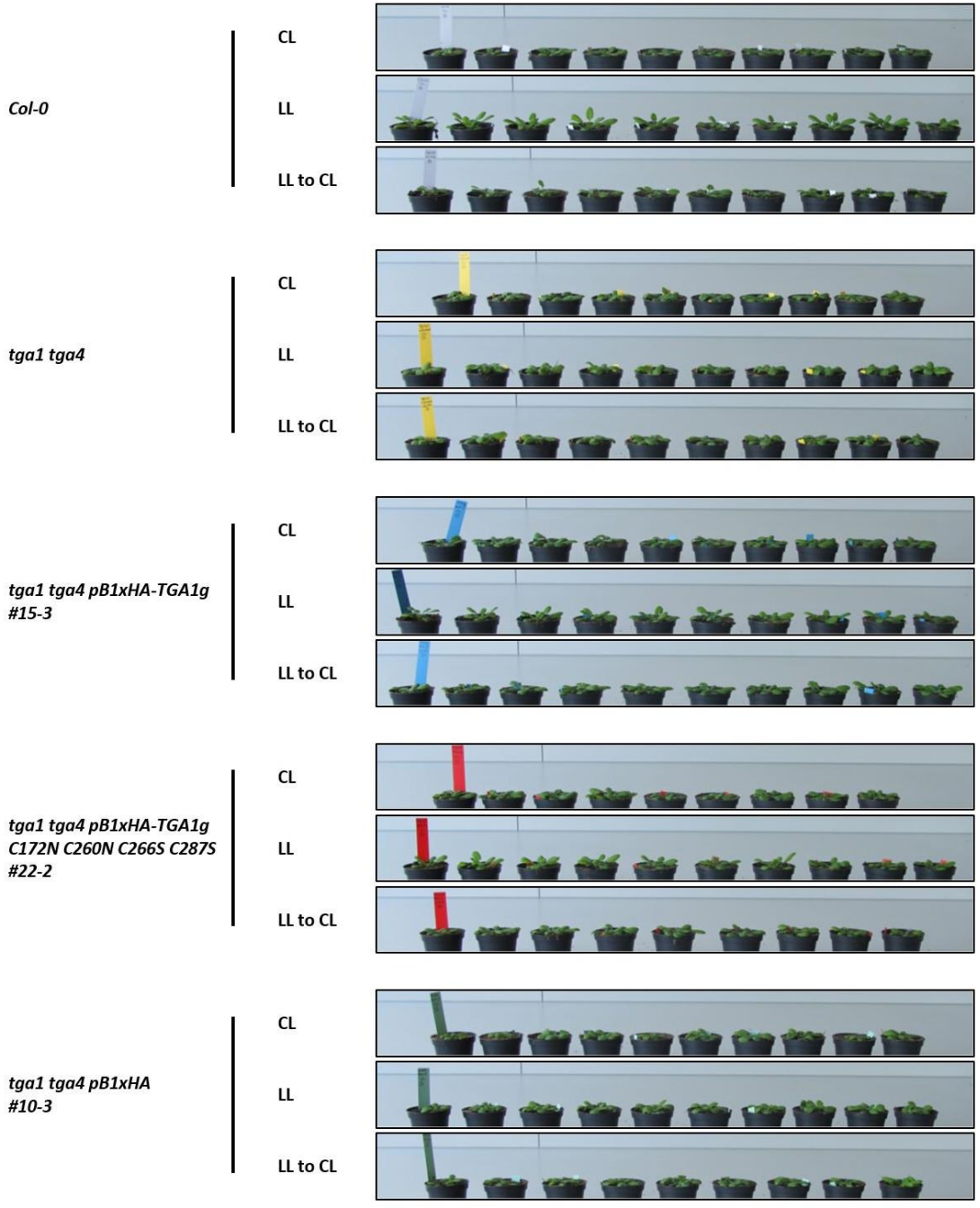

Figure SR9, part I. Analysis of hyponastic growth of the TGA1 complementation lines. Selected complementation lines of the tga 1 tga 4 mutant were grown together with the tga 1 tga 4 mutant and Col-0 wildtype plants for 4 weeks in $12 \mathrm{~h}$ day and then treated with CL, LL or first with LL followed by CL, as described in Methods (page 59). Pictures of the plants were taken with a Canon PowerShot G11 camera. The images above belong to the experiment shown in Figure R25A. $C L$ - control light, $L L-$ low light, $L L$ to $C L-$ shift from low light to control light. tgal tga4 corresponds to tga1 tga4 + pB1xHA-TGA1g \#22-7, which lost its transgene during segregation as a beterosygous plant. 
Supplementary figures

Col-O
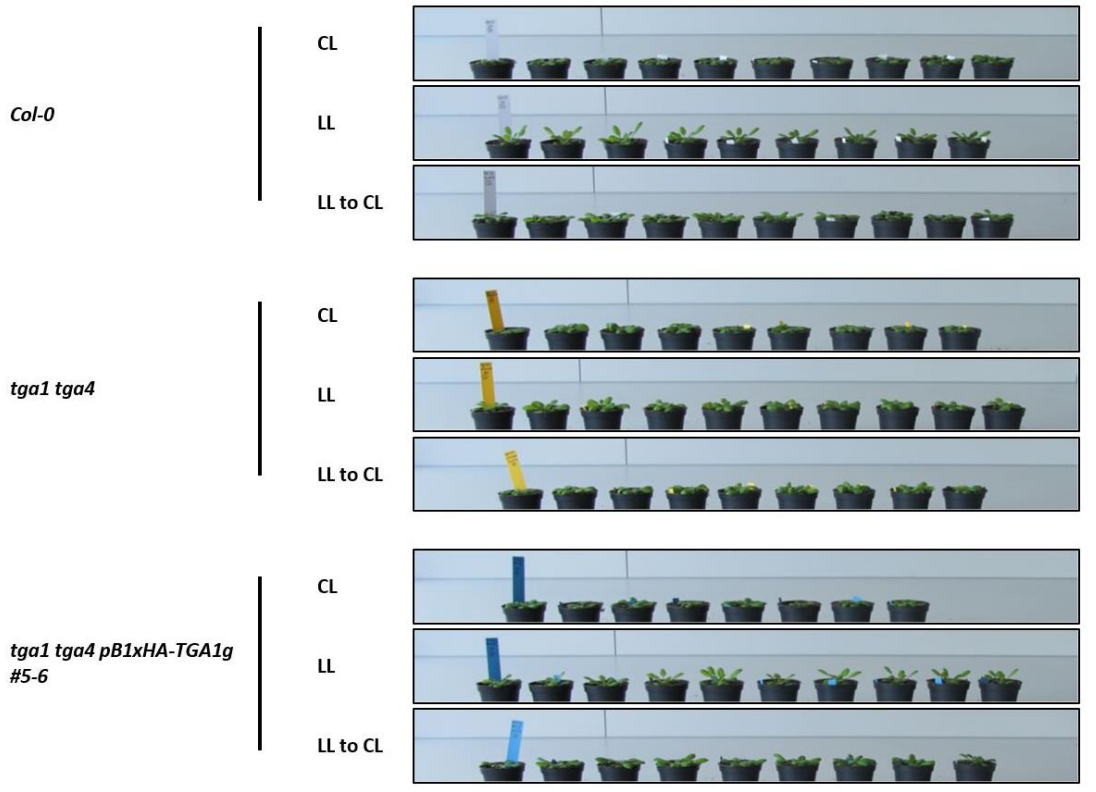

tga1 tga4 pB1xHA-TGA1g
C172N C260N C266S C287S
$\# 15-15$

CL

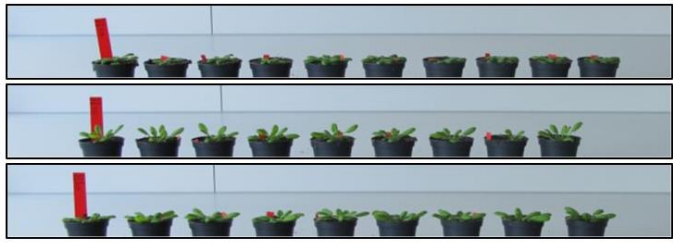

tga1 tga4 PB1xHA

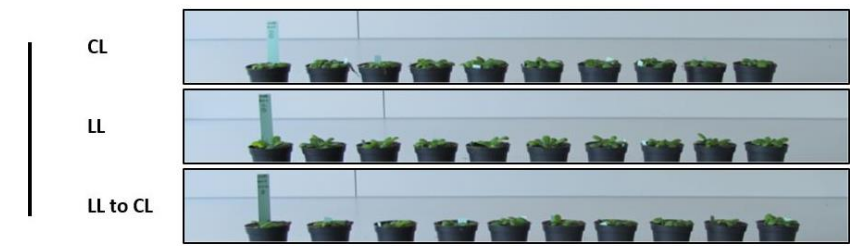

\#11-2

Figure SR9, part II. Analysis of hyponastic growth of the TGA1 complementation lines. Selected complementation lines of the tga 1 tga 4 mutant were grown together with the tga 1 tga4 mutant and Col- 0 wildtype plants for 4 weeks in $12 \mathrm{~h}$ day and then treated with CL, LL or first with LL followed by CL, as described in Methods (page 59). Pictures of the plants were taken with a Canon PowerShot G11 camera. The images above belong to the experiment shown in Figure R25B. CL - control light, $L L$ - low light, LL to CL - shift from low light to control light. tga1 tga4 corresponds to tga1 tga4 $+p B 1 \times H A-T G A 1 g$ \#22-7, which lost its transgene during segregation as a beterozygous plant. 

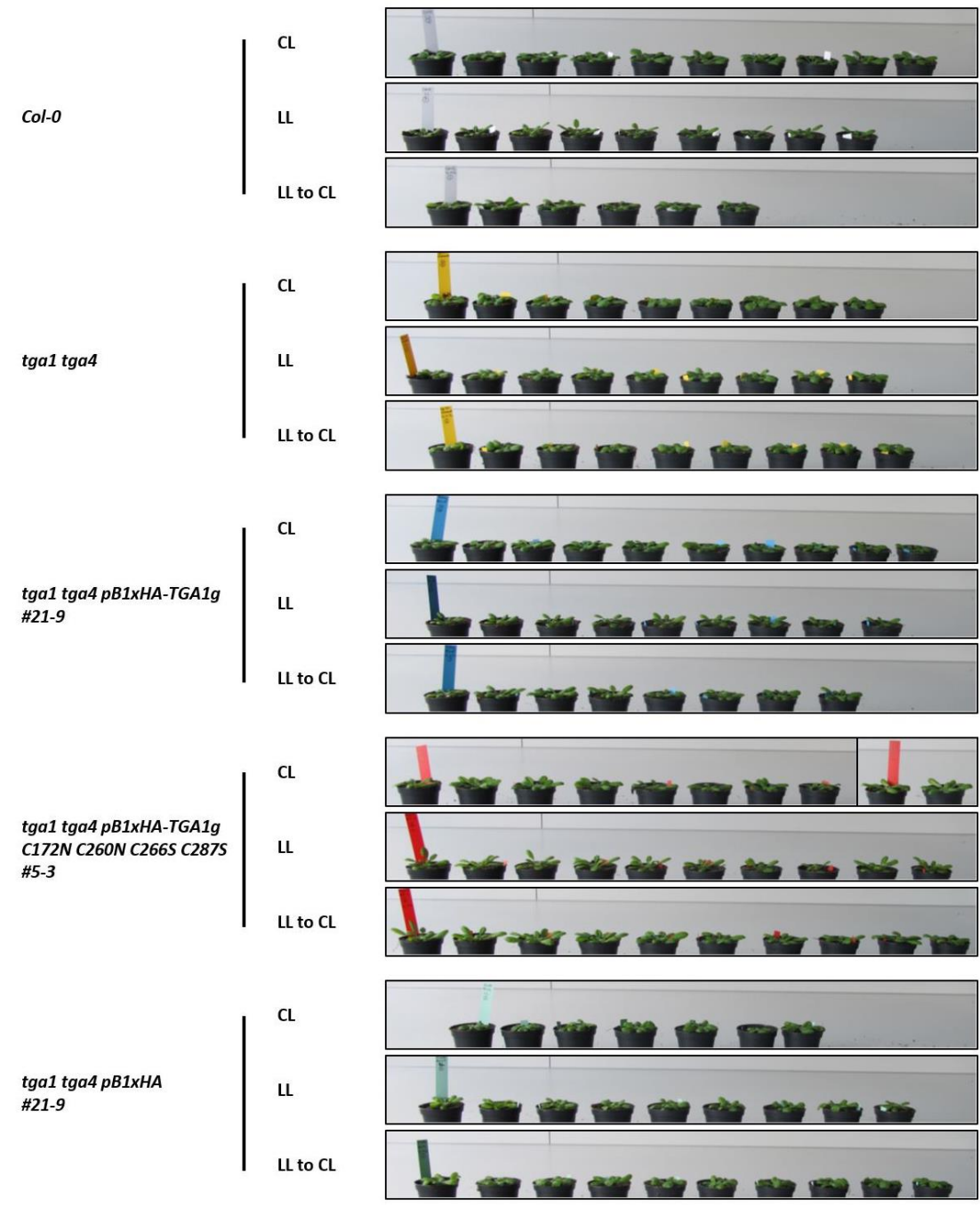

Figure SR9, part III. Analysis of hyponastic growth of the TGA1 complementation lines. Selected complementation lines of the tgal tgat mutant were grown together with the tga 1 tga 4 mutant and Col-0 wildtype plants for 4 weeks in $12 \mathrm{~h}$ day and then treated with CL, LL or first with LL followed by CL, as described in Methods (page 59). Pictures of the plants were taken with a Canon PowerShot G11 camera. The images above belong to the experiment shown in Figure R25C. CL - control light, $L L$ - low light, $L L$ to $C L-$ shift from low light to control light. tga1 tga4 corresponds to tga1 tga4 $+p B 1 \times H A-T G A 1 g$ \#22-7, which lost its transgene during segregation as a heterosygous plant. 
Supplementary figures

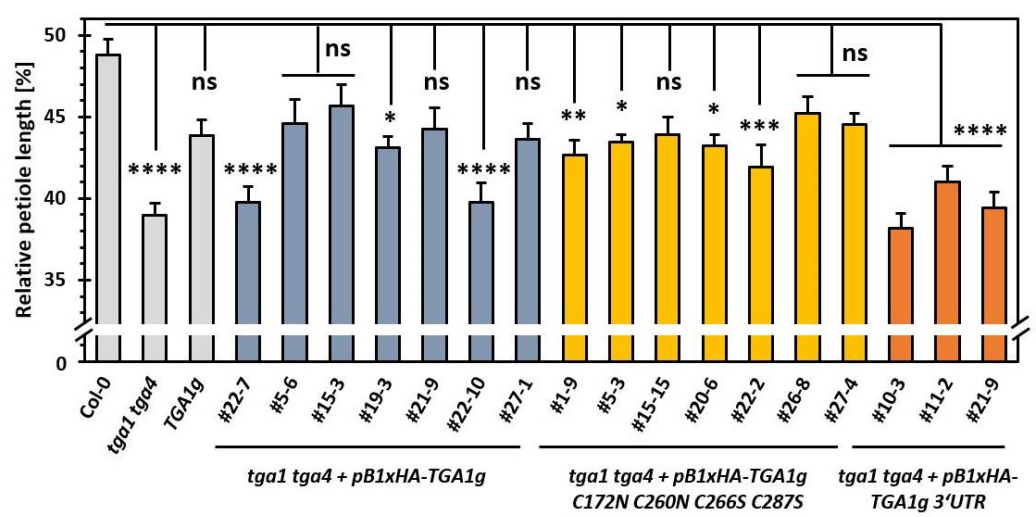

Figure SR10. Measurement of the relative petiole length of the TGA1 complementation lines. Col-0, tga1 tga4, a complementation line (TGA1g) from the Department of Botany (Prof. Dr. Yuelin Zhang, UBC Vancouver [222,223,262]) and selected complementation lines constructed during this work were grown for 4 weeks in $12 \mathrm{~h}$ day. Either leaf 7 or 8 from at least 6 different plants was detached and photographed from the top with a Canon PowerShot G11 camera. The petiole length and the blade length were measured using Image J and the petiole length relative to the entire leaf length computed. Bars represent mean \pm SEM. Statistical analysis (One-way-ANOVA and Tukey test) was performed using GraphPad Prism 8.0.1 assuming normally distributed data. Adjusted $\mathrm{p}$-values in comparison to Col-0 are indicated above the bars: ns - non-significant, $\mathrm{p}>0.05,{ }^{*}$ - $\mathrm{p} \leq 0.05,{ }^{* *}$ - $\mathrm{p}$-value $\leq 0.01$, *** p-value $\leq 0.001, * * * *$ - p-value $\leq 0.0001$. 
A

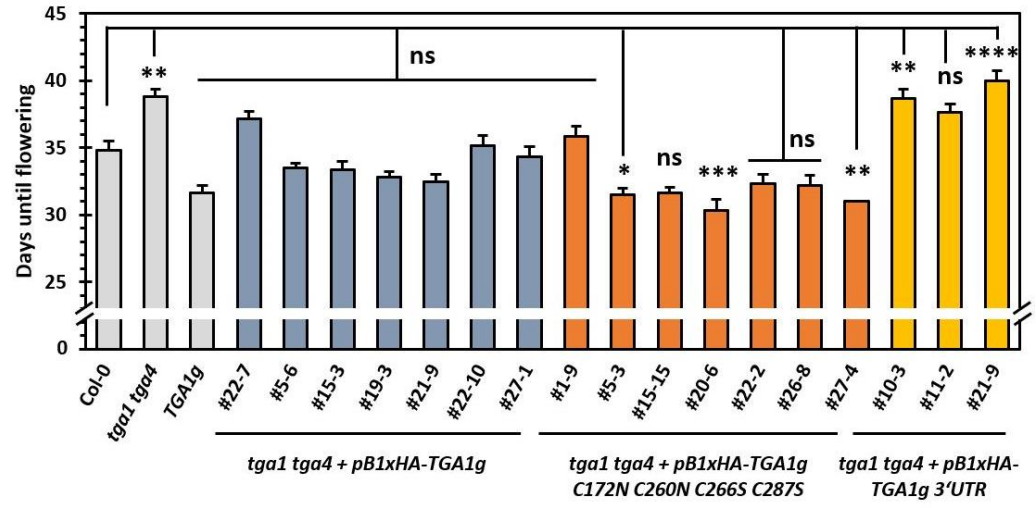

B

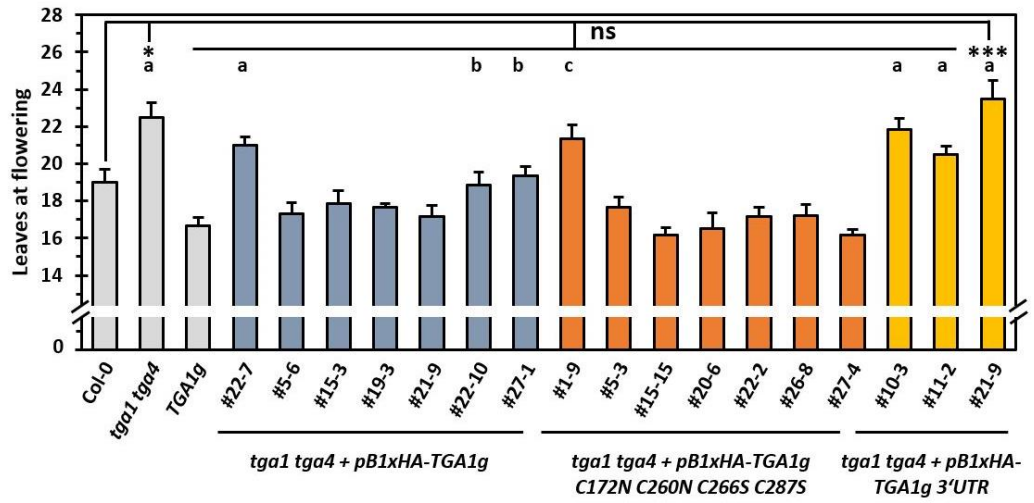

Figure SR11, part I. Flowering time point and leave number at flowering of TGA1 complementation lines. Col-0, tga1 tga4, TGA1g [222,223,262] and tga1 tga4 mutants complemented with the constructs shown in Figure 24A were grown in a long day climate chamber. The number of days was determined until a floral stem of $1 \mathrm{~cm}$ lenght was visible (A). At this time point, the number of leaves was counted, as well (B). Bars are mean \pm SEM from at least 5 replicates. $a$-smaller leaves in between counted leaves for all replicates, $b$ - smaller leaves in between counted leaves for one plant out of six plants, $c$ - smaller leaves in between counted leaves for two or three out of six plants 
Supplementary figures

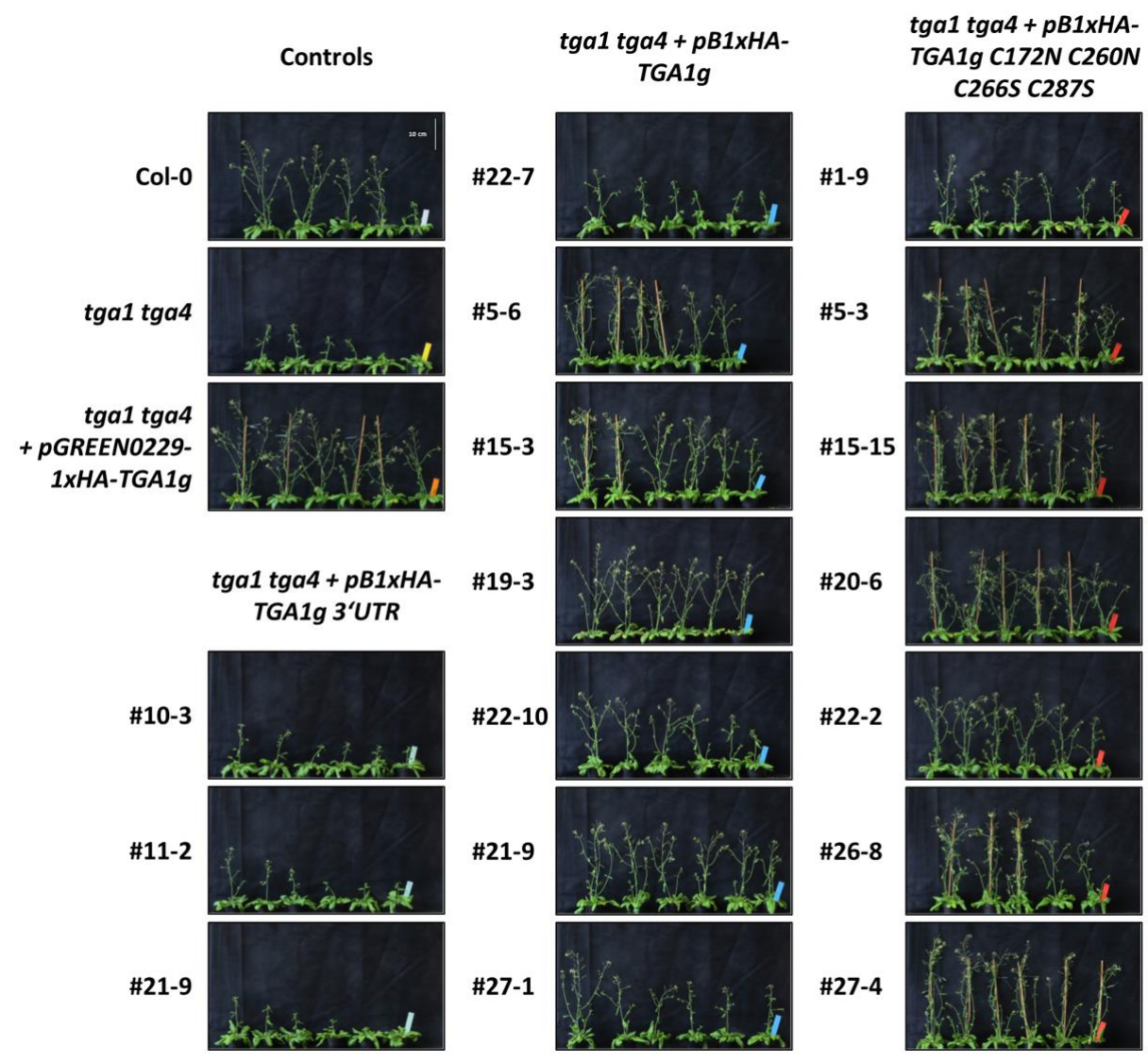

Figure SR11, part II. Flowering time point and leave number at flowering of TGA1 complementation lines. Col-0, tga 1 tga4, TGA1g and tga 1 tga4 mutants complemented with the constructs shown in Figure 24A were grown in a long day climate chamber. As soon as the last plant had flowered, pictures of all plants were taken using a Canon PowerShot G11 camera. Large floral stems were bound to sticks. The scale bar corresponds to roughly $10 \mathrm{~cm}$. 
Supplementary tables 
Supplementary tables

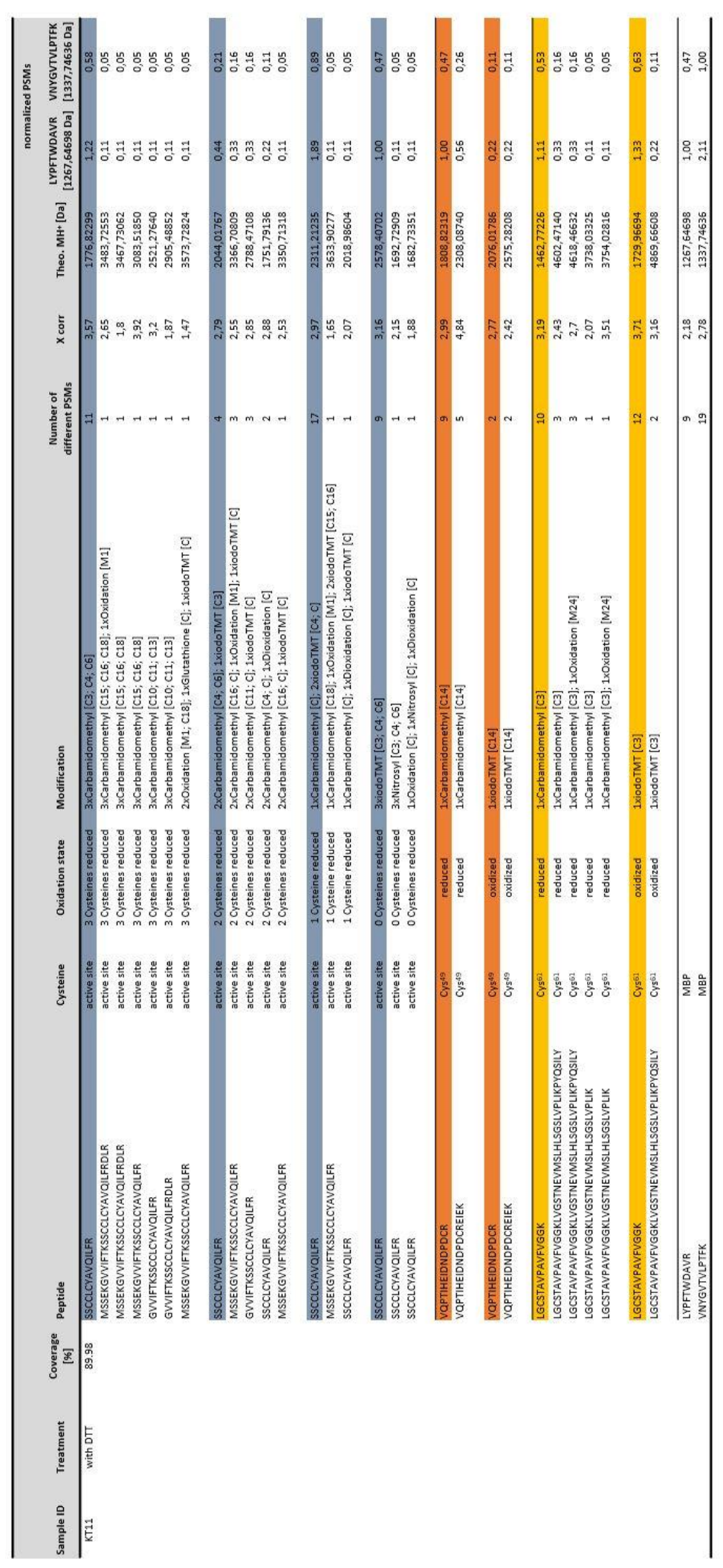


Table SR1. Mass spectrometric analysis of the strep-MBP-ROXY9 oxidation state, part I. Strep-MBP-ROXY9 was purified in the presence of ca. $1 \mathrm{mM}$ DTT. The reduced cysteines were labeled with iodoacetamide, while the oxidized cysteine residues were labeled with iodoTMT ${ }^{0}-126$ and then separated by $10 \%$ non-reducing SDS-PAGE (KT11). The gels were stained with Colloidal Coomassie. Bands at ca. $59 \mathrm{kDa}$ were cut out from the gel and prepared for LC-MS/MS analysis as described in Methods (pages 115-119). The dataset was searched and analyzed using PeptideDiscoverer 2.2 (Thermo Scientific). The modifications searched for were reduced cysteines (Thiol), nitrosylated cysteines (Nitrosyl), glutathionylated cysteines (Glutathione), sulfenic acids (Oxidation), sulfinic acids (Dioxidation), IAM-labelled cysteines (Carbamidomethyl), iodoTMT-labeled cysteines (iodoTMT), and oxidized methionines (Oxidation). The table lists the coverage for each sample, and all identified peptides with their modifications on cysteines $(\mathrm{C})$ and methionines $(\mathrm{M})$ and their mass (Theo. $\left.\mathrm{MH}^{+}[\mathrm{Da}]\right)$. The position of each modified residue in the corresponding peptide is given in brackets (e.g.[C3]). If the position of the modification could not be identified, the residue is only indicated by e.g. $[C]$. The frequency in which a given modification occurs within a peptide is written before the name of the modification (e.g. 3xCarbamidomethyl). The quality of each identification is given by the X corr value. The number of identifications (different PSMs) was normalized to estimate the redox state of the protein. For this, the number of different PSMs for each peptide was divided by the number of different PSMs of two different peptides resulting from the MBP tag. Those peptides plotted in Figure R15 and SR5 are highlighted. 
Supplementary tables

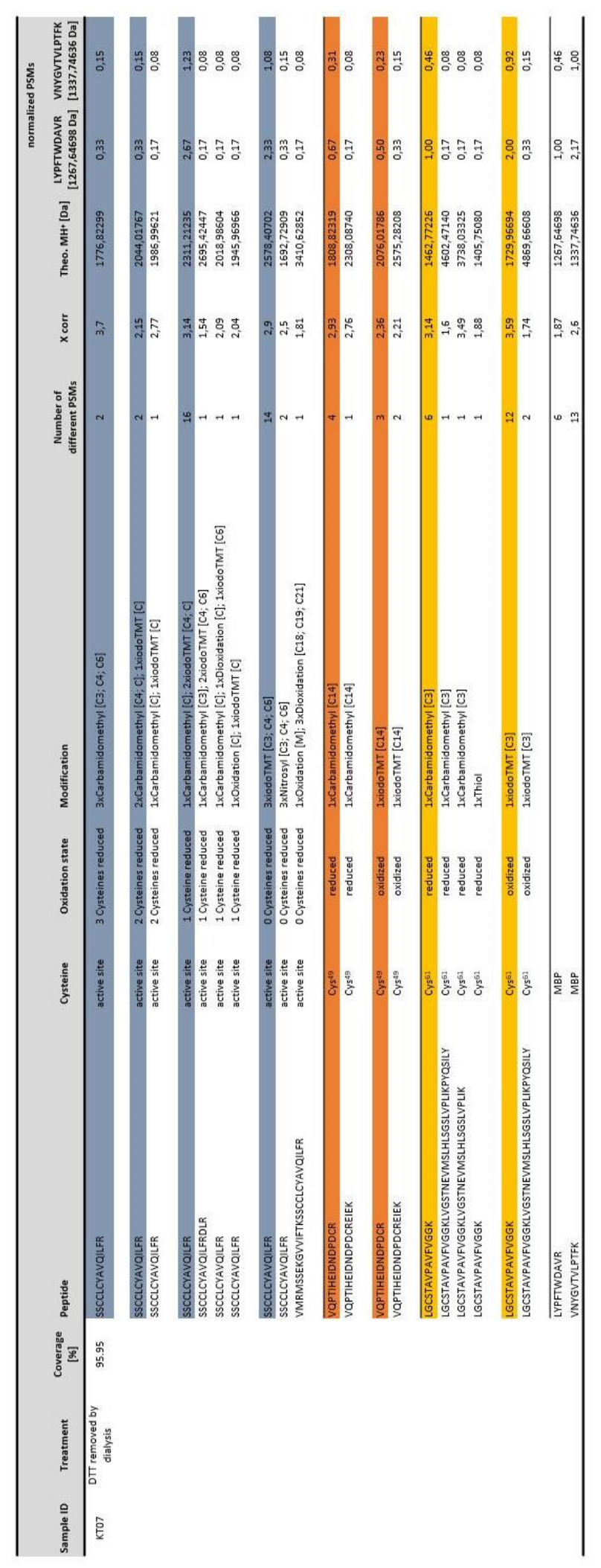


Table SR1. Mass spectrometric analysis of the strep-MBP-ROXY9 oxidation state, part II. Strep-MBP-ROXY9 purified in the presence of ca. $1 \mathrm{mM} \mathrm{DTT}$ was dialyzed to remove DTT. The reduced cysteines were labeled with iodoacetamide, while the oxidized cysteine residues were labeled with iodoTMT ${ }^{0}-126$ and then separated by $10 \%$ non-reducing SDSPAGE (KT07). The gels were stained with Colloidal Coomassie. Bands at ca. $59 \mathrm{kDa}$ were cut out from the gel and prepared for LC-MS/MS analysis as described in Methods (pages 115119). The dataset was searched and analyzed using PeptideDiscoverer 2.2 (Thermo Scientific). The modifications searched for were reduced cysteines (Thiol), nitrosylated cysteines (Nitrosyl), glutathionylated cysteines (Glutathione), sulfenic acids (Oxidation), sulfinic acids (Dioxidation), IAM-labelled cysteines (Carbamidomethyl), iodoTMT-labeled cysteines (iodoTMT), and oxidized methionines (Oxidation). The table lists the coverage for each sample, and all identified peptides with their modifications on cysteines (C) and methionines (M) and their mass (Theo. $\left.\mathrm{MH}^{+}[\mathrm{Da}]\right)$. The position of each modified residue in the corresponding peptide is given in brackets (e.g.[C3]). If the position of the modification could not be identified, the residue is only indicated by e.g. [C]. The frequency in which a given modification occurs within a peptide is written before the name of the modification (e.g. $3 \mathrm{x}$ Carbamidomethyl). The quality of each identification is given by the $\mathrm{X}$ corr value. The number of identifications (different PSMs) was normalized to estimate the redox state of the protein. For this, the number of different PSMs for each peptide was divided by the number of different PSMs of two different peptides resulting from the MBP tag. Those peptides plotted in Figure R15 and SR5 are highlighted. 
Supplementary tables

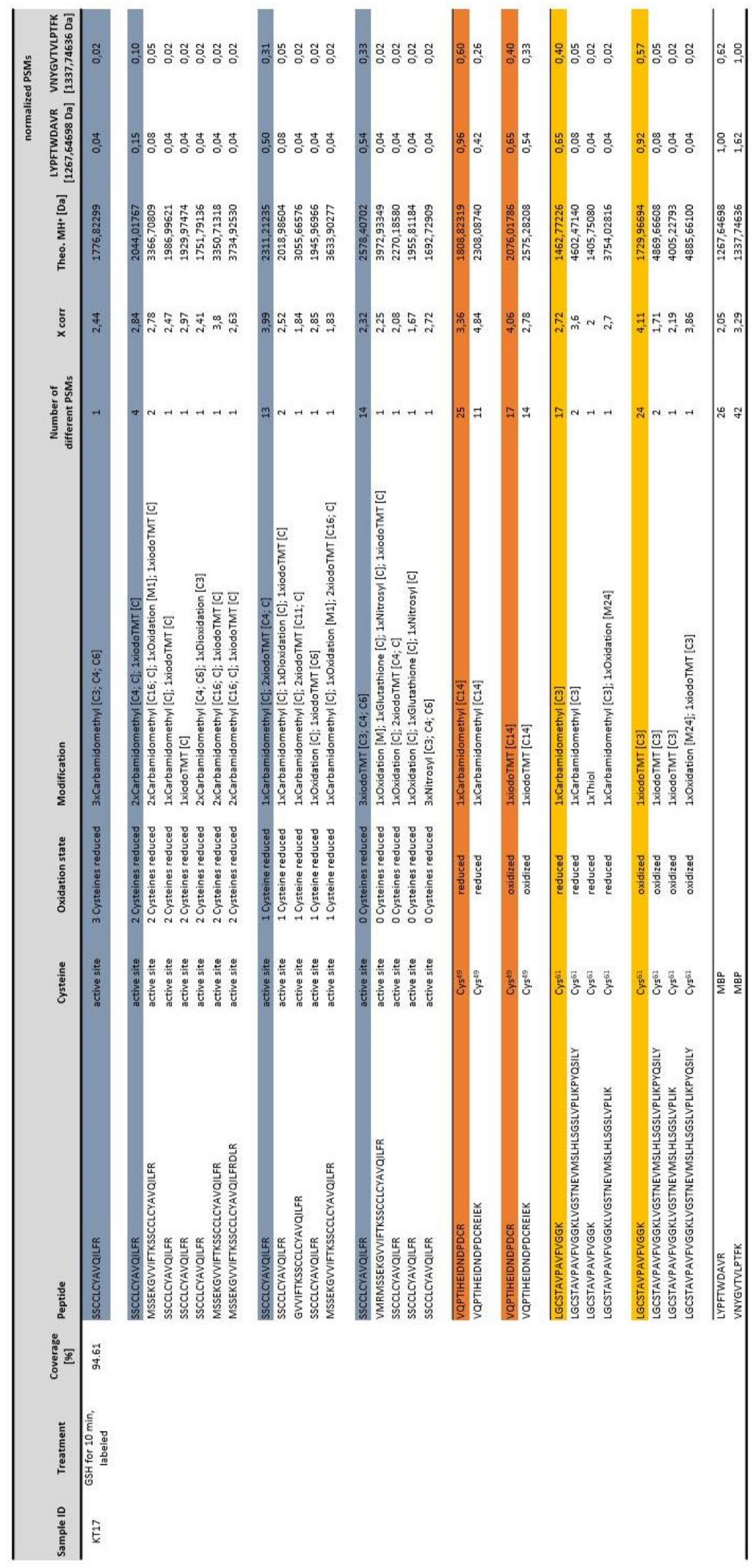


Table SR1. Mass spectrometric analysis of the strep-MBP-ROXY9 oxidation state, part III. Strep-MBP-ROXY9 was purified in the presence of ca. 1 mM DTT. After DTT had been removed by dialysis, the protein was treated with glutathione for $10 \mathrm{~min}$. The reduced cysteines were labeled with iodoacetamide, while the oxidized cysteine residues were labeled with iodoTMT ${ }^{0}-126$ and then separated by $10 \%$ non-reducing SDS-PAGE (KT17). The gels were stained with Colloidal Coomassie. Bands at ca. $59 \mathrm{kDa}$ were cut out from the gel and prepared for LC-MS/MS analysis as described in Methods (pages 115-119). The dataset was searched and analyzed using PeptideDiscoverer 2.2 (Thermo Scientific). The modifications searched for were reduced cysteines (Thiol), nitrosylated cysteines (Nitrosyl), glutathionylated cysteines (Glutathione), sulfenic acids (Oxidation), sulfinic acids (Dioxidation), IAM-labelled cysteines (Carbamidomethyl), iodoTMT-labeled cysteines (iodoTMT), and oxidized methionines (Oxidation). The table lists the coverage for each sample, and all identified peptides with their modifications on cysteines $(\mathrm{C})$ and methionines $(\mathrm{M})$ and their mass (Theo. $\left.\mathrm{MH}^{+}[\mathrm{Da}]\right)$. The position of each modified residue in the corresponding peptide is given in brackets (e.g.[C3]). If the position of the modification could not be identified, the residue is only indicated by e.g. $[C]$. The frequency in which a given modification occurs within a peptide is written before the name of the modification (e.g. 3xCarbamidomethyl). The quality of each identification is given by the $\mathrm{X}$ corr value. The number of identifications (different PSMs) was normalized to estimate the redox state of the protein. For this, the number of different PSMs for each peptide was divided by the number of different PSMs of two different peptides resulting from the MBP tag. Those peptides plotted in Figure R15 and SR5 are highlighted. 
Supplementary tables

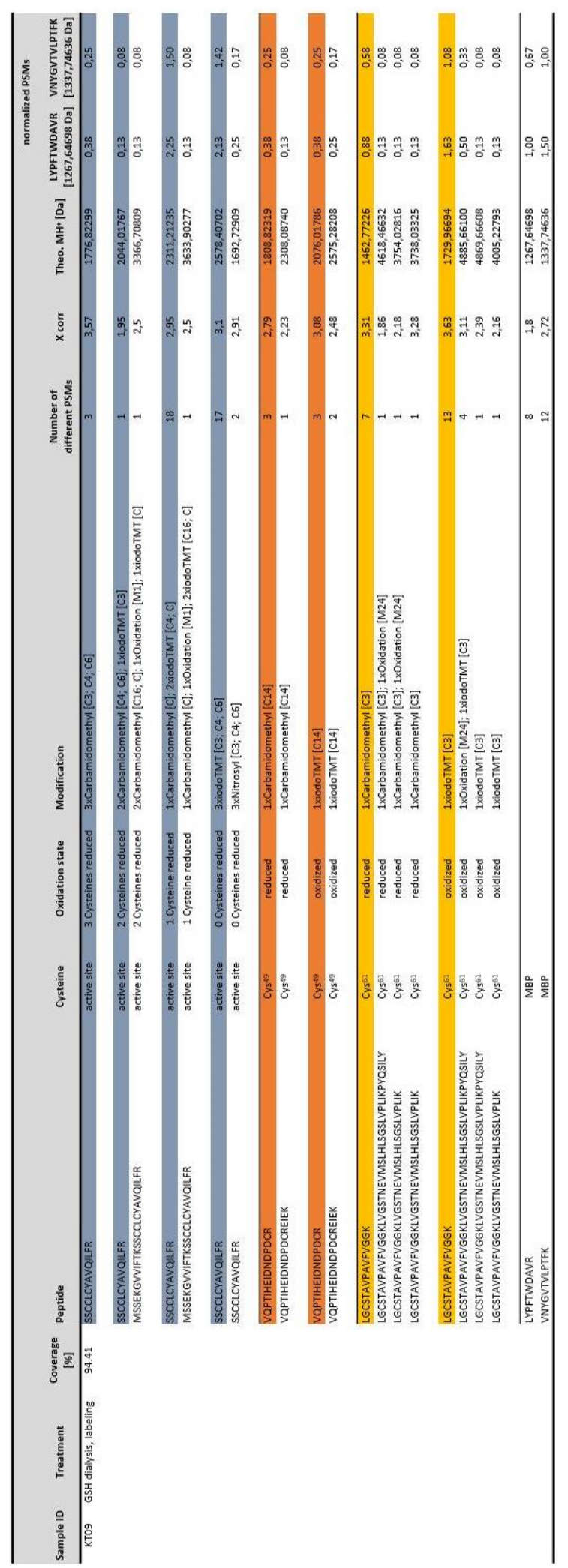


Table SR1. Mass spectrometric analysis of the strep-MBP-ROXY9 oxidation state, part IV. Strep-MBP-ROXY9 was purified in the presence of ca. $1 \mathrm{mM}$ DTT and then dialysed against buffer containing glutathione. This way, DTT was removed while glutathione was introduced into the sample. The reduced cysteines were labeled with iodoacetamide, while the oxidized cysteine residues were labeled with iodoTMT0-126 and then separated by $10 \%$ nonreducing SDS-PAGE (KT09). The gels were stained with Colloidal Coomassie. Bands at ca. $59 \mathrm{kDa}$ were cut out from the gel and prepared for LC-MS/MS analysis as described in Methods (pages 115-119). The dataset was searched and analyzed using PeptideDiscoverer 2.2 (Thermo Scientific). The modifications searched for were reduced cysteines (Thiol), nitrosylated cysteines (Nitrosyl), glutathionylated cysteines (Glutathione), sulfenic acids (Oxidation), sulfinic acids (Dioxidation), IAM-labelled cysteines (Carbamidomethyl), iodoTMT-labeled cysteines (iodoTMT), and oxidized methionines (Oxidation). The table lists the coverage for each sample, and all identified peptides with their modifications on cysteines (C) and methionines $(\mathrm{M})$ and their mass (Theo. $\left.\mathrm{MH}^{+}[\mathrm{Da}]\right)$. The position of each modified residue in the corresponding peptide is given in brackets (e.g.[C3]). If the position of the modification could not be identified, the residue is only indicated by e.g. [C]. The frequency in which a given modification occurs within a peptide is written before the name of the modification (e.g. 3xCarbamidomethyl). The quality of each identification is given by the $\mathrm{X}$ corr value. The number of identifications (different PSMs) was normalized to estimate the redox state of the protein. For this, the number of different PSMs for each peptide was divided by the number of different PSMs of two different peptides resulting from the MBP tag. Those peptides plotted in Figure R15 and SR5 are highlighted. 
Material 
Material

\section{Organisms}

\section{Bacterial strains}

\begin{tabular}{|c|c|c|c|}
\hline Species & Strain & Genotype & Reference \\
\hline $\begin{array}{l}\text { Escherichi } \\
\text { a coli }\end{array}$ & $\mathrm{DH} 5 \alpha$ & $\begin{array}{l}\text { fhuA2 lac(del)U169 pho A glnV44 Ф80' } \\
\text { lacZ(del)M15 gyrA96 recA1 relA1 end A1 thi-1 } \\
\text { hsdR17 }\end{array}$ & $\begin{array}{l}\text { Woodcock et al. } \\
\text { (1989) [276] }\end{array}$ \\
\hline $\begin{array}{l}\text { Escherichi } \\
\text { a coli }\end{array}$ & DB3.1 & $\begin{array}{l}\mathrm{F}-\text { gyrA462 endA1 } \Delta(\mathrm{sr} 1-r e c \mathrm{~A}) \text { mcrB mrr } \\
\text { bsdS20(rB-, mB-) supE } 44 \text { ara-14 galK2 lac } \mathrm{Y} 1 \\
\text { proA2 rpsL20(SmR) xyl-5 } \lambda \text { - leu mtl }\end{array}$ & $\begin{array}{l}\text { Thermo Fisher } \\
\text { Scientific (Bernard et } \\
\text { al. (1993)) [277] }\end{array}$ \\
\hline $\begin{array}{l}\text { Escherichi } \\
\text { a coli }\end{array}$ & $\begin{array}{l}\text { BL21 } \\
\text { (DE3) }\end{array}$ & $B d c m$ omp T hsdS $\left(\mathrm{r}_{\mathrm{B}}^{-} \mathrm{m}_{\mathrm{B}}^{-}\right) \mathrm{gal}$ & $\begin{array}{l}\text { Studier \& Moffatt } \\
\text { (1986) [278] }\end{array}$ \\
\hline $\begin{array}{l}\text { Escherichi } \\
\text { a coli }\end{array}$ & $\begin{array}{l}\text { BL21 } \\
\text { Star } \\
(\mathrm{DE} 3)\end{array}$ & $\mathrm{F}^{-} \operatorname{omp} T$ bsdS ${ }_{B}\left(\mathrm{r}_{\mathrm{B}^{-}} \mathrm{m}_{\mathrm{B}^{-}}\right)$gal dcm rne131 (DE3) & $\begin{array}{l}\text { Thermo Fisher } \\
\text { Scientific }\end{array}$ \\
\hline $\begin{array}{l}\text { Agrobacte } \\
\text { rium } \\
\text { tumefacien } \\
s\end{array}$ & $\begin{array}{l}\text { GV310 } \\
1\end{array}$ & PMP90RK rifr, gmr & $\begin{array}{l}\text { Koncz \& Schell } \\
\text { (1986) [279] }\end{array}$ \\
\hline
\end{tabular}

\section{Insect cells}

\begin{tabular}{llll}
\hline Insect cells & Line & Genotype & Reference \\
\hline Trichoplusia ni & High Five & unknown & Thermo Fisher Scientific
\end{tabular}

\section{Arabidopsis thaliana lines}

\begin{tabular}{ll}
\hline Line & Reference \\
\hline Col-0 & NASC stock no. N1902 \\
Col-0 + HA-ROXY9 \#7-18 & Li, 2017 [155,156]
\end{tabular}




\begin{tabular}{|c|c|}
\hline Line & Reference \\
\hline Col-0 + HA-ROXY9 \#17-4 (genotype: Col-0) & This work \\
\hline $\begin{array}{l}\text { Col-0 + HA-ROXY9 \#2-22, 4-40, 5-18, 7-25, 17-3, 29-1, and } \\
\text { 30-7 }\end{array}$ & This work \\
\hline $\begin{array}{l}\text { Col-0 + HA-ROXY9 CCLCA \#1-3, 9-10, 18-10, 29-2, and 37- } \\
5\end{array}$ & This work \\
\hline $\begin{array}{l}\text { Col-0 + HA-ROXY9 CPYC \#13-8, 18-1, 19-10, 27-3, 29-3, } 31- \\
\text { 4, and 38-5 }\end{array}$ & This work \\
\hline $\operatorname{tg} a 1 \operatorname{tg} a 4$ & $\begin{array}{l}\text { Kesarwani et al. (2007) } \\
\text { [221] }\end{array}$ \\
\hline tga1 tga $4+p B 1 x H A-T G A 1 g \# 22-7$ (genotype: tga1 tga4) & This work \\
\hline tga1 tga4 + & This work \\
\hline $\begin{array}{l}\text { tga1 tga4 }+p B 1 \times H A-T G A 1 g \# 5-6,15-3,19-3,21-9,22-10, \text { and } \\
27-1\end{array}$ & This work \\
\hline $\begin{array}{l}\text { tga1 tga4 }+p B 1 x H A-T G A 1 g C 172 N \text { C260N C266S C287S } \\
\# 1-9,5-3,15-15,20-6,22-2,26-8 \text {, and } 27-4\end{array}$ & This work \\
\hline \multirow[t]{3}{*}{ tga1 tga4 + pGREEN II 0229-HA-TGA1g } & Shearer et al. (2012); \\
\hline & Wang \& Fobert (2013); \\
\hline & $\begin{array}{l}\text { Sun et al. }(2017) \\
{[222,223,262]}\end{array}$ \\
\hline tga1 tga4 + pGREEN II 0229-HA-TGA1g C260N C266S & $\begin{array}{l}\text { Department of Botany, } \\
\text { Prof. Dr. Yuelin Zhang, } \\
\text { UBC Vancouver }\end{array}$ \\
\hline
\end{tabular}


Material

\section{Oligo nucleotides}

\section{Primers}

All primers were ordered from Invitrogen. None of them contained 5' or 3' modifications.

Primers designed by others

\begin{tabular}{|c|c|c|}
\hline Primer name & Sequence $\left(5^{\prime} \rightarrow 3^{\prime}\right)$ & Reference \\
\hline MBP 572 for & CAAGGGTCAACCATCCAAAC & Oberdiek, 2018 [174] \\
\hline NdeI-GFP-for & $\begin{array}{l}\text { GAAGCATATGATGGTGAGCAAGG } \\
\text { GCGAG }\end{array}$ & Oberdiek, 2018 [174] \\
\hline pB2GW7 fwd & САСААТСССАСТАТССТTCGCA & $\begin{array}{l}\text { C. Thurow*, } \\
\text { unpublished }\end{array}$ \\
\hline pB2GW7 rev & CATGAGCGAAACССТАTAAGAACC & $\begin{array}{l}\text { C. Thurow*, } \\
\text { unpublished }\end{array}$ \\
\hline rev23 & TTCACACAGGAAACAGCTATGAC & $\begin{array}{l}\text { C. Thurow*, } \\
\text { unpublished }\end{array}$ \\
\hline SEQ-L1 pDONR & $\begin{array}{l}\text { TCGCGTTAACGCTAGCATGGATCT } \\
\text { C }\end{array}$ & $\begin{array}{l}\text { C. Thurow*, } \\
\text { unpublished }\end{array}$ \\
\hline SEQ-L2 pDONR & GTAACATCAGAGATTTTGAGACAC & $\begin{array}{l}\text { C. Thurow*, } \\
\text { unpublished }\end{array}$ \\
\hline T7termrev & CCT'T'TCGGGCTTT'TGTTAGCAG & $\begin{array}{l}\text { C. Thurow*, } \\
\text { unpublished }\end{array}$ \\
\hline TGA1 FOR & $\begin{array}{l}\text { ATGAATTCGACATCGACACATTTT'T } \\
\text { GTGC }\end{array}$ & Li (2017) [155] \\
\hline TGA1 For Rev & $\begin{array}{l}\text { GCACAAAATGTGTCGATGTCGAAT } \\
\text { TCAT }\end{array}$ & $\mathrm{Li}(2017)$ [155] \\
\hline $\begin{array}{l}\text { TGA1 Promoter term } \\
\text { For }\end{array}$ & $\begin{array}{l}\text { GTGCTCTCCAAGGTCTTAGGGAAT } \\
\text { СTC }\end{array}$ & Li (2017) [155] \\
\hline $\begin{array}{l}\text { TGA1 sequencing } 3 \\
2100 \text { For }\end{array}$ & $\begin{array}{l}\text { CCACAAACAGATAATTAGTCCGGT } \\
\text { G }\end{array}$ & $\mathrm{Li}(2017)$ [155] \\
\hline
\end{tabular}




\begin{tabular}{lll}
\hline Primer name & Sequence $\left(\mathbf{5}^{\prime} \mathbf{\rightarrow} \mathbf{3}^{\prime} \mathbf{)}\right.$ & Reference \\
\hline TGA1 sequencing 4 & TGCTTGTTTGACTTTGTGTGATCT & Li (2017) [155] \\
3500 For & AAC & \\
TGA1 sequencing 4 & CAAGAACTCGATCGTGCTAGACAA & Li (2017) [155] \\
4200 For & C & \\
\hline
\end{tabular}

*Department for Plant Molecular Biology and Physiology, Prof. Dr. Chrisitane Gatz, GeorgAugust-Universität Göttingen

\section{Primers designed during this work}

\begin{tabular}{|c|c|c|}
\hline Primer name & Sequence $\left(5^{\prime} \rightarrow 3^{\prime}\right)$ & Purpose \\
\hline $\begin{array}{l}3 \mathrm{xHA} 3 \mathrm{UTR} \\
\text { fusion fwd }\end{array}$ & $\begin{array}{l}\text { CCCTATGACGTCCCGGACTAT } \\
\text { GCAGTCGACTAGGTTTGAGT } \\
\text { TATTTTGTAACAACCAAATGA } \\
\text { AGAAAATGG }\end{array}$ & $\begin{array}{l}\text { Fwd primer to fuse 3xHA tag } \\
\text { from pUBQ10-GW7-HA to } \\
\text { 3'UTR sequence of TGA1 }\end{array}$ \\
\hline $3 \mathrm{xHA}$ rev & $\begin{array}{l}\text { TGCATAGTCCGGGACGTCAT } \\
\text { AGGGATAGCCC }\end{array}$ & $\begin{array}{l}\text { Rev primer to amplify 3xHA tag } \\
\text { from pUBQ10-GW7-HA }\end{array}$ \\
\hline $\begin{array}{l}3 \times \mathrm{xA} \text { TGA1 } \\
\text { fusion fwd }\end{array}$ & $\begin{array}{l}\text { GTCCCGGACTATGCAGTCGA } \\
\text { CATGAATTCGACATCGACACA } \\
\text { TTTTGTGC }\end{array}$ & $\begin{array}{l}\text { Fwd primer to fuse } 3 \times \mathrm{XHA} \text { tag } \\
\text { from pUBQ10-GW7-HA to } \\
\text { genomic } T G A 1 \text { sequence }\end{array}$ \\
\hline $\begin{array}{l}3 x H A-T G A 1 \\
\text { PmeI fwd }\end{array}$ & $\begin{array}{l}\text { ATTGTTTAAACAATTTGTTCT } \\
\text { TTGTTTTCAGTTGAGGAAAAC } \\
\text { TCGAGATGGCATACCCATAC } \\
\text { GACGTTCCGGACTACGC }\end{array}$ & $\begin{array}{l}\text { Fwd cloning primer binding to } \\
\text { pUBQGW7HA 3xHA tag, for } \\
\text { insertion in pGREENII0229- } \\
\text { HA-TGA1g/PmeI site }\end{array}$ \\
\hline $\begin{array}{l}\text { 3xHA-TGA1 SalI } \\
\text { rev }\end{array}$ & $\begin{array}{l}\text { CATGTCGACTGCATAGTCCG } \\
\text { GGACGTCATAGGGATAGCCC }\end{array}$ & $\begin{array}{l}\text { Rev cloning primer binding to } \\
\text { pUBQGW7HA 3xHA tag, for } \\
\text { insertion in pGREENII0229- } \\
\text { HA-TGA1g/Sall site }\end{array}$ \\
\hline 438 seq rev & $\begin{array}{l}\text { TGTGGTATGGCTGATTATGA } \\
\text { TCC }\end{array}$ & $\begin{array}{l}\text { Rev sequencing primer binding } \\
100 \text { bp before LIC v1 rev site }\end{array}$ \\
\hline CpoI strep tag & $\begin{array}{l}\text { TCCCGGACCGATGGGTTCTTC } \\
\text { TTGGAGCCACCCGCAGTTCG }\end{array}$ & $\begin{array}{l}\text { Fwd primer binding upstream of } \\
\text { strep tag coding sequence in } \\
\text { pGP172-MBP-GW constructs }\end{array}$ \\
\hline Grx1 GW fwd & $\begin{array}{l}\text { GGGGACAAGTTTGTACAAAA } \\
\text { AAGCAGGCTCCATGGCTCAA } \\
\text { GAGTTTGTGAACTGCAAAAT } \\
\text { CC }\end{array}$ & $\begin{array}{l}\text { Fwd primer to amplify human } \\
\text { GRX1 gene from p415-roGFP2 } \\
\text { for later integration in pGP172- } \\
\text { MBP-GW vector }\end{array}$ \\
\hline
\end{tabular}


Material

\begin{tabular}{lll}
\hline Primer name & Sequence $\left(\mathbf{5}^{\prime} \rightarrow \mathbf{3}^{\prime}\right)$ & Purpose \\
& & \\
\hline Grx1 GW rev & GGGGACCACTTTGTACAAGA & Rev primer to amplify human \\
& AAGCTGGGTT'TACTGCAGAG & GRX1 gene from p415-roGFP2 \\
& CTCCAATCTGCT'TAGCC & for later integration in pGP172- \\
& & MBP-GW vector
\end{tabular}

MBP 472 seq rev CAGCAATCAGCGGCCAGG Rev sequencing primer binding after 472 bp of MBP coding sequence

pBGW Acc65I

200 fwd

CTAGCTGATAGTGACCTTAG GC

pBGW EcoRV

$300 \mathrm{rev}$

pGP172 NdeI

fwd

GCACTGAACGTCAGAAGCCG

ATACATATGGCTAGCTGGAG CCACCC

Sequencing of pBGW starting ca. 200 bp upstream of Acc65I site

Sequencing of pBGW starting ca. 300 bp downstream of EcoRV site

Fwd primer binding around $N d e I$ site in front of strep tag in pGP172

pGP172-SUMO- TTTGGATCCCTAGGAGCCTGC Rev primer binding to attB1 site attB1 BamHI rev TTT'TTGTACAAACTTGTGAT and the basepair right before CTG attB1 site in pGP172-SUMOROXY9, contains a stop codon; BamHI site

pGP172-SUMO- TTTGGATCCCTAACCACCAAT Rev primer binding to end of BamHI rev CTGTTCTCTGTGAGCCTC SUMO gene in PGP172-SUMO$\mathrm{GW}$, adds a stopcodon to SUMO gene; Bam HI site

pGTGA1g seq CATACCTCGCTCTGCTAATCC

Sequencing of pGREENII0229HA-TGA1g, starting at basepair 1530

RB fwd

GGTAAACTAAGTCGCTGTAT GTG

Primer binding to right border (RB) of pGREENII0229-HATGA1g

rev23 reversed

GTCATAGCTGT'TCCTGTGTG AA

Reverse complement of rev23 primer for sequencing of pGREENII0229-HA-TGA1g

ROXY19 Lic v1

TACTTCCAATCCAATGCAATG Fwd primer for amplification of For CAAGGAACGATTTCT'TGTGCA ROXY19 with LIC sites to clone it in 438C_strep

ROXY19 Lic v1 TTATCCACTTCCAATGTTATTA Rev primer for amplification of rev TCACAACCACAGAGCCCCAAC ROXY19 with LIC sites to clone it in 438C_strep

ROXY9 CCLCA GTTGTCTCTGCGCCGCCGTTC fwd AAATCCTGTTCCGTGAC

Fwd fusion PCR primer to introduce coding sequence for CCLCA motif in ROXY9 coding sequence 


\begin{tabular}{|c|c|c|}
\hline Primer name & Sequence $\left(5^{\prime} \rightarrow 3^{\prime}\right)$ & Purpose \\
\hline $\begin{array}{l}\text { ROXY9 CCLCA } \\
\text { rev }\end{array}$ & $\begin{array}{l}\text { GATTTGAACGGCGGCGCAGA } \\
\text { GACAACATGAGCTCTTCGTGA } \\
\text { AG }\end{array}$ & $\begin{array}{l}\text { Rev fusion PCR primer to } \\
\text { introduce coding sequence for } \\
\text { CCLCA motif in ROXY9 coding } \\
\text { sequence }\end{array}$ \\
\hline $\begin{array}{l}\text { ROXY9 CPYC } \\
\text { fwd }\end{array}$ & $\begin{array}{l}\text { GAAGAGCTCATGTCCATATTG } \\
\text { CTACGCCGTTCAAATCCTGTT } \\
\text { CCG }\end{array}$ & $\begin{array}{l}\text { Fwd fusion PCR primer to } \\
\text { introduce coding sequence for } \\
\text { CPYC motif in ROXY9 coding } \\
\text { sequence }\end{array}$ \\
\hline $\begin{array}{l}\text { ROXY9 CPYC } \\
\text { rev }\end{array}$ & $\begin{array}{l}\text { GGCGTAGCAATATGGACATG } \\
\text { AGCTCTTCGTGAAGATCACCA } \\
\text { CTCC }\end{array}$ & $\begin{array}{l}\text { Rev fusion PCR primer to } \\
\text { introduce coding sequence for } \\
\text { CPYC motif in ROXY9 coding } \\
\text { sequence }\end{array}$ \\
\hline $\begin{array}{l}\text { ROXY9 Lic v1 } \\
\text { For }\end{array}$ & $\begin{array}{l}\text { TACTTCCAATCCAATGCAATG } \\
\text { GACAAAGTGATGAGAATGTC } \\
\text { TTC }\end{array}$ & $\begin{array}{l}\text { Fwd primer for amplification of } \\
\text { ROXY9 with LIC sites to clone it } \\
\text { in 438C_strep }\end{array}$ \\
\hline $\begin{array}{l}\text { ROXY9 Lic v1 } \\
\text { rev }\end{array}$ & $\begin{array}{l}\text { TTATCCACTTCCAATGTTATTA } \\
\text { CTAGTAAAGGATGGACTGAT } \\
\text { AGG }\end{array}$ & $\begin{array}{l}\text { Rev primer for amplification of } \\
\text { ROXY9 with LIC sites to clone it } \\
\text { in } 438 \text { C_strep }\end{array}$ \\
\hline $\begin{array}{l}\text { TGA1 1.intron } \\
\text { seq } 2361\end{array}$ & $\begin{array}{l}\text { CTTTTAGCCTGTCATTACAAT } \\
\text { ATATG }\end{array}$ & $\begin{array}{l}\text { Sequencing primer starting at } \\
2361 \mathrm{bp} \text { in } 1 \text {. intron of } T G A 1\end{array}$ \\
\hline $\begin{array}{l}\text { TGA1 3UTR SalI } \\
\text { fwd }\end{array}$ & $\begin{array}{l}\text { AAAGTCGACTAGGTTTGAGT } \\
\text { TATTTTGTAACAACCAAATGA } \\
\text { AG }\end{array}$ & $\begin{array}{l}\text { Fwd cloning primer binding to } \\
\text { TGA1 stop codon and } \\
\text { 3'UTR/SalI site }\end{array}$ \\
\hline $\begin{array}{l}\text { TGA1 C172N } \\
\text { fwd }\end{array}$ & $\begin{array}{l}\text { GAACAGAACAGACAGATAAA } \\
\text { TGAACTAAGAACAGTTTTACA } \\
\text { C }\end{array}$ & $\begin{array}{l}\text { Fwd fusion PCR primer to } \\
\text { exchange Cys }{ }^{172} \text { codon in } T G A 1 \\
\text { for Asn codon }\end{array}$ \\
\hline TGA1 C172N rev & $\begin{array}{l}\text { GTGTAAAACTGTTCTTAGTTC } \\
\text { ATTTATCTGTCTGTTCTGTTC }\end{array}$ & $\begin{array}{l}\text { Rev fusion PCR primer to } \\
\text { exchange Cys }{ }^{172} \text { codon in } T G A 1 \\
\text { for Asn codon }\end{array}$ \\
\hline TGA1 C287S fwd & $\begin{array}{l}\text { CCTTGCGGACTCCGTTGCAGC } \\
\text { GGGAC }\end{array}$ & $\begin{array}{l}\text { Fwd fusion PCR primer to } \\
\text { exchange Cys }{ }^{287} \text { codon in } T G A 1 \\
\text { for Ser codon }\end{array}$ \\
\hline TGA1 C287S rev & $\begin{array}{l}\text { GTCCCGCTGCAACGGAGTCC } \\
\text { GCAAGG }\end{array}$ & $\begin{array}{l}\text { Rev fusion PCR primer to } \\
\text { exchange Cys }{ }^{287} \text { codon in } T G A 1 \\
\text { for Ser codon }\end{array}$ \\
\hline TGA1 NcoI fwd & $\begin{array}{l}\text { TTCCCATGGAACAGCAGGAA } \\
\text { СТССТСАС }\end{array}$ & $\begin{array}{l}\text { Fwd cloning primer binding } \\
\text { around } N c o \text { I site within genomic } \\
T G A 1 \text { coding sequence }\end{array}$ \\
\hline $\begin{array}{l}\text { TGA1 seq rev } \\
\text { exon } 8\end{array}$ & $\begin{array}{l}\text { GCCGTTGAAAATACTCACCAA } \\
\text { GAGC }\end{array}$ & $\begin{array}{l}\text { Rev primer for sequencing, binds } \\
\text { in } 8 \text {. exon of } T G A 1\end{array}$ \\
\hline
\end{tabular}


Material

\section{qRT-PCR primers}

These primers were ordered from Invitrogen.

\begin{tabular}{lll}
\hline Primer name & Sequence $\left(\mathbf{5}^{\prime} \rightarrow \mathbf{3}^{\prime}\right)$ & Reference \\
\hline UBQ5 fwd & GACGCTTCATCTCGTCC & Kesarwani et al. (2007) [194] \\
UBQ5 rev & GTAAACGTAGGTGAGTCCA & Kesarwani et al. (2007) [194] \\
TGA1 fwd & ACGAACCTGTCCATCAATTCGG & Muthreich, 2014 [157] \\
TGA1 rev & CCATGGGAAGTATCCTCTGACACG & Muthreich, 2014 [157] \\
\hline
\end{tabular}




\section{Plasmids}

\section{Plasmids generated by others}

\begin{tabular}{|c|c|c|c|}
\hline $\begin{array}{l}\text { Plasmid } \\
\text { name }\end{array}$ & Description & Resistance & Reference \\
\hline $438 \mathrm{C}$ & $\begin{array}{l}\text { Expression vector for insect cells } \\
\text { harboring the coding sequence of } \\
\text { a His-MBP tag for N-terminal } \\
\text { fusion }\end{array}$ & $A m p^{R}$ & $\begin{array}{l}\text { Department for } \\
\text { Molecular Biology, } \\
\text { Prof. Dr. Patrick } \\
\text { Cramer, MPI for } \\
\text { Biophysical } \\
\text { Chemistry, Göttingen }\end{array}$ \\
\hline p415-roGFP2 & $\begin{array}{l}\text { Expression of Homo sapiens Grx1 } \\
\text { fused to roGFP }\end{array}$ & $\begin{array}{c}\operatorname{Amp}^{\mathrm{R}}(\text { E. coli }) \\
\text { LEU2 } \\
(\text { S. cerevisiae })\end{array}$ & $\begin{array}{l}\text { Morgan et al. (2013) } \\
\text { [280] }\end{array}$ \\
\hline $\mathrm{pBGW}$ & $\begin{array}{l}\text { Destination vector for T-DNA } \\
\text { constructs }\end{array}$ & $\begin{array}{c}\operatorname{Spec}^{R}(\text { E. coli }) \\
\text { bar } \\
(\text { A. thaliana })\end{array}$ & $\begin{array}{l}\text { VIB-UGent Center } \\
\text { for Plant Systems } \\
\text { Biology, Ghent } \\
\text { University }\end{array}$ \\
\hline pBGWL7 & $\begin{array}{l}\text { Vector encoding T-DNA with } \\
\text { fLUC gene for fusion to different } \\
\text { promoter regions via Gateway } \\
\text { cloning, } 35 \mathrm{~S} \text { terminator }\end{array}$ & $S p e c^{R}$ & $\begin{array}{l}\text { VIB-UGent Center } \\
\text { for Plant Systems } \\
\text { Biology, Ghent } \\
\text { University }\end{array}$ \\
\hline $\begin{array}{l}\text { pDONR201- } \\
\text { TGA1 }\end{array}$ & $\begin{array}{l}\text { pDONR201 harboring TGA1 } \\
\text { cDNA sequence; the plasmid was } \\
\text { constructed in analogy to the } \\
\text { plasmid pDONR223-TGA1 }\end{array}$ & $\operatorname{Kan}^{R}$ & $\begin{array}{l}\text { M. Zander, } \\
\text { Department for Plant } \\
\text { Molecular Biology } \\
\text { and Physiology, Prof. } \\
\text { Dr. C. Gatz, Georg- } \\
\text { August-Universität } \\
\text { Göttingen, } \\
\text { unpublished }\end{array}$ \\
\hline pDONR207 & $\begin{array}{l}\text { pDONOR plasmid with } \\
\text { gentamicin resistance cassette }\end{array}$ & Gent ${ }^{R}$ & Life Technologies \\
\hline $\begin{array}{l}\text { pDONR207- } \\
\text { GRX480 }\end{array}$ & $\begin{array}{lr}\text { pDONR207 } & \text { harboring } \\
\text { ROXY19/GRX480 } & \text { coding } \\
\text { sequence } & \end{array}$ & Gent ${ }^{R}$ & $\begin{array}{l}\text { Ndamukong et al. } \\
\text { (2007) }\end{array}$ \\
\hline $\begin{array}{l}\text { pDONR207- } \\
\text { ROXY9 }\end{array}$ & $\begin{array}{l}\text { pDONR207 harboring ROXY9 } \\
\text { cDNA sequence }\end{array}$ & Gent $^{R}$ & Muthreich, 2014 [157] \\
\hline
\end{tabular}


Plasmids

\begin{tabular}{|c|c|c|c|}
\hline $\begin{array}{l}\text { Plasmid } \\
\text { name }\end{array}$ & Description & Resistance & Reference \\
\hline $\begin{array}{l}\text { pDONR223- } \\
\text { TGA1 }\end{array}$ & $\begin{array}{l}\text { pDONR223 harboring } T G A 1 \\
\text { cDNA sequence }\end{array}$ & $S_{p p e c}^{R}$ & Li et al. (2018) [156] \\
\hline $\begin{array}{l}\text { pGP172- } \\
\text { GFP-GW }\end{array}$ & $\begin{array}{l}\text { Destination vector for T7- } \\
\text { dependent expression in E. coli, } \\
\text { harbors strep-GFP-tag sequence } \\
\text { for N-terminal fusions }\end{array}$ & $A m p^{R}$ & Oberdiek, 2018 [174] \\
\hline $\begin{array}{l}\text { pGP172- } \\
\text { GFP- } \\
\text { GW } \Delta \text { strep }\end{array}$ & $\begin{array}{l}\text { Destination vector for } \mathrm{T} 7- \\
\text { dependent expression of GFP- } \\
\text { tagged proteins in E. coli }\end{array}$ & $A m p^{R}$ & Oberdiek, 2018 [174] \\
\hline $\begin{array}{l}\text { pGP172- } \\
\text { MBP- } \\
\text { GRX370 }\end{array}$ & $\begin{array}{l}\text { Vector to express a strep-MBP- } \\
\text { fusion of A. thaliana } \\
\text { GRXC2/GRX370 in E. coli under } \\
\text { control of the T7 promoter and } \\
\text { terminator }\end{array}$ & $A m p^{R}$ & Oberdiek, 2018 [174] \\
\hline $\begin{array}{l}\text { pGP172- } \\
\text { SUMO-GW }\end{array}$ & $\begin{array}{l}\text { Destination vector for } \mathrm{T} 7 \text { - } \\
\text { dependent expression of proteins } \\
\text { fused N-terminally to a strep- } \\
\text { SUMO tag }\end{array}$ & $A m p^{R}$ & Oberdiek, 2018 [174] \\
\hline $\begin{array}{l}\text { pGREENII02 } \\
\text { 29-HA- } \\
\text { TGA1g }\end{array}$ & $\begin{array}{l}\text { Vector encoding T-DNA for } \\
\text { insertion of genomic TG } A 1 \text { fused } \\
\text { to } 1 \text { xHA tag coding sequence in } A \text {. } \\
\text { thaliana genome }\end{array}$ & $\begin{array}{c}\operatorname{Kan}^{\mathrm{R}}(\text { E. coli }) \\
\text { bar } \\
(\text { A. thaliana })\end{array}$ & $\begin{array}{l}\text { Shearer et al., (2007) } \\
\text { Wang \& Fobert } \\
(2013) \\
\text { Sun et al. }(2017) \\
{[222,223,262]}\end{array}$ \\
\hline $\begin{array}{l}\mathrm{pMA} / \mathrm{CL}- \\
\mathrm{TEV}-\mathrm{A}\end{array}$ & $\begin{array}{l}\text { Expression of an MBP-His-tagged } \\
\text { TEV protease under control of the } \\
\mathrm{T} 7 \text { promoter, a TEV site is placed } \\
\text { in between the MBP and the His } \\
\text { tag }\end{array}$ & $A m p^{R}$ & $\begin{array}{l}\text { Prof. Dr. Roland Lill, } \\
\text { Institute for } \\
\text { Cytobiology and } \\
\text { Cytopathology, } \\
\text { Philipps-University } \\
\text { Marburg }\end{array}$ \\
\hline $\begin{array}{l}\text { pUBQ10- } \\
\text { GW7-HA }\end{array}$ & $\begin{array}{l}\text { Destination vector for } U B Q 10 \\
\text { promoter-dependent expression } \\
\text { of proteins fused to a } 3 \times \mathrm{xH} \text { tag in } \\
\text { A. thaliana protoplasts }\end{array}$ & Spec ${ }^{R}$ & $\begin{array}{l}\text { Uhrig et al. (2017) } \\
{[177]}\end{array}$ \\
\hline
\end{tabular}


Plasmids generated during this work

The following section shows the maps of all plasmids generated during this work in alphabetical order. Their cloning strategy is described. The maps were prepared in Geneious 5.3.6.

$\underline{\text { List of plasmids }}$

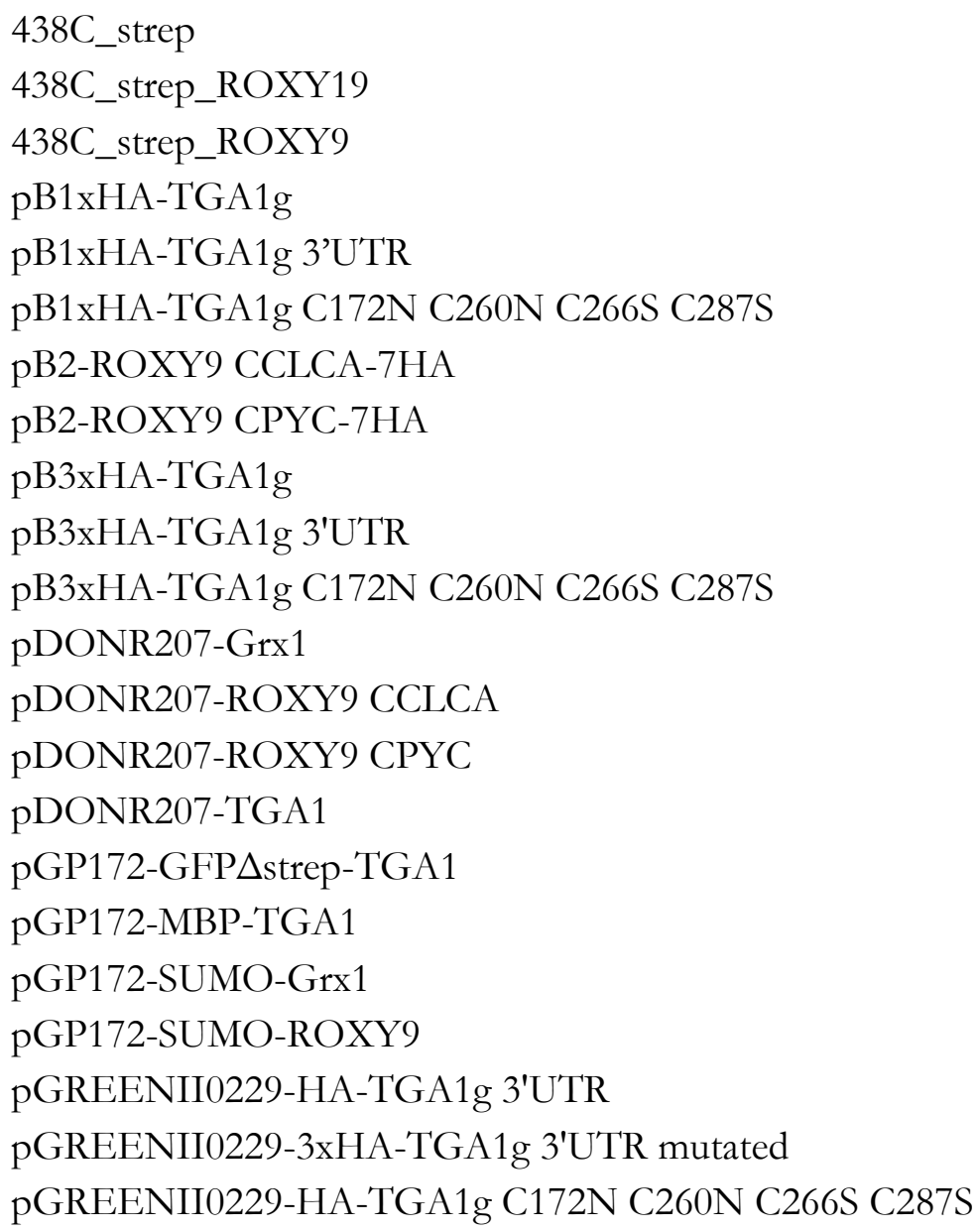


Plasmids

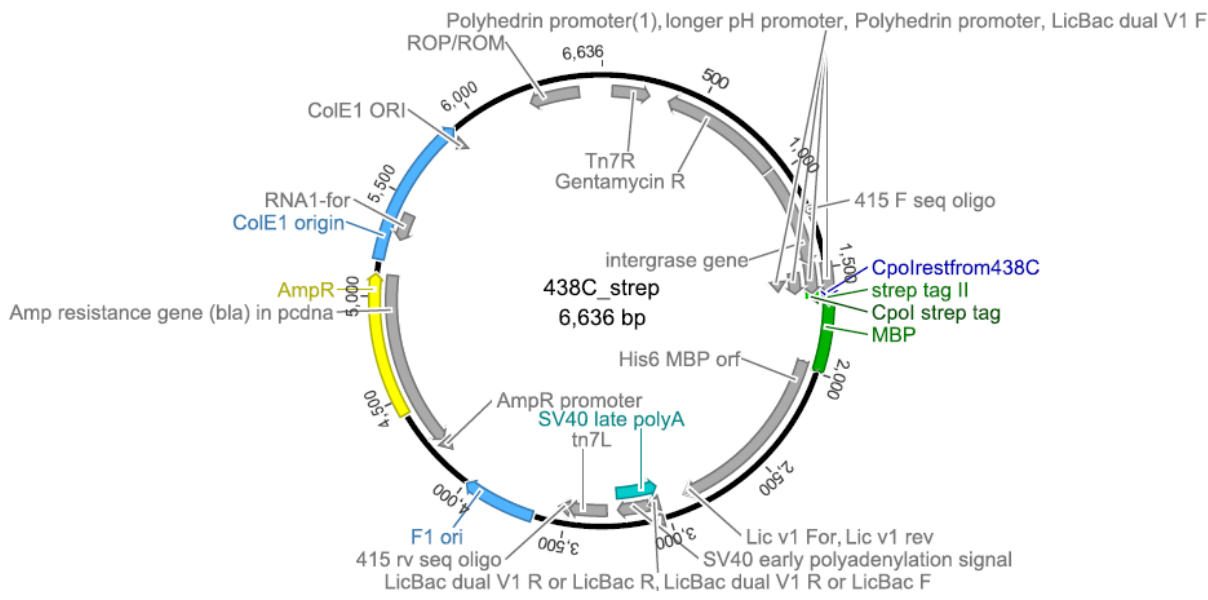

438C_strep. A vector to express a step-MBP fusion protein in insect cells. Strep-MBP-ROXY9 was amplified in a PCR from pGP172-MBP-ROXY9 with primers CpoI strep tag and T7 term rev and digested with CpoI and Pf23II. The 369 bp-fragment was extracted from an agarose gel and ligated with the $6274 \mathrm{bp}$-fragment of 438C digested with CpoI/PfL23II. Resistances: $\operatorname{Kan}^{R}$ (E. coli), bar (A. thaliana).

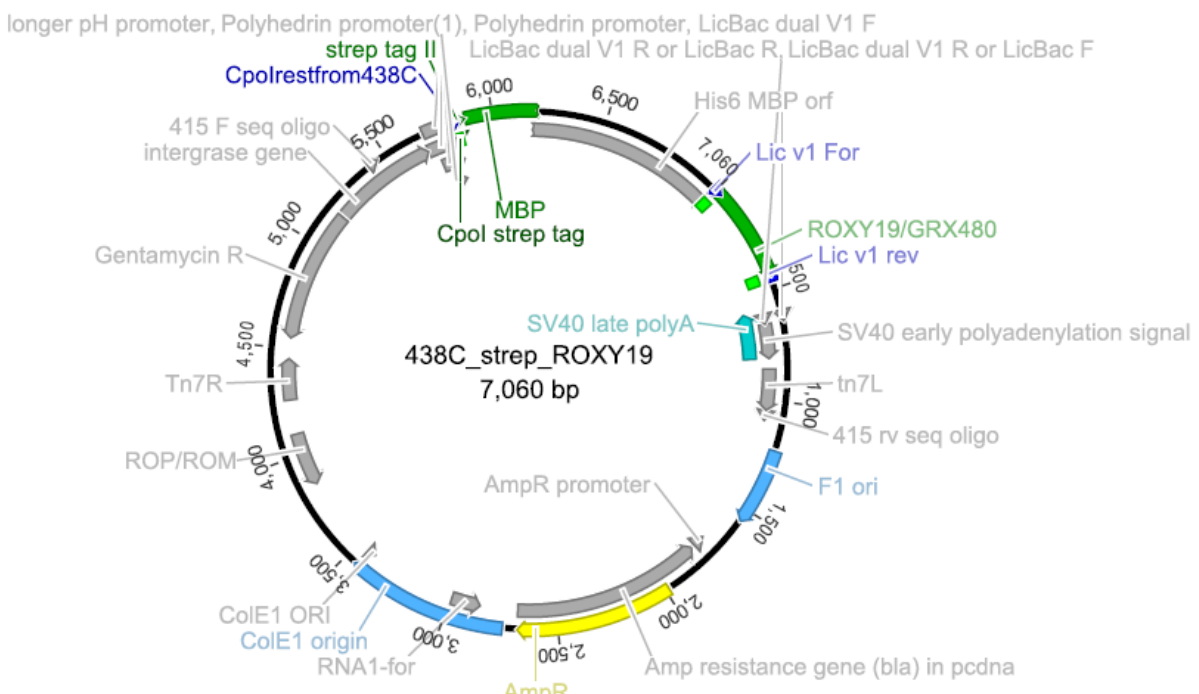

438C_strep_ROXY19. A vector to express a step-MBP fusion of ROXY19 in insect cells. ROXY19 was amplified in a PCR from pDONR207-GRX480 using primers ROXY19 Lic v1 For and ROXY19 Lic v1 rev, and inserted via LIC in 438C_strep using the SspI site. Resistances: $\operatorname{Kan}^{R}$ (E. coli), $\operatorname{bar}$ (A. thaliana). 


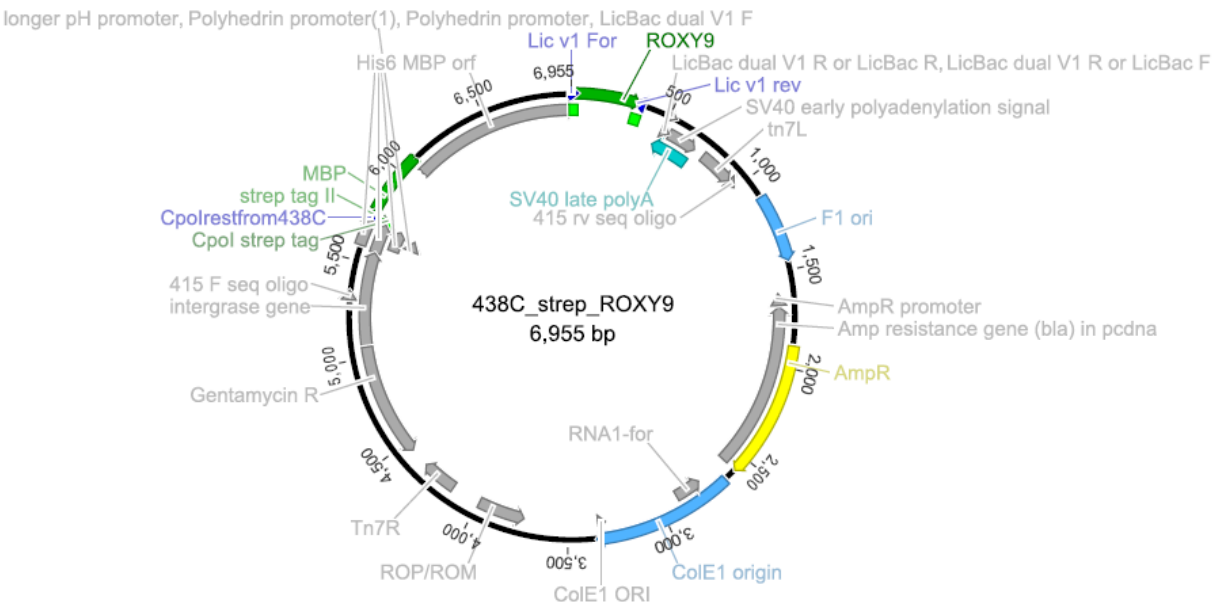

438C_strep_ROXY9. A vector to express a step-MBP fusion of ROXY9 in insect cells. ROXY9 was amplified in a PCR from pDONR207-ROXY9 using primers ROXY9 Lic v1 For and ROXY9 Lic v1 rev, and inserted via LIC in 438C_strep using the $S_{s p} \mathrm{I}$ site. Resistances: $\operatorname{Kan}^{R}$ (E. coli), bar (A. thaliana).

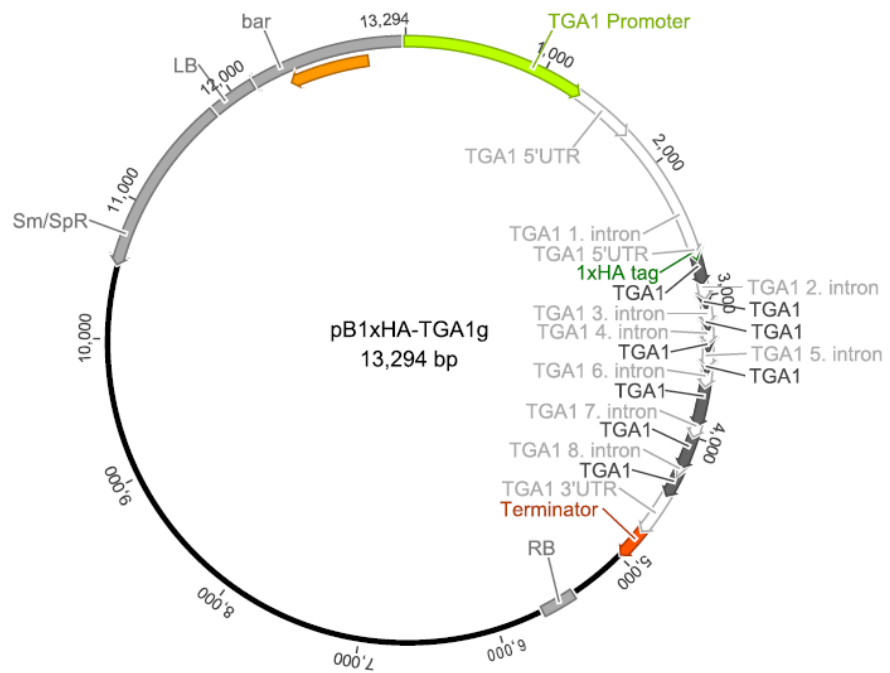

pB1xHA-TGA1g. A vector encoding the T-DNA for insertion of the genomic TGA1 sequence fused to coding sequence for $1 \mathrm{xHA}$ tag in the $A$. thaliana genome. The insert was cut out from pGREENII0229-HA-TGA1g via Acc65I/StuI and ligated in pBGW digested with Acc65I/EcoRV. Resistances: Spec (E. coll), bar (A. thaliana). TGA1 exons are shown in dark grey, introns in light grey. 
Plasmids

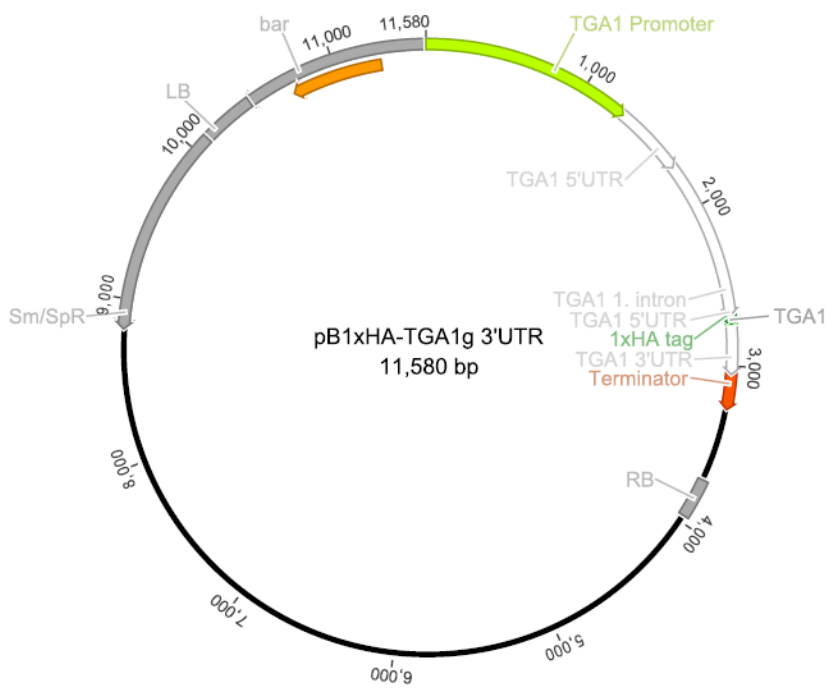

pB1xHA-TGA1g 3'UTR. A vector encoding the T-DNA for insertion of a 1xHA tag gene unter control of the TGA1 promoter (including its 5'UTR) and 3'UTR in the $A$. thaliana genome. The insert was cut out from pGREENII0229-HA-TGA1g 3'UTR via Acc65I/StuI and ligated in pBGW digested with Acc65I/EcoRV. Resistances: Spec ${ }^{R}$ (E. coli), bar (A. thaliana).

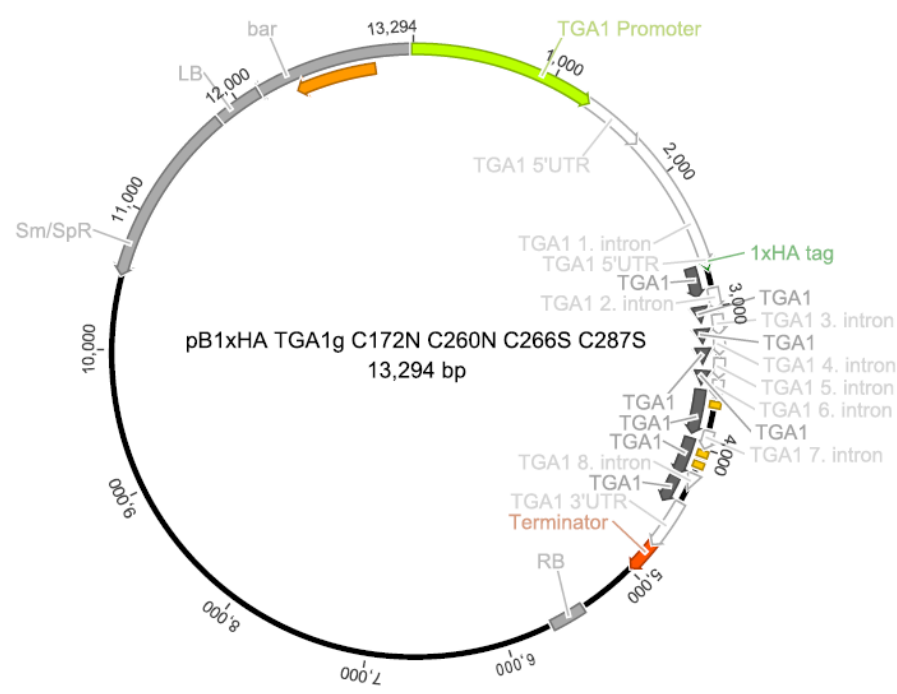

pB1xHA-TGA1g C172N C260N C266S C287S. A vector encoding the T-DNA for insertion of the genomic TGA1 sequence fused to coding sequence of a $1 \mathrm{xHA}$ tag in the $A$. thaliana genome; All critical cysteine residues are mutated to amino acids found in other TGA factors at this site. The insert was cut out from pGREENII0229-HA-TGA1g C172N C260N C266S C287S via Acc65I/StuI and ligated in pBGW digested with Acc65I/EcoRV. Resistances: Spec ${ }^{R}$ (E. coli), bar (A. thaliana). TGA1 exons are shown in dark grey, introns in light grey. 


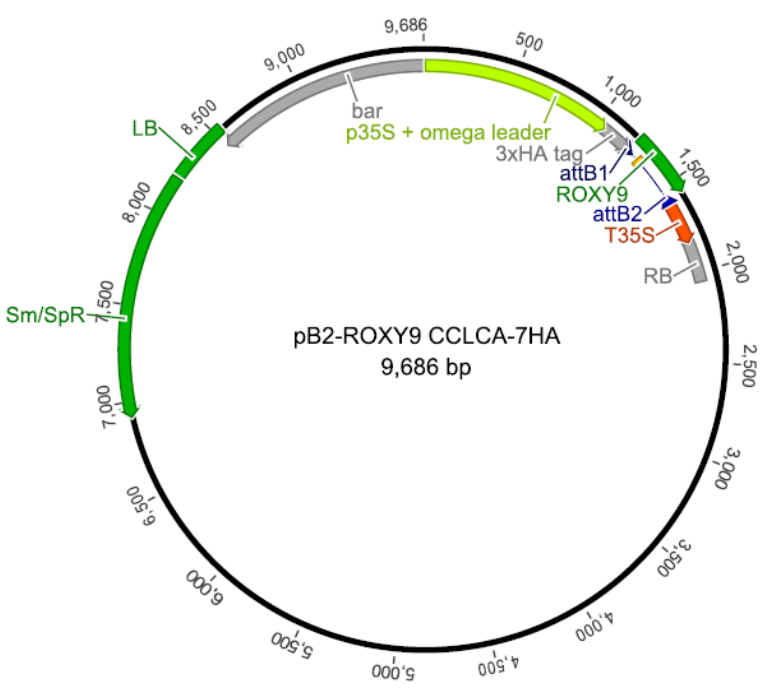

pB2-ROXY9 CCLCA-7HA. A vector encoding the T-DNA for expression of N-terminally HA-tagged ROXY9 CCLCA under control of the $35 \mathrm{~S}$ promoter and terminator. The ROXY9 CCLCA was generated as follows: The first PCR product was amplified using primers SeqL1/ROXY9 CCLCA rev from pDONR207-ROXY9; the second PCR product was amplified using primers ROXY9 CCLCA fwd/SeqL2 from pDONR207-ROXY9. Both PCR products were fused in a PCR containing primers SeqL1/SeqL2. Finally, the fusion PCR product ROXY9 CCLCA was inserted via an LR in pB2GW7HA. Resistances: $S p e c^{R}$ (E. coli), $\operatorname{bar}($ A. thaliana).

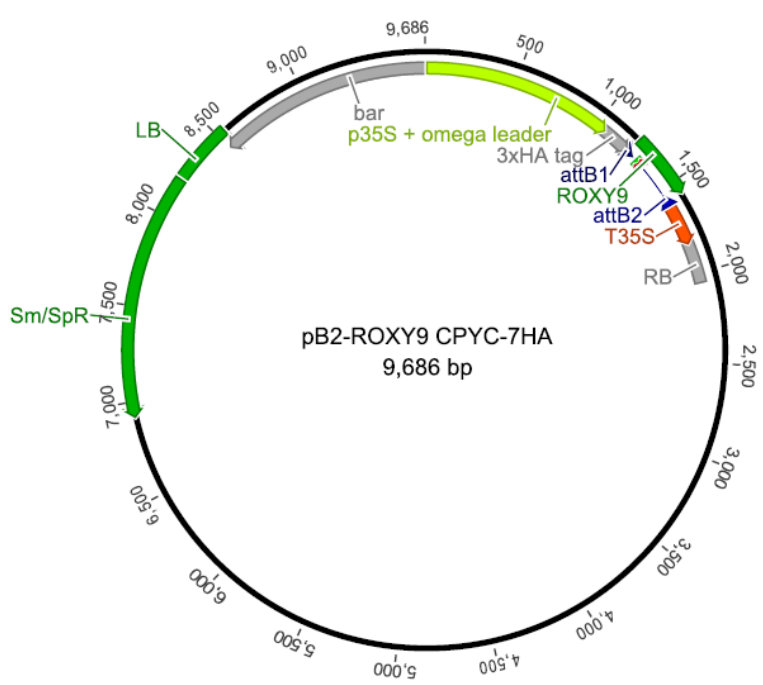

pB2-ROXY9 CPYC-7HA. A vector encoding the T-DNA for expression of N-terminally HA-tagged ROXY9 CPYC under control of $35 \mathrm{~S}$ promoter and terminator. The plasmid was generated via the LR pDONR207-ROXY9 CPYC $\times$ pB2GW7HA. Resistances: Spec ${ }^{R}$ (E. coli), $\operatorname{bar}$ (A. thaliana). 
Plasmids

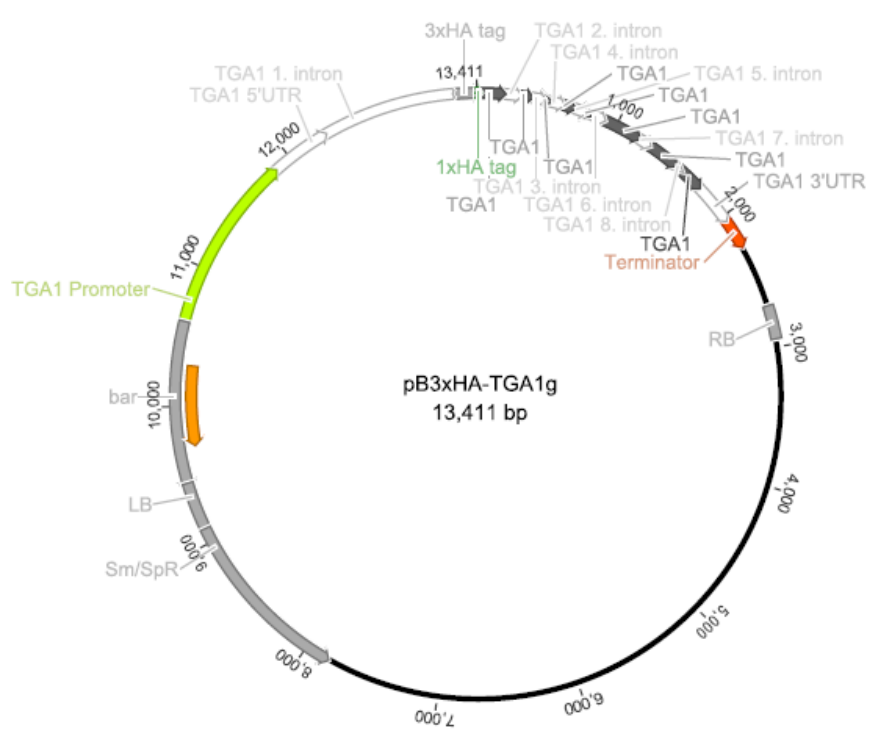

pB3xHA-TGA1g. A vector encoding the T-DNA for the insertion of the genomic TGA1 sequence fused to the coding sequence of a $3 \mathrm{xHA}$ tag in the $A$. thaliana genome. The plasmid

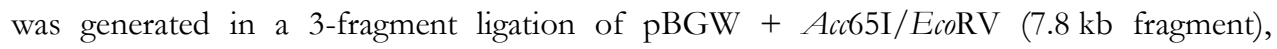
pGREENII0229-HA-TGA1g + SalI/StuI (2.7 kb fragment) and pGREENII0229-3xHATGA1g 3'UTR mutated + Acc65I/SalI (2.8 kb fragment). Resistances: Spec ${ }^{R}$ (E. coli), bar (A. thaliana).

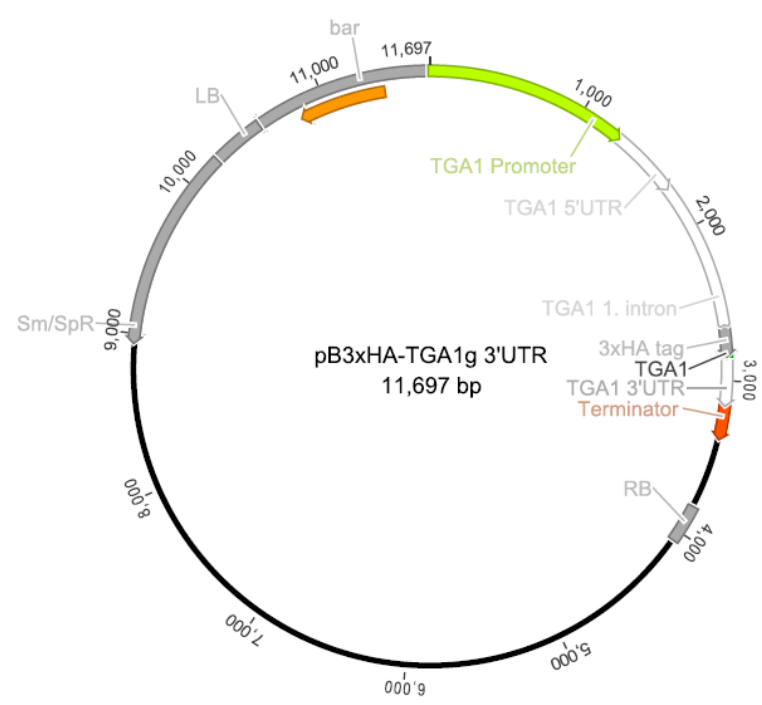

pB3xHA-TGA1g 3'UTR. A vector encoding the T-DNA for insertion of a 3xHA tag coding sequence unter control of the TGA1 promoter (incl. 5'UTR) and 3'UTR in the A. thaliana genome. The plasmid was generated in a 3-fragment ligation of $\mathrm{pBGW}+$ Acc65I/EcoRV (7.8 kb fragment), pGREENII0229-HA-TGA1g 3'UTR + SalI/StuI (1 kb fragment) and pGREENII0229-3xHA-TGA1g 3'UTR mutated + Acc65I/SalI (2.8 kb fragment). Resistances: $\operatorname{Spec}^{R}$ (E. coli), bar (A. thaliana). 


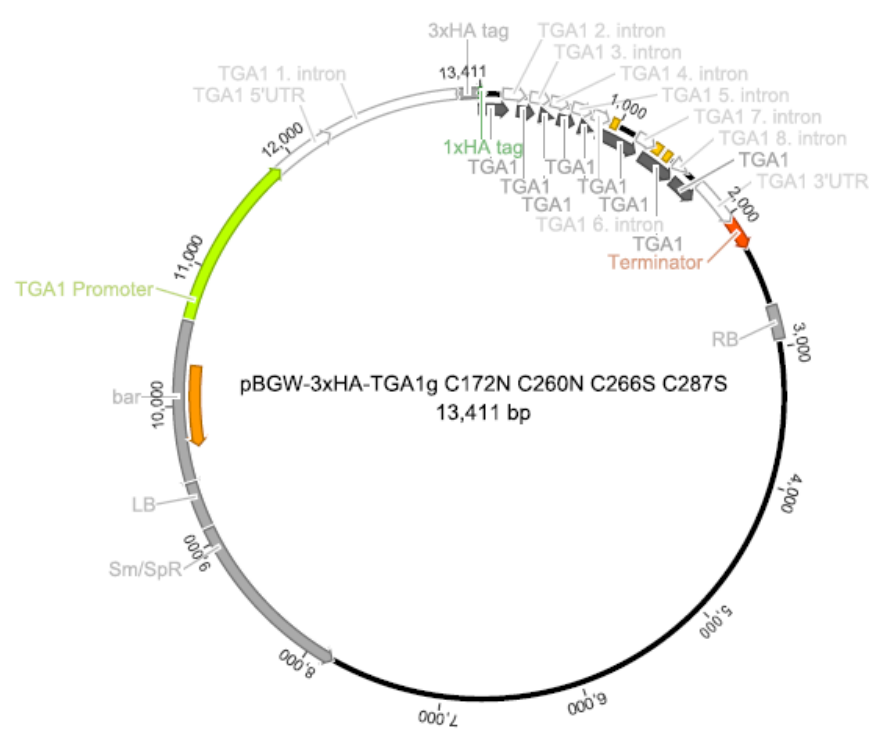

pB3xHA-TGA1g C172N C260N C266S C287S. A vector encoding the T-DNA for the insertion of a genomic TGA1 sequence with a $3 \mathrm{xHA}$ tag coding sequence in the $A$. thaliana genome; All codons for cysteine residues were mutated to codons of amino acids found in other TGA factors at this site. The plasmid was generated in a 3-fragment ligation of $\mathrm{pBGW}$ + Acc65I/EcoRV (7.8 kb fragment), pGREENII0229-HA-TGA1g C172N C260N C266S $\mathrm{C} 287 \mathrm{~S}+$ SalI/StuI (2.7 kb fragment) and pGREENII0229-3xHA-TGA1g 3'UTR mutated + Acc65I/SalI (2.8 kb fragment). Resistances: $\operatorname{Spec}^{R}$ (E. coli), bar (A. thaliana). TGA1 exons are shown in dark grey, introns in light grey.

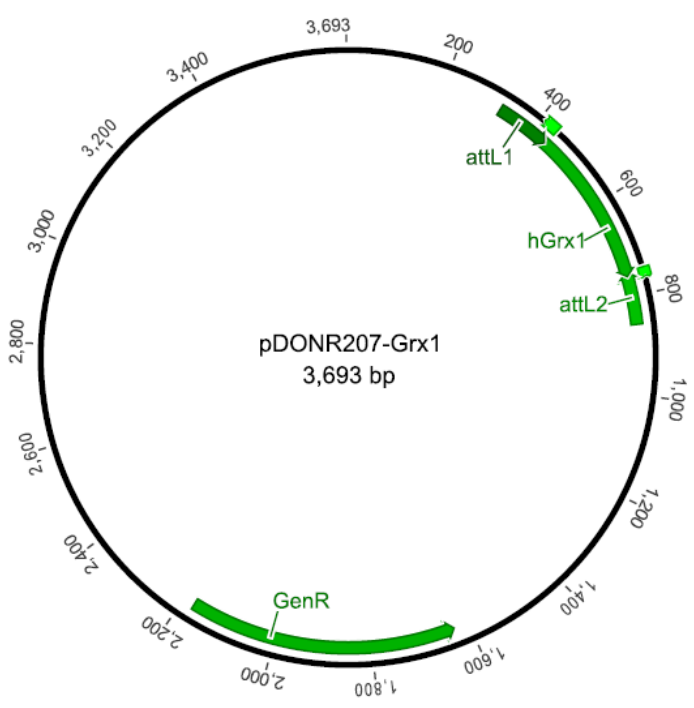

pDONR207-Grx1. pDONR207 harboring the Homo sapiens GRX1 cDNA sequence. The plasmid was generated in a BP reaction of pDONR207 and the PCR product of primers Grx1 GW fwd/Grx1 GW rev on p415-roGFP2. Resistances: Gent ${ }^{R}$. 
Plasmids

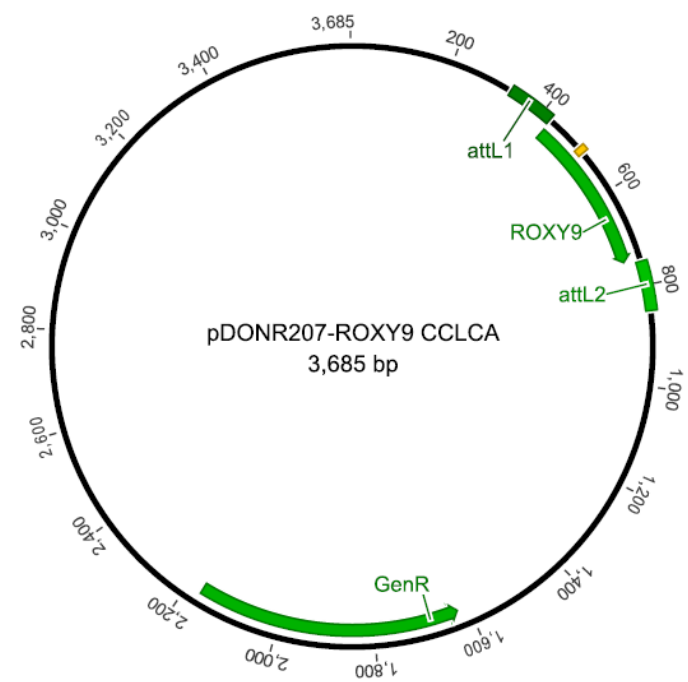

pDONR207-ROXY9 CCLCA. pDONR207 harboring gene encoding ROXY9 with CCLCA active site. The plasmid was generated in a BP reaction using pDONR207 and the fusion PCR product ROXY9 CCLCA. The fusion PCR product was prepared as follows: The first PCR product was amplified using primers T7/ROXY9 CCLCA rev from PGP172-SUMO-ROXY9 and the second PCR product using primers ROXY9 CCLCA fwd/T7termrev from pGP172SUMO-ROXY9. Both fragments were fused in a PCR using primers T7/T7termrev. Resistances: Gent .

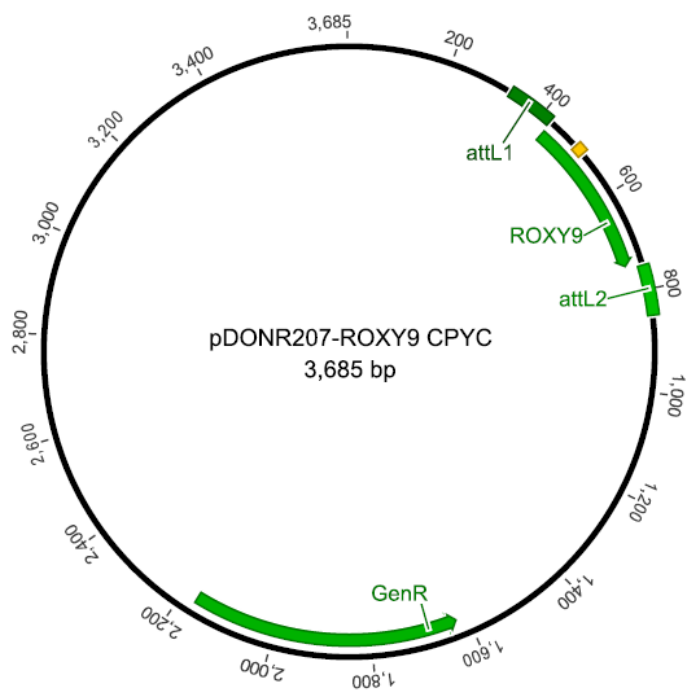

pDONR207-ROXY9 CPYC. pDONR207 harboring gene encoding ROXY9 with CPYC active site. A fusion PCR product resulting from the PCR products ROXY9 CPYC fwd/GB Term-primer and pBD2/ROXY9 CPYC rev (both amplified from pDEST-GBKT7-ROXY9) was inserted in a BP reaction in pDONR207. Resistances: Gent ${ }^{R}$. 


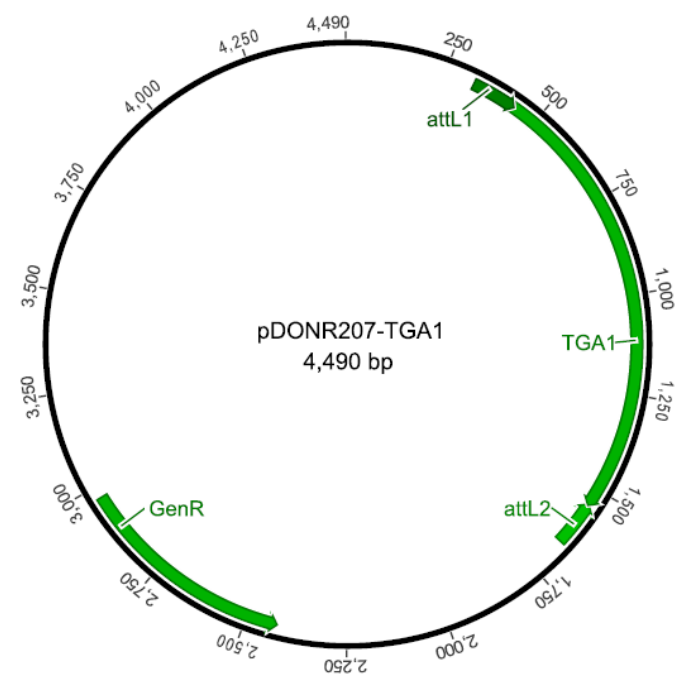

pDONR207-TGA1. pDONR207 harboring TGA1 cDNA sequence. The plasmid was generated in a BP reaction of pDONR207 and pGP172-MBP-TGA1. Resistances: Gent ${ }^{R}$.

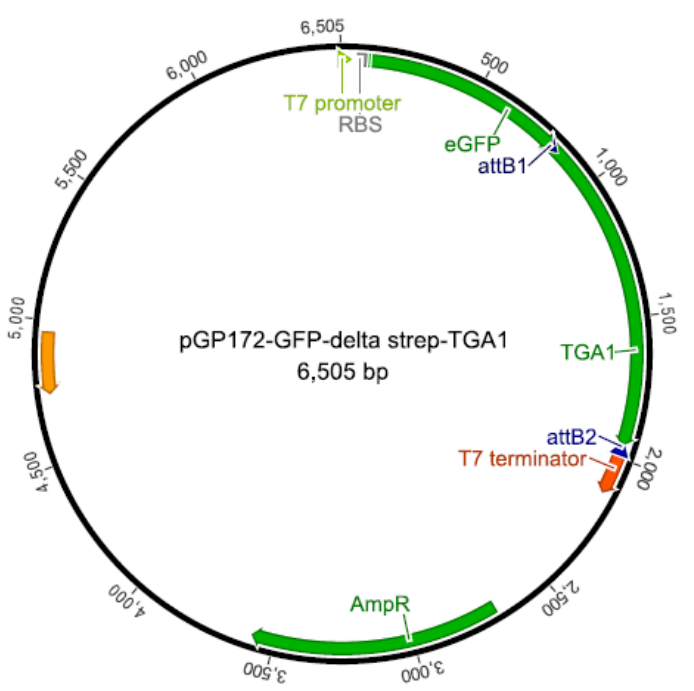

pGP172-GFP $\Delta$ strep-TGA1. A vector to express eGFP-TGA1 in E. coli under control of T7 promoter and terminator. The plasmid was generated in an LR of pGP172-GFP-GW $\Delta$ strep and pDONR207-TGA1. Resistances: $A m p^{\mathrm{R}}$. 
Plasmids

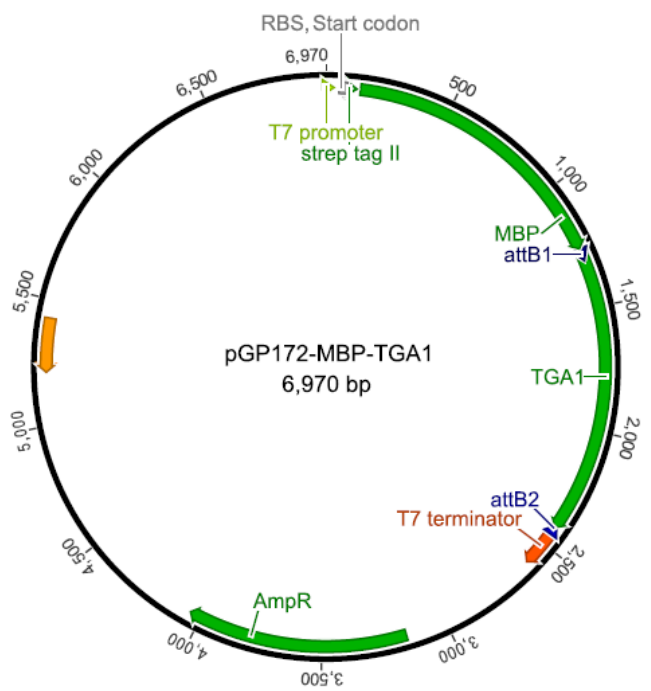

pGP172-MBP-TGA1. An expression plasmid to produce N-terminally strep-MBP-tagged TGA1 in E. coli BL21. The plasmid was generated in an LR using pDONR223-TGA1 and pGP172-MBP-GW. Resistances: $A m p^{R}$.

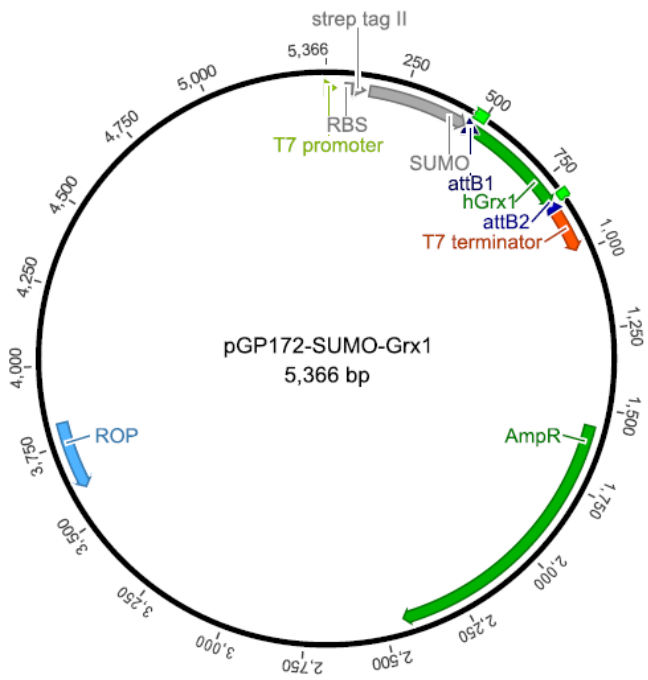

pGP172-SUMO-Grx1. A vector encoding an N-terminal strep-SUMO-fusion of human Grx1 for expression in E. coli via the T7 promoter and terminator. The plasmid was generated in an LR of pDONR207-Grx1 and pGP172-SUMO-GW. Resistances: $A m p^{R}$. 


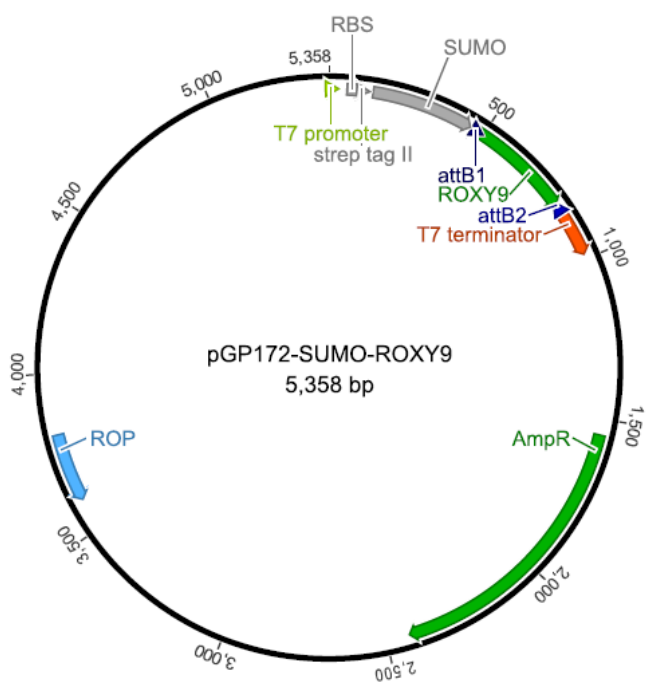

pGP172-SUMO-ROXY9. A vector encoding an N-terminal strep-SUMO-fusion of ROXY9 for expression in E. coli via the T7 promoter and terminator. The plasmid was generated in an LR reaction using pDONR207-ROXY9 and pGP172-SUMP-GW. Resistances: $A m{ }^{\mathrm{R}}$.

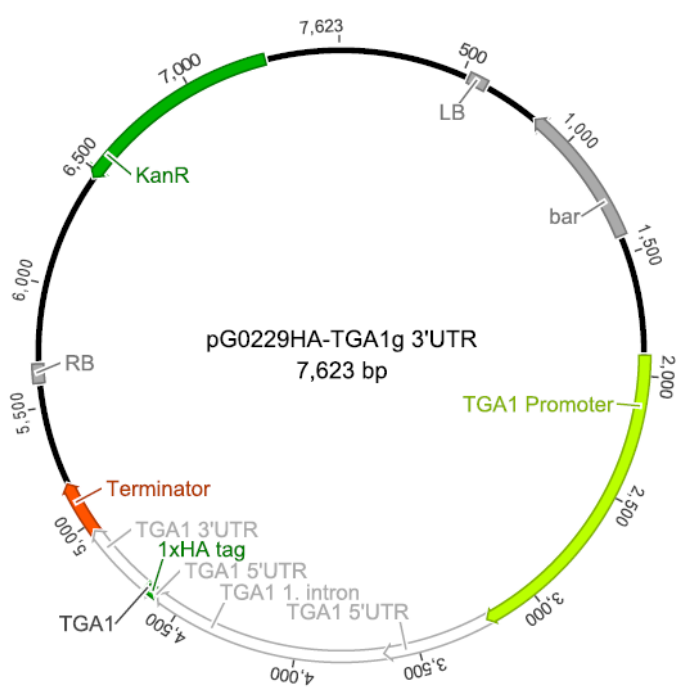

pGREENII0229-HA-TGA1g 3'UTR. A vector encoding the T-DNA for insertion of a 1xHA tag unter control of TGA1 promoter (incl. 5'UTR) and 3'UTR in the $A$. thaliana genome. The PCR product of primers TGA1 3UTR SalI fwd/rev23 on the vector pGREENII0229HA-TGA1g was digested with SalI/BamHI. The ca. $0.5 \mathrm{~kb}$ fragment of this digest was then ligated with the $4.5 \mathrm{~kb}$-fragment of the digest of pGREENII0229-HA-TGA1g with StuI/NheI and the $3.1 \mathrm{~kb}$-fragment of the digest of pGREENII0229-HA-TGA1g with NheI/SalI and the $0.4 \mathrm{~kb}$-fragment of the digest pGREENII0229-HA-TGA1g with BamHI/StuI. Resistances: $\operatorname{Spec}^{R}$ (E. coll), bar (A. thaliana). 
Plasmids

pGREENII0229-3xHA-TGA1g 3'UTR mutated. A vector encoding the T-DNA for insertion of a 3xHA tag unter control of the TGA1 promoter (incl. 5'UTR) and 3'UTR in the A. thaliana genome; the plasmid carries a mutation in the 3'UTR. The PCR products amplified with primers 3xHA-TGA1 Pme1 fwd/3xHA rev from pUBQ10-GW7-HA (ca. $0.2 \mathrm{~kb}$ ) and 3xHA 3UTR fusion fwd/rev23 from pGREENII0229-HA-TGA1g (ca. $0.7 \mathrm{~kb}$ ) were linked in a fusion PCR and cloned in pGREENII0229-HA-TGA1g via PmeI and BamHI (the ca. $7.5 \mathrm{~kb}$ fragment of the vector digest was used for ligation). Resistances: $S p e c^{R}$ (E. coli), bar (A. thaliana).

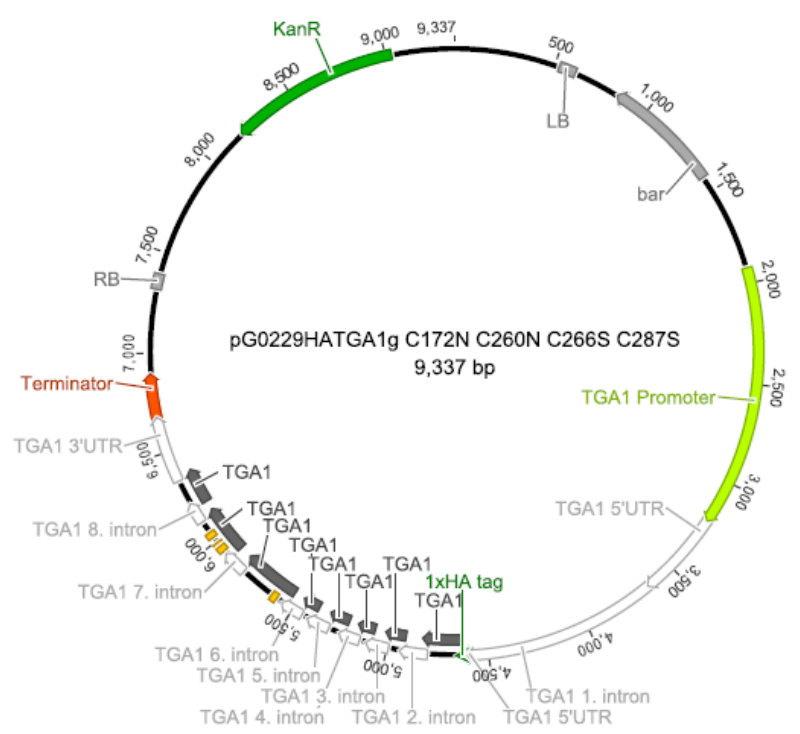

pGREENII0229-HA-TGA1g C172N C260N C266S C287S. A vector encoding the T-DNA for insertion of the genomic TGA1 sequence with a $1 \mathrm{xHA}$ tag coding sequence in the A. thaliana genome; All cysteine codons in TGA1 were mutated to codons of amino acids found in other TGA factors at these sites. First, a TGA1 stretch from total DNA from $A$. thaliana tga1 tga4 + pGTGA1g C260N C266S was amplified using TGA1 C172N fwd/rev23 to obtain the template for a PCR with TGA1 C172N fwd/TGA1 C287S rev. Then, PCRs using primers TGA1 NcoI fwd/TGA1 C172N rev and TGA1 C287S fwd/rev23 on the template pGREENII0229-HA-TGA1g were prepared. All three PCR products were fused by PCR and the product digested with $\mathrm{NcoI} / \mathrm{BamHI}$. The $1.9 \mathrm{~kb}$ fragment from this PCR digest was ligated with the $7.4 \mathrm{~kb}$ fragment of pGREENII0229-HA-TGA1g digested with NcoI/BamHI. Resistances: $\operatorname{Spec}^{R}$ (E. coli), bar (A. thaliana). TGA1 exons are shown in dark grey, introns in white. 


\section{Chemicals}

\begin{tabular}{|c|c|c|}
\hline Chemical & Product name & Company \\
\hline $30 \%(w / w) \mathrm{H}_{2} \mathrm{O}_{2}$ & $\begin{array}{l}\text { Hydrogen peroxide solution } 30 \%(\mathrm{w} / \mathrm{w}) \\
\text { in } \mathrm{H}_{2} \mathrm{O} \text {, contains stabilizer }\end{array}$ & $\begin{array}{l}\text { Sigma-Aldrich } \\
\text { (Madrid, Spain) }\end{array}$ \\
\hline 1,4-Dithiotreit (DTT) & 1,4-Dithiotreit $\geq 99 \%$ p.a. & $\begin{array}{l}\text { Roth (Karlsruhe, } \\
\text { Germany) }\end{array}$ \\
\hline $\begin{array}{l}30 \% \text { Acrylamide- } \\
\text { Bisacrylamide solution } \\
\text { (ratio 37.5:1) }\end{array}$ & $\begin{array}{l}\text { Rotiphorese } \AA \text { Gel } 30 \text { (37.5:1) ready for } \\
\text { use, gas-stabilized, aqueous, } 30 \% \\
\text { acrylamide stock solution with } 0.8 \% \\
\text { Bisacrylamide in a ratio of } 37.5: 1)\end{array}$ & $\begin{array}{l}\text { Roth (Karlsruhe, } \\
\text { Germany) }\end{array}$ \\
\hline $32 \% \mathrm{HCl}$ & $32 \% \mathrm{HCl}$ Rotipuran ${ }^{\circledR}$ p.a. & $\begin{array}{l}\text { Roth (Karlsruhe, } \\
\text { Germany) }\end{array}$ \\
\hline 3-Phosphoglycerate & $\begin{array}{l}\text { D-(-)-3-Phosphoglyceric acid disodium } \\
\geq 93 \%\end{array}$ & $\begin{array}{l}\text { Sigma-Aldrich } \\
\text { (St. Louis, } \\
\text { Missouri, USA) }\end{array}$ \\
\hline $\begin{array}{l}\text { 5,5'-Dithiobis }(2- \\
\text { nitrobenzoic acid) (DTNB) }\end{array}$ & Ellman's Reagent & $\begin{array}{l}\text { Thermo Scientific } \\
\text { (Rockford, } \\
\text { Illinois, USA) }\end{array}$ \\
\hline Acetic acid & Acetic acid Rotipuran ${ }^{\circledR} 100$ \% p.a. & $\begin{array}{l}\text { Roth (Karlsruhe, } \\
\text { Germany) }\end{array}$ \\
\hline Acetone & Acetone $\geq 99.5 \%$ for synthesis & $\begin{array}{l}\text { Roth (Karlsruhe, } \\
\text { Germany) }\end{array}$ \\
\hline Adenosine-5'-triphosphate & Adenosine-5'-triphosphate (ATP) & $\begin{array}{l}\text { Roche } \\
\text { Diagnostics } \\
\text { (Mannheim, } \\
\text { Germany) }\end{array}$ \\
\hline Agar for bacteria & $\begin{array}{l}\text { Agar-Agar, Kobe I, pulv. for microbiology } \\
\text { density } 0.55\end{array}$ & $\begin{array}{l}\text { Roth (Karlsruhe, } \\
\text { Germany) }\end{array}$ \\
\hline Agar for plants & $\begin{array}{l}\text { Agar-Agar, Plant, genetic engineering } \\
\text { quality for plants, density } 0.55\end{array}$ & $\begin{array}{l}\text { Roth (Karlsruhe, } \\
\text { Germany) }\end{array}$ \\
\hline Agarose & $\begin{array}{l}\text { Agarose BioReagent for molecular } \\
\text { biology, low EEO }\end{array}$ & $\begin{array}{l}\text { Sigma-Aldrich } \\
\text { (St. Louis, } \\
\text { Missouri, USA) }\end{array}$ \\
\hline $\begin{array}{l}\text { Aluminium sulfate } \\
\text { Hexadecahydrate }\end{array}$ & $\begin{array}{l}\text { Aluminium sulfate Hexadecahydrate, } \\
\text { purum p.a. } \geq 98.0 \%\end{array}$ & $\begin{array}{l}\text { Fluka (Buchs, } \\
\text { Switzerland) }\end{array}$ \\
\hline
\end{tabular}


Chemicals

\begin{tabular}{|c|c|c|}
\hline Chemical & Product name & Company \\
\hline $\begin{array}{l}\text { Ammoniumperoxodisulfat } \\
\text { e }\end{array}$ & Ammoniumperoxodisulfate $\geq 98 \%$ p.a. & $\begin{array}{l}\text { Roth (Karlsruhe, } \\
\text { Germany) }\end{array}$ \\
\hline Ammonium thiocyanate & $\begin{array}{l}\text { Ammonium thiocyanate ACS reagent, } \geq \\
97.5 \%\end{array}$ & $\begin{array}{l}\text { Sigma-Aldrich } \\
\text { (Steinheim, } \\
\text { Germany) }\end{array}$ \\
\hline Ampicillin & $\begin{array}{l}\text { Ampicilin sodium salt, BioScience Grade } \\
\text { for molecular biology and biochemistry }\end{array}$ & $\begin{array}{l}\text { Roth (Karlsruhe, } \\
\text { Germany) }\end{array}$ \\
\hline Basta & Basta Herbicide & $\begin{array}{l}\text { Bayer } \\
\text { (Leverkusen, } \\
\text { Germany) }\end{array}$ \\
\hline Bromophenol blue & $\begin{array}{l}\text { Bromophenol blue } \mathrm{Na} \text { salt for } \\
\text { electrophoresis }\end{array}$ & $\begin{array}{l}\text { Roth (Karlsruhe, } \\
\text { Germany) }\end{array}$ \\
\hline $\mathrm{CaCl}_{2} \times 2 \mathrm{H}_{2} \mathrm{O}$ & $\mathrm{CaCl}_{2} \times 2 \mathrm{H}_{2} \mathrm{O} \geq 99 \%$ p.a. & $\begin{array}{l}\text { Roth (Karlsruhe, } \\
\text { Germany) }\end{array}$ \\
\hline Chloroform & $\begin{array}{l}\text { Trichlormethane/Chloroform } \\
\text { Rotipuran } ₫ \geq 99 \% \text { p.a. }\end{array}$ & $\begin{array}{l}\text { Roth (Karlsruhe, } \\
\text { Germany) }\end{array}$ \\
\hline Citric acid Monohydrate & $\begin{array}{l}\text { Citric acid Monohydrate Rotipuran } \AA \\
\geq 99.5 \% \text { p.a. }\end{array}$ & $\begin{array}{l}\text { Roth (Karlsruhe, } \\
\text { Germany) }\end{array}$ \\
\hline $\begin{array}{l}\text { Coomassie Brilliant Blue } G \\
250\end{array}$ & $\begin{array}{l}\text { Coomassie Brilliant Blue G250 for } \\
\text { electrophoresis }\end{array}$ & $\begin{array}{l}\text { Merck } \\
\text { (Darmstadt, } \\
\text { Germany) }\end{array}$ \\
\hline D-Desthiobiotin & D-Desthiobiotin & $\begin{array}{l}\text { IBA (Göttingen, } \\
\text { Germany) }\end{array}$ \\
\hline $\begin{array}{l}\text { Desoxyadenosine } \\
\text { triphosphate (dATP) }\end{array}$ & Desoxyadenosine triphosphate & $\begin{array}{l}\text { LifeTechnologies } \\
\text { GmbH } \\
\text { (Darmstadt, } \\
\text { Germany) }\end{array}$ \\
\hline $\begin{array}{l}\text { Desoxycytosine } \\
\text { triphosphate (dCTP) }\end{array}$ & Desoxycytosine triphosphate & $\begin{array}{l}\text { LifeTechnologies } \\
\text { GmbH } \\
\text { (Darmstadt, } \\
\text { Germany) }\end{array}$ \\
\hline $\begin{array}{l}\text { Desoxyguanosine } \\
\text { triphosphate (dGTP) }\end{array}$ & Desoxyguanosine triphosphate & $\begin{array}{l}\text { LifeTechnologies } \\
\text { GmbH } \\
\text { (Darmstadt, } \\
\text { Germany) }\end{array}$ \\
\hline $\begin{array}{l}\text { Desoxythymidine } \\
\text { triphosphate (dTTP) }\end{array}$ & Desoxythymidine triphosphate & $\begin{array}{l}\text { LifeTechnologies } \\
\text { GmbH } \\
\text { (Darmstadt, } \\
\text { Germany) }\end{array}$ \\
\hline Dessicator grease & Dessicator grease white & $\begin{array}{l}\text { Roth (Karlsruhe, } \\
\text { Germany) }\end{array}$ \\
\hline
\end{tabular}




\begin{tabular}{|c|c|c|}
\hline Chemical & Product name & Company \\
\hline Diamide & Diamide & $\begin{array}{l}\text { Sigma-Aldrich } \\
\text { (Rehovot, Israel) }\end{array}$ \\
\hline $\begin{array}{l}\text { Dimethylsulfoxide } \\
\text { (DMSO) }\end{array}$ & $\begin{array}{l}\text { Dimethylsulfoxide } \geq 99.5 \% \text { BioScience- } \\
\text { Grade for molecular biology }\end{array}$ & $\begin{array}{l}\text { Roth (Karlsruhe, } \\
\text { Germany) }\end{array}$ \\
\hline Ethanol & Ethanol & $\begin{array}{l}\text { Nordbrand } \\
\text { GmbH } \\
\text { (Nordhausen, } \\
\text { Germany) }\end{array}$ \\
\hline Ethidium bromide (EtBr) & $\begin{array}{l}\text { Ethidium bromide } \geq 98 \% \text { for DNA } \\
\text { staining }\end{array}$ & $\begin{array}{l}\text { Roth (Karlsruhe, } \\
\text { Germany) }\end{array}$ \\
\hline Gentamicin & $\begin{array}{l}\text { Gentamycin sulfate non-sterile USP } \\
\text { Grade }\end{array}$ & $\begin{array}{l}\text { Amresco (Solon, } \\
\text { USA) }\end{array}$ \\
\hline Glucose Monohydrate & $\begin{array}{l}\mathrm{D}(+) \text {-Glucose Monohydrate } \geq 99.5 \% \text { p.a. } \\
\text { Ph. Eur. }\end{array}$ & $\begin{array}{l}\text { Roth (Karlsruhe, } \\
\text { Germany) }\end{array}$ \\
\hline Glutathione & L-Glutathione reduced & $\begin{array}{l}\text { Sigma-Aldrich } \\
\text { (Tokyo, Japan) }\end{array}$ \\
\hline Glutathione disulfide & L-Glutathione oxidized & $\begin{array}{l}\text { Sigma-Aldrich } \\
\text { (St. Louis, } \\
\text { Missouri, USA) }\end{array}$ \\
\hline Glycerol & $\begin{array}{l}\text { Glycerol Rotipuran } \AA \geq 99.5 \% \text { p.a., } \\
\text { wasserfrei }\end{array}$ & $\begin{array}{l}\text { Roth (Karlsruhe, } \\
\text { Germany) }\end{array}$ \\
\hline Glycerol $86 \%$ & Glycerol $\sim 86 \%$, Ph. Eur., reinst & $\begin{array}{l}\text { Roth (Karlsruhe, } \\
\text { Germany) }\end{array}$ \\
\hline Glycin & Glycin Pufferan ${ }^{\circledR} \geq 99 \%$ p.a. & $\begin{array}{l}\text { Roth (Karlsruhe, } \\
\text { Germany) }\end{array}$ \\
\hline GSNO & S-Nitrosoglutathione $\geq 97 \%$ & $\begin{array}{l}\text { Sigma-Aldrich } \\
\text { (Rehovot, Israel) }\end{array}$ \\
\hline Guanidine thiocyanate & $\begin{array}{l}\text { Guanidine thiocyanate } \geq 99 \% \text {, for } \\
\text { Biochemistry }\end{array}$ & $\begin{array}{l}\text { Roth (Karlsruhe, } \\
\text { Germany) }\end{array}$ \\
\hline HEDS & 2-Hydroxyethlydisulfide technical grade & $\begin{array}{l}\text { Sigma-Aldrich } \\
\text { (Steinheim, } \\
\text { Germany) }\end{array}$ \\
\hline HEPES & $\begin{array}{l}\text { HEPES Pufferan } ₫ \geq 99.5 \% \text { p.a. for } \\
\text { tissue culture }\end{array}$ & $\begin{array}{l}\text { Roth (Karlsruhe, } \\
\text { Germany) }\end{array}$ \\
\hline Imidazol & Imidazol Pufferan ${ }^{\circledR} \geq 99 \%$ p.a. & $\begin{array}{l}\text { Roth (Karlsruhe, } \\
\text { Germany) }\end{array}$ \\
\hline Iodoacetamide & Iodoacetamide & $\begin{array}{l}\text { Sigma-Aldrich } \\
\text { (St. Louis, } \\
\text { Missouri, USA) }\end{array}$ \\
\hline
\end{tabular}




\begin{tabular}{|c|c|c|}
\hline Chemical & Product name & Company \\
\hline iodoTMT $^{0}-126$ & Iodo'TMT0 $^{0}-126^{\mathrm{TM}}$ Label Reagent Set & $\begin{array}{l}\text { Thermo Scientific } \\
\text { (Rockford, } \\
\text { Illinois, USA) }\end{array}$ \\
\hline Isopropanol & 2-propanol Rotipuran ${ }^{\circledR} \geq 99.8 \%$ p.a. & $\begin{array}{l}\text { Roth (Karlsruhe, } \\
\text { Germany) }\end{array}$ \\
\hline $\begin{array}{l}\text { Isopropyl- } \beta-\mathrm{D} \text { - } \\
\text { thiogalactopyranoside } \\
\text { (IPTG) }\end{array}$ & $\begin{array}{l}\text { IPTG } \geq 99 \% \text {, BioScience Grade, dioxan- } \\
\text { free, animal-free }\end{array}$ & $\begin{array}{l}\text { Roth (Karlsruhe, } \\
\text { Germany) }\end{array}$ \\
\hline $\mathrm{K}_{2} \mathrm{HPO}_{4}$ & $\begin{array}{l}\text { di-potassium hydrogenphosphate } \geq 98 \% \text {, } \\
\text { waterfree }\end{array}$ & $\begin{array}{l}\text { Roth (Karlsruhe, } \\
\text { Germany) }\end{array}$ \\
\hline $\mathrm{KH}_{2} \mathrm{PO}_{4}$ & $\mathrm{KH}_{2} \mathrm{PO}_{4}$ (waterfree) $\geq 99 \%$ p.a. & $\begin{array}{l}\text { Roth (Karlsruhe, } \\
\text { Germany) }\end{array}$ \\
\hline $\mathrm{KOH}$ & $\mathrm{KOH} \geq 85 \%$ p.a., in pellets & $\begin{array}{l}\text { Roth (Karlsruhe, } \\
\text { Germany) }\end{array}$ \\
\hline $\mathrm{L}(+)$-Ascorbic acid & $\begin{array}{l}\mathrm{L}(+) \text {-Ascrobic acid p.a. or } \mathrm{L}(+) \text {-Ascorbic } \\
\text { acid } \geq 99 \% \text { p.a. }\end{array}$ & $\begin{array}{l}\text { Roth (Karlsruhe, } \\
\text { Germany) }\end{array}$ \\
\hline Lactose Monohydrate & Lactose Monohydrate & $\begin{array}{l}\text { Merck } \\
\text { (Darmstadt, } \\
\text { Germany) }\end{array}$ \\
\hline L-Cysteine & L-Cysteine 97 \% & $\begin{array}{l}\text { Sigma-Aldrich } \\
\text { (Steinheim, } \\
\text { Germany) }\end{array}$ \\
\hline Maltose Monohydrate & Maltose Monohydrate crystallized & $\begin{array}{l}\text { Merck } \\
\text { (Darmstadt, } \\
\text { Germany) }\end{array}$ \\
\hline Meat extract & LAB-LEMCO' POWDER Meat extract & $\begin{array}{l}\text { Oxoid } \\
\text { (Basingstoke, } \\
\text { England) }\end{array}$ \\
\hline Methanol & Methanol & $\begin{array}{l}\text { Merck } \\
\text { (Darmstadt, } \\
\text { Germany) }\end{array}$ \\
\hline $\mathrm{MgCl}_{2}$ & $\mathrm{MgCl}_{2}$ & $\begin{array}{l}\text { Roth (Karlsruhe, } \\
\text { Germany) }\end{array}$ \\
\hline $\mathrm{MgSO}_{4} \times 7 \mathrm{H}_{2} \mathrm{O}$ & $\mathrm{MgSO}_{4} \times 7 \mathrm{H}_{2} \mathrm{O} \geq 99 \%$ p.a. & $\begin{array}{l}\text { Roth (Karlsruhe, } \\
\text { Germany) }\end{array}$ \\
\hline $\begin{array}{l}\text { Monobromobimane } \\
(\mathrm{mBBr})\end{array}$ & Monobromobimane (mBBr) & $\begin{array}{l}\text { Invitrogen } \\
\text { (Eugene, Oregon, } \\
\text { USA) }\end{array}$ \\
\hline $\begin{array}{l}\text { Murashige \& Skoog (MS) } \\
\text { medium }\end{array}$ & $\begin{array}{l}\text { Murashige \& Skoog medium including } \\
\text { vitamins }\end{array}$ & $\begin{array}{l}\text { Duchefa } \\
\text { Biochemie BV }\end{array}$ \\
\hline
\end{tabular}




\begin{tabular}{|c|c|c|}
\hline Chemical & Product name & Company \\
\hline & & $\begin{array}{l}\text { (Haarlem, The } \\
\text { Netherlands) }\end{array}$ \\
\hline $\mathrm{N}_{2}(\mathrm{l})$ & $\mathrm{N}_{2}(\mathrm{l})$ & $\begin{array}{l}\text { Westfalen AG } \\
\text { (Münster, } \\
\text { Germany) }\end{array}$ \\
\hline $\mathrm{Na}_{3}$-citrate $\times 2 \mathrm{H}_{2} \mathrm{O}$ & Tri-Natriumcitrat Dihydrat p. A. & $\begin{array}{l}\text { Roth (Karlsruhe, } \\
\text { Germany) }\end{array}$ \\
\hline $\mathrm{Na}_{2}-\mathrm{EDTA} \times 2 \mathrm{H}_{2} \mathrm{O}$ & $\begin{array}{l}\text { EDTA Disodium salt: Dihydrate, Sigma } \\
\text { grade }\end{array}$ & $\begin{array}{l}\text { Sigma (Steinheim, } \\
\text { Germany) }\end{array}$ \\
\hline $\mathrm{Na}_{2} \mathrm{HPO}_{4}$ & $\mathrm{Na}_{2} \mathrm{HPO}_{4}$ (waterfree) $\geq 99 \%$ p.a. & $\begin{array}{l}\text { Roth (Karlsruhe, } \\
\text { Germany) }\end{array}$ \\
\hline $\mathrm{Na}_{2} \mathrm{HPO}_{4} \times 2 \mathrm{H}_{2} \mathrm{O}$ & $\mathrm{Na}_{2} \mathrm{HPO}_{4} \times 2 \mathrm{H}_{2} \mathrm{O} \geq 98 \%$ Ph. Eur. & $\begin{array}{l}\text { Roth (Karlsruhe, } \\
\text { Germany) }\end{array}$ \\
\hline $\mathrm{NaCl}$ & $\mathrm{NaCl} \geq 99.5 \%$ p.a. & $\begin{array}{l}\text { Roth (Karlsruhe, } \\
\text { Germany) }\end{array}$ \\
\hline $\mathrm{NADH}$ & $\begin{array}{l}\text { NADH disodium salt, grade II, approx. } \\
98 \%\end{array}$ & $\begin{array}{l}\text { Roche } \\
\text { Diagnostics } \\
\text { (Mannheim, } \\
\text { Germany) }\end{array}$ \\
\hline NADPH & NADPH & $\begin{array}{l}\text { Sigma-Aldrich } \\
\text { (Taufkirchen, } \\
\text { Germany) }\end{array}$ \\
\hline $\mathrm{NaH}_{2} \mathrm{PO}_{4} \times \mathrm{H}_{2} \mathrm{O}$ & $\mathrm{NaH}_{2} \mathrm{PO}_{4} \times \mathrm{H}_{2} \mathrm{O} \geq 98 \%$ p.a. & $\begin{array}{l}\text { Roth (Karlsruhe, } \\
\text { Germany) }\end{array}$ \\
\hline $\mathrm{NaOH}$ & $\mathrm{NaOH} \geq 99 \%$ p.a. in pellets & $\begin{array}{l}\text { Roth (Karlsruhe, } \\
\text { Germany) }\end{array}$ \\
\hline o-Phosphoric acid & $\begin{array}{l}\text { o-Phosphoric acid Rotipuran } \AA \geq 85 \% \\
\text { p.a. }\end{array}$ & $\begin{array}{l}\text { Roth (Karlsruhe, } \\
\text { Germany) }\end{array}$ \\
\hline Phenol & $\begin{array}{l}\text { Phenol solution saturated with } 0.1 \mathrm{M} \\
\text { citrate buffer } \mathrm{pH}=4.3 \pm 0.2 \text { BioReagent } \\
\text { for molecular biology }\end{array}$ & $\begin{array}{l}\text { Sigma (Steinheim, } \\
\text { Germany) }\end{array}$ \\
\hline Phosphinotricine & Phosphinotricine & $\begin{array}{l}\text { Duchefa } \\
\text { Biochemie BV } \\
\text { (Haarlem, The } \\
\text { Netherlands) }\end{array}$ \\
\hline Protease Inhibitor Cocktail & $\begin{array}{l}\text { Protease Inhibitor Cocktail, EDTA-free, } \\
\text { Mini Tablet }\end{array}$ & $\begin{array}{l}\text { Bimake (Munich, } \\
\text { Germany) }\end{array}$ \\
\hline Rifampicin & Rifampicin $>95 \%$ & $\begin{array}{l}\text { Duchefa } \\
\text { Biochemie BV } \\
\text { (Haarlem, The } \\
\text { Netherlands) }\end{array}$ \\
\hline
\end{tabular}


Chemicals

\begin{tabular}{|c|c|c|}
\hline Chemical & Product name & Company \\
\hline SDS & $\begin{array}{l}\text { Sodium lauryl sulfate } \geq 99 \% \text { p.a., for } \\
\text { biochemistry }\end{array}$ & $\begin{array}{l}\text { Roth (Karlsruhe, } \\
\text { Germany) }\end{array}$ \\
\hline Silwet L77 & Silwet L77 & $\begin{array}{l}\text { Kurt Obermeier } \\
\text { GmbH (Bad } \\
\text { Berleburg, } \\
\text { Germany) }\end{array}$ \\
\hline Skimmed milk powder & Skimmed milk powder, easily soluble & $\begin{array}{l}\text { SUCOFIN } \\
\text { (Zeven, } \\
\text { Germany) }\end{array}$ \\
\hline Sodium acetate & Sodium acetate $\geq 99 \%$ p.a. waterfree & $\begin{array}{l}\text { Roth (Karlsruhe, } \\
\text { Germany) }\end{array}$ \\
\hline $\begin{array}{l}\text { Sodium hypochlorite } \\
\text { solution }\end{array}$ & $\begin{array}{l}\text { Sodium hypochlorite solution } 12 \% \mathrm{Cl} \text {. } \\
\text { Techn. }\end{array}$ & $\begin{array}{l}\text { Roth (Karlsruhe, } \\
\text { Germany) }\end{array}$ \\
\hline Spectinomycin & $\begin{array}{l}\text { Spectinomycin Dihydrochloride } \\
\text { Pentahydrate }\end{array}$ & $\begin{array}{l}\text { Duchefa } \\
\text { Biochemie BV } \\
\text { (Haarlem, The } \\
\text { Netherlands) }\end{array}$ \\
\hline Sucrose & Sucrose crystallized & $\begin{array}{l}\text { Duchefa } \\
\text { Biochemie BV } \\
\text { (Haarlem, The } \\
\text { Netherlands) }\end{array}$ \\
\hline TCA & Trichloroacetic acid solution $6.1 \mathrm{~N}$ & $\begin{array}{l}\text { Sigma-Aldrich } \\
\text { (St. Louis, } \\
\text { Missouri, USA) }\end{array}$ \\
\hline TCEP & $\begin{array}{l}\text { Tris(2-carboxyethyl)phosphine } \\
\text { hydrochloride }\end{array}$ & $\begin{array}{l}\text { Sigma-Aldrich } \\
\text { (St. Louis, } \\
\text { Missouri, USA) }\end{array}$ \\
\hline TEMED & $99 \%$ p.a. for electrophoresis & $\begin{array}{l}\text { Roth (Karlsruhe, } \\
\text { Germany) }\end{array}$ \\
\hline $\begin{array}{l}\text { trans-4,5-Dihydroxy-1,2- } \\
\text { dithiane (dithiane, oxidized } \\
\text { DTT) }\end{array}$ & trans-4,5-Dihydroxy-1,2-dithiane $\geq 99 \%$ & $\begin{array}{l}\text { Sigma-Aldrich } \\
\text { (St. Louis, } \\
\text { Missouri, USA) }\end{array}$ \\
\hline TRIS & TRIS Pufferan $® \geq 99.9 \%$ p.a. & $\begin{array}{l}\text { Roth (Karlsruhe, } \\
\text { Germany) }\end{array}$ \\
\hline $\begin{array}{l}\text { Tryptone/peptone from } \\
\text { casein }\end{array}$ & $\begin{array}{l}\text { Tryptone/peptone from casein } \\
\text { (pancreatically digested) }\end{array}$ & $\begin{array}{l}\text { Roth (Karlsruhe, } \\
\text { Germany) }\end{array}$ \\
\hline Tween-20 & Tween-20 & $\begin{array}{l}\text { Roth (Karlsruhe, } \\
\text { Germany) }\end{array}$ \\
\hline Urea & Urea $\geq 99.5 \%$ p.a. BioScience-Grade & $\begin{array}{l}\text { Roth (Karlsruhe, } \\
\text { Germany) }\end{array}$ \\
\hline
\end{tabular}


Material

\begin{tabular}{lll}
\hline Chemical & Product name & Company \\
\hline Yeast extract & Yeast extract, pulv. for bacteriology & $\begin{array}{l}\text { Roth (Karlsruhe, } \\
\text { Germany) }\end{array}$ \\
\end{tabular}


Material

\section{Commercially available reagents}

\begin{tabular}{|c|c|c|}
\hline Reagent & Product name & Company \\
\hline $\begin{array}{l}\text { DNA size } \\
\text { standard }\end{array}$ & $\begin{array}{l}\text { Gene Ruler DNA Ladder Mix } \\
0.5 \mu \mathrm{g} / \mu \mathrm{l}\end{array}$ & $\begin{array}{l}\text { Thermo Scientific (Rockford, } \\
\text { Illinois, USA) }\end{array}$ \\
\hline HENS Buffer & $\begin{array}{l}\text { HENS Buffer }(100 \mathrm{mM} \\
\text { HEPES, pH 8.0, } 1 \mathrm{mM} \text { EDTA, } \\
0.1 \mathrm{mM} \text { Neocuproine, } 1 \% \text { SDS })\end{array}$ & $\begin{array}{l}\text { Thermo Scientific (Rockford, } \\
\text { Illinois, USA) }\end{array}$ \\
\hline \multirow{2}{*}{$\begin{array}{l}\text { HRP substrates } \\
\text { for Western Blot } \\
\text { detection }\end{array}$} & $\begin{array}{l}\text { Luminata }^{\mathrm{TM}} \text { Forte Western HRP } \\
\text { Substrate }\end{array}$ & $\begin{array}{l}\text { Millipore (Billerica, Massachusets, } \\
\text { USA) }\end{array}$ \\
\hline & $\begin{array}{l}\text { SuperSignal }{ }^{\mathrm{TM}} \text { West Femto } \\
\text { Maximum Sensitivity Substrate }\end{array}$ & $\begin{array}{l}\text { Thermo Scientific (Rockford, } \\
\text { Illinois, USA) }\end{array}$ \\
\hline $\begin{array}{l}\text { Protein size } \\
\text { standard for } \\
\text { native PAGE }\end{array}$ & $\begin{array}{l}\text { NativeMark }{ }^{\mathrm{TM}} \text { Unstained } \\
\text { Protein Standard }\end{array}$ & $\begin{array}{l}\text { Invitrogen by Thermo Fisher } \\
\text { Scientific/Life Technologies } \\
\text { Corporation (Carlsbad, California, } \\
\text { USA) }\end{array}$ \\
\hline $\begin{array}{l}\text { Protein size } \\
\text { standard for SDS- } \\
\text { PAGE }\end{array}$ & $\begin{array}{l}\text { Page Ruler }{ }^{\mathrm{TM}} \text { Prestained Protein } \\
\text { Ladder }\end{array}$ & $\begin{array}{l}\text { Thermo Scientific (Vilnius, } \\
\text { Lithuania) }\end{array}$ \\
\hline \multirow{3}{*}{$\begin{array}{l}\text { Chemicals for } \\
\text { protein } \\
\text { concentrations } \\
\text { measurments }\end{array}$} & $\begin{array}{l}\text { Ionic Detergent Compatibility } \\
\text { Reagent (IDCR) }\end{array}$ & $\begin{array}{l}\text { Thermo Scientific (Rockford, } \\
\text { Illinois, USA) }\end{array}$ \\
\hline & $\begin{array}{l}\text { Pierce }{ }^{\circledR} 660 \mathrm{~nm} \text { Protein Assay } \\
\text { Reagent, } 750 \mathrm{ml}\end{array}$ & $\begin{array}{l}\text { Thermo Scientific (Rockford, } \\
\text { Illinois, USA) }\end{array}$ \\
\hline & Roti ${ }^{\circledR}$-Quant 5x Konzentrat & Roth (Karlsruhe, Germany) \\
\hline
\end{tabular}




\section{Proteins}

\begin{tabular}{|c|c|}
\hline Protein & Company \\
\hline $\begin{array}{l}\text { 3-Phosphoglyceric Phosphokinase from baker's yeast } \\
\text { (S. cerevisiae) } 0.9 \mathrm{mg} / \mathrm{ml} 1787 \text { units } / \mathrm{mg} \text { protein }\end{array}$ & $\begin{array}{l}\text { Sigma-Aldrich (St. Louis, Missouri, } \\
\text { USA) }\end{array}$ \\
\hline AcTEVTM Protease $10 \mathrm{U} / \mu \mathrm{l}$ & $\begin{array}{l}\text { Invitrogen by Thermo Fisher } \\
\text { Scientific, Life Technologies } \\
\text { Corporation (Carlsbad, California, } \\
\text { USA) }\end{array}$ \\
\hline $\begin{array}{l}\text { Amersham }{ }^{\mathrm{TM}} \text { ECL }^{\mathrm{TM}} \text { Anti-mouse IgG, Horesradish } \\
\text { Peroxidase-Linked Whole Antibody (from sheep) }\end{array}$ & $\begin{array}{l}\text { GE Healthcare (Buckinghamshire, } \\
\text { UK) }\end{array}$ \\
\hline $\begin{array}{l}\text { Amersham }{ }^{\mathrm{TM}} \text { ECL }{ }^{\mathrm{TM}} \text { Anti-rabbit IgG, Horseradish } \\
\text { Peroxidase-Linked Whole Antibody (from donkey) }\end{array}$ & $\begin{array}{l}\text { GE Healthcare (Buckinghamshire, } \\
\text { UK) }\end{array}$ \\
\hline $\begin{array}{l}\text { Anti-Green Fluorescent Protein stabilized antibody } \\
\text { preparation (mixture of two monoclonal antibodies, } \\
\text { clone } 7.1 \text { and } 13.1 \text {, both clones are Mouse } \operatorname{IgG}_{1 \varkappa} \text { ) }\end{array}$ & Roche (Basel, Switzerland) \\
\hline Anti-HA tag antibody from rabbit, ChIP Grade & Abcam (Cambridge, USA) \\
\hline $\begin{array}{l}\text { BIOTAQ DNA Polymerase } 5 \mathrm{u} / \mu \mathrm{l} \text { with } 10 \mathrm{x} \mathrm{NH} 4 \\
\text { Reaction Buffer }\left(\mathrm{No} M g C l_{2}\right) \text { and } 50 \mathrm{mM} \mathrm{MgCl}_{2} \text { Stock } \\
\text { Solution }\end{array}$ & Bioline (Luckenwalde, Germany) \\
\hline $\begin{array}{l}\text { Calf Intestinal Alkaline Posphatase } 1 \mathrm{U} / \mu \text { l with CIAP } \\
\text { Dilution Buffer }\end{array}$ & $\begin{array}{l}\text { Invitrogen (Carlsbad, California, } \\
\text { USA) }\end{array}$ \\
\hline $\begin{array}{l}\text { DNase I, RNase-free, } 1 \mathrm{U} / \mu \mathrm{l} \text { with } 10 \mathrm{x} \text { Reaction buffer } \\
\text { with } \mathrm{MgCl}_{2} \text { for DNase I and } 50 \mathrm{mM} \text { EDTA }\end{array}$ & $\begin{array}{l}\text { Thermo Scientific (Vilnius, } \\
\text { Lithuania) }\end{array}$ \\
\hline Fluorscein Calibration Dye $1 \mathrm{mM}$ in DMSO & $\begin{array}{l}\text { BioRad Laboratories (Munich, } \\
\text { Germany) }\end{array}$ \\
\hline Gateway cloning system & $\begin{array}{l}\text { LifeTechnologies GmbH } \\
\text { (Darmstadt, Germany) }\end{array}$ \\
\hline $\begin{array}{l}\text { Glutathione Reductase from baker's yeast (S. cerevisiae) } \\
\text { (ammoniumsulfate suspension) } 1.5 \mathrm{mg} \text { protein } / \mathrm{ml} \text {, } \\
170 \text { units } / \mathrm{mg}\end{array}$ & $\begin{array}{l}\text { Sigma-Aldrich (St. Louis, Missouri, } \\
\text { USA) }\end{array}$ \\
\hline $\begin{array}{l}\text { Glyceraldehyde- } 3 \text {-phosphate Dehydrogenase from } \\
\text { rabbit muscle } \geq 75 \text { units } / \mathrm{mg} \text { protein ( } 97 \text { units } / \mathrm{mg} \\
\text { protein) }\end{array}$ & $\begin{array}{l}\text { Sigma-Aldrich (St. Louis, Missouri, } \\
\text { USA) }\end{array}$ \\
\hline Insulin from bovine pancreas Hybri-Max ${ }^{\mathrm{TM}}$ & $\begin{array}{l}\text { Sigma-Aldrich (St. Louis, Missouri, } \\
\text { USA) }\end{array}$ \\
\hline Lysozyme & AGS GmbH \\
\hline
\end{tabular}


Proteins

\begin{tabular}{|c|c|}
\hline Protein & Company \\
\hline $\begin{array}{l}\text { Phusion High-Fidelity DNA Polymerase }(2 \mathrm{U} / \mu \mathrm{l}) \text { with } \\
\left.5 \mathrm{x} \text { Phusion HF Buffer (contains } 7.5 \mathrm{mM} \mathrm{MgCl}_{2}\right)\end{array}$ & $\begin{array}{l}\text { Thermo Scientific (Vilnius, } \\
\text { Lithuania) }\end{array}$ \\
\hline Restriction enzymes with corresponding buffers & $\begin{array}{l}\text { Thermo Scientific (Vilnius, } \\
\text { Lithuania), New England Biolabs } \\
\text { (Frankfurt am Main, Germany), } \\
\text { MBI Fermentas (Vilnius, } \\
\text { Lithuania), Boehringer Mannheim } \\
\text { GmbH (Mannheim, Germany), } \\
\text { GIBCO BRL (Eggenstein, } \\
\text { Germany), Promega (Madison, } \\
\text { Wisconsin, USA) }\end{array}$ \\
\hline $\begin{array}{l}\text { Revert Aid H Minus Reverse Transcriptase } 200 \mathrm{U} / \mu \mathrm{l} \\
\text { with 5x Reaction Buffer for RT }\end{array}$ & $\begin{array}{l}\text { Thermo Scientific (Vilnius, } \\
\text { Lithuania) }\end{array}$ \\
\hline Ribonuclease A & Roth (Karlsruhe, Germany) \\
\hline $\begin{array}{l}\text { StrepMAB classic (IgG1, Anti strep-tag } I I \circledR \\
\text { monoclonal antibody, purified) }\end{array}$ & IBA (Göttingen, Germany) \\
\hline $\begin{array}{l}\text { SYBR }{ }^{\circledR} \text { Green I Nucleic Acid Gel Stain 10000x } \\
\text { concentrate in DMSO }\end{array}$ & $\begin{array}{l}\text { Cambrex BioScience Rockland } \\
\text { (Rockland, Maine, USA) }\end{array}$ \\
\hline $\begin{array}{l}\text { T4 DNA Ligase } 5 \text { Weiss } U / \mu \text { l with 10x T4 DNA } \\
\text { Ligase Buffer }\end{array}$ & $\begin{array}{l}\text { Thermo Scientific (Vilnius, } \\
\text { Lithuania) }\end{array}$ \\
\hline $\begin{array}{l}\text { T4 DNA Polymerase } 5 \mathrm{u} / \mu \mathrm{l} \text { with } 5 \mathrm{x} \text { Buffer for T4 } \\
\text { DNA Polymerase }\end{array}$ & $\begin{array}{l}\text { Fermentas (Waltham, } \\
\text { Massachusetts, USA) }\end{array}$ \\
\hline
\end{tabular}




\section{Kits}

\begin{tabular}{lll}
\hline Kit & Product name & Company \\
\hline $\begin{array}{l}\text { Detection of redox modifications } \\
\text { on proteins }\end{array}$ & $\begin{array}{l}\text { Pierce }^{\text {TM }} \text { S-Nitrosylation } \\
\text { Western Blot Kit }\end{array}$ & $\begin{array}{l}\text { Thermo Scientific } \\
\text { (Rockford, Illinois, } \\
\text { USA) }\end{array}$ \\
DNA extraction from plants & DNeasy Plant Mini Kit & $\begin{array}{l}\text { Qiagen (Hilden, } \\
\text { Germany) }\end{array}$ \\
PCR and gel purification & $\begin{array}{l}\text { NucleoSpin }{ }^{\circledR} \text { Gel and PCR } \\
\text { clean-up }\end{array}$ & $\begin{array}{l}\text { Macherey \& Nagel } \\
\text { (Düren, Germany) }\end{array}$ \\
Plasmid Midi Preparation & $\begin{array}{l}\text { NucleoBond }{ }^{\circledR} \text { Xtra Midi } \\
\text { Plasmid Mini Preparation }\end{array}$ & $\begin{array}{l}\text { Macherey \& Nagel } \\
\text { (Düren, Germany) }\end{array}$ \\
& NucleoSpin ${ }^{\circledR}$ Plasmid & $\begin{array}{l}\text { Macherey \& Nagel } \\
\text { (Düren, Germany) }\end{array}$ \\
\hline
\end{tabular}




\section{Lab material}

\begin{tabular}{|c|c|c|}
\hline Lab material & Product name & Company \\
\hline ÄKTA tubes & $\begin{array}{l}\text { Tube } 12 \mathrm{ml}, \mathrm{PS}, 16 / 100 \mathrm{~mm} \text {, } \\
\text { round bottom, clear }\end{array}$ & $\begin{array}{l}\text { Greiner bio-one } \\
\text { (Frickenhausen, Germany) }\end{array}$ \\
\hline Aluminium foil & $\begin{array}{l}\text { Rotilabo® Aluminiumfolie } \\
\text { Stärke } 30 \mu \mathrm{m}\end{array}$ & Roth (Karlsruhe, Germany) \\
\hline ARACONs (tube and base) & ARACONs & Betatech (Gent, Belgium) \\
\hline Atmosbag glove bag & Atmosbag glove bag & $\begin{array}{l}\text { Sigma-Aldrich (St. Louis, } \\
\text { Missouri, USA) }\end{array}$ \\
\hline \multirow[t]{3}{*}{$\begin{array}{l}\text { Bead material for protein } \\
\text { purification }\end{array}$} & $\begin{array}{l}\text { Strep-Tactin }{ }^{\circledR} \text { Speharose }{ }^{\circledR} \\
50 \% \text { Suspension } 20 \mathrm{ml}\end{array}$ & IBA (Göttingen, Germany) \\
\hline & Amylose Resin $15 \mathrm{ml}$ & $\begin{array}{l}\text { New England Biolabs } \\
\text { (Frankfurt am Main, } \\
\text { Germany) }\end{array}$ \\
\hline & $\begin{array}{l}\text { Profinity }{ }^{\mathrm{TM}} \mathrm{IMAC} \mathrm{Ni}- \\
\text { Charged Resin }\end{array}$ & $\begin{array}{l}\text { BioRad Laboratories } \\
\text { (Munich, Germany) }\end{array}$ \\
\hline Blotting paper & $\begin{array}{l}\text { Blotting-Papier MN218B } 58 \\
\times 60 \mathrm{~cm}\end{array}$ & $\begin{array}{l}\text { Macherey \& Nagel (Düren, } \\
\text { Germany) }\end{array}$ \\
\hline \multirow[t]{2}{*}{$\begin{array}{l}\text { Buffer filtration unit incl. } \\
\text { Filters }\end{array}$} & $\begin{array}{l}\text { Membrane Filter, Non- } \\
\text { sterile, NYLON, Size } 0.2 \\
\text { micron, } 47 \mathrm{~mm}\end{array}$ & $\begin{array}{l}\text { Thermo Fisher Scientific } \\
\text { (Rochester, New York, USA) }\end{array}$ \\
\hline & $\begin{array}{l}\text { Reusable Bottle-Top Filter, } \\
\text { PSF, fits } 45 \mathrm{~mm} \text { neck, size } \\
47 \mathrm{~mm}, 500 \mathrm{ml}\end{array}$ & $\begin{array}{l}\text { Thermo Scientific } \\
\text { Nalgene } \\
\text { (Rochester, New Yoducts }\end{array}$ \\
\hline \multirow[t]{3}{*}{$\begin{array}{l}\text { Columns for protein } \\
\text { purification }\end{array}$} & StrepTrap ${ }^{\mathrm{TM}}$ HP $5 \mathrm{x} 1 \mathrm{ml}$ & $\begin{array}{l}\text { GE Healthcare (Uppsala, } \\
\text { Sweden) }\end{array}$ \\
\hline & MBPTrap $^{\text {TM }}$ HP $5 \times 5 \mathrm{ml}$ & $\begin{array}{l}\text { GE Healthcare (Uppsala, } \\
\text { Sweden) }\end{array}$ \\
\hline & HisTrap $^{\mathrm{TM}} \mathrm{HP}$ 1x 5ml & $\begin{array}{l}\text { GE Healthcare (Uppsala, } \\
\text { Sweden) }\end{array}$ \\
\hline \multirow[t]{2}{*}{ Concentrators } & $\begin{array}{l}\text { Spin-X® UF } 6 ; 30 \text { or } 50 \\
\text { kDa MWCO PES non- } \\
\text { sterile } 6 \mathrm{ml}\end{array}$ & Corning (Flintshire, England) \\
\hline & Vivaspin 20; 3 kDa MWCO & $\begin{array}{l}\text { GE Healthcare } \\
\text { (Buckinghamshire, UK) }\end{array}$ \\
\hline
\end{tabular}




\begin{tabular}{|c|c|c|}
\hline Lab material & Product name & Company \\
\hline & Vivaspin $2 ; 3$ kDa MWCO & $\begin{array}{l}\text { GE Healthcare } \\
\text { (Buckinghamshire, UK) }\end{array}$ \\
\hline Cuvettes & $\begin{array}{l}\text { Cuvettes Polystyrene } 10 \times 4 \\
\times 45 \mathrm{~mm}\end{array}$ & $\begin{array}{l}\text { Sarstedt (Nümbrecht, } \\
\text { Germany) }\end{array}$ \\
\hline \multirow[t]{2}{*}{ Dialysis tubing } & $\begin{array}{l}\text { ZelluTrans regenerated } \\
\text { cellulose Nominal MWCO } \\
12000 \text { - } 14000 \text {, Flat Width } \\
25 \mathrm{~mm} \text {, Length } 15 \mathrm{~m} \text {, Wall } \\
\text { thickness } 20 \mu \mathrm{m}\end{array}$ & Roth (Karlsruhe, Germany) \\
\hline & $\begin{array}{l}\text { Serva } 44310.01 \text { MEMBRA- } \\
\text { CEL } \AA \text { dialysis tubing RC } \\
\text { diameter } 16 \mathrm{~mm}, 5 \mathrm{~m}\end{array}$ & Serva (Heidelberg, Germany) \\
\hline \multirow[t]{4}{*}{ Filter pipette tips } & $\begin{array}{l}96 \text { Biosphere }{ }^{\circledR} \text { Filter Tips } \\
0.1 \text { - } 10 \mu \text { l (Sterile/DNA- } \\
\text { /DNase/ RNase-/PCR } \\
\text { inibitor/ATP-/Pyrogen-free) }\end{array}$ & $\begin{array}{l}\text { Sarstedt (Nümbrecht, } \\
\text { Germany) }\end{array}$ \\
\hline & $\begin{array}{l}\text { Filter Tip } 20 \mu \mathrm{l} \text { (DNA- } \\
\text { /DNase-/RNase-/PCR } \\
\text { inhibitor-free) }\end{array}$ & $\begin{array}{l}\text { Sarstedt (Nümbrecht, } \\
\text { Germany) }\end{array}$ \\
\hline & $\begin{array}{l}\text { Filter Tip } 100 \mu \mathrm{l} \text { (DNA- } \\
\text { /DNase-/RNase-/PCR } \\
\text { inhibitor-free) }\end{array}$ & $\begin{array}{l}\text { Sarstedt (Nümbrecht, } \\
\text { Germany) }\end{array}$ \\
\hline & $\begin{array}{l}\text { Filter Tip } 1000 \mu \mathrm{l}(\mathrm{DNA}- \\
/ \text { DNase-/RNase-/PCR } \\
\text { inhibitor-free) }\end{array}$ & $\begin{array}{l}\text { Sarstedt (Nümbrecht, } \\
\text { Germany) }\end{array}$ \\
\hline \multirow[t]{3}{*}{ Filters } & $\begin{array}{l}\text { Minisart }{ }^{\circledR} \text { syringe filters } \\
\text { NML hydrophilic single use } \\
\text { filter unit, non-pyrogenic } 0.2 \\
\mu \mathrm{m} \text { diameter }\end{array}$ & $\begin{array}{l}\text { Sartorius Stedim Biotech } \\
\text { GmbH (Göttingen, } \\
\text { Germany) }\end{array}$ \\
\hline & $\begin{array}{l}\text { Disposable filter, sterile, } \\
\text { non-pyrogenic, hydrophilic, } \\
0.45 \mu \mathrm{m} \text { diameter }\end{array}$ & $\begin{array}{l}\text { Sartorius Stedim Biotech } \\
\text { GmbH (Göttingen, } \\
\text { Germany) }\end{array}$ \\
\hline & $\begin{array}{l}\text { Filtropur BT25 0.2, } 250 \mathrm{ml} \\
\text { Bottle Top Filter }\end{array}$ & Sarstedt (Newton, USA) \\
\hline Fluorescence 96-well plates & $\begin{array}{l}\text { Greiner Bio-One } 96 \text {-well } \\
\text { (half area) non-sterile } \\
\text { polystyrene plate, medium } \\
\text { binding, colour: black with } \\
\text { transparent bottom }\end{array}$ & $\begin{array}{l}\text { Greiner bio-one } \\
\text { (Frickenhausen, Germany) }\end{array}$ \\
\hline Gel filtration columns & $\begin{array}{l}\text { PD-10 Columns Sephadex } \\
\text { G-25M }\end{array}$ & $\begin{array}{l}\text { GE Healthcare } \\
\text { (Buckinghamshire, UK) }\end{array}$ \\
\hline
\end{tabular}




\begin{tabular}{|c|c|c|}
\hline Lab material & Product name & Company \\
\hline & $\begin{array}{l}\text { ZebaTM Spin Desalting } \\
\text { Columns } 0.5 \mathrm{ml} \text { for } 30-130 \\
\mu \mathrm{l} \text { sample }\end{array}$ & $\begin{array}{l}\text { Thermo Fisher Scientific } \\
\text { (Rochester, New York, USA) }\end{array}$ \\
\hline \multirow[t]{2}{*}{ Glass beads } & Glass globules $\varnothing 0.1 \mathrm{~mm}$ & Roth (Karlsruhe, Germany) \\
\hline & $\begin{array}{l}\text { Glass beads, acid-washed } \\
425-600 \mu \mathrm{m}(30-40 \text { U. S. } \\
\text { sieve })\end{array}$ & $\begin{array}{l}\text { Sigma-Aldrich (Steinheim, } \\
\text { Germany) }\end{array}$ \\
\hline $\begin{array}{l}\text { Glass pipettes }(0.5,1,2,5,10 \\
\text { and } 20 \mathrm{ml})\end{array}$ & $\begin{array}{l}\text { Glass pipettes }(0.5,1,2,5 \text {, } \\
10 \text { and } 20 \mathrm{ml})\end{array}$ & Brand (Wertheim, Germany) \\
\hline $\begin{array}{l}\text { Glass ware (beakers, flasks, } \\
\text { bottles, dessicator) }\end{array}$ & $\begin{array}{l}\text { Glass beakers, glass flasks, } \\
\text { glass bottles, glass dessicator }\end{array}$ & $\begin{array}{l}\text { Schott AG (Mainz, } \\
\text { Germany) }\end{array}$ \\
\hline Laboratory film & $\begin{array}{l}\text { Parafilm }{ }^{\circledR} \text { M Laboratory } \\
\text { Film } 4 \text { IN. } \times 125 \text { FT. Roll }\end{array}$ & $\begin{array}{l}\text { Bemis (Neenah, Wisconsin, } \\
\text { USA) }\end{array}$ \\
\hline Leukopor & Leukopor $2.5 \mathrm{~cm} \times 9.2 \mathrm{~m}$ & $\begin{array}{l}\text { BSN Medical (Hamburg, } \\
\text { Germany) }\end{array}$ \\
\hline Microtiter plates & Mikrotestplatte 96 Well, F & $\begin{array}{l}\text { Sarstedt (Nümbrecht, } \\
\text { Germany) }\end{array}$ \\
\hline Needles & $\begin{array}{l}\text { Sterican® } 1.20 \times 40 \mathrm{~mm} \\
18 \mathrm{G} \times 11 / 2^{\prime \prime}\end{array}$ & $\begin{array}{l}\text { B. Braun (Melsungen, } \\
\text { Germany) }\end{array}$ \\
\hline PCR strips & $\begin{array}{l}\text { Multiply® - } \mu \text { Strip } 0.2 \mathrm{ml} \\
\text { chain (DNA-/DNase- } \\
\text { /RNase-/PCR inhibitor- } \\
\text { free) with 8-Lid chain, flat } \\
\text { (DNA-/DNase-/RNase- } \\
\text { /PCR inhibitor-free) }\end{array}$ & $\begin{array}{l}\text { Sarstedt (Nümbrecht, } \\
\text { Germany) }\end{array}$ \\
\hline PCR tubes & $\begin{array}{l}\text { Ultra Thin-Wall Tubes } 0.2 \\
\text { ml (Free of Dnase, Rnase, } \\
\text { Metal, Pyrogen) }\end{array}$ & $\begin{array}{l}\text { BIOplastics (Landgraaf, The } \\
\text { Netherlands) }\end{array}$ \\
\hline Petri dish (round) & $\begin{array}{l}\text { Petridish } 29 \times 16 \mathrm{~mm} \text { with } \\
\text { cams }\end{array}$ & $\begin{array}{l}\text { Sarstedt (Nümbrecht, } \\
\text { Germany) }\end{array}$ \\
\hline Petri dish (square) & $\begin{array}{l}\text { Petri dish } 100 \times 100 \times 20 \\
\mathrm{~mm}\end{array}$ & $\begin{array}{l}\text { Sarstedt Australia pty Ltd } \\
\text { (Mawson Lakes, South } \\
\text { Australia) }\end{array}$ \\
\hline \multirow[t]{3}{*}{ Pipette tips } & Pipette tip $1000 \mu \mathrm{l}$ & $\begin{array}{l}\text { Sarstedt (Nümbrecht, } \\
\text { Germany) }\end{array}$ \\
\hline & Pipette tip $200 \mu \mathrm{l}$ & $\begin{array}{l}\text { Sarstedt (Nümbrecht, } \\
\text { Germany) }\end{array}$ \\
\hline & Pipette tip $10 \mu \mathrm{l}$ & $\begin{array}{l}\text { Sarstedt (Nümbrecht, } \\
\text { Germany) }\end{array}$ \\
\hline
\end{tabular}




\begin{tabular}{|c|c|c|}
\hline Lab material & Product name & Company \\
\hline & $\begin{array}{l}\text { Gel-Saver II Tip1 - } 200 \mu \mathrm{l} \\
\text { Bulk, } 1000\end{array}$ & Starlab (Hamburg, Germany) \\
\hline Plant pot labels & $\begin{array}{l}\text { Steckettiketten } 10 \times 1.6 \mathrm{~mm} \text {, } \\
\text { unbedruckt, verschiedene } \\
\text { Farben }\end{array}$ & $\begin{array}{l}\text { MEYER-Produckte für den } \\
\text { Gartenbau } \\
\text { (Rellingen/Westerstede/ } \\
\text { Willich/Nossen/Langenau, } \\
\text { Germany) }\end{array}$ \\
\hline $\begin{array}{l}\text { Plastic ware (beakers, } \\
\text { cylinders) }\end{array}$ & $\begin{array}{l}\text { Plastic beakers, plastic } \\
\text { cylinders }\end{array}$ & $\begin{array}{l}\text { Vitlab GmbH } \\
\text { (Grossostheim, Germany) }\end{array}$ \\
\hline \multirow[t]{2}{*}{$\begin{array}{l}\text { Polypropylene columns for } \\
\text { protein purification }\end{array}$} & $\begin{array}{l}\text { Poly-Prep }{ }^{\circledR} \\
\text { Chromatography Columns }\end{array}$ & $\begin{array}{l}\text { BioRad (Hercules, California, } \\
\text { USA) }\end{array}$ \\
\hline & MicroBio-Spin ${ }^{\mathrm{TM}}$ Columns & $\begin{array}{l}\text { BioRad (Hercules, California, } \\
\text { USA) }\end{array}$ \\
\hline Precast PAGE gels & $\begin{array}{l}\text { Mini-PROTEAN® Precast } \\
\text { TGX Gels } 4-20 \% \text {, 10-well } \\
\text { comb, } 50 \mu \mathrm{l}\end{array}$ & $\begin{array}{l}\text { BioRad Laboratories } \\
\text { (Munich, Germany) }\end{array}$ \\
\hline PVDF membrane & $\begin{array}{l}\text { Roti }{ }^{\circledR} \text { PVDF, Pore size } 0.45 \\
\mu \mathrm{m} 375 \times 26.5 \mathrm{~cm}\end{array}$ & Roth (Karlsruhe, Germany) \\
\hline \multirow[t]{5}{*}{ Reaction tubes } & Microtube $1.5 \mathrm{ml}$ & $\begin{array}{l}\text { Sarstedt (Nümbrecht, } \\
\text { Germany) }\end{array}$ \\
\hline & Microtube $2 \mathrm{ml}$ & $\begin{array}{l}\text { Sarstedt (Nümbrecht, } \\
\text { Germany) }\end{array}$ \\
\hline & $\begin{array}{l}\text { Tube } 13 \mathrm{ml} 100 \times 16 \mathrm{~mm} \text {, } \\
\text { PP }\end{array}$ & $\begin{array}{l}\text { Sarstedt (Nümbrecht, } \\
\text { Germany) }\end{array}$ \\
\hline & $\begin{array}{l}\text { Tube } 15 \mathrm{ml} 120 \times 17 \mathrm{~mm} \text {, } \\
\text { PP (DNase-, RNase-free, } \\
\text { non-pyrogenic, non- } \\
\text { cytotoxic) }\end{array}$ & $\begin{array}{l}\text { Sarstedt (Nümbrecht, } \\
\text { Germany) }\end{array}$ \\
\hline & $\begin{array}{l}\text { Tube } 50 \mathrm{ml} 114 \times 28 \mathrm{~mm} \text {, } \\
\text { PP }\end{array}$ & $\begin{array}{l}\text { Sarstedt (Nümbrecht, } \\
\text { Germany) }\end{array}$ \\
\hline Scalpel blades & Bayha ${ }^{\circledR}$ non-sterile & $\begin{array}{l}\text { C. Bruno Bayha GmbH } \\
\text { (Tuttlingen, Germany) }\end{array}$ \\
\hline \multirow[t]{2}{*}{ Serological pipettes } & $\begin{array}{l}\text { Serological Pipette } 10 \mathrm{ml} \text { in } \\
1 / 10 \mathrm{ml} \text { (sterile, non- } \\
\text { cytotoxic, non-pyrogenic) }\end{array}$ & $\begin{array}{l}\text { Sarstedt (Nümbrecht, } \\
\text { Germany) }\end{array}$ \\
\hline & $\begin{array}{l}\text { Serological Pipette } 25 \mathrm{ml} \text { in } \\
2 / 10 \mathrm{ml} \text { (sterile, non- } \\
\text { cytotoxic, non-pyrogenic) }\end{array}$ & $\begin{array}{l}\text { Sarstedt (Nümbrecht, } \\
\text { Germany) }\end{array}$ \\
\hline Stainless Steel beads & $\begin{array}{l}\text { Stainless Steel beads } \varnothing 5 \\
\text { mm G40 Nirosta }\end{array}$ & $\begin{array}{l}\text { Kugel-Rollen UG (Röthlein, } \\
\text { Germany) }\end{array}$ \\
\hline
\end{tabular}


Material

\begin{tabular}{|c|c|c|}
\hline Lab material & Product name & Company \\
\hline Superloop & Superloop $10 \mathrm{ml}, 50 \mathrm{ml}$ & $\begin{array}{l}\text { GE Healthcare (Uppsala, } \\
\text { Sweden) }\end{array}$ \\
\hline \multirow[t]{8}{*}{ Syringes } & 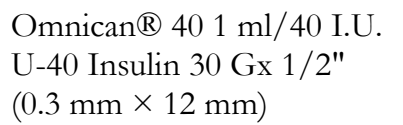 & $\begin{array}{l}\text { B. Braun (Melsungen, } \\
\text { Germany) }\end{array}$ \\
\hline & $\begin{array}{l}\text { Omnifix }{ }^{\circledR} 40 \text { Solo U-40 } \\
\text { Insulin } 1 \mathrm{ml}\end{array}$ & $\begin{array}{l}\text { B. Braun (Melsungen, } \\
\text { Germany) }\end{array}$ \\
\hline & Single-use syringe $2(3) \mathrm{ml}$ & $\begin{array}{l}\text { B. Braun (Melsungen, } \\
\text { Germany) }\end{array}$ \\
\hline & $\begin{array}{l}\text { Disposable syringes 3- } \\
\text { pieced, LUERLOK-slip, } \\
\text { sterile } 50(60) \mathrm{ml}\end{array}$ & $\begin{array}{l}\text { Mediware }{ }^{\circledR} \text { Servoprax }{ }^{\circledR} \\
\text { GmbH (Wesel, Germany) }\end{array}$ \\
\hline & $\begin{array}{l}\text { Disposable syringes 3- } \\
\text { pieced, LUERLOK-slip, } \\
\text { sterile } 30 \mathrm{ml}\end{array}$ & $\begin{array}{l}\text { Mediware }{ }^{\circledR} \text { Servoprax }{ }^{\circledR} \\
\text { GmbH (Wesel, Germany) }\end{array}$ \\
\hline & $\begin{array}{l}\text { Disposable syringes 3- } \\
\text { pieced, LUERLOK-slip, } \\
\text { sterile } 10 \mathrm{ml}\end{array}$ & $\begin{array}{l}\text { Mediware }{ }^{\circledR} \text { Servoprax }{ }^{\circledR} \\
\text { GmbH (Wesel, Germany) }\end{array}$ \\
\hline & $\begin{array}{l}\text { Disposable syringes 3- } \\
\text { pieced, LUERLOK-slip, } \\
\text { sterile } 5 \mathrm{ml}\end{array}$ & $\begin{array}{l}\text { Mediware }{ }^{\circledR} \text { Servoprax }{ }^{\circledR} \\
\text { GmbH (Wesel, Germany) }\end{array}$ \\
\hline & $\begin{array}{l}\text { NORM-JECT® Luer (non- } \\
\text { pyrogenic, non-toxic, sterile) } \\
5 \mathrm{ml}(6 \mathrm{ml})\end{array}$ & $\begin{array}{l}\text { Henke Sass Wolf (Tuttlingen, } \\
\text { Germany) }\end{array}$ \\
\hline Tooth picks & $\begin{array}{l}\text { PAPSTAR PURE } 1000 \\
\text { Toothpicks made of Wood } 8 \\
\mathrm{~cm}\end{array}$ & Papstar (Kall, Germany) \\
\hline
\end{tabular}




\section{Technical devices}

\begin{tabular}{|c|c|c|}
\hline Technical device & Product name & Company \\
\hline \multirow[t]{2}{*}{ 8-Channel pipette } & FINNPIPETTE® F2 1 - $10 \mu \mathrm{l}$ & $\begin{array}{l}\text { Thermo Scientific } \\
\text { (Vantaa, Finland) }\end{array}$ \\
\hline & FINNPIPETTE® F2 $30-300 \mu 1$ & $\begin{array}{l}\text { Thermo Scientific } \\
\text { (Vantaa, Finland) }\end{array}$ \\
\hline \multirow[t]{4}{*}{ Autoclave } & $3870 \mathrm{ELV}$ & $\begin{array}{l}\text { Tuttnauer (Breda, } \\
\text { Netherlands) }\end{array}$ \\
\hline & PSD & $\begin{array}{l}\text { H. Hölzel } ® \mathrm{GmbH} \\
\text { (Hörlkofen, } \\
\text { Germany) }\end{array}$ \\
\hline & Systec VX-150 & $\begin{array}{l}\text { Systec GmbH Labor- } \\
\text { Systemtechnik } \\
\text { (Wettenberg, } \\
\text { Germany) }\end{array}$ \\
\hline & Systec VX-95 & $\begin{array}{l}\text { Systec GmbH Labor- } \\
\text { Systemtechnik } \\
\text { (Wettenberg, } \\
\text { Germany) }\end{array}$ \\
\hline Bead beater/Tissue lyser & Retsch® MM301 Schwingmühle & $\begin{array}{l}\text { Retsch (Haan, } \\
\text { Germany) }\end{array}$ \\
\hline $\begin{array}{l}\text { Blotting chambers } \\
\text { (Western Blot) }\end{array}$ & $\begin{array}{l}\text { V20-SDB 20x } 20 \mathrm{~cm} \text { Semidry Blotter } \\
\text { Unit }\end{array}$ & $\begin{array}{l}\text { SCIE-PLAS LTD } \\
\text { (Cambridge, England) }\end{array}$ \\
\hline Camera & Canon PowerShot G11 & $\begin{array}{l}\text { Canon Deutschland } \\
\text { GmbH (Krefeld, } \\
\text { Germany) }\end{array}$ \\
\hline \multirow[t]{4}{*}{ Centrifuges/Rotors } & Biofuge pico & $\begin{array}{l}\text { Heraeus Instruments } \\
\text { GmbH (Hanau, } \\
\text { Germany) }\end{array}$ \\
\hline & Heraeus Fresco 17 Centrifuge & $\begin{array}{l}\text { Thermo Scientific } \\
\text { (Dreieich, Germany) }\end{array}$ \\
\hline & Heraeus Pico 17 Centrifuge & $\begin{array}{l}\text { Thermo Scientific } \\
\text { (Dreieich, Germany) }\end{array}$ \\
\hline & MICRO STAR 17R & $\begin{array}{l}\text { VWR (Darmstadt, } \\
\text { Germany) }\end{array}$ \\
\hline
\end{tabular}


Technical devices

\begin{tabular}{|c|c|c|}
\hline Technical device & Product name & Company \\
\hline & MIKRO 200 & $\begin{array}{l}\text { Hettich Zentrifugen } \\
\text { (Tuttlingen, } \\
\text { Germany) }\end{array}$ \\
\hline & MIKRO 200R & $\begin{array}{l}\text { Hettich Zentrifugen } \\
\text { (Tuttlingen, } \\
\text { Germany) }\end{array}$ \\
\hline & $\begin{array}{l}\text { Rotina } 380 \mathrm{R} \text { equipped with rotors } \\
\text { 1789-L } 1500 \mathrm{rpm}, 30 \times 4 \mathrm{~g} \text { or } 1798 \\
4000 \mathrm{rpm} 4 \times 300 \mathrm{~g}, 1789-\mathrm{A} 15000 \mathrm{rpm} \\
30 \times 3 \mathrm{~g} \text {, or } 17254000 \mathrm{rpm} \max 4 \times 150\end{array}$ & $\begin{array}{l}\text { Hettich Zentrifugen } \\
\text { (Tuttlingen, } \\
\text { Germany) }\end{array}$ \\
\hline & $g$ & \\
\hline & Rotina 38R & $\begin{array}{l}\text { Hettich Zentrifugen } \\
\text { (Tuttlingen, } \\
\text { Germany) }\end{array}$ \\
\hline & $\begin{array}{l}\text { Sorvall RC6+ Centrifuge equipped with } \\
\text { rotors Sorvall@ Dupont SUPER- } \\
\text { LITETM GS-3 Model SLA-3000, } \\
\text { Sorvall }{ }^{\circledR} \text { SA-600, or Sorvall@ SS-34 } \\
\text { (Dupont) }\end{array}$ & $\begin{array}{l}\text { Thermo Scientific } \\
\text { (Dreieich, Germany) }\end{array}$ \\
\hline \multirow[t]{2}{*}{ Clean benches } & Heraguard & $\begin{array}{l}\text { Thermo Scientific } \\
\text { (Dreieich, Germany) }\end{array}$ \\
\hline & Safe 2020 & $\begin{array}{l}\text { Thermo Scientific } \\
\text { (Dreieich, Germany) }\end{array}$ \\
\hline Climate chambers & Climate chambers & $\begin{array}{l}\text { Johnson Controls } \\
\text { Systems \& Service } \\
\text { GmbH (Essen, } \\
\text { Germany) }\end{array}$ \\
\hline $\begin{array}{l}\text { Cool chamber (cold } \\
\text { room) }\end{array}$ & Cool chamber & $\begin{array}{l}\text { Johnson Controls } \\
\text { Systems \& Service } \\
\text { GmbH (Essen, } \\
\text { Germany) }\end{array}$ \\
\hline \multirow[t]{3}{*}{ Electroporation device } & BioRad capacitance extender plus & $\begin{array}{l}\text { BioRad Laboratories } \\
\text { (Munich, Germany) }\end{array}$ \\
\hline & BioRad Gene Pulser ${ }^{\circledR}$ II & $\begin{array}{l}\text { BioRad Laboratories } \\
\text { (Munich, Germany) }\end{array}$ \\
\hline & BioRad Pulse controller Plus & $\begin{array}{l}\text { BioRad Laboratories } \\
\text { (Munich, Germany) }\end{array}$ \\
\hline \multirow[t]{2}{*}{ Freezer } & Liebherr Medline & $\begin{array}{l}\text { Liebherr (Bulle, } \\
\text { Switzerland) }\end{array}$ \\
\hline & Liebherr Premium & $\begin{array}{l}\text { Liebherr (Bulle, } \\
\text { Switzerland) }\end{array}$ \\
\hline
\end{tabular}




\begin{tabular}{|c|c|c|}
\hline Technical device & Product name & Company \\
\hline & Liebherr Premium NoFrost & $\begin{array}{l}\text { Liebherr (Bulle, } \\
\text { Switzerland) }\end{array}$ \\
\hline & Liebherrr Comfort & $\begin{array}{l}\text { Liebherr (Bulle, } \\
\text { Switzerland) }\end{array}$ \\
\hline & Siemens GS12DA20 & $\begin{array}{l}\text { Siemens (Munich, } \\
\text { Germany) }\end{array}$ \\
\hline & FB $50 \mathrm{I}$ & Electrolux \\
\hline \multirow[t]{4}{*}{ Fridges } & Liebherr Medline & $\begin{array}{l}\text { Liebherr (Bulle, } \\
\text { Switzerland) }\end{array}$ \\
\hline & $\begin{array}{l}\text { Liebherr Drink Refrigerator FK } 2642 \\
\text { Index 20Q/001 }\end{array}$ & Liebherr (Austria) \\
\hline & Liebherr Premium & $\begin{array}{l}\text { Liebherr (Bulle, } \\
\text { Switzerland) }\end{array}$ \\
\hline & Tritec ${ }^{\circledR}$ Hannover & $\begin{array}{l}\text { Tritec (Hannover, } \\
\text { Germany) }\end{array}$ \\
\hline $\begin{array}{l}\text { Gel documentation } \\
\text { system (Agarose gels) }\end{array}$ & $\begin{array}{l}\text { Gel documentation system (Agarose } \\
\text { gels) }\end{array}$ & $\begin{array}{l}\text { Intas (Göttingen, } \\
\text { Germany) }\end{array}$ \\
\hline \multirow[t]{5}{*}{ Heater } & Electric cooker & $\begin{array}{l}\text { Rommelsbacher } \\
\text { (Dinkelsbühl, } \\
\text { Germany) }\end{array}$ \\
\hline & HLC MHR11 & $\begin{array}{l}\text { Ditabis (Pforzheim, } \\
\text { Gemrany) }\end{array}$ \\
\hline & HLC TH26 & $\begin{array}{l}\text { Ditabis (Pforzheim, } \\
\text { Gemrany) }\end{array}$ \\
\hline & MHR13 & $\begin{array}{l}\text { Hettich Lab } \\
\text { Technology } \\
\text { (Geldermalsen, } \\
\text { Netherlands) }\end{array}$ \\
\hline & $\begin{array}{l}\text { Pressure cooking pot WMF } \\
\text { Chromargen }{ }^{\circledR} \text { Stainless steel 18/10 } 8.5 \\
1\end{array}$ & $\begin{array}{l}\text { WMF (Geislingen an } \\
\text { der Steige, Germany) }\end{array}$ \\
\hline \multirow[t]{3}{*}{ Horizontal shakers } & CAT ST5 & $\begin{array}{l}\text { M. Zipper GmbH } \\
\text { (Staufen, Germany) }\end{array}$ \\
\hline & $\begin{array}{l}\text { IKA® Vibrax-VXR equipped with } \\
\text { attachment Typ VX7 }\end{array}$ & $\begin{array}{l}\text { IKA-Werke } \\
\text { (originally Janke \& } \\
\text { Kunkel) (Staufen, } \\
\text { Germany) }\end{array}$ \\
\hline & Mini Gyro-Rocker SSM3 & $\begin{array}{l}\text { Stuart (Staffordshire, } \\
\text { UK) }\end{array}$ \\
\hline
\end{tabular}


Technical devices

\begin{tabular}{|c|c|c|}
\hline Technical device & Product name & Company \\
\hline Ice machine & S-No. 106094 & $\begin{array}{l}\text { Ziegra } \\
\text { Eismaschinenen } \\
\text { GmbH (Isernhagen, } \\
\text { Germany) }\end{array}$ \\
\hline \multirow[t]{2}{*}{ Incubator shaker } & Certomat ${ }^{\circledR}$ BS-1 & $\begin{array}{l}\text { Sartorius Stedim } \\
\text { Biotech (Göttingen, } \\
\text { Germany) }\end{array}$ \\
\hline & Ecotron $\mathrm{H} \mathrm{T}$ & $\begin{array}{l}\text { Infors AG (Einsbach, } \\
\text { Germany) }\end{array}$ \\
\hline \multirow[t]{2}{*}{ Incubators } & Memmert & $\begin{array}{l}\text { Memmert } \\
\text { (Schwabach, } \\
\text { Germany) }\end{array}$ \\
\hline & Memmert Modell 100-800 & $\begin{array}{l}\text { Memmert } \\
\text { (Schwabach, } \\
\text { Germany) }\end{array}$ \\
\hline Lab boy & Boy110 & $\begin{array}{l}\text { Rudolf Cramer AG } \\
\text { (Degersheim, } \\
\text { Switzerland) }\end{array}$ \\
\hline \multirow[t]{2}{*}{ Luminescence imager } & INTAS Chemo cam & $\begin{array}{l}\text { Intas (Göttingen, } \\
\text { Germany) }\end{array}$ \\
\hline & INTAS ChemoStarProfessional & $\begin{array}{l}\text { Intas (Göttingen, } \\
\text { Germany) }\end{array}$ \\
\hline \multirow[t]{7}{*}{ Magnetic stirrers } & $\mathrm{IKA} 囚 \mathrm{RH}$ basic 2 & $\begin{array}{l}\text { IKA-Werke (Staufen, } \\
\text { Germany) }\end{array}$ \\
\hline & IKAMAG® REO & $\begin{array}{l}\text { IKA-Werke (Staufen, } \\
\text { Germany) }\end{array}$ \\
\hline & MR80 & $\begin{array}{l}\text { Heidolph Instruments } \\
\text { (Schwabach, } \\
\text { Germany) }\end{array}$ \\
\hline & RCT basic & $\begin{array}{l}\text { IKA-Werke (Staufen, } \\
\text { Germany) }\end{array}$ \\
\hline & RCT basic & $\begin{array}{l}\text { IKA-Werke (Staufen, } \\
\text { Germany) }\end{array}$ \\
\hline & RSM-01S & $\begin{array}{l}\text { Phoenix Instrument } \\
\text { (Garbsen, Germany) }\end{array}$ \\
\hline & Type RMO Serie 76 & $\begin{array}{l}\text { Gerhardt (Bonn, } \\
\text { West Germany) }\end{array}$ \\
\hline Microscope & $\begin{array}{l}\text { Zeiss Microscope equipped with three } \\
\text { Zeiss objectives (40x Plan 40/0.65 } \\
\text { 160/0.17; 10x 10/0.22 160/-; 3.2x } \\
3.2 / 0.07160 /-)\end{array}$ & $\begin{array}{l}\text { Zeiss (West } \\
\text { Germany) }\end{array}$ \\
\hline
\end{tabular}




\begin{tabular}{|c|c|c|}
\hline Technical device & Product name & Company \\
\hline Microwave & MWD 2923G & $\begin{array}{l}\text { Alaska Elektro } \\
\text { (Viernheim, } \\
\text { Germany) }\end{array}$ \\
\hline \multirow[t]{3}{*}{ Mini centrifuges } & Rotilabo®-mini-centrifuge & $\begin{array}{l}\text { Roth (Karlsruhe, } \\
\text { Germany) }\end{array}$ \\
\hline & $\begin{array}{l}\text { Rotilabo } \AA \text {-mini-centrifuge equipped } \\
\text { with rotor The Butterfly }\end{array}$ & $\begin{array}{l}\text { Roth (Karlsruhe, } \\
\text { Germany) }\end{array}$ \\
\hline & Sprout HS & $\begin{array}{l}\text { Heathrow Scientific } \\
\text { (Vernon Hills, } \\
\text { Illinois, USA) }\end{array}$ \\
\hline PAGE gel chambers & Mini-PROTEAN® Tetra Cell System & $\begin{array}{l}\text { BioRad Laboratories } \\
\text { (Munich, Germany) }\end{array}$ \\
\hline $\mathrm{pH}$ meter & pH211 Microprocessor pH Meter & $\begin{array}{l}\text { HANNA instruments } \\
\text { (Vöhringen, } \\
\text { Germany) }\end{array}$ \\
\hline \multirow[t]{3}{*}{ Photometer } & Libra S11 & $\begin{array}{l}\text { Biochrom (Berlin, } \\
\text { Germany) }\end{array}$ \\
\hline & NanoDrop 2000 Spectrophotometer & $\begin{array}{l}\text { Peqlab (Erlangen, } \\
\text { Germany) }\end{array}$ \\
\hline & NanoDrop ONE & $\begin{array}{l}\text { Thermo Scientific } \\
\text { (Dreieich, Germany) }\end{array}$ \\
\hline $\begin{array}{l}\text { Pipettes (P2N, P10N, } \\
\text { P20N, P200N, P1000N) }\end{array}$ & $\begin{array}{l}\text { Pipettes (P2N, P10N, P20N, P200N, } \\
\text { P1000N) }\end{array}$ & $\begin{array}{l}\text { Gilson (Villiers Le } \\
\text { Bel, France) }\end{array}$ \\
\hline \multirow[t]{2}{*}{ Plant incubators } & Intellus Control system AR-22L & $\begin{array}{l}\text { Percival Scientific } \\
\text { (Perry, Iowa, USA) }\end{array}$ \\
\hline & Intellus Control system AR-66L & $\begin{array}{l}\text { Percival Scientific } \\
\text { (Perry, Iowa, USA) }\end{array}$ \\
\hline Plate reader & Synergy HT & $\begin{array}{l}\text { Bio'Tek (Bad } \\
\text { Friedrichshall, } \\
\text { Germany) }\end{array}$ \\
\hline \multirow[t]{4}{*}{ Power supply } & Consort E323 & $\begin{array}{l}\text { Consort (Turnhout, } \\
\text { Belgium) }\end{array}$ \\
\hline & Consort EV231 & $\begin{array}{l}\text { Consort (Turnhout, } \\
\text { Belgium) }\end{array}$ \\
\hline & Consort EV243 & $\begin{array}{l}\text { Consort (Turnhout, } \\
\text { Belgium) }\end{array}$ \\
\hline & EC 105 & $\begin{array}{l}\text { ECApparatus } \\
\text { Corporation (St. } \\
\text { Petersburg, Florida, } \\
\text { USA) }\end{array}$ \\
\hline
\end{tabular}


Technical devices

\begin{tabular}{|c|c|c|}
\hline Technical device & Product name & Company \\
\hline & PowerPac ${ }^{\mathrm{TM}} \mathrm{HC}$ & $\begin{array}{l}\text { Biorad Laboratories } \\
\text { (Munich, Germany) }\end{array}$ \\
\hline \multirow[t]{2}{*}{$\begin{array}{l}\text { Protein purification } \\
\text { system }\end{array}$} & ÄKTAprime plus & $\begin{array}{l}\text { GE Healthcare } \\
\text { (Uppsala, Sweden) }\end{array}$ \\
\hline & $\begin{array}{l}\text { ÄKTApure equipped with Fraction } \\
\text { Collector F9-R }\end{array}$ & $\begin{array}{l}\text { GE Healthcare } \\
\text { (Uppsala, Sweden) }\end{array}$ \\
\hline \multirow[t]{2}{*}{ qRT PCR thermal cycler } & $\begin{array}{l}\text { BioRad CFX Connect }{ }^{\mathrm{TM}} \text { Real-Time } \\
\text { System }\end{array}$ & $\begin{array}{l}\text { BioRad Laboratories } \\
\text { (Munich, Germany) }\end{array}$ \\
\hline & BioRad iCycler & $\begin{array}{l}\text { BioRad Laboratories } \\
\text { (Munich, Germany) }\end{array}$ \\
\hline $\begin{array}{l}\text { qRT PCR thermal cycler } \\
\text { software }\end{array}$ & BioRad iQ5 & $\begin{array}{l}\text { BioRad Laboratories } \\
\text { (Munich, Germany) }\end{array}$ \\
\hline Quantum Meter & Quantum Flux MQ 200 & $\begin{array}{l}\text { Apogee Electronics } \\
\text { Corporation (Santa } \\
\text { Monica, California, } \\
\text { USA) }\end{array}$ \\
\hline \multirow[t]{2}{*}{ Reaction tube rotor } & Intelli-Mixer RM-2L & $\begin{array}{l}\text { Elmi Laboratory } \\
\text { Equipment (Riga, } \\
\text { Latvia) }\end{array}$ \\
\hline & Intelli-Mixer RM-2L Skyline & $\begin{array}{l}\text { Elmi Laboratory } \\
\text { Equipment (Riga, } \\
\text { Latvia) }\end{array}$ \\
\hline \multirow[t]{3}{*}{ Scales } & Kern 572 Max. $3010 \mathrm{~g} \mathrm{~d}=0.01 \mathrm{~g}$ & $\begin{array}{l}\text { Kern (Balingen, } \\
\text { Germany) }\end{array}$ \\
\hline & $\begin{array}{l}\text { Sartorius Extend Max } 120 \mathrm{~g} \mathrm{~d}=0.1 \mathrm{mg} \\
\text { ED1245 }\end{array}$ & $\begin{array}{l}\text { Sartorius Stedim } \\
\text { (Göttingen, } \\
\text { Germany) }\end{array}$ \\
\hline & Scaltec SPQ51 Max $310 \mathrm{~g} \mathrm{~d}=0.01 \mathrm{~g}$ & $\begin{array}{l}\text { Scaltec Instruments } \\
\text { (Göttingen, } \\
\text { Germany) }\end{array}$ \\
\hline \multirow[t]{2}{*}{ Scanner } & Epson Perfection V700 Photo & $\begin{array}{l}\text { Epson (Meerbusch, } \\
\text { Germany) }\end{array}$ \\
\hline & SilverFast & $\begin{array}{l}\text { LaserSoft Imaging } \\
\text { AG (Kiel, Germany) }\end{array}$ \\
\hline Sonifier & $\begin{array}{l}\text { Sonopuls equipped with an MS73 } \\
\text { sonotrode }\end{array}$ & $\begin{array}{l}\text { Bandelin electronic } \\
\text { (Berlin, Germany) }\end{array}$ \\
\hline Sterilisator & Modell 100-800 & $\begin{array}{l}\text { Memmert } \\
\text { (Schwabach, } \\
\text { Germany) }\end{array}$ \\
\hline
\end{tabular}




\begin{tabular}{|c|c|c|}
\hline Technical device & Product name & Company \\
\hline \multirow[t]{2}{*}{ Thermal cycler } & iCycler & $\begin{array}{l}\text { BioRad Laboratories } \\
\text { (Munich, Germany) }\end{array}$ \\
\hline & MyCyclerTM thermal cycler & $\begin{array}{l}\text { BioRad Laboratories } \\
\text { (Munich, Germany) }\end{array}$ \\
\hline Tripod & ProBlack 2 & $\begin{array}{l}\text { Dörr GmbH (Neu } \\
\text { Ulm, Germany) }\end{array}$ \\
\hline Tube rotator & Stuart ${ }^{\circledR}$ Rotator SB2 & $\begin{array}{l}\text { Stuart (Staffordshire, } \\
\text { UK) }\end{array}$ \\
\hline \multirow[t]{4}{*}{ Ultralow Freezer } & Herafreeze Top $-75^{\circ} \mathrm{C}$ & $\begin{array}{l}\text { Thermo Scientific } \\
\text { (Dreieich, Germany) }\end{array}$ \\
\hline & HeraFreeze HFU T Series & $\begin{array}{l}\text { Thermo Scientific } \\
\text { (Dreieich, Germany) }\end{array}$ \\
\hline & Minus $85^{\circ} \mathrm{C}$ Ultralow Freezer & $\begin{array}{l}\text { Thermo Scientific } \\
\text { (Dreieich, Germany) }\end{array}$ \\
\hline & Minus $85^{\circ} \mathrm{C}$ Ultralow Freezer U57085 & $\begin{array}{l}\text { New Brunswick } \\
\text { Scientific (Nürtingen, } \\
\text { Germany) }\end{array}$ \\
\hline Vaccum pump & Diaphragm Vaccuum Pump MD1C & $\begin{array}{l}\text { Vacuubrand GMbH } \\
\text { \& Co KG } \\
\text { (Werthheim, } \\
\text { Germany) }\end{array}$ \\
\hline \multirow[t]{4}{*}{ Vortexter } & $\begin{array}{l}\text { IKA® Vibrax-VXR basic equipped } \\
\text { with attachment Typ VX2E }\end{array}$ & $\begin{array}{l}\text { IKA-Werke (Staufen, } \\
\text { Germany) }\end{array}$ \\
\hline & LABINCO L46 & $\begin{array}{l}\text { Labinco BV (Breda, } \\
\text { The Netherlands) }\end{array}$ \\
\hline & MS2 Minishaker & $\begin{array}{l}\text { IKA-Werke (Staufen, } \\
\text { Germany) }\end{array}$ \\
\hline & Vortex-Genie 2 & $\begin{array}{l}\text { Scientific Industries } \\
\text { (Bohemia, New York, } \\
\text { USA) }\end{array}$ \\
\hline \multirow[t]{2}{*}{ Water bath } & GFL 1083 & $\begin{array}{l}\text { GFL (Burgwedel, } \\
\text { Germany) }\end{array}$ \\
\hline & GFL® 1092 & $\begin{array}{l}\text { GFL (Burgwedel, } \\
\text { Germany) }\end{array}$ \\
\hline Water desalting device & Arium $\AA$ pro DI & $\begin{array}{l}\text { Sartorius Stedim } \\
\text { Biotech (Göttingen, } \\
\text { Germany) }\end{array}$ \\
\hline
\end{tabular}


Abbreviations 


\section{Abbreviations}

\begin{tabular}{|c|c|}
\hline Abbreviation & Extended spelling \\
\hline (number)-x & (number)-fold \\
\hline 3'UTR & 3'-untranslated region \\
\hline 5'UTR & 5'-untranslated region \\
\hline$\alpha$ & Anti \\
\hline A. thatiana & Arabidopsis thaliana \\
\hline A. tumefaciens & Agrobacterium tumefaciens \\
\hline$A m p^{R}$ & Ampicilin resistance \\
\hline APS & Ammonium peroxodisulfate \\
\hline At & Arabidopsis thaliana \\
\hline ATP & Adenosinetriphosphate \\
\hline BAK1 & Brassinosteroid insensitive 1 (BRI1)-associated receptor kinase \\
\hline bar & Basta resistance \\
\hline BPGA & 1,3-bisphosphoglycerate \\
\hline bp & Base pair \\
\hline BSA & Bovine serum albumin \\
\hline bZIP & Basic leucine zipper \\
\hline $\mathrm{CBB}$ & Coomassie brilliant blue \\
\hline cDNA & Complementary desoxyribonucleic acid \\
\hline CEPD2 & C-terminally encoded peptide downstream 1 \\
\hline CL & Control light \\
\hline $\mathrm{D}$ & Discussion \\
\hline $\mathrm{dH}_{2} \mathrm{O}$ & Distilled water \\
\hline DHA & Dehydroascorbate \\
\hline DMSO & Dimethyl sulfoxide \\
\hline DNA & Desoxyribonucleic acid \\
\hline dNTP & Desoxyribonucleotid triphosphate \\
\hline DTNB & 5,5-dithio-bis-2-nitrobenzoic acid \\
\hline DTT & Dithiothreitol \\
\hline E. coli & Escherichia coli \\
\hline e.g. & Example given \\
\hline EDTA & Ethylenediaminetetraacetic acid \\
\hline $\mathrm{E}_{\mathrm{h}}$ & Abient redox potential \\
\hline Escherichia coli & E. coli \\
\hline ET & Ethylene \\
\hline et al. & et alii \\
\hline
\end{tabular}




\begin{tabular}{|c|c|}
\hline Abbreviation & Extended spelling \\
\hline $\mathrm{EtOH}$ & Ethanol \\
\hline FTR & Ferredoxin thioredoxin reductase \\
\hline fwd & Forward \\
\hline GAP & Glyceraldehyde 3-phosphate \\
\hline GAPDH & Glyceraldehyde 3-phosphate dehydrogenase \\
\hline Gent $^{\mathrm{R}}$ & Gentamicin resistance \\
\hline GRX & Glutaredoxin \\
\hline GSH & Glutathione \\
\hline GSNO & Nitrosoglutathione \\
\hline GSSEtOH & $\begin{array}{l}\text { Mixed disulfide between glutathione and } \\
\beta \text {-mercaptoethanol }\end{array}$ \\
\hline GSSG & Glutathione disulfide \\
\hline HA & Haemagglutinin \\
\hline HEDS & Bis(2-hydroxyethyl)disulfide \\
\hline HEPES & 4-(2-Hydroxyethyl)piperazine-1-ethanesulfonic acid \\
\hline HRP & Horseradish peroxidase \\
\hline I & Introduction \\
\hline i.e. & id est \\
\hline IAM & Iodoacetamide \\
\hline IDCR & Ionic detergent compatability reagent \\
\hline iodoTMT ${ }^{\mathrm{TM}}$ & Iodoacetyl Tandem Mass Tag ${ }^{\mathrm{TM}}$ \\
\hline IPTG & Isopropyl- $\beta$-D-thiogalactopyranoside \\
\hline JA & Jasmonic acid/jasmonate \\
\hline JAZ & $\begin{array}{l}\text { Jasmonate (Zinc-finger inflorescence meristem) ZIM } \\
\text { domain }\end{array}$ \\
\hline $\operatorname{Kan}^{\mathrm{R}}$ & Kanamycin resistance \\
\hline $\mathrm{K}_{\mathrm{av}}$ & Partition coefficient \\
\hline$K_{d}$ & Dissociation constant \\
\hline LB medium & Luria-Bertani medium \\
\hline LC-MS/MS & Liquid chromatography-tandem mass spectrometry \\
\hline LEU2 & Leucine biosynthesis 2 \\
\hline LIC & Ligation-independent cloning \\
\hline LL & Low light \\
\hline $\mathrm{mAU}$ & Milli absorbtion units \\
\hline $\mathrm{mBBr}$ & Monobromobimane \\
\hline MBP & Maltose-binding protein \\
\hline MS medium & Murashige-Skoog medium \\
\hline MS & Mass spectrometry \\
\hline MST & Microscale thermophoresis \\
\hline MW & Molecular weight \\
\hline MWCO & Molecular weight cut-off \\
\hline
\end{tabular}




\begin{tabular}{|c|c|}
\hline Abbreviation & Extended spelling \\
\hline $\mathrm{NAD}^{+}$ & Nicotinamide adenine dinucleotide, oxidized form \\
\hline $\mathrm{NADH}$ & Nicotinamide adenine dinucleotide, reduced form \\
\hline $\mathrm{NADP}^{+}$ & $\begin{array}{l}\text { Nicotinamide adenine dinucleotide phosphate, } \\
\text { oxidized form }\end{array}$ \\
\hline NADPH & $\begin{array}{l}\text { Nicotinamide adenine dinucleotide phosphate, } \\
\text { reduced form }\end{array}$ \\
\hline NHS & N-hydroxysuccinimide \\
\hline NINJA & Novel Interactor of JAZ \\
\hline NMR & Nuclear magnetic resonance \\
\hline NPR1 & Nonexpressor of pathogenesis-related proteins 1 \\
\hline NRT2.1 & Nitrate transporter 2.1 \\
\hline NRT2.2 & Nitrate transporter 2.2 \\
\hline OD Xxxnm & Optical densitiy at XXX nm \\
\hline $\mathrm{ON}$ & Over night \\
\hline ox & Oxidized \\
\hline P. falciparum & Plasmodium falciparum \\
\hline PAGE & Polyacrylamide gel electrophoresis \\
\hline PAN & PERIANTHIA \\
\hline PBS & Phosphate-buffered saline \\
\hline PBS-T & Phosphate-buffered saline-Tween-20 \\
\hline PCR & Polymerase chain reaction \\
\hline PDB & Protein database \\
\hline Pf & Plasmodium falciparum \\
\hline $\mathrm{pI}$ & Isoelectric point \\
\hline PIPES & 1,4-Piperazinediethanesulfonic acid \\
\hline $\mathrm{pK}_{\mathrm{a}}$ value & Acid dissociation constant \\
\hline PSM & Peptide spectrum match \\
\hline PTT & Phosphinotricine \\
\hline PVDF & Polyvinyldiflouride \\
\hline Q1 & $\begin{array}{l}\text { Glutamine-rich region } 1 \text { (domain of TGA } \\
\text { transcription factors) }\end{array}$ \\
\hline Q2 & $\begin{array}{l}\text { Glutamine-rich region } 2 \text { (domain of TGA } \\
\text { transcription factors) }\end{array}$ \\
\hline qRT-PCR & Quantitative realtime PCR \\
\hline $\mathrm{R}$ & Results \\
\hline red & Reduced \\
\hline rev & Reverse \\
\hline $\mathrm{RNA}$ & Ribonucleic acid \\
\hline RNS & Reactive nitrogen species \\
\hline roGFP & Redox-sensitive green fluorescent protein \\
\hline ROS & Reactive oxygen species \\
\hline RT & Room temperature \\
\hline
\end{tabular}




\begin{tabular}{ll}
\hline Abbreviation & Extended spelling \\
\hline S & Supplementary \\
S. cerevisiae & Saccharomyces cerevisiae \\
SA & Salicylic acid/salicylate \\
Sc & Saccharomyces cerevisiae \\
SCL14 & Scarecrow-like 14 \\
SD & Standard deviation \\
SDS & Sodium dodecylsulfate \\
SEM & Standard error of the mean \\
-SG & Glutathione moiety \\
Spec & Spectinomycin resistance \\
SUMO & Small ubiquitin-modifier \\
T. ni & Trichoplusia ni \\
TBS & TRIS-buffered saline \\
TBS-T & TRIS-buffered saline-Tween-20 \\
TCA & Trichloroacetic acid \\
TCEP & Tris(2-carboxyethyl)phosphine \\
T-DNA & Transfer DNA \\
TEMED & Tetramethylethylenediamine \\
TEV & Tobacco etch virus \\
TGA transcription & TGACG-motif binding transcription factor \\
factor & \\
TGA1g & Genomic TGA1 \\
TPL & TOPLESS \\
TR & Thioredoxin reductase \\
TRIS & 2-Amino-2-(hydroxymethyl)-1,3-propanediol \\
TRX & Thioredoxin \\
UV & Ultraviolet \\
UV/Vis & Ultraviolet/Visible light \\
v & Velocity \\
X corr & Cross correlation \\
\hline & \\
&
\end{tabular}




\section{Units and natural constants}

\begin{tabular}{ll}
\hline Unit sign & Extended spelling \\
\hline$\%$ & Percent \\
$\%$ o & Per mil \\
$\%(\mathrm{v} / \mathrm{v})$ & Percent volume per volume \\
$\%(\mathrm{w} / \mathrm{v})$ & Percent weight per volume \\
$\circ$ & Degree \\
${ }^{\circ} \mathrm{C}$ & Degree Celsius \\
$\AA$ & Angström \\
A.U., AU & Absorption units \\
$\mathrm{Da}$ & Dalton \\
$\mathrm{F}$ & Farrad \\
$\mathrm{g}$ & Gramm \\
$\mathrm{h}$ & Hours \\
$\mathrm{L}, \mathrm{l}$ & Litre \\
$\mathrm{M}$ & Molar (mol/l) \\
$\mathrm{m}$ & Metre \\
$\mathrm{min}$ & Minutes \\
$\mathrm{mol}$ & Mol \\
$\mathrm{rpm}$ & Rounds per minute \\
$\mathrm{s}, \mathrm{sec}$ & Seconds \\
$\mathrm{U}, \mathrm{u}$ & Units \\
$\Omega$ & Ohm \\
$\mathrm{V}$ & Volt \\
\hline
\end{tabular}

\begin{tabular}{ccc}
\hline Unit prefix & Extended spelling & Scaling factor \\
\hline $\mathrm{k}$ & kilo & $10^{3}$ \\
$\mathrm{~m}$ & milli & $10^{-3}$ \\
$\mu$ & micro & $10^{-6}$ \\
$\mathrm{n}$ & nano & $10^{-9}$ \\
\hline
\end{tabular}

\begin{tabular}{ll}
\hline Natural constant & Value and unit \\
\hline $\mathrm{g}$ & $9.81 \mathrm{~m} / \mathrm{s}^{2}$ \\
\hline
\end{tabular}




\section{Amino acids and bases}

\begin{tabular}{lcc}
\hline Amino acid & Three letter code & Single letter code \\
\hline Alanine & Ala & $\mathrm{A}$ \\
Cysteine & Cys & $\mathrm{C}$ \\
Aspartate & Asp & $\mathrm{D}$ \\
Glutamate & Glu & $\mathrm{E}$ \\
Phenylalanine & Phe & $\mathrm{F}$ \\
Glycin & Gly & $\mathrm{G}$ \\
Histidine & His & $\mathrm{H}$ \\
Isoleucine & Ile & $\mathrm{I}$ \\
Lysine & Lys & $\mathrm{K}$ \\
Leucine & Leu & $\mathrm{L}$ \\
Methionine & Met & $\mathrm{M}$ \\
Asparagine & Asn & $\mathrm{N}$ \\
Proline & Pro & $\mathrm{P}$ \\
Glutamine & Gln & $\mathrm{Q}$ \\
Arginine & Arg & $\mathrm{R}$ \\
Serine & Ser & $\mathrm{S}$ \\
Threonine & Thr & $\mathrm{T}$ \\
Valine & Val & $\mathrm{V}$ \\
Tryptophane & Trp & $\mathrm{W}$ \\
Tyrosine & Tyr & $\mathrm{Y}$ \\
\hline
\end{tabular}

\begin{tabular}{lc}
\hline Base & Code \\
\hline Adenine & $\mathrm{A}$ \\
Cytosine & $\mathrm{C}$ \\
Guanine & $\mathrm{G}$ \\
Thymine & $\mathrm{T}$ \\
\hline
\end{tabular}


References and Acknowledgements 


\section{References}

1. Rouhier N, Lemaire SD, Jacquot J-P (2008) The role of glutathione in photosynthetic organisms: Emerging functions for glutaredoxins and glutathionylation. Annu Rev Plant Biol 59: 143-166.

2. Rouhier N, Koh C, Gelhaye E, Corbier C, Favier F, Didierjean C, Jacquot J-P (2008) Redox based anti-oxidant systems in plants: Biochemical and structural analyses. Biochim Biophys Acta 1780: 12491260 .

3. Herrera-Vásquez A, Salinas P, Holuigue L (2015) Salicylic acid and reactive oxygen species interplay in the transcriptional control of defense genes expression. Front Plant Sci 6: 1-9.

4. Rouhier N, Cerveau D, Couturier J, Reichheld J-P, Rey P (2015) Involvement of thiol-based mechanisms in plant development. Biochim Biophys Acta 1850: 1479-1496.

5. Grek CL, Zhang J, Manevich Y, Townsend DM, Tew KD (2013) Causes and consequences of cysteine S-glutathionylation. J Biol Chem 288: 26497-26504.

6. Navrot N, Finnie C, Svensson B, Hägglund P (2011) Plant redox proteomics. J Proteomics 74: 1450-1462.

7. Zaffagnini $\mathrm{M}$, Bedhomme $\mathrm{M}$, Marchand $\mathrm{CH}$, Morisse $\mathrm{S}$, Trost $\mathrm{P}$, Lemaire S (2012) Redox regulation in photosynthetic organisms: Focus on glutathionylation. Antioxid Redox Signal 16: 567-586.

8. Cremers CM, Jakob U (2013) Oxidant sensing by reversible disulfide bond formation. J Biol Chem 288: 26489-26496.

9. Deponte M (2013) Glutathione catalysis and the reaction mechanisms of glutathione-dependent enzymes. Biochim Biophys Acta 1830: 32173266.

10. Zagorchev L, Seal CE, Kranner I, Odjakova M (2013) A central role for thiols in plant tolerance to abiotic stress. Int J Mol Sci 14: 7405-7432.

11. Gellert M, Hanschmann EM, Lepka K, Berndt C, Lillig CH (2015) Redox regulation of cytoskeletal dynamics during differentiation and dedifferentiation. Biochim Biophys Acta 1850: 1575-1587.

12. Groitl B, Jakob U (2014) Thiol-based redox switches. Biochim Biophys Acta 1844: 1335-1343.

13. Kalinina E V., Chernov NN, Novichkova MD (2014) Role of 
glutathione, glutathione transferase, and glutaredoxin in regulation of redox-dependent processes. Biochemistry 79: 1562-1583.

14. Zaffagnini M, Bedhomme M, Lemaire SD, Trost P (2012) The emerging roles of protein glutathionylation in chloroplasts. Plant Sci 185-186: 8696.

15. Hill BG, Bhatnagar A (2012) Protein S-glutathionylation: Redoxsensitive regulation of protein function. J Mol Cell Cardiol 52: 559-567.

16. Winterbourn CC, Hampton MB (2008) Thiol chemistry and specificity in redox signaling. Free Radic Biol Med 45: 549-561.

17. Kullik I, Toledano MB, Tartaglia LA, Storz G (1995) Mutational analysis of the redox-sensitive transcriptional regulator OxyR: Regions important for oxidation and transcriptional activation. $J$ Bacteriol 177: 1275-1284.

18. Hausladen A, Privalle CT, Keng T, DeAngelo J, Stamler JS (1996) Nitrosative stress: Activation of the transcription factor OxyR. Cell 86: 719-729.

19. Zheng M, Aslund F, Storz G (1998) Activation of the OxyR transcription factor by reversible disulfide bond formation. Science 279: 1718-1721.

20. Aslund F, Zheng M, Beckwith J, Storz G (1999) Regulation of the OxyR transcription factor by hydrogen peroxide and the cellular thiol-disulfide status. Proc Natl Acad Sci U S A 96: 6161-6165.

21. Tao K (1999) In vivo oxidation-reduction kinetics of OxyR, the transcriptional activator for an oxidative stress-inducible regulon in Escherichia coli. FEBS Lett 457: 90-92.

22. Choi H-J, Kim S-J, Mukhopadhyay P, Cho S, Woo J-R, Storz G, Ryu SE (2001) Structural basis of the redox switch in the OxyR transcription factor. Cell 105: 103-113.

23. Lee C, Lee SM, Mukhopadhyay P, Kim SJ, Lee SC, Ahn W-S, Yu M-H, Storz G, Ryu SE (2004) Redox regulation of OxyR requires specific disulfide bond formation involving a rapid kinetic reaction path. Nat Struct Mol Biol 11: 1179-1185.

24. Kuge S, Jones N, Nomoto A (1997) Regulation of yAP-1 nuclear localization in response to oxidative stress. EMBO J 16: 1710-1720.

25. Kuge S, Arita M, Murayama A, Maeta K, Izawa S, Inoue Y, Nomoto A (2001) Regulation of the yeast Yap1p nuclear export signal is mediated by redox signal-induced reversible disulfide bond formation. Mol Cell Biol 21: 6139-6150. 
26. Wood MJ, Storz G, Tjandra N (2004) Structural basis for redox regulation of Yap1 transcription factor localization. Nature 430: 917921.

27. Pineda-Molina E, Klatt P, Vásquez J, Marina A, García de Lacoba M, Pérez-Sala D, Lamas S (2001) Glutathionylation of the p50 subunit of NF-kappaB: A mechanism for redox-induced inhibition of DNA binding. Biochemistry 40: 14134-14142.

28. Qanungo S, Starke DW, Pai H V, Mieyal JJ, Nieminen A-L (2007) Glutathione supplementation potentiates hypoxic apoptosis by Sglutathionylation of p65-NFkappaB. J Biol Chem 282: 18427-18436.

29. Viola IL, Güttlein LN, Gonzalez DH (2013) Redox modulation of plant developmental regulators from the class I TCP transcription factor family. Plant Physiol 162: 1434-1447.

30. Tron AE, Bertoncini CW, Chan RL, Gonzalez DH (2002) Redox regulation of plant homeodomain transcription factors. J Biol Chem 277: 34800-34807.

31. Mou Z, Fan W, Dong X (2003) Inducers of plant systemic acquired resistance regulate NPR1 function through redox changes. Cell113: 935944.

32. Lindermayr C, Sell S, Müller B, Leister D, Durner J (2010) Redox regulation of the NPR1-TGA1 system of Arabidopsis thaliana by nitric oxide. Plant Cell 22: 2894-2907.

33. Kneeshaw S, Gelineau S, Tada Y, Loake GJ, Spoel SH (2014) Selective protein denitrosylation activity of thioredoxin-h5 modulates plant immunity. Mol Cell 56: 153-162.

34. Tada Y, Spoel SH, Pajerowska-Mukhtar K, Mou Z, Song J, Wang C, Zuo J, Dong X (2008) Plant immunity requires conformational changes of NPR1 via S-nitrosylation and thioredoxins. Science (80- ) 321: 952-956.

35. López-Castillo LM, Jiménez-Sandoval P, Baruch-Torres N, TrasviñaArenas CH, Díaz-Quezada C, Lara-González S, Winkler R, Brieba LG (2016) Structural basis for redox regulation of cytoplasmic and chloroplastic triosephosphate isomerases from Arabidopsis thaliana. Front Plant Sci 7: 1817-1834.

36. Piattoni C V, Guerrero SA, Iglesias AA (2013) A differential redox regulation of the pathways metabolizing glyceraldehyde-3-phosphate tunes the production of reducing power in the cytosol of plant cells. Int J Mol Sci 14: 8073-8092.

37. Zaffagnini M, Fermani S, Costa A, Lemaire SD, Trost P (2013) Plant cytoplasmic GAPDH: Redox post-translational modifications and 
moonlighting properties. Front Plant Sci 4: 450-468.

38. Michelet L, Zaffagnini M, Morisse S, Sparla F, Pérez-Pérez ME, Francia F, Danon A, Marchand CH, Fermani S, Trost P, et al. (2013) Redox regulation of the Calvin-Benson cycle: Something old, something new. Front Plant Sci 4: 470-491.

39. Mailloux RJ, Jin X, Willmore WG (2013) Redox regulation of mitochondrial function with emphasis on cysteine oxidation reactions. Redox Biol 2: 123-139.

40. Irokawa H, Tachibana T, Watanabe T, Matsuyama $\mathrm{Y}$, Motohashi $\mathrm{H}$, Ogasawara A, Iwai K, Naganuma A, Kuge S (2016) Redox-dependent regulation of gluconeogenesis by a novel mechanism mediated by a peroxidatic cysteine of peroxiredoxin. Sci Rep 6: 33536-33552.

41. Danshina P V, Schmalhausen E V, Avetisyan A V, Muronetz VI (2001) Mildly oxidized glyceraldehyde-3-phosphate dehydrogenase as a possible regulator of glycolysis. IUBMB Life 51: 309-314.

42. Queval G, Foyer CH (2012) Redox regulation of photosynthetic gene expression. Philos Trans R Soc B Biol Sci 367: 3475-3485.

43. Allen JF, Alexciev K, Hakansson G (1995) Photosynthesis. Regulation by redox signalling. Curr Biol 5: 869-872.

44. Hancock JT, Henson D, Nyirenda M, Desikan R, Harrison J, Lewis M, Hughes J, Neill SJ (2005) Proteomic identification of glyceraldehyde 3phosphate dehydrogenase as an inhibitory target of hydrogen peroxide in Arabidopsis. Plant Physiol Biochem 43: 828-835.

45. Lindermayr C, Saalbach G, Durner J (2005) Proteomic identification of S-nitrosylated proteins in Arabidopsis. Plant Physiol 137: 921-930.

46. Holtgrefe S, Gohlke J, Starmann J, Druce S, Klocke S, Altmann B, Wojtera J, Lindermayr C, Scheibe R (2008) Regulation of plant cytosolic glyceraldehyde 3-phosphate dehydrogenase isoforms by thiol modifications. Physiol Plant 133: 211-228.

47. Schneider M, Knuesting J, Birkholz O, Heinisch JJ, Scheibe R (2018) Cytosolic GAPDH as a redox-dependent regulator of energy metabolism. BMC Plant Biol 18: 184-198.

48. Bedhomme M, Adamo M, Marchand CH, Couturier J, Rouhier N, Lemaire S, Zaffagnini M, Trost P (2012) Glutathionylation of cytosolic glyceraldehyde-3-phosphate dehydrogenase from the model plant Arabidopsis thaliana is reversed by both glutaredoxins and thioredoxins in vitro. Biochem J 445: 337-347.

49. Berndt C, Lillig CH, Flohé L (2014) Redox regulation by glutathione 
needs enzymes. Front Pharmacol 5: 168-172.

50. Holmgren A (1979) Reduction of disulfides by thioredoxin. Exceptional reactivity of insulin and suggested functions of thioredoxin in mechanism of hormone action. J Biol Chem 254: 9113-9119.

51. Moore EC, Reichard P, Thelander L (1964) Enyzmatic synthesis of deoxyribonucleotides. V. Purification and properties of thioredoxin reductase from Escherichia coli B. J Biol Chem 239: 3445-3452.

52. Axelsson K, Mannervik B (1980) General specificity of cytoplasmic thioltransferase (thiol:disulfide oxidoreductase) from rat liver for thiol and disulfide substrates. Biochim Biophys Acta 613: 324-336.

53. Prinz WA, Aslund F, Holmgren A, Beckwith J (1997) The role of the thioredoxin and glutaredoxin pathways in reducing protein disulfide bonds in the Escherichia coli cytoplasm. J Biol Chem 272: 15661-15667.

54. Laurent TC, Moore CE, Reichard P (1964) Enzymatic synthesis of desoxyribonucleotides. IV. Isolation and characterization of thioredoxin, the hydrogen donor from Escherichia coli B. J Biol Chem 239: 3436-3444.

55. Reichard P (1962) Enzymatic synthesis of deoxyribonucleotides. I. Formation of deoxycytidine diphosphate from cytidine diphosphate with enzymes from Escherichia coli. J Biol Chem 237: 3513-3519.

56. Nagai S, Black S (1968) A thiol-disulfide transhydrogenase from yeast.J Biol Chem 243: 1942-1947.

57. Askelöf P, Axelsson K, Eriksson S, Mannervik B (1974) Mechanism of action of enzymes catalyzing thiol-disulfide interchange. Thioltransferases rather than transhydrogenases. FEBS Lett 38: 263267.

58. Holmgren A (1976) Hydrogen donor system for Escherichia coli ribonucleoside-diphosphate reductase dependent upon glutathione. Proc Natl Acad Sci U S A 73: 2275-2279.

59. Holmgren A (1979) Glutathione-dependent Synthesis of Deoxyribonucleotides. Purification and characterization of glutaredoxin from Escherichia coli. J Biol Chem 254: 3664-3671.

60. Russel M, Holmgren A (1988) Construction and characterization of glutaredoxin-negative mutants of Escherichia coli. Proc Natl Acad Sci USA 85: 990-994.

61. Axelsson K, Eriksson S, Mannervik B (1978) Purification and characterization of cytoplasmic thioltransferase (glutathione:disulfide oxidoreductase) from rat liver. Biochemistry 17: 2978-2984. 
62. Shenton D, Perrone G, Quinn KA, Dawes IW, Grant CM (2002) Regulation of protein S-thiolation by glutaredoxin 5 in the yeast Saccharomyces cerevisiae. J Biol Chem 277: 16853-16859.

63. Trost P, Fermani S, Marri L, Zaffagnini M, Falini G, Scagliarini S, Pupillo P, Sparla F (2006) Thioredoxin-dependent regulation of photosynthetic glyceraldehyde-3- phosphate dehydrogenase: Autonomous vs. CP12-dependent mechanisms. Photosynth Res 89: 263275.

64. Höög J, Jörnvall H, Holmgren A, Carlquist M, Persson M (1983) The primary structure of Escherichia coli glutaredoxin: Distant homology with thioredoxins in a superfamily of small proteins with a redox-active cystine disulfide/cysteine dithiol. Eur J Biochem 136: 223-232.

65. Martin JL (1995) Thioredoxin - a fold for all reasons. Structure 3: 245250.

66. Collet J-F, Messens J (2010) Structure, function, and mechanism of thioredoxin proteins. Antioxid Redox Signal 13: 1205-1216.

67. Qi Y, Grishin N V. (2005) Structural classification of thioredoxin-like fold proteins. Proteins Struct Funct Bioinforma 58: 376-388.

68. Eklund H, Cambillau C, Sjöberg BM, Holmgren A, Jörnvall H, Höög JO, Brändén CI (1984) Conformational and functional similarities between glutaredoxin and thioredoxins. EMBO J 3: 1443-1449.

69. Kallis GB, Holmgren A (1980) Differential reactivity of the functional sulfhydryl groups of cysteine-32 and cysteine-35 present in the reduced form of thioredoxin from Escherichia coli. J Biol Chem 255: 1026110265.

70. Hol WGJ (1985) The role of the $\alpha$-helix dipole in protein function and structure. Prog Biophys Mol Biol 45: 149-195.

71. Dyson JH, Tennant LL, Holmgren A (1991) Proton-transfer effects in the active-site region of Escherichia coli thioredoxin using twodimensional 1H NMR. Biochemistry 30: 4262-4268.

72. Li H, Hanson C, Fuchs JA, Woodward C, Thomas Jr GJ (1993) Determination of the $\mathrm{pKa}$ values of active-center cysteines, cysteines-32 and -35 , in Escherichia coli thioredoxin by Raman spectroscopy. Biochemistry 32: 5800-5808.

73. Sun C, Berardi MJ, Bushweller JH (1998) The NMR solution structure of human glutaredoxin in the fully reduced form. J Mol Biol 280: 687701.

74. Gan ZR, Wells WW (1987) Identification and reactivity of the catalytic 
site of pig liver thioltransferase. J Biol Chem 262: 6704-6707.

75. Begas P, Liedgens L, Moseler A, Meyer AJ, Deponte M (2017) Glutaredoxin catalysis requires two distinct glutathione interaction sites. Nat Commun 8: 14835-14848.

76. Aslund F, Berndt KD, Holmgren A (1997) Redox potentials of glutaredoxins and other thiol-disulfide oxidoreductases of the thioredoxin superfamily determined by direct protein-protein redox equilibria. J Biol Chem 272: 30780-30786.

77. Couturier J, Jacquot J-P, Rouhier N (2013) Toward a refined classification of class I dithiol glutaredoxins from poplar: Biochemical basis for the definition of two subclasses. Front Plant Sci 4: 518-532.

78. Zaffagnini M, Michelet L, Massot V, Trost P, Lemaire SD (2008) Biochemical characterization of glutaredoxins from Chlamydomonas reinhardtii reveals the unique properties of a chloroplastic CGFS-type glutaredoxin. J Biol Chem 283: 8868-8876.

79. Lillig CH, Berndt C, Holmgren A (2008) Glutaredoxin systems. Biochim Biophys Acta 1780: 1304-1317.

80. Gutsche N, Thurow C, Zachgo S, Gatz C (2015) Plant-specific CC-type glutaredoxins: Functions in developmental processes and stress responses. Biol Chem 396: 495-509.

81. Feng Y, Zhong N, Rouhier N, Hase T, Kusunoki M, Jacquot JP, Jin C, Xia B (2006) Structural insight into poplar glutaredoxin C1 with a bridging iron-sulfur cluster at the active site. Biochemistry 45: 7998-8008.

82. Greetham D, Vickerstaff J, Shenton D, Perrone GG, Dawes IW, Grant CM (2010) Thioredoxins function as deglutathionylase enzymes in the yeast Saccharomyces cerevisiae. BMC Biochem 11: 3-13.

83. Benhar M, Forrester MT, Hess DT, Stamler JS (2008) Regulated protein denitrosylation by cytosolic and mitochondrial thioredoxins. Science (80) 320: 1050-1054.

84. Schürmann P, Buchanan BB (2008) The ferredoxin/thioredoxin system of oxygenic photosynthesis. Antioxid Redox Signal 10: 1235-1274.

85. Yoshida K, Hisabori $\mathrm{T}$ (2017) Distinct electron transfer from ferredoxin-thioredoxin reductase to multiple thioredoxin isoforms in chloroplasts. Biochem J 474: 1347-1360.

86. Nikkola M, Gleason FK, Saarinen M, Joelson T, Björnberg O, Eklund $\mathrm{H}$ (1991) A putative glutathione-binding site in T4 glutaredoxin investigated by site-directed mutagenesis. J Biol Chem 266: 16105-16112.

87. Starke DW, Chock PB, Mieyal JJ (2003) Glutathione-thiyl radical 
scavenging and transferase properties of human glutaredoxin (thioltransferase): Potential role in redox signal transduction. J Biol Chem 278: 14607-14613.

88. Bender KW, Wang X, Cheng GB, Kim HS, Zielinski RE, Huber SC (2015) Glutaredoxin AtGRXC2 catalyses inhibitory glutathionylation of Arabidopsis BRI1-associated receptor-like kinase 1 (BAK1) in vitro. Biochem J 467: 399-413.

89. Rodríguez-Manzaneque MT, Tamarit J, Bellí G, Ros J, Herrero E (2002) Grx5 is a mitochondrial glutaredoxin required for the activity of iron/sulfur enzymes. Mol Biol Cell 13: 1109-1121.

90. Zhang B, Bandyopadhyay S, Shakamuri P, Naik SG, Huynh BH, Couturier J, Rouhier N, Johnson MK (2013) Monothiol glutaredoxins can bind linear $[\mathrm{Fe} 3 \mathrm{~S} 4]+$ and $[\mathrm{Fe} 4 \mathrm{~S} 4] 2+$ clusters in addition to [Fe2S2]2+ clusters: Spectroscopic characterization and functional implications. J Am Chem Soc 135: 15153-15164.

91. Fladvad M, Bellanda M, Fernandes AP, Mammi S, Vlamis-Gardikas A, Holmgren A, Sunnerhagen M (2005) Molecular mapping of functionalities in the solution structure of reduced Grx4, a monothiol glutaredoxin from Escherichia coli. J Biol Chem 280: 24553-24561.

92. Iwema T, Picciocchi A, Traore DAK, Ferrer JL, Chauvat F, Jacquamet L (2009) Structural basis for delivery of the intact [Fe2S2] cluster by monothiol glutaredoxin. Biochemistry 48: 6041-6043.

93. Lillig CH, Berndt C (2013) Glutaredoxins in thiol/disulfide exchange. Antioxid Redox Signal 18: 1654-1665.

94. Couturier J, Ströher E, Albetel AN, Roret T, Muthuramalingam M, Tarrago L, Seidel T, Tsan P, Jacquot JP, Johnson MK, et al. (2011) Arabidopsis chloroplastic glutaredoxin $\mathrm{C} 5$ as a model to explore molecular determinants for iron-sulfur cluster binding into glutaredoxins. J Biol Chem 286: 27515-27527.

95. Wang L, Li Y, Jacquot JP, Rouhier N, Xia B (2014) Characterization of poplar GrxS14 in different structural forms. Protein Cell 5: 329-333.

96. Couturier J, Koh CS, Zaffagnini M, Winger AM, Gualberto JM, Corbier C, Decottignies P, Jacquot JP, Lemaire SD, Didierjean C, et al. (2009) Structure-function relationship of the chloroplastic glutaredoxin S12 with an atypical WCSYS active site. J Biol Chem 284: 9299-9310.

97. Moseler A, Aller I, Wagner S, Nietzel T, Przybyla-Toscano J, Mühlenhoff U, Lill R, Berndt C, Rouhier N, Schwarzländer M, et al. (2015) The mitochondrial monothiol glutaredoxin S15 is essential for iron-sulfur protein maturation in Arabidopsis thaliana. Proc Natl Acad Sci US A 112: 13735-13740. 
98. Li L, Cheng N, Hirschi KD, Wang X (2010) Structure of Arabidopsis chloroplastic monothiol glutaredoxin AtGRXcp. Acta Crystallogr Sect D Biol Crystallogr 66: 725-732.

99. Bushweller JH, Holmgren A, Wüthrich K (1993) Biosynthetic $15 \mathrm{~N}$ and 13C isotope labelling of glutathione in the mixed disulfide with Escherichia coli glutaredoxin documented by sequence-specific NMR assignments. Eur J Biochem 218: 327-334.

100. Bushweller JH, Billeter M, Holmgren A, Wüthrich K (1994) The nuclear magnetic resonance solution structure of the mixed disulfide between Escherichia coli glutaredoxin(C14S) and glutathione. J Mol Biol 235: 1585.1597.

101. Yang Y, Wells WW (1991) Catalytic mechanism of thioltransferase.J Biol Chem 266: 12766-12771.

102. Ukuwela AA, Bush AI, Wedd AG, Xiao Z (2018) Glutaredoxins employ parallel monothiol-dithiol mechanisms to catalyze thiol-disulfide exchanges with protein disulfides. Chem Sci 9: 1173-1183.

103. Brose J, La Fontaine S, Wedd AG, Xiao Z (2014) Redox sulfur chemistry of the copper chaperone Atox1 is regulated by the enzyme glutaredoxin 1 , the reduction potential of the glutathione couple GSSG/2GSH and the availability of $\mathrm{Cu}(\mathrm{I})$. Metallomics 6: 793-808.

104. Tamarit J, Bellí G, Cabiscol E, Herrero E, Ros J (2003) Biochemical characterization of yeast mitochondrial Grx5 monothiol glutaredoxin. $J$ Biol Chem 278: 25745-25751.

105. Fernandes AP, Fladvad M, Berndt C, Andrésen C, Lillig CH, Neubauer P, Sunnerhagen M, Holmgren A, Vlamis-Gardikas A (2005) A novel monothiol glutaredoxin (Grx4) from Escherichia coli can serve as a substrate for thioredoxin reductase. J Biol Chem 280: 24544-24552.

106. Johansson C, Lillig CH, Holmgren A (2004) Human mitochondrial glutaredoxin reduces S-glutathionylated proteins with high affinity accepting electrons from either glutathione or thioredoxin reductase. $J$ Biol Chem 279: 7537-7543.

107. Bushweller JH, Åslund F, Wüthrich K, Holmgren A (1992) Structural and functional characterization of the mutant Escherichia coli glutaredoxin (C14---S) and its mixed disulfide with glutathione. Biochemistry 31: 9288-9293.

108. Luo M, Jiang YL, Ma XX, Tang YJ, He YX, Yu J, Zhang RG, Chen Y, Zhou CZ (2010) Structural and biochemical characterization of yeast monothiol glutaredoxin Grx6. J Mol Biol 398: 614-622.

109. Eckers E, Bien M, Stroobant V, Herrmann JM, Deponte M (2009) 
Biochemical characterization of dithiol glutaredoxin 8 from Saccharomyces cerevisiae: The catalytic redox mechanism redux. Biochemistry 48: 1410-1423.

110. Li WF, Yu J, Ma XX, Teng Y Bin, Luo M, Tang YJ, Zhou CZ (2010) Structural basis for the different activities of yeast Grx1 and Grx2. Biochim Biophys Acta 1804: 1542-1547.

111. Nordstrand K, Sandström A, Åslund F, Holmgren A, Otting G, Berndt KD (2000) NMR structure of oxidized glutaredoxin 3 from Escherichia coli. J Mol Biol 303: 423-432.

112. Riondet C, Desouris JP, Montoya JG, Chartier Y, Meyer Y, Reichheld JP (2012) A dicotyledon-specific glutaredoxin GRXC1 family with dimer-dependent redox regulation is functionally redundant with GRXC2. Plant Cell Env 35: 360-373.

113. Rouhier N, Unno H, Bandyopadhyay S, Masip L, Kim S-K, Hirasawa M, Gualberto JM, Lattard V, Kusunoki M, Knaff DB, et al. (2007) Functional, structural, and spectroscopic characterization of a glutathione-ligated [2Fe-2S] cluster in poplar glutaredoxin C1. Proc Natl Acad Sci U S A 104: 7379-7384.

114. Bandyopadhyay S, Gama F, Molina-Navarro MM, Gualberto JM, Claxton R, Naik SG, Huynh BH, Herrero E, Jacquot JP, Johnson MK, et al. (2008) Chloroplast monothiol glutaredoxins as scaffold proteins for the assembly and delivery of [2Fe-2S] clusters. EMBO J 27: 1122 1133.

115. Picciocchi A, Saguez C, Boussac A, Cassier-Chauvat C, Chauvat F (2007) CGFS-type monothiol glutaredoxins from the cyanobacterium Synechocystis PCC6803 and other evolutionary distant model organisms possess a glutathione-ligated [2Fe-2S] cluster. Biochemistry 46: 15018-15026.

116. Ströher E, Grassl J, Carrie C, Fenske R, Whelan J, Millar AH (2016) Glutaredoxin S15 is involved in Fe-S cluster transfer in mitochondria influencing lipoic acid-dependent enzymes, plant growth and arsenic tolerance in Arabidopsis. Plant Physiol 170: 1284-1299.

117. Cheng NH (2008) AtGRX4, an Arabidopsis chloroplastic monothiol glutaredoxin, is able to suppress yeast grx 5 mutant phenotypes and respond to oxidative stress. FEBS Lett 582: 848-854.

118. Balk J, Schaedler TA (2014) Iron cofactor assembly in plants. Annu Rev Plant Biol 65: 125-153.

119. Beinert H, Holm RH, Münck E (1997) Iron-sulfur clusters : Nature's modular, multipurpose structures. Science (80- ) 277: 653-659. 
120. Hoffman BM, Lukoyanov D, Yang Z-Y, Dean DR, Seefeldt LC (2014) Mechanism of nitrogen fixation by nitrogenase: The next stage. Chem Rev 114: 4041-4062.

121. Imsande J (1999) Iron-sulfur clusters: Formation, perturbation, and physiological functions. Plant Physiol Biochem 37: 87-97.

122. Banci L, Brancaccio D, Ciofi-Baffoni S, Del Conte R, Gadepalli R, Mikolajczyk M, Neri S, Piccioli M, Winkelmann J (2014) [2Fe-2S] cluster transfer in iron-sulfur protein biogenesis. Proc Natl Acad Sci 111: 62036208.

123. Knuesting J, Riondet C, Maria C, Kruse I, Bécuwe N, König N, Berndt C, Tourrette S, Guilleminot-Montoya J, Herrero E, et al. (2015) Arabidopsis glutaredoxin S17 and its partner, the nuclear factor $\mathrm{Y}$ subunit $\mathrm{C} 11 /$ negative cofactor $2 \alpha$, contribute to maintenance of the shoot apical meristem under long-day photoperiod. Plant Physiol 167: 1643-1658.

124. Couturier J, Przybyla-Toscano J, Roret T, Didierjean C, Rouhier N (2015) The roles of glutaredoxins ligating Fe-S clusters: Sensing, transfer or repair functions? Biocbim Biophys Acta 1853: 1513-1527.

125. Johansson C, Roos AK, Montano SJ, Sengupta R, Filippakopoulos P, Guo K, von Delft F, Holmgren A, Oppermann U, Kavanagh KL (2011) The crystal structure of human GLRX5: Iron-sulfur cluster coordination, tetrameric assembly and monomer activity. Biochem J 433: 303-311.

126. Bräutigam L, Johansson C, Kubsch B, McDonough MA, Bill E, Holmgren A, Berndt C (2013) An unusual mode of iron-sulfur-cluster coordination in a teleost glutaredoxin. Biochem Biophys Res Commun 436: 491-496.

127. Dhalleine T, Rouhier N, Couturier J (2014) Putative roles of glutaredoxin-BolA holo-heterodimers in plants. Plant Signal Behav 9: e28564.

128. Inigo S, Nagels Durand A, Ritter A, Le Gall S, Termathe M, Klassen R, Tohge T, De Coninck B, Van Leene J, De Clercq R, et al. (2016) Glutaredoxin GRXS17 associates with the cytosolic iron-sulfur cluster assembly pathway. Plant Physiol 172: 858-873.

129. Gao H, Subramanian S, Couturier J, Naik SG, Kim SK, Leustek T, Knaff DB, Wu HC, Vignols F, Huynh BH, et al. (2013) Arabidopsis thaliana Nfu2 accommodates [2Fe-2S] or [4Fe-4S] clusters and is competent for in vitro maturation of chloroplast [2Fe-2S] and [4Fe-4S] cluster-containing proteins. Biochemistry 52: 6633-6645.

130. Roret T, Tsan P, Couturier J, Zhang B, Johnson MK, Rouhier N, 
Didierjean C (2014) Structural and spectroscopic insights into BolAGlutaredoxin complexes. J Biol Chem 289: 24588-24598.

131. Rey P, Becuwe N, Tourrette S, Rouhier N (2017) Involvement of Arabidopsis glutaredoxin S14 in the maintenance of chlorophyll content. Plant Cell Environ 40: 2319-2332.

132. Rouhier N, Gelhaye E, Jacquot J-P (2004) Plant glutaredoxins: Still mysterious reducing systems. Cell Mol Life Sci 61: 1266-1277.

133. Lemaire SD (2004) The glutaredoxin family in oxygenic photosynthetic organisms. Photosynth Res 79: 305-318.

134. Rouhier N, Couturier J, Jacquot J-P (2006) Genome-wide analysis of plant glutaredoxin systems. J Exp Bot 57: 1685-1696.

135. Xing S, Lauri A, Zachgo S (2006) Redox regulation and flower development: A novel function for glutaredoxins. Plant Biol 8: 547-555.

136. Ziemann M, Bhave M, Zachgo S (2009) Origin and diversification of land plant CC-type glutaredoxins. Genome Biol Evol 1: 265-277.

137. Zaffagnini M, Bedhomme M, Marchand CH, Couturier J, Gao X-H, Rouhier N, Trost P, Lemaire SD (2012) Glutaredoxin S12: Unique properties for redox signaling. Antioxid Redox Signal 16: 17-32.

138. Lillig CH, Berndt C, Vergnolle O, Lönn ME, Hudemann C, Bill E, Holmgren A (2005) Characterization of human glutaredoxin 2 as ironsulfur protein: A possible role as redox sensor. Proc Natl Acad Sci US A 102: 8168-8173.

139. Liu X, Liu S, Feng Y, Liu J-Z, Chen Y, Pham K, Deng H, Hirschi KD, Wang X, Cheng N (2013) Structural insights into the N-terminal GIYYIG endonuclease activity of Arabidopsis glutaredoxin AtGRXS16 in chloroplasts. Proc Natl Acad Sci U S A 110: 9565-9570.

140. Guo Y, Huang C, Xie Y, Song F, Zhou X (2010) A tomato glutaredoxin gene SIGRX1 regulates plant responses to oxidative, drought and salt stresses. Planta 232: 1499-1509.

141. Cheng NH, Liu JZ, Liu X, Wu Q, Thompson SM, Lin J, Chang J, Whitham SA, Park S, Cohen JD, et al. (2011) Arabidopsis monothiol glutaredoxin, AtGRXS17, is critical for temperature-dependent postembryonic growth and development via modulating auxin response. J Biol Chem 286: 20398-20406.

142. Wu Q, Lin J, Liu JZ, Wang X, Lim W, Oh M, Park J, Rajashekar CB, Whitham SA, Cheng NH, et al. (2012) Ectopic expression of Arabidopsis glutaredoxin AtGRXS17 enhances thermotolerance in tomato. Plant Biotechnol J 10: 945-955. 
143. Hu Y, Wu Q, Peng Z, Sprague SA, Wang W, Park J, Akhunov E, Jagadish KSV, Nakata PA, Cheng N, et al. (2017) Silencing of OsGRXS17 in rice improves drought stress tolerance by modulating ROS accumulation and stomatal closure. Sci Rep 7: 15950-15964.

144. Wu Q, Hu Y, Sprague SA, Kakeshpour T, Park J, Nakata PA, Cheng N, Hirschi KD, White FF, Park S (2017) Expression of a monothiol glutaredoxin, AtGRXS17, in tomato (Solanum lycopersicum) enhances drought tolerance. Biochem Biophys Res Commun 491: 1034-1039.

145. Li S, Lauri A, Ziemann M, Busch A, Bhave M, Zachgo S (2009) Nuclear activity of ROXY1, a glutaredoxin interacting with TGA factors, is required for petal development in Arabidopsis thaliana. Plant Cell 21: 429-441.

146. Xing S, Rosso MG, Zachgo S (2005) ROXY1, a member of the plant glutaredoxin family, is required for petal development in Arabidopsis thaliana. Development 132: 1555-1565.

147. Ndamukong I, Abdallat A Al, Thurow C, Fode B, Zander M, Weigel R, Gatz C (2007) SA-inducible Arabidopsis glutaredoxin interacts with TGA factors and suppresses JA-responsive PDF1.2 transcription. Plant J 50: 128-139.

148. La Camera S, L'Haridon F, Astier J, Zander M, Abou-Mansour E, Page G, Thurow C, Wendehenne D, Gatz C, Métraux J-P, et al. (2011) The glutaredoxin ATGRXS13 is required to facilitate Botrytis cinerea infection of Arabidopsis thaliana plants. Plant J 68: 507-519.

149. Laporte D, Olate E, Salinas P, Salazar M, Jordana X, Holuigue L (2012) Glutaredoxin GRXS13 plays a key role in protection against photooxidative stress in Arabidopsis. J Exp Bot 63: 503-515.

150. Lai Z, Schluttenhofer CM, Bhide K, Shreve J, Thimmapuram J, Lee SY, Yun D-J, Mengiste T (2014) MED18 interaction with distinct transcription factors regulates multiple plant functions. Nat Commun 5: 3064-3078.

151. Herrera-Vásquez A, Carvallo L, Blanco F, Tobar M, Villarroel-Candia E, Vicente-Carbajosa J, Salinas P, Holuigue L (2015) Transcriptional control of glutaredoxin GRXC9 expression by a salicylic acid-dependent and NPR1-independent pathway in Arabidopsis. Plant Mol Biol Report 33: 624-637.

152. Huang L-J, Li N, Thurow C, Wirtz M, Hell R, Gatz C (2016) Ectopically expressed glutaredoxin ROXY19 negatively regulates the detoxification pathway in Arabidopsis thaliana. BMC Plant Biol 16: 200-212.

153. El-Kereamy A, Bi Y-M, Mahmood K, Ranathunge K, Yaish MW, Nambara E, Rothstein SJ (2015) Overexpression of the CC-type 
glutaredoxin, OsGRX6 affects hormone and nitrogen status in rice plants. Front Plant Sci 6: 934-946.

154. Ohkubo Y, Tanaka M, Tabata R, Ogawa-Ohnishi M, Matsubayashi Y (2017) Shoot-to-root mobile polypeptides involved in systemic regulation of nitrogen acquisition. Nat Plants 3:.

155. Li N (2017) Plant-specific glutaredoxin ROXY9 regulates hyponastic growth by inhibiting TGA1 function. PhD thesis, Göttingen.

156. Li N, Muthreich M, Huang L-J, Thurow C, Sun T, Zhang Y, Gatz C (2018) TGACG-BINDING FACTORs (TGAs) and TGA-interacting CC-type glutaredoxins modulate hyponastic growth in Arabidopsis thaliana. New Phytol doi: 10.1111.

157. Muthreich M (2014) Characterization of clade I TGA transcription factors in Arabidopsis thaliana with respect to biotic stress. PhD thesis, Göttingen.

158. Xing S, Zachgo S (2008) ROXY1 and ROXY2, two Arabidopsis glutaredoxin genes, are required for anther development. Plant J 53: 790_ 801.

159. Jung FM (2016) Analysis of the defense responses of Arabidopsis thaliana tga1,4 knockout mutants and plants ectopically expressing ROXY9 and establishment of a roxy9 knockout mutant. Master thesis, Göttingen.

160. Gutsche N, Holtmannspötter M, Maß L, O’Donoghue M, Busch A, Lauri A, Schubert V, Zachgo S (2017) Conserved redox-dependent DNA binding of ROXY glutaredoxins with TGA transcription factors. Plant Direct 1: doi: 10.1002.

161. Wang Z, Xing S, Birkenbihl RP, Zachgo S (2009) Conserved functions of Arabidopsis and rice CC-type glutaredoxins in flower development and pathogen response. Mol Plant 2: 323-335.

162. Hong L, Tang D, Zhu K, Wang K, Li M, Cheng Z (2012) Somatic and reproductive cell development in rice anther is regulated by a putative glutaredoxin. Plant Cell 24: 577-588.

163. Yang L, Qian X, Chen M, Fei Q, Meyers BC, Liang W, Zhang D (2016) Regulatory role of a receptor-like kinase in specifying anther cell identity. Plant Physiol 171: 2085-2100.

164. Yang F, Bui HT, Pautler M, Llaca V, Johnston R, Lee B, Kolbe A, Sakai H, Jackson D (2015) A maize glutaredoxin gene, Abphyl2, regulates shoot meristem size and phyllotaxy. Plant Cell 27: 121-131.

165. Kelliher T, Walbot V (2012) Hypoxia triggers meiotic fate acquisition in 
References

maize. Science (80- ) 337: 345-348.

166. Patterson K, Walters LA, Cooper AM, Olvera JG, Rosas MA, Rasmusson AG, Escobar MA (2016) Nitrate-regulated glutaredoxins control Arabidopsis primary root growth. Plant Physiol 170: 989-999.

167. Wydro MM, Sharma P, Foster JM, Bych K, Meyer EH, Balk J (2013) The evolutionarily conserved iron-sulfur protein INDH is required for complex I assembly and mitochondrial translation in Arabidopsis. Plant Cell 25: 4014-4027.

168. Zander M, Chen S, Imkampe J, Thurow C, Gatz C (2012) Repression of the Arabidopsis thaliana jasmonic acid/ethylene-induced defense pathway by TGA-interacting glutaredoxins depends on their C-terminal ALWL motif. Mol Plant 5: 831-840.

169. Murmu J, Bush MJ, DeLong C, Li S, Xu M, Khan M, Malcolmson C, Fobert PR, Zachgo S, Hepworth SR (2010) Arabidopsis basic leucinezipper transcription factors TGA9 and TGA10 interact with floral glutaredoxins ROXY1 and ROXY2 and are redundantly required for anther development. Plant Physiol 154: 1492-1504.

170. Li S, Gutsche N, Zachgo S (2011) The ROXY1 C-terminal L**LL motif is essential for the interaction with TGA transcription factors. Plant Physiol 157: 2056-2068.

171. Ziemann M, Bhave M, Zachgo S (2011) Bioinformatic studies of the wheat glutaredoxin gene family and functional analysis of the ROXY1 orthologues. Funct Plant Biol 38: 25-34.

172. Gutsche N, Zachgo S (2016) The N-terminus of the floral Arabidopsis TGA transcription factor PERIANTHIA mediates redox-sensitive DNA-binding. PLoS One 11: e0153810.

173. Quon T, Lampugnani ER, Smyth DR (2017) PETAL LOSS and ROXY1 interact to limit growth within and between sepals but to promote petal initiation in Arabidopsis thaliana. Front Plant Sci 8: 152166.

174. Oberdiek J (2018) Funktionale Analyse des CC-Typ Glutaredoxin ROXY19 in Arabidopsis thaliana. PhD thesis, Göttingen.

175. Ziemann M (2010) Glutaredoxin family genes in plant development. PhD thesis, Melb.

176. Couturier J, Didierjean C, Jacquot JP, Rouhier N (2010) Engineered mutated glutaredoxins mimicking peculiar plant class III glutaredoxins bind iron-sulfur centers and possess reductase activity. Biochem Biophys Res Commun 403: 435-441. 
177. Uhrig JF, Huang L-J, Barghahn S, Willmer M, Thurow C, Gatz C (2017) CC-type glutaredoxins recruit the transcriptional co-repressor TOPLESS to TGA-dependent target promoters in Arabidopsis thaliana. Biochim Biophys Acta Gene Regul Mech 1860: 218-226.

178. Willmer M (2014) Struktur-Funktions-Analyse von ROXYGlutaredoxinen und TIFY-Domänen-Proteinen in Arabidopsis thaliana. Bachelorarbeit, Göttingen.

179. Pauwels L, Goossens A (2011) The JAZ proteins: A crucial interface in the jasmonate signaling cascade. Plant Cell 23: 3089-3100.

180. Bai Y, Meng Y, Huang D, Qi Y, Chen M (2011) Origin and evolutionary analysis of the plant-specific TIFY transcription factor family. Genomics 98: 128-136.

181. Pauwels L, Fernández Barbero G, Geerinck J, Tilleman S, Grunewald W, Cuéllar Pérez A, Chico JM, Vanden Bossche R, Sewell J, Gil E, et al. (2010) NINJA connects the co-repressor TOPLESS to jasmonate signalling. Nature 464: 788-791.

182. Jakoby M, Weisshaar B, Dröge-Laser W, Vicente-Carbajosa J, Tiedemann J, Kroj T, Parcy F (2002) bZIP transcription factors in Arabidopsis. Trends Plant Sci 7: 106-111.

183. Qin XF, Holuigue L, Horvath DM, Chua N-H (1994) Immediate early transcription activation by salicylic acid via the cauliflower mosaic virus as-1 element. Plant Cell 6: 863-874.

184. Katagiri F, Lam E, Chua NH (1989) Two tobacco DNA-binding proteins with homology to the nuclear factor CREB. Nature 340: 727730.

185. Lam E, Benfey PN, Gilmartin PM, Fang RX, Chua NH (1989) Sitespecific mutations alter in vitro factor binding and change promoter expression pattern in transgenic plants. Proc Natl Acad Sci US A 86: 7890-7894.

186. Fan W, Dong X (2002) In vivo interaction between NPR1 and transcription factor TGA2 leads to salicylic acid-mediated gene activation in Arabidopsis. Plant Cell 14: 1377-1389.

187. Pascuzzi P, Hamilton D, Bodily K, Arias J (1998) Auxin-induced stress potentiates trans-activation by a conserved plant basic/leucine-zipper factor. J Biol Chem 273: 26631-26637.

188. Neuhaus G, Neuhaus-Url G, Katagiri F, Seipel K, Chua N (1994) Tissue-Specific Expression of as-1 in Transgenic Tobacco. Plant Cell 6: 827-834. 
189. Boyle P, Le Su E, Rochon A, Shearer HL, Murmu J, Chu JY, Fobert PR, Després C (2009) The BTB/POZ Domain of the Arabidopsis Disease Resistance Protein NPR1 Interacts with the Repression Domain of TGA2 to Negate Its Function. Plant Cell 21: 3700-3713.

190. Gatz C (2013) From pioneers to team players: TGA transcription factors provide a molecular link between different stress pathways. Mol PlantMicrobe Interact 26: 151-159.

191. Gemayel R, Chavali S, Pougach K, Legendre M, Zhu B, Boeynaems S, van der Zande E, Gevaert K, Rousseau F, Schymkowitz J, et al. (2015) Variable glutamine-rich repeats modulate transcription factor activity. Mol Cell 59: 615-627.

192. Zhang Y, Fan W, Kinkema M, Li X, Dong X (1999) Interaction of NPR1 with basic leucine zipper protein transcription factors that bind sequences required for salicylic acid induction of the PR-1 gene. Proc Natl Acad Sci U S A 96: 6523-6528.

193. Zhou JM, Trifa Y, Silva H, Pontier D, Lam E, Shah J, Klessig DF (2000) NPR1 differentially interacts with members of the TGA/OBF family of transcription factors that bind an element of the PR-1 gene required for induction by salicylic acid. Mol Plant Microbe Interact 13: 191-202.

194. Kesarwani M, Yoo J, Dong X (2007) Genetic interactions of TGA transcription factors in the regulation of pathogenesis-related genes and disease resistance in Arabidopsis. Plant Physiol 144: 336-346.

195. Fujii Y, Shimizu T, Toda T, Yanagida M, Hakoshima T (2000) Structural basis for the diversity of DNA recognition by bZIP transcription factors. Nat Struct Biol 7: 889-893.

196. Zhang Y, Tessaro MJ, Lassner M, Li X (2003) Knockout analysis of Arabidopsis transcription factors TGA2, TGA5, and TGA6 reveals their redundant and essential roles in systemic acquired resistance. Plant Cell 15: 2647-2653.

197. Cao H, Glazebrook J, Clarke JD, Volko S, Dong X (1997) The Arabidopsis NPR1 gene that controls systemic acquired resistance encodes a novel protein containing ankyrin repeats. Cell 88: 57-63.

198. Ryals J, Weymann K, Lavvton K, Friedrich L, Ellis D, Steiner H-Y, Johnson J, Delaney TP, Jesse T, Vos P, et al. (1997) The Arabidopsis NIMl protein shows homology to the mammalian transcription factor inhibitor IkappaB. Plant Cell 9: 425-439.

199. Fu ZQ, Yan S, Saleh A, Wang W, Ruble J, Oka N, Mohan R, Spoel SH, Tada Y, Zheng N, et al. (2012) NPR3 and NPR4 are receptors for the immune signal salicylic acid in plants. Nature 486: 228-232. 
200. Wu Y, Zhang D, Chu JY, Boyle P, Wang Y, Brindle ID, De Luca V, Després C (2012) The Arabidopsis NPR1 protein is a receptor for the plant defense hormone salicylic acid. Cell Rep 1: 639-647.

201. Ding Y, Sun T, Ao K, Peng Y, Zhang Y, Li X, Zhang Y (2018) Opposite roles of salicylic acid receptors NPR1 and NPR3/NPR4 in transcriptional regulation of plant immunity. Cell 173: 1454-1467.

202. Subramaniam R, Desveaux D, Spickler C, Michnick SW, Brisson N (2001) Direct visualization of protein interactions in plant cells. Nat Biotechnol 19: 769-772.

203. Rochon A, Boyle P, Wignes T, Fobert PR, Després C (2006) The coactivator function of Arabidopsis NPR1 requires the core of its BTB/POZ domain and the oxidation of C-terminal cysteines. Plant Cell 18: $3670-3685$.

204. Malamy J, Carr JP, Klessig DF, Raskin I (1990) Salicylic acid: A likely endogenous signal in the resistance response of tobacco to viral infection. Science (80- ) 250: 1002-1004.

205. Pieterse CMJ, Van der Does D, Zamioudis C, Leon-Reyes A, Van Wees SCM (2012) Hormonal modulation of plant immunity. Annu Rev Cell Dev Biol 28: 489-521.

206. Fode B, Siemsen T, Thurow C, Weigel R, Gatz C (2008) The Arabidopsis GRAS protein SCL14 interacts with class II TGA transcription factors and is essential for the activation of stress-inducible promoters. Plant Cell 20: 3122-3135.

207. Running MP, Meyerowitz EM (1996) Mutations in the PERIANTHIA gene of Arabidopsis specifically alter floral organ number and initiation pattern. Development 122: 1261-1269.

208. Noshi M, Mori D, Tanabe N, Maruta T, Shigeoka S (2016) Arabidopsis clade IV TGA transcription factors, TGA10 and TGA9, are involved in ROS-mediated responses to bacterial PAMP flg22. Plant Sci 252: 12-21.

209. Johnson C, Boden E, Arias J (2003) Salicylic acid and NPR1 induce the recruitment of trans- activating TGA factors to a defense gene promoter in Arabidopsis. Plant Cell 15: 1846-1858.

210. Sarkar S, Das A, Khandagale P, Maiti IB, Chattopadhyay S, Dey N (2018) Interaction of Arabidopsis TGA3 and WRKY53 transcription factors on Cestrum yellow leaf curling virus (CmYLCV) promoter mediates salicylic acid - dependent gene expression in planta. Planta 247: 181-199.

211. Gangadharan A, Sreerekha M-V, Whitehill J, Ham J, Mackey D (2013) The Pseudomonas syringae pv. tomato type III effector HopM1 
suppresses Arabidopsis defenses independent of suppressing salicylic acid signaling and of targeting AtMIN7. PLoS One 8: e82032.

212. Windram O, Madhou P, McHattie S, Hill C, Hickman R, Cooke E, Jenkins DJ, Penfold CA, Baxter L, Breeze E, et al. (2012) Arabidopsis defense against Botrytis cinerea: chronology and regulation deciphered by high-resolution temporal transcriptomic analysis. Plant Cell 24: 3530 3557.

213. Choi J, Huh SU, Kojima M, Sakakibara H, Paek K, Hwang I (2010) The cytokinin-activated transcription factor ARR 2 promotes plant immunity via TGA3/NPR1-dependent salicylic acid signaling in Arabidopsis. Dev Cell 19: 284-295.

214. Saleh A, Withers J, Mohan R, Marqués J, Gu Y, Yan S, Zavaliev R, Nomoto M, Tada Y, Dong X (2015) Posttranslational modifications of the master transcriptional regulator NPR1 enable dynamic but tight control of plant immune responses. Cell Host Microbe 18: 169-182.

215. Farinati S, DalCorso G, Varotto S, Furini A (2010) The Brassica juncea BjCdR15, an ortholog of Arabidopsis TGA3, is a regulator of cadmium uptake, transport and accumulation in shoots and confers cadmium tolerance in transgenic plants. New Phytol 185: 964-978.

216. Fang H, Liu Z, Long Y, Liang Y, Jin Z, Zhang L, Liu D, Li H, Zhai J, Pei Y (2017) The Ca2+/calmodulin2-binding transcription factor TGA3 elevates LCD expression and H2S production to bolster Cr6+ tolerance in Arabidopsis. Plant J 91: 1038-1050.

217. Després C, DeLong C, Glaze S, Liu E, Fobert PR (2000) The Arabidopsis NPR1/NIM1 protein enhances the DNA binding activity of a subgroup of the TGA family of bZIP transcription factors. Plant Cell 12: 279-290.

218. Shearer HL, Wang L, DeLong C, Després C, Fobert PR (2009) NPR1 enhances the DNA binding activity of the Arabidopsis bZIP transcription factor TGA7. Botany 87: 561-570.

219. Jones JDG, Dangl JL (2006) The plant immune system. Nature 444: 323 329.

220. Kushalappa AC, Yogendra KN, Karre S (2016) Plant innate immune response: Qualitative and quantitative resistance. CRC Crit Rev Plant Sci 35: $38-55$.

221. Kesarwani M, Yoo J, Dong X (2007) Genetic interactions of TGA transcription factors in the regulation of pathogenesis-related genes and disease resistance in Arabidopsis. Plant Physiol 144: 336-346.

222. Wang L, Fobert PR (2013) Arabidopsis clade I TGA factors regulate 
apoplastic defences against the bacterial pathogen Pseudomonas syringae through endoplasmic reticulum-based processes. PLoS One 8: e77378.

223. Shearer HL, Cheng YT, Wang L, Liu J, Boyle P, Després C, Zhang Y, Li X, Fobert PR (2012) Arabidopsis clade I TGA transcription factors regulate plant defenses in an NPR1-independent fashion. Mol PlantMicrobe Interact 25: 1459-1468.

224. Després C, Chubak C, Rochon A, Clark R, Bethune T, Desveaux D, Fobert PR (2003) The Arabidopsis NPR1 disease resistance protein is a novel cofactor that confers redox regulation of DNA binding activity to the basic domain/leucine zipper transcription factor TGA1. Plant Cell 15: 2181-2191.

225. Corpas FJ, Alché JD, Barroso JB (2013) Current overview of Snitrosoglutathione (GSNO) in higher plants. Front Plant Sci 4: 126-129.

226. Martínez-Ruiz A, Lamas S (2007) Signalling by NO-induced protein Snitrosylation and S-glutathionylation: Convergences and divergences. Cardiovasc Res 75: 220-228.

227. van Zanten M, Pons TL, Janssen JAM, Voesenek LACJ, Peeters AJM (2010) On the relevance and control of leaf angle. CRC Crit Rev Plant Sci 29: 300-316.

228. Polko JK, Voesenek LACJ, Peeters AJM, Pierik R (2011) Petiole hyponasty: An ethylene-driven, adaptive response to changes in the environment. AoB Plants doi: 10.1093.

229. Alvarez JM, Riveras E, Vidal EA, Gras DE, Contreras-López O, Tamayo KP, Aceituno F, Gómez I, Ruffel S, Lejay L, et al. (2014) Systems approach identifies TGA1 and TGA4 transcription factors as important regulatory components of the nitrate response of Arabidopsis thaliana roots. Plant J 80: 1-13.

230. Canales J, Contreras-López O, Álvarez JM, Gutiérrez RA (2017) Nitrate induction of root hair density is mediated by TGA1/TGA4 and CPC transcription factors in Arabidopsis thaliana. Plant J 92: 305-316.

231. Walters LA, Escobar MA (2016) The AtGRXS3/4/5/7/8 glutaredoxin gene cluster on Arabidopsis thaliana chromosome 4 is coordinately regulated by nitrate and appears to control primary root growth. Plant Signal Behav 11: e1171450.

232. Inoue H, Nojima H, Okayama H (1990) High efficiency transformation of Escherichia coli with plasmids. Gene 96: 23-28.

233. Clough SJ, Bent AF (1998) Floral dip: A simplified method for Agrobacterium-mediated transformation of Arabidopsis thaliana. Plant 
J 16: $735-743$.

234. Schneider CA, Rasband WS, Eliceiri KW (2012) NIH Image to ImageJ: 25 years of image analysis. Nat Methods 9: 671-675.

235. Sanger F, Nicklen S, Coulson A (1977) DNA sequencing with chainterminating inhibitors. Proc Natl Acad Sci U S A 74: 5463-5467.

236. Rozen S, Skaletsky H (2000) Primer 3 on the WWW for general users and for biologist programmers. Methods Mol Biol 132: 365-386.

237. Bradford MM (1976) A rapid and sensitive method for the quantitation of microgram quantities of protein utilizing the principle of protein-dye binding. Anal Biochem 72: 248-254.

238. Kang D, Gho YS, Suh M, Kang C (2002) Highly sensitive and fast protein detection with Coomassie brilliant blue in sodium dodecyl sulfate-polyacrylamide gel electrophoresis. Bull Korean Chem Soc 23: 1511-1512.

239. Studier F (2014) Stable expression clones and auto-induction for protein production in E. coli. Methods Mol Biol 1091: 17-32.

240. Begas P, Staudacher V, Deponte M (2015) Systematic re-evaluation of the bis(2-hydroxyethyl)disulfide (HEDS) assay reveals an alternative mechanism and activity of glutaredoxins. Chem Sci 6: 3788-3796.

241. Mieyal JJ, Starke DW, Gravina SA, Hocevar BA (1991) Thioltransferase in human red blood cells: Kinetics and equilibrium. Biochemistry 30: 8883-8891.

242. Gallogly MM, Starke DW, Leonberg AK, Ospina SM, Mieyal JJ (2008) Kinetic and mechanistic characterization and versatile catalytic properties of mammalian glutaredoxin 2: Implications for intracellular roles. Biochemistry 47: 11144-11157.

243. Holmgren A (1979) Thioredoxin catalyzes the reduction of insulin disulfides by dithiothreitol and dihydrolipoamide. J Biol Chem 254: $9627-$ 9632.

244. Hanson GT, Aggeler R, Oglesbee D, Cannon M, Capaldi RA, Tsien RY, Remington SJ (2004) Investigating mitochondrial redox potential with redox-sensitive green fluorescent protein indicators. J Biol Chem 279: 13044-13053.

245. Hennessy DJ, Reid GR, Smith FE, Thompson SL (1984) Ferene - a new spectrophotometric reagent for iron. Can J Chem 62: 721-724.

246. Moest RR (1975) Hydrogen sulfide determination by the methylene blue method. Anal Chem 47: 1204-1205. 
247. Vollheyde K (2014) Etablierung einer Methode zur Identifizierung von Cysteinmodifikationen am Beispiel des SCL14-Proteins aus Arabidopsis thaliana Wurzeln. Bachelor thesis, Göttingen.

248. Ellman G, Lysko H (1979) A precise method for the determination of whole blood and plasma sulfhydryl groups. Anal Biochem 93: 98-102.

249. Kosower NS, Kosower EM, Newton GL, Ranney HM (1979) Bimane fluorescent labels: Labeling of normal human red cells under physiological conditions. Proc Natl Acad Sci 76: 3382-3386.

250. Kozuka T, Kobayashi J, Horiguchi G, Demura T, Sakakibara H, Tsukaya H, Nagatani A (2010) Involvement of auxin and brassinosteroid in the regulation of petiole elongation under the shade. Plant Physiol 153: 1608-1618.

251. Tsukaya H, Kozuka T, Kim GT (2002) Genetic control of petiole length in Arabidopsis thaliana. Plant Cell Physiol 43: 1221-1228.

252. Tsukaya H (2005) Leaf shape: Genetic controls and environmental factors. Int J Dev Biol 49: 547-555.

253. Sasidharan R, Keuskamp DH, Kooke R, Voesenek LACJ, Pierik R (2014) Interactions between auxin, microtubules and XTHs mediate green shade- induced petiole elongation in Arabidopsis. PLoS One 9: e90587.

254. Marblestone J, Edavettal S, Lim Y, Lim P, Zuo X, Butt T (2006) Comparison of SUMO fusion technology with traditional gene fusion systems: Enhanced expression and solubility with SUMO. Protein Sci 15: 182-189.

255. Srinivasan U, Mieyal PA, Mieyal JJ (1997) pH profiles indicative of ratelimiting nucleophilic displacement in thioltransferase catalysis. Biochemistry 36: 3199-3206.

256. Yang Y, Jao SC, Nanduri S, Starke DW, Mieyal JJ, Qin J (1998) Reactivity of the human thioltransferase (glutaredoxin) C7S, C25S, C78S, C82S mutant and NMR solution structure of its glutathionyl mixed disulfide intermediate reflect catalytic specificity. Biochemistry 37: 17145-17156.

257. Gravina SA, Mieyal JJ (1993) Thioltransferase is a specific glutathionyl mixed disulfide oxidoreductase. Biochemistry 32: 3368-3376.

258. Agnetti G, Lindsey ML, Foster DB (2016) Manual of cardiovascular proteomics. Springer.

259. Couturier J, Wu HC, Dhalleine T, Pégeot H, Sudre D, Gualberto JM, Jacquot JP, Gaymard F, Vignols F, Rouhier N (2014) Monothiol 
glutaredoxin-BolA interactions: Redox control of Arabidopsis thaliana BolA2 and SufE1. Mol Plant 7: 187-205.

260. Cheng NH, Liu JZ, Brock A, Nelson RS, Hirschi KD (2006) AtGRXcp, an Arabidopsis chloroplastic glutaredoxin, is critical for protection against protein oxidative damage. J Biol Chem 281: 26280-26288.

261. Freibert SA, Weiler BD, Bill E, Pierik AJ, Mühlenhoff U, Lill R (2018) Biochemical reconstitution and spectroscopic analysis of iron-sulfur proteins. Methods Enzymol 599: 197-226.

262. Sun T, Busta L, Zhang Q, Ding P, Jetter R, Zhang Y (2017) TGACGBINDING FACTOR 1 (TGA1) and TGA4 regulate salicylic acid and pipecolic acid biosynthesis by modulating the expression of SYSTEMIC ACQUIRED RESISTANCE DEFICIENT 1 (SARD1) and CALMODULIN-BINDING PROTEIN 60g (CBP60g). New Phytol217: 344-354.

263. Zannini F, Moseler A, Bchini R, Dhalleine T, Meyer AJ, Rouhier N, Couturier J (2019) The thioredoxin-mediated recycling of Arabidopsis thaliana GRXS16 relies on a conserved C-terminal cysteine. Biochim Biophys Acta 1863: 426-436.

264. Tomanek L (2015) Proteomic responses to environmentally induced oxidative stress. J Exp Biol 218: 1867-1879.

265. Tripathy BC, Oelmüller R (2012) Reactive oxygen species generation and signaling in plants. Plant Signal Behav 7: 1621-1633.

266. Gallogly MM, Starke DW, Mieyal JJ (2009) Mechanistic and kinetic details of catalysis of thiol-disulfide exchange by glutaredoxins and potential mechanisms of regulation. Antioxid Redox Signal 11: 1059-1081.

267. Barinova K V., Serebryakova M V., Muronetz VI, Schmalhausen E V. (2017) S-glutathionylation of glyceraldehyde-3-phosphate dehydrogenase induces formation of C150-C154 intrasubunit disulfide bond in the active site of the enzyme. Biochim Biophys Acta - Gen Subj 1861: 3167-3177.

268. Meyer AJ, Dick TP (2010) Fluorescent protein-based redox probes. Antioxid Redox Signal 13: 621-650.

269. Morgan B (2014) Reassessing cellular glutathione homoeostasis: novel insights revealed by genetically encoded redox probes. Biochem Soc Trans 42: 979-984.

270. Outten FW, Theil EC (2009) Iron-based redox switches in biology. Antioxid Redox Signal 11: 1029-1046.

271. Landry AP, Cheng Z, Ding H (2013) Iron binding activity is essential 
for the function of IscA in iron-sulphur cluster biogenesis. Dalt Trans 42: 3100-3106.

272. Song YH, Song NY, Shin SY, Kim HJ, Yun D-J, Lim CO, Lee SY, Kang KY, Hong JC (2008) Isolation of CONSTANS as a TGA4/OBF4 interacting protein. Mol Cells 25: 559-565.

273. Michaud O, Fiorucci A-S, Xenarios I, Fankhauser C (2017) Local auxin production underlies a spatially restricted neighbor-detection response in Arabidopsis. Proc Natl Acad Sci 114: 7444-7449.

274. Ritsema T, van Zanten M, Leon-Reyes A, Voesenek LACJ, Millenaar FF, Pieterse CMJ, Peeters AJM (2010) Kinome profiling reveals an interaction between jasmonate, salicylate and light control of hyponastic petiole growth in Arabidopsis thaliana. PLoS One 5: e14255.

275. Cuéllar Pérez A, Nagels Durand A, Vanden Bossche R, De Clercq R, Persiau G, Van Wees SCM, Pieterse CMJ, Gevaert K, De Jaeger G, Goossens A, et al. (2014) The non-JAZ TIFY protein TIFY8 from Arabidopsis thaliana is a transcriptional repressor. PLoS One 9: e84891.

276. Woodcock DM, Crowther PJ, Doherty J, Jefferson S, Decruz E, NoyerWeidner M, Smith SS, Michael MZ, Graham MW (1989) Quantitative evaluation of Escherichia coli host strains for tolerance to cytosine methylation in plasmid and phage recombinants. Nucleic Acids Res 17: 3469-3478.

277. Bernard P, Couturier M (1992) Cell killing by the F plasmid CcdB protein involves poisoning of DNA-topoisomerase II complexes. J Mol Biol 226: 735-745.

278. Studier FW, Moffatt BA (1986) Use of bacteriophage T7 RNA polymerase to direct selective high-level expression of cloned genes. $J$ Mol Biol 189: 113-130.

279. Koncz C, Schell J (1986) The promoter of TL-DNA gene 5 controls the tissue-specific expression of chimaeric genes carried by a novel type of Agrobacterium binary vector. MGG Mol Gen Genet 204: 383-396.

280. Morgan B, Ezeriņa D, Amoako TNE, Riemer J, Seedorf M, Dick TP (2013) Multiple glutathione disulfide removal pathways mediate cytosolic redox homeostasis. Nat Chem Biol 9: 119-125. 


\section{Acknowledgments}

The years until the submission and defense of a $\mathrm{PhD}$ thesis can be fascinating, funny, successful or stressful and desparating or a mixture of all. The odysee with ROXY9 was in the beginning of the most frustful sort - however, it changed suddenly, when Isaac Fianu (AG Cramer, MPI for Biophysical Chemistry, Göttingen) managed to express monomeric ROXY9 in insect cells. After this breakthrough, many helpful cooperation partners contributed to the characterization of this protein: Dr. Viktor Sautner and Lisa-Marie Funk (AG Tittmann, University of Göttingen) showed me how to use an ÄKTA purifier and accompanied me through gel filtrations. They were always open for discussions and to give advice regarding protein biochemistry. Lara Ostermann (AG Schwarzländer, University of Bonn) kindly performed the roGFP assays for us, while Dr. Sven Freibert (AG Lill, University of Marburg) reconstituted ROXY9 and measured the glutathione binding. The exchange with all these groups contributed significantly to the progress of the ROXY9 project.

Within the department for Plant Molecular Biology and Physiology, I would like to thank Prof. Dr. Christiane Gatz for employing me - at that time, a desperate PhD student left suddenly without a $\mathrm{PhD}$ position. In this context, I would also like to thank the Dorothea-Schlözer-Stiftung for funding. Prof. Gatz, Dr. Corinna Thurow, and PD Dr. Joachim Uhrig guided me through the project and supported me constantly with their knowledge and experience, allowing "out-of-order" discussions when needed. My thesis committee members Prof. Dr. Ivo Feußner and Dr. Marcel Wiermer contributed their advice, as well.

In the lab, Dr. Guido Kriete and the technicians Anna Hermann, Ronald Scholz and Katharina Dworak were always there, when help was needed. Together with my doctoral collegues Jan Oberdiek, Jelena Budimir, Aswin Nair and Louisa Ulrich, they provided an enjoyable atmosphere. Unfortunately, all the master and bachelor students I came to know are too many to be listed here (but you know who you are (-)). A big thanks goes to Annika Gibron, who helped me during her lab rotation in the ROXY9 project. The gardeners Felicitas Glasenapp and Susanne Mester are acknowledged for preparing hundreds of pots for hyponastic growth and flowering experiments.

Despite all these friendly and tolerant people, I felt mainly tired and sad during my time in the lab - thanks to my "own special fate". For this reason, I would like to thank deeply all those people who supported me during this tough time. 